FLÁVIO PROTÁSIO RIBEIRO

\title{
ARRAYS DE MICROFONES PARA MEDIDA DE CAMPOS ACÚSTICOS
}

São Paulo 

FLÁVIO PROTÁSIO RIBEIRO

\section{ARRAYS DE MICROFONES PARA MEDIDA DE CAMPOS ACÚSTICOS}

Tese apresentada à Escola Politécnica da Universidade de São Paulo para a obtenção do título de Doutor em Engenharia.

Área de Concentração: Sistemas Eletrônicos Orientador: Prof. Dr. Vítor Heloiz Nascimento

São Paulo

2012 
Autorizo a reprodução e divulgação total ou parcial deste trabalho, por qualquer meio convencional ou eletrônico, para fins de estudo e pesquisa, desde que citada a fonte.

Este exemplar foi revisado e alterado em relação à versão original, sob responsabilidade única do autor e com a anuência de seu orientador.

São Paulo, 6 de fevereiro de 2012.

Assinatura do autor

Assinatura do orientador

\section{FICHA CATALOGRÁFICA}

Ribeiro, Flavio Protasio

Arrays de microfones para medida de campos acústicos / F.P. Ribeiro. - ed. rev. - São Paulo, 2012.

$218 \mathrm{p}$.

Tese (Doutorado) - Escola Politécnica da Universidade de São Paulo. Departamento de Engenharia de Sistemas Eletrônicos.

1. Processamento digital de sinais 2. Acústica 3. Estimação de parâmetros 4. Transformadas rápidas 5. Mínimos quadrados regularizados I.Universidade de São Paulo. Escola Politécnica. Departamento de Engenharia de Sistemas Eletrônicos II.t. 
Aos meus pais. 



\section{Agradecimentos}

A todos que me ajudaram, e que me permitiram ir muito mais longe do que teria ido sozinho.

Ao Prof. Vítor Nascimento, por ter me orientado desde os tempos da graduação, por ter depositado sua confiança em mim, e por sua generosidade.

Aos professores e colegas do LPS. Em particular, aos professores Magno da Silva, Miguel Ramirez, Cristiano Panazio, Phillip Burt e Cassio Lopes, e ao colega João Mendes.

Aos pesquisadores da MSR, pelas oportunidades que me deram: Dinei Florêncio, Cha Zhang, Phil Chou, Mike Seltzer e Zhengyou Zhang. E aos amigos que fiz lá: Sven Seuken, Julia Ruscher, Vanessa Testoni e Demba Ba.

Aos meus pais, que sempre me deram apoio incondicional. 



\section{Resumo}

Imageamento acústico é um problema computacionalmente caro e mal-condicionado, que envolve estimar distribuições de fontes com grandes arranjos de microfones. O método clássico para imageamento acústico utiliza beamforming, e produz a distribuição de fontes de interesse convoluída com a função de espalhamento do arranjo. Esta convolução borra a imagem ideal, significativamente diminuindo sua resolução. Convoluções podem ser evitadas com técnicas de ajuste de covariância, que produzem estimativas de alta resolução. Porém, estas têm sido evitadas devido ao seu alto custo computacional. Nesta tese, admitimos um arranjo bidimensional com geometria separável, e desenvolvemos transformadas rápidas para acelerar imagens acústicas em várias ordens de grandeza. Estas transformadas são genéricas, e podem ser aplicadas para acelerar beamforming, algoritmos de deconvolução e métodos de mínimos quadrados regularizados. Assim, obtemos imagens de alta resolução com algoritmos estado-da-arte, mantendo baixo custo computacional. Mostramos que arranjos separáveis produzem estimativas competitivas com as de geometrias espirais logaritmicas, mas com enormes vantagens computacionais. Finalmente, mostramos como estender este método para incorporar calibração, um modelo para propagação em campo próximo e superfícies focais arbitrárias, abrindo novas possibilidades para imagens acústicas.

Palavras-chave: array processing, imagens acústicas, transformadas rápidas, aproximação de Kronecker, mínimos quadrados regularizados, reconstrução esparsa. 



\section{Abstract}

Acoustic imaging is a computationally intensive and ill-conditioned inverse problem, which involves estimating high resolution source distributions with large microphone arrays. The classical method for acoustic imaging consists of beamforming, and produces the source distribution of interest convolved with the array point spread function. This convolution smears the image of interest, significantly reducing its effective resolution. Convolutions can be avoided with covariance fitting methods, which have been known to produce robust high-resolution estimates. However, these have been avoided due to prohibitive computational costs. In this thesis, we assume a 2D separable array geometry, and develop fast transforms to accelerate acoustic imaging by several orders of magnitude with respect to previous methods. These transforms are very generic, and can be applied to accelerate beamforming, deconvolution algorithms and regularized least-squares solvers. Thus, one can obtain high-resolution images with state-of-the-art algorithms, while maintaining low computational cost. We show that separable arrays deliver accuracy competitive with multi-arm spiral geometries, while producing huge computational benefits. Finally, we show how to extend this approach with array calibration, a near-field propagation model and arbitrary focal surfaces, opening new and exciting possibilities for acoustic imaging.

Keywords: array processing, acoustic imaging, fast transform, Kronecker approximation, regularized least squares, sparse reconstruction. 



\section{Lista de Ilustrações}

2.1 Sistema de coordenadas esféricas . . . . . . . . . . . . . 6

2.2 Array genérico . . . . . . . . . . . . . . . . . . 7

2.3 Espectro de um sinal $f(t)$ com banda estreita . . . . . . . . . . . . 11

2.4 Array linear uniforme $(\mathrm{ULA}) \ldots \ldots \ldots \ldots$. . . . . . . . . . . . . 12

2.5 Resposta em frequência-número de onda . . . . . . . . . . . . . . . 14

2.6 Resposta em frequência-número de onda $(\mathrm{dB}) \ldots \ldots \ldots \ldots$

2.7 Diagrama de radiação $(\mathrm{dB}) \ldots \ldots \ldots \ldots \ldots \ldots$

2.8 Respostas espaciais na presença de aliasing . . . . . . . . . . . . 16

2.9 Direcionamento de arrays . . . . . . . . . . . . . . 17

3.1 Exemplos de janelas e seus respectivos padrões de radiação . . . . . . . . . 24

3.2 Base do espaço de feixes . . . . . . . . . . . . . . . . . . . 32

3.3 Respostas de um ULA e de um array não-redundante . . . . . . . . . . 35

5.1 Exemplos de respostas MVDR . . . . . . . . . . . . . 55

5.2 Generalized sidelobe canceller (GSC) . . . . . . . . . . 58

7.1 Exemplos de imagens acústicas . . . . . . . . . . . . 76

7.2 Imagem acústica panorâmica de uma sala de concertos . . . . . . . . . 79

7.3 Exemplo da organização de uma imagem acústica com amostragem uniforme no espaço $\mathrm{U} \ldots \ldots \ldots \ldots . \ldots . \ldots . \ldots . \ldots$

8.1 Exemplo de implementação rápida para $\boldsymbol{\Xi}_{s}$ e $\boldsymbol{\Xi}_{s}^{T} \ldots \ldots$. . . . . . . 100

8.2 Exemplo de implementação rápida para $\boldsymbol{\Xi}_{u}$. . . . . . . . . . . . . . 104

8.3 Relação entre $G_{x}(i), G_{y}(j)$ e os elementos de $\overline{\mathbf{S}} \ldots \ldots$. . . . . . . . . 110

8.4 Exemplo de implementação rápida para $\Xi_{u}^{T} \ldots \ldots$. . . . . . . . 111

8.5 Tempos de execução para a transformada direta . . . . . . . . . . . . . 122

8.6 Tempos de execução para a transformada adjunta . . . . . . . . . . . . 122

8.7 Tempos de execução para a transformada direta-adjunta . . . . . . . . . . 122

8.8 Geometria separável simulada . . . . . . . . . . . . . . 126

8.9 Distribuições ideais para o padrão de calibração . . . . . . . . . . . 127

8.10 Reconstrução delay-and-sum, padrão de calibração, geometria separável 128

8.11 Reconstrução DAMAS2, padrão de calibração, geometria separável . . . 128 
8.12 Reconstrução regularizada $\ell_{1}$, padrão de calibração, geometria separável 129

8.13 Reconstrução TV, padrão de calibração, geometria separável . . . . . . . 129

8.14 Distribuições ideais para o padrão impulsivo . . . . . . . . . . . . . 130

8.15 Reconstrução delay-and-sum, padrão impulsivo, geometria separável . . 131

8.16 Reconstrução DAMAS2, padrão impulsivo, geometria separável . . . . . . 131

8.17 Reconstrução regularizada $\ell_{1}$, padrão impulsivo, geometria separável . 132

8.18 Reconstrução regularizada TV, padrão impulsivo, geometria separável 132

8.19 Padrão de testes não-esparso . . . . . . . . . . . . . . . . . 133

8.20 Reconstrução delay-and-sum, padrão não-esparso, geometria separável 134

8.21 Reconstrução DAMAS2, padrão não-esparso, geometria separável . . . . . 134

8.22 Reconstrução regularizada $\ell_{1}$, padrão não-esparso, geometria separável . 135

8.23 Reconstrução regularizada TV, padrão não-esparso, geometria separável 135

8.24 Geometria espiral logarítmica com 63 elementos . . . . . . . . . . . . . 136

8.25 Reconstrução delay-and-sum, padrão não-esparso, geometria espiral . . . . 137

8.26 Reconstrução DAMAS2, padrão não-esparso, geometria espiral . . . . . 137

8.27 Reconstrução regularizada $\ell_{1}$, padrão não-esparso, geometria espiral . . . 138

8.28 Reconstrução regularizada TV, padrão não-esparso, geometria espiral . . 138

9.1 Reconstruções TV para campo próximo $(1 \mathrm{kHz}-3 \mathrm{kHz})$. . . . . . . . 145

9.2 Reconstruções TV para campo próximo $(4 \mathrm{kHz}-6 \mathrm{kHz}) \ldots$. . . . . . . 146

9.3 Reconstruções TV para campo próximo $(7 \mathrm{kHz}-9 \mathrm{kHz}) \ldots$. . . . . . . 147

9.4 Primeiros 100 valores singulares de $\mathcal{R}(\mathbf{A})$ e $\mathcal{R}\left(\boldsymbol{\Xi}^{T} \mathbf{A}\right)$ (normalizados) . . 148

9.5 Erros de reconstrução para campo próximo, em função de K . . . . . . . 148

A.1 Geometria do array projetado . . . . . . . . . . . . . 167

A.2 Layout do array de microfones . . . . . . . . . . . . . . . 167

A.3 Placa de desenvolvimento Altera DE3 . . . . . . . . . . . . . 168

A.4 Diagrama de blocos do sistema de aquisição . . . . . . . . . . . . 168 


\section{Lista de Tabelas}

3.1 Medidas de desempenho para diferentes janelas . . . . . . . . . . . 25 



\section{Lista de Algoritmos}

7.1 CLEAN para deconvolução em banda estreita, para PSF normalizada . . . 86

8.1 Computação rápida de $\overline{\mathbf{W}}$ a partir de $\overline{\mathbf{S}} \ldots$. . . . . . . . . . . . . 111

8.2 Implementação genérica do algoritmo matching pursuit . . . . . . . . . . 123 



\section{Lista de Símbolos}

\begin{tabular}{ll}
\hline Símbolo & Significado \\
\hline \hline$T$ & transposta de matriz ou vetor \\
\hline$H$ & transposta Hermitiana de matriz ou vetor \\
\hline mod $(a, b)$ & conjugado complexo \\
\hline$\lfloor x\rfloor$ & arredondamento de $x \in \mathbb{R}$ em direção a $-\infty$ \\
\hline vec $\{\cdot\}$ & operador de vetorização \\
\hline$\otimes$ & produto de Kronecker \\
\hline$\odot$ & produto de Hadamard \\
\hline$\omega$ & frequência de operação no domínio de Fourier \\
\hline $\mathbf{q}_{m}$ & coordenadas de uma fonte em coordenadas Cartesianas \\
\hline $\mathbf{u}_{m}$ & coordenadas de uma fonte parametrizada no espaço U \\
\hline $\mathbf{p}_{n}$ & coordenadas de um sensor em coordenadas Cartesianas \\
\hline $\mathbf{v}(\omega)$ & vetor diretor de um array \\
\hline $\mathbf{Y}(\omega)$ & imagem acústica na frequência $\omega$ \\
\hline $\mathbf{S}(\omega)$ & matriz espectral na frequência $\omega$ \\
\hline $\mathbf{B}_{i, j}$ & elemento $(i, j)$ de uma matriz $\mathbf{B}$ genérica \\
\hline $\mathbf{0}$ & matriz ou vetor de zeros \\
\hline $\mathbb{1}$ & matriz ou vetor de uns \\
\hline
\end{tabular}





\section{Sumário}

1 Introdução 1

2 Arrays e filtros espaciais 5

2.1 Introdução . . . . . . . . . . . . . . . . . . 5 5

2.2 Respostas tempo-frequência e padrões de radiação . . . . . . . . . . . 7

2.3 Considerações sobre banda passante . . . . . . . . . . . . . . . . 11

2.4 Arrays lineares uniformes (ULAs) . . . . . . . . . . . . . 12

2.5 Direcionamento de arrays . . . . . . . . . . . . . 16

2.6 Diretividade . . . . . . . . . . . . . . . . . 17

2.7 Ganho . . . . . . . . . . . . . . . . . . . 18

2.8 Sensibilidade a perturbações . . . . . . . . . . . . . . . 20

3 Fundamentos de síntese e implementação 23

3.1 Métodos clássicos . . . . . . . . . . . . . . . . . . 23

3.1 .1 Janelas . . . . . . . . . . . . . . . . 23

3.1 .2 Amostragem em $\psi \ldots \ldots \ldots . \ldots \ldots 26$

3.1.3 Síntese por mínimos quadrados . . . . . . . . . . . . 27

3.1 .4 Outros métodos . . . . . . . . . . . . . . . 28

3.2 Restrições de zeros . . . . . . . . . . . . . . . . . . . . . 28

3.3 Realizações em espaços de feixes (beamspace processing) . . . . . . . . . 31

3.4 Arrays não-uniformes, bidimensionais e tridimensionais . . . . . . . . . . . 34

4 Processos aleatórios temporais-espaciais $\quad 37$

4.1 Introdução . . . . . . . . . . . . . . . . . . . . 37

4.2 Representação em frequência . . . . . . . . . . . . . . . . 37

4.3 Vetores aleatórios Gaussianos . . . . . . . . . . . . . . 39

4.4 Modelos de ondas planas . . . . . . . . . . . . . . . . 40

4.4.1 Caso 1: um único sinal determinístico . . . . . . . . . . . . . . . 40

4.4.2 Caso 2: um sinal desejado, $M$ interferências, todos determinísticos . 40

4.4.3 Caso 3: um sinal desejado determinístico, $M$ interferências Gaussianas 40

4.4.4 Caso 4: um sinal desejado, $M$ interferências, todos Gaussianos . . . 41

4.5 Representação de processos em frequência-número de onda . . . . . . . . . 42 
4.6 Representação de processos tridimensionais . . . . . . . . . . . . . 45

4.7 Filtragem em frequência-número de onda . . . . . . . . . . . . . 47

5 Beamformers ótimos $\quad 49$

5.1 Introdução . . . . . . . . . . . . . . . . . . . . . . . 49

5.2 Beamformer MVDR . . . . . . . . . . . . . . . 49

5.3 Beamformer MMSE (minimum mean-square error) . . . . . . . . . . 51

5.4 Beamformer MVDR submetido a múltiplas interferências . . . . . . . . . . 52

5.5 Sensibilidade . . . . . . . . . . . . . . . . 54

5.6 Beamformer MVDR ou MPDR com restrições lineares . . . . . . . . 56

5.7 Realização GSC (generalized sidelobe canceller) . . . . . . . . . . . 57

5.8 Regularização (diagonal loading) _ . . . . . . . . . . . . . 59

5.9 Realizações em espaços de dimensões reduzidas . . . . . . . . . . . . 60

5.9 .1 Cenário 1: alta SNR/INR . . . . . . . . . . . 60

5.9 .2 Cenário 2: baixa SNR e alta INR . . . . . . . . . . . . 62

5.10 Desempenho para sinais correlacionados . . . . . . . . . . . 63

5.11 Calibração . . . . . . . . . . . . . . . . . 6 66

6 Estimação de direção de chegada $\quad 69$

6.1 Introdução . . . . . . . . . . . . . . . . . . . . 69

6.2 Métodos de varredura . . . . . . . . . . . . . . 69

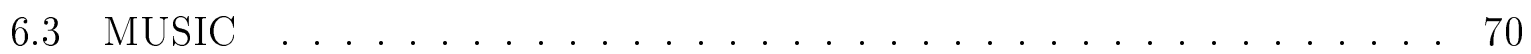

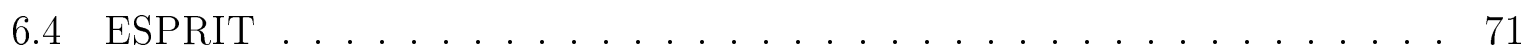

$\begin{array}{lll}7 & \text { Imagens acústicas } & 75\end{array}$

7.1 Introdução . . . . . . . . . . . . . . . . . . . 75

7.2 Parametrização no espaço U . . . . . . . . . . . . . . 78

7.3 Decomposição em fontes descorrelacionadas . . . . . . . . . . . 80

7.4 Invariância translacional de beamformers . . . . . . . . . . . . . . . 82

7.5 Deconvolução de imagens acústicas . . . . . . . . . . . . 85

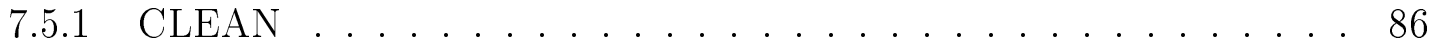

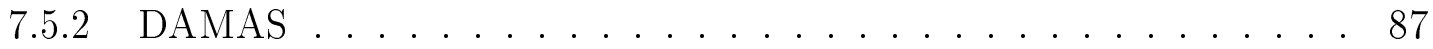

7.6 Covariance fitting . . . . . . . . . . . . . . . 89

8 Transformadas rápidas para imagens acústicas $\quad 93$

8.1 Transformada rápida direta . . . . . . . . . . . . . . . 95

8.1.1 Caso 1: geometria separável . . . . . . . . . . . . 96

8.1.2 Caso 2: geometria uniforme . . . . . . . . . . . . . 101

8.2 Transformada rápida transposta . . . . . . . . . . . . . . . . 104

8.2.1 Caso 1: geometria separável . . . . . . . . . . . . 105 
8.2.2 Caso 2: geometria uniforme . . . . . . . . . . . . 108

8.3 Transformada rápida adjunta . . . . . . . . . . . . . . . 112

8.4 Transformada rápida direta-adjunta . . . . . . . . . . . . . . 113

8.5 Conexões . . . . . . . . . . . . . . . . . . . . . 114

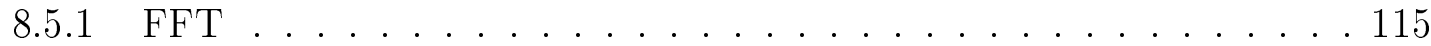

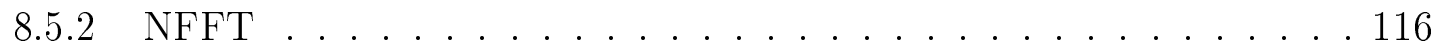

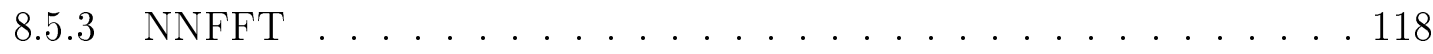

8.5.4 Beamformer delay-and-sum . . . . . . . . . . . . . 119

8.5.5 Beamformer MPDR . . . . . . . . . . . . . . . 119

8.6 Desempenho . . . . . . . . . . . . . . . . . 120

8.7 Aplicações . . . . . . . . . . . . . . . . . . . . 123

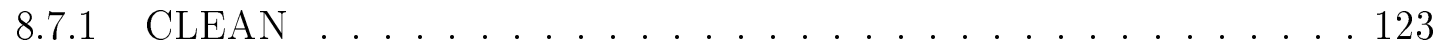

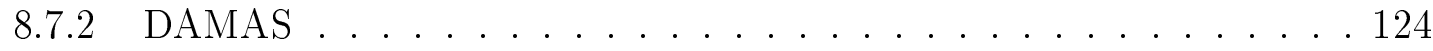

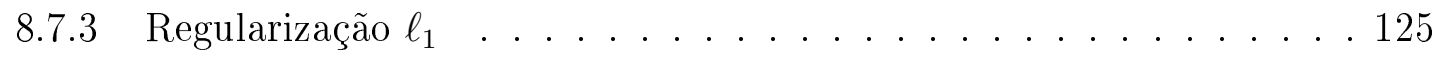

8.7.4 Regularização TV . . . . . . . . . . . . . . . . . 125

8.8 Exemplos de aplicação . . . . . . . . . . . . . . . . 126

8.8.1 Padrões tabuleiro de xadrez . . . . . . . . . . . . . 127

8.8.2 Padrões esparsos . . . . . . . . . . . . . . . 130

8.8.3 Padrão não-esparso . . . . . . . . . . . . . . . . . . . . 130

8.9 Comparação com geometrias espirais . . . . . . . . . . . . . 133

9 Transformadas rápidas para campo próximo 139

9.1 Transformadas rápidas como aproximações de posto K . . . . . . . 139

9.2 Calibração e foco . . . . . . . . . . . . . . . . . . . . 142

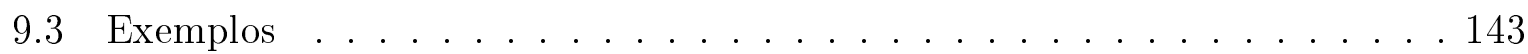

10 Transformadas rápidas para imagens correlacionadas 149

10.1 Transformada rápida direta . . . . . . . . . . . . . . . . 150

10.2 Transformada rápida transposta . . . . . . . . . . . . . . . 152

10.3 Transformada rápida adjunta . . . . . . . . . . . . . . . 153

10.4 Transformada rápida direta-adjunta . . . . . . . . . . . . . . . 153

10.5 Aplicações . . . . . . . . . . . . . . . . . . . . 154

11 Conclusão $\quad 155$

$\begin{array}{lr}\text { Referências Bibliográficas } & 157\end{array}$

A Projeto de uma plataforma de referência $\quad 165$

A.1 Introdução . . . . . . . . . . . . . . . . . . . . . 165

A.2 Hardware projetado . . . . . . . . . . . . 166 
B Publicações relevantes 


\section{Capítulo 1}

\section{Introdução}

Nesta tese desenvolvemos métodos para mapear as direções de chegada e intensidades de fontes acústicas localizadas em uma região de interesse. Para isto, utilizamos arranjos espaciais (arrays) de sensores para amostrar campos arbitrários. Utilizando algoritmos de reconstrução, estimamos distribuições de fontes a partir de um número relativamente pequeno de amostras discretas de um campo. Este sistema de medida é conceitualmente análogo a um sensor fotográfico, porém capaz de operar com ondas sonoras. Por isso, as distribuições estimadas recebem o nome de imagens acústicas.

Imagens acústicas apresentam aplicações em problemas de análise e redução de ruído, que tipicamente estão presentes na fase de prototipagem de máquinas e veículos. Por exemplo, um array de microfones pode ser posicionado em um túnel de vento para determinar a distribuição de ruído sobre um modelo devido ao fluxo de ar em alta velocidade. Este tipo de medida é rotineiramente usado para desenvolver carros, trens e aviões mais silenciosos e aerodinamicamente eficientes.

Arrays de antenas são conhecidos há muitas décadas e são usados amplamente em telecomunicações, rádio astronomia e radares de alta resolução. Seu uso permite a construção de filtros espaciais e temporais, conferindo ao sistema resultante resolução e imunidade a ruído muito superior à que seria possível com um único receptor ou transmissor.

Arrays de microfones têm gradualmente ganhado popularidade. Durante décadas, sua aplicação mais notável se restringiu a submarinos, cujos sonares são implementados na forma de arrays lineares. Mais recentemente, equipamentos de ultrassonografia com matrizes de transdutores possibilitaram visualização tridimensional não invasiva. Apesar disso, aplicações de arrays com ondas sonoras propagantes no ar ainda são relativamente raras, e restritas a cenários onde seu custo elevado possa ser justificado. Isso pode se explicado em parte pelas seguintes dificuldades: sinais de áudio têm banda larga, as medições são frequentemente feitas no campo próximo (onde a hipótese de ondas planas não é válida com boa aproximação) e microfones são construídos com tolerâncias muito piores do que antenas. Estas características implicam em uma maior complexidade algorítmica, e um alto custo de processamento.

Para lidar com estas dificuldades técnicas, propomos técnicas de reconstrução usando 
mínimos quadrados regularizados. Como veremos, estes métodos produzem reconstruções com resolução muito superior à de técnicas tradicionais, como beamforming. Por outro lado, seu custo computacional também é muito maior. Para viabilizar seu uso, desenvolvemos transformadas rápidas que relacionam imagens acústicas e as matrizes de covariância amostradas por arrays. Estas transformadas permitem acelerar métodos tradicionais e de mínimos quadrados regularizados em várias ordens de grandeza, obtendo imagens com precisão muito superior à obtenível com técnicas tradicionais, e em tempo comparável.

Com o aumento da resolução e redução do custo computacional, podemos produzir imagens acústicas com um número menor de sensores, e com processadores de propósito geral. Com o objetivo de reduzir ainda mais os custos, propomos um projeto de referência para arrays de microfones, que tem o potencial de transformar arrays de microfones bidimensionais em produtos de prateleira.

A seguir resumimos a organização deste texto. No Capítulo 2 introduzimos o tópico de filtragem espacial usando arrays de elementos discretos. Definimos o conceito de resposta espacial, apresentamos o array linear uniforme, mostramos como arrays equivalem a antenas eletronicamente direcionáveis, definimos algumas medidas de desempenho e ilustramos dificuldades decorrentes de imperfeições presentes em arrays reais.

O Capítulo 3 apresenta técnicas clássicas para projeto de filtros espaciais (ou beamformers). Mostramos a analogia existente entre filtros FIR e beamformers, e mostramos como técnicas de projetos de filtros se aplicam a arrays clássicos.

A linguagem de processos aleatórios temporais-espaciais é apresentada no Capítulo 4. Esta descrição é usada recorrentemente ao longo do texto para a caracterização de campos de ondas não-determinísticos ou desconhecidos. Neste capítulo discutimos a decomposição de processos de interesse em modelos de ondas planas, e a representação e estimação das estatísticas de segunda ordem de processos estacionários no tempo e espaço.

No Capítulo 5 descrevemos beamformers ótimos no sentido estatístico, utilizando a linguagem de processos aleatórios. Comentamos também a sensibilidade destas técnicas frente a erros de construção e estimação.

Alguns métodos celebrados para estimação sub-ótima de direção de chegada são descritos no Capítulo 6. Estes métodos são particularmente relevantes para aplicações práticas, pois algoritmos ótimos são intratáveis para casos não triviais.

A teoria de imagens acústicas é o tópico do Capítulo 7. Descrevemos métodos clássicos, e apresentamos uma revisão do estado da arte. Finalmente, motivamos a necessidade de técnicas computacionalmente mais eficientes para permitir a aplicação de técnicas de alta resolução.

No Capítulo 8 desenvolvemos transformadas rápidas para obter matrizes de covariância a partir de distribuições de fontes descorrelacionadas e vice-versa, sob a hipótese de fontes no campo distante. Mostramos como estas transformadas se relacionam à transformada de Fourier de tempo contínuo, à FFT, à DFT com amostragem não uniforme e a diversas 
formas de beamforming. Utilizamos estas transformadas para significativamente acelerar métodos existentes, e também para viabilizar a reconstrução de imagens acústicas com mínimos quadrados regularizados.

Nos Capítulos 9 e 10 retiramos as hipóteses de campo distante e fontes descorrelacionadas, respectivamente. No Capítulo 9, mostramos que a transformada para campo distante é equivalente a uma aproximação de posto 1 da transformada exata, usando uma permutação convenientemente escolhida. Para fontes em campo próximo, usamos aproximações de posto K (para K pequeno), obtendo transformadas com precisão controlável e baixo custo computacional. No Capítulo 10, admitimos a possibilidade de correlações entre pares de fontes, e obtemos as transformadas rápidas correspondentes.

Finalmente, o Capítulo 11 apresenta nossas conclusões. 



\section{Capítulo 2}

\section{Arrays e filtros espaciais}

\subsection{Introdução}

Arranjos ou arrays de sensores são conjuntos de transdutores espacialmente espalhados, projetados para amostrar campos de ondas. Seu uso permite explorar a diversidade espacial de um sinal e extrair informação que não estaria disponível caso fosse usado um único sensor.

O processamento de um array pode ser caracterizado como um processo de filtragem, onde os sinais amostrados são processados simultaneamente nos domínios do tempo e do espaço (ou em seus domínios conjugados, frequência e número de onda). A linguagem para caracterização de sinais espaciais é uma generalização da linguagem tradicional para processamento de sinais temporais, onde as dimensões espaciais são consideradas, e fenômenos particulares à propagação (por exemplo, a não homogeneidade de um meio ou a propagação em campo próximo) são tratados.

Arrays de sensores têm aplicações praticamente em qualquer campo que envolva detecção e estimação de sinais ondulatórios. Algumas aplicações atuais são: aquisição de imagens médicas por ultrassom; interligação de rádio-telescópios; detecção e rastreamento de fenômenos meteorológicos; controle de tráfego aéreo; telefonia celular; medição de ondas sísmicas para prospecção subterrânea ou subaquática; detecção e rastreamento de objetos subaquáticos; medições de campo acústico; aquisição de sinais de voz.

Através do uso de diversos sensores é possível obter resoluções muito superiores às que seriam possíveis com um único elemento. Um exemplo dramático é encontrado com rádio-telescópios. A resolução angular $\alpha$ de um único telescópio é bem aproximada pelo critério de Rayleigh, que sugere $\alpha \approx 1.22 \lambda / D$, onde $\lambda$ é o comprimento de onda e $D$ é o diâmetro da antena. Considerando que um sinal de $100 \mathrm{GHz}$ tem comprimento de onda aproximadamente 5 mil vezes maior do que o da luz visível, rádio-telescópios formados por uma única antena têm resoluções aproximadamente 5 mil vezes piores do que as de telescópios ópticos de tamanhos comparáveis. Este efeito é compensado interligando rádio telescópios localizados ao redor do mundo, criando arrays de telescópios ${ }^{1}$ com dimensões

\footnotetext{
${ }^{1}$ Por exemplo, www.vlba.nrao.edu, www.vla.nrao.edu e www.lofar.org.
} 


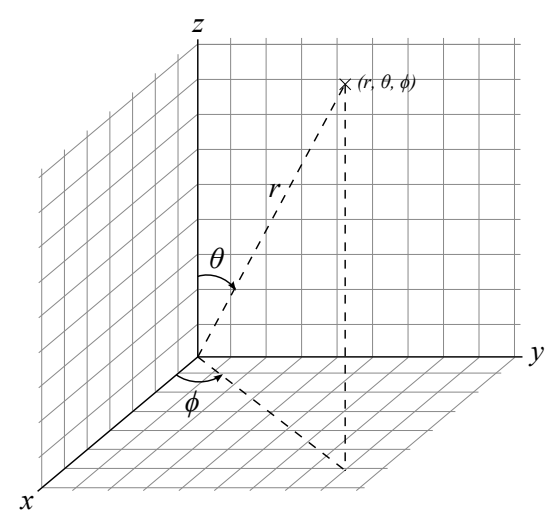

Figura 2.1: Sistema de coordenadas esféricas

de dezenas de milhares de km, e resoluções competitivas com as de grandes telescópios ópticos.

Arrays oferecem recursos de filtragem espacial, onde o domínio espacial (ou, mais precisamente, o domínio do número de onda) é tratado de forma análoga ao domínio da frequência no processamento de sinais temporais. Da mesma forma que filtros temporais permitem a extração ou rejeição de sinais com frequências específicas, arrays permitem aceitar ou rejeitar sinais com dependência em seu ângulo de chegada. Este recurso permite obter relações sinal-ruído consideravelmente superiores às que seriam possíveis apenas com filtros temporais.

Alguns recursos disponibilizados por arrays são específicos do domínio espacial. Em um sistema de aquisição tradicional, o receptor deve ser apontado mecanicamente para a direção do sinal de interesse. Arrays permitem o direcionamento eletrônico, tal que a resposta angular do array é alterada exclusivamente através da filtragem no domínio do espaço. Isso permite a detecção e rastreamento de múltiplas fontes simultaneamente, e a estimação da direção de fontes desconhecidas.

O processamento espacial-temporal exige dois tipos de projeto. O primeiro decide a geometria do array, que por sua vez estabelece limites em relação à detecção e estimação do sistema. Por exemplo, arrays lineares só podem resolver uma componente angular, dando origem a um cone de ambiguidade. A escolha entre diferentes geometrias tipicamente é motivada pelos recursos disponíveis para a aplicação. O segundo projeto define o processador usado para filtrar os sinais amostrados.

No tratamento a seguir usaremos frequentemente o sistema de coordenadas esféricas (Figura 2.1). As relações entre coordenadas esféricas e cartesianas é dada por:

$$
\begin{aligned}
& x=r \sin \theta \cos \phi \\
& y=r \sin \theta \sin \phi, \\
& z=r \cos \theta .
\end{aligned}
$$




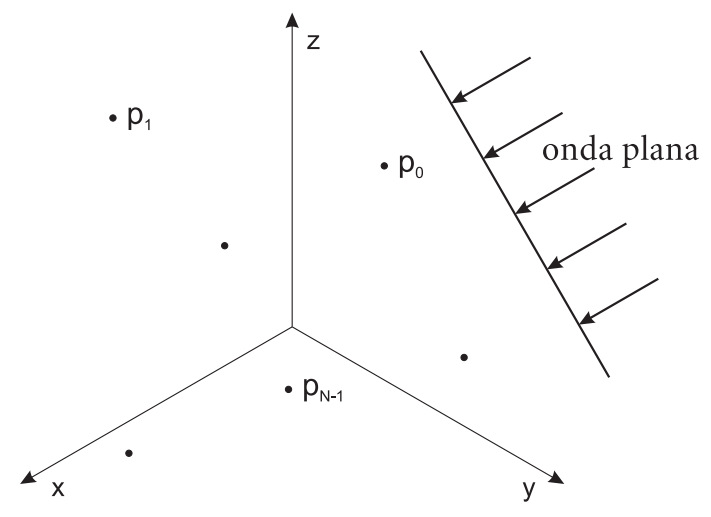

Figura 2.2: Array genérico

As ondas de interesse serão soluções da chamada equação de onda

$$
\frac{\partial^{2} f}{\partial x^{2}}+\frac{\partial^{2} f}{\partial y^{2}}+\frac{\partial^{2} f}{\partial z^{2}}=\frac{1}{c^{2}} \frac{\partial^{2} f}{\partial t^{2}}
$$

onde $f(t, \mathbf{x})$ é um campo escalar, com $\mathbf{x}=x \vec{i}+y \vec{j}+z \vec{k}$ o vetor de coordenadas espaciais, e $c$ é a velocidade de propagação da onda no meio. As soluções da equação de onda são combinações lineares de

$$
\begin{aligned}
f(t, x, y, z) & =A e^{j\left(\omega t-k_{x} x-k_{y} y-k_{z} z\right)} \\
& =A e^{j\left(\omega t-\mathbf{k}^{T} \mathbf{x}\right)}
\end{aligned}
$$

onde

$$
k_{x}^{2}+k_{y}^{2}+k_{z}^{2}=\frac{\omega^{2}}{c^{2}} .
$$

Chamaremos $\mathbf{k}=k_{x} \vec{i}+k_{y} \vec{j}+k_{z} \vec{k}$ de vetor número de onda.

Os planos de fase constante são regiões onde $\mathbf{k}^{T} \mathbf{x}=c$, com $c$ constante. Logo, são perpendiculares a $\mathbf{k}$. Se $f(t, \mathbf{x})$ for de fato uma onda propagante, então planos de fase constante se movem uma distância $\Delta \mathbf{x}$ a cada incremento de tempo $\Delta t$, tal que $f(t+\Delta t, \mathbf{x}+\Delta \mathbf{x})=f(t, \mathbf{x})$ e $\omega \Delta t-\mathbf{k}^{T} \Delta \mathbf{x}=0$. Usando $\|\mathbf{k}\|=\frac{\omega}{c}, \frac{\Delta \mathbf{x}}{\Delta t}=c$ e a desigualdade de Cauchy-Schwarz é possível concluir que $\Delta \mathbf{x}$ e $\mathbf{k}$ têm a mesma direção.

Sempre consideraremos meios não-dispersivos (i.e., com velocidades de propagação independentes da frequência), homogêneos e sem perdas. Essas suposições são razoáveis para ondas acústicas ou eletromagnéticas ao ar livre, mas são falsas em outros cenários (por exemplo, ondas eletromagnéticas em guias de onda ou ondas acústicas no oceano).

\subsection{Respostas tempo-frequência e padrões de radiação}

Consideremos um array genérico com $N$ elementos, conforme ilustrado na Figura 2.2. Os sensores amostram o campo nas coordenadas $\mathbf{p}_{n}$, com $n \in\{0, \ldots, N-1\}$, tal que $f\left(t, \mathbf{p}_{i}\right)$ é a saída do i-ésimo sensor. 


\section{Definimos}

$$
\mathbf{f}(t, \mathbf{p})=\left[\begin{array}{c}
f\left(t, \mathbf{p}_{0}\right) \\
f\left(t, \mathbf{p}_{1}\right) \\
\vdots \\
f\left(t, \mathbf{p}_{N-1}\right)
\end{array}\right]
$$

Cada sensor é processado por um filtro linear invariante no tempo com resposta impulsiva $h_{n}(t)$. As saídas dos filtros são somadas, produzindo o sinal $y(t)$. Podemos representar $y(t)$ usando uma integral de convolução, tal que

$$
y(t)=\sum_{n=0}^{N-1} \int_{-\infty}^{+\infty} h_{n}(t-\tau) f\left(\tau, \mathbf{p}_{n}\right) d \tau .
$$

Podemos reescrever $y(t)$ como

$$
y(t)=\int_{-\infty}^{+\infty} \mathbf{h}^{T}(t-\tau) \mathbf{f}(\tau, \mathbf{p}) d \tau
$$

onde

$$
\mathbf{h}(t)=\left[\begin{array}{c}
h_{0}(t) \\
h_{1}(t) \\
\vdots \\
h_{N-1}(t)
\end{array}\right]
$$

Este resultado pode ser reescrito no domínio da frequência, tal que

$$
\begin{aligned}
\hat{y}(\omega) & =\int_{-\infty}^{+\infty} y(t) e^{-j \omega t} d t \\
& =\hat{\mathbf{h}}^{T}(\omega) \hat{\mathbf{f}}(\omega),
\end{aligned}
$$

com

$$
\begin{aligned}
\hat{\mathbf{h}}(\omega) & =\int_{-\infty}^{+\infty} \mathbf{h}(t) e^{-j \omega t} d t \\
\hat{\mathbf{f}}(\omega) & =\int_{-\infty}^{+\infty} \mathbf{f}(t, \mathbf{p}) e^{-j \omega t} d t .
\end{aligned}
$$

Consideremos o caso de um array genérico submetido a uma onda plana com direção a e velocidade de propagação $c$. Definimos a como um vetor unitário da forma

$$
\mathbf{a}=\left[\begin{array}{c}
-\sin \theta \cos \phi \\
-\sin \theta \sin \phi \\
-\cos \theta
\end{array}\right]
$$

onde o sinal se deve à orientação do vetor. Definimos também a direção de visada $\mathbf{u}=-\mathbf{a}$. 
O sinal recebido nos $N$ sensores é dado por

$$
\mathbf{f}(t, \mathbf{p})=\left[\begin{array}{c}
f\left(t-\tau_{0}\right) \\
f\left(t-\tau_{1}\right) \\
\vdots \\
f\left(t-\tau_{N-1}\right)
\end{array}\right]
$$

onde

$$
\tau_{n}=\frac{\mathbf{a}^{T} \mathbf{p}_{n}}{c}=-\frac{\mathbf{u}^{T} \mathbf{p}_{n}}{c}
$$

é o atraso do sinal recebido no i-ésimo sensor em relação ao centro de coordenadas.

O n-ésimo elemento de $\hat{\mathbf{f}}(\omega)$ pode ser escrito como

$$
\hat{f}_{n}(\omega)=\int_{-\infty}^{+\infty} e^{-j \omega t} f\left(t-\tau_{n}\right) d t=e^{-j \omega \tau_{n}} \hat{f}(\omega)
$$

Como $\|\mathbf{k}\|=\frac{\omega}{c}$ e $\mathbf{k}$ têm a direção do vetor de propagação, podemos reescrever o vetor número de onda como

$$
\mathbf{k}=\frac{\omega}{c} \mathbf{a}=\frac{2 \pi}{\lambda} \mathbf{a}
$$

onde $\lambda$ é o comprimento de onda correspondente à frequência $\omega$. Substituindo (2.2.11) em (2.2.9), obtemos

$$
\omega \tau_{n}=\mathbf{k}^{T} \mathbf{p}_{n}
$$

Definindo

$$
\mathbf{v}_{\mathbf{k}}(\mathbf{k})=\left[\begin{array}{c}
e^{-j \mathbf{k}^{T} \mathbf{p}_{0}} \\
e^{-j \mathbf{k}^{T} \mathbf{p}_{1}} \\
\vdots \\
e^{-j \mathbf{k}^{T} \mathbf{p}_{N-1}}
\end{array}\right]
$$

podemos reescrever $\hat{\mathbf{f}}(\omega)$ como

$$
\hat{\mathbf{f}}(\omega)=\hat{f}(\omega) \mathbf{v}_{\mathbf{k}}(\mathbf{k}) .
$$

$\mathbf{v}_{\mathbf{k}}(\mathbf{k})$ é chamado vetor de resposta, vetor diretor ou array manifold vector ${ }^{2}$, e representa completamente a geometria do array. O índice ${ }_{k}$ indica que o vetor de resposta é dado em função do vetor número de onda. Em alguns cenários pode ser conveniente reescrever $\mathbf{v}_{\mathbf{k}}(\mathbf{k})$ em função de outros parâmetros - por exemplo, dos ângulos de azimute e elevação. Em cenários mais sofisticados (por exemplo, em que a velocidade de propagação é dependente da frequência), $\mathbf{v}_{\mathbf{k}}$ é função de parâmetros adicionais.

\footnotetext{
${ }^{2} \mathrm{~A}$ rigor, o array manifold para uma dada frequência $\omega_{0}$ é dado por $\mathcal{M}\left(\omega_{0}\right)=$ $\left\{\mathbf{v}_{\mathbf{k}}(\mathbf{k}): \mathbf{k} \in \mathbb{R}^{3} \wedge\|\mathbf{k}\|=\frac{\omega_{0}}{c}\right\}$. É fácil mostrar que $\mathcal{M}\left(\omega_{0}\right)$ tem as propriedades esperadas de um $m a-$ nifold (variedade).
} 
Uma possível estratégia de processamento consiste em maximizar a potência do sinal incidente. Isso pode ser feito compensando os atrasos $\tau_{i}$ de cada sensor, de forma a alinhar no tempo cada sinal recebido. Este tipo de compensação dá origem ao conformador de feixes atrasa-e-soma, usualmente chamado de delay-and-sum beamformer. Assim, temos

$$
h_{n}(t)=\frac{1}{N} \delta\left(t+\tau_{n}\right)
$$

onde $\frac{1}{N}$ é um fator de normalização para que obtenhamos

$$
y(t)=f(t) .
$$

Definindo $\mathbf{k}_{s}$ como o vetor número de onda do sinal do interesse, podemos expressar este beamformer no domínio da frequência:

$$
\hat{\mathbf{h}}^{T}(\omega)=\frac{1}{N} \mathbf{v}_{\mathbf{k}}^{H}\left(\mathbf{k}_{s}\right)
$$

Se o sinal incidente for uma onda plana monocromática com amplitude unitária, frequência $\omega$ e número de onda $\mathbf{k}$, teremos $f_{n}\left(t, \mathbf{p}_{n}\right)=e^{j\left(\omega t-\mathbf{k}^{T} \mathbf{p}_{n}\right)}$. Podemos representar $\mathbf{f}(t, \mathbf{p})$ usando o array manifold vector, tal que

$$
\mathbf{f}(t, \mathbf{p})=\mathbf{v}_{\mathbf{k}}(\mathbf{k}) e^{j \omega t} .
$$

A saída do array quando submetido a esta excitação é dada por

$$
y(t, \mathbf{k})=\hat{\mathbf{h}}^{T}(\omega) \mathbf{v}_{\mathbf{k}}(\mathbf{k}) e^{j \omega t}
$$

Definimos a resposta em frequência-número de onda como a função

$$
\Upsilon(\omega, \mathbf{k})=\hat{\mathbf{h}}^{T}(\omega) \mathbf{v}_{\mathbf{k}}(\mathbf{k})
$$

O padrão de radiação de um array é definido como a resposta em frequência-número de onda em função do ângulo de visada, ou

$$
B_{\theta, \phi}(\omega, \theta, \phi)=\left.\hat{\mathbf{h}}^{T}(\omega) \mathbf{v}_{\mathbf{k}}(\mathbf{k})\right|_{\mathbf{k}=\frac{2 \pi}{\lambda} \mathbf{r}(\theta, \phi)},
$$

onde $\mathbf{r}(\theta, \phi)$ é o vetor unitário com ângulos $\theta$ e $\phi$ em coordenadas esféricas. Os subíndices $\theta, \phi$ indicam que o padrão de radiação está parametrizado em função de $(\theta, \phi)$.

Consideremos uma realização do beamformer no caso particular de onda plana monocromática com frequência $\omega_{c}$. Como $\hat{\mathbf{h}}(\omega)$ só será excitado em $\omega=\omega_{c}$, podemos impor

$$
\mathbf{w}^{H}=\hat{\mathbf{h}}^{T}\left(\omega_{c}\right)
$$




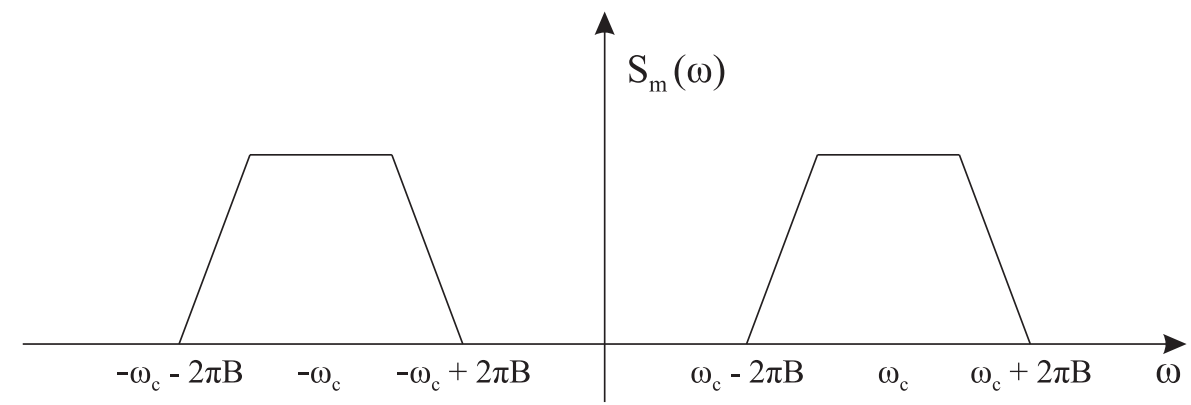

Figura 2.3: Espectro de um sinal $f(t)$ com banda estreita

que equivale a substituir cada filtro $h_{n}(t)$ por um único coeficiente complexo. Assim, as expressões equivalentes para $y(t, \mathbf{k})$ e $\Upsilon(\omega, \mathbf{k})$ são

$$
\begin{aligned}
y(t, \mathbf{k}) & =\mathbf{w}^{H} \mathbf{v}_{\mathbf{k}}(\mathbf{k}) e^{j \omega_{c} t} \\
\Upsilon(\omega, \mathbf{k}) & =\mathbf{w}^{H} \mathbf{v}_{\mathbf{k}}(\mathbf{k}) .
\end{aligned}
$$

\subsection{Considerações sobre banda passante}

O caso monocromático é uma boa aproximação para entradas de banda suficientemente estreita. De fato, consideremos uma onda plana gerada por uma fonte $f(t)=$ $R e\left\{m(t) e^{j \omega t}\right\}$, onde $m(t)$ é real e tem potência concentrada em $|\omega|<2 \pi B$ (ver a Figura 2.3). O campo medido no ponto $\mathbf{p}_{n}$ é dado por

$$
f\left(t, \mathbf{p}_{n}\right)=\operatorname{Re}\left\{m\left(t-\tau_{n}\right) e^{j \omega\left(t-\tau_{n}\right)}\right\}
$$

onde $\tau_{n}$ é o atraso de propagação dado por (2.2.9).

Se não impusermos nenhuma restrição sobre a posição do array em relação ao sistema de coordenadas, os atrasos $\tau_{n}$ podem ter valores arbitrariamente grandes, o que torna a expressão acima inconveniente para as considerações que seguem. Seja

$$
\Delta T_{\max }=\max _{i, j} \frac{\left\|\mathbf{p}_{i}-\mathbf{p}_{j}\right\|}{c}
$$

o máximo tempo de propagação entre quaisquer dois elementos do array. Se a origem do sistema de coordenadas estiver em algum dos elementos do array ou em seu baricentro, automaticamente temos para todo $n \in\{0, \ldots, N-1\}$,

$$
\tau_{n} \leq \Delta T_{\max }
$$

Sob esta condição, dizemos informalmente que um sinal tem banda estreita se suas variações são lentas o suficiente para que

$$
m\left(t-\tau_{n}\right) \approx m(t)
$$




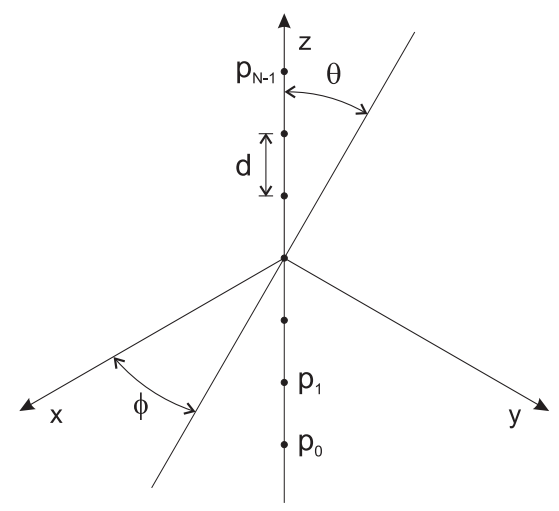

Figura 2.4: Array linear uniforme (ULA)

para todo $n \in\{0, \ldots, N-1\}$. Neste caso, a expressão (2.3.1) pode ser aproximada por

$$
f\left(t, \mathbf{p}_{n}\right)=\operatorname{Re}\left\{m(t) e^{j \omega\left(t-\tau_{n}\right)}\right\} .
$$

Se satisfeita, a hipótese de banda estreita tem a vantagem de permitir a substituição de atrasos puros por deslocamentos de fase, que podem ser implementados em hardware com boa resolução e baixo custo. O mesmo não pode ser dito sobre a implementação de atrasos puros variáveis. Arrays implementados mediante deslocamentos de fase são chamados de phased arrays.

Para que (2.3.4) seja válida, [1] verificou que o sinal deve satisfazer

$$
B \cdot \Delta T_{\max } \ll 1
$$

No Capítulo 4 revisitaremos esta condição, e definiremos condições precisas para processamento em banda estreita.

No texto que segue frequentemente simplificaremos a notação, omitindo a variável $\omega$. Alternativamente, muitos algoritmos serão descritos substituindo $\hat{\mathbf{h}}^{T}(\omega)$ por $\mathbf{w}^{H}$. Esta troca de notação corresponde a uma realidade de implementação, visto que beamformers banda larga são frequentemente implementados como bancos de beamformers de banda estreita. Neste caso, cada beamformer opera sobre um vetor $\hat{\mathbf{x}}\left(\omega_{m}\right)$, equivalente à m-ésima raia de uma transformada de Fourier de tempo curto do sinal de entrada $\mathbf{x}(t)$.

\subsection{Arrays lineares uniformes (ULAs)}

Consideremos o array linear uniforme (ou uniform linear array) da Figura 2.4. Seus elementos estão dispostos ao longo do eixo $z$, equi-espaçados por uma distância $d$. O array tem centro na origem do sistema de coordenadas. 
As coordenadas de cada sensor são dadas por

$$
\begin{aligned}
& p_{x_{n}}=0, \\
& p_{y_{n}}=0, \\
& p_{z_{n}}=\left(n-\frac{N-1}{2}\right) d .
\end{aligned}
$$

Substituindo estas coordenadas na expressão de $\mathbf{v}_{\mathbf{k}}(\mathbf{k})$, obtemos

$$
\mathbf{V}_{\mathbf{k}}(\mathbf{k})=\left[\begin{array}{c}
e^{j\left(\frac{N-1}{2}\right) k_{z} d} \\
e^{j\left(\frac{N-1}{2}-1\right) k_{z} d} \\
\vdots \\
e^{-j\left(\frac{N-1}{2}\right) k_{z} d}
\end{array}\right],
$$

onde $k_{z}=-\frac{2 \pi}{\lambda} \cos \theta=-\|\mathbf{k}\| \cos \theta$.

Para simplificar as expressões que seguem, definimos

$$
\psi=-k_{z} d=\frac{2 \pi}{\lambda} \cos \theta \cdot d=\frac{2 \pi}{\lambda} u_{z} d
$$

Como admitimos $\theta \in[0, \pi]$, consideraremos $u_{z} \in[-1,1], k_{z} \in\left[-\frac{2 \pi}{\lambda}, \frac{2 \pi}{\lambda}\right]$ e $\psi \in\left[-\frac{2 \pi}{\lambda} d, \frac{2 \pi}{\lambda} d\right]$. Estes intervalos são conhecidos como a região visivel do array. Usando estas definições, a resposta em frequência-número de onda e o padrão de radiação de um array linear uniforme se tornam

$$
\begin{aligned}
\Upsilon\left(\omega, k_{z}\right) & =\mathbf{w}^{H} \mathbf{v}_{\mathbf{k}}\left(k_{z}\right) \\
& =\sum_{n=0}^{N-1} w_{n}^{*} e^{-j\left(n-\frac{N-1}{2}\right) k_{z} d} \\
B_{\psi}(\psi) & =e^{-j \frac{N-1}{2} \psi} \sum_{n=0}^{N-1} w_{n}^{*} e^{j n \psi} .
\end{aligned}
$$

Note que suprimimos a variável $\omega$. Consideremos o caso particular do delay-and-sum beamformer, com

$$
\mathbf{w}=\frac{1}{N}\left[\begin{array}{llll}
1 & 1 & \cdots & 1
\end{array}\right]^{T}=\frac{1}{N} \mathbf{1}
$$




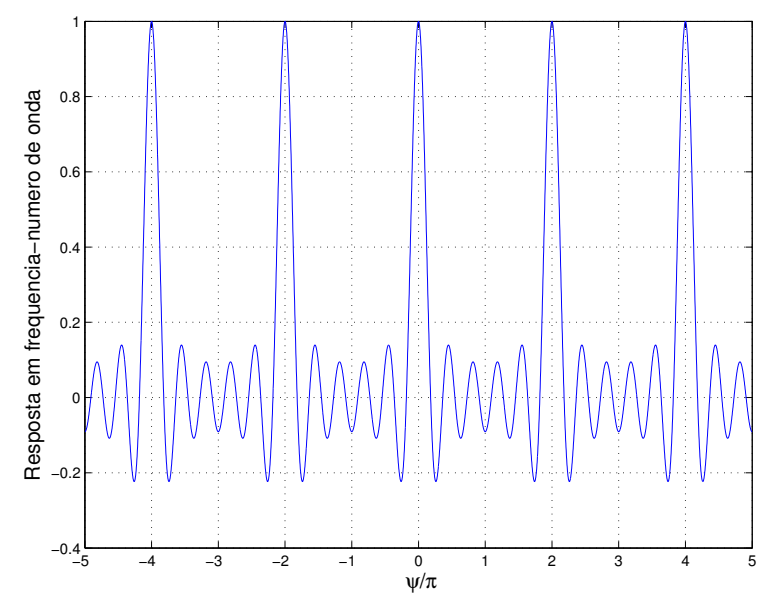

Figura 2.5: $\Upsilon(\psi) ; \psi=\frac{2 \pi}{\lambda} d \cos \theta, N=11$

Substituindo w na expressão da resposta em frequência-número de onda,

$$
\begin{aligned}
\Upsilon(\psi) & =\frac{1}{N} \sum_{n=0}^{N-1} e^{j\left(n-\frac{N-1}{2}\right) \psi} \\
& =\frac{1}{N} e^{-j\left(\frac{N-1}{2}\right) \psi} \sum_{n=0}^{N-1} e^{j n \psi} \\
& =\frac{1}{N} e^{-j\left(\frac{N-1}{2}\right) \psi} \frac{1-e^{j N \psi}}{1-e^{j \psi}} \\
& =\frac{1}{N} \frac{\sin \left(N \frac{\psi}{2}\right)}{\sin \frac{\psi}{2}} .
\end{aligned}
$$

Substituindo $u=\cos \theta$ e $\psi=\frac{2 \pi}{\lambda} d \cos \theta$, temos padrões de radiação em função de $u$ e $\theta$ :

$$
\begin{gathered}
B_{u}(u)=\frac{1}{N} \frac{\sin \left(\frac{\pi N d}{\lambda} u\right)}{\sin \left(\frac{\pi d}{\lambda} u\right)} . \\
B_{\theta}(\theta)=\frac{1}{N} \frac{\sin \left(\frac{N}{2} \frac{2 \pi}{\lambda} d \cos \theta\right)}{\sin \left(\frac{1}{2} \frac{2 \pi}{\lambda} d \cos \theta\right)} .
\end{gathered}
$$

As Figuras 2.5, 2.6 e 2.7 mostram gráficos da resposta em frequência-número de onda e o diagrama de radiação para o caso particular $N=11$ e $d=\lambda / 2$.

Consideremos o que ocorre quando $d \neq \lambda / 2$. Como já observamos, as respostas $\Upsilon(\psi)$ são periódicas em $\psi$. Em particular, (2.4.6) apresenta seus máximos quando seu numerador e denominador se anulam. Isso ocorre quando $\psi / 2=m \pi$, ou $\psi=2 m \pi$, com $m \in \mathbb{Z}$. Usando (2.4.3), vemos que os máximos ocorrem para $u=m \cdot \frac{\lambda}{d}$.

Se $d>\lambda / 2$, máximos consecutivos estarão a menos que duas unidades de distância no espaço $u$. Como veremos na seção seguinte, isso terá o efeito de inserir mais de um máximo 


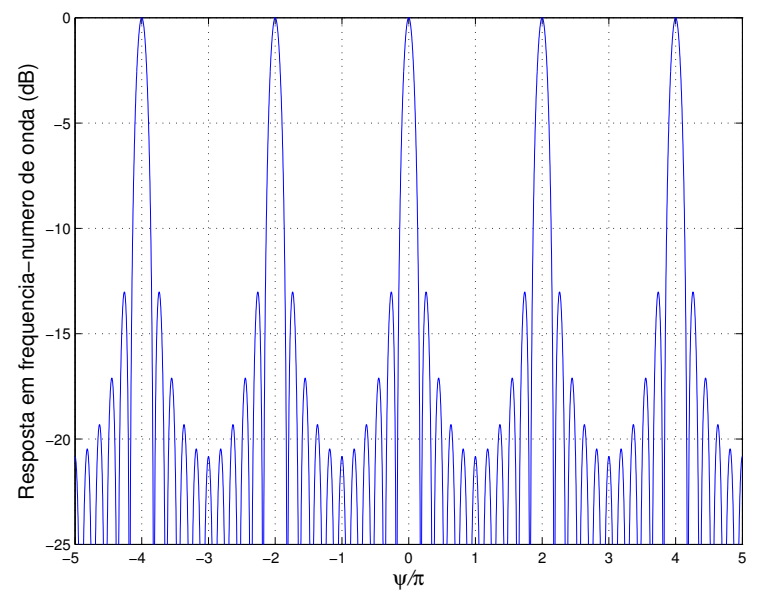

Figura 2.6: $20 \log _{10}|\Upsilon(\psi)| ; \psi=\frac{2 \pi}{\lambda} d \cos \theta, N=11$

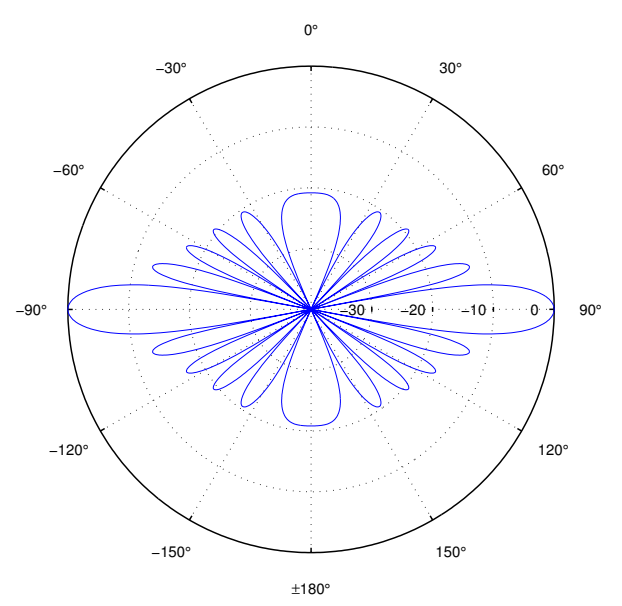

Figura 2.7: Diagrama de radiação para $B_{\theta}(\theta)(\mathrm{em} \mathrm{dB}) ; N=11, d=\lambda / 2$ 

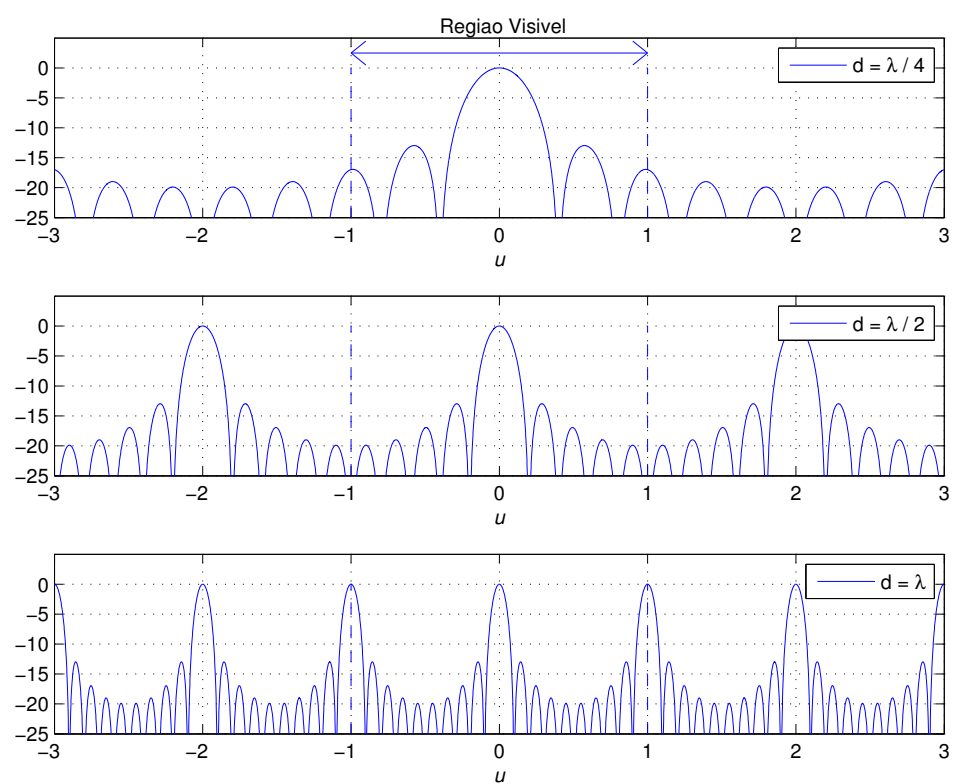

Figura 2.8: $20 \log _{10}\left|B_{u}(u)\right|$ na presença de aliasing; $N=11$

na região visível $(u \in[-1,1])$ quando redirecionarmos o lóbulo principal da resposta. Este efeito pode ser interpretado como aliasing espacial, e é visível na Figura 2.8.

Para evitar que ocorra aliasing espacial, sempre impomos a condição $d \leq \lambda / 2$.

\subsection{Direcionamento de arrays}

Uma vantagem proporcionada por arrays é a possibilidade de direcionar sua resposta eletronicamente. Isso elimina restrições de direcionamento mecânico como o tempo de varredura, e também permite a aquisição e rastreamento simultâneo de múltiplos objetos.

Seja $\mathbf{k}_{T}$ a direção desejada para o lóbulo principal da resposta, também conhecido como eixo principal da resposta. Consideremos que sobre o array incide uma onda plana com direção de chegada $\mathbf{k}$ arbitrária, dada por $\mathbf{f}(t, \mathbf{p})=e^{j \omega t} \mathbf{v}_{\mathbf{k}}(\mathbf{k})$. Desejamos que a saída $y(t)$ do processador tenha potência máxima quando $\mathbf{k}=\mathbf{k}_{T}$.

Seja $\mathbf{I}_{s}\left(\mathbf{k}_{T}\right)$ a matriz diretora dada por

$$
\mathbf{I}_{s}\left(\mathbf{k}_{T}\right)=\left[\begin{array}{cccc}
e^{j \mathbf{k}_{T}^{T} \mathbf{p}_{0}} & 0 & \cdots & 0 \\
0 & e^{j \mathbf{k}_{T}^{T} \mathbf{p}_{1}} & \cdots & 0 \\
\vdots & \vdots & \ddots & \vdots \\
0 & 0 & \cdots & e^{j \mathbf{k}_{T}^{T} \mathbf{p}_{N-1}}
\end{array}\right]
$$

O direcionamento de arrays consiste em usar $\mathbf{I}_{s}\left(\mathbf{k}_{T}\right)$ como preprocessador, conforme ilustrado na Figura 2.9. De fato, $\mathbf{I}_{s}\left(\mathbf{k}_{T}\right) \mathbf{f}(t, \mathbf{p})=e^{j \omega t} \mathbf{v}_{\mathbf{k}}\left(\mathbf{k}-\mathbf{k}_{T}\right)$, tal que para $\mathbf{k}=\mathbf{k}_{T}$ a saída do preprocessador equivale a um sinal com direção de chegada coincidente com o eixo principal de um processador não direcionado.

Sabemos que a resposta em frequência-número de onda para o caso monocromático é 


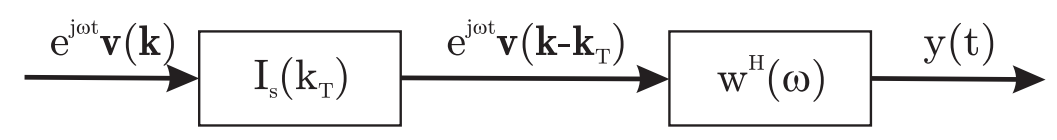

Figura 2.9: Direcionamento de arrays

dada por (2.2.23), que repetimos aqui por conveniência:

$$
\Upsilon(\omega, \mathbf{k})=\mathbf{w}^{H} \mathbf{v}_{\mathbf{k}}(\mathbf{k})
$$

Se $\Upsilon\left(\omega, \mathbf{k} \mid \mathbf{k}_{T}\right)$ é a resposta direcionada, o uso de $\mathbf{I}_{s}\left(\mathbf{k}_{T}\right)$ resulta em

$$
\begin{aligned}
\Upsilon\left(\omega, \mathbf{k} \mid \mathbf{k}_{T}\right) & =\mathbf{w}^{H} \mathbf{I}_{s}\left(\mathbf{k}_{T}\right) \mathbf{v}_{\mathbf{k}}(\mathbf{k}) \\
& =\mathbf{w}^{H} \mathbf{v}_{\mathbf{k}}\left(\mathbf{k}-\mathbf{k}_{T}\right) \\
& =\Upsilon\left(\omega, \mathbf{k}-\mathbf{k}_{T}\right) .
\end{aligned}
$$

Em particular, para o array linear uniforme com pesos uniformes temos

$$
\mathbf{w}^{H} \mathbf{I}_{s}\left(\mathbf{k}_{T}\right)=\frac{1}{N} \mathbf{v}_{\mathbf{k}}^{H}\left(\mathbf{k}_{T}\right)
$$

tal que

$$
\begin{aligned}
B\left(\mathbf{k} \mid \mathbf{k}_{T}\right) & =\frac{1}{N} \mathbf{v}_{\mathbf{k}}^{H}\left(\mathbf{k}_{T}\right) \mathbf{v}_{\mathbf{k}}(\mathbf{k}) \\
B_{u}\left(u \mid u_{T}\right) & =\frac{1}{N} \frac{\sin \left(\frac{\pi N d}{\lambda}\left(u-u_{T}\right)\right)}{\sin \left(\frac{\pi d}{\lambda}\left(u-u_{T}\right)\right)} \\
B_{\psi}\left(\psi \mid \psi_{T}\right) & =\frac{1}{N} \frac{\sin \left(N \frac{\psi-\psi_{T}}{2}\right)}{\sin \left(\frac{\psi-\psi_{T}}{2}\right)} .
\end{aligned}
$$

\subsection{Diretividade}

Seja $P(\theta, \phi)$ o padrão de potência de um array, definido como

$$
P(\theta, \phi)=|B(\theta, \phi)|^{2}
$$

A diretividade é definida por

$$
D=\frac{P\left(\theta_{T}, \phi_{T}\right)}{\frac{1}{4 \pi} \int_{0}^{2 \pi} \int_{0}^{\pi} P(\theta, \phi) \sin \theta d \theta d \phi},
$$

onde $\left(\theta_{T}, \phi_{T}\right)$ é a direção de máxima radiação (i.e., o eixo principal da resposta). Se admitirmos que o padrão de potência está normalizado tal que $P\left(\theta_{T}, \phi_{T}\right)=1, D$ se reduz a

$$
D=\left[\frac{1}{4 \pi} \int_{0}^{2 \pi} \int_{0}^{\pi} P(\theta, \phi) \sin \theta d \theta d \phi\right]^{-1} .
$$


Em um array linear uniforme temos $P(\theta, \phi)=P(\theta)$, tal que

$$
\begin{aligned}
D & =\left[\frac{1}{2} \int_{0}^{\pi} P(\theta) \sin \theta d \theta\right]^{-1} \\
& =\left[\frac{1}{2} \int_{-1}^{+1}\left|B_{u}(u)\right|^{2} d u\right]^{-1}
\end{aligned}
$$

Expandindo (2.6.4) em função do vetor de pesos w,

$$
\begin{aligned}
D & =\left[\frac{1}{2} \int_{-1}^{+1} \sum_{n=0}^{N-1} w_{n}^{*} e^{j n\left(\frac{2 \pi d}{\lambda}\right)\left(u-u_{T}\right)} \sum_{m=0}^{N-1} w_{m} e^{-j m\left(\frac{2 \pi d}{\lambda}\right)\left(u-u_{T}\right)} d u\right]^{-1} \\
& =\left[\sum_{n=0}^{N-1} \sum_{m=0}^{N-1} w_{m} w_{n}^{*} e^{j\left(\frac{2 \pi d}{\lambda}\right)(m-n) u_{T}} \operatorname{sinc}\left(\frac{2 \pi d}{\lambda}(n-m)\right)\right]^{-1}
\end{aligned}
$$

Para obter uma expressão mais compacta, definimos a matriz sinc e a matriz diretora $\mathbf{I}_{s}$ :

$$
\begin{aligned}
{[\text { sinc }]_{n m}=} & \operatorname{sinc}\left(\frac{2 \pi d}{\lambda}(n-m)\right) \\
\mathbf{I}_{s}= & {\left[\begin{array}{cccc}
1 & 0 & \cdots & 0 \\
0 & e^{j \frac{2 \pi d}{\lambda} u_{T}} & \cdots & 0 \\
\vdots & \vdots & \ddots & \vdots \\
0 & 0 & \cdots & e^{j \frac{2 \pi d}{\lambda}(N-1) u_{T}}
\end{array}\right] }
\end{aligned}
$$

onde $\operatorname{sinc}(x)=\frac{\sin x}{x}$. Com essas definições,

$$
D=\left[\mathbf{w}^{H} \mathbf{I}_{s}^{H}[\mathbf{s i n c}] \mathbf{I}_{s} \mathbf{w}\right]^{-1}
$$

Um caso de interesse é o array linear uniforme padrão, onde $d=\lambda / 2$. Neste cenário, $[$ sinc $]=\mathbf{I}$, tal que

$$
\begin{aligned}
D & =\left[\mathbf{w}^{H} \mathbf{w}\right]^{-1} \\
& =\|\mathbf{w}\|^{-2} .
\end{aligned}
$$

Para o caso de pesos uniformes, $\|\mathbf{w}\|^{2}=\frac{1}{N}$, e $D=N$. É fácil mostrar usando multiplicadores de Lagrange que o vetor de pesos que maximiza a diretividade sob a restrição $\sum_{n=0}^{N-1} w_{n}=1$ (equivalente a ganho unitário para $u_{T}=0$ ) é o vetor de pesos uniforme.

\subsection{Ganho}

O ganho de um array é definido como a razão entre a relação sinal-ruído obtida pelo array e a relação sinal-ruído obtida por um só sensor. Consideremos que a entrada de cada sensor corresponda a uma medida de onda plana incidente mais as amostras de um processo de ruído espacialmente branco, descorrelacionado com a onda plana:

$$
x_{n}(t)=f\left(t-\tau_{n}\right)+n_{n}(t) .
$$


A relação sinal-ruído medida por um único sensor na frequência $\omega$ é dada por

$$
S N R_{i}(\omega)=\frac{S_{f}(\omega)}{S_{n}(\omega)}
$$

De $(2.2 .3)$,

$$
y(t)=\int_{-\infty}^{+\infty} \mathbf{h}^{T}(t-\tau) \mathbf{x}(\tau) d \tau .
$$

A correlação $R_{y}(\tau)$ e o espectro $S_{y}(\omega)$ da saída $y(t)$ são dados por

$$
\begin{aligned}
& R_{y}(\tau)=\operatorname{E}\left\{y(t) y^{*}(t-\tau)\right\} \\
& S_{y}(\omega)=\int_{-\infty}^{+\infty} e^{-j \omega t} R_{y}(\tau) d \tau .
\end{aligned}
$$

Substituindo (2.7.3) em (2.7.4),

$$
\begin{aligned}
S_{y}(\omega) & =\int_{-\infty}^{+\infty} e^{-j \omega \tau} \int_{-\infty}^{+\infty} \int_{-\infty}^{+\infty} \mathbf{h}^{T}(\alpha) \mathrm{E}\left\{\mathbf{x}(t-\alpha) \mathbf{x}^{H}(t-\tau-\beta)\right\} \mathbf{h}^{*}(\beta) d \alpha d \beta \\
& =\int_{-\infty}^{+\infty} e^{-j \omega \alpha} \mathbf{h}^{T}(\alpha) d \alpha \int_{-\infty}^{+\infty} e^{-j \omega \gamma} \mathbf{R}_{\mathbf{x}}(\gamma) d \gamma \int_{-\infty}^{+\infty} e^{+j \omega \beta} \mathbf{h}^{*}(\beta) d \beta \\
& =\hat{\mathbf{h}}^{T}(\omega) \mathbf{S}_{\mathbf{x}}(\omega) \hat{\mathbf{h}}^{*}(\omega) \\
& =\mathbf{w}^{H} \mathbf{S}_{\mathbf{x}}(\omega) \mathbf{w} .
\end{aligned}
$$

Para calcular o ganho do array é necessário normalizar sua saída na direção do sinal $\mathbf{k}_{s}$. A restrição a seguir é conhecida como distortionless constraint, pois é usada para garantir uma resposta unitária na direção de interesse:

$$
\mathbf{w}^{H} \mathbf{v}_{\mathbf{k}}\left(\mathbf{k}_{s}\right)=1
$$

Na ausência de ruído, o espectro do sinal medido por todos os elementos do array é dado pela matriz

$$
\mathbf{S}_{f}(\omega)=\mathbf{v}_{\mathbf{k}}\left(\mathbf{k}_{s}\right) S_{f}(\omega) \mathbf{v}_{\mathbf{k}}^{H}\left(\mathbf{k}_{s}\right) .
$$

Note que em (2.7.7) admitimos que se conhece exatamente a direção de chegada do sinal.

Substituindo (2.7.7) em (2.7.5), temos o espectro de saída devido somente ao sinal de interesse,

$$
\begin{aligned}
S_{y_{s}}(\omega) & =\mathbf{w}^{H} \mathbf{v}_{\mathbf{k}}\left(\mathbf{k}_{s}\right) S_{f}(\omega) \mathbf{v}_{\mathbf{k}}^{H}\left(\mathbf{k}_{s}\right) \mathbf{w} \\
& =S_{f}(\omega) .
\end{aligned}
$$

O espectro de saída devido ao ruído é dado por

$$
S_{y_{n}}(\omega)=\mathbf{w}^{H} \mathbf{S}_{\mathbf{n}}(\omega) \mathbf{w} .
$$


Para o caso de ruído isotrópico espacialmente branco, $\mathbf{S}_{\mathbf{n}}(\omega)=S_{n}(\omega) \mathbf{I}$, tal que

$$
S_{y_{n}}(\omega)=S_{n}(\omega)\|\mathbf{w}\|^{2} .
$$

Logo,

$$
S N R_{o}(\omega)=\frac{1}{\|\mathbf{w}\|^{2}} \frac{S_{f}(\omega)}{S_{n}(\omega)}
$$

Finalmente, o ganho do array é

$$
\begin{aligned}
A_{w} & =\frac{S N R_{o}(\omega)}{S N R_{i}(\omega)} \\
& =\|\mathbf{w}\|^{-2} .
\end{aligned}
$$

Para o caso particular de pesos uniformes, $A_{w}=N$. Em geral, $A_{w} \leq N$. De fato, da desigualdade de Cauchy-Schwarz decorre que $\left|\mathbf{w}^{H} \mathbf{v}_{\mathbf{k}}\left(\mathbf{k}_{s}\right)\right| \leq\|\mathbf{w}\|\left\|\mathbf{v}_{\mathbf{k}}\left(\mathbf{k}_{s}\right)\right\|$, tal que $1 \leq\|\mathbf{w}\| \sqrt{N}$ e $A_{w}=\|\mathbf{w}\|^{-2} \leq N$.

\subsection{Sensibilidade a perturbações}

Um array real está sujeito a perturbações nas coordenadas $\mathbf{p}_{i}$ de seus sensores, seja por imprecisões mecânicas de construção ou por imprecisões decorrentes do processo de calibração. Seus coeficientes $\mathbf{w}_{i}$ também estão sujeitos a erros, uma vez que transdutores sempre apresentam variações de ganho e fase.

Um aspecto prático que não deve ser ignorado é o desempenho de um array quando submetido a perturbações. A optimalidade de um projeto pode se tornar irrelevante se as tolerâncias necessárias não puderem ser atendidas na prática.

Consideremos um array com coordenadas nominais $\mathbf{p}_{i}^{n}$ e pesos nominais $\mathbf{w}_{i}^{n}=g_{i}^{n} e^{j \phi_{i}^{n}}$, para $i \in\{0, \ldots, N-1\}$. Sejam suas coordenadas e pesos reais

$$
\begin{aligned}
\mathbf{p}_{i} & =\mathbf{p}_{i}^{n}+\Delta \mathbf{p}_{i} \\
g_{i} & =g_{i}^{n}\left(1+\Delta g_{i}\right) \\
\phi_{i} & =\phi_{i}^{n}+\Delta \phi_{i},
\end{aligned}
$$

onde $\Delta \mathbf{p}_{i}, \Delta g_{i}$ e $\Delta \phi_{i}$ são variáveis aleatórias Gaussianas independentes e com média zero. Suponhamos que $\Delta g_{i}$ e $\Delta \phi_{i}$ tenham variâncias $\sigma_{g}^{2}$ e $\sigma_{\phi}^{2}$, e que cada componente de $\Delta \mathbf{p}_{i}$ tenha variância $\sigma_{p}^{2}$. Substituindo as definições de $\mathbf{p}_{i}, g_{i}$ e $\phi_{i}$ e usando a independência das perturbações, [2] mostra que

$$
\mathrm{E}\left\{|B(\mathbf{k})|^{2}\right\}=\left|B^{n}(\mathbf{k})\right|^{2} e^{-\left(\sigma_{\phi}^{2}+\sigma_{\lambda}^{2}\right)}+\sum_{i=0}^{N-1}\left(g_{i}^{n}\right)^{2}\left\{\left(1+\sigma_{g}^{2}\right)-e^{-\left(\sigma_{\phi}^{2}+\sigma_{\lambda}^{2}\right)}\right\},
$$

onde $\left|B^{n}(\mathbf{k})\right|^{2}$ é o padrão de potência nominal e $\sigma_{\lambda}=2 \pi \sigma_{p} / \lambda$. 
O primeiro termo indica que o padrão nominal é atenuado devido às perturbações. Este comportamento é tolerável, uma vez que a atenuação é uniforme ao longo de todas as direções de visada. O segundo termo é problemático, pois envolve a distorção do padrão de radiação. Usando que $\sum_{i=0}^{N-1}\left(g_{i}^{n}\right)^{2}=\|\mathbf{w}\|^{2}$ e supondo que $\sigma_{g}^{2}, \sigma_{\phi}^{2}$ e $\sigma_{\lambda}^{2}$ são pequenos, o segundo termo pode ser aproximado por

$$
\mathrm{E}\left\{\Delta|B(\mathbf{k})|^{2}\right\} \approx\|\mathbf{w}\|^{2}\left\{\sigma_{g}^{2}+\sigma_{\phi}^{2}+\sigma_{\lambda}^{2}\right\}
$$

De (2.7.12) e (2.8.3), temos que a sensibilidade é inversamente proporcional ao ganho para ruído branco. Em particular, para o caso de pesos uniformes o ganho para ruído branco é máximo e a sensibilidade é mínima.

O termo $\mathrm{E}\left\{\Delta|B(\mathbf{k})|^{2}\right\}$ tem o efeito de elevar o valor de rejeição mínima do array. Um array ideal seria capaz de ter resposta nula em direções correspondentes a interferências, algo impossível na presença de perturbações não compensadas.

Para limitar a sensibilidade de arrays, um procedimento de projeto é o uso da restrição $\|\mathbf{w}\|^{2} \leq T_{0}$, com $T_{0}$ constante. Uma consequência inevitável desta imposição é a limitação do quão abruptas podem ser variações de ganho do padrão de radiação. Isto impede que respostas nulas sejam colocadas arbitrariamente próximas de sinais de interesse, seja por métodos de projeto offline ou por algoritmos adaptativos. Esta característica é simultaneamente inconveniente e desejável. De fato, na presença de erros de calibração, um algoritmo adaptativo poderia incorretamente classificar o sinal de interesse como um sinal de interferência próximo ao eixo principal da resposta. $\mathrm{O}$ algoritmo automaticamente tentaria anular a suposta interferência, e consequentemente anularia o sinal de interesse.

Para ilustrar este comportamento, consideremos $\mathbf{v}_{\mathbf{k}}(\mathbf{k})$ e $\mathbf{v}_{\mathbf{k}}(\tilde{\mathbf{k}})$ vetores diretores tais que $\left\|\mathbf{v}_{\mathbf{k}}(\mathbf{k})-\mathbf{v}_{\mathbf{k}}(\tilde{\mathbf{k}})\right\| \leq \varepsilon$. Assim, $\|\Upsilon(\mathbf{k})-\Upsilon(\tilde{\mathbf{k}})\|=\left\|\mathbf{w}^{H}\left(\mathbf{v}_{\mathbf{k}}(\mathbf{k})-\mathbf{v}_{\mathbf{k}}(\tilde{\mathbf{k}})\right)\right\| \leq T_{0} \varepsilon$, que implica $|\|\Upsilon(\mathbf{k})\|-\|\Upsilon(\tilde{\mathbf{k}})\|| \leq T_{0} \varepsilon$. Além disso, como $\mathbf{v}_{\mathbf{k}}(\mathbf{k})$ é Lipschitz contínua [3], temos que $\exists M \in \mathbb{R}$ tal que $\left\|\mathbf{v}_{\mathbf{k}}(\mathbf{k})-\mathbf{v}_{\mathbf{k}}(\tilde{\mathbf{k}})\right\| \leq M\|\mathbf{k}-\tilde{\mathbf{k}}\|, \forall \mathbf{k}, \tilde{\mathbf{k}}$. Se $\|\mathbf{k}-\tilde{\mathbf{k}}\| \leq$ $\varepsilon$, podemos aplicar o resultado anterior e concluir que $\|\Upsilon(\mathbf{k})\|-\|\Upsilon(\tilde{\mathbf{k}})\| \mid \leq T_{0} M \varepsilon$. Portanto, a restrição de projeto $\|\mathbf{w}\|^{2} \leq T_{0}$ permite o controle da sensibilidade de $\Upsilon(\mathbf{k})$. 



\section{Capítulo 3}

\section{Fundamentos de síntese e implementação}

\subsection{Métodos clássicos}

Os métodos clássicos para projeto de arrays lineares e retangulares foram derivados diretamente da teoria de filtros digitais. A seguir mencionaremos brevemente algumas técnicas na linguagem característica de arrays, e que podem ser encontradas na linguagem de filtros digitais em textos como $[4,5]$.

\subsubsection{Janelas}

O projeto mediante janelas é uma técnica heurística que atrai interesse prático devido à sua simplicidade e robustez. No campo de filtros digitais, janelas são usadas para minimizar os efeitos do truncamento de respostas impulsivas. Neste contexto, a escolha de uma janela para o projeto de filtros ou análise espectral envolve um compromisso entre resolução espectral e faixa dinâmica. Analogamente, janelas são usadas para minimizar os efeitos das aberturas finitas de arrays. O compromisso passa a ser entre resolução espacial e faixa dinâmica.

A relação entre filtros e arrays pode ser formalizada substituindo $z=e^{j \psi}$ no padrão de radiação (2.4.4):

$$
\begin{aligned}
B_{z}(z) & =z^{-\frac{N-1}{2}} \sum_{n=0}^{N-1} w_{n}^{*} z^{n} \\
& =z^{-\frac{N-1}{2}}\left(\sum_{n=0}^{N-1} w_{n} z^{-n}\right)^{*}
\end{aligned}
$$

Lembrando que o termo entre parênteses é a transformada $Z W(z)$ de $\left\{w_{n}\right\}_{n=0}^{N-1}$,

$$
B_{z}(z)=z^{-\frac{N-1}{2}} W^{*}(z)
$$

Portanto, o mesmo comportamento esperado no domínio da frequência em filtros digitais pode ser esperado no domínio $\psi$ em ULAs.

A Figura 3.1 apresenta exemplos de padrões de radiação para diferentes janelas, cujas expressões analíticas podem ser verificadas em [2,4,5]. A Tabela 3.1 apresenta medidas de desempenho para as mesmas janelas (onde HPBW é a largura de feixe a meia-potência e $B W_{N N}$ é a distância entre os zeros que definem o lóbulo principal; ambas as medidas 

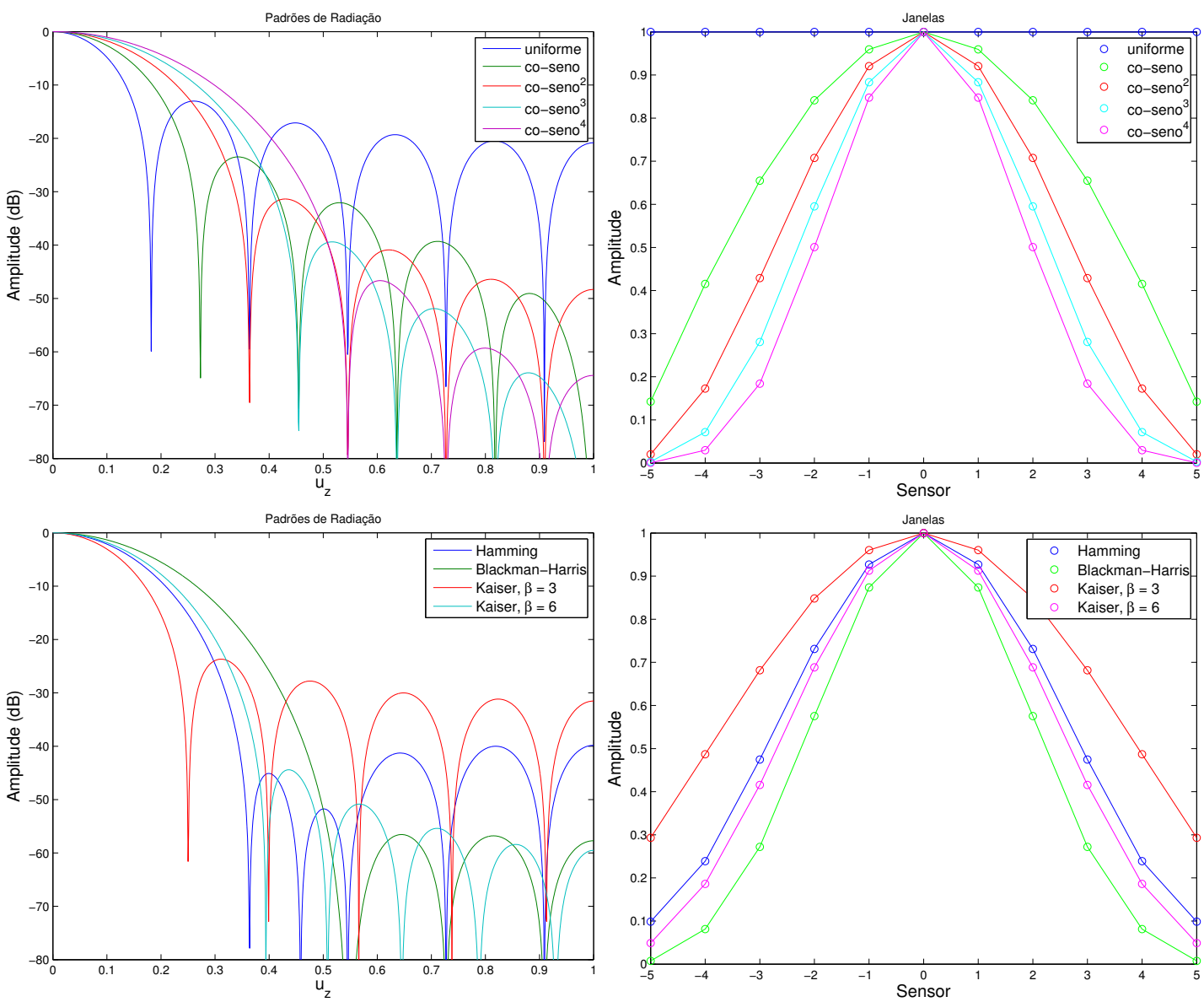

Figura 3.1: Exemplos de janelas e seus respectivos padrões de radiação; $N=11, d=\lambda / 2$

são apresentadas no espaço u).

Um método menos heurístico consiste em projetar pesos que maximizem a potência recebida em um setor angular. Os correspondentes padrões de radiação são as chamadas funções esferoidais prolatas discretas, cujo uso em filtros FIR [6,7] antecede sua aplicação em beamformers. O tratamento a seguir é o mesmo dado em $[2,8]$.

O objetivo de projeto é maximizar a função

$$
\alpha=\frac{\iint_{\Omega}|B(\theta, \phi)|^{2} \sin \theta d \theta d \phi}{\int_{0}^{\pi} \int_{0}^{2 \pi}|B(\theta, \phi)|^{2} \sin \theta d \theta d \phi}
$$

onde $\Omega$ representa um setor angular ao redor do eixo principal. Para o caso particular de um ULA padrão (i.e., $\operatorname{com} d=\lambda / 2$ ),

$$
\alpha=\frac{\int_{-\psi_{0}}^{+\psi_{0}}|B(\psi)|^{2} d \psi}{\int_{-\pi}^{+\pi}|B(\psi)|^{2} d \psi} .
$$


Tabela 3.1: Medidas de desempenho para diferentes janelas [2]; $N=11, d=\lambda / 2$. HPBW é a largura de feixe a meia-potência e $B W_{N N}$ é a distância entre os zeros que definem o lóbulo principal.

\begin{tabular}{|c|c|c|c|c|}
\hline Janela & HPBW & $B W_{N N}$ & Altura Lóbulo Sec. & $\mathrm{D}$ \\
\hline \hline Uniforme & $0.89 \frac{2}{N}$ & $2.0 \frac{2}{N}$ & $-13.0 d B$ & 1 \\
\hline Co-seno $^{2}$ & $1.18 \frac{2}{N}$ & $3.0 \frac{2}{N}$ & $-23.5 d B$ & 0.816 \\
\hline Co-seno $^{2}$ & $1.44 \frac{2}{N}$ & $4.0 \frac{2}{N}$ & $-31.4 d B$ & 0.667 \\
\hline Co-seno $^{3}$ & $1.66 \frac{2}{N}$ & $5.0 \frac{2}{N}$ & $-39.4 d B$ & 0.576 \\
\hline Co-seno $^{4}$ & $1.85 \frac{2}{N}$ & $6.0 \frac{2}{N}$ & $-46.7 d B$ & 0.514 \\
\hline Hamming & $1.31 \frac{2}{N}$ & $4.0 \frac{2}{N}$ & $-39.5 d B$ & 0.730 \\
\hline Blackman-Harris & $1.65 \frac{2}{N}$ & $6.0 \frac{2}{N}$ & $-56.6 d B$ & 0.577 \\
\hline Kaiser $\beta=3$ & $2.18 / N$ & $1.75 \pi / N$ & $-23.7 d B$ & 0.882 \\
\hline Kaiser $\beta=6$ & $2.80 / N$ & $2.76 \pi / N$ & $-44.4 d B$ & 0.683 \\
\hline
\end{tabular}

Substituindo $\psi=-k_{z} d$ na definição de $\mathbf{v}_{\mathbf{k}}(\mathbf{k})$ em (2.4.2), obtemos o vetor diretor

$$
\mathbf{v}_{\psi}(\psi)=\left[\begin{array}{c}
e^{-j\left(\frac{N-1}{2}\right) \psi} \\
e^{-j\left(\frac{N-1}{2}-1\right) \psi} \\
\vdots \\
e^{j\left(\frac{N-1}{2}\right) \psi}
\end{array}\right]
$$

Usando (2.2.23), podemos escrever o numerador de $\alpha$ como

$$
\begin{aligned}
\alpha_{N} & =\int_{-\psi_{0}}^{+\psi_{0}} \mathbf{w}^{H} \mathbf{v}_{\psi}(\psi) \mathbf{v}_{\psi}^{H}(\psi) \mathbf{w} d \psi \\
& =\mathbf{w}^{H}\left[\int_{-\psi_{0}}^{+\psi_{0}} \mathbf{v}_{\psi}(\psi) \mathbf{v}_{\psi}^{H}(\psi) d \psi\right] \mathbf{w} \\
& =\mathbf{w}^{H} \mathbf{A} \mathbf{w}
\end{aligned}
$$

onde

$$
\mathbf{A}=\int_{-\psi_{0}}^{+\psi_{0}} \mathbf{v}_{\psi}(\psi) \mathbf{v}_{\psi}^{H}(\psi) d \psi
$$

Podemos verificar que

$$
[\mathbf{A}]_{m n}=\frac{2 \sin \left((m-n) \psi_{0}\right)}{(m-n)} .
$$

Analogamente, o denominador é dado por

$$
\begin{aligned}
\alpha_{D} & =\int_{-\pi}^{+\pi} \mathbf{w}^{H} \mathbf{v}_{\psi}(\psi) \mathbf{v}_{\psi}^{H}(\psi) \mathbf{w} d \psi \\
& =\mathbf{w}^{H} \mathbf{B} \mathbf{w}
\end{aligned}
$$

onde

$$
\begin{aligned}
\mathbf{B} & =\int_{-\pi}^{+\pi} \mathbf{v}_{\psi}(\psi) \mathbf{v}_{\psi}^{H}(\psi) d \psi \\
& =2 \pi \mathbf{I}
\end{aligned}
$$


Logo, a função objetivo se escreve como

$$
\alpha=\frac{\mathbf{w}^{H} \mathbf{A} \mathbf{w}}{2 \pi \mathbf{w}^{H} \mathbf{w}}
$$

que pode ser maximizada escolhendo o autovetor correspondente ao maior autovalor de

$$
2 \pi \lambda \mathbf{w}=\mathbf{A} \mathbf{w}
$$

Note que no caso mais geral $d \neq \lambda / 2$ temos $\mathbf{B} \neq \mathbf{I}$, tal que devemos escolher o autovetor correspondente ao maior autovalor de

$$
\lambda \mathbf{B w}=\mathbf{A w} .
$$

\subsubsection{Amostragem em $\psi$}

Uma técnica tradicional de projeto de filtros FIR consiste em amostrar uma resposta em frequência desejada, e transformá-la para o domínio do tempo através da antitransformada discreta de Fourier. O cenário é análogo para arrays lineares uniformes, onde trabalhamos sobre o domínio $\psi$. No entanto, um ajuste é necessário devido ao formato da expressão (3.1.2), uma vez que $B_{z}(z)$ não corresponde exatamente a $W(z)$, a transformada $\mathrm{Z}$ do vetor de pesos. De (3.1.2), temos

$$
W(z)=B_{z}^{*}(z) z^{-\frac{N-1}{2}}
$$

Substituindo $z=e^{j \omega}$,

$$
W\left(e^{j \omega}\right)=B_{\psi}^{*}(\psi) e^{-j \frac{N-1}{2} \psi} .
$$

Amostramos em $\psi_{k}=\left(k-\frac{N-1}{2}\right) \frac{2 \pi}{N}$ (i.e., $z_{k}=e^{j\left(k-\frac{N-1}{2}\right) \frac{2 \pi}{N}}$ ), para $k \in\{0, \ldots, N-1\}$, que correspondem a $N$ pontos simétricos em relação à origem, com $\psi \in[-\pi, \pi]$.

$$
\begin{aligned}
B_{\psi}^{*}\left(\psi_{k}\right) e^{-j \frac{N-1}{2} \psi_{k}} & =\sum_{n=0}^{N-1} w_{n} z_{k}^{-n} \\
& =\sum_{n=0}^{N-1} w_{n} e^{-j\left(k-\frac{N-1}{2}\right) \frac{2 \pi}{N} n} \\
& =\sum_{n=0}^{N-1} w_{n} e^{j n \pi\left(\frac{N-1}{N}\right)} e^{-j k n \frac{2 \pi}{N}}
\end{aligned}
$$

Definindo

$$
\begin{aligned}
b_{n} & =w_{n} e^{j n \pi\left(\frac{N-1}{N}\right)} \\
B(k) & =B_{\psi}^{*}\left(\psi_{k}\right) e^{-j \frac{N-1}{2} \psi_{k}},
\end{aligned}
$$

temos

$$
B(k)=\sum_{n=0}^{N-1} b_{n} e^{-j k n \frac{2 \pi}{N}}
$$


tal que $B(k)$ é a transformada discreta de Fourier (DFT) de $\left\{b_{n}\right\}_{n=0}^{N-1}$. Denotando os elementos $B(k)$ e $\left\{b_{n}\right\}_{n=0}^{N-1}$ como os vetores $\mathbf{B}$ e b, e definindo a matriz da DFT como

$$
[F]_{m n}=e^{-j \frac{2 \pi}{N} m n}
$$

temos

$$
\mathbf{B}=\mathbf{F b}
$$

Da definição de $\mathbf{F}$, vale

$$
\begin{aligned}
\mathbf{b} & =\mathbf{F}^{-1} \mathbf{B} \\
& =\frac{1}{N} \mathbf{F}^{H} \mathbf{B} .
\end{aligned}
$$

Portanto, o projeto por amostragem em $\psi$ consiste em (1) obter os valores $B_{\psi}\left(\psi_{k}\right) ;(2)$ obter B através de (3.1.17); (3) aplicar $\mathbf{b}=\frac{1}{N} \mathbf{F}^{H} \mathbf{B}$; (4) obter $w_{n}$ através de (3.1.17).

\subsubsection{Síntese por mínimos quadrados}

Seja $B_{d}(\psi)$ um padrão desejado, sintetizável ou não. Para um array ULA com vetor de pesos $\mathbf{w}$, definimos o erro quadrático da síntese por

$$
\begin{aligned}
\xi & =\int_{-\pi}^{+\pi}\left|B_{d}(\psi)-\mathbf{w}^{H} \mathbf{v}_{\psi}(\psi)\right|^{2} d \psi \\
& =\int_{-\pi}^{+\pi}\left(B_{d}(\psi)-\mathbf{w}^{H} \mathbf{v}_{\psi}(\psi)\right)\left(B_{d}^{*}(\psi)-\mathbf{v}_{\psi}^{H}(\psi) \mathbf{w}\right) d \psi
\end{aligned}
$$

Tratando $\xi\left(\mathbf{w}, \mathbf{w}^{H}\right)$ como independentemente analítica em relação a $\mathbf{z}$ e $\mathbf{z}^{H}$, definindo $\boldsymbol{\nabla}_{\mathbf{w}^{H}}$ como o gradiente complexo [9] em relação a $\mathbf{w}^{H}$, e usando o fato que $\boldsymbol{\nabla}_{\mathbf{w}^{H}} \xi\left(\mathbf{w}_{o}, \mathbf{w}^{H}\right)=$ 0 é condição necessária para optimalidade de $\mathbf{w}_{o}$,

$$
-\int_{-\pi}^{+\pi} \mathbf{v}_{\psi}(\psi) B_{d}^{*}(\psi) d \psi+\left[\int_{-\pi}^{+\pi} \mathbf{v}_{\psi}(\psi) \mathbf{v}_{\psi}^{H}(\psi) d \psi\right] \mathbf{w}_{o}=0 .
$$

Definindo

$$
\mathbf{A}=\int_{-\pi}^{+\pi} \mathbf{v}_{\psi}(\psi) \mathbf{v}_{\psi}^{H}(\psi) d \psi
$$

e substituindo na expressão acima,

$$
\mathbf{w}_{o}=\mathbf{A}^{-1} \int_{-\pi}^{+\pi} \mathbf{v}_{\psi}(\psi) B_{d}^{*}(\psi) d \psi
$$

Para o ULA com $d=\lambda / 2$, novamente temos que

$$
\mathbf{A}=2 \pi \mathbf{I}
$$

Substituindo $\left[\mathbf{v}_{\psi}(\psi)\right]_{n}=e^{j\left(n-\frac{N-1}{2}\right) \psi}$ na expressão de $\mathbf{w}_{o}$, temos

$$
\left[\mathbf{w}_{o}\right]_{n}=\frac{1}{2 \pi} \int_{-\pi}^{+\pi} e^{j\left(n-\frac{N-1}{2}\right) \psi} B_{d}^{*}(\psi) d \psi
$$


Este resultado pode ser interpretado como a expansão em série de Fourier de $B_{d}(\psi)$, onde o termo $n-\frac{N-1}{2}$ varia de forma simétrica em torno de zero.

\subsubsection{Outros métodos}

Qualquer outro método para projeto de filtros FIR pode ser adaptado para beamformers. Beamformers Chebyshev, Villeneuve, Taylor estão convenientemente formalizados em [2]. O algoritmo de Parks-McClellan para otimização minimax pode ser derivado para padrões de radiação, definindo uma função de erro $\xi(\psi)=W(\psi)\left[B_{d}(\psi)-B(\psi)\right]$ (onde $W(\psi)$ é uma função peso, $B_{d}(\psi)$ é a resposta desejada e $B(\psi)$ é a resposta a ser otimizada) e aplicando as mesmas considerações decorrentes do teorema da alternância [5].

\subsection{Restrições de zeros}

Assim como filtros FIR podem ser projetados com zeros em frequências pré-definidas, arrays podem ser projetados com respostas espaciais nulas em direções pré-determinadas. Este recurso permite o cancelamento de interferências com direção conhecida, aumentando o ganho sinal-ruído muito além do que seria possível somente com filtros temporais.

Para garantir que o sinal de interesse com direção $\mathbf{k}_{T}$ não será atenuado ou distorcido seja lá qual for o método usado, aplicamos também a restrição (2.7.6), repetida a seguir:

$$
B\left(\mathbf{k}_{T}\right)=\mathbf{w}^{H} \mathbf{v}_{\mathbf{k}}\left(\mathbf{k}_{\mathbf{T}}\right)=1 .
$$

Suponhamos que as direções $\mathbf{k}_{i}$ para $i \in\{1, \ldots, M\}=S_{0}$ contenham interferências. Isso nos motiva a impor as restrições

$$
B\left(\mathbf{k}_{i}\right)=\mathbf{w}^{H} \mathbf{v}_{\mathbf{k}}\left(\mathbf{k}_{i}\right)=0
$$

Definimos $C_{0}$, a matriz de restrições de ordem 0:

$$
\mathbf{C}_{0}=\left[\begin{array}{lll}
\mathbf{v}_{\mathbf{k}}\left(\mathbf{k}_{1}\right) & \cdots & \mathbf{v}_{\mathbf{k}}\left(\mathbf{k}_{\left|S_{0}\right|}\right)
\end{array}\right]
$$

A partir da expansão em série de Taylor da resposta em frequência-número de onda podemos concluir que zeros nas derivadas da resposta produzem vales mais largos. Este resultado nos motiva a impor restrições de ordem superior, com a desvantagem de exigir graus de liberdade adicionais ${ }^{1}$ :

$$
\frac{d}{d k} B\left(\mathbf{k}_{i}\right)=\mathbf{w}^{H} \frac{d}{d k} \mathbf{v}_{\mathbf{k}}\left(\mathbf{k}_{i}\right)=\mathbf{w}^{H} \mathbf{d}_{1}\left(\mathbf{k}_{i}\right)=0,
$$

\footnotetext{
${ }^{1}$ Uma questão é como representar derivadas de ordem superior. Neste caso usamos $\frac{d}{d k}$, a derivada em relação a um número de onda escalar. No caso de ULAs, uma alternativa seria $\frac{d}{d \psi}$ ou $\frac{d}{d u}$. No caso geral, poderíamos impor $\nabla_{\mathbf{k}} B\left(\mathbf{k}_{i}\right)=0$.
} 
para $i \in S_{1} \subseteq S_{0}$. Definimos $C_{1}$, a matriz de restrições de ordem 1:

$$
\mathbf{C}_{1}=\left[\begin{array}{lll}
\mathbf{d}_{1}\left(\mathbf{k}_{1}\right) & \cdots & \mathbf{d}_{1}\left(\mathbf{k}_{\left|S_{1}\right|}\right)
\end{array}\right]
$$

Repetindo para ordens superiores,

$$
\frac{d^{n}}{d k^{n}} B\left(\mathbf{k}_{i}\right)=\mathbf{w}^{H} \frac{d^{n}}{d k^{n}} \mathbf{v}_{\mathbf{k}}\left(\mathbf{k}_{i}\right)=\mathbf{w}^{H} \mathbf{d}_{j}\left(\mathbf{k}_{i}\right)=0,
$$

para $i \in S_{j} \subseteq S_{j-1} \subseteq \cdots \subseteq S_{0}$. Definimos $C_{j}$, a matriz de restrições de ordem $j$ :

$$
\mathbf{C}_{j}=\left[\begin{array}{lll}
\mathbf{d}_{j}\left(\mathbf{k}_{1}\right) & \cdots & \mathbf{d}_{j}\left(\mathbf{k}_{\left|S_{j}\right|}\right)
\end{array}\right] .
$$

Supondo que só sejam usadas derivadas até ordem 2, o conjunto as restrições de zeros pode ser denotado por

$$
\mathbf{w}^{H} \mathbf{C}=\mathbf{w}^{H}\left[\begin{array}{lll}
\mathbf{C}_{0} & \mathbf{C}_{1} & \mathbf{C}_{2}
\end{array}\right]=\mathbf{0} .
$$

A proposta a seguir para síntese com restrições de zeros foi proposta pela primeira vez em [10] e está apresentada na linguagem de [2].

Seja $B_{d}=\mathbf{w}_{d}^{H} \mathbf{v}_{\mathbf{k}}(\mathbf{k})$ uma resposta que se deseja aproximar com as restrições acima. Se a resposta desejada não for realizável, deve-se obter uma aproximação realizável usando, por exemplo, o procedimento por mínimos quadrados descrito anteriormente. Para projetar o vetor de coeficientes, minimizaremos

$$
\begin{aligned}
\xi & =\int\left|B_{d}(\mathbf{k})-\mathbf{w}^{H} \mathbf{v}_{\mathbf{k}}(\mathbf{k})\right|^{2} d \mathbf{k} \\
& =\int\left|\mathbf{w}_{d}^{H} \mathbf{v}_{\mathbf{k}}(\mathbf{k})-\mathbf{w}^{H} \mathbf{v}_{\mathbf{k}}(\mathbf{k})\right|^{2} d \mathbf{k}
\end{aligned}
$$

sob a restrição $\mathbf{w}^{H} \mathbf{C}=\mathbf{0}$.

Usando multiplicadores de Lagrange e admitindo que $\int \mathbf{v}_{\mathbf{k}}(\mathbf{k}) \mathbf{v}_{\mathbf{k}}^{H}(\mathbf{k})=\mathbf{I}$, temos a função objetivo

$$
F\left(\mathbf{w}, \mathbf{w}^{H}\right)=\left(\mathbf{w}_{d}^{H}-\mathbf{w}^{H}\right)\left(\mathbf{w}_{d}-\mathbf{w}\right)+\mathbf{w}^{H} \mathbf{C} \boldsymbol{\lambda}+\boldsymbol{\lambda}^{H} \mathbf{C}^{H} \mathbf{w} .
$$

Avaliando o gradiente complexo em relação a w $\left(\mathrm{ou} \mathbf{w}^{H}\right)$ e igualando a $\mathbf{0}$ temos

$$
-\mathbf{w}_{d}^{H}+\mathbf{w}_{o}^{H}+\boldsymbol{\lambda}^{H} \mathbf{C}^{H}=\mathbf{0} \Rightarrow \mathbf{w}_{o}^{H}=\mathbf{w}_{d}^{H}-\boldsymbol{\lambda}^{H} \mathbf{C}^{H} .
$$

Usando que $\mathbf{w}^{H} \mathbf{C}=\mathbf{0}$, obtemos $\mathbf{w}_{d}^{H} \mathbf{C}-\boldsymbol{\lambda}^{H} \mathbf{C}^{H} \mathbf{C}=\mathbf{0}$.

Suponha que as colunas de $\mathbf{C}$ sejam suficientemente independentes, tal que $\mathbf{C}^{H} \mathbf{C}$ seja bem condicionada. Se este não for o caso, uma possibilidade é computar a SVD de $\mathbf{C}$, eliminar os valores e vetores singulares menos significativos, e obter uma aproximação de 
$\mathbf{C}$ com colunas suficientemente independentes. Usando a não-singularidade de $\mathbf{C}^{H} \mathbf{C}$,

$$
\boldsymbol{\lambda}^{H}=\mathbf{w}_{d}^{H} \mathbf{C}\left[\mathbf{C}^{H} \mathbf{C}\right]^{-1} .
$$

Finalmente,

$$
\begin{aligned}
\mathbf{w}_{o}^{H} & =\mathbf{w}_{d}^{H}-\mathbf{w}_{d}^{H} \mathbf{C}\left[\mathbf{C}^{H} \mathbf{C}\right]^{-1} \mathbf{C}^{H} \\
& =\mathbf{w}_{d}^{H}\left(\mathbf{I}-\mathbf{C}\left[\mathbf{C}^{H} \mathbf{C}\right]^{-1} \mathbf{C}^{H}\right) .
\end{aligned}
$$

Observe que $\mathbf{P}_{\mathbf{C}}=\mathbf{C}\left[\mathbf{C}^{H} \mathbf{C}\right]^{-1} \mathbf{C}^{H}$ é a matriz de projeção sobre o sub-espaço das restrições. Assim,

$$
\mathbf{w}_{o}^{H}=\mathbf{w}_{d}^{H} \mathbf{P}_{\mathbf{C}}^{\perp}
$$

onde $\mathbf{P}_{\mathbf{C}}^{\perp}=\mathbf{I}-\mathbf{P}_{\mathbf{C}}$ é a matriz de projeção sobre o sub-espaço ortogonal às restrições.

Uma segunda interpretação pode ser obtida escrevendo

$$
\begin{aligned}
\mathbf{w}_{o}^{H} & =\mathbf{w}_{d}^{H}-\mathbf{w}_{d}^{H} \mathbf{C}\left[\mathbf{C}^{H} \mathbf{C}\right]^{-1} \mathbf{C}^{H} \\
& =\mathbf{w}_{d}^{H}-\mathbf{a} \mathbf{C}^{H}
\end{aligned}
$$

$\operatorname{com} \mathbf{a}=\mathbf{w}_{d}^{H} \mathbf{C}\left[\mathbf{C}^{H} \mathbf{C}\right]^{-1}$. Multiplicando pela direita por $\mathbf{v}_{\mathbf{k}}(\mathbf{k})$, obtemos

$$
\begin{aligned}
B_{o}(\mathbf{k}) & =\left[\mathbf{w}_{d}^{H}-\mathbf{a} \mathbf{C}^{H}\right] \mathbf{v}_{\mathbf{k}}(\mathbf{k}) \\
& =B_{d}(\mathbf{k})-\mathbf{a} \mathbf{C}^{H} \mathbf{v}_{\mathbf{k}}(\mathbf{k}) .
\end{aligned}
$$

As linhas de $\mathbf{C}^{H}$ são da forma $\mathbf{v}_{\mathbf{k}}^{H}\left(\mathbf{k}_{i}\right), \frac{d}{d k} \mathbf{v}_{\mathbf{k}}^{H}\left(\mathbf{k}_{i}\right)$ ou $\frac{d^{2}}{d k^{2}} \mathbf{v}_{\mathbf{k}}^{H}\left(\mathbf{k}_{i}\right)$, para vetores $\mathbf{k}_{i}$ apropriados. Note que

$$
\mathbf{v}_{\mathbf{k}}^{H}\left(\mathbf{k}_{i}\right) \mathbf{v}_{\mathbf{k}}(\mathbf{k})=B_{c}\left(\mathbf{k}-\mathbf{k}_{i}\right)
$$

onde $B_{c}\left(\mathbf{k}-\mathbf{k}_{i}\right)$ é o padrão de radiação convencional que aponta para $\mathbf{k}_{i}$. Analogamente, observe que

$$
\begin{aligned}
{\left[\frac{d}{d k} \mathbf{v}_{\mathbf{k}}^{H}\left(\mathbf{k}_{i}\right)\right] \mathbf{v}_{\mathbf{k}}(\mathbf{k})=-\mathbf{v}_{\mathbf{k}}^{H}\left(\mathbf{k}_{i}\right)\left[\frac{d}{d k} \mathbf{v}_{\mathbf{k}}(\mathbf{k})\right]=-\frac{d}{d k} B_{c}\left(\mathbf{k}-\mathbf{k}_{i}\right) } \\
{\left[\frac{d^{2}}{d k^{2}} \mathbf{v}_{\mathbf{k}}^{H}\left(\mathbf{k}_{i}\right)\right] \mathbf{v}_{\mathbf{k}}(\mathbf{k})=\mathbf{v}_{\mathbf{k}}^{H}\left(\mathbf{k}_{i}\right)\left[\frac{d^{2}}{d k^{2}} \mathbf{v}_{\mathbf{k}}(\mathbf{k})\right]=\frac{d^{2}}{d k^{2}} B_{c}\left(\mathbf{k}-\mathbf{k}_{i}\right) . }
\end{aligned}
$$

Portanto, o produto $\mathbf{a C}^{H} \mathbf{v}_{\mathbf{k}}(\mathbf{k})$ se escreve como uma soma ponderada de padrões convencionais e derivadas de padrões convencionais que apontam para as direções dos zeros. Temos então

$$
\begin{aligned}
B_{o}(\mathbf{k})= & {\left[\mathbf{w}_{d}^{H}-\mathbf{a} \mathbf{C}^{H}\right] \mathbf{v}_{\mathbf{k}}(\mathbf{k}) } \\
= & B_{d}(\mathbf{k})-\sum_{n=1}^{\left|S_{0}\right|} a_{n} B_{c}\left(\mathbf{k}-\mathbf{k}_{i}\right)+\sum_{n=\left|S_{0}\right|+1}^{\left|S_{0}\right|+\left|S_{1}\right|} a_{n} \dot{B}_{c}\left(\mathbf{k}-\mathbf{k}_{i}\right) \\
& \quad-\sum_{n=\left|S_{0}\right|+\left|S_{1}\right|+1}^{\left|S_{n}\right|+\left|S_{1}\right|+\left|S_{2}\right|} a_{n} \ddot{B}_{c}\left(\mathbf{k}-\mathbf{k}_{i}\right) .
\end{aligned}
$$




\subsection{Realizações em espaços de feixes (beamspace processing)}

Os beamformers desenvolvidos até este ponto podem operar sobre sinais $\mathbf{x}$ no domínio do tempo ou $\hat{\mathbf{x}}$ no domínio da frequência. Comparando as expressões (2.2.20) e (2.2.23), é fácil ver que beamformers banda estreita para $\mathbf{x}$ e $\hat{\mathbf{x}}$ têm estruturas equivalentes. Já beamformers banda larga podem ser implementados usando bancos de filtros FIR no domínio do tempo ou bancos de beamformers banda estreita.

Seja qual for a realização escolhida, o custo de processamento será na melhor das hipóteses proporcional a $N$, o número de elementos do array. O processamento no espaço de feixes é uma proposta para reduzir este custo através da redução da dimensão do espaço de entradas. Para isso, a entrada $\mathbf{x}$ ou $\hat{\mathbf{x}}$ é mapeada em um domínio onde as características espaciais do sinal são claramente identificáveis, permitindo que somente sinais vindos de certas regiões designadas a priori como importantes sejam processados. Este espaço tipicamente terá dimensão reduzida, tal que o esforço computacional será menor e o desempenho potencialmente será maior, desde que o sub-espaço desprezado tenha ruído ou interferências como componentes dominantes.

A estratégia de processamento em espaços transformados é bem estabelecida na literatura de codificação e filtragem, tal que muitos resultados obtidos em arrays são aplicações de uma teoria mais geral. Dirigimos o leitor a [11,12] para detalhes sobre codificação por transformadas.

Ilustremos o processamento em um espaço de feixes através de um exemplo. Como já vimos, a resposta de um ULA padrão com vetor de pesos uniforme $\mathbf{w}=\frac{1}{N} \mathbf{1}$ é dada por (2.4.7). Esta resposta pode ser dirigida para qualquer direção $u \in[-1,1]$, usando (2.5.4). Para um array com $N$ elementos, formamos um conjunto de $N$ feixes com eixos principais que amostram uniformemente o espaço $u$. A seguir, denotaremos por $\mathbf{w}(u)=$ $\frac{1}{N} \mathbf{v}_{u}(u)$ o vetor de pesos com padrão uniforme que aponta na direção $u$. Aplicando (2.5.6), concluímos que esses feixes têm respostas

$$
B_{i}(u)=\frac{1}{N} \frac{\sin \left(\frac{\pi N}{2}\left(u-u_{i}\right)\right)}{\sin \left(\frac{\pi}{2}\left(u-u_{i}\right)\right)},
$$

para $\left\{u_{i}\right\}_{i=0}^{N-1}$ escolhidos de forma a amostrar uniformemente e simetricamente o intervalo $[-1,1]$. Na Figura 3.2 estão plotados feixes da família $\mathbf{w}=\frac{1}{N} \mathbf{1}$ para o caso $N=11$. Esses feixes herdam propriedades da função sinc - em particular, o eixo principal de qualquer feixe coincide com os zeros de todos os outros, e suas respostas são ortogonais.

É fácil verificar que

$$
\mathbf{w}^{H}\left(u_{i}\right) \mathbf{w}\left(u_{j}\right)=\frac{1}{N} \delta_{i j} .
$$

A ortogonalidade dos feixes pode ser verificada diretamente sem recorrer à integração da 

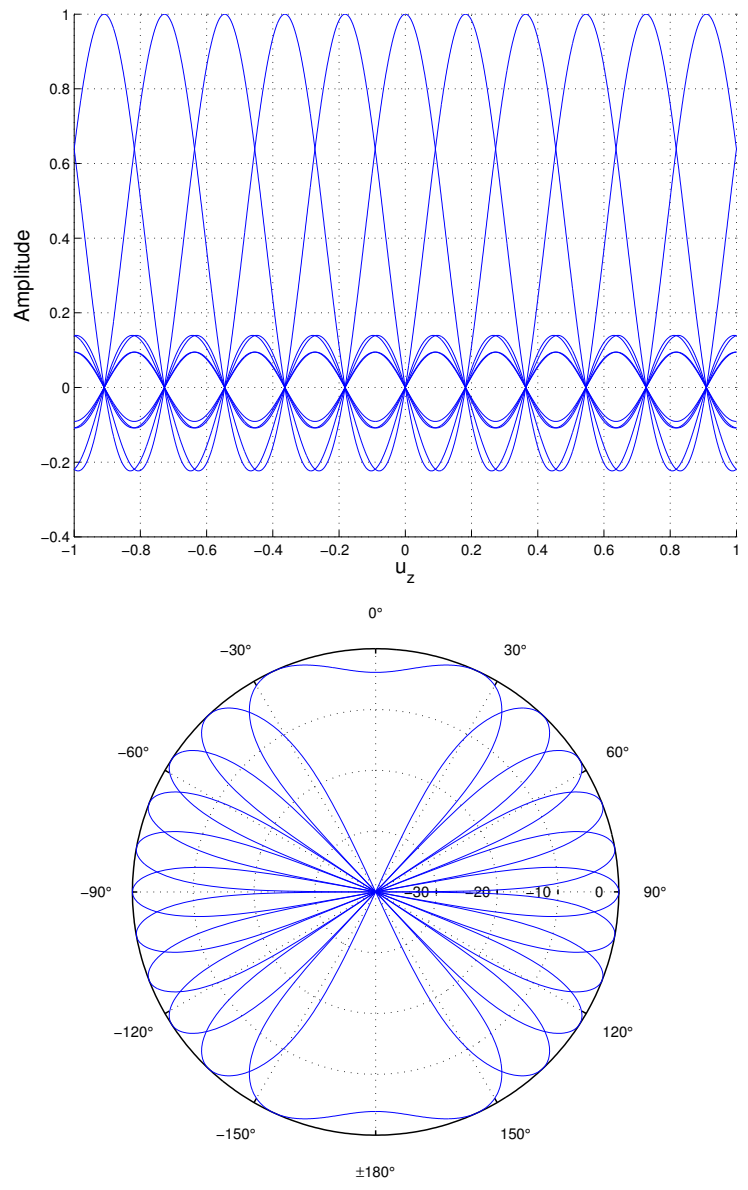

Figura 3.2: Base do espaço de feixes; $\mathbf{w}(u=0)=\frac{1}{N} \mathbf{1}, N=11, d=\lambda / 2$ 
função sinc, usando

$$
\begin{aligned}
\int_{-1}^{1} B_{i}(u) B_{j}^{*}(u) d u & =\mathbf{w}^{H}\left(u_{i}\right)\left[\int_{-1}^{1} \mathbf{v}(u) \mathbf{v}^{H}(u) d u\right] \mathbf{w}\left(u_{j}\right) \\
& =\mathbf{w}^{H}\left(u_{i}\right) \mathbf{I} \mathbf{w}\left(u_{j}\right) \\
& =\frac{1}{N} \delta_{i j}
\end{aligned}
$$

Devido à ortogonalidade dos vetores $\mathbf{w}\left(u_{i}\right)$, é natural que sejam interpretados como uma base de $\mathbb{C}^{\mathrm{n}}$. A transformação entre domínios é dada por

$$
\mathbf{x}_{b s}=\mathbf{B}_{b s}^{H} \mathbf{x}
$$

onde o índice bs indica beamspace, e a matriz de transformação $\mathbf{B}_{b s}^{H}$ tem $\mathbf{w}^{H}\left(u_{i}\right)$ em sua i-ésima linha. Da definição de $\mathbf{w}^{H}\left(u_{i}\right)$, verifica-se que $\mathbf{B}_{b s}^{H}$ é a matriz da DFT.

Evidentemente nosso objetivo é processar $\mathbf{x}_{b s}$ de forma a obter uma saída escalar. Para isso, definimos o vetor resposta no espaço dos feixes

$$
\mathbf{v}_{b s}(\psi)=\mathbf{B}_{b s}^{H} \mathbf{v}_{\psi}(\psi)
$$

Com $\mathbf{v}_{b s}(\psi)$ no lugar de $\mathbf{v}_{\psi}(\psi)$, é possível usar qualquer algoritmo já conhecido para projetar um processador $\mathbf{w}_{b s}$, cuja resposta será dada por

$$
\mathbf{B}_{\psi}(\psi)=\mathbf{w}_{b s}^{H} \mathbf{v}_{b s}(\psi)
$$

No caso geral, um espaço de feixes é um espaço vetorial cuja base é formada por vetores de pesos linearmente independentes, que automaticamente geram feixes linearmente independentes. Os vetores da base normalmente são derivados de um vetor cuja resposta tem máximo em $u=0$ ( $\mathbf{w}=\frac{1}{N} \mathbf{1}$, no exemplo), tal que suas respostas no espaço $u$ são versões deslocadas da resposta de $\mathbf{w}$.

Muito algoritmos exigem a ortogonalidade dos feixes. Dada uma matriz de transformação não ortogonal $\tilde{\mathbf{B}}^{H}$, podemos ortogonalizá-la usando $\mathbf{B}_{b s}=\tilde{\mathbf{B}}\left[\tilde{\mathbf{B}}^{H} \tilde{\mathbf{B}}\right]^{-1 / 2}$. Um efeito colateral será a modificação dos feixes originais, tal que propriedades como diretividade e altura de lóbulos laterais serão inevitavelmente alteradas.

Quando a dimensão do espaço de feixes é inferior a $N$, temos os chamados espaços de dimensões reduzidas. Nestes casos, os feixes estarão concentrados em somente algumas regiões de interesse. Qualquer interferência vinda de outras regiões será automaticamente atenuada, resultando em melhores relações sinal-ruído e sinal-interferência. Por outro lado, um erro na definição das regiões de cobertura pode causar perda irreversível do sinal de interesse. 


\subsection{Arrays não-uniformes, bidimensionais e tridimensionais}

A literatura contém numerosos exemplos de arranjos além do linear uniforme. Arrays não-uniformes têm interesse prático em cenários onde é impossível obter um arranjo regular devido à falta de controle sobre a disposição ou orientação dos sensores. Uma aplicação notável é a de boias oceânicas para aplicações militares e meteorológicas.

Uma vantagem de arrays não uniformes é a possibilidade de obter melhores resultados com menos elementos. Isso pode ser intuitivamente compreendido considerando a operação na ausência de ruído em um meio de propagação homogêneo e isotrópico. Neste caso, as características de uma onda plana (direção, amplitude, fase e frequência) podem ser estimadas por quaisquer dois elementos. De forma mais geral, consideremos a autocorrelação de um processo aleatório $f(t, \mathbf{p})$ temporalmente estacionário, espacialmente homogêneo e de média zero, dada por

$$
\begin{aligned}
K_{f}\left(t_{1}, t_{2}, \mathbf{p}_{1}, \mathbf{p}_{2}\right) & =\mathrm{E}\left\{f\left(t_{1}, \mathbf{p}_{1}\right) f^{*}\left(t_{2}, \mathbf{p}_{2}\right)\right\} \\
& =K_{f}\left(t_{1}-t_{2}, \mathbf{p}_{1}-\mathbf{p}_{2}\right) .
\end{aligned}
$$

Um array linear uniforme com $N$ elementos, espaçamento $d$ e orientação a oferece $N-n$ formas de calcular a autocorrelação espacial com $\mathbf{p}_{1}-\mathbf{p}_{2}=n d \mathbf{a}$, com $n \in\{1, \ldots, N-1\}$. Logo, na ausência de ruído, uma estratégia ótima de projeto buscaria posicionar os elementos de forma a eliminar redundâncias, tal que cada par de elementos fosse responsável por estimar uma autocorrelação única.

Veremos no Capítulo 7 que os métodos clássicos de filtragem e estimação espacial utilizam beamformers. Para problemas de imagens acústicas, as figuras de mérito mais relevantes são a largura do lóbulo principal e a atenuação na banda de rejeição. Para uma mesma largura de banda, arrays com maiores dimensões possuem lóbulos principais mais estreitos. Portanto, para um número de elementos fixo e mínima distância entre elementos fixa, temos que um array de mínima redundância sempre terá dimensões superiores a um array uniforme (e portanto, terá um lóbulo principal mais estreito). Por outro lado, um array uniforme produz máxima atenuação na banda de rejeição.

A Figura 3.3 mostra um exemplo comparando um ULA com $d=\lambda / 2$ e um array não-redundante com $N=9$ [13]. A menor distância entre pares de elementos é a mesma para ambos arrays. Portanto, eles têm a mesma frequência máxima de operação. Apesar do lóbulo principal do array não-redundante ser muito mais estreito, sua atenuação na banda de rejeição é de $\approx 10 \mathrm{~dB}$, contra uma atenuação máxima de quase $20 \mathrm{~dB}$ para o ULA.

Como também veremos no Capítulo 7, uma imagem acústica produzida por beamforming corresponde à distribuição de fontes que se deseja estimar, convoluída com o beampattern do array. Existe um compromisso claro entre resolução (dada pela largura do lóbulo principal) e atenuação na banda de rejeição (dada pela altura dos lóbulos se- 

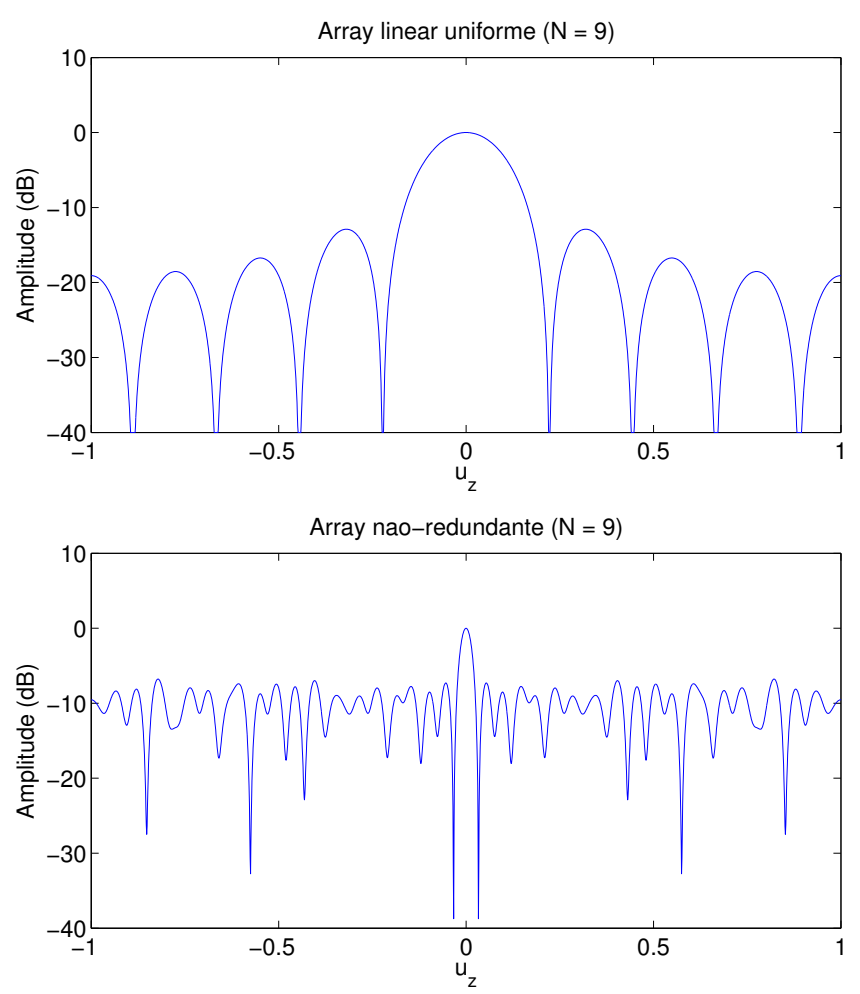

Figura 3.3: Respostas de um ULA com $d=\lambda / 2$ e de um array não-redundante [13], ambos com $N=9$

cundários). Na prática, arrays com grandes lóbulos laterais não são utilizados para beamforming, pois é preferível ter baixa resolução do que imagens contendo fontes fantasmas.

No entanto, fixada uma frequência máxima de operação, arrays com geometrias nãoredundantes permitem a amostragem de $K_{f}\left(t_{1}-t_{2}, \mathbf{p}_{1}-\mathbf{p}_{2}\right)$ para um número elevado de linhas de base $\mathbf{p}_{1}-\mathbf{p}_{2}$. Infelizmente, devido à presença de lóbulos laterais, o uso de beamformers não permite explorar essa diversidade e obter imagens de alta resolução. Isso nos motiva a buscar métodos alternativos para reconstrução de imagens, que possam compensar o efeito dos lóbulos laterais.

Finalmente, observamos que apesar de arranjos minimamente redundantes serem altamente desejáveis, geometrias bidimensionais com esta propriedade podem apresentar dificuldades mecânicas de produção. Além disso, algoritmos rápidos tipicamente exploram estruturas convenientes (por exemplo, matrizes unitárias e aritmética real) que são produzidas por regularidades na geometria dos arrays (por exemplo, invariâncias por isometrias). Portanto, a definição de uma geometria envolve múltiplos requisitos conflitantes.

Entre os arranjos simétricos, são de especial interesse os arrays retangulares uniformes, circulares uniformes e esféricos. Arrays retangulares uniformes são extensões naturais dos ULAs já vistos. Seus diagramas de radiação passam a ser parametrizados em duas variáveis, por exemplo $\psi_{x}$ e $\psi_{y}$ ou $u_{x}$ e $u_{y}$. Seus vetores de resposta podem ser escritos 
empilhando vetores de resposta correspondentes às linhas do array, tal que

$$
\mathbf{v}_{m}(\boldsymbol{\psi})=\left[\begin{array}{c}
e^{-j m \psi_{y}} \\
e^{-j\left(\psi_{x}+m \psi_{y}\right)} \\
\vdots \\
e^{-j\left((N-1) \psi_{x}+m \psi_{y}\right)}
\end{array}\right]
$$

corresponde ao vetor resposta da m-ésima linha do array retangular, e

$$
\mathbf{V}_{\psi}(\psi)=\left[\begin{array}{c}
\mathbf{v}_{0}(\psi) \\
\vdots \\
\mathbf{v}_{M-1}(\boldsymbol{\psi})
\end{array}\right]
$$

corresponde ao vetor resposta de um array com $N \times M$ elementos.

O projeto de respostas bidimensionais pode ser realizado usando pesos separáveis, tal que $w_{i j}=w_{i} \tilde{w}_{j}$ e $B\left(\psi_{x}, \psi_{y}\right)=B_{\psi_{x}}\left(\psi_{x}\right) \cdot B_{\psi_{y}}\left(\psi_{y}\right)$, onde $\left\{w_{i}\right\}_{i=0}^{N-1}$ e $\left\{\tilde{w}_{i}\right\}_{i=0}^{M-1}$ são pesos de ULAs com respectivas respostas $B_{\psi_{x}}(\psi)$ e $B_{\psi_{y}}(\psi)$. Transformadas de Fourier e transformadas $\mathrm{Z}$ devem ser tratadas de forma bidimensional, e projetos de filtros específicos devem ser adaptados (por exemplo, filtros Chebyshev devem usar polinômios de Chebyshev bidimensionais). Com as devidas adaptações, o projeto de arrays retangulares uniformes se reduz ao projeto de ULAs.

Arrays circulares são especialmente importantes por permitirem uma cobertura de um ângulo de $360^{\circ}$ sem perda de resolução, ao passo que arrays lineares apresentam resolução ótima para sinais com direção de propagação perpendicular ao array (broadside) e resolução pior para sinais com propagação paralela ao array (endfire). Arrays esféricos possuem a mesma capacidade de resolução para qualquer ângulo de chegada, além de não apresentarem regiões de ambiguidade. Apesar da importância de arrays circulares e esféricos, não trataremos de suas técnicas de projeto, por serem altamente específicas e pouco generalizáveis. 


\section{Capítulo 4}

\section{Processos aleatórios temporais-espaciais}

\subsection{Introdução}

Até este ponto realizamos projetos sob condições determinísticas, onde o objetivo era obter uma aproximação para uma resposta em frequência-número de onda especificada $a$ priori. Esta metodologia é razoavelmente simples e demonstra-se robusta, desde que as direções de chegada do sinal de interesse e de possíveis interferências sejam conhecidas e que os sinais a serem recebidos ou cancelados estejam espacialmente concentrados. No entanto, nada garante a optimalidade desses métodos. Pudemos mostrar que no caso particular de ruído espacialmente branco, a expressão do ganho (2.7.12) atinge seu máximo quando $\mathbf{w}=\frac{1}{N} \mathbf{1}$, o que apesar de interessante é um resultado de escopo extremamente limitado. A partir da descrição de processos aleatórios em tempo-espaço e frequêncianúmero de onda, teremos uma linguagem para expressar condições de optimalidade em cenários muito mais gerais, e assim sintetizar arrays rigorosamente ótimos.

\subsection{Representação em frequência}

Na Seção 2.3, mencionamos que um beamformer banda-estreita no domínio da frequência opera sobre $\hat{\mathbf{x}}\left(\omega_{m}\right)$, equivalente à m-ésima raia de uma transformada de Fourier de tempo curto do sinal de entrada $\mathbf{x}(t)$. Dado um sinal em banda base com potência na faixa $[-\pi B,+\pi B]$, procedemos como Hodgkiss e Nolte em [1] e definimos

$$
\hat{\mathbf{x}}_{\Delta T}\left(\omega_{m}, k\right)=\frac{1}{\sqrt{\Delta T}} \int_{0}^{\Delta T} \mathbf{x}(t+k \Delta T) e^{-j m \omega_{0} t} d t
$$

onde

$$
\omega_{0}=\frac{2 \pi}{\Delta T}
$$

e $k \in \mathbb{Z}_{+}$representa o bloco (snapshot) de comprimento $\Delta T$ usado para estimar esta transformada. Frequentemente omitiremos a variável $k$ para simplificar a notação.

Para um caso mais geral com potência concentrada no intervalo $\left[\omega_{c}-\pi B, \omega_{c}+\pi B\right]$, usamos

$$
\hat{\mathbf{x}}_{\Delta T}\left(\omega_{m}, k\right)=\frac{1}{\sqrt{\Delta T}} \int_{0}^{\Delta T} \mathbf{x}(t+k \Delta T) e^{-j\left(\omega_{c}+m \omega_{\Delta}\right) t}
$$


onde

$$
\omega_{\Delta}=\frac{2 \pi}{\Delta T} .
$$

Estamos efetivamente amostrando o espectro de $\mathbf{x}$ uniformemente, tal que a quantidade de raias $\omega_{m}$ depende do intervalo de observação $\Delta T$ escolhido e da largura de faixa $B$ do processo. Usamos $m \in\{-(M-1) / 2, \ldots,(M-1) / 2\}$, com

$$
M=\lfloor B \cdot \Delta T\rfloor+1
$$

e sob a imposição de $M$ ímpar, para que a amostragem em frequência seja simétrica. Para que as definições acima tenham sentido físico, exigimos que $\Delta T \gg \Delta T_{\max }$, onde $\Delta T_{\max }$ está definido em (2.3.2) como o máximo tempo de propagação entre elementos do array.

Definimos a correlação de $\hat{\mathbf{x}}_{\Delta T}\left(\omega_{m}\right)$ e $\hat{\mathbf{x}}_{\Delta T}\left(\omega_{n}\right)$ como

$$
\mathbf{S}_{\mathbf{x}, \Delta T}(m, n)=\mathrm{E}\left\{\hat{\mathbf{x}}_{\Delta T}\left(\omega_{m}\right) \hat{\mathbf{x}}_{\Delta T}^{H}\left(\omega_{n}\right)\right\}
$$

De acordo com o teorema de Wiener-Khinchin [14],

$$
\lim _{\Delta T \rightarrow \infty}\left[\mathbf{S}_{\mathbf{x}, \Delta T}(m, m)\right]_{n n}=\left[\mathbf{S}_{\mathbf{x}}\left(\omega_{c}+m \omega_{\Delta}\right)\right]_{n n}
$$

onde $\mathbf{S}_{\mathbf{x}}(\omega)=\mathcal{F}\left\{\mathbf{R}_{\mathbf{x}}(\tau)\right\}$ é a matriz densidade espectral de potência do processo. O desenvolvimento de [1] mostra que um resultado similar também vale para termos fora da diagonal, tal que

$$
\lim _{\Delta T \rightarrow \infty} \mathbf{S}_{\mathbf{x}, \Delta T}(m, m)=\mathbf{S}_{\mathbf{x}}\left(\omega_{c}+m \omega_{\Delta}\right) .
$$

Logo, $\mathbf{S}_{\mathbf{x}, \Delta T}$ pode ser usada como aproximação arbitrariamente precisa de $\mathbf{S}_{\mathbf{x}}$, mediante uma escolha adequada do produto $B \cdot \Delta T$. Esta escolha deve tornar $\mathbf{S}_{\mathbf{x}}(\omega)$ aproximadamente constante em um intervalo de $\pm 2 \omega_{\Delta}$ ou $\pm 3 \omega_{\Delta}$ ao redor de $\omega_{c}+m \omega_{\Delta}$. (Na prática, valores de $B \cdot \Delta T$ entre 16 e 512 são típicos.) Para este caso, Hodgkiss e Nolte também mostraram que para $m \neq n$,

$$
\mathbf{S}_{\mathbf{x}, \Delta T}(m, n)=\mathbf{0},
$$

e para $k \neq l$,

$$
\mathrm{E}\left\{\hat{\mathbf{x}}_{\Delta T}\left(\omega_{m}, k\right) \hat{\mathbf{x}}_{\Delta T}^{H}\left(\omega_{n}, l\right)\right\}=\mathbf{0}
$$

Portanto, se cada bloco for suficientemente longo para garantir $\mathbf{S}_{\mathbf{x}}(\omega)$ aproximadamente constante na vizinhança de cada tap $\omega_{m}$, os snapshots para diferentes frequências e diferentes blocos serão descorrelacionados com boa aproximação. Se $\mathbf{x}(t)$ for modelado como um processo Gaussiano, snapshots para diferentes frequências e blocos serão variáveis aleatórias conjuntamente Gaussianas, tal que poderão ser consideradas estatisticamente independentes, e processados isoladamente sem perda de optimalidade. 


\subsection{Vetores aleatórios Gaussianos}

Praticamente toda a literatura de array processing recorre a processos aleatórios Gaussianos para modelar sinais, interferências e ruído. Nosso tratamento não é exceção, e supomos que $\mathbf{x}(t)$, o sinal medido pelo array, representa um processo estocástico Gaussiano real. Como $\hat{\mathbf{x}}_{\Delta T}\left(\omega_{m}\right)$ é um funcional linear de $\mathbf{x}(t)$, suas amostras são variáveis aleatórias conjuntamente Gaussianas. Se obedecidas as hipóteses descritas na seção anterior sobre a escolha de $\Delta T$, verifica-se com boa aproximação que

$$
\mathrm{E}\left\{\hat{\mathbf{x}}_{\Delta T}\left(\omega_{m}, k\right) \hat{\mathbf{x}}_{\Delta T}^{T}\left(\omega_{m}, k\right)\right\}=\mathbf{0}
$$

Como (4.3.1) equivale às condições

$$
\mathrm{E}\left\{\operatorname{Re}\left\{\hat{\mathbf{x}}_{\Delta T}\left(\omega_{m}, k\right)\right\}^{2}\right\}=\mathrm{E}\left\{\operatorname{Im}\left\{\hat{\mathbf{x}}_{\Delta T}\left(\omega_{m}, k\right)\right\}^{2}\right\}
$$

$\mathrm{e}$

$$
\mathrm{E}\left\{\operatorname{Re}\left\{\hat{\mathbf{x}}_{\Delta T}\left(\omega_{m}, k\right)\right\} \operatorname{Im}\left\{\hat{\mathbf{x}}_{\Delta T}\left(\omega_{m}, k\right)\right\}^{T}\right\}=\mathbf{0},
$$

$\hat{\mathbf{x}}_{\Delta T}\left(\omega_{m}\right)$ é por definição um vetor de variáveis aleatórias circulares complexas $[15,16]$.

Sejam

$$
\begin{aligned}
\mathbf{m}_{\Delta T}\left(\omega_{m}, k\right) & =\mathrm{E}\left\{\hat{\mathbf{x}}_{\Delta T}\left(\omega_{m}, k\right)\right\} \\
\mathbf{S}_{\mathbf{x}, \Delta T}\left(\omega_{m}, k\right) & =\mathrm{E}\left\{\hat{\mathbf{x}}_{\Delta T}\left(\omega_{m}, k\right) \hat{\mathbf{x}}_{\Delta T}^{H}\left(\omega_{n}, k\right)\right\} \\
\mathbf{K}_{\mathbf{x}, \Delta T}\left(\omega_{m}, k\right) & =\mathrm{E}\left\{\left[\hat{\mathbf{x}}_{\Delta T}\left(\omega_{m}, k\right)-\mathbf{m}_{\Delta T}\left(\omega_{m}, k\right)\right]\left[\hat{\mathbf{x}}_{\Delta T}^{H}\left(\omega_{n}, k\right)-\mathbf{m}_{\Delta T}^{H}\left(\omega_{m}, k\right)\right]\right\}
\end{aligned}
$$

a média, correlação e covariância do vetor aleatório $\hat{\mathbf{x}}_{\Delta T}\left(\omega_{m}, k\right)$. Sua distribuição para os casos de média nula e média não-nula é dada por

$$
\begin{aligned}
& p_{\hat{\mathbf{x}}, \Delta T}\left(\hat{\mathbf{x}}_{\Delta T}\right)=\frac{1}{\pi^{N}\left|\mathbf{S}_{\mathbf{x}, \Delta T}\right|} \exp \left\{-\hat{\mathbf{x}}_{\Delta T}^{H} \mathbf{S}_{\mathbf{x}, \Delta T}^{-1} \hat{\mathbf{x}}_{\Delta T}\right\} \\
& p_{\hat{\mathbf{x}}, \Delta T}\left(\hat{\mathbf{x}}_{\Delta T}\right)=\frac{1}{\pi^{N}\left|\mathbf{K}_{\mathbf{x}, \Delta T}\right|} \exp \left\{-\left[\hat{\mathbf{x}}_{\Delta T}-\mathbf{m}_{\Delta T}\right]^{H} \mathbf{K}_{\mathbf{x}, \Delta T}^{-1}\left[\hat{\mathbf{x}}_{\Delta T}-\mathbf{m}_{\Delta T}\right]\right\},
\end{aligned}
$$

onde as variáveis $\omega_{m}$ e $k$ foram omitidas para simplificar a notação. Nos desenvolvimentos que seguem, consideraremos que $B \cdot \Delta T$ foi adequadamente escolhido, tal que todas as aproximações descritas anteriormente são válidas. Por isso, omitiremos o índice $\Delta T$ das variáveis e funções estimadas através da expressão (4.2.3). Para aliviar a notação, omitiremos sempre o indexador de bloco $k$ e ocasionalmente a frequência de operação $\omega_{m}$. 


\subsection{Modelos de ondas planas}

\subsubsection{Caso 1: um único sinal determinístico}

Suponhamos que a saída do array seja dada por

$$
\begin{aligned}
\mathbf{x}(t) & =\mathbf{x}_{s}(t)+\mathbf{n}(t) \\
\hat{\mathbf{x}}\left(\omega_{m}\right) & =\hat{\mathbf{x}}_{s}\left(\omega_{m}\right)+\hat{\mathbf{n}}\left(\omega_{m}\right),
\end{aligned}
$$

onde $\mathbf{x}_{s}(t)$ representa um sinal determinístico desejado, e $\mathbf{n}(t)$ representa ruído, modelado como um processo aleatório Gaussiano. Admitindo que o sinal foi gerado por uma fonte com espectro $\hat{f}_{s}\left(\omega_{m}\right)$ e que seu campo é descrito por um modelo de onda plana com propagação na direção $\mathbf{k}_{s}$, temos

$$
\begin{aligned}
\hat{\mathbf{x}}_{s}\left(\omega_{m}\right) & =\mathbf{v}\left(\omega_{m}, \mathbf{k}_{s}\right) \hat{f}_{s}\left(\omega_{m}\right) \\
p_{\hat{\mathbf{x}}}(\hat{\mathbf{x}}) & =\frac{1}{\pi^{N}\left|\mathbf{S}_{n}\right|} \exp \left\{-\left[\hat{\mathbf{x}}-\mathbf{v}\left(\mathbf{k}_{s}\right) \hat{f}_{s}\right]^{H} \mathbf{S}_{n}^{-1}\left[\hat{\mathbf{x}}-\mathbf{v}\left(\mathbf{k}_{s}\right) \hat{f}_{s}\right]\right\}
\end{aligned}
$$

onde $\mathbf{S}_{\mathbf{n}}\left(\omega_{m}\right)$ é a matriz da densidade espectral de potência de $\mathbf{n}(t)$.

\subsubsection{Caso 2: um sinal desejado, $M$ interferências, todos determinísticos}

Neste caso,

$$
\hat{\mathbf{x}}\left(\omega_{m}\right)=\mathbf{v}\left(\omega_{m}, \mathbf{k}_{s}\right) \hat{f}_{s}\left(\omega_{m}\right)+\sum_{i=1}^{M} \mathbf{v}\left(\omega_{m}, \mathbf{k}_{i}\right) \hat{f}_{i}\left(\omega_{m}\right)+\hat{\mathbf{n}}\left(\omega_{m}\right)
$$

Definimos uma matriz de respostas $\mathbf{V}\left(\omega_{m}, \mathbf{k}\right)$ e um vetor de fontes $\hat{\mathbf{f}}\left(\omega_{m}\right)$ como

$$
\begin{aligned}
\mathbf{V}\left(\omega_{m}, \mathbf{k}\right) & =\left[\begin{array}{llll}
\mathbf{v}\left(\omega_{m}, \mathbf{k}_{s}\right) & \mathbf{v}\left(\omega_{m}, \mathbf{k}_{1}\right) & \cdots & \mathbf{v}\left(\omega_{m}, \mathbf{k}_{M}\right)
\end{array}\right] \\
\hat{\mathbf{f}}\left(\omega_{m}\right) & =\left[\begin{array}{llll}
\hat{f}_{s}\left(\omega_{m}\right) & \hat{f}_{1}\left(\omega_{m}\right) & \cdots & \hat{f}_{M}\left(\omega_{m}\right)
\end{array}\right]^{T},
\end{aligned}
$$

que nos permite escrever

$$
\begin{aligned}
\hat{\mathbf{x}}\left(\omega_{m}\right) & =\mathbf{V}\left(\omega_{m}, \mathbf{k}\right) \hat{\mathbf{f}}\left(\omega_{m}\right)+\hat{\mathbf{n}}\left(\omega_{m}\right) \\
p_{\hat{\mathbf{x}}}(\hat{\mathbf{x}}) & =\frac{1}{\pi^{N}\left|\mathbf{S}_{n}\right|} \exp \left\{-[\hat{\mathbf{x}}-\mathbf{V} \hat{\mathbf{f}}]^{H} \mathbf{S}_{n}^{-1}[\hat{\mathbf{x}}-\mathbf{V} \hat{\mathbf{f}}]\right\} .
\end{aligned}
$$

\subsubsection{Caso 3: um sinal desejado determinístico, $M$ interferências Gaussianas}

Assim como no caso anterior,

$$
\hat{\mathbf{x}}\left(\omega_{m}\right)=\mathbf{v}\left(\omega_{m}, \mathbf{k}_{s}\right) \hat{f}_{s}\left(\omega_{m}\right)+\sum_{i=1}^{M} \mathbf{v}\left(\omega_{m}, \mathbf{k}_{i}\right) \hat{f}_{i}\left(\omega_{m}\right)+\hat{\mathbf{n}}\left(\omega_{m}\right)
$$


Definimos uma matriz de respostas $\mathbf{V}_{I}\left(\omega_{m}, \mathbf{k}\right)$ e um vetor de fontes $\hat{\mathbf{f}}_{I}\left(\omega_{m}\right)$ para as interferências como

$$
\begin{aligned}
\mathbf{V}_{I}\left(\omega_{m}, \mathbf{k}\right) & =\left[\begin{array}{llll}
\mathbf{v}\left(\omega_{m}, \mathbf{k}_{1}\right) & \cdots & \mathbf{v}\left(\omega_{m}, \mathbf{k}_{M}\right)
\end{array}\right] \\
\hat{\mathbf{f}}\left(\omega_{m}\right) & =\left[\begin{array}{llll}
\hat{f}_{1}\left(\omega_{m}\right) & \cdots & \hat{f}_{M}\left(\omega_{m}\right)
\end{array}\right]^{T} .
\end{aligned}
$$

Podemos escrever a matriz espectral das fontes interferentes como

$$
\mathbf{S}_{f}=\mathrm{E}\left\{\hat{\mathbf{f}} \hat{\mathbf{f}}^{H}\right\}
$$

Devido à independência de $\hat{f}_{i}\left(\omega_{m}\right)$ e $\hat{n}\left(\omega_{i}\right)$, a matriz espectral das interferências e ruído é dada por

$$
\mathbf{S}_{I+N}=\mathbf{V}_{I} \mathbf{S}_{f} \mathbf{V}_{I}^{H}+\mathbf{S}_{n}
$$

Finalmente,

$$
p_{\hat{\mathbf{x}}}(\hat{\mathbf{x}})=\frac{1}{\pi^{N}\left|\mathbf{S}_{I+N}\right|} \exp \left\{-\left[\hat{\mathbf{x}}-\mathbf{v}(\mathbf{k}) \hat{f}_{s}\right]^{H} \mathbf{S}_{I+N}^{-1}\left[\hat{\mathbf{x}}-\mathbf{v}(\mathbf{k}) \hat{f}_{s}\right]\right\}
$$

\subsubsection{Caso 4: um sinal desejado, $M$ interferências, todos Gaussianos}

Novamente,

$$
\hat{\mathbf{x}}\left(\omega_{m}\right)=\mathbf{v}\left(\omega_{m}, \mathbf{k}_{s}\right) \hat{f}_{s}\left(\omega_{m}\right)+\sum_{i=1}^{M} \mathbf{v}\left(\omega_{m}, \mathbf{k}_{i}\right) \hat{f}_{i}\left(\omega_{m}\right)+\hat{\mathbf{n}}\left(\omega_{m}\right)
$$

Assim como no caso 2,

$$
\begin{aligned}
\mathbf{V}\left(\omega_{m}, \mathbf{k}\right) & =\left[\begin{array}{llll}
\mathbf{v}\left(\omega_{m}, \mathbf{k}_{s}\right) & \mathbf{v}\left(\omega_{m}, \mathbf{k}_{1}\right) & \cdots & \mathbf{v}\left(\omega_{m}, \mathbf{k}_{M}\right)
\end{array}\right] \\
\hat{\mathbf{f}}\left(\omega_{m}\right) & =\left[\begin{array}{llll}
\hat{f}_{s}\left(\omega_{m}\right) & \hat{f}_{1}\left(\omega_{m}\right) & \cdots & \hat{f}_{M}\left(\omega_{m}\right)
\end{array}\right]^{T},
\end{aligned}
$$

tal que

$$
\hat{\mathbf{x}}\left(\omega_{m}\right)=\mathbf{V}\left(\omega_{m}, \mathbf{k}\right) \hat{\mathbf{f}}\left(\omega_{m}\right)+\hat{\mathbf{n}}\left(\omega_{m}\right) .
$$

A matriz espectral da fonte desejada e das fontes interferentes é

$$
\mathbf{S}_{f}=\mathrm{E}\left\{\hat{\mathbf{f}} \hat{\mathbf{f}}^{H}\right\}
$$

A matriz espectral de todos os sinais é

$$
\mathbf{S}_{S+I+N}=\mathbf{V} \mathbf{S}_{f} \mathbf{V}^{H}+\mathbf{S}_{n} .
$$


E a função densidade de probabilidade é

$$
p_{\hat{\mathbf{x}}}(\hat{\mathbf{x}})=\frac{1}{\pi^{N}\left|\mathbf{S}_{S+I+N}\right|} \exp \left\{-\hat{\mathbf{x}}^{H} \mathbf{S}_{S+I+N}^{-1} \hat{\mathbf{x}}\right\}
$$

\subsection{Representação de processos em frequência-número de onda}

Consideraremos a seguir um processo aleatório escalar e complexo $f(t, \mathbf{p})$ definido sobre o espaço e o tempo. No caso geral, $t \in \mathbb{R}$ e $\mathbf{p} \in \mathbb{R}^{3}$. Definimos sua média $m_{f}(t, \mathbf{p})$ e correlação temporal-espacial $K_{f}\left(t_{1}, t_{2}, \mathbf{p}_{1}, \mathbf{p}_{2}\right)$ como

$$
\begin{aligned}
m_{f}(t, \mathbf{p}) & =\mathrm{E}\{f(t, \mathbf{p})\} \\
K_{f}\left(t_{1}, t_{2}, \mathbf{p}_{1}, \mathbf{p}_{2}\right) & =\mathrm{E}\left\{\left[f\left(t_{1}, \mathbf{p}_{1}\right)-m_{f}\left(t_{1}, \mathbf{p}_{1}\right)\right]\left[f^{*}\left(t_{2}, \mathbf{p}_{2}\right)-m_{f}^{*}\left(t_{2}, \mathbf{p}_{2}\right)\right]\right\}
\end{aligned}
$$

Admitiremos que $f(t, \mathbf{p})$ é estacionário no tempo e homogêneo no espaço, tal que

$$
\begin{aligned}
K_{f}\left(t_{1}, t_{2}, \mathbf{p}_{1}, \mathbf{p}_{2}\right) & =K_{f}\left(t_{1}-t_{2}, \mathbf{p}_{1}-\mathbf{p}_{2}\right) \\
& =K_{f}(\tau, \Delta \mathbf{p})
\end{aligned}
$$

Usando a transformada de Fourier, podemos calcular funções transformadas em relação a $\tau$ e $\Delta \mathbf{p}$, dando origem a funções nas variáveis conjugadas $\omega$ e $\mathbf{k}$.

A seguir definimos as funções espectro em frequência-correlação espacial $S_{f}(\omega, \Delta \mathbf{p})$, correlação temporal-espectro em número de onda $F_{f}(\tau, \mathbf{k})$ e espectro em frequência-número de onda $P_{f}(\omega, \mathbf{k})$ :

$$
\begin{aligned}
S_{f}(\omega, \Delta \mathbf{p}) & =\int_{-\infty}^{+\infty} K_{f}(\tau, \Delta \mathbf{p}) e^{-j \omega \tau} d \tau \\
F_{f}(\tau, \mathbf{k}) & =\int_{\mathbb{R}^{3}} K_{f}(\tau, \Delta \mathbf{p}) e^{+j \mathbf{k}^{T} \Delta \mathbf{p}} d \Delta \mathbf{p} \\
P_{f}(\omega, \mathbf{k}) & =\int_{\mathbb{R}^{3}} S_{f}(\omega, \Delta \mathbf{p}) e^{+j \mathbf{k}^{T} \Delta \mathbf{p}} d \Delta \mathbf{p} .
\end{aligned}
$$

Suas inversas são respectivamente

$$
\begin{aligned}
K_{f}(\tau, \Delta \mathbf{p}) & =\frac{1}{2 \pi} \int_{-\infty}^{+\infty} S_{f}(\omega, \Delta \mathbf{p}) e^{+j \omega \tau} d \omega \\
K_{f}(\tau, \Delta \mathbf{p}) & =\frac{1}{(2 \pi)^{3}} \int_{\mathbb{R}^{3}} F_{f}(\tau, \mathbf{k}) e^{-j \mathbf{k}^{T} \Delta \mathbf{p}} d \mathbf{k} \\
S_{f}(\omega, \Delta \mathbf{p}) & =\frac{1}{(2 \pi)^{3}} \int_{\mathbb{R}^{3}} P_{f}(\omega, \mathbf{k}) e^{-j \mathbf{k}^{T} \Delta \mathbf{p}} d \mathbf{k} .
\end{aligned}
$$

A equação de onda (2.1.2) impõe a restrição (2.1.4) sobre valores admissíveis de $\mathbf{k}$. Isso implica que o espectro em frequência-número de onda $P_{f}\left(\omega_{0}, \mathbf{k}\right)$ de um campo propagante sobre um meio homogêneo será não-nulo apenas sobre a esfera $k_{x}^{2}+k_{y}^{2}+k_{z}^{2}=\frac{\omega_{0}^{2}}{c^{2}}$. Esta 
consideração nos permite representar qualquer número de onda tridimensional através de sua projeção sobre um plano qualquer, com apenas uma possível ambiguidade de sinal.

Para arrays com geometrias que não oferecem resolução em certas direções, podemos representar $\mathbf{k}$ através de sua projeção sobre um único eixo de um sistema de coordenadas adequadamente escolhido. Para esses casos de dimensão reduzida, temos os seguintes espectros em frequência-número de onda:

$$
\begin{aligned}
P_{2 f}\left(\omega, k_{x}, k_{y}\right) & =\frac{1}{2 \pi} \int_{-\infty}^{+\infty} P_{f}\left(\omega, k_{x}, k_{y}, k_{z}\right) d k_{z} \\
P_{1 f}\left(\omega, k_{x}\right) & =\frac{1}{2 \pi} \int_{-\infty}^{+\infty} P_{2 f}\left(\omega, k_{x}, k_{y}\right) d k_{y} .
\end{aligned}
$$

As respostas em frequência-correlação espacial projetadas são dadas por

$$
\begin{aligned}
S_{2 f}\left(\omega, \Delta p_{x}, \Delta p_{y}\right) & =\frac{1}{(2 \pi)^{2}} \int_{-\infty}^{+\infty} \int_{-\infty}^{+\infty} P_{2 f}\left(\omega, k_{x}, k_{y}\right) e^{-j k_{x} \Delta p_{x}} e^{-j k_{y} \Delta p_{y}} d k_{x} d k_{y} \\
S_{1 f}\left(\omega, \Delta p_{x}\right) & =\frac{1}{2 \pi} \int_{-\infty}^{+\infty} P_{1 f}\left(\omega, k_{x}\right) e^{-j k_{x} \Delta p_{x}} d k_{x}
\end{aligned}
$$

Substituindo (4.5.10) em (4.5.12), obtemos a relação

$$
\begin{aligned}
S_{2 f}\left(\omega, \Delta p_{x}, \Delta p_{y}\right) & =\frac{1}{(2 \pi)^{3}} \int_{-\infty}^{+\infty} \int_{-\infty}^{+\infty} \int_{-\infty}^{+\infty} P_{f}\left(\omega, k_{x}, k_{y}, k_{z}\right) e^{-j\left(k_{x} \Delta p_{x}+k_{y} \Delta p_{y}\right)} d k_{x} d k_{y} d k_{z} \\
& =S_{f}\left(\omega, \Delta p_{x}, \Delta p_{y}, 0\right)
\end{aligned}
$$

Analogamente,

$$
S_{1 f}\left(\omega, \Delta p_{x}\right)=S_{f}\left(\omega, \Delta p_{x}, 0,0\right)
$$

A seguir demonstramos uma generalização do teorema de Wiener-Khinchin para processos aleatórios espaciais-temporais. Resultados análogos são apresentados para processos unidimensionais em [14]. Seja

$$
F(\omega, \mathbf{k})=\int_{\mathbb{R}^{3}} \int_{-\infty}^{+\infty} f(t, \mathbf{p}) e^{-j \omega t} e^{+j \mathbf{k}^{T} \mathbf{p}} d t d \mathbf{p}
$$

a transformada para o domínio frequência-número de onda do processo $f(t, \mathbf{p})$. Usando $\tau=t_{1}-t_{2}, \Delta \mathbf{p}=\mathbf{p}_{1}-\mathbf{p}_{2}$, a identidade $\int_{-\infty}^{+\infty} e^{-j \omega t} d t=2 \pi \delta(\omega)$ e a definição $\delta(\mathbf{k})=$ $\delta\left(k_{x}\right) \delta\left(k_{y}\right) \delta\left(k_{z}\right)$ 


$$
\begin{aligned}
\mathrm{E}\{F & \left.\left(\omega_{1}, \mathbf{k}_{1}\right) F^{*}\left(\omega_{2}, \mathbf{k}_{2}\right)\right\}= \\
& =\iiint \int \mathrm{E}\left\{f\left(t_{1}, \mathbf{p}_{1}\right) f^{*}\left(t_{2}, \mathbf{p}_{2}\right)\right\} e^{-j \omega_{1} t_{1}} e^{+j \omega_{2} t_{2}} e^{+j \mathbf{k}_{1}^{T} \mathbf{p}_{1}} e^{-j \mathbf{k}_{2}^{T} \mathbf{p}_{2}} d t_{1} d t_{2} d \mathbf{p}_{1} d \mathbf{p}_{2} \\
& =\iiint \int K_{f}\left(t_{1}-t_{2}, \mathbf{p}_{1}-\mathbf{p}_{2}\right) e^{-j\left(\omega_{1} t_{1}-\omega_{2} t_{2}\right)} e^{+j\left(\mathbf{k}_{1}^{T} \mathbf{p}_{1}-\mathbf{k}_{2}^{T} \mathbf{p}_{2}\right)} d t_{1} d t_{2} d \mathbf{p}_{1} d \mathbf{p}_{2} \\
& =\iiint \int K_{f}(\tau, \Delta \mathbf{p}) e^{-j \omega_{1} \tau} e^{-j \mathbf{k}_{1}^{T} \Delta \mathbf{p}} e^{-j\left(\omega_{1}-\omega_{2}\right) t_{2}} e^{+j\left(\mathbf{k}_{1}^{T}-\mathbf{k}_{2}^{T}\right) \mathbf{p}_{2}} d \tau d t_{2} d \Delta \mathbf{p} d \mathbf{p}_{2} \\
& =(2 \pi)^{4} P_{f}(\omega, \mathbf{k}) \delta\left(\omega_{1}-\omega_{2}\right) \delta\left(\mathbf{k}_{1}-\mathbf{k}_{2}\right) .
\end{aligned}
$$

Definimos o espectro integrado de $f(t, \mathbf{p})$ em relação a $\omega$ e $\mathbf{k}$ como

$$
Z_{\omega, \mathbf{k}}(\omega, \mathbf{k})=\int_{-\infty}^{\mathbf{k}} \int_{-\infty}^{\omega} F(\alpha, \boldsymbol{\beta}) d \alpha d \boldsymbol{\beta}
$$

onde a integral em relação a $\mathbf{k}$ é avaliada no retângulo aberto $\left(-\infty, k_{x}\right) \times\left(-\infty, k_{y}\right) \times$ $\left(-\infty, k_{z}\right)$. O uso de espectros integrados torna desnecessário o uso de funções generalizadas para representar singularidades. De fato, se $F(\omega, \mathbf{k})$ contiver impulsos da forma $\delta\left(\omega-\omega_{i}\right)$ ou $\delta\left(\mathbf{k}-\mathbf{k}_{j}\right)$, então $Z_{\omega, \mathbf{k}}(\omega, \mathbf{k})$ torna-se simplesmente descontínua em $\omega_{i}$ ou $\mathbf{k}_{j}$.

Usando (4.5.17) e (4.5.18), resulta que $\forall \omega_{1}, \omega_{2}, \mathbf{k}_{1}, \mathbf{k}_{2}$,

$$
\mathrm{E}\left\{\left|Z_{\omega, \mathbf{k}}\left(\omega_{1}, \mathbf{k}_{1}\right)-Z_{\omega, \mathbf{k}}\left(\omega_{2}, \mathbf{k}_{2}\right)\right|^{2}\right\}=(2 \pi)^{4} \int_{\mathbf{k}_{1}}^{\mathbf{k}_{2}} \int_{\omega_{1}}^{\omega_{2}} P_{f}(\omega, \mathbf{k}) d \omega d \mathbf{k} .
$$

De forma similar verificamos que para $\left(\omega_{1}, \omega_{2}\right) \times\left(\mathbf{k}_{1}, \mathbf{k}_{2}\right) \cap\left(\omega_{3}, \omega_{4}\right) \times\left(\mathbf{k}_{3}, \mathbf{k}_{4}\right)=\emptyset$,

$$
\mathrm{E}\left\{\left[Z_{\omega, \mathbf{k}}\left(\omega_{1}, \mathbf{k}_{1}\right)-Z_{\omega, \mathbf{k}}\left(\omega_{2}, \mathbf{k}_{2}\right)\right]\left[Z_{\omega, \mathbf{k}}\left(\omega_{3}, \mathbf{k}_{3}\right)-Z_{\omega, \mathbf{k}}\left(\omega_{4}, \mathbf{k}_{4}\right)\right]\right\}=0
$$

ou seja, o espectro em frequência-número de onda sobre retângulos disjuntos é descorrelacionado. Isso nos permitirá representar processos estacionários no tempo e homogêneos no espaço como a superposição de ondas planas descorrelacionadas.

Substituindo

$$
\begin{array}{llll}
\omega_{1}=\widetilde{\omega}, & \omega_{2}=\widetilde{\omega}+d \omega, & \omega_{3}=\widetilde{\widetilde{\omega}}, & \omega_{4}=\widetilde{\widetilde{\omega}}+d \omega \\
\mathbf{k}_{1}=\widetilde{\mathbf{k}}, & \mathbf{k}_{2}=\widetilde{\mathbf{k}}+d \mathbf{k}, & \mathbf{k}_{3}=\widetilde{\widetilde{k}}, & \mathbf{k}_{4}=\widetilde{\widetilde{\mathbf{k}}}+d \mathbf{k}
\end{array}
$$

nas expressões (4.5.19) e (4.5.20), obtemos

$$
\begin{aligned}
& \mathrm{E}\left\{\left|d Z_{\omega, \mathbf{k}}(\widetilde{\omega}, \widetilde{\mathbf{k}})\right|^{2}\right\}=(2 \pi)^{4} P_{f}(\widetilde{\omega}, \widetilde{\mathbf{k}}) d \omega d \mathbf{k} \\
& \mathrm{E}\left\{d Z_{\omega, \mathbf{k}}(\widetilde{\omega}, \widetilde{\mathbf{k}}) d Z_{\omega, \mathbf{k}}^{*}(\widetilde{\widetilde{\omega}}, \widetilde{\widetilde{\mathbf{k}}})\right\}=0 \quad \widetilde{\omega} \neq \widetilde{\widetilde{\omega}} \vee \widetilde{\mathbf{k}} \neq \widetilde{\widetilde{\mathbf{k}}}
\end{aligned}
$$


$\operatorname{com} d Z_{\omega, \mathbf{k}}(\widetilde{\omega}, \widetilde{\mathbf{k}})=F(\widetilde{\omega}, \widetilde{\mathbf{k}}) d \omega d \mathbf{k}$

Note que o espectro integrado pode ser definido em relação a somente uma das variáveis. Por exemplo, se

$$
F(\omega, \mathbf{p})=\int_{-\infty}^{+\infty} f(t, \mathbf{p}) e^{-j \omega t} d t
$$

definimos o espectro integrado em relação a $\omega$ como

$$
Z_{\omega}(\omega, \mathbf{p})=\int_{-\infty}^{\omega} F(\alpha, \mathbf{p}) d \alpha
$$

Analogamente, para

$$
F(\omega, \mathbf{k})=\int_{\mathbb{R}^{3}} \int_{-\infty}^{+\infty} f(t, \mathbf{p}) e^{-j \omega t} e^{+j \mathbf{k}^{T} \mathbf{p}} d t d \mathbf{p}
$$

o espectro integrado em relação a $\omega$ é dado por

$$
Z_{\omega}(\omega, \mathbf{k})=\int_{-\infty}^{\omega} F(\alpha, \mathbf{k}) d \alpha
$$

Todos os resultados desta seção continuam válidos independentemente para $\omega$ e p. Por exemplo, temos

$$
\mathrm{E}\left\{d Z_{\omega}(\widetilde{\omega}, \mathbf{p}) d Z_{\omega}^{*}(\widetilde{\omega}, \mathbf{p}-\Delta \mathbf{p})\right\}=2 \pi \cdot S_{f}(\widetilde{\omega}, \Delta \mathbf{p}) d \omega .
$$

\subsection{Representação de processos tridimensionais}

Em contextos práticos, teremos a descrição de um processo na forma de uma distribuição espacial $S_{o}(\omega, \theta, \phi)$, que é um espectro em frequência-número de onda parametrizado em coordenadas esféricas. Nosso objetivo nesta seção é determinar como esta descrição mais natural se relaciona com $S_{f}(\omega, \Delta \mathbf{p})$ e $P_{f}(\omega, \mathbf{k})$. Para isso seguiremos [17], com as devidas adaptações de notação.

Os resultados (4.5.19) e (4.5.20) nos permitem modelar um campo arbitrário como a superposição de processos de onda plana infinitesimais, cada um com sua específica frequência e direção de chegada. Podemos considerar que cada um desses processos foi gerado sobre a superfície de uma esfera com raio suficientemente grande, centrada na origem do sistema de coordenadas.

Sejam $Z_{\omega}(\omega, \mathbf{p})$ o espectro integrado dado por (4.5.24) e $Z_{\omega}(\omega, \theta, \phi)$ o espectro integrado dado por (4.5.26), parametrizado em coordenadas esféricas. Note que $d Z_{\omega}\left(\omega_{0}, \theta, \phi\right)$ modela uma onda plana infinitesimal com número de onda $\mathbf{k}(\theta, \phi)=k_{0} \mathbf{a}_{r}(\theta, \phi)$, onde $\mathbf{a}_{r}(\theta, \phi)$ é o vetor unitário que aponta na direção radial. Temos então que

$$
d Z_{\omega}\left(\omega_{0}, \mathbf{p}\right)=\int_{0}^{\pi} \int_{0}^{2 \pi} d Z_{\omega}\left(\omega_{0}, \theta, \phi\right) e^{-j k_{0} \mathbf{a}_{r}^{T}(\theta, \phi)} \frac{\sin \theta}{4 \pi} d \phi d \theta
$$


A função espectro em frequência-correlação espacial é dada por (4.5.27), que repetimos a seguir:

$$
\mathrm{E}\left\{d Z_{\omega}(\widetilde{\omega}, \mathbf{p}) d Z_{\omega}^{*}(\widetilde{\omega}, \mathbf{p}-\Delta \mathbf{p})\right\}=2 \pi \cdot S_{f}(\widetilde{\omega}, \Delta \mathbf{p}) d \omega
$$

Admitindo que regiões disjuntas da esfera irradiem de forma descorrelacionada,

$$
\begin{aligned}
\mathrm{E}\{d & \left.d Z_{\omega}\left(\omega_{0}, \theta_{1}, \phi_{1}\right) d Z_{\omega}^{*}\left(\omega_{0}, \theta_{2}, \phi_{2}\right)\right\}= \\
& =2 \pi \cdot S_{o}\left(\omega_{0}, \theta_{1}, \phi_{1}\right)\left(\frac{\delta\left(\theta_{1}-\theta_{2}\right) \delta\left(\phi_{1}-\phi_{2}\right)}{\frac{\sin \theta_{1}}{4 \pi}}\right) d \omega,
\end{aligned}
$$

onde o termo $\sin \theta_{1}$ é referente ao Jacobiano da transformação para coordenadas esféricas.

Substituindo (4.6.1) em (4.6.2),

$$
\begin{aligned}
2 \pi \cdot S_{f} & \left(\omega_{0}, \Delta \mathbf{p}\right) d \omega= \\
& =\int_{0}^{\pi} \int_{0}^{2 \pi} \int_{0}^{\pi} \int_{0}^{2 \pi} \mathrm{E}\left\{d Z_{\omega}\left(\omega_{0}, \theta_{1}, \phi_{1}\right) d Z_{\omega}^{*}\left(\omega_{0}, \theta_{2}, \phi_{2}\right)\right\} \\
& =e^{-j k_{0} \mathbf{a}_{r}^{T}\left(\theta_{1}, \phi_{1}\right) \mathbf{p}+j k_{0} \mathbf{a}_{r}^{T}\left(\theta_{2}, \phi_{2}\right)(\mathbf{p}-\Delta \mathbf{p})} \frac{\sin \theta_{1}}{4 \pi} \frac{\sin \theta_{2}}{4 \pi} d \phi_{1} d \theta_{1} d \phi_{2} d \theta_{2} .
\end{aligned}
$$

Usando (4.6.3),

$$
S_{f}\left(\omega_{0}, \Delta \mathbf{p}\right)=\int_{0}^{\pi} \int_{0}^{2 \pi} S_{o}\left(\omega_{0}, \theta, \phi\right) e^{-j k_{0} \mathbf{a}_{r}^{T}(\theta, \phi) \Delta \mathbf{p}} \frac{\sin \theta}{4 \pi} d \phi d \theta .
$$

A partir de $S_{f}\left(\omega_{0}, \Delta \mathbf{p}\right)$ é possível obter $P_{f}\left(\omega_{0}, \mathbf{k}\right)$. Por definição,

$$
\begin{aligned}
P_{f}(\omega, \mathbf{k}) & =\int_{\mathbb{R}^{3}} S_{f}(\omega, \Delta \mathbf{p}) e^{+j \mathbf{k}^{T} \Delta \mathbf{p}} d \Delta \mathbf{p} \\
& =\int_{\mathbb{R}^{3}} \int_{0}^{\pi} \int_{0}^{2 \pi} S_{o}\left(\omega_{0}, \theta, \phi\right) e^{-j k_{0} \mathbf{a}_{r}^{T}(\theta, \phi) \Delta \mathbf{p}} e^{+j \mathbf{k}^{T} \Delta \mathbf{p}} \frac{\sin \theta}{4 \pi} d \phi d \theta d \Delta \mathbf{p} .
\end{aligned}
$$

Substituindo $\mathbf{k}=k_{r} \mathbf{a}_{r}\left(\theta_{k}, \phi_{k}\right)$,

$$
\begin{aligned}
P_{f}\left(\omega_{0}, \mathbf{k}\right) & =\int_{\mathbb{R}^{3}} \int_{0}^{\pi} \int_{0}^{2 \pi} S_{o}\left(\omega_{0}, \theta, \phi\right) e^{j\left(k_{r} \mathbf{a}_{r}\left(\theta_{k}, \phi_{k}\right)-k_{0} \mathbf{a}_{r}(\theta, \phi)\right)^{T} \Delta \mathbf{p}} \frac{\sin \theta}{4 \pi} d \phi d \theta d \Delta \mathbf{p} \\
& =\int_{0}^{\pi} \int_{0}^{2 \pi} S_{o}\left(\omega_{0}, \theta, \phi\right)\left[\int_{\mathbb{R}^{3}} e^{j\left(k_{r} \mathbf{a}_{r}\left(\theta_{k}, \phi_{k}\right)-k_{0} \mathbf{a}_{r}(\theta, \phi)\right)^{T} \Delta \mathbf{p}} d \Delta \mathbf{p}\right] \frac{\sin \theta}{4 \pi} d \phi d \theta .
\end{aligned}
$$

A integral entre colchetes leva a um impulso em coordenadas esféricas:

$$
\int_{\mathbb{R}^{3}} e^{j\left(k_{r} \mathbf{a}_{r}\left(\theta_{k}, \phi_{k}\right)-k_{0} \mathbf{a}_{r}(\theta, \phi)\right)^{T} \Delta \mathbf{p}} d \Delta \mathbf{p}=(2 \pi)^{3} \frac{\delta\left(k_{r}-k_{0}\right) \delta\left(\theta_{k}-\theta\right) \delta\left(\phi_{k}-\phi\right)}{k_{0}^{2} \sin \theta},
$$


que quando substituído em (4.6.7) leva a

$$
P_{f}\left(\omega_{0}, \mathbf{k}\right)=(2 \pi)^{3} S_{o}\left(\omega_{0}, \theta_{k}, \phi_{k}\right) \frac{\delta\left(k_{r}-k_{0}\right)}{4 \pi k_{0}^{2}}
$$

\subsection{Filtragem em frequência-número de onda}

Consideremos um processo $x(t, \mathbf{p})$ estacionário e homogêneo. Se medirmos este processo com um array de $\mathrm{N}$ elementos, teremos o vetor de saídas

$$
\mathbf{x}(t, \mathbf{p})=\left[\begin{array}{c}
x\left(t, \mathbf{p}_{1}\right) \\
x\left(t, \mathbf{p}_{2}\right) \\
\vdots \\
x\left(t, \mathbf{p}_{N}\right)
\end{array}\right]
$$

Por definição, a matriz espectral do array é dada por

$$
\left[\mathbf{S}_{\mathbf{x}}(\omega)\right]_{i j}=S_{x}\left(\omega, \mathbf{p}_{i}-\mathbf{p}_{j}\right)
$$

Em uma implementação prática, estimamos $\mathbf{S}_{\mathbf{x}}(\omega)$ a partir de seus snapshots $\hat{\mathbf{x}}_{\Delta T}\left(\omega_{m}, k\right)$. O limite (4.2.8) garante que para $\Delta T$ suficientemente grande,

$$
\mathrm{E}\left[\hat{\mathbf{x}}_{\Delta T}\left(\omega_{m}, k\right) \hat{\mathbf{x}}_{\Delta T}^{H}\left(\omega_{m}, k\right)\right] \approx \mathbf{S}_{\mathbf{x}}\left(\omega_{m}\right)
$$

Seja $\mathbf{w}\left(\omega_{n}\right)$ o vetor de coeficientes usado para processar a entrada $\hat{\mathbf{x}}_{\Delta T}\left(\omega_{m}, k\right)$. De (2.7.5), temos

$$
S_{y}\left(\omega_{m}\right)=\mathbf{w}^{H}\left(\omega_{m}\right) \mathbf{S}_{\mathbf{x}}\left(\omega_{m}\right) \mathbf{w}\left(\omega_{m}\right) .
$$

Expandindo as multiplicações, usando (4.5.9) e a definição do padrão de radiação,

$$
\begin{aligned}
S_{y}\left(\omega_{m}\right) & =\sum_{i=1}^{N} \sum_{j=1}^{N} w_{i}^{*}\left(\omega_{m}\right) S_{x}\left(\omega_{m}, \mathbf{p}_{i}-\mathbf{p}_{j}\right) w_{j}\left(\omega_{m}\right) \\
& =\frac{1}{(2 \pi)^{3}} \int_{\mathbb{R}^{3}} \sum_{i=1}^{N} \sum_{j=1}^{N} w_{i}^{*}\left(\omega_{m}\right) w_{j}\left(\omega_{m}\right) P_{x}(\omega, \mathbf{k}) e^{-j \mathbf{k}^{T}\left(\mathbf{p}_{i}-\mathbf{p}_{j}\right)} d \mathbf{k} \\
& =\frac{1}{(2 \pi)^{3}} \int_{\mathbb{R}^{3}} P_{x}(\omega, \mathbf{k})\left|B\left(\omega_{m}, \mathbf{k}\right)\right|^{2} d \mathbf{k} .
\end{aligned}
$$

Logo, o processamento de um array corresponde a uma filtragem no domínio frequêncianúmero de onda. 



\section{Capítulo 5}

\section{Beamformers ótimos}

\subsection{Introdução}

O objetivo desta seção é realizar o projeto de processadores ótimos. Os critérios de optimalidade serão definidos usando a linguagem de processos aleatórios espaciaistemporais, tal que o projeto dependerá das estatísticas dos processos amostrados. Em um cenário real essas estatísticas são desconhecidas e devem ser estimadas.

Os métodos expostos a seguir admitem que os processos envolvidos são estacionários para o intervalo de medida. Na prática, poucos processos práticos podem ser considerados absolutamente estacionários, e algum mecanismo de atualização é necessário para manter a optimalidade. Uma estratégia de atualização óbvia consiste em repetir os algoritmos de projeto periodicamente, substituindo os parâmetros do processador a cada iteração. Uma alternativa consiste no uso de algoritmos adaptativos [2,18-20], que estão além do escopo deste texto.

Os resultados desta seção podem ser usados diretamente ou indiretamente para separar espacialmente sinais de interesse, possibilitando a estimação de parâmetros como potência, forma de onda, espectro e direção de chegada.

\subsection{Beamformer MVDR}

Suponhamos que a entrada do array seja dada conforme na Seção 4.4.1, onde tratamos um único sinal determinístico e desconhecido com direção de propagação $\mathbf{k}_{s}$, submetido a ruído Gaussiano de média zero. Omitindo a variável $\omega$, o sinal de entrada é dado por

$$
\begin{aligned}
\hat{\mathbf{x}} & =\hat{\mathbf{x}}_{s}+\hat{\mathbf{n}} \\
& =\mathbf{v}\left(\mathbf{k}_{s}\right) \hat{f}_{s}+\hat{\mathbf{n}} .
\end{aligned}
$$

Seja $y$ a saída do processador. Na ausência de ruído, gostaríamos que $y=\hat{f}$. Assim, impo$\operatorname{mos} \mathbf{w}^{H} \mathbf{v}\left(\mathbf{k}_{s}\right)=1$. Usamos como função objetivo a variância de $y$, tal que o processador 
ótimo será aquele que minimiza

$$
\begin{aligned}
\mathrm{E}\left\{|y-\bar{y}|^{2}\right\} & =\mathrm{E}\left\{\left|\mathbf{w}^{H} \hat{\mathbf{n}}\right|^{2}\right\} \\
& =\mathbf{w}^{H} \mathrm{E}\left\{\hat{\mathbf{n}} \hat{\mathbf{n}}^{H}\right\} \mathbf{w} \\
& =\mathbf{w}^{H} \mathbf{S}_{\mathbf{n}} \mathbf{w} .
\end{aligned}
$$

Usando multiplicadores de Lagrange, buscamos

$$
\mathbf{w}_{o}=\underset{\mathbf{w}}{\operatorname{argmin}} \mathbf{w}^{H} \mathbf{S}_{\mathbf{n}} \mathbf{w}+\lambda\left[\mathbf{w}^{H} \mathbf{v}\left(\mathbf{k}_{s}\right)-1\right]+\lambda^{*}\left[\mathbf{v}^{H}\left(\mathbf{k}_{s}\right) \mathbf{w}-1\right] .
$$

Aplicando o gradiente complexo em relação a w e igualando a zero, temos

$$
\mathbf{w}_{o}^{H}=-\lambda \mathbf{v}^{H}\left(\mathbf{k}_{s}\right) \mathbf{S}_{\mathbf{n}}^{-1}
$$

Impondo que $\mathbf{w}^{H} \mathbf{v}(\mathbf{k})=1$, resulta

$$
\lambda=-\frac{1}{\mathbf{v}^{H}\left(\mathbf{k}_{s}\right) \mathbf{S}_{\mathbf{n}}^{-1} \mathbf{v}\left(\mathbf{k}_{s}\right)} .
$$

Portanto,

$$
\mathbf{w}_{o}^{H}=\frac{\mathbf{v}^{H}\left(\mathbf{k}_{s}\right) \mathbf{S}_{\mathbf{n}}^{-1}}{\mathbf{v}^{H}\left(\mathbf{k}_{s}\right) \mathbf{S}_{\mathbf{n}}^{-1} \mathbf{v}\left(\mathbf{k}_{s}\right)} .
$$

Este processador é conhecido como minimum variance distortionless response, ou MVDR. Na literatura também é chamado de beamformer Capon, pois foi proposto pela primeira vez em [21]. Note que sua construção exige $\mathbf{S}_{\mathbf{n}}$ não-singular. Em casos práticos isso ocorre, pois $\mathbf{S}_{\mathbf{n}}$ sempre tem uma componente espacialmente branca (por exemplo, devido a ruído térmico).

Consideremos a função densidade de probabilidade encontrada na Seção 4.4.1, repetida a seguir:

$$
p_{\hat{\mathbf{x}}}(\hat{\mathbf{x}})=\frac{1}{\pi^{N}\left|\mathbf{S}_{n}\right|} \exp \left\{-\left[\hat{\mathbf{x}}-\mathbf{v}\left(\mathbf{k}_{s}\right) \hat{f}_{s}\right]^{H} \mathbf{S}_{n}^{-1}\left[\hat{\mathbf{x}}-\mathbf{v}\left(\mathbf{k}_{s}\right) \hat{f}_{s}\right]\right\}
$$

Desprezando constantes multiplicativas, sua função log-verossimilhança é

$$
l\left(f_{s}\right)=-\left[\hat{\mathbf{x}}^{H}-\mathbf{v}^{H}\left(\mathbf{k}_{s}\right) \hat{f}_{s}^{*}\right] \mathbf{S}_{n}^{-1}\left[\hat{\mathbf{x}}-\mathbf{v}\left(\mathbf{k}_{s}\right) \hat{f}_{s}\right] .
$$

Avaliando seu gradiente complexo em relação a $f_{s}^{*}$ e igualando a zero, resulta

$$
\hat{f}_{s}=\frac{\mathbf{v}^{H}\left(\mathbf{k}_{s}\right) \mathbf{S}_{\mathbf{n}}^{-1} \hat{\mathbf{x}}}{\mathbf{v}^{H}\left(\mathbf{k}_{s}\right) \mathbf{S}_{\mathbf{n}}^{-1} \mathbf{v}\left(\mathbf{k}_{s}\right)}
$$

que é exatamente a estimativa que seria obtida com o processador MVDR. Note que a estimativa ML acima foi obtida admitindo conhecidas a direção de chegada $\mathbf{k}_{s}$ e a matriz 
espectral do ruído $\mathbf{S}_{\mathbf{n}}^{-1}$. Para casos mais gerais, o processador MVDR não coincide com o estimador ML.

Determinemos o ganho do beamformer MVDR. Devido a (2.7.6), o componente de sinal de $y$ tem espectro

$$
S_{y_{s}}=S_{f}
$$

O componente de ruído de $y$ tem espectro

$$
\begin{aligned}
S_{y_{n}} & =\mathbf{w}_{o}^{H} \mathbf{S}_{\mathbf{n}} \mathbf{w}_{o} \\
& =\frac{\mathbf{v}^{H}\left(\mathbf{k}_{s}\right) \mathbf{S}_{\mathbf{n}}^{-1} \mathbf{S}_{\mathbf{n}} \mathbf{S}_{\mathbf{n}}^{-1} \mathbf{v}\left(\mathbf{k}_{s}\right)}{\left(\mathbf{v}^{H}\left(\mathbf{k}_{s}\right) \mathbf{S}_{\mathbf{n}}^{-1} \mathbf{v}\left(\mathbf{k}_{s}\right)\right)^{2}} \\
& =\frac{1}{\mathbf{v}^{H}\left(\mathbf{k}_{s}\right) \mathbf{S}_{\mathbf{n}}^{-1} \mathbf{v}\left(\mathbf{k}_{s}\right)} .
\end{aligned}
$$

Portanto, o ganho é dado por

$$
\begin{aligned}
A_{\mathrm{MVDR}} & =\frac{S_{f} \mathbf{v}^{H}\left(\mathbf{k}_{s}\right) \mathbf{S}_{\mathbf{n}}^{-1} \mathbf{v}\left(\mathbf{k}_{s}\right)}{S_{f} / S_{n}} \\
& =S_{n} \mathbf{v}^{H}\left(\mathbf{k}_{s}\right) \mathbf{S}_{\mathbf{n}}^{-1} \mathbf{v}\left(\mathbf{k}_{s}\right) .
\end{aligned}
$$

Para o caso de ruído espacialmente branco, $\mathbf{S}_{\mathbf{n}}=\sigma_{n}^{2} \mathbf{I}$ e

$$
A_{\mathrm{MVDR}}=N
$$

que é exatamente $A_{c}$, o ganho do beamformer convencional (delay-and-sum), que mostramos ser ótimo para este caso. No caso geral, $A_{M V D R} \geq A_{c}$.

\subsection{Beamformer MMSE (minimum mean-square error)}

Consideremos agora uma variação do cenário visto na Seção 4.4.4, com

$$
\hat{\mathbf{x}}=\mathbf{v}\left(\mathbf{k}_{s}\right) \hat{f}_{s}+\hat{\mathbf{n}},
$$

onde $\hat{f}_{s}$ é não-determinístico com média zero. Como desejamos obter $\hat{f}_{s}$ a partir de $\hat{\mathbf{x}}$, definimos o erro quadrático médio da estimação como

$$
\begin{aligned}
\xi & =\mathrm{E}\left\{\left|\hat{f}_{s}-\mathbf{w}^{H} \hat{\mathbf{x}}\right|^{2}\right\} \\
& =\mathrm{E}\left\{\left(\hat{f}_{s}-\mathbf{w}^{H} \hat{\mathbf{x}}\right)\left(\hat{f}_{s}^{*}-\hat{\mathbf{x}}^{H} \mathbf{w}\right)\right\} .
\end{aligned}
$$

Avaliando o gradiente complexo em relação a w e igualando a zero, temos

$$
\mathrm{E}\left\{\hat{f}_{s} \hat{\mathbf{x}}^{H}\right\}-\mathrm{E}\left\{\mathbf{w}_{o}^{H} \hat{\mathbf{x}} \hat{\mathbf{x}}^{H}\right\}=\mathbf{0}
$$


que reescrevemos como

$$
\mathbf{S}_{f \mathbf{x}^{H}}=\mathbf{w}_{o}^{H} \mathbf{S}_{\mathbf{x}}
$$

Portanto,

$$
\mathbf{w}_{o}^{H}=\mathbf{S}_{f \mathbf{x}^{H}} \mathbf{S}_{\mathbf{x}}^{-1} .
$$

Como $\hat{f}_{s}$ e o ruído são descorrelacionados,

$$
\mathbf{w}_{o}^{H}=S_{f} \mathbf{v}^{H}\left(\mathbf{k}_{s}\right) \mathbf{S}_{\mathbf{x}}^{-1} .
$$

A matriz espectral de $\hat{\mathbf{x}}$ é dada por

$$
\mathbf{S}_{\mathbf{x}}=S_{f} \mathbf{v}\left(\mathbf{k}_{s}\right) \mathbf{v}^{H}\left(\mathbf{k}_{s}\right)+\mathbf{S}_{\mathbf{n}}
$$

Usando o lema de inversão de matrizes,

$$
\mathbf{S}_{\mathbf{x}}^{-1}=\mathbf{S}_{\mathbf{n}}^{-1}-S_{f} \mathbf{S}_{\mathbf{n}}^{-1} \mathbf{v}\left(\mathbf{k}_{s}\right)\left[1+S_{f} \mathbf{v}^{H}\left(\mathbf{k}_{s}\right) \mathbf{S}_{\mathbf{n}}^{-1} \mathbf{v}\left(\mathbf{k}_{s}\right)\right]^{-1} \mathbf{v}^{H}\left(\mathbf{k}_{s}\right) \mathbf{S}_{\mathbf{n}}^{-1}
$$

Substituindo (5.3.7) em (5.3.5) resulta

$$
\mathbf{w}_{o}^{H}=\frac{S_{f}}{S_{f}+\left[\mathbf{v}^{H}\left(\mathbf{k}_{s}\right) \mathbf{S}_{\mathbf{n}}^{-1} \mathbf{v}\left(\mathbf{k}_{s}\right)\right]^{-1}} \frac{\mathbf{v}^{H}\left(\mathbf{k}_{s}\right) \mathbf{S}_{\mathbf{n}}^{-1}}{\mathbf{v}^{H}\left(\mathbf{k}_{s}\right) \mathbf{S}_{\mathbf{n}}^{-1} \mathbf{v}\left(\mathbf{k}_{s}\right)} .
$$

Logo, o processador MMSE difere do processador MVDR apenas por uma constante multiplicativa.

Para filtros escalares e sinais Gaussianos é possível provar que o processador ótimo no sentido quadrático médio é linear [15]. A prova se estende para o caso de processos temporais-espaciais Gaussianos, tal que o processador ótimo MMSE será dado por (5.3.8).

Outro critério de interesse é a maximização da relação sinal-ruído. É possível mostrar usando multiplicadores de Lagrange [2] que o processador ótimo novamente tem a forma dos processadores MVDR e MMSE. No texto que segue, o termo "beamformer MVDR" fará referência a toda esta classe de beamformers ótimos.

\subsection{Beamformer MVDR submetido a múltiplas interferências}

Os resultados desta seção são devidos a [2].

Consideremos o cenário da Seção 4.4.3, no qual um processador MVDR está submetido a $M$ interferências planas. Neste caso,

$$
\hat{\mathbf{x}}=\mathbf{v}\left(\mathbf{k}_{s}\right) \hat{f}_{s}+\sum_{i=1}^{M} \mathbf{v}\left(\mathbf{k}_{i}\right) \hat{f}_{i}+\hat{\mathbf{n}}
$$


Definimos

$$
\begin{aligned}
\mathbf{V}_{I}(\mathbf{k}) & =\left[\begin{array}{llll}
\mathbf{v}\left(\mathbf{k}_{1}\right) & \mathbf{v}\left(\mathbf{k}_{2}\right) & \cdots & \mathbf{v}\left(\mathbf{k}_{M}\right)
\end{array}\right] \\
\hat{\mathbf{f}}_{I} & =\left[\begin{array}{llll}
\hat{f}_{1} & \hat{f}_{2} & \cdots & \hat{f}_{M}
\end{array}\right]^{T},
\end{aligned}
$$

tal que

$$
\begin{aligned}
\mathbf{S}_{I} & =\mathrm{E}\left\{\hat{\mathbf{f}}_{I} \hat{\mathbf{f}}_{I}^{H}\right\} \\
\mathbf{S}_{I+N} & =\mathbf{V}_{I} \mathbf{S}_{I} \mathbf{V}_{I}^{H}+\sigma_{n}^{2} \mathbf{I} .
\end{aligned}
$$

Usando o lema de inversão de matrizes,

$$
\mathbf{S}_{I+N}^{-1}=\frac{1}{\sigma_{n}^{2}}\left[\mathbf{I}-\mathbf{V}_{I}\left(\mathbf{I}+\frac{\mathbf{S}_{I}}{\sigma_{n}^{2}} \mathbf{V}_{I}^{H} \mathbf{V}_{I}\right)^{-1} \frac{\mathbf{S}_{I}}{\sigma_{n}^{2}} \mathbf{V}_{I}^{H}\right]
$$

Substituindo (5.4.6) em (5.2.5) temos

$$
\mathbf{w}_{\mathrm{MVDR}}^{H} \propto \mathbf{v}^{H}\left(\mathbf{k}_{s}\right)-\mathbf{v}^{H}\left(\mathbf{k}_{s}\right) \mathbf{V}_{I}\left(\mathbf{I}+\frac{\mathbf{S}_{I}}{\sigma_{n}^{2}} \mathbf{V}_{I}^{H} \mathbf{V}_{I}\right)^{-1} \frac{\mathbf{S}_{I}}{\sigma_{n}^{2}} \mathbf{V}_{I}^{H}
$$

Como $B_{\mathrm{MVDR}}\left(\mathbf{k} \mid \mathbf{k}_{s}\right)=\mathbf{w}_{\mathrm{MVDR}}^{H} \mathbf{v}(\mathbf{k})$,

$$
B_{\mathrm{MVDR}}\left(\mathbf{k} \mid \mathbf{k}_{s}\right) \propto \mathbf{v}^{H}\left(\mathbf{k}_{s}\right) \mathbf{v}(\mathbf{k})-\mathbf{v}^{H}\left(\mathbf{k}_{s}\right) \mathbf{V}_{I}\left(\mathbf{I}+\frac{\mathbf{S}_{I}}{\sigma_{n}^{2}} \mathbf{V}_{I}^{H} \mathbf{V}_{I}\right)^{-1} \frac{\mathbf{S}_{I}}{\sigma_{n}^{2}} \mathbf{V}_{I}^{H} \mathbf{v}(\mathbf{k})
$$

Note que $\mathbf{v}^{H}\left(\mathbf{k}_{s}\right) \mathbf{v}(\mathbf{k})=B_{c}\left(\mathbf{k} \mid \mathbf{k}_{s}\right)$, onde $B_{c}\left(\mathbf{k} \mid \mathbf{k}_{s}\right)$ é a resposta de um beamformer convencional com eixo principal em $\mathbf{k}_{s}$. Analogamente,

$$
\mathbf{V}_{I}^{H} \mathbf{v}(\mathbf{k})=\left[\begin{array}{c}
B_{c}\left(\mathbf{k} \mid \mathbf{k}_{1}\right) \\
B_{c}\left(\mathbf{k} \mid \mathbf{k}_{2}\right) \\
\vdots \\
B_{c}\left(\mathbf{k} \mid \mathbf{k}_{M}\right)
\end{array}\right]=\mathbf{B}_{c}\left(\mathbf{k} \mid \mathbf{k}_{I}\right)
$$

tal que

$$
B_{\mathrm{MVDR}}\left(\mathbf{k} \mid \mathbf{k}_{s}\right) \propto B_{c}\left(\mathbf{k} \mid \mathbf{k}_{s}\right)-\mathbf{v}^{H}\left(\mathbf{k}_{s}\right) \mathbf{V}_{I}\left(\mathbf{I}+\frac{\mathbf{S}_{I}}{\sigma_{n}^{2}} \mathbf{V}_{I}^{H} \mathbf{V}_{I}\right)^{-1} \frac{\mathbf{S}_{I}}{\sigma_{n}^{2}} \mathbf{B}_{c}\left(\mathbf{k} \mid \mathbf{k}_{I}\right) .
$$

Portanto, a resposta do beamformer MVDR é dada por uma resposta convencional direcionada para o sinal de interesse, menos uma ponderação de respostas convencionais apontadas para as interferências. Se as interferências forem descorrelacionadas, $\mathbf{S}_{I}$ é dia- 
gonal e (5.4.7) se reduz a

$$
\mathbf{w}_{\mathrm{MVDR}}^{H} \propto \mathbf{v}^{H}\left(\mathbf{k}_{s}\right)\left[\mathbf{I}-\mathbf{V}_{I}\left(\sigma_{n}^{2} \mathbf{S}_{I}^{-1}+\mathbf{V}_{I}^{H} \mathbf{V}_{I}\right)^{-1} \mathbf{V}_{I}^{H}\right]
$$

Se a potência das interferências for muito maior que $\sigma_{n}^{2}$,

$$
\mathbf{w}_{\mathrm{MVDR}}^{H} \propto \mathbf{v}^{H}\left(\mathbf{k}_{s}\right)\left[\mathbf{I}-\mathbf{V}_{I}\left(\mathbf{V}_{I}^{H} \mathbf{V}_{I}\right)^{-1} \mathbf{V}_{I}^{H}\right]
$$

que pode ser escrito como

$$
\mathbf{w}_{\mathrm{MVDR}}^{H} \propto \mathbf{v}^{H}\left(\mathbf{k}_{s}\right) \mathbf{P}_{I}^{\perp}
$$

onde $\mathbf{P}_{I}^{\perp}$ é a matriz de projeção sobre o subespaço ortogonal ao subespaço das interferências. Logo, neste caso o beamformer ótimo insere zeros nas direções das interferências.

Expressões analíticas para o ganho podem ser obtidas substituindo $\mathbf{S}_{n}^{-1}$ em (5.2.11).

\subsection{Sensibilidade}

A Figura 5.1 apresenta padrões de radiação MVDR calculados para uma única interferência, sob uma relação interferência-ruído (INR) de $20 \mathrm{~dB}$. Conforme a interferência se aproxima do eixo principal, mais abrupta deve ser a variação angular de ganho para que $\mathbf{w}^{H} \mathbf{v}\left(\mathbf{k}_{s}\right)=1$ seja atendida e a interferência seja cancelada. Como na realidade $\mathbf{v}\left(\mathbf{k}_{s}\right)$ e $\mathbf{S}_{\mathbf{n}}$ estão sempre sujeitos a erros, uma sensibilidade excessiva tipicamente implica em ganho não-unitário na direção real do sinal (que será diferente de $\mathbf{k}_{s}$ ) e atenuação insuficiente da interferência.

A sensibilidade de um processador tem consequências ainda mais sérias. De fato, consideremos que em muitas situações reais a matriz espectral $\mathbf{S}_{\mathbf{n}}$ não pode ser diretamente estimada. Neste caso poderíamos substituir $\mathbf{S}_{\mathbf{n}}$ por $\mathbf{S}_{\mathbf{x}}$ no beamformer MVDR. Consideremos como critério de projeto a minimização de $\mathrm{E}\left\{|y|^{2}\right\}$ (a potência quadrática média da saída) sob a restrição $\mathbf{w}^{H} \mathbf{v}\left(\mathbf{k}_{s}\right)=1$. A derivação do beamformer ótimo é idêntica à vista para o MVDR, e resulta no processador MPDR (minimum power distortionless response):

$$
\mathbf{w}_{\mathrm{MPDR}}^{H}=\frac{\mathbf{v}^{H}\left(\mathbf{k}_{s}\right) \mathbf{S}_{\mathbf{x}}^{-1}}{\mathbf{v}^{H}\left(\mathbf{k}_{s}\right) \mathbf{S}_{\mathbf{x}}^{-1} \mathbf{v}\left(\mathbf{k}_{s}\right)} .
$$

Devido à restrição (2.7.6), a saída deste processador é (em média) igual a $\hat{f}_{s}$ quando $\mathbf{S}_{\mathbf{x}} \mathrm{e}$ $\mathbf{k}_{s}$ são perfeitamente estimados. No entanto, na prática isso nunca ocorre. O beamformer MPDR torna-se então equivalente a um beamformer MVDR com uma interferência muito próxima do eixo principal, uma vez que o processador incorretamente classifica o sinal de interesse como uma interferência a ser cancelada. Um sinal com SNR alta é interpretado por um beamformer MPDR mal projetado como uma interferência com INR alta, implicando em grande atenuação do sinal de interesse.

Logo, a sensibilidade de um processador pode causar considerável degradação de sua 

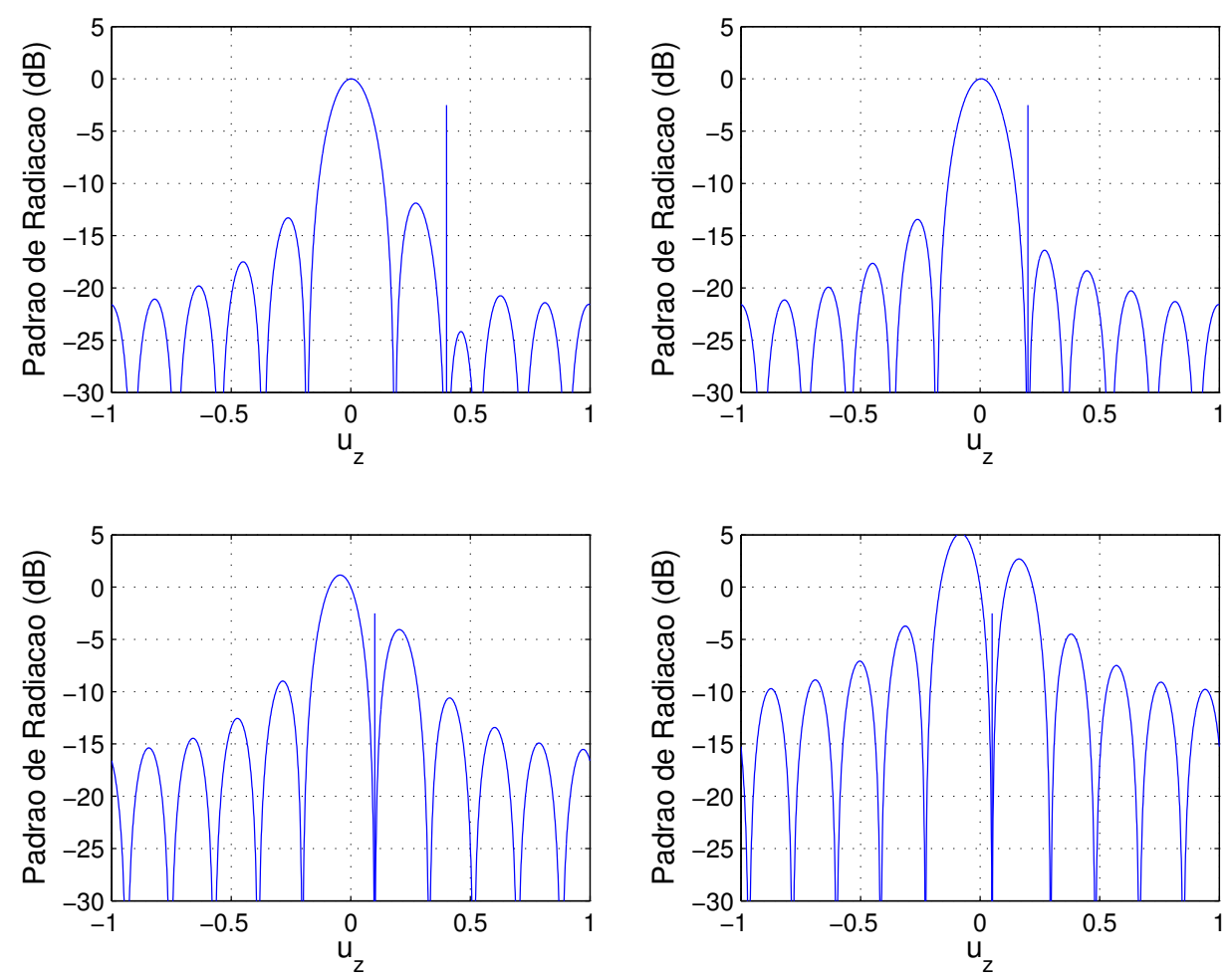

Figura 5.1: Respostas MVDR para $N=11, d=\lambda / 2, I N R=20 d B$, e $u_{I} \in$ $\{0.05,0.1,0.2,0.4\}$

resposta em cenários práticos. Já encontramos esta sensibilidade na Seção 2.8, quando foi discutido o efeito de perturbações. Naquele instante concluímos que a restrição $\|\mathbf{w}\|^{2} \leq T_{0}$ poderia ser usada para controlar variações da resposta em frequência-número de onda. Quando imposta, esta restrição torna processadores mais robustos a estimativas de $\mathbf{S}_{\mathbf{n}}$, $\mathbf{S}_{\mathbf{x}}$ ou $\mathbf{k}_{s}$ realizadas com poucas amostras, perturbações dos elementos do array e erros numéricos.

Consideremos o problema de minimizar $\mathbf{P}_{y}=\mathbf{w}^{H} \mathbf{S}_{\mathbf{x}} \mathbf{w}$ sob as restrições $\mathbf{w}^{H} \mathbf{v}\left(\mathbf{k}_{s}\right)=1$ e $\|\mathbf{w}\|^{2}=T_{0}$. Usando multiplicadores de Lagrange, a função objetivo é dada por

$$
\mathbf{w}^{H} \mathbf{S}_{\mathbf{x}} \mathbf{w}+\lambda_{1}\left[\mathbf{w}^{H} \mathbf{w}-T_{0}\right]+\lambda_{2}\left[\mathbf{w}^{H} \mathbf{v}\left(\mathbf{k}_{s}\right)-1\right]+\lambda_{2}^{*}\left[\mathbf{v}^{H}\left(\mathbf{k}_{s}\right) \mathbf{w}-1\right] .
$$

Avaliando $\nabla_{\mathbf{w}}$ e igualando a zero,

$$
\mathbf{w}_{o}^{H} \mathbf{S}_{\mathbf{x}}+\lambda_{1} \mathbf{w}_{o}^{H}+\lambda_{2}^{*} \mathbf{v}^{H}\left(\mathbf{k}_{s}\right)=\mathbf{0} .
$$

Isolando $\mathbf{w}_{o}^{H}$ e usando $\mathbf{w}_{o}^{H} \mathbf{v}\left(\mathbf{k}_{s}\right)=1$, obtemos

$$
\mathbf{w}_{o}^{H}=\frac{\mathbf{v}^{H}\left(\mathbf{k}_{s}\right)\left[\mathbf{S}_{\mathbf{x}}+\lambda_{1} \mathbf{I}\right]^{-1}}{\mathbf{v}^{H}\left(\mathbf{k}_{s}\right)\left[\mathbf{S}_{\mathbf{x}}+\lambda_{1} \mathbf{I}\right]^{-1} \mathbf{v}\left(\mathbf{k}_{s}\right)} .
$$

Esta expressão equivale a (5.5.1) $\operatorname{com} \mathbf{S}_{\mathbf{x}}+\lambda_{1} \mathbf{I}$ no lugar de $\mathbf{S}_{\mathbf{x}}$, e corresponde a uma solução 
regularizada [22,23] do processador MPDR. Usando esta interpretação, frequentemente o parâmetro $\lambda_{1}$ é escolhido diretamente sem considerar sua relação com $T_{0}$. Veremos na Seção 5.7 como obter uma solução ótima para $\|\mathbf{w}\|^{2} \leq T_{0}$.

\subsection{Beamformer MVDR ou MPDR com restrições lineares}

Até agora usamos somente a restrição $\mathbf{w}^{H} \mathbf{v}\left(\mathbf{k}_{s}\right)=1$ para o projeto ótimo. Assim como fizemos na Seção 3.2, podemos inserir restrições adicionais para o padrão de resposta e suas derivadas. Na Seção 3.2 queríamos aproximar uma resposta desejada $B_{d}(\mathbf{k})$ sob um conjunto de restrições $\mathbf{w}^{H} \mathbf{C}=\mathbf{0}$. Nesta seção desejamos minimizar

$$
\mathbf{P}_{n}=\mathbf{w}^{H} \mathbf{S}_{\mathbf{n}} \mathbf{w}
$$

no caso MVDR ou

$$
\mathbf{P}_{y}=\mathbf{w}^{H} \mathbf{S}_{\mathbf{x}} \mathbf{w}
$$

no caso MPDR, sob as restrições $\mathbf{w}^{H} \mathbf{C}=\mathbf{g}^{H}$ (onde $\mathbf{g}$ pode ser não nulo).

Através de restrições pretendemos aproveitar conhecimento disponível a priori sobre a localização de sinais de interesse e interferências. Por exemplo, restrições de zeros podem ser usadas para garantir boa atenuação em regiões populadas por interferências, independentemente da qualidade da estimação de $\mathbf{S}_{\mathbf{n}}$ ou $\mathbf{S}_{\mathbf{x}}$. Analogamente, restrições podem ser usadas para preservar um formato desejado de lóbulo principal, aliviando os problemas de sensibilidade descritos na seção anterior. Os graus de liberdade não empregados para atender às restrições são utilizados na minimização de (5.6.1) ou (5.6.2).

Claramente ainda é possível aproximar qualquer resposta desejada, desde que sejam impostas restrições suficientes. Por outro lado, cada restrição consome um grau de liberdade que o beamformer MVDR/MPDR poderia usar para atenuar ou anular interferências. A seguir mostramos como aproximar uma resposta desejada $B_{d}(\mathbf{k})$ de forma ótima, de forma a não desperdiçar restrições.

Seja $\xi$ o erro de aproximação definido sobre uma região $\mathbf{K}$ do espaço frequência-número de onda:

$$
\begin{aligned}
\xi & =\int_{\mathbf{k} \in \mathbf{K}}\left|B_{d}(\mathbf{k})-\mathbf{w}^{H} \mathbf{v}(\mathbf{k})\right|^{2} d \mathbf{k} \\
& =\int_{\mathbf{k} \in \mathbf{K}}\left|\mathbf{w}_{d}^{H} \mathbf{v}(\mathbf{k})-\mathbf{w}^{H} \mathbf{v}(\mathbf{k})\right|^{2} d \mathbf{k} .
\end{aligned}
$$

Definimos $\mathbf{w}_{p}=\mathbf{w}_{d}-\mathbf{w}$, tal que

$$
\xi=\mathbf{w}_{p}^{H} \mathbf{A} \mathbf{w}_{p},
$$

com

$$
\mathbf{A}=\int_{\mathbf{k} \in \mathbf{K}} \mathbf{v}(\mathbf{k}) \mathbf{v}^{H}(\mathbf{k}) d \mathbf{k} .
$$

Consideremos uma decomposição de $\mathbf{A}$ em seus autovalores $\lambda_{1} \geq \cdots \geq \lambda_{N}$ e autovetores 
$\Phi_{i}$, tal que

$$
A=\sum_{i=1}^{N} \lambda_{i} \boldsymbol{\Phi}_{i} \boldsymbol{\Phi}_{i}^{H} .
$$

Portanto,

$$
\xi=\sum_{i=1}^{N} \lambda_{i}\left|\mathbf{w}_{p}^{H} \boldsymbol{\Phi}_{i}\right|^{2}
$$

e para minimizar $\xi$ devemos impor $\mathbf{w}_{p}^{H} \boldsymbol{\Phi}_{i}=0 \Leftrightarrow \mathbf{w}^{H} \boldsymbol{\Phi}_{i}=\mathbf{w}_{d}^{H} \boldsymbol{\Phi}_{i}$, para $i \in\left\{1, \ldots, N_{\max }\right\}$, onde $N_{\text {max }}$ é a quantidade máxima de restrições que desejamos empregar para atender esta aproximação.

Para minimizar (5.6.1) sujeito a $\mathbf{w}^{H} \mathbf{C}=\mathbf{g}^{H}$, reescrevemos a função objetivo usando multiplicadores de Lagrange:

$$
\mathbf{w}_{o}=\underset{\mathbf{w}}{\operatorname{argmin}} \mathbf{w}^{H} \mathbf{S}_{\mathbf{n}} \mathbf{w}+\left[\mathbf{w}^{H} \mathbf{C}-\mathbf{g}^{H}\right] \boldsymbol{\lambda}+\boldsymbol{\lambda}^{H}\left[\mathbf{C}^{H} \mathbf{w}-\mathbf{g}\right]
$$

Avaliando o gradiente complexo em relação a $\mathbf{w}^{H}$ e igualando a zero,

$$
\mathbf{S}_{\mathbf{n}} \mathbf{w}_{o}+\mathbf{C} \boldsymbol{\lambda}=\mathbf{0} \Rightarrow \mathbf{w}_{o}=-\mathrm{S}_{\mathbf{n}}^{-1} \mathbf{C} \boldsymbol{\lambda}
$$

Usando $\mathbf{w}^{H} \mathbf{C}=\mathbf{g}^{H}$, obtemos

$$
\mathbf{w}_{o}^{H}=\mathbf{g}^{H}\left[\mathbf{C}^{H} \mathbf{S}_{\mathbf{n}}^{-1} \mathbf{C}\right]^{-1} \mathbf{C}^{H} \mathbf{S}_{\mathbf{n}}^{-1} .
$$

O processador com restrições obtido acima é conhecido como LCMV (linearly constrained minimum variance) ou LCMP (linearly constrained minimum power) [24,25], dependendo da função objetivo usada.

\subsection{Realização GSC (generalized sidelobe canceller)}

O uso de restrições lineares para aproximar respostas desejadas e preservar a forma do lóbulo principal tem grande interesse prático. A realização mais usada de beamformers LCMV ou LCMP é conhecida como generalized sidelobe canceller (GSC), foi proposta inicialmente por Applebaum e Chapman em [26] e popularizada por Griffiths e Jim [27] no contexto de arrays adaptativos.

A maior motivação para o uso de restrições continua sendo a resistência a degradações do padrão de radiação. Na ausência de interferências, o comportamento do array deve estar bem definido, e é desejável que seu padrão de radiação se aproxime de uma resposta definida a priori. Como grande parte dos cenários práticos envolvem campos não-estacionários, algoritmos adaptativos são praticamente imprescindíveis.

A estrutura GSC permite a realização de beamformers LCMV ou LCMP adaptativos de forma computacionalmente eficiente. Isso é possível graças à partição do espaço do sinal 


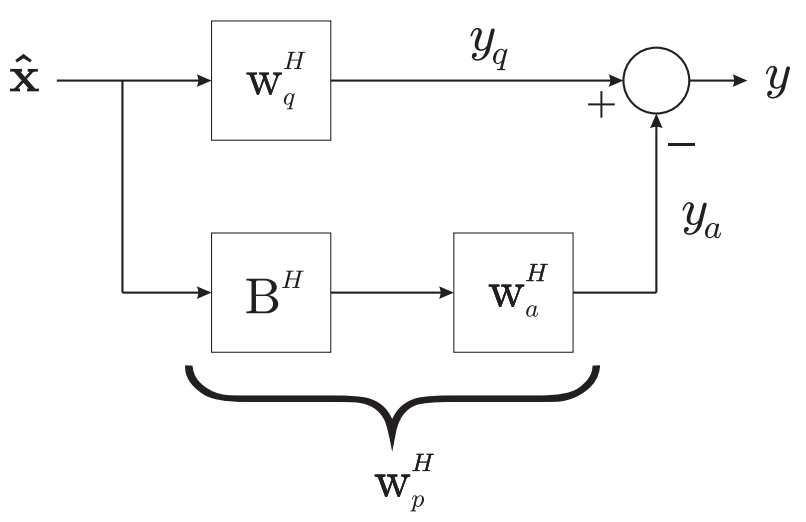

Figura 5.2: Generalized sidelobe canceller (GSC)

de entrada em um subespaço de restrições e um subespaço ortogonal. Cada vetor de entrada é decomposto em componentes ortogonais, que são processadas independentemente e então recombinadas através de uma soma. O processador adaptativo é colocado no subespaço ortogonal, tal que sua resposta sempre atende às restrições. Como este espaço tem dimensão reduzida, a adaptação exige menos recursos do que uma implementação no espaço original.

Seja $\mathbf{C}$ a matriz $N \times M$ de restrições, e $\mathbf{B}$ uma matriz $N \times(N-M)$ tal que $\mathbf{C}^{H} \mathbf{B}=\mathbf{0}$. (Note que $\mathbf{B}$ em geral não é única). Seja $\mathbf{P}_{C}=\mathbf{C}\left[\mathbf{C}^{H} \mathbf{C}\right]^{-1} \mathbf{C}^{H}$ o operador de projeção sobre o subespaço de restrições, e $\mathbf{P}_{C}^{\perp}$ seu operador ortogonal. Podemos decompor $\mathbf{w}_{o}$ em componentes ortogonais, tal que

$$
\mathbf{w}_{o}^{H}=\mathbf{w}_{q}^{H}-\mathbf{w}_{p}^{H}
$$

$\operatorname{com} \mathbf{w}_{q}^{H}=\mathbf{w}_{o}^{H} \mathbf{P}_{C}$ e $\mathbf{w}_{p}^{H}=-\mathbf{w}_{o}^{H} \mathbf{P}_{C}^{\perp}$. Por definição,

$$
\begin{aligned}
\mathbf{w}_{q}^{H} & =\mathbf{g}^{H}\left[\mathbf{C}^{H} \mathbf{S}_{\mathbf{n}}^{-1} \mathbf{C}\right]^{-1} \mathbf{C}^{H} \mathbf{S}_{\mathbf{n}}^{-1} \mathbf{C}\left[\mathbf{C}^{H} \mathbf{C}\right]^{-1} \mathbf{C}^{H} \\
& =\mathbf{g}^{H}\left[\mathbf{C}^{H} \mathbf{C}\right]^{-1} \mathbf{C}^{H}
\end{aligned}
$$

Portanto, $\mathbf{w}_{q}^{H}$ independe de $\mathbf{S}_{\mathbf{n}}$. É possível demonstrar que se o ruído for branco, $\mathbf{S}_{\mathbf{n}} \propto \mathbf{I}$ $\mathrm{e} \mathbf{w}_{q}^{H}=\mathbf{w}_{o}^{H}$. Por isso $\mathbf{w}_{q}^{H}$ é frequentemente chamado de componente quiescente de $\mathbf{w}_{o}^{H}$.

Por construção, $\mathbf{w}_{o}^{H} \mathbf{C}=\mathbf{g}^{H}$. Por outro lado, $\mathbf{w}_{o}^{H} \mathbf{C}=\mathbf{w}_{q}^{H} \mathbf{C}-\mathbf{w}_{p}^{H} \mathbf{C}=\mathbf{w}_{q}^{H} \mathbf{C}$. Logo, $\mathbf{w}_{p}^{H}$ pode variar livremente dentro de seu sub-espaço sem violar as restrições. Esta característica e a invariância $\mathbf{w}_{q}^{H}$ tornam a realização GSC popular para a implementação de algoritmos adaptativos. Como $\mathbf{w}_{p}$ pertence ao subespaço gerado pelas colunas de $\mathbf{B}$, podemos escrever $\mathbf{w}_{p}^{H}=\mathbf{w}_{a}^{H} \mathbf{B}^{H}$, onde $\mathbf{w}_{a}$ é o vetor $(N-M) \times 1$ que deve ser atualizado pelo algoritmo adaptativo. Esta estrutura de processamento está representada na Figura 5.2 . 
Para obter $\mathbf{w}_{a}^{H}$, note que a função objetivo LCMP é dada por

$$
\min \left[\mathbf{w}_{q}^{H}-\mathbf{w}_{a}^{H} \mathbf{B}^{H}\right] \mathbf{S}_{\mathbf{x}}\left[\mathbf{w}_{q}-\mathbf{B} \mathbf{w}_{a}\right]
$$

onde $\mathbf{w}_{q}^{H}$ é conhecido, pois depende somente das restrições. Avaliando $\nabla_{\mathbf{w}}$ e igualando a zero, obtemos

$$
\mathbf{w}_{a}^{H}=\mathbf{w}_{q}^{H} \mathbf{S}_{\mathbf{x}} \mathbf{B}\left[\mathbf{B}^{H} \mathbf{S}_{\mathbf{x}} \mathbf{B}\right]^{-1} .
$$

Na Seção 5.6 usamos restrições de autovetores para construir uma resposta desejada. Usando a estrutura GSC podemos fazer o caminho inverso, isto é, dada uma resposta $\mathbf{w}_{d}$ desejada, usamos restrições para impedir que ela seja distorcida. Primeiro mostremos que uma resposta $\mathbf{w}_{d}$ arbitrária pode ser transformada em resposta quiescente. Seja

$$
\widetilde{\mathbf{w}}_{d}=\frac{\mathbf{w}_{d}}{\left\|\mathbf{w}_{d}\right\|^{2}}
$$

Consideremos a restrição $\mathbf{w}_{o}^{H} \widetilde{\mathbf{w}}_{d}=1$. Usando (5.7.2) temos

$$
\mathbf{w}_{q}=\frac{\mathbf{w}_{d}}{\left\|\mathbf{w}_{d}\right\|^{2}}\left(\frac{\mathbf{w}_{d}^{H} \mathbf{w}_{d}}{\left\|\mathbf{w}_{d}\right\|^{4}}\right)^{-1}=\mathbf{w}_{d} .
$$

Devemos aumentar $\mathbf{C}$ para impedir que o filtro adaptativo modifique esta resposta em regiões de interesse. Ou seja, as restrições devem prevenir o cancelamento do sinal desejado e garantir a atenuação das interferências. Seja $\mathbf{K}$ uma região do espaço frequência-número de onda para a qual a resposta de $\mathbf{w}_{d}$ deve ser preservada. Procedendo como na Seção 5.6 , concluímos que para minimizar $\xi$ devemos impor $\mathbf{w}_{o}^{H} \boldsymbol{\Phi}_{i}=\mathbf{w}_{d}^{H} \boldsymbol{\Phi}_{i}$ para os autovetores dominantes $\boldsymbol{\Phi}_{i}$ de $\mathbf{A}=\int_{\mathbf{k} \in \mathbf{K}} \mathbf{v}(\mathbf{k}) \mathbf{v}^{H}(\mathbf{k}) d \mathbf{k}$. Porém, se $\widetilde{\mathbf{w}}_{d}$ estiver no subespaço formado pelos autovetores dominantes $\boldsymbol{\Phi}_{i}$, as restrições $\mathbf{w}_{o}^{H} \widetilde{\mathbf{w}}_{d}=1$ e $\mathbf{w}_{o}^{H} \boldsymbol{\Phi}_{i}=\mathbf{w}_{d}^{H} \boldsymbol{\Phi}_{i}$ podem ser conflitantes. Para evitar este problema e dar prioridade à restrição $\mathbf{w}_{o}^{H} \widetilde{\mathbf{w}}_{d}=1$, impomos $\mathbf{w}_{o}^{H} \widetilde{\boldsymbol{\Phi}}_{i}=\mathbf{w}_{d}^{H} \widetilde{\boldsymbol{\Phi}}_{i}$ para os autovetores dominantes $\widetilde{\boldsymbol{\Phi}}_{i}$ de $\tilde{\mathbf{A}}=\mathbf{P}_{\mathbf{w}_{d}}^{\perp} \mathbf{A} \mathbf{P}_{\mathbf{w}_{d}}^{\perp}$, com $\mathbf{P}_{\mathbf{w}_{d}}^{\perp}=\mathbf{I}-\mathbf{w}_{d}\left[\mathbf{w}_{d}^{H} \mathbf{w}_{d}\right]^{-1} \mathbf{w}_{d}^{H}$. De fato, neste caso temos $\mathbf{w}_{d}^{H} \widetilde{\boldsymbol{\Phi}}_{i}=0$, e é possível verificar por substituição direta que $\mathbf{w}_{q}=\mathbf{w}_{d}$.

\subsection{Regularização (diagonal loading)}

Na Seção 5.5 vimos que a minimização de $\mathbf{P}_{y}=\mathbf{w}^{H} \mathbf{S}_{\mathbf{x}} \mathbf{w}$ sob as restrições $\mathbf{w}^{H} \mathbf{v}\left(\mathbf{k}_{s}\right)=1$ e $\|\mathbf{w}\|^{2}=T_{0}$ leva a uma solução regularizada para o processador MPDR. A realização GSC permite-nos resolver de maneira elegante o problema equivalente submetido à restrição $\|\mathbf{w}\|^{2} \leq T_{0}$.

Consideremos primeiro o problema de minimizar $\mathbf{P}_{y}=\mathbf{w}^{H} \mathbf{S}_{\mathbf{x}} \mathbf{w}$ sob as restrições $\mathbf{C}^{H} \mathbf{w}=\mathbf{g}$ e $\|\mathbf{w}\|^{2}=T_{0}$. Usando multiplicadores de Lagrange, a função objetivo é dada por

$$
\mathbf{w}^{H} \mathbf{S}_{\mathbf{x}} \mathbf{w}+\lambda_{1}\left[\mathbf{w}^{H} \mathbf{w}-T_{0}\right]+\boldsymbol{\lambda}_{2}\left[\mathbf{C}^{H} \mathbf{w}-\mathbf{g}\right]+\left[\mathbf{w}^{H} \mathbf{C}-\mathbf{g}^{H}\right] \boldsymbol{\lambda}_{2}^{H} .
$$


Avaliando $\nabla_{\mathbf{w}}$ e igualando a zero,

$$
\mathbf{w}_{o}^{H} \mathbf{S}_{\mathbf{x}}+\lambda_{1} \mathbf{w}_{o}^{H}+\boldsymbol{\lambda}_{2} \mathbf{C}^{H}=\mathbf{0} .
$$

Isolando $\mathbf{w}_{o}^{H}$ e usando $\mathbf{C}^{H} \mathbf{w}_{o}=\mathbf{g}$, obtemos

$$
\mathbf{w}_{o}^{H}=\mathbf{g}^{H}\left[\mathbf{C}^{H}\left(\mathbf{S}_{\mathbf{x}}+\lambda_{1} \mathbf{I}\right)^{-1} \mathbf{C}\right]^{-1} \mathbf{C}^{H}\left(\mathbf{S}_{\mathbf{x}}+\lambda_{1} \mathbf{I}\right)^{-1},
$$

que é uma generalização de (5.5.4).

Consideremos a realização GSC da expressão (5.8.3). Consideremos que $\mathbf{B}^{H} \mathbf{B}=\mathbf{I}$ (por exemplo, escolhendo as colunas de $\mathbf{B}$ como $N-M$ vetores ortonormais do espaço imagem de $\mathbf{P}_{C}^{\perp}=\mathbf{I}-\mathbf{P}_{C}$ ). Na configuração GSC,

$$
\mathbf{w}^{H}=\mathbf{w}_{q}^{H}-\mathbf{w}_{a}^{H} \mathbf{B}^{H} .
$$

Logo,

$$
\begin{aligned}
\|\mathbf{w}\|^{2} & =\mathbf{w}^{H} \mathbf{w} \\
& =\mathbf{w}_{q}^{H} \mathbf{w}_{q}+\mathbf{w}_{a}^{H} \mathbf{B}^{H} \mathbf{B} \mathbf{w}_{a} \\
& =\mathbf{w}_{q}^{H} \mathbf{w}_{q}+\mathbf{w}_{a}^{H} \mathbf{w}_{a} \leq T_{0} .
\end{aligned}
$$

Como $\mathbf{w}_{q}^{H}$ independe de $T_{0}$ e é conhecido, podemos reescrever (5.8.5) como

$$
\mathbf{w}_{a}^{H} \mathbf{w}_{a} \leq T_{0}-\mathbf{w}_{q}^{H} \mathbf{w}_{q}=K
$$

Usando (5.7.4) e substituindo $\mathbf{S}_{\mathbf{x}} \leftarrow \mathbf{S}_{\mathbf{x}}+\lambda_{1} \mathbf{I}$, obtemos a expressão de $\mathbf{w}_{a}^{H}$. Analisando $\frac{d}{d \lambda_{1}} \mathbf{w}_{a}^{H} \mathbf{w}_{a}$, é possível mostrar que $\left\|\mathbf{w}_{a}\right\|$ é função monótona decrescente de $\lambda_{1}$. Logo, (5.8.6) pode ser resolvido por qualquer método tradicional de aproximações sucessivas.

\subsection{Realizações em espaços de dimensões reduzidas}

\subsubsection{Cenário 1: alta SNR/INR}

Consideremos a decomposição de $\mathbf{S}_{\mathbf{x}}$ em seus autovalores $\lambda_{1} \geq \cdots \geq \lambda_{N}$ e autovetores $\boldsymbol{\Phi}_{i}$, tal que

$$
\mathbf{S}_{\mathbf{x}}=\sum_{i=1}^{N} \lambda_{i} \boldsymbol{\Phi}_{i} \boldsymbol{\Phi}_{i}^{H}=\mathbf{U} \boldsymbol{\Lambda} \mathbf{U}^{H}
$$

onde

$$
\begin{aligned}
\mathbf{U} & =\left[\begin{array}{llll}
\boldsymbol{\Phi}_{1} & \boldsymbol{\Phi}_{2} & \cdots & \boldsymbol{\Phi}_{N}
\end{array}\right] \\
\mathbf{\Lambda} & =\operatorname{diag}\left[\begin{array}{llll}
\lambda_{1} & \lambda_{2} & \cdots & \lambda_{N}
\end{array}\right] .
\end{aligned}
$$


Assim como na Seção 3.3, desejamos processar o sinal de entrada em um subespaço de dimensão reduzida. Para isso, primeiro consideramos o sub-espaço gerado pelos $M$ autovalores dominantes de $\mathbf{S}_{\mathbf{x}}$. Usamos como motivação para esta escolha o fato de que para sinais com alta SNR ou INR, os autovalores dominantes são com boa aproximação uma base para o sub-espaço gerado por seus vetores diretores. Denotando por $\mathbf{U}_{S+I}$ e $\Lambda_{S+I}$ os $M$ autovetores e autovalores dominantes, e por $\mathbf{U}_{N}$ e $\Lambda_{N}$ os autovetores e autovalores restantes, podemos escrever

$$
\begin{aligned}
\mathbf{S}_{\mathbf{x}} & =\mathbf{U}_{S+I} \boldsymbol{\Lambda}_{S+I} \mathbf{U}_{S+I}^{H}+\mathbf{U}_{N} \boldsymbol{\Lambda}_{N} \mathbf{U}_{N}^{H} \\
\mathbf{S}_{\mathbf{x}}^{-1} & =\mathbf{U}_{S+I} \boldsymbol{\Lambda}_{S+I}^{-1} \mathbf{U}_{S+I}^{H}+\mathbf{U}_{N} \boldsymbol{\Lambda}_{N}^{-1} \mathbf{U}_{N}^{H} .
\end{aligned}
$$

Em cenários de ruído espacialmente branco com $\mathbf{S}_{\mathbf{n}}=\sigma_{n}^{2} \mathbf{I}$,

$$
\lambda_{i}= \begin{cases}\lambda_{s, i}+\sigma_{n}^{2} & i \leq M \\ \sigma_{n}^{2} & i>M\end{cases}
$$

onde $\lambda_{s, i}$ é a componente de sinal do i-ésimo autovetor. Os subespaços gerados pelas colunas de $\mathbf{U}_{S+I}$ e $\mathbf{U}_{N}$ são chamados respectivamente de subespaço do sinal e subespaço do ruído. (Apesar disso, por definição o subespaço do sinal contém interferências, e a observação acima mostra que também contém ruído.)

Seja $\mathbf{v}\left(\mathbf{k}_{s}\right)$ o vetor diretor do sinal de interesse. Como $\mathbf{U}_{N}^{H} \mathbf{v}\left(\mathbf{k}_{s}\right)=\mathbf{0}$, o processador MPDR é dado por

$$
\mathbf{w}^{H}=\frac{\mathbf{v}^{H}\left(\mathbf{k}_{s}\right) \mathbf{U}_{S+I} \boldsymbol{\Lambda}_{S+I}^{-1} \mathbf{U}_{S+I}^{H}}{\mathbf{v}^{H}\left(\mathbf{k}_{s}\right) \mathbf{U}_{S+I} \boldsymbol{\Lambda}_{S+I}^{-1} \mathbf{U}_{S+I}^{H} \mathbf{v}\left(\mathbf{k}_{s}\right)} .
$$

Na linguagem de processadores em espaços de feixes, temos $\mathbf{B}_{b s}=\mathbf{U}_{S+I}^{H}$. De fato, usando que

$$
\begin{aligned}
\hat{\mathbf{x}}_{b s} & =\mathbf{B}_{b s} \hat{\mathbf{x}} \\
\mathbf{v}_{b s}\left(\mathbf{k}_{s}\right) & =\mathbf{B}_{b s} \mathbf{v}\left(\mathbf{k}_{s}\right),
\end{aligned}
$$

então

$$
\mathbf{w}_{b s}^{H}=\frac{\mathbf{v}_{b s}^{H}\left(\mathbf{k}_{s}\right) \Lambda_{S+I}^{-1}}{\mathbf{v}_{b s}^{H}\left(\mathbf{k}_{s}\right) \boldsymbol{\Lambda}_{S+I}^{-1} \mathbf{v}_{b s}\left(\mathbf{k}_{s}\right)}
$$

Na Seção 3.3 alertamos que a construção incorreta do sub-espaço poderia causar perda irreversível de informação. Neste caso, isso ocorre se a dimensão $M$ for exageradamente pequena, tal que contribuições de sinal ou de interferências serão erroneamente desprezadas. 


\subsubsection{Cenário 2: baixa SNR e alta INR}

Para sinais com baixa SNR e interferências com alta INR, [28] propõe o beamformer dominant mode rejection (DMR). Novamente partimos de uma decomposição em autovalores e autovetores, e denotamos por $\mathbf{U}_{d m}$ e $\Lambda_{d m}$ os $M$ autovetores e autovalores dominantes, que dão origem ao chamado subespaço de modos dominantes. Organizamos os autovalores restantes em uma matriz $\mathbf{U}_{d m}^{\perp}$ (que não deve ser confundida com a matriz de projeção). Geramos então uma matriz espectral $\widetilde{\mathbf{S}}_{\mathbf{x}}$ para a qual os $(N-M)$ autovalores menos significativos são substituídos por sua média

$$
\sigma_{n}^{2}=\frac{1}{N-M} \sum_{i=M+1}^{N} \lambda_{i}
$$

Portanto,

$$
\begin{aligned}
\widetilde{\mathbf{S}}_{\mathbf{x}} & =\mathbf{U}_{d m} \boldsymbol{\Lambda}_{d m} \mathbf{U}_{d m}^{H}+\sigma_{n}^{2} \mathbf{U}_{d m}^{\perp}\left(\mathbf{U}_{d m}^{\perp}\right)^{H} \\
\widetilde{\mathbf{S}}_{\mathbf{x}}^{-1} & =\mathbf{U}_{d m} \boldsymbol{\Lambda}_{d m}^{-1} \mathbf{U}_{d m}^{H}+\sigma_{n}^{-2} \mathbf{U}_{d m}^{\perp}\left(\mathbf{U}_{d m}^{\perp}\right)^{H}
\end{aligned}
$$

O beamformer DMR é por definição um beamformer MPDR construído usando $\widetilde{\mathbf{S}}_{\mathbf{x}}^{-1}$.

Consideremos o que ocorre quando $\mathbf{v}\left(\mathbf{k}_{s}\right)$ pertence ao subespaço de modos dominantes (i.e., o cenário é de alta SNR). Na ausência de erros de estimação, a resposta do processador ao sinal de interesse é dada por

$$
\mathbf{w}^{H} \mathbf{v}\left(\mathbf{k}_{s}\right)=\frac{\sum_{i=1}^{M} \frac{1}{\lambda_{i}}\left|\mathbf{v}^{H}\left(\mathbf{k}_{s}\right) \boldsymbol{\Phi}_{i}\right|^{2}}{\sum_{i=1}^{M} \frac{1}{\lambda_{i}}\left|\mathbf{v}^{H}\left(\mathbf{k}_{s}\right) \boldsymbol{\Phi}_{i}\right|^{2}}=1 .
$$

A potência de saída do array quando submetido a uma entrada com espectro $\lambda_{k} \boldsymbol{\Phi}_{k} \boldsymbol{\Phi}_{k}^{H}$, para $k \in\{1, \ldots, M\}$ é

$$
P_{y}\left(\boldsymbol{\Phi}_{i}\right)=\frac{\frac{1}{\lambda_{k}}\left|\mathbf{v}^{H}\left(\mathbf{k}_{s}\right) \boldsymbol{\Phi}_{k}\right|^{2}}{\left[\sum_{i=1}^{M} \frac{1}{\lambda_{i}}\left|\mathbf{v}^{H}\left(\mathbf{k}_{s}\right) \boldsymbol{\Phi}_{i}\right|^{2}\right]^{2}} .
$$

Como

$$
\frac{1}{\lambda_{i}}=\frac{1}{\lambda_{s, i}+\sigma_{n}^{2}}=\frac{1 / \sigma_{n}^{2}}{\lambda_{s, i} / \sigma_{n}^{2}+1}
$$

então

$$
P_{y}\left(\boldsymbol{\Phi}_{i}\right)=\sigma_{n}^{2} \frac{\frac{1}{\lambda_{s, k} / \sigma_{n}^{2}+1}\left|\mathbf{v}^{H}\left(\mathbf{k}_{s}\right) \boldsymbol{\Phi}_{k}\right|^{2}}{\left[\sum_{i=1}^{M} \frac{1}{\lambda_{s, i} / \sigma_{n}^{2}+1}\left|\mathbf{v}^{H}\left(\mathbf{k}_{s}\right) \boldsymbol{\Phi}_{i}\right|^{2}\right]^{2}} .
$$

Se $\lambda_{s, k} / \sigma_{n}^{2} \gg 1$, então $P_{y}\left(\boldsymbol{\Phi}_{i}\right)$ é pequeno e o k-ésimo modo dominante é rejeitado. Quando $M$ é igual à quantidade de interferências com alta INR, o processador DMR tem ganho comparável ao MVDR, com a vantagem de operar sobre um espaço de apenas $M$ dimensões e exigir somente a determinação de autovalores e autovetores dominantes. 
No entanto, destacamos que a análise acima foi realizada sob a hipótese de alta SNR e estimação perfeita de $\mathbf{v}\left(\mathbf{k}_{s}\right)$. Na presença de erros de estimação o beamformer DMR apresenta séria degradação de desempenho. De fato, quanto maior for a SNR, pior será o ganho do array. Para mitigar esses problemas, [29] propõe reduzir a contribuição de autovetores que apresentam alta correlação com o sinal de interesse, de forma a preservar o lóbulo principal. Esta técnica tem efeito similar ao uso de restrições, porém com custo computacional inferior. A regularização de $\widetilde{\mathbf{S}}_{\mathbf{x}}$ também apresenta bons resultados.

O beamformer DMR tem aplicação de maior interesse em cenários de SNR baixa, quando o sinal desejado não pertence ao subespaço de modos dominantes. Nestes casos, o ganho do processador DMR se aproxima do ganho MVDR com estimação perfeita.

\subsection{Desempenho para sinais correlacionados}

Até este ponto admitimos que os sinais emitidos pelas fontes eram descorrelacionados. Veremos a seguir que os beamformers ótimos apresentados sofrem considerável degradação de desempenho na presença de correlações. Em particular, para SNRs e INRs altas a presença de interferências coerentes tornará nula a saída do processador.

Consideremos um sinal determinístico da forma

$$
\hat{\mathbf{x}}=\mathbf{v}\left(\mathbf{k}_{s}\right) \hat{f}_{s}+\sum_{i=1}^{M} \mathbf{v}\left(\mathbf{k}_{i}\right) \hat{f}_{i}+\hat{\mathbf{n}}
$$

onde $\hat{f}_{s}$ é um sinal de interesse, $\hat{f}_{i}$ são interferências e $\hat{f}_{i}=\gamma_{i} \hat{f}_{s}$, com $\gamma_{i} \in \mathbb{C}$. Admitimos que os sinais e as interferências têm potências altas em relação ao ruído $\mathbf{N}$. Definindo

$$
\begin{aligned}
\mathbf{V} & =\left[\begin{array}{llll}
\mathbf{v}\left(\mathbf{k}_{s}\right) & \mathbf{v}\left(\mathbf{k}_{1}\right) & \cdots & \mathbf{v}\left(\mathbf{k}_{M}\right)
\end{array}\right] \\
\hat{\mathbf{f}} & =\left[\begin{array}{llll}
\hat{f}_{s} & \gamma_{1} \hat{f}_{s} & \cdots & \gamma_{M} \hat{f}_{s}
\end{array}\right]^{T},
\end{aligned}
$$

podemos escrever

$$
\begin{aligned}
\hat{\mathbf{x}} & =\mathbf{V} \hat{\mathbf{f}}+\hat{\mathbf{n}} . \\
\mathbf{S}_{\mathbf{x}} & =\mathbf{V S}_{\mathbf{f}} \mathbf{V}^{H}+\mathbf{S}_{\mathbf{n}} .
\end{aligned}
$$

$\mathbf{S}_{\mathbf{f}}=\mathrm{E}\left\{\hat{\mathbf{f}} \hat{\mathbf{f}}^{H}\right\}$ tem posto 1, tal que o subespaço do sinal de $\mathbf{S}_{\mathbf{x}}$ também tem (aproximadamente) posto 1 .

É fácil verificar que $\boldsymbol{\Phi}_{1}=\mathbf{v}\left(\mathbf{k}_{s}\right)+\sum_{i=1}^{M} \gamma_{i} \mathbf{v}\left(\mathbf{k}_{i}\right)$. Portanto, o processador MPDR tem 
a forma

$$
\begin{aligned}
\mathbf{w}^{H} & \propto \mathbf{v}^{H}\left(\mathbf{k}_{s}\right) \mathbf{S}_{\mathbf{x}}^{-1} \\
& =\mathbf{v}^{H}\left(\mathbf{k}_{s}\right)\left[\frac{1}{\lambda_{s, 1}+\sigma_{n}^{2}} \boldsymbol{\Phi}_{1} \boldsymbol{\Phi}_{1}^{H}+\sum_{i=2}^{N} \frac{1}{\sigma_{n}^{2}} \boldsymbol{\Phi}_{i} \boldsymbol{\Phi}_{i}^{H}\right] \\
& \approx \mathbf{v}^{H}\left(\mathbf{k}_{s}\right)\left[\sum_{i=2}^{N} \frac{1}{\sigma_{n}^{2}} \boldsymbol{\Phi}_{i} \boldsymbol{\Phi}_{i}^{H}\right] .
\end{aligned}
$$

Como $\hat{\mathbf{x}}=\boldsymbol{\Phi}_{1} \hat{f}_{s}+\hat{\mathbf{n}}$ e $\boldsymbol{\Phi}_{i}^{H} \boldsymbol{\Phi}_{1}=0$ para $i \geq 2$, então

$$
\mathbf{w}^{H} \hat{\mathbf{x}}=\mathbf{w}^{H} \hat{\mathbf{n}} .
$$

Portanto, para sinais e interferências coerentes a saída do processador é uma combinação do ruído incidente.

Consideremos agora um cenário com correlação arbitrária entre um sinal desejado e uma interferência. A seguir resumimos um resultado de [30]. Seja

$$
\hat{\mathbf{x}}=\mathbf{v}_{s} \hat{f}_{s}+\gamma \mathbf{v}_{I} \hat{f}_{I}
$$

$\operatorname{com} \mathrm{E}\left\{\hat{f}_{s}\right\}=\mathrm{E}\left\{\hat{f}_{I}\right\}=1,\left\|\mathbf{v}\left(\mathbf{k}_{s}\right)\right\|=\left\|\mathbf{v}\left(\mathbf{k}_{I}\right)\right\|=1, \mathbf{v}_{s}^{H} \mathbf{v}_{I}=0$ e $\rho=\mathrm{E}\left\{\hat{f}_{I} \hat{f}_{s}^{*}\right\}$. Então

$$
\mathbf{S}_{\mathbf{x}}=\mathbf{v}_{s} \mathbf{v}_{s}^{H}+\gamma \rho \mathbf{v}_{I} \mathbf{v}_{s}^{H}+\gamma^{*} \rho^{*} \mathbf{v}_{s} \mathbf{v}_{I}^{H}+|\gamma|^{2} \mathbf{v}_{I} \mathbf{v}_{I}^{H} .
$$

O beamformer MPDR, a potência na saída do array e a resposta na direção $\mathbf{v}_{I}$ são dados respectivamente por

$$
\begin{aligned}
\mathbf{w}^{H} & =\mathbf{v}_{s}^{H}-\frac{\rho}{\gamma} \mathbf{v}_{I}^{H} \\
P_{y} & =1-|\rho|^{2} \\
\mathbf{w}^{H} \mathbf{v}_{I} & =-\frac{\rho}{\gamma} .
\end{aligned}
$$

Portanto, a correlação entre sinais causa uma queda na potência de saída do array com uma correspondente perda de ganho. Isso ocorre porque o array permite a combinação destrutiva do sinal desejado com a interferência correlacionada.

O problema de fontes correlacionadas foi percebido com as primeiras implementações de arrays ótimos, e foi amplamente estudado. Referências notáveis são [30-34]. Uma proposta para reduzir a correlação e remover a singularidade de sinais coerentes foi apresentada pela primeira vez por Evans em [35] sob o nome spatial smoothing. A técnica consiste em particionar um ULA de $N$ elementos em $L=N-M+1$ subarrays lineares uniformes, cada um com $M$ elementos. A matriz espectral usada é a média das matrizes 
espectrais dos subarrays.

Se um número grande de subarrays for usado, obtém-se bons resultados de descorrelação. Por outro lado, a resolução angular do processador será pior. Como a escolha de $M$ é dependente das correlações encontradas, em alguns cenários pode ser difícil estimar a priori qual valor usar. [36] propõe escolher $M$ que maximiza a distância entre os dois autovalores menos significativos do subespaço do sinal.

A versão de spatial smoothing apresentada é também conhecida como forward spatial smoothing. Se $\mathbf{S}_{\mathbf{x}, i}=\hat{\mathbf{x}}_{i} \hat{\mathbf{x}}_{i}^{H}$ for a matriz espectral do i-ésimo sub-array (para $1 \leq i \leq L$ ), usamos a estimativa

$$
\widetilde{\mathbf{S}}_{\mathbf{x}}=\frac{1}{L} \sum_{i=1}^{L} \mathbf{S}_{\mathbf{x}, i}
$$

Uma modificação proposta por [34] que produz estimativas menos correlacionadas é chamada de forward-backward spatial smoothing e consiste em usar

$$
\widetilde{\mathbf{S}}_{\mathbf{x}}=\frac{1}{2 L} \sum_{i=1}^{L}\left(\mathbf{S}_{\mathbf{x}, i}+\mathbf{J}\left[\mathbf{S}_{\mathbf{x}, i}\right]^{*} \mathbf{J}\right),
$$

onde $J$ é a matriz de permutação

$$
J=\left[\begin{array}{cccc}
0 & 0 & 0 & 1 \\
\vdots & \vdots & 1 & 0 \\
0 & . \cdot & \vdots & \vdots \\
1 & 0 & 0 & 0
\end{array}\right]
$$

Para o caso particular de dois sinais separados por uma distância $\Delta \psi$ (com $\psi$ dado por (2.4.3)) e com coeficiente de correlação $\rho$, seu coeficiente de correlação $\rho_{F}$ após o procedimento de forward smoothing será

$$
\rho_{F}=\frac{\rho}{L} \frac{\sin \left(\frac{L \Delta \psi}{2}\right)}{\sin \left(\frac{\Delta \psi}{2}\right)} .
$$

Usando forward/backward smoothing, o coeficiente de correlação se torna

$$
\rho_{F B}=\frac{\operatorname{Re}\{\rho\}}{L} \frac{\sin \left(\frac{L \Delta \psi}{2}\right)}{\sin \left(\frac{\Delta \psi}{2}\right)} .
$$

O bom desempenho da técnica forward-backward smoothing merece uma justificativa. Para arrays com simetria em relação a um ponto,

$$
\left[\mathbf{S}_{\mathbf{x}}\right]_{i j}=\left[\mathbf{S}_{\mathbf{x}}\right]_{N-i+1, N-j+1}^{*}=\left[\mathbf{S}_{\mathbf{x}}\right]_{j i}^{*}
$$


ou seja, $\mathbf{S}_{\mathbf{x}}$ apresenta simetria complexa em relação à diagonal principal e à diagonal cruzada. Isso implica que $\mathbf{S}_{\mathbf{x}}=\mathbf{J S}_{\mathbf{x}}^{*} \mathbf{J}$. Conforme demonstra [37] para o contexto de arrays, a média forward-backward gera a estimativa ML sujeita à restrição $\widetilde{\mathbf{S}}_{\mathbf{x}}=\mathbf{J} \widetilde{\mathbf{S}}_{\mathbf{x}}^{*} \mathbf{J}$ quando as amostras $\left\{\hat{\mathbf{x}}_{i}\right\}_{i=1}^{L}$ adquiridas pelos subarrays são vetores aleatórios Gaussianos independentes e identicamente distribuídos.

\subsection{Calibração}

A calibração de arrays é uma etapa imprescindível em aplicações reais, mas que recebe relativamente pouca atenção na literatura. Erros de calibração podem comprometer completamente o desempenho de um array de baixo custo, potencialmente inviabilizando seu uso. Se os parâmetros físicos de um array forem conhecidos com boa precisão, processadores menos tolerantes a erros podem ser usados, melhorando o desempenho do conjunto.

Nosso objetivo nesta seção é apresentar ideias gerais sobre como compensar erros de posicionamento, ganho, fase e acoplamento mútuo entre sensores. É importante destacar que para arrays banda larga, os métodos a seguir devem ser implementados para todas as raias da transformada de Fourier do sinal de entrada.

Consideremos um array ideal (i.e., sem perturbações) sobre o qual incide um sinal determinístico $\hat{f}_{s}$ com direção $\mathbf{k}_{0}$, tal que o sinal recebido é dado por $\hat{\mathbf{x}}=\mathbf{v}\left(\mathbf{k}_{0}\right) \hat{f}_{s}$. Consideremos que este array foi perturbado, e denotemos por $\breve{\mathrm{x}}$ o sinal recebido e por $\breve{\mathbf{v}}\left(\mathbf{k}_{0}\right)$ o vetor diretor na presença de imperfeições. Seja $\mathbf{Q}$ uma matriz de correção tal que

$$
\breve{\mathbf{v}}\left(\mathbf{k}_{0}\right)=\mathbf{Q v}\left(\mathbf{k}_{0}\right) .
$$

Observe que na ausência de ruído temos $\hat{\mathbf{x}}=\mathbf{Q}^{-1} \breve{\mathbf{x}}$. Através de $\mathbf{Q}$ podemos transformar os vetores diretores de um array imperfeito, de forma a obter um array virtual com as características esperadas. Este procedimento é conhecido na literatura como interpolação, e tem aplicações que vão além da calibração. Por exemplo, uma aplicação consiste em interpolar setores de arrays circulares, transformando-os em arrays virtuais com estrutura linear uniforme, para os quais existem algoritmos computacionalmente eficientes para estimação de direção.

Dizemos que Q é global se independe da direção de chegada, e local se é função de $\mathbf{k}$. Para obter matrizes de correção, primeiro coletamos dados de calibração. Um transmissor é movido sobre um grid de calibração ao longo das direções $\mathbf{k}_{1}, \ldots, \mathbf{k}_{M}$. Para cada direção $\mathbf{k}_{i}$, o vetor diretor $\breve{\mathbf{v}}\left(\mathbf{k}_{i}\right)$ é escolhido como o autovetor dominante de $\mathbf{S}_{\mathbf{x}}$. Sejam $\mathbf{V}$ a matriz com os vetores diretores ideais e $\breve{\mathbf{V}}$ a matriz com os vetores diretores estimados. A matriz de correção global é dada por

$$
\mathbf{Q}=\underset{\mathbf{Q}}{\operatorname{argmin}}\|\breve{\mathbf{V}}-\mathbf{Q} \mathbf{V}\|_{F}
$$


onde $\|\cdot\|_{F}$ é a norma de Frobenius. A matriz de correção local é dada por [38]

$$
\mathbf{Q}(\mathbf{k})=\underset{\mathbf{Q}}{\operatorname{argmin}}\|(\breve{\mathbf{V}}-\mathbf{Q V}) \mathbf{W}(\mathbf{k})\|_{F},
$$

onde $\mathbf{W}(\mathbf{k})$ é uma matriz diagonal de pesos, com

$$
[\mathbf{W}(\mathbf{k})]_{j j}=e^{-h\left\|\mathbf{k}-\mathbf{k}_{i}\right\|}
$$

O parâmetro $h$ controla o quão local a matriz de correção deve ser. [39] impõe que matrizes de correção locais devem ser diagonais, e mesmo assim obtém melhores resultados para localização de fontes do que usando matrizes de correção globais.

A técnica acima é suficientemente simples, mas depende do posicionamento correto de uma fonte ao longo de um grid. Erros de posicionamento são inevitáveis, e implicam em erros de calibração. Como alternativa temos a estimação ML conjunta dos parâmetros do array e das fontes de sinal [40]. Sob a suposição de que os vetores diretores $\breve{\mathbf{v}}\left(\mathbf{k}_{i}\right)$ apresentam uma distribuição Gaussiana em torno de seus valores verdadeiros, a estimativa ML de $\mathbf{Q}$ é dada por

$$
\mathbf{Q}=\underset{\mathbf{Q}, \mathbf{k}_{1}, \ldots, \mathbf{k}_{M}}{\operatorname{argmin}}\|\breve{\mathbf{V}}-\mathbf{Q V}\|_{F}
$$

Um procedimento de otimização iterativa é proposto em [40], onde a estimação de $\mathbf{Q}$ e de $\mathbf{k}_{1}, \ldots, \mathbf{k}_{M}$ é desacoplada, e a otimização de $\mathbf{k}_{1}, \ldots, \mathbf{k}_{M}$ é feita pelo método de Newton.

Um estimador ML é proposto em [41], para o caso em que somente as coordenadas dos sensores são desejadas. Um conjunto com pelo menos 5 fontes com localizações desconhecidas é usado, e os atrasos de propagação entre todas as fontes e sensores são estimados usando o algoritmo GCC-PHAT (generalized cross-correlation with phase transform). 



\section{Capítulo 6}

\section{Estimação de direção de chegada}

\subsection{Introdução}

Para que os algoritmos explorados até agora possam ser projetados, devemos ser capazes de estimar o número de sinais e a direção de chegada $\mathbf{v}\left(\mathbf{k}_{s}\right)$ do sinal desejado. No caso geral, gostaríamos de estimar um vetor $\boldsymbol{\theta}$ com todas as direções de sinais incidentes. Para os casos em que $\boldsymbol{\theta}$ é determinístico, sob condições fracas de regularidade demonstra-se que o estimador ML assintoticamente tende ao estimador ótimo da família dos estimadores não-viesados [15]. Para o caso em que $\boldsymbol{\theta}$ é um vetor aleatório, resultados análogos de eficiência podem ser obtidos para o estimador MAP.

Na prática, estimadores MAP e ML são pouco usados em aplicações de processamento de arrays. A quantidade de parâmetros a serem estimados é tipicamente grande, tornando proibitivo o custo computacional das buscas multidimensionais envolvidas. Os algoritmos descritos nesta seção tornaram-se alternativas populares por apresentarem um bom equilíbrio entre desempenho e complexidade computacional.

\subsection{Métodos de varredura}

A forma mais simples e tradicional de estimar a magnitude de um campo consiste em sintetizar uma resposta fixa com um único lóbulo principal e usá-la para amostrar uma região espacial de interesse. Quanto mais estreito for o lóbulo principal, mais precisa será a estimação.

Usando uma resposta delay-and-sum, a potência de saída para uma direção $(\theta, \phi)$ é dada por

$$
P(\omega, \theta, \phi)=\mathbf{v}^{H}(\omega, \theta, \phi) \mathbf{S}_{\mathbf{x}}(\omega) \mathbf{v}(\omega, \theta, \phi),
$$

onde $\mathbf{S}_{\mathbf{x}}(\omega)$ é a matriz espectral estimada para a frequência $\omega$. Esta resposta pode ser generalizada para uma resposta sintetizada através da matriz diretora $\mathbf{I}_{s}(\omega, \theta, \phi)$, tal que

$$
P(\omega, \theta, \phi)=\mathbf{w}^{H}(\omega) \mathbf{I}_{s}(\omega, \theta, \phi) \mathbf{S}_{\mathbf{x}}(\omega) \mathbf{I}_{s}^{H}(\omega, \theta, \phi) \mathbf{w}(\omega)
$$

Se usarmos $\mathbf{w}^{H}=\mathbf{w}_{\text {MPDR }}^{H}$, teremos a estimativa apresentada por Capon em [21]. A 
potência de saída para este caso é dada por

$$
P(\omega, \theta, \phi)=\frac{1}{\mathbf{v}^{H}(\omega, \theta, \phi) \mathbf{S}_{\mathbf{x}}^{-1}(\omega) \mathbf{v}(\omega, \theta, \phi)}
$$

A análise do beamformer Capon revela que sua resolução é consideravelmente superior à de beamformers convencionais, na presença de fontes pontuais. Uma forma de obter desempenho ainda melhor para o problema de identificação de fontes consiste em analisar o denominador $\mathbf{v}^{H}(\mathbf{k}) \mathbf{S}_{\mathbf{x}}^{-1} \mathbf{v}(\mathbf{k})$, que no caso de ULAs pode ser escrito como

$$
Q_{\psi}(\psi)=\mathbf{v}^{H}(\psi) \mathbf{S}_{\mathbf{x}}^{-1} \mathbf{v}(\psi)
$$

Escolhendo um sistema de coordenadas conveniente, podemos escrever

$$
\mathbf{v}(\psi)=\left[\begin{array}{llll}
1 & e^{j \psi} & \cdots & e^{j(N-1) \psi}
\end{array}\right]^{T} .
$$

Substituindo $z=e^{j \psi}$, podemos transformar $\mathbf{v}^{H}(\psi) \mathbf{S}_{\mathbf{x}}^{-1} \mathbf{v}(\psi)$ em um polinômio em $z$. Determinar os máximos de $P(\omega, \theta, \phi)$ equivale a detectar os mínimos de $Q(z)=\mathbf{v}^{H}(z) \mathbf{S}_{\mathbf{x}}^{-1} \mathbf{v}(z)$ sobre a circunferência unitária. Graças à simetria Hermitiana de $\mathbf{S}_{\mathbf{x}}^{-1}, Q(z)$ possui coeficientes complexos conjugados e pode ser fatorado na forma $Q(z)=H(z) H^{*}\left(1 / z^{*}\right)$. Para cada raiz $z_{i}$ de $Q(z)$ próxima da circunferência unitária podemos determinar com boa aproximação um mínimo $\hat{\psi}_{i}$ com a forma

$$
\hat{\psi}_{i}=\frac{\arg z_{i}}{\pi}
$$

que representa a direção de chegada de um sinal.

\subsection{MUSIC}

O algoritmo MUSIC (multiple signal classification) [42] para detecção de ângulos de chegada é a solução mais tradicional para obter estimativas de super-resolução, ou seja, com resolução superior à dos beamformers da seção anterior.

Seja $\hat{\mathbf{x}}$ formado por $M$ ondas planas com vetores diretores $\mathbf{v}_{i}=\mathbf{v}\left(\mathbf{k}_{i}\right)$ e ruído espacialmente branco. Sua matriz espectral é dada por

$$
\mathbf{S}_{\mathbf{x}}=\mathbf{V S}_{\mathbf{f}} \mathbf{V}^{H}+\sigma_{n}^{2} \mathbf{I}
$$

Consideremos a decomposição de $\mathbf{S}_{\mathbf{x}}$ em subespaços do sinal e do ruído, conforme a Seção 5.9:

$$
\mathbf{S}_{\mathbf{x}}=\mathbf{U}_{S} \boldsymbol{\Lambda}_{S} \mathbf{U}_{S}^{H}+\mathbf{U}_{N} \boldsymbol{\Lambda}_{N} \mathbf{U}_{N}^{H}
$$

Seja $\mathbf{v}_{i}$ o vetor diretor da i-ésima fonte. $\mathbf{v}_{i}$ pertence ao espaço do sinal, tal que é combina- 
ção linear das colunas de $\mathbf{U}_{S}$. Como $\mathbf{U}_{S}^{H} \mathbf{U}_{N}=\mathbf{0}$, temos $\mathbf{v}_{i}^{H} \mathbf{U}_{N}=\mathbf{0}$ ou alternativamente, $\mathbf{v}_{i}^{H} \mathbf{U}_{N} \mathbf{U}_{N}^{H} \mathbf{v}_{i}=0$.

Para o caso particular de um array linear uniforme, o algoritmo MUSIC consiste em determinar os mínimos de

$$
Q_{\psi}(\psi)=\mathbf{v}^{H}(\psi) \mathbf{U}_{N} \mathbf{U}_{N}^{H} \mathbf{v}(\psi)
$$

Note que (6.3.3) e (6.2.4) têm a mesma forma. Portanto, a mesma estratégia de fatoração e busca de raízes usada na seção anterior se aplica aqui. Este algoritmo é conhecido como root-MUSIC [43], e tem ampla difusão na literatura. Uma versão mais apropriada para implementação foi proposta em [44] sob o nome unitary root-MUSIC, e tem as vantagens de só exigir aritmética real e automaticamente aplicar forward-backward averaging.

\subsection{ESPRIT}

A seguir descrevemos o algoritmo ESPRIT (estimation of signal parameters via rotational invariance techniques), que foi desenvolvido por Roy em $[45,46]$.

Consideremos um ULA com elementos nas coordenadas $\mathbf{p}_{i}$, com $1 \leq i \leq N$. Consideremos dois subarrays que também sejam ULAs, cada um com $N_{s}$ elementos e com a propriedade que um subarray pode ser obtido a partir de uma translação do outro. Seja $d_{s} \cdot d$ a medida da translação, onde $d$ é o espaçamento entre elementos do array principal. Por exemplo, dado um array inicial com $N=10$ elementos, $\left\{\mathbf{p}_{i}\right\}_{i=1}^{9}$ e $\left\{\mathbf{p}_{i}\right\}_{i=2}^{10}$ são possíveis subarrays com $d_{s}=1$. Outro exemplo seria $\left\{\mathbf{p}_{1}, \mathbf{p}_{3}, \mathbf{p}_{5}, \mathbf{p}_{7}\right\}$ e $\left\{\mathbf{p}_{4}, \mathbf{p}_{6}, \mathbf{p}_{8}, \mathbf{p}_{10}\right\}$, com $d_{s}=3$. Seja $M$ o número de sinais incidentes, com $M<N_{s}$ e vetores diretores $\mathbf{v}\left(\mathbf{k}_{1}\right), \ldots, \mathbf{v}\left(\mathbf{k}_{M}\right)$. A matriz $V$ de vetores diretores é dada por

$$
\mathbf{V}=\left[\begin{array}{llll}
\mathbf{v}\left(\mathbf{k}_{1}\right) & \mathbf{v}\left(\mathbf{k}_{2}\right) & \cdots & \mathbf{v}\left(\mathbf{k}_{M}\right)
\end{array}\right]
$$

Sejam $\mathbf{V}_{1}$ e $\mathbf{V}_{2}$ as matrizes diretoras dos subarrays. Podemos escrever

$$
\begin{aligned}
\mathbf{V}_{1} & =\mathbf{J}_{s 1} \mathbf{V} \\
\mathbf{V}_{2} & =\mathbf{J}_{s 2} \mathbf{V},
\end{aligned}
$$

onde $\mathbf{J}_{s 1}$ e $\mathbf{J}_{s 2}$ são matrizes de seleção. Por exemplo, para $N=10$ o subarray $\left\{\mathbf{p}_{1}, \mathbf{p}_{3}, \mathbf{p}_{5}, \mathbf{p}_{7}\right\}$ tem matriz de seleção

$$
\mathbf{J}_{s}=\left[\begin{array}{llllllllll}
1 & 0 & 0 & 0 & 0 & 0 & 0 & 0 & 0 & 0 \\
0 & 0 & 1 & 0 & 0 & 0 & 0 & 0 & 0 & 0 \\
0 & 0 & 0 & 0 & 1 & 0 & 0 & 0 & 0 & 0 \\
0 & 0 & 0 & 0 & 0 & 0 & 1 & 0 & 0 & 0
\end{array}\right]
$$


Como os subarrays diferem apenas por um deslocamento, podemos escrever

$$
\mathbf{V}_{2}=\mathbf{V}_{1} \Phi
$$

onde

$$
\boldsymbol{\Phi}=\operatorname{diag}\left[\begin{array}{lll}
e^{j d_{s} \psi_{1}} & \cdots & e^{j d_{s} \psi_{M}}
\end{array}\right]
$$

e $\psi_{1}, \ldots, \psi_{M}$ são as direções de chegada dos sinais expressadas no espaço $\psi$.

Seja $\mathbf{U}_{S}$ uma representação matricial do espaço do sinal, conforme medido pelo array principal. Como as colunas de $\mathbf{U}_{\mathbf{S}}$ e de $\mathbf{V}$ geram o mesmo espaço, existe $\mathbf{T}$ não-singular tal que

$$
\mathbf{U}_{S}=\mathbf{V T}
$$

Definimos

$$
\begin{aligned}
\mathbf{U}_{S 1} & =\mathbf{J}_{s 1} \mathbf{U}_{S} \\
\mathbf{U}_{S 2} & =\mathbf{J}_{s 2} \mathbf{U}_{S} .
\end{aligned}
$$

Usando as relações acima,

$$
\begin{gathered}
\mathbf{U}_{S 1}=\mathbf{J}_{s 1} \mathbf{U}_{S}=\mathbf{J}_{s 1} \mathbf{V} \mathbf{T}=\mathbf{V}_{1} \mathbf{T} \Rightarrow \mathbf{V}_{1}=\mathbf{U}_{S 1} \mathbf{T}^{-1}, \\
\mathbf{U}_{S 2}=\mathbf{J}_{s 2} \mathbf{U}_{S}=\mathbf{J}_{s 2} \mathbf{V} \mathbf{T}=\mathbf{V}_{2} \mathbf{T}=\mathbf{V}_{1} \mathbf{\Phi} \mathbf{T} \Rightarrow \mathbf{U}_{S 2}=\mathbf{U}_{S 1} \mathbf{T}^{-1} \mathbf{\Phi} \mathbf{T} .
\end{gathered}
$$

Definimos

$$
\Psi=\mathrm{T}^{-1} \boldsymbol{\Phi} \mathrm{T}
$$

tal que

$$
\mathbf{U}_{S 2}=\mathbf{U}_{S 1} \Psi
$$

Note que $\mathbf{U}_{S}$ pode ser estimado a partir do array principal. Então $\hat{\mathbf{U}}_{S 1}$ e $\hat{\mathbf{U}}_{S 2}$ podem ser obtidos usando (6.4.7) e (6.4.8). Devido a erros de estimação, (6.4.12) não terá solução com probabilidade 1, mas podemos obter $\hat{\Psi}$ usando uma otimização por mínimos quadrados (ou de preferência, mínimos quadrados totais). Finalmente, $\hat{\boldsymbol{\Phi}}$ é a matriz dos autovalores de $\hat{\Psi}$, e as direções de chegada podem ser estimadas usando

$$
\hat{\psi}_{i}=\frac{1}{d_{s}} \arg \hat{\lambda}_{i}
$$

[47] apresenta uma realização ESPRIT unitária com forward-backward averaging, onde só é necessária aritmética real e os valores de $\boldsymbol{\Phi}$ são restritos ao círculo unitário. [48] propõe uma implementação unitária beamspace usando feixes DFT, e também uma 
implementação unitária para arrays circulares uniformes.

A maior vantagem do estimador ESPRIT é sua menor complexidade computacional. A busca do mínimo para o algoritmo MUSIC é inconveniente para arrays bidimensionais, enquanto que a estimação das direções de chegada pelo estimador ESPRIT exige somente a determinação de SVDs (mesmo no caso bidimensional). Por outro lado, o algoritmo MUSIC não faz suposições sobre a geometria do array, e pode ser implementado em qualquer cenário. 



\section{Capítulo 7}

\section{Imagens acústicas}

\subsection{Introdução}

Consideremos um processo aleatório estacionário no tempo e homogêneo no espaço, com correlação temporal-espacial dada por $K(\tau, \Delta \mathbf{p})$. Seu espectro em frequência-correlação espacial $S(\omega, \Delta \mathbf{p})$ e espectro em frequência-número de onda $P(\omega, \mathbf{k})$ são dados por (4.5.4), (4.5.6), (4.5.7) e (4.5.9) que repetimos a seguir:

$$
\begin{aligned}
S(\omega, \Delta \mathbf{p}) & =\int_{-\infty}^{+\infty} K(\tau, \Delta \mathbf{p}) e^{-j \omega \tau} d \tau \\
P(\omega, \mathbf{k}) & =\int_{\mathbb{R}^{3}} S(\omega, \Delta \mathbf{p}) e^{+j \mathbf{k}^{T} \Delta \mathbf{p}} d \Delta \mathbf{p} \\
K(\tau, \Delta \mathbf{p}) & =\frac{1}{2 \pi} \int_{-\infty}^{+\infty} S(\omega, \Delta \mathbf{p}) e^{+j \omega \tau} d \omega \\
S(\omega, \Delta \mathbf{p}) & =\frac{1}{(2 \pi)^{3}} \int_{\mathbb{R}^{3}} P(\omega, \mathbf{k}) e^{-j \mathbf{k}^{T} \Delta \mathbf{p}} d \mathbf{k} .
\end{aligned}
$$

Dado um processo com correlação temporal-espacial $K(\tau, \Delta \mathbf{p})$, chamamos de imagem acústica uma versão discretizada de $P(\omega, \mathbf{k})$, para $\omega$ fixo e $\mathbf{k}$ amostrado sobre uma região de interesse. Note que para $\omega$ fixo, $\mathbf{k}$ pode ser parametrizado em apenas duas coordenadas (por exemplo, usando coordenadas esféricas), tal que a imagem acústica seja de fato bidimensional. Intuitivamente, uma imagem acústica é a imagem que seria enxergada por um indivíduo se seus olhos fossem sensíveis à potência e direção de chegada de ondas sonoras. Exemplos de imagem acústicas estão apresentados na Figura 7.1.

Para obter uma interpretação mais rigorosa, note que $P(\omega, \mathbf{k})$ é real, não negativa e representa a densidade espectral de potência do processo. Como demonstrado na Seção 4.5, processos estacionários e homogêneos podem ser representados por uma superposição de ondas planas descorrelacionadas em frequência e direção de propagação. Usando (4.6.9), segue que $P(\omega, \mathbf{k})$ é proporcional à potência por frequência e por unidade de ângulo sólido que deve ser emitida por uma distribuição de fontes descorrelacionadas e localizadas no campo distante para recriar o campo de ondas do processo. Portanto, uma versão discretizada de $P(\omega, \mathbf{k})$ (uma imagem acústica) é uma discretização da distribuição 

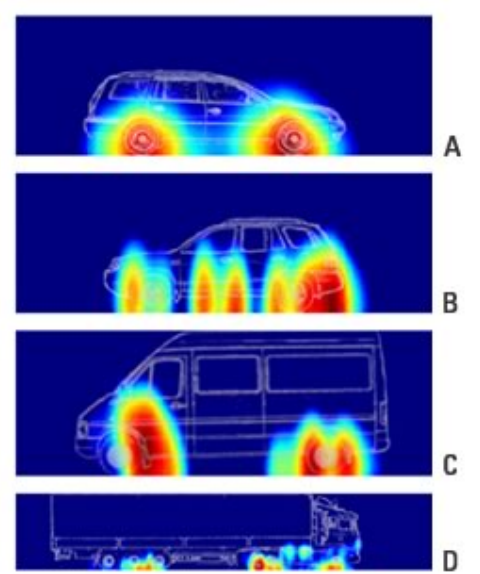

Figura 7.1: Exemplos de imagens acústicas (National Instruments, Nordborg Acoustics $\mathrm{AB})$

de fontes de um processo estacionário espacial-temporal, sob a aproximação de que estas estejam no campo distante.

Esta seção trata do problema de geração de imagens acústicas usando arrays de sensores. Seja $\hat{\mathbf{x}}(\omega)$ o sinal medido por um array de $N$ sensores, e seja $\mathbf{S}_{\mathbf{x}}(\omega)=$ $\mathrm{E}\left\{\hat{\mathbf{x}}(\omega) \hat{\mathbf{x}}^{H}(\omega)\right\}$ sua correspondente matriz espectral. Veremos a seguir que $\mathbf{S}_{\mathbf{x}}(\omega)$ contém uma versão amostrada de $S(\omega, \Delta \mathbf{p})$. Logo, a forma direta de obter uma imagem acústica consiste em usar transformadas de Fourier discretas para obter uma amostragem de $P(\omega, \mathbf{k})$ a partir de uma amostragem de $S(\omega, \Delta \mathbf{p}$ ) (que por sua vez, pode ser obtida a partir de $\left.\mathbf{S}_{\mathbf{x}}(\omega)\right)$. No entanto, esta implementação não é trivial como pode parecer à primeira vista, pois uma transformada de Fourier discreta exige amostragem retangular de $S(\omega, \Delta \mathbf{p})$ em $\Delta \mathbf{p}$, e retorna uma amostragem também retangular de $P(\omega, \mathbf{k})$ em $\mathbf{k}$, com intervalo de amostragem fixo e dependente do intervalo de amostragem de $\Delta \mathbf{p}$. Assim, veremos que na sua forma tradicional, uma transformada de Fourier somente pode ser aplicada para arrays retangulares uniformes com espaçamento $d=\frac{\lambda}{2}$ e amostragem uniforme em k. Esta restrição é forte demais, pois impede que a transformada de Fourier seja usada para mais de uma frequência de operação.

A forma clássica de produzir imagens acústicas com amostragem arbitrária em $\mathbf{k}$ e arrays com geometria arbitrária consiste em usar beamforming. Consideremos, por exemplo, o beamformer MPDR projetado no Capítulo 5. Seu vetor de pesos é dado por (5.5.4), $\mathrm{Ou}$

$$
\mathbf{w}_{\text {MPDR }}^{H}\left(\omega \mid \mathbf{k}_{s}\right)=\frac{\mathbf{v}^{H}\left(\omega, \mathbf{k}_{s}\right)\left[\mathbf{S}_{\mathbf{x}}(\omega)+\lambda_{1} \mathbf{I}\right]^{-1}}{\mathbf{v}^{H}\left(\omega, \mathbf{k}_{s}\right)\left[\mathbf{S}_{\mathbf{x}}(\omega)+\lambda_{1} \mathbf{I}\right]^{-1} \mathbf{v}\left(\omega, \mathbf{k}_{s}\right)},
$$

onde $\mathbf{k}_{s}$ é a direção de chegada de interesse e $\lambda_{1}$ é o parâmetro de regularização. Por construção, o beamformer MPDR é projetado sob a restrição de não distorção para sinais com direção $\mathbf{k}_{s}$. Portanto, se o processo de interesse for caracterizado por um única fonte com potência $\left|Y\left(\omega, \mathbf{k}_{s}\right)\right|^{2}$ emitindo ondas planas com direção de propagação $\mathbf{k}_{s}$, na 
ausência de erros, a potência na saída do beamformer será

$$
\left|Y\left(\omega, \mathbf{k}_{s}\right)\right|^{2}=\mathbf{w}_{\mathrm{MPDR}}^{H}\left(\omega, \mathbf{k}_{s}\right) \mathbf{S}_{\mathbf{x}}(\omega) \mathbf{w}_{\mathrm{MPDR}}\left(\omega, \mathbf{k}_{s}\right) .
$$

Consideremos agora um processo gerado por uma coleção de $M$ fontes discretas, irradiando ondas planas com direções de propagação $\left\{\mathbf{k}_{i}\right\}_{i=0}^{M-1}$ e potências $\left\{\left|Y\left(\omega, \mathbf{k}_{i}\right)\right|^{2}\right\}_{i=0}^{M-1}$. Dado um beamformer genérico com vetor de pesos $\mathbf{w}(\omega, \mathbf{k})$, o procedimento para gerar uma imagem acústica consiste em usar a aproximação

$$
\left|Y\left(\omega, \mathbf{k}_{i}\right)\right|^{2} \approx \mathbf{w}^{H}\left(\omega, \mathbf{k}_{i}\right) \mathbf{S}_{\mathbf{x}}(\omega) \mathbf{w}\left(\omega, \mathbf{k}_{i}\right)
$$

para $0 \leq i<M$. Note que esta aproximação pode ser grosseira mesmo na ausência de ruído, pois qualquer beamformer possui lóbulos laterais. Portanto, $\mathbf{w}^{H}\left(\mathbf{k}_{i}\right) \mathbf{S}_{\mathbf{x}} \mathbf{w}\left(\mathbf{k}_{i}\right)$ contém contribuições de fontes com $\mathbf{k} \neq \mathbf{k}_{i}$, tal que uma imagem gerada por um beamformer sempre superestima a distribuição $P(\omega, \mathbf{k})$ exata. Veremos que sob a aproximação de campo distante, a imagem produzida por um beamformer na ausência de ruído é igual à distribuição de fontes que se deseja obter, convoluída com o padrão de potência do array.

Ao longo desta seção, descreveremos com maiores detalhes a teoria de imagens acústicas e algumas formas de reconstrução, incluindo custos computacionais, vantagens e desvantagens. Mas antes disso é importante mencionar uma forma alternativa de reconstrução de campos acústicos, chamada holografia acústica de campo próximo [49] (NAH nearfield acoustic holography). Esta técnica propõe estimar o campo tridimensional no interior de um volume, a partir de medidas feitas sobre uma superfície que o envolva. A proposta consiste em resolver a equação de derivadas parciais que descreve campos de ondas em campo próximo, de forma a obter soluções sobre superfícies paralelas à superfície de medida, usando os dados coletados como condição de contorno.

A partir da amostragem adequada do campo de pressão sonora sobre uma superfície fechada é possível reconstruir os campos de pressão e velocidade desde a fonte até o campo distante. Os campos de pressão e velocidade podem ser usados para reconstruir o campo vetorial de intensidades, que quando integrado sobre uma superfície revela a potência irradiada. O campo de velocidades também pode ser usado para estimar os modos de vibração sobre estruturas presentes no problema.

Para que bons resultados sejam obtidos, o campo de pressão deve ser amostrado sobre uma malha suficientemente fina. A amostragem deve ser feita o mais próximo possível das fontes, de forma a capturar a componente evanescente do campo próximo (se a amostragem for realizada no campo distante, a resolução da holografia estará limitada por $\lambda / 2$ ). Um número relativamente grande de amostras deve ser coletado ao longo de uma superfície que envolva o objeto de interesse, e as coordenadas de todos os sensores devem ser registradas com boa precisão ao longo de todo este trajeto. A dificuldade em registrar coordenadas com boa precisão usando arrays móveis limita o uso de holografias 
para campos de baixa frequência (até algumas centenas de Hz). Finalmente, o processo deve ser estacionário para a duração da medição.

As exigências acima são indispensáveis, e demonstram-se bastante inconvenientes. Se o campo de interesse for gerado por um objeto grande, a quantidade de medidas pode consumir tempo suficiente para violar a hipótese de estacionariedade, produzindo resultados com validade questionável. A dificuldade em precisar as coordenadas dos sensores pode também comprometer a repetibilidade do método. Evidentemente, técnicas de holografia de campo próximo não podem ser aplicadas a objetos móveis e para estimação de processos variantes no tempo. Em aplicações de análise e controle de ruído, holografias devem ser refeitas frequentemente para validar resultados, consumindo tempo e recursos.

Em contraste com NAH, as imagens acústicas que descreveremos representam a distribuição de fontes estimada na superfície do array. Para estas técnicas, o array pode ser posicionado tanto em campo próximo como em campo distante, pois o objetivo não é reconstruir o campo volumétrico (ainda que isto possa ser feito, se for usado um array com geometria conveniente). Assim, a região amostrada pelos sensores é análoga ao filme de uma câmera fotográfica, onde o registro de ondas incidentes em um intervalo de tempo dá origem a uma imagem. Alguns trabalhos recentes [50-53] mostram resultados usando arrays esféricos e decomposição em ondas planas [54], permitindo a visualização de ondas propagantes na forma de diagramas de intensidade. A apresentação das respostas acústicas espaciais torna-se especialmente atraente se os diagramas de intensidade forem sobrepostos a fotografias do ambiente, permitindo a visualização dos caminhos percorridos por ondas sonoras (Figura 7.2). Este recurso permite a interpretação de cenas e a localização dinâmica de fontes, incluindo análises de espalhamentos devidos a reflexões. Uma aplicação é a caracterização acústica de ambientes (por exemplo, salas de concerto, estúdios e auditórios). Claramente não é possível utilizar NAH para este tipo de aplicação.

Imagens acústicas também encontram muitas aplicações industriais, para as quais NAH seria inconveniente ou impossível de ser usada. Por exemplo, arrays de microfones podem ser posicionados em túneis de vento para determinar a distribuição de ruído sobre modelos devido ao fluxo de ar em alta velocidade [55,56]. Estas medidas são frequentemente utilizadas para desenvolver carros, trens e aviões mais silenciosos para observadores e passageiros. Arrays de microfones também são usados para medir o ruído gerado por turbinas de avião [57] e turbinas de vento [58], para aplicações semelhantes de redução de ruído. Finalmente, arrays de grandes dimensões já foram usados para medidas de aviões em vôo, para estudos de ruído e detecção de turbulências [59,60].

\subsection{Parametrização no espaço U}

Ao longo das seções anteriores, tipicamente descrevemos arrays usando seus manifold vectors $\mathbf{v}_{\mathbf{k}}(\mathbf{k})$, com $\mathbf{k}$ dado por (2.2.11). Em algumas aplicações das Seções 2 e 3, consideramos o caso particular de arrays unidimensionais, e utilizamos $k_{z}=-\|\mathbf{k}\| \cos \theta$, 


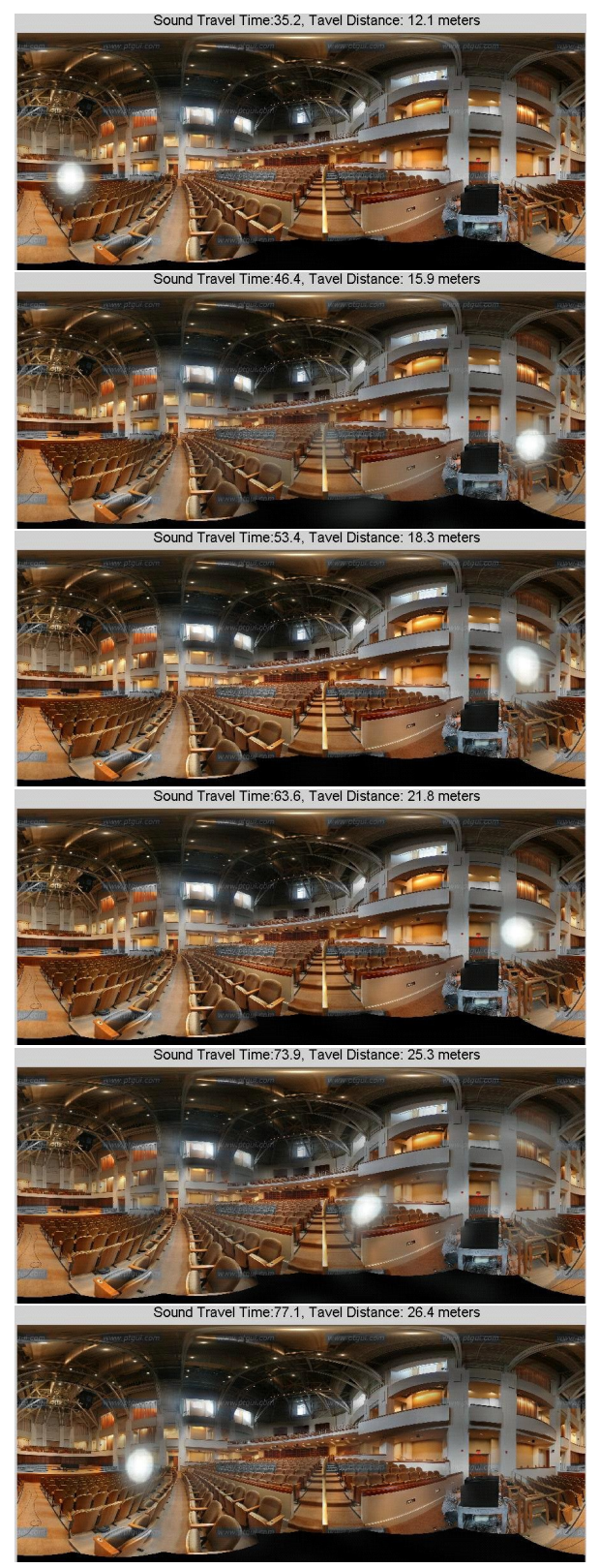

Figura 7.2: Imagem acústica panorâmica de uma sala de concertos, representando sucessivas reflexões através de 5 imagens acústicas [53]. As imagens foram geradas usando um array esférico de $10 \mathrm{~cm}$ de raio, com 60 elementos. 
$\psi=-k_{z} d$ ou $u=\cos \theta$ para parametrizar manifold vectors e padrões de potência.

Para a aplicação de imagens acústicas, estamos interessados em uma parametrização bidimensional, de forma a obter uma correspondência biunívoca entre a parametrização de $\mathbf{k}$ e as coordenadas de um pixel da imagem acústica. No Capítulo 2, definimos

$$
\mathbf{u}=\left[\begin{array}{c}
\sin \theta \cos \phi \\
\sin \theta \sin \phi \\
\cos \theta
\end{array}\right]
$$

a direção de visada (look direction) do array, parametrizada em coordenadas esféricas, onde $\theta$ e $\phi$ são ângulos de azimute e elevação. É possível parametrizar a semi-esfera definindo

$$
\begin{aligned}
& u_{x}(\theta, \phi)=\sin \phi \cos \theta \\
& u_{y}(\theta, \phi)=\sin \phi \sin \theta
\end{aligned}
$$

tal que

$$
\mathbf{u}=\left[\begin{array}{c}
u_{x} \\
u_{y} \\
\sqrt{1-u_{x}^{2}-u_{y}^{2}}
\end{array}\right]
$$

para $u_{x}^{2}+u_{y}^{2} \leq 1$. Note que esta parametrização permite representar qualquer direção de chegada com as coordenadas $\left(u_{x}, u_{y}\right) \in[-1,1]^{2}$. A seguir, diremos que qualquer função de $\left(u_{x}, u_{y}\right) \in[-1,1]^{2}$ estará parametrizada no espaço $\mathrm{U}$, para $\mathrm{U}=[-1,1]^{2}$.

Consideremos um array de $N$ microfones com coordenadas $\mathbf{p}_{0}, \ldots, \mathbf{p}_{N-1} \in \mathbb{R}^{3}$. O array manifold vector para fontes no campo distante é dado por (2.2.13), ou

$$
\mathbf{v}_{\mathbf{k}}(\mathbf{k})=\left[\begin{array}{c}
e^{-j \mathbf{k}^{T} \mathbf{p}_{0}} \\
e^{-j \mathbf{k}^{T} \mathbf{p}_{1}} \\
\vdots \\
e^{-j \mathbf{k}^{T} \mathbf{p}_{N-1}}
\end{array}\right]
$$

Usando $\mathbf{k}=-\frac{\omega}{c} \mathbf{u}$, temos

$$
\mathbf{v}_{\mathbf{u}}(\omega, \mathbf{u})=\left[\begin{array}{c}
\mathrm{e}^{j \omega \mathbf{u}^{T} \mathbf{p}_{0} / c} \\
\mathrm{e}^{j \omega \mathbf{u}^{T} \mathbf{p}_{1} / c} \\
\vdots \\
\mathrm{e}^{j \omega \mathbf{u}^{T} \mathbf{p}_{N-1} / c}
\end{array}\right]
$$

\subsection{Decomposição em fontes descorrelacionadas}

Consideremos agora um campo de ondas arbitrário. Suponhamos que este campo possa ser modelado através da superposição de $M$ fontes pontuais localizadas nas dire- 
ções $\left\{\mathbf{u}_{i}\right\}_{i=0}^{M-1}$, com $M$ suficientemente grande de forma a garantir um pequeno erro de discretização. Os sinais recebidos por cada microfone são segmentados em quadros com $K$ amostras, e cada quadro é transformado para o domínio da frequência. Na presença de ruído aditivo, o vetor $N \times 1$ de saída do array para uma única frequência $\omega_{k}$ pode ser escrito como

$$
\hat{\mathbf{x}}\left(\omega_{k}\right)=\mathbf{V}\left(\omega_{k}\right) \hat{\mathbf{f}}\left(\omega_{k}\right)+\hat{\boldsymbol{\eta}}\left(\omega_{k}\right),
$$

onde $0 \leq k<K, \mathbf{V}\left(\omega_{k}\right)=\left[\begin{array}{llll}\mathbf{v}_{\mathbf{u}}\left(\omega_{k}, \mathbf{u}_{0}\right) & \mathbf{v}_{\mathbf{u}}\left(\omega_{k}, \mathbf{u}_{1}\right) & \cdots & \mathbf{v}_{\mathbf{u}}\left(\omega_{k}, \mathbf{u}_{M-1}\right)\end{array}\right]$ é a matriz de vetores diretores, $\hat{\mathbf{f}}\left(\omega_{k}\right)=\left[\begin{array}{llll}\hat{f}_{0}\left(\omega_{k}\right) & \hat{f}_{1}\left(\omega_{k}\right) & \cdots & \hat{f}_{M-1}\left(\omega_{k}\right)\end{array}\right]^{T}$ é o sinal emitido pelas fontes no domínio da frequência e $\hat{\boldsymbol{\eta}}\left(\omega_{k}\right)$ é o ruído aditivo medido por cada sensor no domínio da frequência.

Seja

$$
\mathbf{S}_{\mathbf{x}}\left(\omega_{k}\right)=\mathrm{E}\left\{\hat{\mathbf{x}}\left(\omega_{k}\right) \hat{\mathbf{x}}^{H}\left(\omega_{k}\right)\right\}
$$

a matriz espectral do array para $0 \leq k<K$. Se $\hat{\mathbf{x}}_{0}\left(\omega_{k}\right), \ldots, \hat{\mathbf{x}}_{L-1}\left(\omega_{k}\right)$ correspondem a $L$ quadros no domínio da frequência, a matriz espectral pode ser estimada usando

$$
\hat{\mathbf{S}}_{\mathbf{x}}\left(\omega_{k}\right)=\frac{1}{L} \sum_{l=0}^{L-1} \hat{\mathbf{x}}_{l}\left(\omega_{k}\right) \hat{\mathbf{x}}_{l}^{H}\left(\omega_{k}\right) .
$$

É mais conveniente processar $\hat{\mathbf{S}}_{\mathbf{x}}\left(\omega_{k}\right)$ ao invés de cada $\hat{\mathbf{x}}_{l}\left(\omega_{k}\right)$ isoladamente, pois $\hat{\mathbf{S}}_{\mathbf{x}}\left(\omega_{k}\right)$ contém somente os atrasos de fase relativos entre microfones, e também porque contém menos ruído, já que é o resultado de uma média. De fato, para cada $0 \leq l<L, \hat{\mathbf{x}}_{l}\left(\omega_{k}\right)$ tem um atraso de fase que é igual para cada elemento, porém desconhecido, e que desaparece ao computar $\hat{\mathbf{S}}_{\mathbf{x}}\left(\omega_{k}\right)$. Para simplificar a notação, ocasionalmente ignoraremos o argumento $\omega_{k}$ (sob a hipótese que o processamento é feito em banda estreita) e o subíndice $\mathbf{x}$. Assim, $\mathbf{S}_{\mathbf{x}}\left(\omega_{k}\right)$ será frequentemente denotado $\mathbf{S}$.

Substituindo (7.3.1) em (7.3.2) e admitindo que o ruído seja espacialmente branco e descorrelacionado com fontes de interesse, temos

$$
\mathbf{S}_{\mathbf{x}}\left(\omega_{k}\right)=\mathbf{V}\left(\omega_{k}\right) E\left\{\hat{\mathbf{f}}\left(\omega_{k}\right) \hat{\mathbf{f}}^{H}\left(\omega_{k}\right)\right\} \mathbf{V}^{H}\left(\omega_{k}\right)+\sigma^{2} \mathbf{I}
$$

onde $\sigma^{2}=\mathrm{E}\left\{\hat{\eta}_{i}\left(\omega_{k}\right) \hat{\eta}_{i}^{*}\left(\omega_{k}\right)\right\}, 0 \leq i<N$.

Suponhamos que o campo de ondas incidente no array possa ser modelado como gerado pela superposição de fontes pontuais descorrelacionadas no campo distante. Podemos representar a direção destas fontes através de uma coleção de coordenadas $\left\{\mathbf{u}_{i}\right\}_{i=0}^{M-1}$ localizadas sobre um grid suficientemente fino no espaço U. Esta representação é efetivamente uma imagem bi-dimensional, onde coordenadas de pixels correspondem a direções de chegada no espaço $U$, e valores de pixels correspondem a potências de fontes. Note que em (7.3.4), admitir que as fontes são descorrelacionadas é equivalente a admitir que 
$\mathrm{E}\left\{\hat{\mathbf{f}}\left(\omega_{k}\right) \hat{\mathbf{f}}^{H}\left(\omega_{k}\right)\right\}$ é diagonal. Além disso, os elementos da diagonal de $\mathrm{E}\left\{\hat{\mathbf{f}}\left(\omega_{k}\right) \hat{\mathbf{f}}^{H}\left(\omega_{k}\right)\right\}$ correspondem a uma versão vetorizada da imagem acústica.

Dada uma imagem acústica, é simples obter a matriz espectral correspondente usando (7.3.4), desde que suponhamos que as fontes (pixels) sejam descorrelacionadas. Na presença de correlações, devemos abandonar a hipótese de que $\mathrm{E}\left\{\hat{\mathbf{f}}\left(\omega_{k}\right) \hat{\mathbf{f}}^{H}\left(\omega_{k}\right)\right\}$ é diagonal, e precisamos da correlação entre cada par de fontes, cuja determinação é claramente impraticável até mesmo para imagens pequenas (por exemplo, uma imagem $64 \times 64$ teria $64^{2}=4096$ pixels (fontes) e $\frac{1}{2} 4096 \cdot 4097 \approx 8$ milhões de correlações únicas). Por esse motivo, ao longo das Seções 7 e 8 admitiremos que as fontes são descorrelacionadas. Somente eliminaremos esta hipótese no Capítulo 10, onde estabeleceremos alguns resultados para fontes correlacionadas.

Consideremos uma imagem acústica com $M_{x} \times M_{y}$ pixels. Defina $M=M_{x} M_{y}$ e seja $\mathbf{u}_{0}, \ldots, \mathbf{u}_{M-1}$ uma enumeração de todas as coordenadas de pixels no espaço U. Seja $\mathbf{v}\left(\mathbf{u}_{m}\right)$ o array manifold vector quando direcionado para $\mathbf{u}_{m}$. Para $0 \leq m<M$, seja $\left|Y\left(\mathbf{u}_{m}\right)\right|^{2}$ a potência da fonte com direção $\mathbf{u}_{m}$. Aplicando (7.3.4), temos que

$$
\mathbf{S}=\sum_{m=0}^{M-1}\left|Y\left(\mathbf{u}_{m}\right)\right|^{2} \mathbf{v}\left(\mathbf{u}_{m}\right) \mathbf{v}^{H}\left(\mathbf{u}_{m}\right)
$$

\subsection{Invariância translacional de beamformers}

Consideremos um array com geometria plana e com $N$ microfones nas coordenadas $\mathbf{p}_{0}, \ldots, \mathbf{p}_{N-1} \in \mathbb{R}^{3}$, posicionado tal que $\left[\mathbf{p}_{i}\right]_{3}=0$ para $0 \leq i<N$.

Suponha que $\mathbf{Y}$ seja uma imagem digital com $M_{x} \times M_{y}$ pixels ( $\mathbf{Y}$ tem $M_{x}$ colunas e $M_{y}$ linhas). Sejam $\left\{u_{x_{m}}\right\}_{0 \leq m<M_{x}}$ e $\left\{u_{y_{n}}\right\}_{0 \leq n<M_{y}}$ pontos que amostram o espaço $\mathrm{U}$ ao longo dos eixos x e y, ordenados da esquerda para a direita e de cima para baixo. Definimos $\mathbf{u}_{0}, \ldots, \mathbf{u}_{M-1}$ tal que

$$
\mathbf{u}_{m}=\left[\begin{array}{ll}
u_{\left.x_{\lfloor m / M y}\right\rfloor} & u_{y_{\bmod \left(m, M_{y}\right)}}
\end{array}\right]^{T},
$$

e ordenamos os pixels de $\mathbf{Y}$ tal que

$$
\operatorname{vec}\{\mathbf{Y}\}=\left[\begin{array}{c}
\left|Y\left(\mathbf{u}_{0}\right)\right|^{2} \\
\left|Y\left(\mathbf{u}_{1}\right)\right|^{2} \\
\vdots \\
\left|Y\left(\mathbf{u}_{M-1}\right)\right|^{2}
\end{array}\right]
$$

Note que isto implica $\mathbf{Y}_{n, m}=\left|Y\left(u_{x_{m}}, u_{y_{n}}\right)\right|^{2}$. A Figura 7.3 mostra um exemplo para $M_{x}=M_{y}=21$.

Seja $\mathbf{Y}$ a distribuição exata de fontes que se deseja reconstruir, e $\mathbf{S}$ a matriz espectral gerada pela distribuição dada por $\mathbf{Y}$. Consideremos o problema de obter $\breve{Y}$, uma esti- 


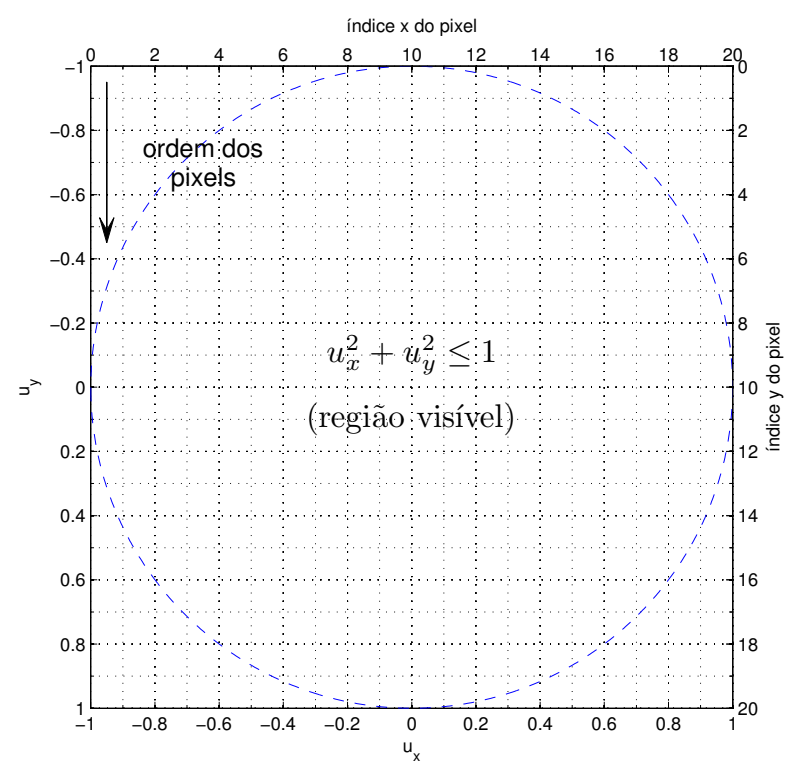

Figura 7.3: Exemplo da organização de uma imagem acústica para $M_{x}=M_{y}=21$, com amostragem uniforme no espaço U.

mativa de $\mathbf{Y}$, utilizando um beamformer fixo com vetor de pesos $\mathbf{w}_{0}^{H}$. Por definição, $\mathbf{w}_{0}^{H}$ produz um padrão de potência com máximo em $\left(u_{x}, u_{y}\right)=(0,0)$. No caso geral, $\mathbf{w}^{H}(\mathbf{u})$ se escreve como a composição de uma matriz diretora $\mathbf{I}_{s}(\mathbf{u}) \operatorname{com} \mathbf{w}_{0}^{H}$, análoga a (2.5.1) mas parametrizada em $\mathbf{u}$, tal que

$$
\begin{aligned}
\mathbf{w}^{H}(\mathbf{u}) & =\mathbf{w}_{0}^{H} \mathbf{I}_{s}(\mathbf{u}) \\
& =\mathbf{w}_{0}^{H} \odot \mathbf{v}^{H}(\mathbf{u}),
\end{aligned}
$$

onde $\odot$ é o produto de Hadamard. Note que se o beamformer for delay-and-sum, esta expressão se reduz a $\mathbf{w}^{H}(\mathbf{u})=\frac{1}{N} \mathbf{v}^{H}(\mathbf{u})$.

Como visto em (7.1.7), a reconstrução utiliza a aproximação

$$
\begin{aligned}
\mathbf{Y}_{n, m} & \approx \breve{\mathbf{Y}}_{n, m} \\
& =\mathbf{w}^{H}\left(u_{x_{m}}, u_{y_{n}}\right) \mathbf{S w}\left(u_{x_{m}}, u_{y_{n}}\right)
\end{aligned}
$$

para $0 \leq m<M$.

Mostremos que se a amostragem em $\mathrm{U}$ for uniforme, um deslocamento de $\mathbf{Y}$ produz um deslocamento idêntico em $\breve{Y}$ (ou seja, beamforming é invariante por deslocamentos). Usando (7.3.5), podemos escrever $\mathbf{S}$ como a superposição da contribuição de cada fonte. Como (7.4.6) é linear em $\mathbf{S}$, basta mostrar que beamforming é invariante por deslocamentos para distribuições impulsivas da forma $\mathbf{Y}\left(n_{0}, m_{0}\right)$, definidas por

$$
\left[\mathbf{Y}\left(n_{0}, m_{0}\right)\right]_{n, m}=\delta\left(m-m_{0}\right) \delta\left(n-n_{0}\right),
$$


para $0 \leq m_{0}<M_{x}$ e $0 \leq n_{0}<M_{y}$ arbitrários. Ou seja, devemos mostrar que

$$
\left[\breve{\mathbf{Y}}\left(n_{0}+\Delta n, m_{0}+\Delta m\right)\right]_{n+\Delta n, m+\Delta m}=\left[\breve{\mathbf{Y}}\left(n_{0}, m_{0}\right)\right]_{n, m},
$$

para quaisquer $\Delta n, \Delta m \in \mathbb{Z}$ que produzam índices válidos.

Por definição,

$$
\begin{aligned}
{\left[\breve{\mathbf{Y}}\left(n_{0}, m_{0}\right)\right]_{n, m} } & =\mathbf{w}^{H}\left(u_{x_{m}}, u_{y_{n}}\right) \mathbf{S w}\left(u_{x_{m}}, u_{y_{n}}\right) \\
& =\left[\mathbf{w}_{0}^{H} \odot \mathbf{v}^{H}\left(u_{x_{m}}, u_{y_{n}}\right)\right] \mathbf{S}\left[\mathbf{v}\left(u_{x_{m}}, u_{y_{n}}\right) \odot \mathbf{w}_{0}\right]
\end{aligned}
$$

e

$$
\begin{aligned}
\mathbf{S} & =\sum_{i, j}\left[\mathbf{Y}\left(n_{0}, m_{0}\right)\right]_{j, i} \mathbf{v}\left(u_{x_{i}}, u_{y_{j}}\right) \mathbf{v}^{H}\left(u_{x_{i}}, u_{y_{j}}\right) \\
& =\mathbf{v}\left(u_{x_{m_{0}}}, u_{y_{n_{0}}}\right) \mathbf{v}^{H}\left(u_{x_{m_{0}}}, u_{y_{n_{0}}}\right)
\end{aligned}
$$

tal que

$$
\left[\breve{\mathbf{Y}}\left(n_{0}, m_{0}\right)\right]_{n, m}=\left|\left[\mathbf{w}_{0}^{H} \odot \mathbf{v}^{H}\left(u_{x_{m}}, u_{y_{n}}\right)\right] \mathbf{v}\left(u_{x_{m_{0}}}, u_{y_{n_{0}}}\right)\right|^{2}
$$

Usando

$$
\mathbf{v}\left(\mathbf{u}_{m}\right)=\left[\begin{array}{c}
\mathrm{e}^{j \omega \mathbf{u}_{m}^{T} \mathbf{p}_{0} / c} \\
\mathrm{e}^{j \omega \mathbf{u}_{m}^{T} \mathbf{p}_{1} / c} \\
\vdots \\
\mathrm{e}^{j \omega \mathbf{u}_{m}^{T} \mathbf{p}_{N-1} / c}
\end{array}\right]
$$

temos que para $0 \leq i<N$,

$$
\left[\mathbf{v}\left(u_{x}, u_{y}\right)\right]_{i}=\mathrm{e}^{j \frac{\omega}{c}\left(u_{x} p_{x_{i}}+u_{y} p_{y_{i}}\right)}
$$

Portanto,

$$
\begin{aligned}
{\left[\breve{\mathbf{Y}}\left(n_{0}, m_{0}\right)\right]_{n, m} } & =\left|\left[\mathbf{w}_{0}^{H} \odot \mathbf{v}^{H}\left(u_{x_{m}}, u_{y_{n}}\right)\right] \mathbf{v}\left(u_{x_{m_{0}}}, u_{y_{n_{0}}}\right)\right|^{2} \\
& =\mid \sum_{i=0}^{N-1}\left[\mathbf{w}_{0}^{H}\right]_{i} \mathrm{e}^{\left.j \frac{\omega}{c}\left(\left(u_{x_{m_{0}}}-u_{x_{m}}\right) p_{x_{i}}+\left(u_{y_{n_{0}}}-u_{y_{n}}\right) p_{y_{i}}\right)\right|^{2}} \\
& =\mid \sum_{i=0}^{N-1}\left[\mathbf{w}_{0}^{H}\right]_{i} \mathrm{e}^{\left.j \frac{\omega}{c}\left(\left(u_{x_{m_{0}}+\Delta m}-u_{x_{m+\Delta m}}\right) p_{x_{i}}+\left(u_{y_{n_{0}}+\Delta n}-u_{y_{n+\Delta n}}\right) p_{y_{i}}\right)\right|^{2}} \\
& =\left[\breve{\mathbf{Y}}\left(n_{0}+\Delta n, m_{0}+\Delta m\right)\right]_{n+\Delta n, m+\Delta m} .
\end{aligned}
$$


A penúltima igualdade é válida, pois como a amostragem em U é uniforme,

$$
\begin{gathered}
u_{x_{m_{0}}}-u_{x_{m}}=u_{x_{m_{0}+\Delta m}}-u_{x_{m+\Delta m}} \\
u_{y_{n_{0}}}-u_{y_{n}}=u_{y_{n_{0}+\Delta n}}-u_{y_{n+\Delta n}} .
\end{gathered}
$$

Portanto, se considerarmos fontes descorrelacionadas em campo distante e usarmos amostragem uniforme no espaço U, a reconstrução de imagens por beamforming é uma operação linear e invariante a deslocamentos.

Como todo sistema linear e invariante a deslocamentos pode ser representado pela convolução de um sinal de entrada com uma resposta impulsiva [5,61,62], a imagem reconstruída por um beamformer consiste da distribuição de fontes de interesse convoluída com o padrão de potência do beamformer (que é sua resposta impulsiva espacial, ou point spread function (PSF)).

\subsection{Deconvolução de imagens acústicas}

Como um array de microfones construído com a tecnologia atual terá no máximo algumas centenas de elementos, sua PSF não será compacta. Consequentemente, as imagens acústicas produzidas por beamformers parecem desfocadas, pois PSFs típicas têm aspecto similar ao de filtros passa-baixas. Esta característica é altamente indesejável, pois reduz a resolução das imagens reconstruídas e impede a determinação de níveis de potência na presença de múltiplas fontes, ou de fontes distribuídas.

Técnicas específicas para projeto de beamformers foram propostas para lidar com os efeitos da convolução. Alguns autores sugerem projetar beamformers com largura de feixe invariante com respeito à frequência [63,64]. A motivação para esta proposta vem do fato que beamformers típicos (por exemplo, delay-and-sum ou MVDR) têm largura de feixe inversamente proporcional à frequência. Como o padrão de potência é normalizado em amplitude, a integral de uma PSF para baixas frequências é muito maior que a integral de uma PSF para altas frequências. Logo, beamformers tradicionais produzem estimativas de potência dependentes da frequência. Este efeito dificulta a interpretação de imagens acústicas obtidas para fontes iguais e frequências diferentes, pois impede a determinação das potências relativas entre as imagens.

Note, porém, que devido aos efeitos da convolução, a determinação de potências absolutas é essencialmente impossível para casos não triviais, seja lá qual for o beamformer usado. Além disso, a única forma de obter largura de feixe constante é aumentando a largura de feixe para altas frequências, pois para baixas frequências ela é limitada pelas dimensões do array. Portanto, estas técnicas efetivamente reduzem a resolução das imagens reconstruídas, e nada contribuem para desfazer os efeitos da convolução.

Para melhorar a qualidade das imagens, várias técnicas de deconvolução foram propostas [65-69]. Elas utilizam como entrada a imagem produzida por beamforming (tipi- 


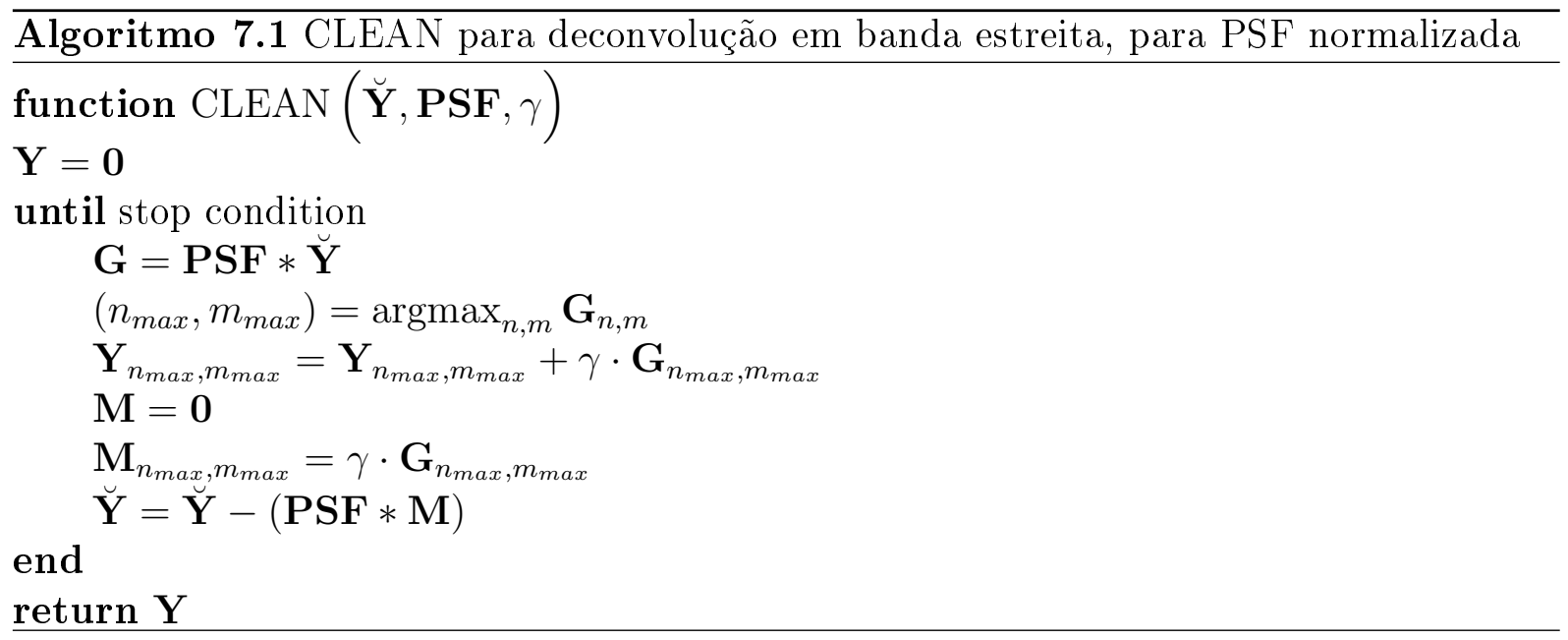

camente delay-and-sum) e a PSF do array, e retornam uma aproximação mais fidedigna da distribuição de fontes verdadeira. A seguir, descrevemos as técnicas de deconvolução mais populares para imagens acústicas.

\subsubsection{CLEAN}

CLEAN [65] é um algoritmo celebrado para deconvolução de imagens geradas com arrays, e foi proposto pela primeira vez para reconstrução de imagens radio-astronômicas. CLEAN foi estendido para o caso banda larga com múltiplos snapshots em [66]. Como descrito anteriormente, admite-se que a imagem de interesse (chamada imagem limpa) foi convoluída com a PSF do array, produzindo uma chamada imagem suja. Uma versão do algoritmo em pseudocódigo está apresentada no Algoritmo 7.1.

CLEAN recebe como entrada a imagem suja $\breve{\mathbf{Y}}$, a PSF do array e um escalar $0<\gamma \ll 1$ (sobre o qual comentaremos a seguir), e retorna uma estimativa $\mathbf{Y}$ da imagem deconvoluída. Para isto, CLEAN iterativamente estima as coordenadas da fonte dominante utilizando o máximo da convolução entre a PSF e $\breve{\mathbf{Y}}$, dada por $\mathbf{G}=\mathbf{P S F} * \breve{\mathbf{Y}}$ (que, usando a simetria da PSF, equivale a um filtro casado). A cada iteração, uma fração $\gamma$ do valor máximo $\mathbf{G}_{n_{\max }, m_{\max }}$ é adicionada a $\mathbf{Y}_{n_{\max }, m_{\max }}$. A fração $\gamma$ recebe o nome de ganho do loop, e deve ser mantida pequena para evitar divergência. A imagem suja é atualizada, subtraindo a contribuição de uma fonte pontual localizada em $\left(n_{\max }, m_{\max }\right)$ com potência $\gamma \cdot \mathbf{G}_{n_{\text {max }}, m_{\max }}$, convoluída pela PSF.

Apesar de CLEAN ter bom desempenho para fontes pontuais, imagens acústicas são caracterizadas por fontes distribuídas. A mesma característica que o torna eficiente para sinais esparsos o torna sub-ótimo, pois na presença de fontes distribuídas, a contribuição de uma grande quantidade de lóbulos laterais produz máximos onde nenhuma fonte significativa existe. O filtro casado incorretamente identifica estes máximos como fontes. Além disso, como cada iteração trata apenas uma fração $\gamma$ da potência de uma única fonte, um número muito grande de iterações torna-se necessário. O fato da PSF não ser compacta também cria artefatos para fontes próximas às bordas da região visível. Como 
o filtro casado implicitamente requer o deslocamento de PSFs e a imagem de interesse não é circularmente periódica e tem tamanho finito, CLEAN pode somente ser aplicado com confiabilidade a fontes que estejam na região central da imagem. Para fontes próximas às bordas, uma parte significativa da PSF é deslocada para fora da imagem, resultando em artefatos. Finalmente, imagens acústicas são obrigatoriamente não-negativas, e CLEAN produz pixels negativos mesmo na ausência de ruído, produzindo também artefatos indesejáveis.

\subsubsection{DAMAS}

Um método popular para deconvolução de imagens acústicas é DAMAS (deconvolution approach for the mapping of acoustic sources) [67,70]. Sob a hipótese de fontes descorrelacionadas, é possível escrever um sistema linear que relaciona a imagem suja obtida com um beamformer delay-and-sum e a imagem limpa formada por fontes pontuais. De fato, se $\mathbf{Y}$ for uma distribuição arbitrária de fontes no campo distante, com

$$
\mathbf{y}=\operatorname{vec}\{\mathbf{Y}\}=\left[\begin{array}{c}
\left|Y\left(\mathbf{u}_{0}\right)\right|^{2} \\
\left|Y\left(\mathbf{u}_{1}\right)\right|^{2} \\
\vdots \\
\left|Y\left(\mathbf{u}_{M-1}\right)\right|^{2}
\end{array}\right]
$$

então a matriz espectral correspondente é dada por

$$
\begin{aligned}
\mathbf{S} & =\sum_{m=0}^{M-1}\left|Y\left(\mathbf{u}_{m}\right)\right|^{2} \mathbf{v}\left(\mathbf{u}_{m}\right) \mathbf{v}^{H}\left(\mathbf{u}_{m}\right) \\
& =\sum_{m=0}^{M-1} \mathbf{y}_{m} \mathbf{v}\left(\mathbf{u}_{m}\right) \mathbf{v}^{H}\left(\mathbf{u}_{m}\right) .
\end{aligned}
$$

Seja $\breve{Y}$ a imagem estimada por delay-and-sum, com

$$
\breve{\mathbf{y}}=\operatorname{vec}\{\breve{\mathbf{Y}}\}=\left[\begin{array}{c}
\left|\breve{Y}\left(\mathbf{u}_{0}\right)\right|^{2} \\
\left.\breve{Y}\left(\mathbf{u}_{1}\right)\right|^{2} \\
\vdots \\
\left|\breve{Y}\left(\mathbf{u}_{M-1}\right)\right|^{2}
\end{array}\right] .
$$

Então

$$
\begin{aligned}
\breve{\mathbf{y}}_{m^{\prime}} & =\mathbf{v}^{H}\left(\mathbf{u}_{m^{\prime}}\right) \mathbf{S v}\left(\mathbf{u}_{m^{\prime}}\right) \\
& =\mathbf{v}^{H}\left(\mathbf{u}_{m^{\prime}}\right)\left[\sum_{m=0}^{M-1} \mathbf{y}_{m} \mathbf{v}\left(\mathbf{u}_{m}\right) \mathbf{v}^{H}\left(\mathbf{u}_{m}\right)\right] \mathbf{v}\left(\mathbf{u}_{m^{\prime}}\right)
\end{aligned}
$$


tal que

$$
\left[\begin{array}{c}
\breve{\mathbf{y}}_{0} \\
\breve{\mathbf{y}}_{1} \\
\vdots \\
\breve{\mathbf{y}}_{M-1}
\end{array}\right]=\left[\begin{array}{cccc}
B_{0,0} & B_{0,1} & \cdots & B_{0, M-1} \\
B_{1,0} & B_{1,1} & \cdots & B_{1, M-1} \\
\vdots & \vdots & & \vdots \\
B_{M-1,0} & B_{M-1,1} & \cdots & B_{M-1, M-1}
\end{array}\right]\left[\begin{array}{c}
\mathbf{y}_{0} \\
\mathbf{y}_{1} \\
\vdots \\
\mathbf{y}_{M-1}
\end{array}\right]
$$

com

$$
B_{m^{\prime}, m}=\left|\mathbf{v}^{H}\left(\mathbf{u}_{m^{\prime}}\right) \mathbf{v}\left(\mathbf{u}_{m}\right)\right|^{2} .
$$

DAMAS iterativamente resolve (7.5.7) usando o método de Gauss-Seidel, com

$$
\begin{aligned}
\mathbf{y}_{i}^{(k+1)} & =\max \left\{\frac{1}{B_{i, i}}\left[\breve{\mathbf{y}}_{i}-\left(\sum_{j=0}^{i-1} B_{i, j} \mathbf{y}_{j}^{(k+1)}+\sum_{j=i+1}^{M-1} B_{i, j} \mathbf{y}_{j}^{(k)}\right)\right], 0\right\} \\
& =\max \left\{\mathbf{y}_{i}^{(k)}+\frac{1}{B_{i, i}}\left[\breve{\mathbf{y}}_{i}-\left(\sum_{j=0}^{i-1} B_{i, j} \mathbf{y}_{j}^{(k+1)}+\sum_{j=i}^{M-1} B_{i, j} \mathbf{y}_{j}^{(k)}\right)\right], 0\right\},
\end{aligned}
$$

onde $\mathbf{y}^{(0)}=\mathbf{0}$ e $\mathbf{y}^{(k+1)} \rightarrow \mathbf{y}$ se $\mathbf{B}$ tiver diagonal dominante ou for positiva definida. Note que é necessário forçar $\mathbf{y}^{(k+1)}$ a ser não negativa a cada iteração, pois tipicamente B é mal condicionada, tal que (7.5.7) não tem solução única ou estável na ausência de regularização.

DAMAS produz melhoras significativas em relação a beamforming convencional, pois retorna uma imagem deconvoluída. Por outro lado, ele ainda apresenta várias desvantagens. Se a imagem de interesse tiver $M_{x} \times M_{y}$ pixels, DAMAS exige a solução de um sistema com $M_{x} M_{y}$ equações, o que significativamente limita a resolução das imagens que podem ser reconstruídas. Além disso, o método de Gauss-Seidel não tem convergência garantida, pois $\mathbf{B}$ pode não ter diagonal dominante ou ser positiva definida. Por conta desta falta de condicionamento, $\mathbf{y}^{(k+1)}$ frequentemente depende da ordem dos pixels usada ao resolver (7.5.10), a imagem reconstruída pode ser muito ruidosa e a convergência é tipicamente lenta. A dependência da ordem dos pixels e do ruído podem ser melhorados aplicando um filtro passa-baixas após cada iteração externa Gauss-Seidel, mas isto não alivia o custo computacional do método.

DAMAS2 [68] apresenta uma otimização notável de DAMAS. Usando uma aproximação de campo distante, ele assume que a imagem convoluída produzida por delay-and-sum é igual à imagem limpa convoluída com a PSF do beamformer. Estas convoluções são o gargalo do algoritmo, mas se for usada amostragem uniforme em U, elas podem ser significativamente aceleradas com FFTs bidimensionais. Como a imagem delay-and-sum e a PSF são as únicas entradas, a matriz $\mathbf{B}$ torna-se desnecessária.

Seja $\breve{Y}$ a imagem obtida com delay-and-sum, $\mathbf{P}_{d s}$ a PSF para o beamformer delay- 
and-sum, $\mathbf{Y}$ a imagem limpa e $\mathbf{Y}^{(k)}$ a imagem reconstruída na iteração $k$. Por definição,

$$
\breve{\mathbf{Y}}=\mathbf{P}_{d s} * \mathbf{Y}
$$

onde $*$ representa convolução $2 \mathrm{D}$.

DAMAS2 obtém uma aproximação de $\mathbf{Y}$ iterando

$$
\hat{\mathbf{Y}}^{(k+1)}=\max \left\{\hat{\mathbf{Y}}^{(k)}+\frac{1}{a}\left[\breve{\mathbf{Y}}-\left(\mathbf{P}_{d s} * \hat{\mathbf{Y}}^{(k)}\right)\right], \mathbf{0}\right\}
$$

onde $\max \{\cdot, \cdot\}$ retorna o máximo ponto a ponto, $a=\sum_{i, j}\left[\mathbf{P}_{d s}\right]_{i, j}, \hat{\mathbf{Y}}^{(0)}=\mathbf{0}$ e a convolução é implementada usando uma FFT bidimensional e zero padding.

Note que (7.5.10) e (7.5.12) têm a mesma forma, exceto que DAMAS atualiza uma variável por vez, enquanto DAMAS2 atualiza todas as variáveis simultaneamente. Ao atualizar uma variável, DAMAS sempre utiliza os valores mais recentes para todas as outras variáveis, então tende a convergir mais rápido para imagens simples. No entanto, como estes problemas inversos tendem a ser mal condicionados, o método de Gauss-Seidel também produz resultados muito ruidosos, tal que a convergência em cenários realistas é mais lenta e menos confiável do que usando DAMAS2. Como DAMAS2 atualiza todas as variáveis simultaneamente, ele não depende da ordem dos pixels e tende a produzir resultados muito mais limpos. Ele também é muito mais eficiente computacionalmente, pois DAMAS exige um produto matriz-vetor para atualizar cada variável, enquanto DAMAS2 requer (no espaço u) apenas uma convolução acelerada por uma FFT para atualizar o sistema inteiro. Portanto, DAMAS2 pode eficientemente produzir uma imagem deconvoluída, que permite a determinação precisa de pressão sonora em uma escala absoluta (algo que não pode ser feito com beamformers de qualquer tipo e fontes distribuídas).

\subsection{Covariance fitting}

Os métodos mais populares para reconstrução de imagens acústicas utilizam beamforming para obter uma imagem convoluída, e algum método de deconvolução para obter uma aproximação da distribuição de fontes verdadeira. CLEAN e DAMAS são exemplos de algoritmos de deconvolução desenvolvidos para aplicações com phased arrays, mas nada impede que sejam usados métodos desenvolvidos para imagens ópticas (por exemplo, [69] compara DAMAS, DAMAS2, mínimos quadrados não-negativos e o algoritmo de Richardson-Lucy [71,72]). No entanto, estes métodos estão sempre limitados pelo fato de que arrays têm PSFs grandes, que tornam deconvoluções muito mal condicionadas, especialmente para fontes próximas às bordas da região visível. Para evitar o processo de deconvolução, [73] propôs uma técnica de ajuste de matriz de covariância (covariance matrix fitting). 
Dada uma distribuição Y com

$$
\mathbf{y}=\operatorname{vec}\{\mathbf{Y}\}=\left[\begin{array}{c}
\left|Y\left(\mathbf{u}_{0}\right)\right|^{2} \\
\left|Y\left(\mathbf{u}_{1}\right)\right|^{2} \\
\vdots \\
\left|Y\left(\mathbf{u}_{M-1}\right)\right|^{2}
\end{array}\right]
$$

podemos obter $\mathbf{S}$ usando

$$
\begin{aligned}
\mathbf{S} & =\sum_{m=0}^{M-1}\left|Y\left(\mathbf{u}_{m}\right)\right|^{2} \mathbf{v}\left(\mathbf{u}_{m}\right) \mathbf{v}^{H}\left(\mathbf{u}_{m}\right) \\
& =\sum_{m=0}^{M-1} \mathbf{y}_{m} \mathbf{v}\left(\mathbf{u}_{m}\right) \mathbf{v}^{H}\left(\mathbf{u}_{m}\right)
\end{aligned}
$$

Logo,

$$
\operatorname{vec}\{\mathbf{S}\}=\left[\operatorname{vec}\left\{\mathbf{v}\left(\mathbf{u}_{0}\right) \mathbf{v}^{H}\left(\mathbf{u}_{0}\right)\right\} \quad \cdots \quad \operatorname{vec}\left\{\mathbf{v}\left(\mathbf{u}_{M-1}\right) \mathbf{v}^{H}\left(\mathbf{u}_{M-1}\right)\right\}\right] \operatorname{vec}\{\mathbf{Y}\} .
$$

Seja

$$
\mathbf{A}=\left[\operatorname{vec}\left\{\mathbf{v}\left(\mathbf{u}_{0}\right) \mathbf{v}^{H}\left(\mathbf{u}_{0}\right)\right\} \quad \cdots \quad \operatorname{vec}\left\{\mathbf{v}\left(\mathbf{u}_{M-1}\right) \mathbf{v}^{H}\left(\mathbf{u}_{M-1}\right)\right\}\right],
$$

tal que

$$
\operatorname{vec}\{\mathbf{S}\}=\operatorname{Avec}\{\mathbf{Y}\} \text {. }
$$

Os autores de [73] propõem resolver

$$
\min _{\hat{\mathbf{Y}}, \sigma^{2}}\left\|\operatorname{vec}\{\mathbf{S}\}-\mathbf{A} \operatorname{vec}\{\hat{\mathbf{Y}}\}-\sigma^{2} \operatorname{vec}\{\mathbf{I}\}\right\|_{2},
$$

sujeito a $\hat{\mathbf{Y}}_{i, j} \geq 0, \sigma^{2} \geq 0$ e $\|\operatorname{vec}\{\hat{\mathbf{Y}}\}\|_{1} \leq \lambda$, onde $\sigma$ é o nível de ruído branco e $\|\operatorname{vec}\{\hat{\mathbf{Y}}\}\|_{1} \leq \lambda$ controla a esparsidade da solução. Este método supõe que a distribuição de fontes seja esparsa, ou seja, que apenas uma pequena quantidade de pontos no espaço U possua fontes irradiantes. (7.6.7) é um problema de otimização convexa, e pode ser resolvido usando métodos numéricos razoavelmente eficientes (pelo menos no que diz respeito à sua taxa de convergência). A restrição $\ell_{1}$ serve para regularizar o problema, e permitir a obtenção de uma solução estável para um sistema mal condicionado. Evidentemente, esta restrição somente é razoável se a distribuição de fontes for de fato esparsa, o que pode não ser o caso. Além disso, A é uma matriz grande, mesmo para imagens de baixa resolução, tal que resolver (7.6.7) com uma representação matricial de A é um problema computacionalmente intensivo. Os autores de [73] também estenderam seu método para o caso de fontes correlacionadas, onde $\mathbf{A}$ torna-se rapidamente intratável conforme o tamanho do problema aumenta. 
Graças à regularização $\ell_{1}$, os autores de [73] mostram através de exemplos numéricos que (7.6.7) pode de fato produzir soluções esparsas de excelente qualidade. Sua proposta supera DAMAS devido ao uso de regularização, e porque não envolve uma convolução intermediária. No entanto, o custo computacional permanece um problema, pois a representação explícita de $\mathbf{A}$ tem custo muito alto em tempo e memória. Esta observação motiva o capítulo seguinte, onde desenvolvemos implementações rápidas para $\mathbf{A}$. 



\section{Capítulo 8}

\section{Transformadas rápidas para imagens acústicas}

Conforme apresentado no capítulo anterior, as técnicas para reconstrução de imagens acústicas se dividem em 3 categorias: (i) beamforming; (ii) beamforming seguida de deconvolução; (iii) covariance fitting. Beamforming apresenta a pior qualidade de reconstrução, mas o menor custo computacional. Deconvolução melhora significativamente a qualidade das imagens, mas requer mais processamento, exige amostragem uniforme no espaço $\mathrm{U}$ e envolve perda de informação, devido à etapa de convolução. Além disso, como os métodos de deconvolução não envolvem formas explícitas de regularização (mas apenas soluções ad hoc, como a imposição de não-negatividade, e filtragem passa-baixas), as soluções encontradas podem não ser estáveis ou ótimas. Covariance fitting conforme [73] produz os melhores resultados, pois encontra uma solução ótima e estável mediante o uso de regularização, e não envolve perda de informação, pois dispensa a convolução intermediária. Por outro lado, tem custo computacional extremamente alto.

Recentemente, problemas de otimização regularizados tornaram-se populares em aplicações de processamento de sinais, graças ao surgimento de uma teoria rigorosa de compressive sensing [74]. Esta estabelece condições sob as quais é possível recuperar sinais esparsos a partir de um número de amostras menor do que exigido pelo critério de NyquistShannon. Muitos problemas de reconstrução de imagens podem ser expressados como problemas de otimização convexa, e várias contribuições recentes produziram métodos iterativos computacionalmente eficientes para resolvê-los. Ainda que muitas destas técnicas tenham sido desenvolvidas para aplicações de imagens, seu uso permaneceu limitado a áreas específicas. Em particular, a maior parte destas técnicas não foi aplicada a imagens acústicas.

Para entender a barreira de entrada ao campo de imagens acústicas, consideremos o problema genérico de reconstrução não-linear dado por

$$
\hat{\mathbf{x}}=\underset{\mathbf{x}}{\operatorname{argmin}}\|\mathbf{\Psi} \mathbf{x}\| \text { sujeito a } \mathbf{\Phi} \mathbf{x}=\mathbf{y}
$$

onde $\hat{\mathbf{x}}$ é o sinal reconstruído, $\mathbf{y}$ é o sinal medido, $\Psi$ é uma transformada que torna $\mathbf{x}$ esparso quando medido com a norma $\|\cdot\|$, e $\Phi$ é a transformada que modela o processo de 
medida. Tipicamente, y é uma versão significativamente subamostrada de x. Para uma imagem acústica, $\mathbf{x}$ seria uma versão vetorizada da imagem descrevendo a distribuição de fontes verdadeira, e $\mathbf{y}$ seria uma versão vetorizada da matriz espectral medida pelo array.

Métodos iterativos e computacionalmente eficientes dependem de implementações rápidas de $\boldsymbol{\Phi}$ e $\boldsymbol{\Phi}^{H}$ para resolver (8.0.1). Na ausência de implementações rápidas, a avaliação de $\boldsymbol{\Phi} \mathbf{u}$ e $\boldsymbol{\Phi}^{H} \mathbf{v}$ para $\mathbf{u}, \mathbf{v}$ arbitrários inevitavelmente se torna o gargalo do solver. Por exemplo, em aplicações de ressonância magnética, temos $\mathbf{\Phi}=\mathbf{P} \mathcal{F}$, onde $\mathcal{F}$ é uma FFT e $\mathbf{P}$ é um operador de subamostragem, e $\boldsymbol{\Psi}$ é tipicamente uma transformada wavelet rápida. Acreditamos que este é o primeiro trabalho a propor uma implementação rápida de $\mathbf{\Phi}$ para imagens acústicas.

Para motivar a necessidade de uma transformada rápida, considere uma representação matricial de $\boldsymbol{\Phi}$. Dado um array de $N$ sensores e uma imagem com $M$ pixels, $\boldsymbol{\Phi}$ tem $N^{2}$ linhas e $M$ colunas. Para $N^{2}=M=256^{2}, \boldsymbol{\Phi}$ tem 4 bilhões de elementos e os produtos $\boldsymbol{\Phi} \mathbf{u}$ e $\boldsymbol{\Phi}^{H} \mathbf{v}$ são computacionalmente muito caros, tornando qualquer algoritmo de otimização convexa intratável com recursos computacionais atuais. Portanto, uma implementação matricial de $\boldsymbol{\Phi}$ é somente prática para imagens de baixa resolução e arrays com poucos elementos, motivando o desenvolvimento de uma transformada rápida.

Até o momento, propostas para acelerar imagens acústicas foram baseadas em beamforming. Zimmerman e Studer [75] propuseram usar uma FPGA para realizar a computação associada ao beamforming, e assim desenhar imagens acústicas sobre um framebuffer. Ainda que esta proposta reduza o tempo computacional em relação a um processador de propósito geral, ela não reduz o custo computacional. Huang [76] propõe um método para recursivamente obter uma aproximação da imagem acústica durante a aquisição de dados (em contraste com outros algoritmos, que computam a imagem a partir de uma matriz de covariância). Apesar deste método ter a vantagem de retornar resultados incrementais, ele tem o mesmo custo computacional de beamforming, e resolução semelhante. Em contraste, as transformadas propostas a seguir reduzem dramaticamente o custo computacional de imagens acústicas, permitindo reconstruções com super-resolução.

A seguir, apresentamos transformadas rápidas para implementar $\boldsymbol{\Phi}, \boldsymbol{\Phi}^{T}, \boldsymbol{\Phi}^{H}$ e $\boldsymbol{\Phi}^{H} \boldsymbol{\Phi}$ para geometrias separáveis e uniformes. Obtemos tempos de execução que são ordens de magnitude menores que aqueles obtidos com representações matriciais. Estes resultados podem ser aplicados para acelerar algoritmos existentes, como beamforming, CLEAN e DAMAS. A existência de uma transformada rápida também permite o uso de solvers de propósito geral para resolver problemas de imagem acústicas, evitando o uso de implementações ad hoc que tipicamente produzem resultados inferiores. De fato, com transformadas rápidas torna-se possível utilizar a maior parte dos solvers desenvolvidos para mínimos quadrados regularizados, usados frequentemente para reconstrução de imagens médicas e compressive sensing. 


\subsection{Transformada rápida direta}

Defina $\mathbf{y}=\left[\begin{array}{lll}\left|Y\left(\mathbf{u}_{0}\right)\right|^{2} & \ldots & \left|Y\left(\mathbf{u}_{M-1}\right)\right|^{2}\end{array}\right]^{T}$. Escrevamos (7.3.5) como a transformada linear $\mathbf{A}$ tal que vec $\{\mathbf{S}\}=\mathbf{A y}$. Para economizar espaço, escreveremos $\mathbf{v}\left(\mathbf{u}_{m}\right)$ como $\mathbf{v}_{\mathbf{u}_{m}}$, e denotaremos seu $i$-ésimo elemento por $\mathrm{v}_{u_{m}}^{i}$ (elementos de array manifold vectors serão indicados usando superíndices). Seja $N$ o número de microfones do array. Note que

$$
\operatorname{vec}\left\{\mathbf{v}_{\mathbf{u}_{m}} \mathbf{v}_{\mathbf{u}_{m}}^{H}\right\}=\left[\begin{array}{c}
\mathbf{v}_{\mathbf{u}_{m}} \mathbf{v}_{\mathbf{u}_{m}}^{0 *} \\
\mathbf{v}_{\mathbf{u}_{m}} \mathbf{v}_{\mathbf{u}_{m}}^{1 *} \\
\vdots \\
\mathbf{v}_{\mathbf{u}_{m}} \mathbf{v}_{\mathbf{u}_{m}}^{(N-1) *}
\end{array}\right] \text {. }
$$

Portanto,

$$
\begin{aligned}
\operatorname{vec}\{\mathbf{S}\}= & \mathbf{A y} \\
= & {\left[\begin{array}{cccc}
\mathbf{v}_{\mathbf{u}_{0}} \mathrm{v}_{\mathbf{u}_{0}}^{0 *} & \mathbf{v}_{\mathbf{u}_{1}} \mathbf{v}_{\mathbf{u}_{1}}^{0 *} & \cdots & \mathbf{v}_{\mathbf{u}_{M-1}} \mathbf{v}_{\mathbf{u}_{M-1}}^{0 *} \\
\mathbf{v}_{\mathbf{u}_{0}} \mathrm{v}_{\mathbf{u}_{0}}^{1 *} & \mathbf{v}_{\mathbf{u}_{1}} \mathbf{v}_{\mathbf{u}_{1}}^{1 *} & \cdots & \mathbf{v}_{\mathbf{u}_{M-1}} \mathbf{v}_{\mathbf{u}_{M-1}}^{1 *} \\
\vdots & \vdots & & \vdots \\
\mathbf{v}_{\mathbf{u}_{0}} \mathbf{v}_{\mathbf{u}_{0}}^{(N-1) *} & \mathbf{v}_{\mathbf{u}_{1}} \mathbf{v}_{\mathbf{u}_{1}}^{(N-1) *} & \cdots & \mathbf{v}_{\mathbf{u}_{M-1}} \mathbf{v}_{\mathbf{u}_{M-1}}^{(N-1) *}
\end{array}\right] \mathbf{y} } \\
= & {\left[\begin{array}{llll}
\mathbf{v}_{\mathbf{u}_{0}}^{*} \otimes \mathbf{v}_{\mathbf{u}_{0}} & \mathbf{v}_{\mathbf{u}_{1}}^{*} \otimes \mathbf{v}_{\mathbf{u}_{1}} & \cdots & \mathbf{v}_{\mathbf{u}_{M-1}}^{*} \otimes \mathbf{v}_{\mathbf{u}_{M-1}}
\end{array}\right] \mathbf{y}, }
\end{aligned}
$$

onde $\otimes$ é o produto de Kronecker.

Dado um array bidimensional, seu array manifold vector $\mathbf{v}(\mathbf{u})=\mathbf{v}\left(u_{x}, u_{y}\right)$ é dito separável se existirem $\mathbf{a}\left(u_{x}\right)$ e $\mathbf{b}\left(u_{y}\right)$ tais que $\mathbf{v}\left(u_{x}, u_{y}\right)=\mathbf{a}\left(u_{x}\right) \otimes \mathbf{b}\left(u_{y}\right)$ para todos $u_{x}, u_{y}$ válidos. Destacamos que $\mathbf{a}\left(u_{x}\right)$ e $\mathbf{b}\left(u_{y}\right)$ não precisam ser manifold vectors de subarrays.

A seguir obteremos primeiro transformadas rápidas supondo somente $\mathbf{v}(\mathbf{u})$ separável. Mostraremos que em campo distante, esta hipótese equivale ao uso de uma geometria Cartesiana. Então admitiremos uma geometria uniforme, que levará a uma otimização adicional. A motivação para apresentar ambas realizações ao invés de supor diretamente a separabilidade e a uniformidade vem de aplicações de reconstruções de imagens. Arrays uniformes podem ser muito convenientes de um ponto de vista computacional, mas sua maior frequência de operação está limitada pelo teorema de amostragem de NyquistShannon. Portanto, para evitar aliasing espacial, o menor comprimento de onda mensurável fica limitado à metade da distância interelementos. Para eliminar esta restrição, uma geometria não uniforme deve ser usada, o que aumenta o custo computacional da transformada.

Em uma aplicação prática, a escolha da geometria do array envolve um compromisso entre qualidade de reconstrução e complexidade computacional. Para otimizar a qualidade da reconstrução, um array aleatório seria desejável, pois permitiria a reconstru- 
ção de imagens em comprimentos de onda consideravelmente menores que sua distância interelementos média (veja a Seção 3.4). Por outro lado, as transformadas rápidas que descreveremos não se aplicariam, e o algoritmo de reconstrução seria computacionalmente muito mais caro. Portanto, admitir somente a separabilidade confere ao usuário a opção de usar uma transformada acelerada, sem perda significativa de diversidade espacial.

Para simplificar a notação que segue, usaremos a enumeração de $\mathbf{u}_{0}, \ldots, \mathbf{u}_{M-1}$ descrita na Seção 7.4. Ou seja, sejam $\left\{u_{x_{m}}\right\}_{0 \leq m<M_{x}}$ e $\left\{u_{y_{n}}\right\}_{0 \leq n<M_{y}}$ os pontos de amostragem de $\mathrm{U}$ ao longo dos eixos x e y, ordenados da esquerda para a direita e de cima para baixo. Definimos $\mathbf{u}_{0}, \ldots, \mathbf{u}_{M-1}$ tal que

$$
\mathbf{u}_{m}=\left[\begin{array}{ll}
u_{x_{\left\lfloor m / M_{y}\right\rfloor}} & u_{y_{\bmod \left(m, M_{y}\right)}}
\end{array}\right]^{T} .
$$

Seja Y uma imagem com $M_{x} \times M_{y}$ pixels. Ordenamos os pixels de $\mathbf{Y}$ tal que

$$
\operatorname{vec}\{\mathbf{Y}\}=\left[\begin{array}{c}
\left|Y\left(\mathbf{u}_{0}\right)\right|^{2} \\
\left|Y\left(\mathbf{u}_{1}\right)\right|^{2} \\
\vdots \\
\left|Y\left(\mathbf{u}_{M-1}\right)\right|^{2}
\end{array}\right]
$$

o que implica $\mathbf{Y}_{n, m}=\left|Y\left(u_{x_{m}}, u_{y_{n}}\right)\right|^{2}$.

\subsubsection{Caso 1: geometria separável}

Mostremos inicialmente que sob uma parametrização em campo distante dada por (7.2.6), um array tem $\mathbf{v}(\mathbf{u})$ separável se e somente se possui elementos localizados sobre um grid Cartesiano.

Consideremos um array com geometria Cartesiana, com sensores nas coordenadas $\mathbf{p}_{i} \in \mathbb{R}^{3}$ para $0 \leq i<N$. Suponhamos que as coordenadas $\mathrm{x}$ e y destes sensores foram escolhidas de $\left\{p_{x_{i}}\right\}_{i=0}^{N_{x}-1}$ e $\left\{p_{y_{i}}\right\}_{i=0}^{N_{y}-1}$, tal que

$$
\mathbf{p}_{i}=\left[\begin{array}{lll}
p_{x_{\left\lfloor i / N_{y}\right\rfloor}} & p_{y_{\bmod \left(i, N_{y}\right)}} & 0
\end{array}\right]^{T} .
$$

Definimos um array horizontal com sensores em $\mathbf{p}_{x_{i}} \in \mathbb{R}^{3}$, para $0 \leq i<N_{x}$, e um array vertical com sensores em $\mathbf{p}_{y_{j}} \in \mathbb{R}^{3}$, para $0 \leq j<N_{y}$, tal que

$$
\begin{aligned}
& \mathbf{p}_{x_{i}}=\left[\begin{array}{lll}
p_{x_{i}} & 0 & 0
\end{array}\right]^{T} \\
& \mathbf{p}_{y_{j}}=\left[\begin{array}{lll}
0 & p_{y_{j}} & 0
\end{array}\right]^{T} .
\end{aligned}
$$

Sejam $\mathbf{v}_{x}$ e $\mathbf{v}_{y}$ os manifold vectors com dimensões $N_{x} \times 1$ e $N_{y} \times 1$ correspondentes a estes arrays. Então para $0 \leq i<N_{x}$ e $0 \leq j<N_{y}$, 


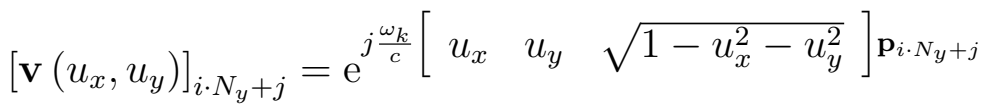

$$
\begin{aligned}
& =\mathrm{e}^{j \frac{\omega_{k}}{c}\left[\begin{array}{lll}
u_{x} & u_{y} & \sqrt{1-u_{x}^{2}-u_{y}^{2}}
\end{array}\right]\left(\mathbf{p}_{x_{i}}+\mathbf{p}_{y_{j}}\right)}
\end{aligned}
$$

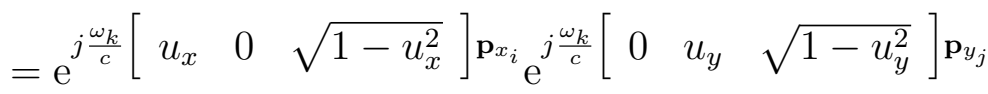

$$
\begin{aligned}
& =\left[\mathbf{v}_{x}\left(u_{x}\right)\right]_{i}\left[\mathbf{v}_{y}\left(u_{y}\right)\right]_{j},
\end{aligned}
$$

o que por definição é equivalente a $\mathbf{v}\left(u_{x}, u_{y}\right)=\mathbf{v}_{x}\left(u_{x}\right) \otimes \mathbf{v}_{y}\left(u_{y}\right)$. Portanto, arrays com geometrias Cartesianas são separáveis sob uma parametrização no espaço U.

Para provar a recíproca, note que

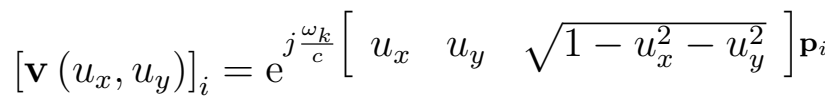

$$
\begin{aligned}
& =\mathrm{e}^{j \frac{\omega_{k}}{c}\left(u_{x} \bar{p}_{x_{i}}+u_{y} \bar{p}_{y_{i}}\right)} \\
& =\mathrm{e}^{j \frac{\omega_{k}}{c} u_{x} \bar{p}_{x_{i}}} \mathrm{e}^{j \frac{\omega_{k}}{c} u_{y} \bar{p}_{y_{i}},}
\end{aligned}
$$

onde $\mathbf{p}_{i}=\left[\begin{array}{lll}\bar{p}_{x_{i}} & \bar{p}_{y_{i}} & 0\end{array}\right]^{T}$. Por hipótese, existem $\mathbf{a}\left(u_{x}\right)$ e $\mathbf{b}\left(u_{x}\right)$ tais que $\mathbf{v}\left(u_{x}, u_{y}\right)=$ $\mathbf{a}\left(u_{x}\right) \otimes \mathbf{b}\left(u_{y}\right)$. O termo $u_{x} \bar{p}_{x_{i}}$ de (8.1.16) deve pertencer a $\mathbf{a}\left(u_{x}\right)$, pois é uma função de $u_{x}$ e $\bar{p}_{x_{i}}$ é constante. Segue que $\mathbf{a}\left(u_{x}\right)=\mathbf{v}_{x}\left(u_{x}\right)$ e $\mathbf{b}\left(u_{y}\right)=\mathbf{v}_{y}\left(u_{y}\right), \operatorname{com} \mathbf{v}_{x}\left(u_{x}\right)$ e $\mathbf{v}_{y}\left(u_{y}\right)$ definidos acima, implicando uma geometria Cartesiana.

A seguir, desenvolveremos uma transformada rápida admitindo a separabilidade de $\mathbf{v}(\mathbf{u})$. Para economizar espaço, usaremos a notação

$$
\begin{aligned}
& \mathbf{v}_{x}\left(u_{x_{m}}\right)=\mathbf{v}_{x_{m}}=\left[\begin{array}{llll}
\mathrm{v}_{x_{m}}^{0} & \mathrm{v}_{x_{m}}^{1} & \cdots & \mathrm{v}_{x_{m}}^{N_{x}-1}
\end{array}\right]^{T} \\
& \mathbf{v}_{y}\left(u_{y_{n}}\right)=\mathbf{v}_{y_{n}}=\left[\begin{array}{llll}
\mathrm{v}_{y_{n}}^{0} & \mathrm{v}_{y_{n}}^{1} & \cdots & \mathrm{v}_{y_{n}}^{N_{y}-1}
\end{array}\right]^{T} .
\end{aligned}
$$

Usando a separabilidade do array em (8.1.4), obtemos

$$
\mathbf{A}=\left[\begin{array}{lll}
\left(\mathbf{v}_{x_{0}}^{*} \otimes \mathbf{v}_{y_{0}}^{*}\right) \otimes\left(\mathbf{v}_{x_{0}} \otimes \mathbf{v}_{y_{0}}\right) & \cdots & \left.\left(\mathbf{v}_{x_{M-1}}^{*} \otimes \mathbf{v}_{y_{M-1}}^{*}\right) \otimes\left(\mathbf{v}_{x_{M-1}} \otimes \mathbf{v}_{y_{M-1}}\right)\right]
\end{array}\right]
$$

Para $0 \leq m, n<N_{x} N_{y}$, a separabilidade permite escrever a linha $m \cdot N_{x} N_{y}+n$ de $\mathbf{A}$ como

$$
\left[\begin{array}{lll}
\mathrm{v}_{x_{0}}^{i *} \mathrm{v}_{x_{0}}^{j} & \cdots & \mathrm{v}_{x_{M_{x}-1}}^{i *} \mathrm{v}_{x_{M_{x}-1}}^{j}
\end{array}\right] \otimes\left[\begin{array}{lll}
\mathrm{v}_{y_{0}}^{k *} \mathrm{v}_{y_{0}}^{l} & \cdots & \mathrm{v}_{y_{M_{y}-1}}^{k *} \mathrm{v}_{y_{M_{y}-1}}^{l}
\end{array}\right]
$$

onde $i=\left\lfloor\frac{m}{N_{y}}\right\rfloor, j=\left\lfloor\frac{n}{N_{y}}\right\rfloor, k=\bmod \left(m, N_{y}\right), l=\bmod \left(n, N_{y}\right)$. 
Para $0 \leq i, j<N_{x}$ e $0 \leq k, l<N_{y}$, defina

$$
\begin{aligned}
c_{m}(i, j) & =\mathrm{v}_{x_{m}}^{i *} \mathrm{v}_{x_{m}}^{j} \\
d_{n}(k, l) & =\mathrm{v}_{y_{n}}^{k *} \mathrm{v}_{y_{n}}^{l} .
\end{aligned}
$$

Para $0 \leq m, n<N_{x} N_{y}$, um elemento arbitrário $\mathbf{S}_{n, m}$ de $\mathbf{S}$ pode ser escrito como o produto da linha $m \cdot N_{x} N_{y}+n$ de $\mathbf{A}$ e vec $\{\mathbf{Y}\}$. Defina

$$
\begin{aligned}
\mathbf{c}(i, j) & =\left[\begin{array}{lll}
c_{0}(i, j) & \cdots & c_{M_{x}-1}(i, j)
\end{array}\right]^{T} \\
& =\left[\begin{array}{lll}
\mathrm{v}_{x_{0}}^{i *} \mathrm{v}_{x_{0}}^{j} & \cdots & \mathrm{v}_{x_{M_{x}-1}}^{i *} \mathrm{v}_{x_{M_{x}-1}}^{j}
\end{array}\right]^{T} \\
\mathbf{d}(k, l) & =\left[\begin{array}{lll}
d_{0}(k, l) & \cdots & d_{M_{y}-1}(k, l)
\end{array}\right]^{T} \\
& =\left[\begin{array}{lll}
\mathrm{v}_{y_{0}}^{k *} \mathrm{v}_{y_{0}}^{l} & \cdots & \mathrm{v}_{y_{M_{y}-1}}^{k *} \mathrm{v}_{y_{M_{y}-1}}^{l}
\end{array}\right]^{T} .
\end{aligned}
$$

Usando (8.1.19), temos

$$
\begin{aligned}
\mathbf{S}_{n, m} & =\left[\mathbf{c}^{T}(i, j) \otimes \mathbf{d}^{T}(k, l)\right] \operatorname{vec}\{\mathbf{Y}\} \\
& =\mathbf{d}^{T}(k, l) \mathbf{Y} \mathbf{c}(i, j)
\end{aligned}
$$

onde $i=\left\lfloor\frac{m}{N_{y}}\right\rfloor, j=\left\lfloor\frac{n}{N_{y}}\right\rfloor, k=\bmod \left(m, N_{y}\right), l=\bmod \left(n, N_{y}\right)$. Além disso, (8.1.21) e (8.1.22) são equivalentes porque $\left(\mathbf{A}^{T} \otimes \mathbf{B}\right) \operatorname{vec}\{\mathbf{C}\}=\operatorname{vec}\{\mathbf{B C A}\}$ sempre que BCA estiver definido [77].

Para $0 \leq i, j<N_{x}$ e $0 \leq k, l<N_{y}$, defina

$$
(i, j) \diamond(k, l)=\mathbf{d}^{T}(k, l) \mathbf{Y c}(i, j)
$$

e

$$
\mathbf{T}_{j, i}=\left[\begin{array}{ccc}
(i, j) \diamond(0,0) & \cdots & (i, j) \diamond\left(N_{y}-1,0\right) \\
(i, j) \diamond(0,1) & \cdots & (i, j) \diamond\left(N_{y}-1,1\right) \\
\vdots & & \vdots \\
(i, j) \diamond\left(0, N_{y}-1\right) & \cdots & (i, j) \diamond\left(N_{y}-1, N_{y}-1\right)
\end{array}\right]
$$

Usando os resultados acima, é fácil mostrar que

$$
\mathbf{S}=\left[\begin{array}{cccc}
\mathbf{T}_{0,0} & \mathbf{T}_{0,1} & \cdots & \mathbf{T}_{0, N_{x}-1} \\
\mathbf{T}_{1,0} & \mathbf{T}_{1,1} & \cdots & \mathbf{T}_{1, N_{x}-1} \\
\vdots & \vdots & & \vdots \\
\mathbf{T}_{N_{x}-1,0} & \mathbf{T}_{N_{x}-1,1} & \cdots & \mathbf{T}_{N_{x}-1, N_{x}-1}
\end{array}\right]
$$


Ainda que seja possível obter $(i, j) \diamond(k, l)$ para $0 \leq i, j<N_{x}$ e $0 \leq k, l<N_{y}$ através da avaliação direta de (8.1.23), deve-se organizar as operações de forma a eliminar redundâncias. Além disso, em arquiteturas de computador modernas, as unidades aritméticas podem processar operandos mais rápido que a memória principal pode fornecê-los. Portanto, deve-se maximizar a localidade de referência para garantir que os operandos estejam frequentemente no cache de dados. Em particular, o algoritmo deve promover acessos sequenciais à memória, para que as unidades aritméticas não parem de trabalhar à espera de um operando localizado na memória principal. A seguir apresentaremos uma implementação com estas características.

Seja

$$
\begin{aligned}
\mathbf{t}_{i, j} & =\operatorname{vec}\left\{\mathbf{T}_{i, j}\right\} \\
\mathbf{Z} & =\left[\begin{array}{llll}
\mathbf{t}_{0,0} & \mathbf{t}_{1,0} & \ldots & \mathbf{t}_{N_{x}-1, N_{x}-1}
\end{array}\right] .
\end{aligned}
$$

Dado $\mathbf{Z}$, é fácil obter $\mathbf{S}$, pois cada bloco $\mathbf{T}_{i, j}$ de $\mathbf{S}$ pode ser obtido desempilhando $\mathbf{t}_{i, j}$.

Defina

$$
\begin{array}{r}
\mathbf{V}_{\mathbf{x}}=\left[\begin{array}{ccc}
c_{0}(0,0) & \cdots & c_{M_{x}-1}(0,0) \\
c_{0}(0,1) & \cdots & c_{M_{x}-1}(0,1) \\
\vdots & & \vdots \\
c_{0}\left(N_{x}-1, N_{x}-1\right) & \cdots & c_{M_{x}-1}\left(N_{x}-1, N_{x}-1\right)
\end{array}\right] \\
\mathbf{V}_{\mathbf{y}}=\left[\begin{array}{ccc}
d_{0}(0,0) & \cdots & d_{M_{y}-1}(0,0) \\
d_{0}(0,1) & \cdots & d_{M_{y}-1}(0,1) \\
\vdots & & \vdots \\
d_{0}\left(N_{y}-1, N_{y}-1\right) & \cdots & d_{M_{y}-1}\left(N_{y}-1, N_{y}-1\right)
\end{array}\right] .
\end{array}
$$

Comparando com (8.1.23), pode-se verificar que

$$
\mathbf{Z}=\mathbf{V}_{\mathbf{y}} \mathbf{Y} \mathbf{V}_{\mathbf{x}}^{T}
$$

Defina $\boldsymbol{\Xi}_{s}$ tal que vec $\{\mathbf{S}\}=\boldsymbol{\Xi}_{s} \operatorname{vec}\{\mathbf{Z}\}$. Note que $\boldsymbol{\Xi}_{s}$ é uma permutação (a Figura 8.1 mostra como $\boldsymbol{\Xi}_{s}$ opera sobre $\mathbf{Z}$, para um caso particular). Portanto, vec $\{\mathbf{S}\}=$ $\boldsymbol{\Xi}_{s}\left(\mathbf{V}_{\mathbf{x}} \otimes \mathbf{V}_{\mathbf{y}}\right) \operatorname{vec}\{\mathbf{Y}\}$ e

$$
\mathbf{A}=\boldsymbol{\Xi}_{s}\left(\mathbf{V}_{\mathbf{x}} \otimes \mathbf{V}_{\mathbf{y}}\right)
$$

Como $\boldsymbol{\Xi}_{s}$ é computacionalmente simples e $\left(\mathbf{V}_{\mathbf{x}} \otimes \mathbf{V}_{\mathbf{y}}\right) \operatorname{vec}\{\mathbf{Y}\}=\operatorname{vec}\left\{\mathbf{V}_{\mathbf{y}} \mathbf{Y} \mathbf{V}_{\mathbf{x}}^{T}\right\},(8.1 .31)$ pode ser implementada como uma transformada rápida.

De (8.1.25) pode ser visto que $\mathbf{T}_{i, j}$ contém as covariâncias cruzadas entre pares de colunas com $N_{y} \times 1$ sensores. Logo, $\mathbf{Z}$ é uma reorganização de $\mathbf{S}$ que empilha estas covariâncias cruzadas com a regularidade que coincide com a ordem das linhas de $\mathbf{V}_{\mathbf{x}} \otimes \mathbf{V}_{\mathbf{y}}$ 

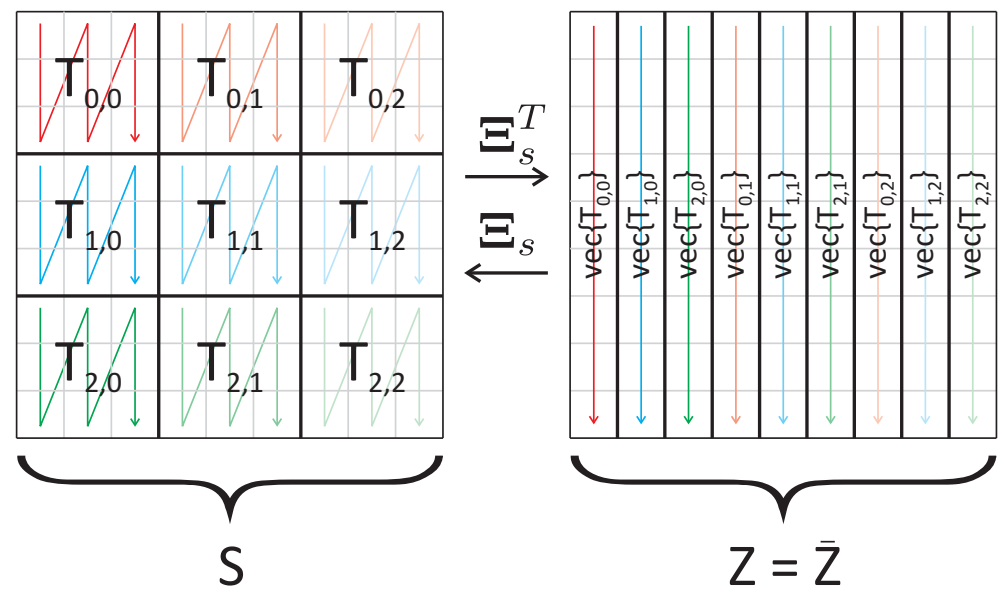

Figura 8.1: Exemplo de implementação rápida para $\boldsymbol{\Xi}_{s}$ e $\boldsymbol{\Xi}_{s}^{T}$, supondo $N_{x}=N_{y}=3$.

(pois vec $\left.\{\mathbf{Z}\}=\left(\mathbf{V}_{\mathbf{x}} \otimes \mathbf{V}_{\mathbf{y}}\right) \operatorname{vec}\{\mathbf{Y}\}\right)$.

O produto direto Ay de (8.1.2) requer aproximadamente $\frac{1}{2} M_{x} M_{y} N_{x}^{2} N_{y}^{2}$ acumulaçõesmultiplicações (MACs) complexas se implementado explorando a simetria Hermitiana de A. Avaliar $\left(\mathbf{V}_{\mathbf{y}} \mathbf{Y}\right) \mathbf{V}_{\mathbf{x}}^{T}$ e $\mathbf{V}_{\mathbf{y}}\left(\mathbf{Y} \mathbf{V}_{\mathbf{x}}^{T}\right)$ requer $N_{y}^{2} M_{x} M_{y}+N_{x}^{2} N_{y}^{2} M_{x}$ e $N_{x}^{2} M_{x} M_{y}+N_{x}^{2} N_{y}^{2} M_{y}$ MACs complexos, respectivamente. Como $\mathrm{Y}$ é real, o primeiro produto pode ser otimizado e o custo cai para $\frac{1}{2} N_{y}^{2} M_{x} M_{y}+N_{x}^{2} N_{y}^{2} M_{x}$ e $\frac{1}{2} N_{x}^{2} M_{x} M_{y}+N_{x}^{2} N_{y}^{2} M_{y}$ MACs complexos, respectivamente. Usando a primeira expressão e desprezando o tempo para obter $\mathbf{S}$ a partir de $\mathbf{Z}$, a aceleração relativa em termos de MACs é

$$
\begin{aligned}
\frac{\frac{1}{2} M_{x} M_{y} N_{x}^{2} N_{y}^{2}}{\frac{1}{2} N_{y}^{2} M_{x} M_{y}+N_{x}^{2} N_{y}^{2} M_{x}} & =\frac{M_{y} N_{x}^{2}}{M_{y}+2 N_{x}^{2}} \\
& \geq \frac{M_{y} N_{x}^{2}}{2 \cdot \max \left\{M_{y}, 2 N_{x}^{2}\right\}} \\
& =\min \left\{\frac{M_{y}}{4}, \frac{N_{x}^{2}}{2}\right\}
\end{aligned}
$$

Se a geometria do array for simétrica em relação ao eixo y, então $\mathbf{V}_{x}$ terá simetria conjugada em relação à sua linha central. Uma consideração análoga vale para $\mathbf{V}_{y}$. Se aplicáveis, estas simetrias podem ser usadas para reduzir ainda mais o custo computacional.

Recorde que introduzimos $\mathbf{Y}$ tendo linhas que realizam uma amostragem separável arbitrária de U. Se $\left\{u_{x_{i}}\right\}$ e $\left\{u_{y_{i}}\right\}$ amostrarem uniformemente o espaço U, então $\mathbf{V}_{\mathbf{x}}$ e $\mathbf{V}_{\mathbf{y}}$ podem ser interpretadas como matrizes de DFT com amostragem não uniforme em frequência (este fato pode ser verificado escrevendo $\mathbf{V}_{\mathbf{x}}$ e $\mathbf{V}_{\mathbf{y}}$ usando exponenciais complexas). Portanto, para $N_{x}$ e $N_{y}$ suficientemente grandes, uma otimização consiste em utilizar uma transformada rápida de Fourier não-uniforme (NFFT) [78] ao invés de cada produto matricial. Uma regra aproximada obtida de experimentos numéricos é usar a NFFT para $N_{x}>8$ ou $N_{y}>8$ e $M_{x}>2^{8}$ ou $M_{y}>2^{8}$. Detalhes referentes ao desempenho com e sem 
a NFFT estão apresentados na Seção 8.6.

\subsubsection{Caso 2: geometria uniforme}

Para obter uma otimização adicional, suponha que o array seja retangular, com sensores uniformemente espaçados ao longo dos eixos x e y (o espaçamento interelementos horizontal pode diferir do espaçamento interelementos vertical).

A uniformidade do array implica

$$
\begin{aligned}
& \forall u \exists \alpha \in \mathbb{C} \forall i, k \mathrm{v}_{x}^{i+k}(u)=\alpha^{k} \mathrm{v}_{x}^{i}(u) \\
& \forall u \exists \alpha \in \mathbb{C} \forall i, k \mathrm{v}_{y}^{i+k}(u)=\alpha^{k} \mathrm{v}_{y}^{i}(u)
\end{aligned}
$$

Por exemplo, dado um espaçamento interelementos horizontal $\Delta p_{x}$ e uma frequência de operação $\omega$, referindo a (7.2.6) é possível verificar que $\alpha=\mathrm{e}^{j \omega u \Delta p_{x} / c}$.

Note que como $\mathrm{v}_{x_{m}}^{i}=\mathrm{e}^{-\sqrt{-1} \omega \mathbf{u}_{x_{m}}^{T} \mathbf{p}_{x_{i}} / c}$ e $\mathrm{v}_{y_{m}}^{j}=\mathrm{e}^{-\sqrt{-1} \omega \mathbf{u}_{y_{m}}^{T} \mathbf{p}_{y_{j}} / c}$, então

$$
\begin{aligned}
\mathrm{v}_{x_{m}}^{i *} & =\frac{1}{\mathrm{v}_{x_{m}}^{i}} \\
\mathrm{v}_{y_{n}}^{j *} & =\frac{1}{\mathrm{v}_{y_{n}}^{j}} .
\end{aligned}
$$

Usando (8.1.33) e (8.1.32), então para todos $i, j, k$ que resultem em índices válidos,

$$
\begin{aligned}
\mathrm{v}_{x_{m}}^{i *} \mathrm{v}_{x_{m}}^{j} & =\frac{1}{\mathrm{v}_{x_{m}}^{i}} \mathrm{v}_{x_{m}}^{j} \\
& =\frac{1}{\mathrm{v}_{m}^{i+k}} \mathrm{v}_{x_{m}}^{j+k} \\
& =\mathrm{v}_{x_{m}}^{(i+k) *} \mathrm{v}_{x_{m}}^{j+k} \\
\mathrm{v}_{y_{n}}^{i *} \mathrm{v}_{y_{n}}^{j} & =\mathrm{v}_{y_{n}}^{(i+k) *} \mathrm{v}_{y_{n}}^{j+k} .
\end{aligned}
$$

Portanto,

$$
\begin{aligned}
& c_{m}(i, j)=c_{m}(i+k, j+k) \\
& d_{n}(i, j)=d_{n}(i+k, j+k)
\end{aligned}
$$

para todos $i, j, k$ que resultem em índices válidos. Isto implica que a maior parte dos valores de $\mathbf{V}_{\mathbf{x}}$ e $\mathbf{V}_{\mathbf{y}}$ são repetidos. De fato, cada coluna de $\mathbf{V}_{\mathbf{x}}$ tem $N_{x}^{2}$ elementos, e segue da identidade acima que no máximo $\left(2 N_{x}-1\right)$ deles são únicos. Um resultado análogo é válido para $\mathbf{V}_{\mathbf{y}}$. Usaremos este fato para obter versões menores e não redundantes de $\mathbf{V}_{\mathbf{x}}$ e $\mathbf{V}_{\mathbf{y}}$, que levarão a produtos matriciais mais rápidos.

Para $-N_{x}<i<N_{x}$ e $-N_{y}<j<N_{y}$, defina

$$
\begin{aligned}
& c_{m}(i)=\left\{\begin{array}{ll}
c_{m}(0,|i|) & \text { se } i \geq 0 \\
c_{m}(|i|, 0) & \text { se } i<0
\end{array}= \begin{cases}\mathrm{v}_{x_{m}}^{0 *} \mathrm{v}_{x_{m}}^{i} & \text { se } i \geq 0 \\
{\left[\mathrm{v}_{x_{m}}^{0 *} \mathrm{v}_{x_{m}}^{-i}\right]^{*}} & \text { se } i<0\end{cases} \right. \\
& d_{n}(j)=\left\{\begin{array}{ll}
d_{n}(0,|j|) & \text { se } j \geq 0 \\
d_{n}(|j|, 0) & \text { se } j<0
\end{array}= \begin{cases}\mathrm{v}_{y_{n}}^{0 *} \mathrm{v}_{y_{n}}^{j} & \text { se } j \geq 0 \\
{\left[\mathrm{v}_{y_{n}}^{0 *} \mathrm{v}_{y_{n}}^{-j}\right]^{*}} & \text { se } j<0\end{cases} \right.
\end{aligned}
$$


É fácil verificar que para todo $0 \leq i, j<N_{x}$ e $0 \leq k, l<N_{y}$,

$$
\begin{aligned}
\mathrm{v}_{x_{m}}^{i *} \mathrm{v}_{x_{m}}^{j} & =c_{m}(j-i) \\
\mathrm{v}_{y_{n}}^{k *} \mathrm{v}_{y_{n}}^{l} & =d_{n}(l-k) .
\end{aligned}
$$

Para $-N_{x}<k<N_{x}$ e $-N_{y}<l<N_{y}$, defina

$$
k \diamond l=\left[\begin{array}{llll}
d_{0}(l) & d_{1}(l) & \cdots & d_{M_{y}-1}(l)
\end{array}\right] \mathbf{Y}\left[\begin{array}{c}
c_{0}(k) \\
c_{1}(k) \\
\vdots \\
c_{M_{x}-1}(k)
\end{array}\right] .
$$

Para $0 \leq i, j<N_{x}, \mathbf{T}_{j, i}$ de (8.1.24) se torna

$$
\mathbf{T}_{j, i}=\left[\begin{array}{cccc}
(j-i) \diamond 0 & (j-i) \diamond-1 & \cdots & (j-i) \diamond 1-N_{y} \\
(j-i) \diamond 1 & (j-i) \diamond 0 & \cdots & (j-i) \diamond 2-N_{y} \\
\vdots & \vdots & & \vdots \\
(j-i) \diamond N_{y}-1 & (j-i) \diamond N_{y}-2 & \cdots & (j-i) \diamond 0
\end{array}\right] .
$$

Dos resultados anteriores, é fácil mostrar que

$$
\mathbf{S}=\left[\begin{array}{cccc}
\mathbf{T}_{0,0} & \mathbf{T}_{0,1} & \cdots & \mathbf{T}_{0, N_{x}-1} \\
\mathbf{T}_{1,0} & \mathbf{T}_{1,1} & \cdots & \mathbf{T}_{1, N_{x}-1} \\
\vdots & \vdots & & \vdots \\
\mathbf{T}_{N_{x}-1,0} & \mathbf{T}_{N_{x}-1,1} & \cdots & \mathbf{T}_{N_{x}-1, N_{x}-1}
\end{array}\right]
$$

Por exemplo, para $N_{x}=8$ e $N_{y}=4$ temos

$$
\mathbf{S}=\left[\begin{array}{cccc|c|cccc}
0 \diamond 0 & 0 \diamond-1 & \cdots & 0 \diamond-3 & \cdots & -7 \diamond 0 & -7 \diamond-1 & \cdots & -7 \diamond-3 \\
0 \diamond 1 & 0 \diamond 0 & \cdots & 0 \diamond-2 & \cdots & -7 \diamond 1 & -7 \diamond 0 & \cdots & -7 \diamond-2 \\
\vdots & \vdots & & \vdots & \cdots & \vdots & \vdots & & \vdots \\
0 \diamond 3 & 0 \diamond 2 & \cdots & 0 \diamond 0 & \cdots & -7 \diamond 3 & -7 \diamond 2 & \cdots & -7 \diamond 0 \\
\hline \vdots & \vdots & \vdots & \vdots & & \vdots & \vdots & \vdots & \vdots \\
\hline 7 \diamond 0 & 7 \diamond-1 & \cdots & 7 \diamond-3 & \cdots & 0 \diamond 0 & 0 \diamond-1 & \cdots & 0 \diamond-3 \\
7 \diamond 1 & 7 \diamond 0 & \cdots & 7 \diamond-2 & \cdots & 0 \diamond 1 & 0 \diamond 0 & \cdots & 0 \diamond-2 \\
\vdots & \vdots & & \vdots & \cdots & \vdots & \vdots & & \vdots \\
7 \diamond 3 & 7 \diamond 2 & \cdots & 7 \diamond 0 & \cdots & 0 \diamond 3 & 0 \diamond 2 & \cdots & 0 \diamond 0
\end{array}\right] .
$$

De (8.1.25), podemos ver que $\mathbf{S}$ tem uma estrutura bloco-Toeplitz e segue de (8.1.39) que agora cada bloco também é Toeplitz. Portanto, dada uma imagem $\mathbf{Y}$, a matriz espectral 
$\mathbf{S}$ pode ser determinada de forma extremamente eficiente. Como antes, podemos obter uma formulação matricial para avaliar $k \diamond l$, para todos $k$ e $l$, como mostramos a seguir.

Defina

$$
\mathbf{W}=\left[\begin{array}{cccc}
-N_{x}+1 \diamond-N_{y}+1 & -N_{x}+2 \diamond-N_{y}+1 & \cdots & N_{x}-1 \diamond-N_{y}+1 \\
-N_{x}+1 \diamond-N_{y}+2 & -N_{x}+2 \diamond-N_{y}+2 & \cdots & N_{x}-1 \diamond-N_{y}+2 \\
\vdots & \vdots & & \vdots \\
-N_{x}+1 \diamond N_{y}-1 & -N_{x}+2 \diamond N_{y}-1 & \cdots & N_{x}-1 \diamond N_{y}-1
\end{array}\right]
$$

Dado $\mathbf{W}$, é fácil obter $\mathbf{S}$, pois cada bloco Toeplitz de $\mathbf{S}$ pode ser obtido a partir de uma coluna de $\mathbf{W}$ sem computações adicionais.

Defina

$$
\begin{array}{r}
\mathbf{V}_{\mathbf{x}}=\left[\begin{array}{cccc}
c_{0}\left(-N_{x}+1\right) & c_{1}\left(-N_{x}+1\right) & \cdots & c_{M_{x}-1}\left(-N_{x}+1\right) \\
c_{0}\left(-N_{x}+2\right) & c_{1}\left(-N_{x}+2\right) & \cdots & c_{M_{x}-1}\left(-N_{x}+2\right) \\
\vdots & \vdots & & \vdots \\
c_{0}\left(N_{x}-1\right) & c_{1}\left(N_{x}-1\right) & \cdots & c_{M_{x}-1}\left(N_{x}-1\right)
\end{array}\right] \\
\mathbf{V}_{\mathbf{y}}=\left[\begin{array}{cccc}
d_{0}\left(-N_{y}+1\right) & d_{1}\left(-N_{y}+1\right) & \cdots & d_{M_{y}-1}\left(-N_{y}+1\right) \\
d_{0}\left(-N_{y}+2\right) & d_{1}\left(-N_{y}+2\right) & \cdots & d_{M_{y}-1}\left(-N_{y}+2\right) \\
\vdots & \vdots & & \vdots \\
d_{0}\left(N_{y}-1\right) & d_{1}\left(N_{y}-1\right) & \cdots & d_{M_{y}-1}\left(N_{y}-1\right)
\end{array}\right] .
\end{array}
$$

Comparando com (8.1.38), podemos verificar que

$$
\mathbf{W}=\mathbf{V}_{\mathbf{y}} \mathbf{Y} \mathbf{V}_{\mathbf{x}}^{T}
$$

Defina $\boldsymbol{\Xi}_{u}$ tal que vec $\{\mathbf{S}\}=\boldsymbol{\Xi}_{u}$ vec $\{\mathbf{W}\}$ (a Figura 8.2 mostra como $\boldsymbol{\Xi}_{u}$ gera $\mathbf{S}$ a partir de $\mathbf{W}$, para um caso particular). Portanto, vec $\{\mathbf{S}\}=\mathbf{\Xi}_{u}\left(\mathbf{V}_{\mathbf{x}} \otimes \mathbf{V}_{\mathbf{y}}\right) \operatorname{vec}\{\mathbf{Y}\}$ e

$$
\mathbf{A}=\boldsymbol{\Xi}_{u}\left(\mathbf{V}_{\mathbf{x}} \otimes \mathbf{V}_{\mathbf{y}}\right)
$$

Como $\boldsymbol{\Xi}_{u}$ é computacionalmente simples, (8.1.45) também pode ser implementada como uma transformada rápida.

Para avaliar $\left(\mathbf{V}_{\mathbf{y}} \mathbf{Y}\right) \mathbf{V}_{\mathbf{x}}^{T} \quad$ e $\quad \mathbf{V}_{\mathbf{y}}\left(\mathbf{Y V}_{\mathbf{x}}^{T}\right)$ são necessários $\left(2 N_{y}-1\right) M_{x} M_{y}+$ $\left(2 N_{x}-1\right)\left(2 N_{y}-1\right) M_{x}$ e $\left(2 N_{x}-1\right) M_{x} M_{y}+\left(2 N_{x}-1\right)\left(2 N_{y}-1\right) M_{y}$ MACs complexos, respectivamente. Usando que $\mathbf{Y}$ é real, a complexidade cai para $\frac{1}{2}\left(2 N_{y}-1\right) M_{x} M_{y}+$ $\left(2 N_{x}-1\right)\left(2 N_{y}-1\right) M_{x}$ e $\frac{1}{2}\left(2 N_{x}-1\right) M_{x} M_{y}+\left(2 N_{x}-1\right)\left(2 N_{y}-1\right) M_{y}$ MACs complexos, respectivamente. Usando a primeira expressão e admitindo $M_{x} \gg\left(2 N_{x}-1\right)$, a aceleração 


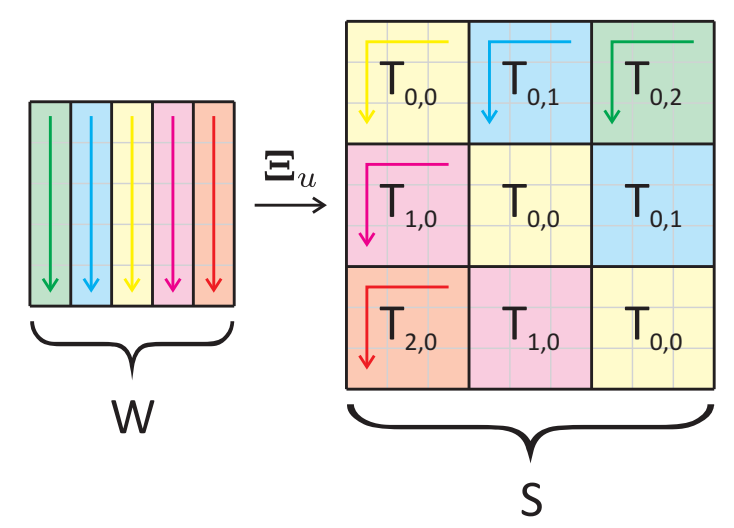

Figura 8.2: Exemplo de implementação rápida para $\boldsymbol{\Xi}_{u}$, supondo $N_{x}=N_{y}=3$.

relativa em termos de MACs é

$$
\begin{aligned}
\frac{\frac{1}{2} M_{x} M_{y} N_{x}^{2} N_{y}^{2}}{\frac{1}{2}\left(2 N_{y}-1\right) M_{x} M_{y}+\left(2 N_{x}-1\right)\left(2 N_{y}-1\right) M_{x}} & \approx \frac{M_{x} M_{y} N_{x}^{2} N_{y}^{2}}{\left(2 N_{y}-1\right) M_{x} M_{y}} \\
& =\frac{N_{x}^{2} N_{y}^{2}}{\left(2 N_{y}-1\right)} \\
& \approx \frac{1}{2} N_{x}^{2} N_{y}
\end{aligned}
$$

Como a geometria é simétrica em relação ao eixo y, $\mathbf{V}_{x}$ tem simetria conjugada em relação à sua linha central. Uma consideração análoga vale para $\mathbf{V}_{y}$. Estas simetrias podem ser aplicadas para reduzir ainda mais o custo computacional.

Para arrays retangulares uniformes que amostrem o espaço $\mathbf{U}$ uniformemente, $\mathbf{V}_{\mathbf{x}}$ e $\mathbf{V}_{\mathbf{y}}$ podem ser interpretados como matrizes DFT para amostragem não uniforme em frequência. Poderíamos suspeitar que uma otimização adicional seria possível se utilizássemos NFFTs ao invés de produtos matriciais. No entanto, devido aos valores relativamente pequenos de $N_{x}$ e $N_{y}$, os produtos matriciais são mais rápidos que as NFFTs. Uma NFFT somente seria interessante se $N_{x}$ e $N_{y}$ tivessem valores da ordem de centenas (correspondendo a um array com pelo menos dezenas de milhares de elementos), o que é claramente impraticável com a tecnologia atual.

\subsection{Transformada rápida transposta}

Como veremos nos exemplos da Seção 8.7, dada uma matriz espectral medida S, muitos algoritmos eficientes para reconstrução de imagens exigem apenas implementações de $\mathbf{A}$ e $\mathbf{A}^{H}$ para estimar uma distribuição de fontes. Um algoritmo eficiente para reconstrução deve possuir implementações eficientes de ambas transformadas, caso contrário a transformada mais lenta se tornará o gargalo do solver. Como visto antes, a implementação rápida de $\mathbf{A}$ requer reescrever cada linha de $\mathbf{A}$ como um produto de Kronecker, e cada elemento se refere a um par $\left(u_{x}, u_{y}\right)$ único. Por outro lado, a implementação rápida de $\mathbf{A}^{T}$ requer reescrever cada coluna de $\mathbf{A}$ como um produto de Kronecker diferente, e 
cada elemento agora se refere a um elemento único do array, mas ao mesmo par $\left(u_{x}, u_{y}\right)$. Por isso, o método apresentado na seção anterior não pode ser modificado diretamente para obter uma implementação rápida de $\mathbf{A}^{H}$. A seguir obtemos uma implementação rápida de $\mathbf{A}^{T}$, como passo intermediário para obter uma implementação rápida de $\mathbf{A}^{H}$, e mostramos que ela apresenta a mesma complexidade computacional que a implementação rápida de $\mathbf{A}$.

\subsubsection{Caso 1: geometria separável}

Sejam $\overline{\mathbf{S}} \in \mathbb{C}^{N_{x} N_{y} \times N_{x} N_{y}}$ e $\overline{\mathbf{Y}} \in \mathbb{C}^{M_{y} \times M_{x}}$ tal que vec $\{\overline{\mathbf{Y}}\}=\mathbf{A}^{T}$ vec $\{\overline{\mathbf{S}}\}$. Dados vetores $\mathbf{u}, \mathbf{v} \in \mathbb{C}^{n}$, definimos o produto escalar $\mathbf{u} \cdot \mathbf{v}=\mathbf{u}^{T} \mathbf{v}$ (observamos que esta não é a definição usual do produto escalar, pois este não é um produto interno).

Para $0 \leq m<M_{x}$ e $0 \leq n<M_{y}$, um elemento arbitrário $\overline{\mathbf{Y}}_{n, m}$ de $\overline{\mathbf{Y}}$ pode ser escrito como o produto escalar da coluna $m \cdot M_{y}+n$ de $\mathbf{A}$ e vec $\{\overline{\mathbf{S}}\}$. Usando (8.1.18), temos

$$
\begin{aligned}
\overline{\mathbf{Y}}_{n, m} & =\left[\left(\mathbf{v}_{x_{m}} \otimes \mathbf{v}_{y_{n}}\right)^{*} \otimes\left(\mathbf{v}_{x_{m}} \otimes \mathbf{v}_{y_{n}}\right)\right] \cdot \operatorname{vec}\{\overline{\mathbf{S}}\} \\
& =\operatorname{vec}\left\{\left(\mathbf{v}_{x_{m}} \otimes \mathbf{v}_{y_{n}}\right)^{H} \otimes\left(\mathbf{v}_{x_{m}} \otimes \mathbf{v}_{y_{n}}\right)\right\} \cdot \operatorname{vec}\{\overline{\mathbf{S}}\} \\
& =\operatorname{vec}\left\{\left(\mathbf{v}_{x_{m}}^{H} \otimes \mathbf{v}_{x_{m}}\right) \otimes\left(\mathbf{v}_{y_{n}}^{H} \otimes \mathbf{v}_{y_{n}}\right)\right\} \cdot \operatorname{vec}\{\overline{\mathbf{S}}\},
\end{aligned}
$$

onde a última igualdade pode ser verificada expandindo o produto de Kronecker.

Por definição,

$$
\begin{aligned}
& \mathbf{v}_{x_{m}}^{H} \otimes \mathbf{v}_{x_{m}}=\left[\begin{array}{cccc}
\mathrm{v}_{x_{m}}^{0 *} \mathrm{v}_{x_{m}}^{0} & \mathrm{v}_{x_{m}}^{1 *} \mathrm{v}_{x_{m}}^{0} & \cdots & \mathrm{v}_{x_{m}}^{\left(N_{x}-1\right) *} \mathrm{v}_{x_{m}}^{0} \\
\mathrm{v}_{x_{m}}^{0 *} \mathrm{v}_{x_{m}}^{1} & \mathrm{v}_{x_{m}}^{1 *} \mathrm{v}_{x_{m}}^{1} & \cdots & \mathrm{v}_{x_{m}}^{\left(N_{x}-1\right) *} \mathrm{v}_{x_{m}}^{1} \\
\vdots & \vdots & & \vdots \\
\mathrm{v}_{x_{m}}^{0 *} \mathrm{v}_{x_{m}}^{N_{x_{x}-1}} & \mathrm{v}_{x_{m}}^{1 *} \mathrm{v}_{x_{m}}^{N_{x_{x}-1}} & \cdots & \mathrm{v}_{x_{m}}^{\left(N_{x}-1\right) *} \mathrm{v}_{x_{m}}^{N_{x_{m}-1}}
\end{array}\right] \\
& =\left[\begin{array}{cccc}
c_{m}(0,0) & c_{m}(1,0) & \cdots & c_{m}\left(N_{x}-1,0\right) \\
c_{m}(0,1) & c_{m}(1,1) & \cdots & c_{m}\left(N_{x}-1,1\right) \\
\vdots & \vdots & & \vdots \\
c_{m}\left(0, N_{x}-1\right) & c_{m}\left(1, N_{x}-1\right) & \cdots & c_{m}\left(N_{x}-1, N_{x}-1\right)
\end{array}\right] \\
& \mathbf{v}_{y_{n}}^{H} \otimes \mathbf{v}_{y_{n}}=\left[\begin{array}{cccc}
\mathrm{v}_{y_{n}}^{0 *} \mathrm{v}_{y_{n}}^{0} & \mathrm{v}_{y_{n}}^{1 *} \mathrm{v}_{y_{n}}^{0} & \cdots & \mathrm{v}_{y_{n}}^{\left(N_{y}-1\right) *} \mathrm{v}_{y_{n}}^{0} \\
\mathrm{v}_{y_{n}}^{0 *} \mathrm{v}_{y_{n}}^{1} & \mathrm{v}_{y_{n}}^{1 *} \mathrm{v}_{y_{n}}^{1} & \cdots & \mathrm{v}_{y_{n}}^{\left(N_{y}-1\right) *} \mathrm{v}_{y_{n}}^{1} \\
\vdots & \vdots & & \vdots \\
\mathrm{v}_{y_{n}}^{0 *} \mathrm{v}_{y_{n}}^{N_{y}-1} & \mathrm{v}_{y_{n}}^{1 *} \mathrm{v}_{y_{n}}^{N_{y_{n}-1}} & \cdots & \mathrm{v}_{y_{n}}^{\left(N_{y}-1\right) *} \mathrm{v}_{y_{n}}^{N_{y}-1}
\end{array}\right] \\
& =\left[\begin{array}{cccc}
d_{n}(0,0) & d_{n}(1,0) & \cdots & d_{n}\left(N_{y}-1,0\right) \\
d_{n}(0,1) & d_{n}(1,1) & \cdots & d_{n}\left(N_{y}-1,1\right) \\
\vdots & \vdots & & \vdots \\
d_{n}\left(0, N_{y}-1\right) & d_{n}\left(1, N_{y}-2\right) & \cdots & d_{n}\left(N_{y}-1, N_{y}-1\right)
\end{array}\right] \text {. }
\end{aligned}
$$


Seja

$$
\begin{aligned}
\overline{\mathbf{S}} & =\left[\begin{array}{cccc}
\overline{\mathbf{T}}_{0,0} & \overline{\mathbf{T}}_{0,1} & \cdots & \overline{\mathbf{T}}_{0, N_{x}-1} \\
\overline{\mathbf{T}}_{1,0} & \overline{\mathbf{T}}_{1,1} & \cdots & \overline{\mathbf{T}}_{1, N_{x}-1} \\
\vdots & \vdots & & \vdots \\
\overline{\mathbf{T}}_{N_{x}-1,0} & \overline{\mathbf{T}}_{N_{x}-1,1} & \cdots & \overline{\mathbf{T}}_{N_{x}-1, N_{x}-1}
\end{array}\right] \\
\mathbf{C}_{m} & =\mathbf{v}_{x_{m}}^{H} \otimes \mathbf{v}_{x_{m}} \\
\mathbf{D}_{n} & =\mathbf{v}_{y_{n}}^{H} \otimes \mathbf{v}_{y_{n}},
\end{aligned}
$$

onde cada $\overline{\mathbf{T}}_{i, j}$ é um bloco $N_{y} \times N_{y}$ de $\overline{\mathbf{S}}$. Defina

$$
\begin{aligned}
& \overline{\mathbf{t}}_{i, j}=\operatorname{vec}\left\{\overline{\mathbf{T}}_{i, j}\right\} \\
& \mathbf{c}_{m}=\operatorname{vec}\left\{\mathbf{C}_{m}\right\} \\
& \mathbf{d}_{n}=\operatorname{vec}\left\{\mathbf{D}_{n}\right\}
\end{aligned}
$$

Usando estas definições em (8.2.1),

$$
\begin{aligned}
& \overline{\mathbf{Y}}_{n, m}=\operatorname{vec}\left\{\mathbf{C}_{m} \otimes \mathbf{D}_{n}\right\} \cdot \operatorname{vec}\left\{\left[\begin{array}{cccc}
\overline{\mathbf{T}}_{0,0} & \overline{\mathbf{T}}_{0,1} & \cdots & \overline{\mathbf{T}}_{0, N_{x}-1} \\
\overline{\mathbf{T}}_{1,0} & \overline{\mathbf{T}}_{1,1} & \cdots & \overline{\mathbf{T}}_{1, N_{x}-1} \\
\vdots & \vdots & & \vdots \\
\overline{\mathbf{T}}_{N_{x}-1,0} & \overline{\mathbf{T}}_{N_{x}-1,1} & \cdots & \overline{\mathbf{T}}_{N_{x}-1, N_{x}-1}
\end{array}\right]\right\} \\
& =\operatorname{vec}\left\{\mathbf{C}_{m} \otimes \mathbf{d}_{n}\right\} \cdot \operatorname{vec}\left\{\left[\begin{array}{cccc}
\overline{\mathbf{t}}_{0,0} & \overline{\mathbf{t}}_{0,1} & \cdots & \overline{\mathbf{t}}_{0, N_{x}-1} \\
\overline{\mathbf{t}}_{1,0} & \overline{\mathbf{t}}_{1,1} & \cdots & \overline{\mathbf{t}}_{1, N_{x}-1} \\
\vdots & \vdots & & \vdots \\
\overline{\mathbf{t}}_{N_{x}-1,0} & \overline{\mathbf{t}}_{N_{x}-1,1} & \cdots & \overline{\mathbf{t}}_{N_{x}-1, N_{x}-1}
\end{array}\right]\right\} \\
& =\left(\mathbf{c}_{m} \otimes \mathbf{d}_{n}\right) \cdot \operatorname{vec}\left\{\left[\begin{array}{llll}
\overline{\mathbf{t}}_{0,0} & \overline{\mathbf{t}}_{1,0} & \cdots & \overline{\mathbf{t}}_{N_{x}-1, N_{x}-1}
\end{array}\right]\right\} \\
& =\left(\mathbf{c}_{m}^{T} \otimes \mathbf{d}_{n}^{T}\right) \operatorname{vec}\left\{\left[\begin{array}{llll}
\overline{\mathbf{t}}_{0,0} & \overline{\mathbf{t}}_{1,0} & \cdots & \overline{\mathbf{t}}_{N_{x}-1, N_{x}-1}
\end{array}\right]\right\} \\
& =\mathbf{d}_{n}^{T}\left[\begin{array}{llll}
\overline{\mathbf{t}}_{0,0} & \overline{\mathbf{t}}_{1,0} & \cdots & \overline{\mathbf{t}}_{N_{x}-1, N_{x}-1}
\end{array}\right] \mathbf{c}_{m} .
\end{aligned}
$$

Para verificar que (8.2.12) e (8.2.13) são iguais, note que $\mathbf{D}_{n}$ e cada bloco $\overline{\mathbf{T}}_{i, j}$ têm as mesmas dimensões. Se a mesma reorganização for aplicada a $\mathbf{D}_{n}$ e a cada $\overline{\mathbf{T}}_{i, j}$, o produto escalar (8.2.12) é preservado. A equivalência de (8.2.15) e (8.2.16) segue da identidade $\left(\mathbf{A}^{T} \otimes \mathbf{B}\right) \operatorname{vec}\{\mathbf{C}\}=\operatorname{vec}\{\mathbf{B C A}\}$.

De (8.1.28) e (8.1.29),

$$
\begin{aligned}
& \mathbf{V}_{\mathbf{x}}=\left[\begin{array}{llll}
\mathbf{c}_{0} & \mathbf{c}_{1} & \cdots & \mathbf{c}_{M_{x}-1}
\end{array}\right] \\
& \mathbf{V}_{\mathbf{y}}=\left[\begin{array}{llll}
\mathbf{d}_{0} & \mathbf{d}_{1} & \cdots & \mathbf{d}_{M_{y}-1}
\end{array}\right] .
\end{aligned}
$$


Definimos

$$
\overline{\mathbf{Z}}=\left[\begin{array}{llll}
\overline{\mathbf{t}}_{0,0} & \overline{\mathbf{t}}_{1,0} & \cdots & \overline{\mathbf{t}}_{N_{x}-1, N_{x}-1}
\end{array}\right]
$$

tal que

$$
\overline{\mathbf{Y}}=\mathbf{V}_{\mathbf{y}}^{T} \overline{\mathbf{Z}} \mathbf{V}_{\mathbf{x}}
$$

Segue que

$$
\mathbf{A}^{T}=\left(\mathbf{V}_{\mathbf{x}}^{T} \otimes \mathbf{V}_{\mathbf{y}}^{T}\right) \mathbf{\Xi}_{s}^{T}
$$

que tem o mesmo custo computacional de $\mathbf{A}$.

Deve estar claro que $\overline{\mathbf{Z}}$ pode ser facilmente obtido, pois cada coluna de $\overline{\mathbf{Z}}$ corresponde a um bloco $N_{y} \times N_{y}$ de $\overline{\mathbf{S}}$, quanto vetorizado. Note que se $\overline{\mathbf{S}}=\mathbf{S}$, então por definição, $\overline{\mathbf{Z}}=\mathbf{Z}$. Usaremos este fato na Seção 8.4.

A computação direta de $\mathbf{A}^{T}$ vec $\{\overline{\mathbf{S}}\}$ requer $\frac{1}{2} M_{x} M_{y} N_{x}^{2} N_{y}^{2}$ MACs. O produto matricial $\left(\mathbf{V}_{\mathbf{y}}^{T} \overline{\mathbf{Z}}\right) \mathbf{V}_{\mathbf{x}}$ requer $M_{y} N_{x}^{2} N_{y}^{2}+M_{y} N_{x}^{2} M_{x}$ MACs, enquanto $\mathbf{V}_{\mathbf{y}}^{T}\left(\overline{\mathbf{Z}} \mathbf{V}_{\mathbf{x}}\right)$ requer $M_{x} N_{y}^{2} N_{x}^{2}+$ $M_{x} N_{y}^{2} M_{y}$ MACs. Em muitas aplicações de reconstrução de imagens, espera-se que o resultado da transformada transposta seja real. Ao computar somente a parte real do segundo produto matricial, o custo da transformada cai para $M_{y} N_{x}^{2} N_{y}^{2}+\frac{1}{2} M_{y} N_{x}^{2} M_{x}$ e $M_{x} N_{y}^{2} N_{x}^{2}+\frac{1}{2} M_{x} N_{y}^{2} M_{y}$ MACs complexos, respectivamente. Usando a segunda expressão e ignorando o tempo necessário para computar $\overline{\mathbf{Z}}$, a aceleração relativa em termos de MACs é

$$
\begin{aligned}
\frac{\frac{1}{2} M_{x} M_{y} N_{x}^{2} N_{y}^{2}}{M_{x} N_{y}^{2} N_{x}^{2}+\frac{1}{2} M_{x} N_{y}^{2} M_{y}} & =\frac{M_{y} N_{x}^{2}}{2 N_{x}^{2}+M_{y}} \\
& \geq \frac{M_{y} N_{x}^{2}}{2 \cdot \max \left\{M_{y}, 2 N_{x}^{2}\right\}} \\
& =\min \left\{\frac{M_{y}}{4}, \frac{N_{x}^{2}}{2}\right\} .
\end{aligned}
$$

Se a amostragem em $\mathrm{U}$ for uniforme em relação ao eixo $\mathbf{y}, \mathbf{V}_{\mathbf{x}}$ terá simetria conjugada em relação à sua coluna central. Uma afirmação análoga vale para $\mathbf{V}_{\mathbf{y}}$. Se aplicáveis, estas simetrias podem ser usadas para reduzir o custo computacional.

Para arrays separáveis e amostragem uniforme no espaço $\mathbf{U}$, os produtos por $\mathbf{V}_{\mathbf{x}} \mathrm{e}$ $\mathbf{V}_{\mathbf{y}}$ podem novamente ser otimizados usando NFFTs, com resultados semelhantes aos da transformada direta.

É possível obter a forma da transformada transposta de uma maneira mais simples, que apresentamos a seguir. Esta dedução seria suficiente se só estivéssemos interessados no caso separável. No entanto, para obter otimizações para geometrias uniformes é preciso recorrer à dedução detalhada que foi apresentada acima. 
Na seção anterior mostramos que vec $\{\mathbf{S}\}=\operatorname{Avec}\{\mathbf{Y}\}$ pode ser determinado computando

$$
\mathbf{Z}=\mathbf{V}_{\mathbf{y}} \mathbf{Y} \mathbf{V}_{\mathbf{x}}^{T}
$$

e então reorganizando cada uma das $N_{x}^{2}$ colunas de $\mathbf{Z}$ para formar um bloco $N_{y} \times N_{y}$ de S. Seja $\boldsymbol{\Xi}_{s}$ a transformada que realiza esta reorganização, tal que vec $\{\mathbf{S}\}=\boldsymbol{\Xi}_{s} \operatorname{vec}\{\mathbf{Z}\}$.

Por definição,

$$
\begin{aligned}
\operatorname{vec}\{\mathbf{S}\} & =\operatorname{Avec}\{\mathbf{Y}\} \\
& =\boldsymbol{\Xi}_{s} \operatorname{vec}\left\{\mathbf{V}_{\mathbf{y}} \mathbf{Y} \mathbf{V}_{\mathbf{x}}^{T}\right\} \\
& =\boldsymbol{\Xi}_{s}\left(\mathbf{V}_{\mathbf{x}} \otimes \mathbf{V}_{\mathbf{y}}\right) \operatorname{vec}\{\mathbf{Y}\}
\end{aligned}
$$

Como $\boldsymbol{\Xi}_{s}$ é uma permutação, temos que $\boldsymbol{\Xi}_{s}^{T}=\boldsymbol{\Xi}_{s}^{-1}$.

Portanto,

$$
\begin{aligned}
\operatorname{vec}\{\overline{\mathbf{Y}}\} & =\mathbf{A}^{T} \operatorname{vec}\{\overline{\mathbf{S}}\} \\
& =\left(\mathbf{V}_{\mathbf{x}}^{T} \otimes \mathbf{V}_{\mathbf{y}}^{T}\right) \boldsymbol{\Xi}_{s}^{T} \operatorname{vec}\{\overline{\mathbf{S}}\} \\
& =\left(\mathbf{V}_{\mathbf{x}}^{T} \otimes \mathbf{V}_{\mathbf{y}}^{T}\right) \boldsymbol{\Xi}_{s}^{-1} \operatorname{vec}\{\overline{\mathbf{S}}\} \\
& =\left(\mathbf{V}_{\mathbf{x}}^{T} \otimes \mathbf{V}_{\mathbf{y}}^{T}\right) \operatorname{vec}\{\overline{\mathbf{Z}}\} \\
& =\operatorname{vec}\left\{\mathbf{V}_{\mathbf{y}}^{T} \overline{\mathbf{Z}} \mathbf{V}_{\mathbf{x}}\right\}
\end{aligned}
$$

Tal que

$$
\overline{\mathbf{Y}}=\mathbf{V}_{\mathbf{y}}^{T} \overline{\mathbf{Z}} \mathbf{V}_{\mathbf{x}}
$$

que é o resultado obtido anteriormente.

\subsubsection{Caso 2: geometria uniforme}

Usando a uniformidade do array, (8.2.2) e (8.2.4) podem ser escritas como

$$
\begin{aligned}
\mathbf{v}_{x_{m}}^{H} \otimes \mathbf{v}_{x_{m}}= & {\left[\begin{array}{cccc}
\mathrm{v}_{x_{m}}^{0 *} \mathrm{v}_{x_{m}}^{0} & \mathrm{v}_{x_{m}}^{1 *} \mathrm{v}_{x_{m}}^{0} & \cdots & \mathrm{v}_{x_{m}}^{\left(N_{x}-1\right) *} \mathrm{v}_{x_{m}}^{0} \\
\mathrm{v}_{x_{m}}^{0 *} \mathrm{v}_{x_{m}}^{1} & \mathrm{v}_{x_{m}}^{1 *} \mathrm{v}_{x_{m}}^{1} & \cdots & \mathrm{v}_{x_{m}}^{\left(N_{x}-1\right) *}{ }^{1} \\
\vdots & \vdots & & \vdots \\
\mathrm{v}_{x_{m}}^{0 *} \mathrm{v}_{x_{m}}^{N_{x}-1} & \mathrm{v}_{x_{m}}^{1 *} \mathrm{v}_{x_{m}}^{N_{x_{x}-1}} & \cdots & \mathrm{v}_{x_{m}}^{\left(N_{x}-1\right) *}{ }^{N_{x_{m}}-1}
\end{array}\right] } \\
= & {\left[\begin{array}{ccccc}
c_{m}(0) & c_{m}(-1) & \cdots & c_{m}\left(-N_{x}+1\right) \\
c_{m}(1) & c_{m}(0) & \cdots & c_{m}\left(-N_{x}+2\right) \\
\vdots & \vdots & & \vdots \\
c_{m}\left(N_{x}-1\right) & c_{m}\left(N_{x}-2\right) & \cdots & c_{m}(0)
\end{array}\right] }
\end{aligned}
$$




$$
\begin{aligned}
\mathbf{v}_{y_{n}}^{H} \otimes \mathbf{v}_{y_{n}}= & {\left[\begin{array}{cccc}
\mathrm{v}_{y_{n}}^{0 *} \mathrm{v}_{y_{n}}^{0} & \mathrm{v}_{y_{n}}^{1 *} \mathrm{v}_{y_{n}}^{0} & \cdots & \mathrm{v}_{y_{n}}^{\left(N_{y}-1\right) *} \mathrm{v}_{y_{n}}^{0} \\
\mathrm{v}_{y_{n}}^{0 *} \mathrm{v}_{y_{n}}^{1} & \mathrm{v}_{y_{n}}^{1 *} \mathrm{v}_{y_{n}}^{1} & \cdots & \mathrm{v}_{y_{n}}^{\left(N_{y}-1\right) *} \mathrm{v}_{y_{n}}^{1} \\
\vdots & \vdots & & \vdots \\
\mathrm{v}_{y_{n}}^{0 *} \mathrm{v}_{y_{n}}^{N_{y}-1} & \mathrm{v}_{y_{n}}^{1 *} \mathrm{v}_{y_{n}}^{N_{y_{n}}-1} & \cdots & \mathrm{v}_{y_{n}}^{\left(N_{y}-1\right) *} \mathrm{v}_{y_{n}}^{N_{y}-1}
\end{array}\right] } \\
= & {\left[\begin{array}{ccccc}
d_{n}(0) & d_{n}(-1) & \cdots & d_{n}\left(-N_{y}+1\right) \\
d_{n}(1) & d_{n}(0) & \cdots & d_{n}\left(-N_{y}+2\right) \\
\vdots & \vdots & & \vdots \\
d_{n}\left(N_{y}-1\right) & d_{n}\left(N_{y}-2\right) & \cdots & d_{n}(0)
\end{array}\right] . }
\end{aligned}
$$

Note que $\mathbf{v}_{x_{m}}^{H} \otimes \mathbf{v}_{x_{m}}$ e $\mathbf{v}_{y_{n}}^{H} \otimes \mathbf{v}_{y_{n}}$ têm no máximo $\left(2 N_{x}-1\right)$ e $\left(2 N_{y}-1\right)$ valores distintos, respectivamente. Portanto, para $0 \leq m<M_{x}$ e $0 \leq n<M_{y},\left(\mathbf{v}_{x_{m}}^{H} \otimes \mathbf{v}_{x_{m}}\right) \otimes\left(\mathbf{v}_{y_{n}}^{H} \otimes \mathbf{v}_{y_{n}}\right)$ tem no máximo $\left(2 N_{x}-1\right)\left(2 N_{y}-1\right)$ valores distintos. Para otimizar o produto matricial usando as versões pequenas de $\mathbf{V}_{\mathbf{x}}$ e $\mathbf{V}_{\mathbf{y}}$ dadas por (8.1.42) e (8.1.43), devemos primeiro acumular os elementos de $\overline{\mathbf{S}}$ que seriam multiplicados pelos mesmos valores se fossem usadas as versões grandes de $\mathbf{V}_{\mathbf{x}}$ e $\mathbf{V}_{\mathbf{y}}$ dadas por (8.1.28) e (8.1.29).

$$
\begin{aligned}
& \text { Para }-N_{x}<i<N_{x} \text { e }-N_{y}<j<N_{y} \text {, defina } \\
& \qquad \begin{aligned}
E_{x}(i)=\left\{(k, l) \in \mathbb{Z}^{2}: 0 \leq k, l<N_{x}, k-l=i\right\} \\
E_{y}(j)=\left\{(k, l) \in \mathbb{Z}^{2}: 0 \leq k, l<N_{y}, k-l=j\right\} .
\end{aligned}
\end{aligned}
$$

Segue que $\left\{E_{x}(i)\right\}_{i}$ e $\left\{E_{y}(j)\right\}_{j}$ particionam $\mathbf{v}_{x_{m}}^{H} \otimes \mathbf{v}_{x_{m}}$ e $\mathbf{v}_{y_{n}}^{H} \otimes \mathbf{v}_{y_{n}}$ em diagonais, e para $-N_{x}<i<N_{x}$ e $-N_{y}<j<N_{y}$,

$$
\begin{aligned}
& (k, l) \in E_{x}(i) \Rightarrow\left[\mathbf{v}_{x_{m}}^{H} \otimes \mathbf{v}_{x_{m}}\right]_{k, l}=c_{m}(i) \\
& (k, l) \in E_{y}(j) \Rightarrow\left[\mathbf{v}_{y_{n}}^{H} \otimes \mathbf{v}_{y_{n}}\right]_{k, l}=d_{n}(j) .
\end{aligned}
$$

Para $-N_{x}<i<N_{x}$ e $-N_{y}<j<N_{y}$, defina

$$
\begin{aligned}
G_{x}(i) & =\left\{(k, l) \in \mathbb{Z}^{2}: 0 \leq k, l<N_{x} N_{y},\left(\left\lfloor\frac{k}{N_{y}}\right\rfloor,\left\lfloor\frac{l}{N_{y}}\right\rfloor\right) \in E_{x}(i)\right\} \\
G_{y}(j) & =\left\{(k, l) \in \mathbb{Z}^{2}: 0 \leq k, l<N_{x} N_{y},\left(\bmod \left(k, N_{y}\right), \bmod \left(l, N_{y}\right)\right) \in E_{y}(j)\right\} \\
G_{y x}(i, j) & =G_{y}(i) \cap G_{x}(j) .
\end{aligned}
$$

Note que $\left\{G_{y x}(i, j)\right\}_{i, j}$ é uma partição dos índices de $\left(\mathbf{v}_{x_{m}}^{H} \otimes \mathbf{v}_{x_{m}}\right) \otimes\left(\mathbf{v}_{y_{n}}^{H} \otimes \mathbf{v}_{y_{n}}\right)$ tal que para $-N_{x}<i<N_{x} \mathrm{e}-N_{y}<j<N_{y}$,

$$
(k, l) \in G_{y x}(i, j) \Rightarrow\left[\left(\mathbf{v}_{x_{m}}^{H} \otimes \mathbf{v}_{x_{m}}\right) \otimes\left(\mathbf{v}_{y_{n}}^{H} \otimes \mathbf{v}_{y_{n}}\right)\right]_{k, l}=c_{m}(j) d_{n}(i) .
$$




$$
\left[\begin{array}{rrr|rrr|rrr}
0 & 0 & 0 & -1 & -1 & -1 & -2 & -2 & -2 \\
0 & 0 & 0 & -1 & -1 & -1 & -2 & -2 & -2 \\
0 & 0 & 0 & -1 & -1 & -1 & -2 & -2 & -2 \\
\hline 1 & 1 & 1 & 0 & 0 & 0 & -1 & -1 & -1 \\
1 & 1 & 1 & 0 & 0 & 0 & -1 & -1 & -1 \\
1 & 1 & 1 & 0 & 0 & 0 & -1 & -1 & -1 \\
\hline 2 & 2 & 2 & 1 & 1 & 1 & 0 & 0 & 0 \\
2 & 2 & 2 & 1 & 1 & 1 & 0 & 0 & 0 \\
2 & 2 & 2 & 1 & 1 & 1 & 0 & 0 & 0
\end{array}\right]
$$

pertinência a $G_{x}(i)$

$\left[\begin{array}{rrr|rrr|rrr}0 & -1 & -2 & 0 & -1 & -2 & 0 & -1 & -2 \\ 1 & 0 & 1 & 1 & 0 & 1 & 1 & 0 & 1 \\ 2 & 1 & 0 & 2 & 1 & 0 & 2 & 1 & 0 \\ \hline 0 & -1 & -2 & 0 & -1 & -2 & 0 & -1 & -2 \\ 1 & 0 & 1 & 1 & 0 & 1 & 1 & 0 & 1 \\ 2 & 1 & 0 & 2 & 1 & 0 & 2 & 1 & 0 \\ \hline 0 & -1 & -2 & 0 & -1 & -2 & 0 & -1 & -2 \\ 1 & 0 & 1 & 1 & 0 & 1 & 1 & 0 & 1 \\ 2 & 1 & 0 & 2 & 1 & 0 & 2 & 1 & 0\end{array}\right]$

pertinência a $G_{y}(j)$

Figura 8.3: Ilustração de $G_{x}(i)$ e $G_{y}(j)$, para $N_{x}=N_{y}=3$. $\overline{\mathbf{S}}$ é uma matriz $N_{x} N_{y} \times N_{x} N_{y}$ formada por blocos $N_{y} \times N_{y}$. Os diagramas acima indicam a quais $G_{x}(i)$ e $G_{y}(j)$ cada elemento de $\overline{\mathbf{S}}$ pertence.

Antes de proceder, é importante mostrar intuitivamente a relação entre $G_{x}(i), G_{y}(j)$ e os elementos de $\overline{\mathbf{S}}$. A Figura 8.3 mostra um exemplo visual para $N_{x}=N_{y}=3$.

Defina $\overline{\mathbf{W}} \in \mathbb{C}^{\left(2 N_{y}-1\right) \times\left(2 N_{x}-1\right)}$ tal que para $-N_{y}<i<N_{y}$ e $-N_{x}<j<N_{x}$,

$$
\overline{\mathbf{W}}_{i+N_{y}-1, j+N_{x}-1}=\sum_{(k, l) \in G_{y x}(i, j)} \overline{\mathbf{S}}_{k, l} .
$$

Então

$$
\overline{\mathbf{Y}}_{n, m}=\sum_{i=-N_{y}+1}^{N_{y}-1} \sum_{j=-N_{x}+1}^{N_{x}-1} c_{m}(j) d_{n}(i) \overline{\mathbf{W}}_{i+N_{y}-1, j+N_{x}-1}
$$

Tal que usando (8.1.42) e (8.1.43),

$$
\overline{\mathbf{Y}}=\mathbf{V}_{\mathbf{y}}^{T} \overline{\mathbf{W}} \mathbf{V}_{\mathbf{x}}
$$

Por definição, segue que vec $\{\overline{\mathbf{W}}\}=\boldsymbol{\Xi}_{u}^{T} \operatorname{vec}\{\overline{\mathbf{S}}\}$ e

$$
\mathbf{A}^{T}=\left(\mathbf{V}_{\mathbf{x}}^{T} \otimes \mathbf{V}_{\mathbf{y}}^{T}\right) \mathbf{\Xi}_{u}^{T}
$$

Usando o Algoritmo 8.1, $\Xi_{u}^{T}$ pode ser aplicada com baixo custo. Note que o loop externo de acumulação pode ser executado em paralelo para todos $-N_{x}<i<N_{x}$, acumulando colunas de blocos $N_{y} \times N_{y}$, para produzir uma matriz temporária $\left(2 N_{x}-1\right) N_{y} \times N_{y}$ formada por $2 N_{x}-1$ blocos empilhados (veja a Figura 8.4). Analogamente, o loop interno de acumulação pode ser executado em paralelo para todos $-N_{y}<j<N_{y}$, acumulando as colunas de $B(i)$ para produzir a coluna $i+N_{x}-1$ de $\overline{\mathbf{W}}$. Assim, $\overline{\mathbf{W}}$ pode ser obtido com uma única leitura sequencial de $\overline{\mathbf{S}}$ (exigindo $N_{x}^{2} N_{y}^{2} \mathrm{MACs}$ ), seguida de $2 N_{x}-1$ leituras sequenciais de $N_{y}^{2}$ palavras de ponto flutuante (exigindo $\left(2 N_{x}-1\right) N_{y}^{2}$ MACs).

O produto matricial $\left(\mathbf{V}_{\mathbf{y}}^{T} \overline{\mathbf{W}}\right) \mathbf{V}_{\mathbf{x}}$ requer $M_{y}\left(2 N_{x}-1\right)\left(2 N_{y}-1+M_{x}\right)$ MACs, enquanto 

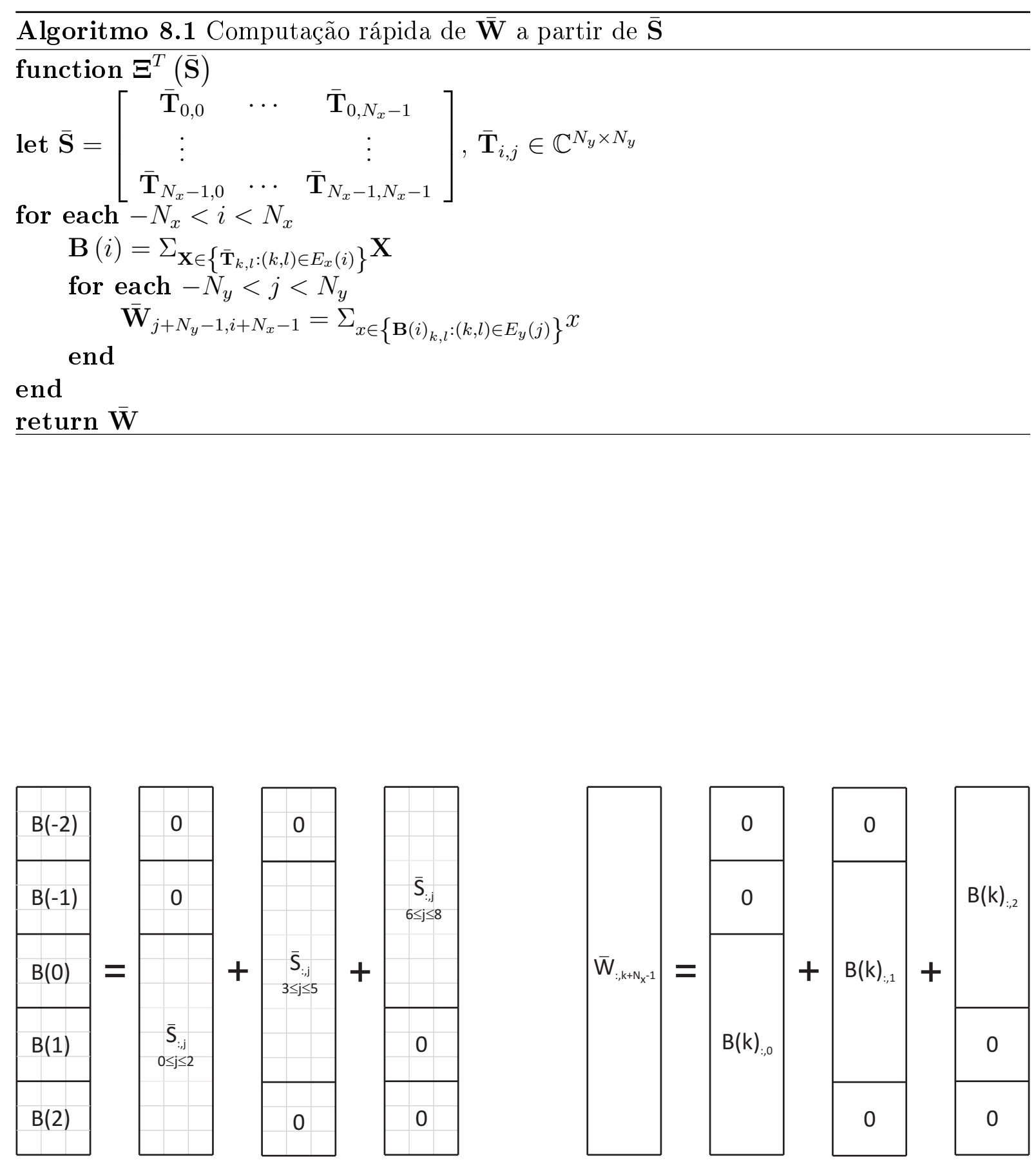

Figura 8.4: Exemplo de implementação rápida para $\boldsymbol{\Xi}_{u}^{T}$, supondo $N_{x}=N_{y}=3$. O símbolo ':' representa a seleção de todas os índices em uma determinada dimensão. 
$\mathbf{V}_{\mathbf{y}}^{T}\left(\overline{\mathbf{W}} \mathbf{V}_{\mathbf{x}}\right)$ requer $M_{x}\left(2 N_{y}-1\right)\left(2 N_{x}-1+M_{y}\right)$ MACs. Computando somente a parte real do segundo produto matricial, as complexidades caem para $M_{y}\left(2 N_{x}-1\right)$ $\left(2 N_{y}-1+\frac{1}{2} M_{x}\right)$ e $M_{x}\left(2 N_{y}-1\right)\left(2 N_{x}-1+\frac{1}{2} M_{y}\right)$ MACs complexos, respectivamente. Se $\mathbf{A}^{H}$ fosse representada na forma de uma matriz, seriam necessários $\frac{1}{2} M_{x} M_{y} N_{x}^{2} N_{y}^{2}$ MACs complexos. Usando a segunda expressão, supondo que $M_{y} \gg\left(2 N_{x}-1\right)$ e desprezando o tempo necessário para obter $\overline{\mathbf{W}}$, a aceleração relativa em termos de MACs é

$$
\begin{aligned}
\frac{\frac{1}{2} M_{x} M_{y} N_{x}^{2} N_{y}^{2}}{M_{x}\left(2 N_{y}-1\right)\left(2 N_{x}-1+\frac{1}{2} M_{y}\right)} & \approx \frac{N_{x}^{2} N_{y}^{2}}{2 N_{y}-1} \\
& \approx \frac{1}{2} N_{x}^{2} N_{y} .
\end{aligned}
$$

Com as definições acima, é possível mostrar que se $\overline{\mathbf{S}}=\mathbf{S}$, então

$$
\overline{\mathbf{W}}=\left(\mathbf{n}_{y} \mathbf{n}_{x}^{T}\right) \odot \mathbf{W}
$$

onde

$$
\begin{aligned}
& \mathbf{n}_{x}=\left[\begin{array}{lllllll}
1 & 2 & \cdots & N_{x} & \cdots & 2 & 1
\end{array}\right]^{T} \\
& \mathbf{n}_{y}=\left[\begin{array}{lllllll}
1 & 2 & \cdots & N_{y} & \cdots & 2 & 1
\end{array}\right]^{T} .
\end{aligned}
$$

Usaremos esta identidade na Seção 8.4.

\subsection{Transformada rápida adjunta}

Seja A a transformada direta, tal que $\mathbf{A}^{T}$ e $\mathbf{A}^{H}$ sejam sua transposta e adjunta, respectivamente. Como visto na seção anterior, podemos implementar vec $\{\overline{\mathbf{Y}}\}=\mathbf{A}^{T} \operatorname{vec}\{\overline{\mathbf{S}}\}$ $\operatorname{com} \overline{\mathbf{Y}}=\mathbf{V}_{\mathbf{y}}^{T} \overline{\mathbf{Z}} \mathbf{V}_{\mathbf{x}}$ no caso separável, e $\overline{\mathbf{Y}}=\mathbf{V}_{\mathbf{y}}^{T} \overline{\mathbf{W}} \mathbf{V}_{\mathbf{x}}$ no caso uniforme (para $\mathbf{V}_{\mathbf{x}}$ e $\mathbf{V}_{\mathbf{y}}$ apropriadas). Portanto, como $\mathbf{A}^{H}=\left[\mathbf{A}^{T}\right]^{*}$, a adjunta rápida vec $\{\breve{\mathbf{Y}}\}=\mathbf{A}^{H} \operatorname{vec}\{\overline{\mathbf{S}}\}$ pode ser determinada com

$$
\breve{\mathbf{Y}}=\mathbf{V}_{\mathbf{y}}^{H} \overline{\mathbf{Z}} \mathbf{V}_{\mathbf{x}}^{*}
$$

no caso separável, e

$$
\breve{\mathbf{Y}}=\mathbf{V}_{\mathbf{y}}^{H} \overline{\mathbf{W}} \mathbf{V}_{\mathbf{x}}^{*}
$$

no caso uniforme. 


\subsection{Transformada rápida direta-adjunta}

Dada a transformada direta $\mathbf{A}$ e sua adjunta $\mathbf{A}^{H}$, consideremos a transformada dada por $\mathbf{A}^{H} \mathbf{A}$. Esta composição será usada na Seção 8.7 para reconstrução de imagens, e nesta seção apresentamos um método para otimizá-la ainda mais.

Dada uma entrada $\mathbf{Y}$, o procedimento óbvio para obter uma versão rápida de vec $\{\breve{\mathbf{Y}}\}=$ $\mathbf{A}^{H} \mathbf{A v e c}\{\mathbf{Y}\}$ para uma geometria separável consiste em:

1. avaliar $\mathbf{Z}=\mathbf{V}_{\mathbf{y}} \mathbf{Y} \mathbf{V}_{\mathbf{x}}^{T}$;

2. obter $\mathbf{S}$ a partir de $\mathbf{Z}$ utilizando $\Xi_{s}$;

3. obter $\overline{\mathbf{Z}}$ a partir de $\mathbf{S}$ utilizando $\Xi_{s}^{H}=\Xi_{s}^{T}$;

4. avaliar $\breve{\mathbf{Y}}=\mathbf{V}_{\mathbf{y}}^{H} \overline{\mathbf{Z}} \mathbf{V}_{\mathbf{x}}^{*}$.

Os passos (1) e (2) implementam A, e os passos (3) e (4) implementam $\mathbf{A}^{H}$.

Como observado anteriormente, temos $\overline{\mathbf{Z}}=\mathbf{Z}$, tal que os passos (2) e (3) podem ser omitidos e temos

$$
\breve{\mathbf{Y}}=\mathbf{V}_{\mathbf{y}}^{H} \mathbf{V}_{\mathbf{y}} \mathbf{Y} \mathbf{V}_{\mathbf{x}}^{T} \mathbf{V}_{\mathbf{x}}^{*}
$$

Esta implementação é especialmente interessante quando $N_{x}$ e $N_{y}$ são suficientemente grandes em comparação a $M_{x}$ e $M_{y}$, pois permite-nos usar

$$
\breve{\mathbf{Y}}=\left(\mathbf{V}_{\mathbf{y}}^{H} \mathbf{V}_{\mathbf{y}}\right) \mathbf{Y}\left(\mathbf{V}_{\mathbf{x}}^{T} \mathbf{V}_{\mathbf{x}}^{*}\right)
$$

usando versões pré-computadas de $\mathbf{V}_{\mathbf{y}}^{H} \mathbf{V}_{\mathbf{y}}$ e $\mathbf{V}_{\mathbf{x}}^{T} \mathbf{V}_{\mathbf{x}}^{*}$, que têm valores reais.

Para geometrias uniformes, o procedimento é análogo, e consiste em:

1. avaliar $\mathbf{W}=\mathbf{V}_{\mathbf{y}} \mathbf{Y} \mathbf{V}_{\mathbf{x}}^{T}$;

2. obter $\mathbf{S}$ a partir de $\mathbf{W}$ utilizando $\Xi_{u}$;

3. obter $\overline{\mathbf{W}}$ a partir de $\mathbf{S}$ utilizando $\Xi_{u}^{H}=\Xi_{u}^{T}$;

4. avaliar $\breve{\mathbf{Y}}=\mathbf{V}_{\mathbf{y}}^{H} \overline{\mathbf{W}} \mathbf{V}_{\mathbf{x}}^{*}$.

Como demonstrado anteriormente, temos que $\mathbf{W}=\left(\mathbf{n}_{y} \mathbf{n}_{x}^{T}\right) \odot \mathbf{W}$. Sejam

$$
\begin{aligned}
& \breve{\mathbf{V}}_{\mathbf{x}}=\underbrace{\left[\begin{array}{lll}
\mathbf{n}_{x} & \cdots & \mathbf{n}_{x}
\end{array}\right]}_{M_{x} \text { vezes }} \odot \mathbf{V}_{\mathbf{x}} \\
& \breve{\mathbf{V}}_{\mathbf{y}}=\underbrace{\left[\begin{array}{ccc}
\mathbf{n}_{y} & \cdots & \mathbf{n}_{y}
\end{array}\right]}_{M_{y} \text { vezes }} \odot \mathbf{V}_{\mathbf{y}} .
\end{aligned}
$$


É possível verificar que

$$
\breve{\mathbf{Y}}=\breve{\mathbf{V}}_{\mathbf{y}}^{H} \mathbf{V}_{\mathbf{y}} \mathbf{Y} \mathbf{V}_{\mathbf{x}}^{T} \breve{\mathbf{V}}_{\mathbf{x}}^{*}
$$

tal que as mesmas otimizações do caso separável podem ser usadas.

É sempre mais rápido implementar a transformada direta-adjunta usando (8.4.1) e (8.4.5) do que com uma composição das transformadas diretas e adjunta, principalmente porque para problemas grandes torna-se vantajoso pré-computar $\breve{\mathbf{V}}_{\mathbf{y}}^{H} \mathbf{V}_{\mathbf{y}}$ e $\mathbf{V}_{\mathbf{x}}^{T} \breve{\mathbf{V}}_{\mathbf{x}}^{*}$. Além disso, (8.4.1) e (8.4.5) podem ser paralelizadas com maior eficiência, pois dispensam a aplicação de $\Xi_{s}$ e $\Xi_{u}$.

As implementações que usam a NFFT estão em desvantagem para a transformada direta-adjunta, pois não permitem que uma versão equivalente de $\breve{\mathbf{V}}_{\mathbf{y}}^{H} \mathbf{V}_{\mathbf{y}}$ e $\mathbf{V}_{\mathbf{x}}^{T} \breve{\mathbf{V}}_{\mathbf{x}}^{*}$ seja precomputada. Portanto, com a implementação baseada na NFFT somos forçados a usar a composição das transformadas apresentadas anteriormente, que para a maior parte dos casos é significativamente mais lenta do que (8.4.1) e (8.4.5). Como mostrado na Seção 8.6, a implementação acelerada por NFFTs supera o produto matricial apenas para imagens muito grandes.

\subsection{Conexões}

Nesta seção descrevemos como as transformadas propostas se relacionam com versões bidimensionais da FFT, NFFT e NNFFT ${ }^{1}$. Com a exceção da NNFFT, cada transformada se aplica somente a geometrias específicas ou amostragens específicas do espaço U. Transformadas que fazem hipóteses mais restritas sobre a geometria ou amostragem em U tendem a ser computacionalmente mais eficientes. Além disso, algumas transformadas resolvem problemas diferentes, tornando a sua escolha dependente do problema que se deseja resolver, e não somente da qualidade de reconstrução desejada e do custo computacional. Por exemplo, a NFFT pode ser usada com geometrias arbitrárias e amostragens retangulares de $\mathrm{U}$, enquanto as transformadas propostas podem ser usadas com geometrias separáveis arbitrárias e amostragens separáveis arbitrárias do espaço U.

Suponhamos que o campo de ondas amostrado seja um processo aleatório com média zero, estacionário no tempo e homogêneo no espaço. Consideremos um array de $N$ microfones com coordenadas cartesianas $\mathbf{p}_{0}, \ldots, \mathbf{p}_{N-1} \in \mathbb{R}^{3}$. Para uma frequência fixa $\omega$, sua matriz espectral $\mathbf{S}(\omega)=\mathrm{E}\left\{\mathbf{x}(\omega) \mathbf{x}^{H}(\omega)\right\}$ é por definição uma matriz de covariância. Para $0 \leq m, n<N,[\mathbf{S}(\omega)]_{m, n}$ contém a covariância cruzada no domínio da frequência entre quaisquer dois pontos cujas coordenadas difiram por $\mathbf{p}_{m}-\mathbf{p}_{n}$. Seja $P(\omega, \mathbf{k})=|Y(\omega, \mathbf{k})|^{2}$ a densidade espectral de potência quando parametrizada em função do número de onda $\mathbf{k}=-\frac{\omega}{c} \mathbf{u} \in \mathbb{R}^{3}$, e seja $S(\omega, \Delta \mathbf{p})$ a covariância espectral entre dois

\footnotetext{
${ }^{1}$ Como mencionado anteriormente, NFFTs são transformadas rápidas de Fourier com amostragem não-uniforme em um dos domínios [78]. NNFFTs são generalizações onde a amostragem não-uniforme também se aplica ao domínio conjugado.
} 
pontos cujas coordenadas difiram por $\Delta$ p. Como visto anteriormente,

$$
\begin{aligned}
P(\omega, \mathbf{k}) & =\int_{\mathbb{R}^{3}} S(\omega, \Delta \mathbf{p}) e^{+j \mathbf{k}^{T} \Delta \mathbf{p}} d \Delta \mathbf{p} \\
S(\omega, \Delta \mathbf{p}) & =\frac{1}{(2 \pi)^{3}} \int_{\mathbb{R}^{3}} P(\omega, \mathbf{k}) e^{-j \mathbf{k}^{T} \Delta \mathbf{p}} d \mathbf{k} .
\end{aligned}
$$

Portanto, o conhecimento de $S(\omega, \Delta \mathbf{p})$, limitado a uma coleção de vetores $\Delta \mathbf{p}$, permite a obtenção de uma versão aproximada e discretizada de $P(\omega, \mathbf{k})$, que é a imagem de interesse. As conexões abaixo decorrem naturalmente de diferentes formas de amostrar estas relações, e avaliá-las numericamente para espaço discreto e espaço U discreto.

\subsubsection{FFT}

A seguir, omitiremos a variável $\omega$ para tornar a notação mais compacta. Seja $\mathbf{H} \in$ $\mathbb{C}^{M \times M}$ para $M$ par, e defina a DFT e IDFT bidimensional de $\mathbf{H}$ como

$$
\begin{aligned}
\mathcal{F}\{\mathbf{H}\}_{l, k} & =\sum_{n=0}^{M-1} \sum_{m=0}^{M-1} e^{-j\left(\frac{2 \pi l n}{M}+\frac{2 \pi k m}{M}\right)} \mathbf{H}_{n, m} \\
\mathcal{F}^{-1}\{\mathbf{H}\}_{l, k} & =\frac{1}{M^{2}} \sum_{n=0}^{M-1} \sum_{m=0}^{M-1} e^{j\left(\frac{2 \pi l n}{M}+\frac{2 \pi k m}{M}\right)} \mathbf{H}_{n, m} .
\end{aligned}
$$

Note que esta definição coloca as frequências baixas na vizinhança de $\mathcal{F}\{\mathbf{H}\}_{0,0}$. Para uma matriz arbitrária $\mathbf{H} \in \mathbb{C}^{M \times M} \mathrm{e}-\frac{M}{2} \leq m, n<\frac{M}{2}$ defina $\mathbf{H}(n, m)=\mathbf{H}_{n+\frac{M}{2}, m+\frac{M}{2}}$. Então para $-\frac{M}{2} \leq k, l<\frac{M}{2}$,

$$
\begin{aligned}
\mathcal{F}\{\mathbf{H}\}(l, k) & =\sum_{n=-M / 2}^{M / 2-1} \sum_{m=-M / 2}^{M / 2-1} e^{-j\left(\frac{2 \pi l n}{M}+\frac{2 \pi k m}{M}\right)} \mathbf{H}(n, m) \\
\mathcal{F}^{-1}\{\mathbf{H}\}(l, k) & =\frac{1}{M^{2}} \sum_{n=-M / 2}^{M / 2-1} \sum_{m=-M / 2}^{M / 2-1} e^{j\left(\frac{2 \pi l n}{M}+\frac{2 \pi k m}{M}\right)} \mathbf{H}(n, m) .
\end{aligned}
$$

Esta definição coloca colocas as frequências baixas na vizinhança de $\mathcal{F}\{\mathbf{H}\}(0,0)$.

Consideremos primeiro o caso de um array retangular uniforme com espaçamento interelementos $d_{x}=d_{y}=\lambda / 2$, onde $\lambda=\frac{2 \pi c}{\omega}$ é o comprimento de onda do sinal. Seja $\mathrm{Y}$ a imagem obtida por amostragem retangular do espaço $\mathrm{U}$, com $M_{x}=M_{y}$, e com coordenadas pertencentes a

$$
\tilde{\mathrm{U}}=\left\{\frac{2 i}{M_{x}}\right\}_{i=-M_{x} / 2}^{M_{x} / 2-1} \times\left\{\frac{2 j}{M_{y}}\right\}_{j=-M_{y} / 2}^{M_{y} / 2-1} .
$$

Mostremos agora que $\mathbf{S}$ pode ser obtido a partir de $\mathbf{Y}$ usando uma FFT bidimensional. Dada a linearidade da FFT, é suficiente mostrar que esta transformada é correta para 
uma imagem contendo apenas um impulso unitário com coordenadas arbitrárias dadas por $\mathbf{u}_{0} \in \tilde{\mathrm{U}}$.

Usando (7.2.4), para $-\frac{M_{x}}{2} \leq m_{0}<\frac{M_{x}}{2}$ e $-\frac{M_{y}}{2} \leq n_{0}<\frac{M_{y}}{2}$ arbitrários, definimos

$$
\mathbf{u}_{0}=\left[\begin{array}{c}
\frac{2 m_{0}}{M_{x}} \\
\frac{2 n_{0}}{M_{y}} \\
\sqrt{1-\left(\frac{2 m_{0}}{M_{x}}\right)^{2}-\left(\frac{2 n_{0}}{M_{y}}\right)^{2}}
\end{array}\right]
$$

$\mathrm{e}$

$$
\mathbf{Y}(n, m)= \begin{cases}1 & \text { se } n=n_{0}, m=m_{0} \\ 0 & \text { caso contrário. }\end{cases}
$$

Por definição,

$$
\begin{aligned}
\mathbf{S}_{r, s} & =\left[\mathbf{v}\left(\mathbf{u}_{0}\right) \mathbf{v}^{H}\left(\mathbf{u}_{0}\right)\right]_{r, s} \\
& =\mathrm{e}^{j \frac{\omega}{c} \mathbf{u}_{0}^{T}\left(\mathbf{p}_{r}-\mathbf{p}_{s}\right)} \\
& =\mathrm{e}^{j 2 \pi \mathbf{u}_{0}^{T} \frac{\left(\mathbf{p}_{r}-\mathbf{p}_{s}\right)}{\lambda}} \\
& =\mathrm{e}^{j 2 \pi \frac{\mathbf{u}_{0}^{T}}{2} \frac{2\left(\mathbf{p}_{r}-\mathbf{p}_{s}\right)}{\lambda}}
\end{aligned}
$$

Como $d_{x}=d_{y}=\lambda / 2$, para $0 \leq r, s<N$ arbitrários temos $\frac{2\left(\mathbf{p}_{r}-\mathbf{p}_{s}\right)}{\lambda}=\left[\begin{array}{lll}k & l & 0\end{array}\right]^{T} \in$ $\mathbb{Z}^{2} \times\{0\}$.

Comparando (8.5.10) com (8.5.6), podemos escrever

$$
\begin{aligned}
\mathbf{S}_{r, s} & =\mathrm{e}^{j 2 \pi \frac{\mathbf{u}_{0}^{T}}{2} \frac{2\left(\mathbf{p}_{r}-\mathbf{p}_{s}\right)}{\lambda}} \\
& =\mathrm{e}^{j\left(\frac{2 \pi m_{0} k}{M_{x}}+\frac{2 \pi n_{0} l}{M_{y}}\right)} \\
& =M^{2} \mathcal{F}^{-1}\{\mathbf{Y}\}(l, k) .
\end{aligned}
$$

Portanto, para uma distribuição de fontes arbitrária $\mathbf{Y}$, a covariância cruzada $\mathbf{S}_{r, s}$ para $0 \leq r, s<N$ é dada por um elemento de $\mathcal{F}^{-1}\{\mathbf{Y}\}$.

Ainda que esta transformada seja computacionalmente eficiente, a restrição $d_{x}=d_{y}=$ $\lambda / 2$ a torna quase nada prática. Além disso, ela requer $-\frac{M_{x}}{2} \leq k, l<\frac{M_{x}}{2}$. Como tipicamente o número de pixels excede a quantidade de termos de covariância cruzada, somos obrigados a descartar termos que não correspondam a elementos físicos do array. Portanto, a FFT calcula termos que não são usados.

\subsubsection{NFFT}

Para remover a restrição $d_{x}=d_{y}=\lambda / 2$ e obter uma transformada mais útil, reescreveremos (8.5.10) usando a NDFT. Antes disso, revisaremos sua definição. A seguir, usamos a linguagem de [78]. Uma NDFT d-dimensional é definida usando uma coleção 
de pontos espaciais arbitrários $\mathcal{X}$ e um vetor largura de banda $\mathbf{M} \in \mathbb{N}^{d}$. Cada ponto $x_{j}$ pertence ao conjunto de amostragem $\mathcal{X}=\left\{x_{i} \in\left[-\frac{1}{2}, \frac{1}{2}\right)^{d}: 0 \leq i<N\right\}$ tal que $|\mathcal{X}|=N$, onde $|\cdot|$ indica a cardinalidade do conjunto. O conjunto de índices

$$
I_{\mathbf{N}}=\mathbb{Z}^{d} \cap \prod_{t=0}^{d-1}\left[-\frac{M_{t}}{2}, \frac{M_{t}}{2}\right),
$$

define um grid retangular sobre o qual a função de interesse será amostrada.

A entrada é dada por um conjunto de amostras $h_{\mathbf{k}} \in \mathbb{C}$ para $\mathbf{k} \in I_{\mathbf{N}}$, e a NDFT é definida por

$$
\hat{h}_{i}=\sum_{\mathbf{k} \in I_{\mathbf{N}}} h_{\mathbf{k}} e^{-j 2 \pi \mathbf{k}^{T} \mathbf{x}_{i}}
$$

para $0 \leq i<N$. Sua adjunta (dada pela transposta Hermitiana se escrita em forma matricial, e que em geral não coincide com a inversa) é dada por

$$
g_{\mathbf{k}}=\sum_{i=0}^{N-1} \hat{h}_{i} e^{+j 2 \pi \mathbf{k}^{T} \mathbf{x}_{i}}
$$

A NFFT é uma versão rápida aproximada da NDFT, calculada interpolando uma FFT sobre-amostrada, e que obtém um boa combinação de precisão numérica e complexidade computacional.

Para obter (8.5.10) usando a NFFT, amostragem retangular em U e uma geometria arbitrária de $N$ microfones, usamos

$$
\begin{aligned}
\mathbf{M} & =\left[\begin{array}{ll}
M_{x} & M_{y}
\end{array}\right]^{T} \\
I_{\mathbf{N}} & =\mathbb{Z}^{2} \cap\left[-\frac{M_{x}}{2}, \frac{M_{x}}{2}\right) \times\left[-\frac{M_{y}}{2}, \frac{M_{y}}{2}\right) \\
\mathcal{X} & =\left\{\mathbf{x}_{i}=\frac{2}{\lambda}\left(\mathbf{p}_{\lfloor i / N\rfloor}-\mathbf{p}_{\bmod (i, N)}\right) \odot\left[\begin{array}{ll}
M_{x}^{-1} & M_{y}^{-1}
\end{array}\right]^{T}: 0 \leq i<N^{2}\right\},
\end{aligned}
$$

onde $\odot$ representa o produto ponto a ponto (ou produto de Hadamard) e as linhas de base $\mathbf{p}_{r}-\mathbf{p}_{s}$ são representadas somente por suas coordenadas x e y (pois o array é plano, e está orientado em relação ao sistema de coordenadas tal que a terceira coordenada espacial seja sempre nula). Mostremos que esta parametrização da NFFT produz a transformada direta.

Novamente,

$$
\begin{aligned}
\mathbf{S}_{r, s} & =\mathrm{e}^{j 2 \pi \frac{\mathbf{u}_{0}^{T} \frac{2\left(\mathbf{p}_{r}-\mathbf{p}_{s}\right)}{\lambda}}{\lambda}} \\
& =\mathrm{e}^{j 2 \pi\left(\mathbf{u}_{0} \odot\left[\begin{array}{ll}
\frac{M_{x}}{2} & \frac{M_{y}}{2}
\end{array}\right]^{T}\right)^{T}\left(\frac{2}{\lambda}\left(\mathbf{p}_{r}-\mathbf{p}_{s}\right) \odot\left[\begin{array}{ll}
M_{x}^{-1} & M_{y}^{-1}
\end{array}\right]^{T}\right)} .
\end{aligned}
$$


Comparando (8.5.19) com (8.5.14), o primeiro termo entre parênteses claramente pertence a $I_{\mathbf{N}}$. Como para $0 \leq i<N^{2},\left(\mathbf{p}_{\lfloor i / N\rfloor}-\mathbf{p}_{\bmod (i, N)}\right)$ cobre todas as possíveis linhas de base $\mathbf{p}_{r}-\mathbf{p}_{s}$, o segundo termo entre parênteses pertence a $\mathcal{X}$. A enumeração dada por $(\lfloor i / N\rfloor, \bmod (i, N))$ cobre todos os elementos de $\mathbf{S}_{r, s}$, de linha em linha. Dada a simetria Hermitiana de $\mathbf{S}$, isto é equivalente a conjugar (8.5.19) e cobrir os elementos de $\mathcal{X}$ de coluna em coluna, tornando (8.5.19) equivalente a (8.5.13).

A NFFT tem a vantagem de permitir geometrias arbitrárias, mas é aproximadamente uma ordem de magnitude mais lenta que a transformada proposta para geometrias separáveis, e também exige amostragem uniforme em U. A restrição de amostragem uniforme em U é inconveniente, pois imagens acústicas tipicamente são formadas por grupos de fontes distribuídas e grandes regiões sem fontes significativas. A transformada proposta permite sobre-amostrar regiões com fontes e sub-amostrar regiões vazias, sem aumento de custo computacional. Além disso, como veremos, a transformada proposta pode ser generalizada para modelar frentes de onda esféricas devidas a fontes em campo próximo. Em contraste, a FFT, NFFT e NNFFT (vista a seguir) requerem uma hipótese de campo distante.

\subsubsection{NNFFT}

Abandonando a restrição de amostragem uniforme em U, obtemos a transformada em sua máxima generalidade. Esta versão pode ser acelerada com a NNFFT. Apesar da NNFFT ser consideravelmente mais lenta que as transformadas propostas e a NFFT, ela requer muito menos memória que uma representação matricial de $\mathbf{A}$.

A entrada da NNFFT é um conjunto de amostras $h_{l} \in \mathbb{C}$ para $0 \leq l<L$, e a NNFFT é definida por

$$
\hat{h}_{i}=\sum_{l=0}^{L-1} h_{l} e^{-j 2 \pi\left(\mathbf{v}_{l} \odot \mathbf{M}\right)^{T} \mathbf{x}_{i}}
$$

para $0 \leq i<N$, e $\mathbf{v}_{l}, \mathbf{x}_{i} \in\left[-\frac{1}{2}, \frac{1}{2}\right)^{d}$ arbitrários. Para obter (8.5.10) usando a NNFFT, amostragem arbitrária em U e geometria arbitrária com $N$ microfones, usamos $x_{j} \in \mathcal{X}$ e $v_{l} \in \mathcal{V}, \mathrm{com}$

$$
\begin{aligned}
\mathbf{M} & =\left[\begin{array}{ll}
M_{x} & M_{y}
\end{array}\right]^{T} \\
\mathcal{V} & =\left\{v_{l}=\left(\frac{u_{x_{l}}}{2}, \frac{u_{y_{l}}}{2}\right) \in\left[-\frac{1}{2}, \frac{1}{2}\right)^{2}: l=0, \ldots, M_{x} M_{y}-1\right\} \\
\mathcal{X} & =\left\{\mathbf{x}_{i}=\frac{2}{\lambda}\left(\mathbf{p}_{\lfloor i / N\rfloor}-\mathbf{p}_{\bmod (i, N)}\right) \odot\left[\begin{array}{ll}
M_{x}^{-1} & M_{y}^{-1}
\end{array}\right]^{T}: 0 \leq i<N^{2}\right\}
\end{aligned}
$$

que tem a mesma forma de (8.5.15)-(8.5.17), mas permite amostragem arbitrária em U. 


\subsubsection{Beamformer delay-and-sum}

Dada uma matriz espectral $\mathbf{S}$, sua imagem correspondente é tipicamente aproximada usando um beamformer delay-and-sum usando

$$
\left|Y\left(u_{x_{m}}, u_{y_{n}}\right)\right|^{2} \approx \frac{\mathbf{v}^{H}\left(u_{x_{m}}, u_{y_{n}}\right) \mathbf{S v}\left(u_{x_{m}}, u_{y_{n}}\right)}{\left[\mathbf{v}^{H}\left(u_{x_{m}}, u_{y_{n}}\right) \mathbf{v}\left(u_{x_{m}}, u_{y_{n}}\right)\right]^{2}}
$$

onde a aproximação é devida a efeitos de convolução.

Podemos escrever

$$
\begin{aligned}
\mathbf{v}^{H}\left(u_{x_{m}}, u_{y_{n}}\right) \mathbf{S v}\left(u_{x_{m}}, u_{y_{n}}\right) & =\left(\mathbf{v}_{x_{m}}^{H} \otimes \mathbf{v}_{y_{n}}^{H}\right) \mathbf{S}\left(\mathbf{v}_{x_{m}} \otimes \mathbf{v}_{y_{n}}\right) \\
& =\left[\left(\mathbf{v}_{x_{m}} \otimes \mathbf{v}_{y_{n}}\right) \otimes\left(\mathbf{v}_{x_{m}}^{*} \otimes \mathbf{v}_{y_{n}}^{*}\right)\right] \cdot \operatorname{vec}\{\mathbf{S}\} \\
& =\left[\mathbf{A}^{H} \operatorname{vec}\{\mathbf{S}\}\right]_{m \cdot M_{y}+n} .
\end{aligned}
$$

Onde a última igualdade segue da comparação de (8.5.26) com (8.2.1).

Portanto, a reconstrução de imagens via delay-and-sum pode ser implementada usando a transformada rápida adjunta, derivada na Seção 8.3. Além disso, a composição diretaadjunta $\mathbf{A}^{H} \mathbf{A}$ é a transformada que obtém uma imagem delay-and-sum a partir de uma imagem limpa (ideal). Se admitirmos que as fontes estão no campo distante e que o espaço U é amostrado uniformemente, esta imagem delay-and-sum é simplesmente a imagem limpa convoluída com a PSF do beamformer. Portanto, sob estas hipóteses, $\mathbf{A}^{H} \mathbf{A}$ também pode ser obtida com uma convolução acelerada por uma FFT. Como veremos na Seção 8.6, a transformada proposta sempre pode ser utilizada para avaliar $\mathbf{A}^{H} \mathbf{A}$ em menos tempo que uma FFT.

\subsubsection{Beamformer MPDR}

Imagens obtidas com beamformers MPDR são na prática mais populares que aquelas obtidas por delay-and-sum, pois o beamformer MPDR pode obter melhor resolução para fontes pontuais (desde que o ruído não seja excessivo, e que o parâmetro de regularização seja escolhido corretamente). Recorde que o processador MVDR direcionado para $\mathbf{v}_{T}=$ $\mathbf{v}\left(u_{x_{T}}, u_{y_{T}}\right)$ é dado por

$$
\mathbf{w}_{\mathrm{MVDR}}^{H}\left(u_{x_{T}}, u_{y_{T}}\right)=\frac{\mathbf{v}_{T}^{H} \mathbf{S}_{\mathbf{n}}^{-1}}{\mathbf{v}_{T}^{H} \mathbf{S}_{\mathbf{n}}^{-1} \mathbf{v}_{T}}
$$

onde $\mathbf{S}_{\mathbf{n}}$ é a matriz espectral do ruído.

Uma forma de se obter $\mathbf{S}_{\mathbf{n}}$ para imagens acústicas é através de uma medida adicional, sem o sinal de interesse (por exemplo, com o modelo removido do túnel de vento) [79]. Se isto não for possível, pode-se usar um beamformer MPDR com regularização, usando $\mathbf{S}+\lambda \mathbf{I}$ no lugar de $\mathbf{S}_{\mathbf{n}}$, onde $\lambda$ é um parâmetro de regularização adequadamente escolhido e $\mathbf{S}$ é a matriz espectral que inclui as fontes de interesse e o ruído. Assim, 


$$
\mathbf{w}_{\mathrm{MPDR}}^{H}\left(u_{x_{T}}, u_{y_{T}}\right)=\frac{\mathbf{v}_{T}^{H}[\mathbf{S}+\lambda \mathbf{I}]^{-1}}{\mathbf{v}_{T}^{H}[\mathbf{S}+\lambda \mathbf{I}]^{-1} \mathbf{v}_{T}} .
$$

Seja $\hat{\mathbf{x}}$ a saída do array no domínio da frequência, tal que $\mathbf{S}=\mathrm{E}\left\{\hat{\mathbf{x}} \hat{\mathbf{x}}^{H}\right\}$. O valor de cada pixel da imagem acústica pode ser aproximado pela potência na saída do beamformer, tal que para $\mathbf{w}=\mathbf{w}_{\mathrm{MVDR}}$,

$$
\begin{aligned}
\left|Y\left(u_{x_{T}}, u_{y_{T}}\right)\right|^{2} & \approx \mathrm{E}\left\{\left|\mathbf{w}^{H}\left(u_{x_{T}}, u_{y_{T}}\right) \hat{\mathbf{x}}\right|^{2}\right\} \\
& =\mathbf{w}^{H}\left(u_{x_{T}}, u_{y_{T}}\right) \mathrm{E}\left\{\hat{\mathbf{x}} \hat{\mathbf{x}}^{H}\right\} \mathbf{w}\left(u_{x_{T}}, u_{y_{T}}\right) \\
& =\frac{\mathbf{v}_{T}^{H} \mathbf{S}_{\mathbf{n}}{ }^{-1} \mathbf{S S}_{\mathbf{n}}{ }^{-1} \mathbf{v}_{T}}{\left(\mathbf{v}_{T}^{H} \mathbf{S}_{\mathbf{n}}{ }^{-1} \mathbf{v}_{T}\right)^{2}} .
\end{aligned}
$$

Dos resultados anteriores, segue que podemos obter $\mathbf{v}_{T}^{H} \mathbf{S}_{\mathbf{n}}{ }^{-1} \mathbf{S S}_{\mathbf{n}}{ }^{-1} \mathbf{v}_{T}$ para todas as direções de interesse simultaneamente avaliando $\mathbf{A}^{H}$ vec $\left\{\mathbf{S}_{\mathbf{n}}{ }^{-1} \mathbf{S S}_{\mathbf{n}}{ }^{-1}\right\}$. Analogamente, podemos computar $\left[\mathbf{v}_{T}^{H} \mathbf{S}_{\mathbf{n}}{ }^{-1} \mathbf{v}_{T}\right]^{2}$ para todas as direções de interesse com o quadrado ponto a ponto de $\mathbf{A}^{H}$ vec $\left\{\mathbf{S}_{\mathbf{n}}{ }^{-1}\right\}$. Dividindo um pelo outro, podemos eficientemente obter uma imagem acústica com um beamformer MVDR. Resultados análogos são válidos para beamformers MPDR.

\subsection{Desempenho}

Esta seção apresenta resultados experimentais que permitem comparar os tempos de execução das transformadas propostas, da NFFT e da NNFFT. Apesar de ser fácil estimar o desempenho relativo em termos de MACs para os algoritmos implementados com produtos matriciais, na prática os tempos de execução podem desviar consideravelmente para problemas de certos tamanhos. De fato, em arquiteturas modernas, o desempenho é fortemente dependente da interação de unidades aritméticas paralelas, largura de banda da memória, tamanho do cache e predição de desvios, tal que a quantidade de operações de ponto flutuante serve somente como uma aproximação grosseira do custo computacional.

Os tempos de execução apresentados nas Figuras 8.5 e 8.6 são médias coletadas em loops com duração de 10 segundos para cada algoritmo e tamanho de problema. Todas as simulações foram executadas em um processador Intel Core 2 Duo T9400 em modo de 64-bits, usando apenas um core. As funções $\boldsymbol{\Xi}_{u}, \boldsymbol{\Xi}_{u}^{T}, \boldsymbol{\Xi}_{s}$ e $\boldsymbol{\Xi}_{s}^{T}$ foram escritas em ANSI C, a biblioteca NFFT foi compilada com otimizações padrão e todas as outras funções foram escritas em M-code para o MATLAB R2008b. Como o código não possui loops significativos e o MATLAB utiliza a Intel Math Kernel Library para aritmética de vetores e matrizes, as transformadas propostas executam praticamente como código ajustado à arquitetura. O MATLAB e a NFFT utilizam a biblioteca FFTW [80] para computação de FFTs, tal que estas também executam com desempenho praticamente ótimo.

A implementação da transformada proposta supõe um grid de amostragem separável 
em U. Para a NFFT, este grid é obrigatoriamente uniforme, pois a NFFT é não-uniforme apenas em relação ao domínio da frequência. Para NNFFT, não realizamos nenhuma suposição sobre o grid de amostragem ou sobre a geometria do array. A implementação explícita na forma da matriz A não é apresentada, pois requer uma quantidade proibitiva de memória para problemas com $M_{x}>32$ ou $M_{y}>32$. Se fosse implementada, as restrições de largura de banda da memória a tornariam a transformada mais lenta para problemas de praticamente todos os tamanhos. Para todos os algoritmos, tempos de inicialização foram desconsiderados.

A transformada proposta para geometria uniforme é claramente a mais eficiente. De fato, ela é tão rápida que para os casos em que $N_{x}=N_{y} \geq 20$ e $M_{x}=M_{y} \leq 256$, o gargalo do algoritmo é a alocação de memória, pois cada transformada retorna uma matriz $N_{x} N_{y} \times N_{x} N_{y}$. A transformada proposta para a geometria separável é a segunda mais rápida. A implementação com produto matricial por $\mathbf{V}_{\mathbf{x}} \mathrm{e} \mathbf{V}_{\mathbf{y}}$ apresenta desempenho muito bom para imagens pequenas e médias. Para arrays e imagens grandes, é melhor substituir o produto matricial por NFFTs. A implementação que utiliza diretamente a NFFT é útil se for desejável ter uma geometria arbitrária, mas também tem a desvantagem de exigir amostragem uniforme em U. A implementação utiliza diretamente a NNFFT é a mais lenta por uma margem significativa. Para todas as implementações, as transformadas direta e transposta têm desempenhos semelhantes.

A Figura 8.7 apresenta tempos de execução para a transformada direta-adjunta. As implementações rápidas utilizando (8.4.1) e (8.4.5) analisam o tamanho do problema e automaticamente selecionam a ordem ótima para os produtos matriciais. Para valores grandes de $N_{x}$ e $N_{y}$, elas também utilizam versões precomputadas de $\mathbf{V}_{\mathbf{y}}^{H} \mathbf{V}_{\mathbf{y}}$ e $\mathbf{V}_{\mathbf{x}}^{T} \mathbf{V}_{\mathbf{x}}^{*}$, o que faz a complexidade computacional depender apenas de $M_{x}$ e $M_{y}$. A implementação acelerada pela NFFT utiliza a composição das transformadas direta e adjunta, sem nenhuma otimização adicional. A convolução acelerada pela FFT acrescenta zeros para prevenir efeitos de borda. Isto é sempre necessário com arrays acústicos, pois a PSF não tem suporte compacto. As implementações da transformada direta-adjunta utilizando (8.4.1) e (8.4.5) são significativamente mais rápidas, exceto para o caso $M_{x}=M_{y}=1024$, onde a versão acelerada pela NFFT é mais rápida.

As transformadas propostas têm a vantagem adicional de serem simples de implementar e facilmente paralelizáveis, pois só exigem produtos matriciais. Em arquiteturas similares a DSPs, onde a penalidade para acessar a memória local é pequena ou inexistente, a transformada proposta para geometrias uniformes terá desempenho ainda melhor. De fato, $\mathbf{V}_{\mathbf{x}}$ e $\mathbf{V}_{\mathbf{y}}$ podem ser matrizes muito largas, para as quais o produto matricial apresenta desempenho sub-ótimo em arquiteturas de propósito geral, mas que são triviais de implementar eficientemente usando DSPs. 

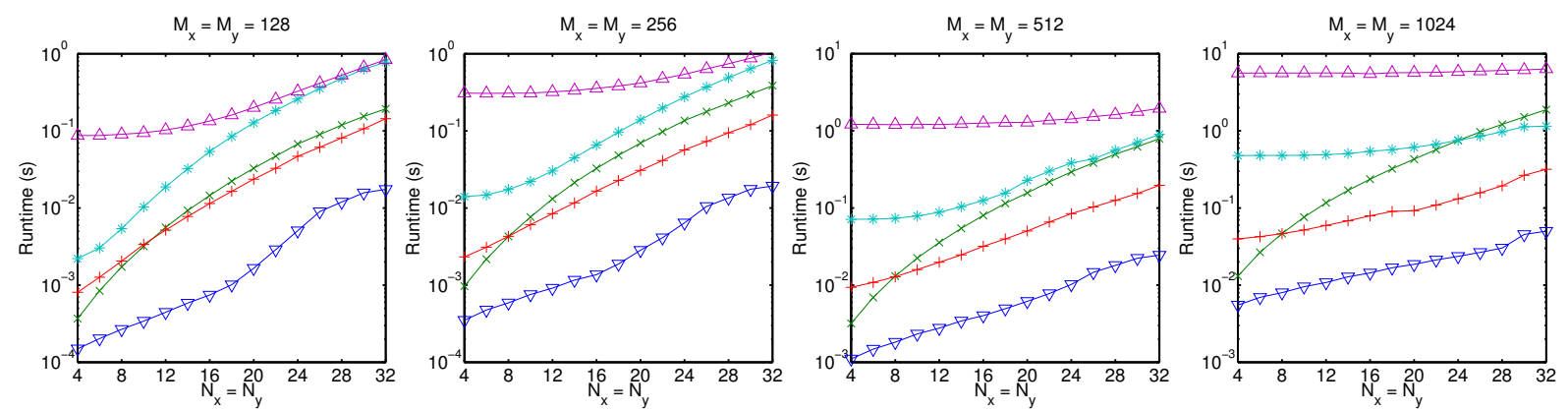

Figura 8.5: Tempos de execução para a transformada direta. $\nabla$ : transformada proposta, geometria uniforme, $\times$ : transformada proposta, geometria separável com produto matricial, + : transformada proposta, geometria separável com NFFT, $*$ : implementação direta com NFFT, $\triangle$ : implementação direta com NNFFT.
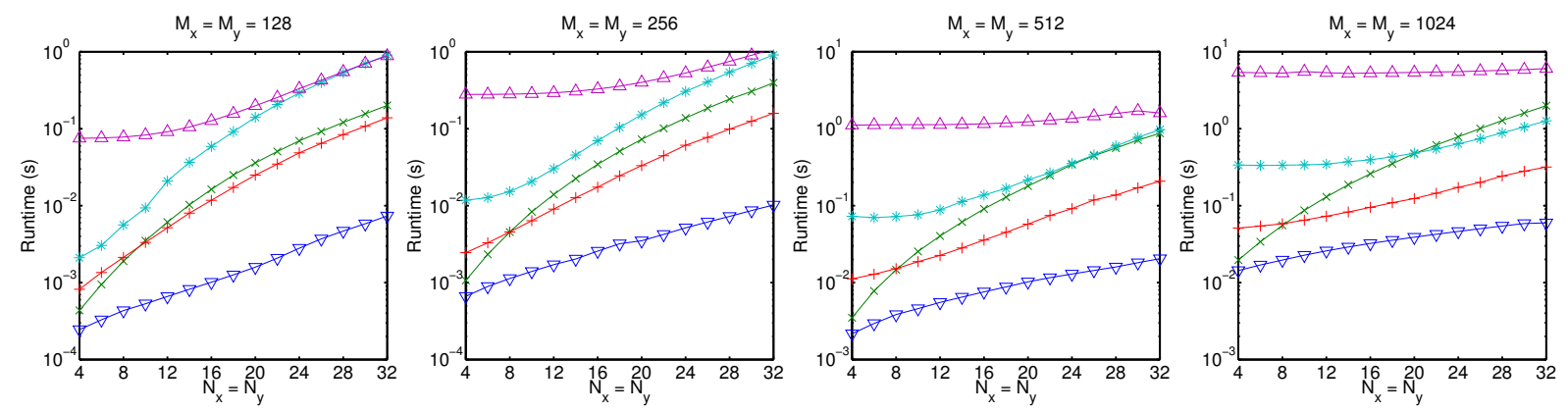

Figura 8.6: Tempos de execução para a transformada adjunta. $\nabla$ : transformada proposta, geometria uniforme, $\times$ : transformada proposta, geometria separável com produto matricial, + : transformada proposta, geometria separável com NFFT, $*$ : implementação direta com NFFT, $\triangle$ : implementação direta com NNFFT.
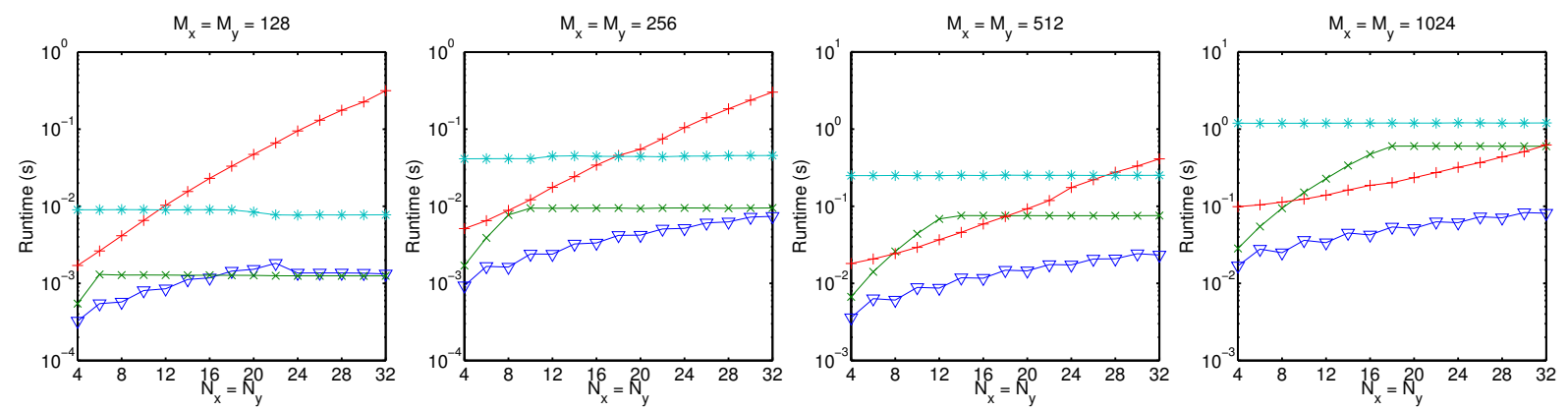

Figura 8.7: Tempos de execução para a transformada direta-adjunta. $\nabla$ : transformada proposta, geometria uniforme, implementada com (8.4.5), $\times$ : transformada proposta, geometria separável, implementada com $(8.4 .1),+$ : transformada proposta, geometria separável, implementada com NFFTs, $*$ : convolução $2 \mathrm{D}$ implementada com FFT 


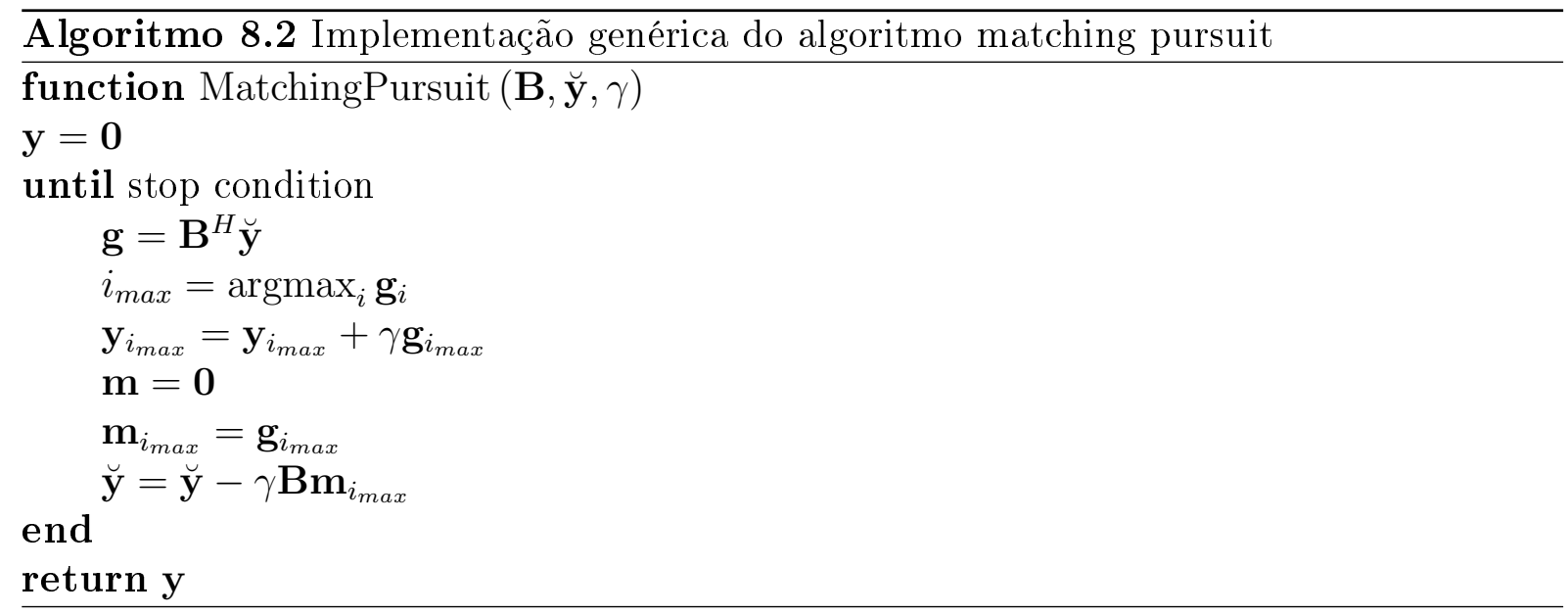

\subsection{Aplicações}

\subsubsection{CLEAN}

Na Seção 7.5.1, CLEAN foi apresentado usando beamforming e convoluções. Esta é a linguagem em que foi descrito pela primeira vez em [65], e estendido ao caso de imagens acústicas em [66]. Nesta seção, apresentamos uma formulação equivalente, tratando CLEAN como uma instância do algoritmo matching pursuit [81]. Esta formulação naturalmente leva ao uso da transformada rápida, permitindo a aceleração do método.

Sejam Y uma distribuição arbitrária em campo distante, $\mathbf{S}$ a matriz espectral gerada por $\mathbf{Y}, \mathbf{s}=\operatorname{vec}\{\mathbf{S}\}$ e $\mathbf{y}=\operatorname{vec}\{\mathbf{Y}\}$. Sob condições ideais, $\mathbf{s}=\mathbf{A y}$, onde $\mathbf{A}$ é a transformada direta. Conforme visto na Seção 8.5.4, se $\breve{\mathbf{Y}}$ for a imagem obtida com delay-and-sum e $\breve{\mathbf{y}}=\operatorname{vec}\{\breve{\mathbf{Y}}\}$, então $\breve{\mathbf{y}}=\mathbf{A}^{H} \mathbf{s}=\mathbf{A}^{H} \mathbf{A y}$.

CLEAN tenta obter $\mathbf{y}$ (a imagem limpa) a partir de $\breve{\mathbf{y}}$ (a imagem suja). Na linguagem de matching pursuit, dado $\mathbf{B}=\mathbf{A}^{H} \mathbf{A}$, o algoritmo decompõe $\breve{\mathbf{y}}$ em uma soma ponderada de colunas de $\mathbf{B}$, com pesos dados por $\mathbf{y}$. Como as colunas de $\mathbf{B}$ são linearmente dependentes, matching pursuit utiliza uma heurística para escolher a melhor coluna para atualizar primeiro. Esta melhor coluna é escolhida a cada iteração, e é aquela que tem o maior produto interno com $\breve{\mathbf{y}}$. A cada iteração, uma fração $\gamma$ da contribuição da melhor coluna é descontada de $\breve{\mathbf{y}}$.

O Algoritmo 8.2 descreve uma implementação genérica do algoritmo matching pursuit. A condição de parada pode depender do número de iterações, número de coeficientes recuperados, erro quadrático médio, etc. Para implementar o método CLEAN, basta usar $\mathbf{B}=\mathbf{B}^{H}=\mathbf{A}^{H} \mathbf{A}$. A aplicação de $\mathbf{B}$ é claramente o gargalo do algoritmo, mas pode ser acelerada com a transformada rápida, que executa em menos tempo que uma convolução acelerada por uma FFT.

No entanto, esta implementação acelerada apresenta exatamente a mesma velocidade de convergência que a implementação tradicional. Portanto, não é capaz de produzir reconstruções de melhor qualidade. Por isso, sequer apresentaremos exemplos de recons- 
trução usando CLEAN.

\subsubsection{DAMAS}

Usando a transformada rápida, é quase trivial implementar uma versão rápida do algoritmo DAMAS2. De fato, das Seções 8.4 e 8.5.4 temos que

$$
\begin{aligned}
\breve{\mathbf{y}} & =\operatorname{vec}\{\breve{\mathbf{Y}}\} \\
& =\operatorname{vec}\left\{\mathbf{P}_{d s} * \mathbf{Y}\right\} \\
& =\mathbf{A}^{H} \mathbf{A} \operatorname{vec}\{\mathbf{Y}\} \\
& =\mathbf{A}^{H} \mathbf{A y}
\end{aligned}
$$

e assim,

$$
\operatorname{vec}\left\{\mathbf{P}_{d s} * \hat{\mathbf{Y}}^{(k)}\right\}=\mathbf{A}^{H} \mathbf{A} \hat{\mathbf{y}}^{(k)}
$$

onde $\mathbf{A}^{H} \mathbf{A}$ pode ser implementado com a transformada rápida.

Portanto, (7.5.12) se torna

$$
\hat{\mathbf{y}}^{(k+1)}=\max \left\{\hat{\mathbf{y}}^{(k)}+\frac{1}{a}\left[\breve{\mathbf{y}}-\mathbf{A}^{H} \mathbf{A} \hat{\mathbf{y}}^{(k)}\right], \mathbf{0}\right\}
$$

onde $a$ tem o mesmo significado que antes.

Como as convoluções são o gargalo de DAMAS2, a aceleração devida a (8.7.6) em relação a (7.5.12) é devida ao tempo de execução de $\mathbf{A}^{H} \mathbf{A}$ quando comparado ao de uma convolução acelerada por uma FFT. Referindo à Figura 8.7, pode-se ver que o algoritmo proposto é consideravelmente mais rápido para problemas de todos os tamanhos. Além disso, as transformadas rápidas permitem o uso de amostragens separáveis em U, o que previamente não era possível com DAMAS2.

Apesar de DAMAS2 produzir melhoras significativas se comparado com os métodos que o antecederam, ele não utiliza nenhuma regularização além da exigência de nãonegatividade da solução. Portanto, ele não incorpora um modelo que caracterize a distribuição. Além disso, DAMAS2 é um método de deconvolução que tem como entrada as imagens delay-and-sum. Como mostramos, beamforming delay-and-sum é equivalente à aplicação de $\mathbf{A}^{H} \mathbf{A}$ ou a uma convolução pela PSF do array, que é um filtro passa-baixas. A característica passa-baixas implica que $\mathbf{A}^{H} \mathbf{A}$ tem muitos valores singulares pequenos. Portanto, a aplicação de $\mathbf{A}^{H} \mathbf{A}$ atenua significativamente os componentes do espaço vetorial correspondente a estes valores singulares, dificultando a inversão de $\breve{\mathbf{y}}=\mathbf{A}^{H} \mathbf{A y}$ (como proposta por DAMAS2). Por outro lado, os valores singulares de $\mathbf{A}$ são a raiz quadrada dos valores singulares de $\mathbf{A}^{H} \mathbf{A}$. Logo, a aplicação de $\mathbf{A}$ somente atenua componentes pela raiz quadrada dos fatores anteriores, tornando preferível a inversão de $\mathbf{s}=\mathbf{A y}$ (como proposta por formulações de mínimos quadrados). Por estes motivos, métodos de 
mínimos quadrados regularizados são mais estáveis, como veremos a seguir.

\subsubsection{Regularização $\ell_{1}$}

As transformadas rápidas podem ser usadas para acelerar a solução de problemas inversos com regularização $\ell_{1}$, desde que o método de otimização convexa utilizado dependa somente de implementações de $\mathbf{A}$ e $\mathbf{A}^{H}$. Assim, problemas de reconstrução se tornam ordens de magnitude mais rápidos, e permitem a reconstrução de imagens com resoluções maiores.

Para obter uma implementação rápida que seja tratável com solvers existentes, propomos reescrever (7.6.7) como um problema do tipo basis pursuit com redução de ruído $(\mathrm{BPDN})$, que tem a forma

$$
\min _{\hat{\mathbf{Y}}}\|\hat{\mathbf{Y}}\|_{1} \text { sujeito a }\|\operatorname{vec}\{\mathbf{S}\}-\mathbf{A v e c}\{\hat{\mathbf{Y}}\}\|_{2} \leq \sigma
$$

Nos exemplos, resolveremos (8.7.7) com o solver SPGL1 [82], que pode eficientemente resolver problemas de grande escala.

\subsubsection{Regularização TV}

Como arrays acústicos amostram campos de onda com uma quantidade relativamente pequena de elementos, os problemas inversos de reconstrução de imagens tendem a ser muito mal condicionados. Para obter reconstruções precisas, algum tipo de regularização é necessária para reduzir o espaço de possíveis soluções que se ajustam ao sinal medido. Ainda que a regularização $\ell_{1}$ tenha sido usada com sucesso em aplicações de compressive sensing, campos acústicos normalmente não são esparsos em suas representações canônicas, e regularização $\ell_{1}$ deve ser usada com uma transformada esparsificante. Para lidar com este problema, propomos reconstruir imagens acústicas usando a variação total (TV) para regularização.

Para $\mathbf{Y} \in \mathbb{C}^{M_{y} \times M_{x}}$, defina sua variação total isotrópica como

$$
\|\mathbf{Y}\|_{B V}=\sum_{i, j} \sqrt{\left[\nabla_{x} \mathbf{Y}\right]_{i, j}^{2}+\left[\nabla_{y} \mathbf{Y}\right]_{i, j}^{2}}
$$

onde $\nabla_{x}$ e $\nabla_{y}$ são os operadores de primeira diferença ao longo das dimensões x e y com fronteiras periódicas, para $0 \leq i<M_{y}$ e $0 \leq j<M_{x}$. $\|\cdot\|_{B V}$ é a semi-norma de variação limitada (BV).

Propomos resolver

$$
\min _{\hat{\mathbf{Y}}}\|\hat{\mathbf{Y}}\|_{B V}+\frac{\mu}{2}\|\operatorname{vec}\{\mathbf{S}\}-\mathbf{A v e c}\{\hat{\mathbf{Y}}\}\|_{2}^{2}
$$

sujeito a $\hat{\mathbf{Y}}_{i, j} \geq 0$. O primeiro termo mede o quanto a imagem oscila. Portanto, é menor para imagens com plateaus e transições monótonas, e tende a privilegiar soluções simples 
e com pequena quantidade de ruído. O segundo termo garante um bom ajuste entre a imagem reconstruída e os dados medidos. Esta formulação foi primeiro proposta para redução de ruído de imagens por Rudin, Osher e Fatemi [83], para $\mathbf{A}=\mathbf{I}$. Ela foi depois generalizada e aplicada com sucesso a muitos problemas de reconstrução de imagens.

Enquanto problemas de minimização $\ell_{1}$ podem ser resolvidos com programação linear ou com algoritmos sub-ótimos, minimização TV é consideravelmente mais complexa por causa da maior não-linearidade de $\|\cdot\|_{B V}$. Para resolver (8.7.9) escolhemos o solver TVAL3 [84], que utiliza o método de multiplicadores de Lagrange aumentado e separação de variáveis para desacoplar a minimização TV do problema de ajuste de covariância. TVAL3 se compara favoravelmente a outros solvers em termos de tempo de execução e qualidade de reconstrução.

\subsection{Exemplos de aplicação}

A seguir apresentamos exemplos de reconstrução de imagens ilustrando o uso de delayand-sum, DAMAS2, regularização $\ell_{1}$ e regularização $\mathrm{TV}$, todos implementados com a transformada rápida proposta. Para relaxar a restrição de amostragem abaixo da frequência de Nyquist, utilizamos uma geometria separável não uniforme. Simulamos uma geometria com 64 elementos, com $N_{x}=N_{y}=8$, e com dimensões horizontais e verticais de $30 \mathrm{~cm}$. Cada subarray linear com $N_{x} \times 1$ e $N_{y} \times 1$ elementos é um array não-redundante com um mínimo de diferenças ausentes (minimum missing lags) [13] e espaçamento interelementos dado por 1.3.5.6.7.10.2. (onde os pontos representam elementos, e os números indicam distâncias entre elementos). Esta geometria está plotada na Figura 8.8.

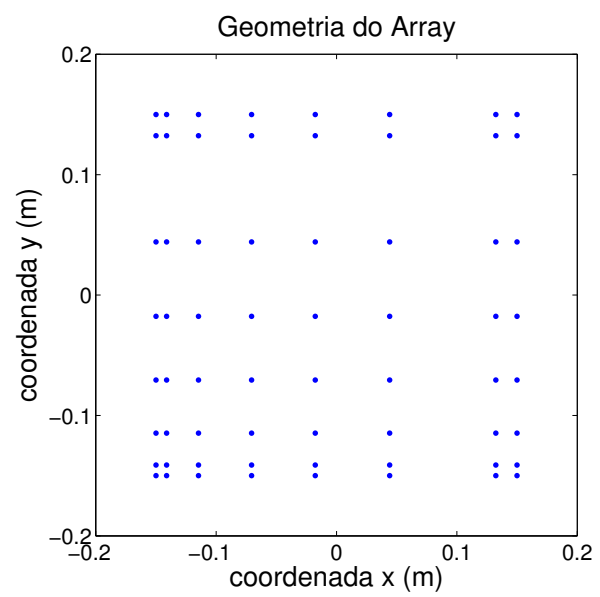

Figura 8.8: Geometria separável simulada

A seguir comparamos resultados obtidos com delay-and-sum, DAMAS2, reconstrução regularizada $\ell_{1}$ dada por (8.7.7) com o solver SPGL1 [82], e reconstrução regularizada TV dada por (8.7.9) com o solver TVAL3 [84]. Todos os métodos foram acelerados com as versões exatas das transformadas rápidas (sem uso da NFFT), e as imagens foram reconstruídas com $M_{x}=M_{y}=256$. DAMAS2, SPGL1 e TVAL3 usaram 1000, 200 e 100 


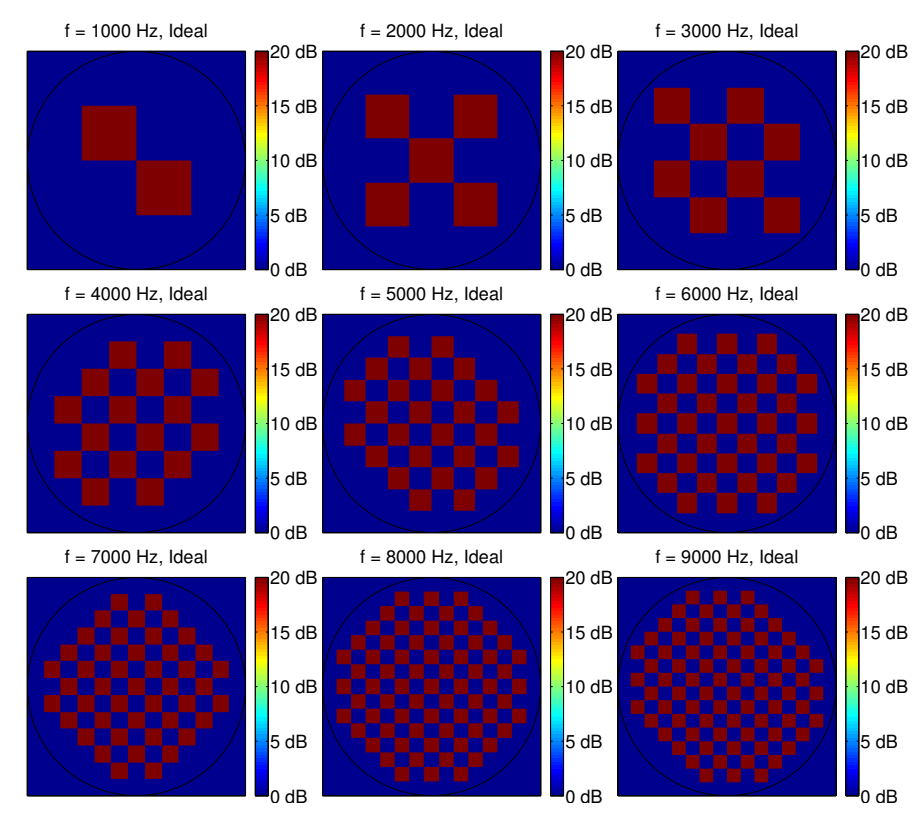

Figura 8.9: Distribuições ideais para o padrão de calibração

iterações, respectivamente, o que produz um bom equilíbrio entre custo computacional e qualidade de imagem. Os tempos de reconstrução para delay-and-sum, DAMAS2, regularização $\ell_{1}$ e regularização TV foram de aproximadamente $10 \mathrm{~ms}, 1.5 \mathrm{~s}, 8 \mathrm{~s}$ e $4 \mathrm{~s}$ por imagem, respectivamente. DAMAS2 não requer parâmetros. Usamos $\sigma=0.01\|\mathbf{S}\|_{F}$ em (8.7.7), e $\mu=10^{3}$ em (8.7.9). O modelo do sinal é dado por $\mathbf{S}=\mathbf{V}_{n} \mathrm{E}\left\{\mathrm{ff}^{H}\right\} \mathbf{V}_{n}^{H}+\sigma^{2} \mathbf{I}$, com $\sigma^{2}$ escolhido para obter uma SNR de $20 \mathrm{~dB}$. Como a intenção destas simulações não é analisar a sensibilidade a ruído de cada algoritmo, somente uma SNR foi usada.

\subsubsection{Padrões tabuleiro de xadrez}

As Figuras 8.10-8.13 mostram padrões de tabuleiro de xadrez reconstruídos usando delay-and-sum, DAMAS2, regularização $\ell_{1}$ com (8.7.7), e regularização TV com (8.7.9). DAMAS convencional não é apresentado, pois é muito lento, exige muita memória (pois requer a representação explícita de uma matriz com $M_{x}^{2} M_{y}^{2}$ elementos) e produz imagens ruidosas na ausência de filtragem passa baixas.

A reconstrução dos padrões de tabuleiro de xadrez mostra claramente as deficiências de reconstrução com delay-and-sum. As imagens parecem muito borradas, com vazamento significativo para fora da região visível. DAMAS2 reconstrói os padrões de teste corretamente, com a exceção de alguns artefatos. Regularização $\ell_{1}$ não apresenta reconstruções tão precisas, pois as imagens não são esparsas. Em várias imagens, os centros dos quadrados são estimados com pressão sonora inferior à real. Estes artefatos são comuns quando a regularização $\ell_{1}$ é usada para reconstruir sinais com plateaus, pois corresponde ao melhor compromisso entre esparsidade da solução e ajuste à matriz espectral medida. Regularização TV apresenta resultados similares ao DAMAS2, e com menos artefatos fora da região visível. 


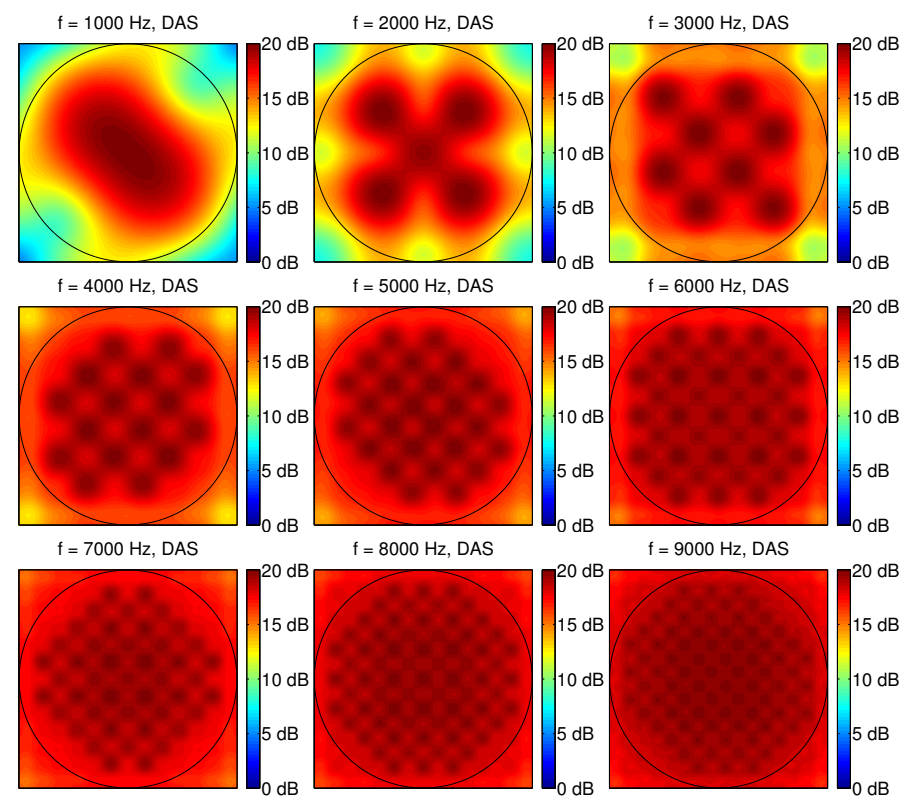

Figura 8.10: Reconstrução delay-and-sum, padrão de calibração, geometria separável

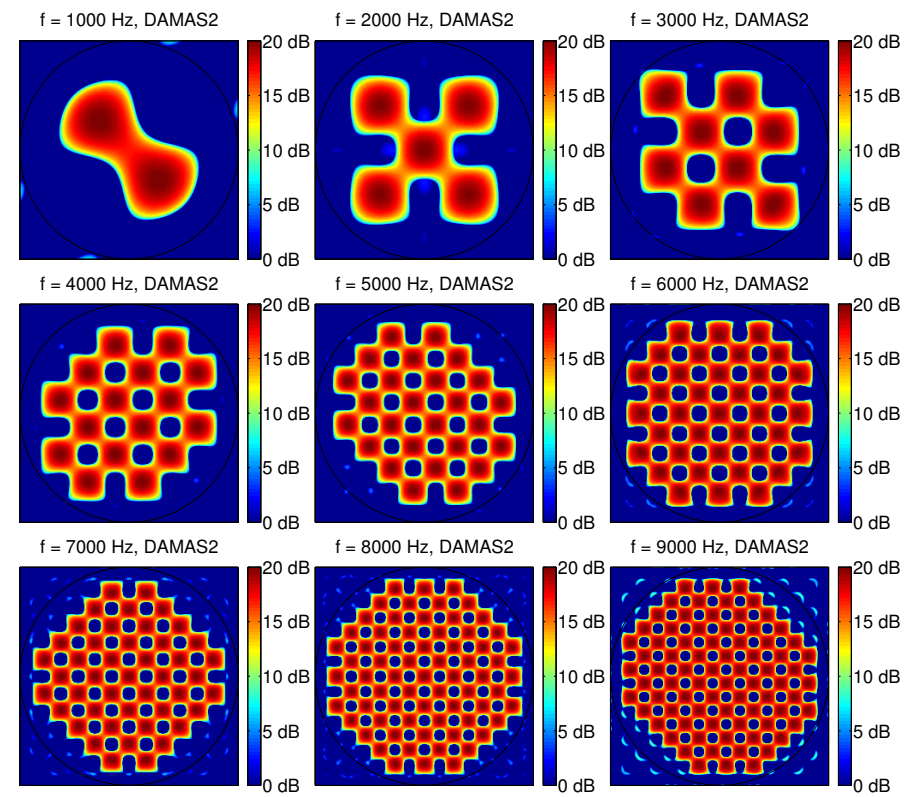

Figura 8.11: Reconstrução DAMAS2, padrão de calibração, geometria separável 


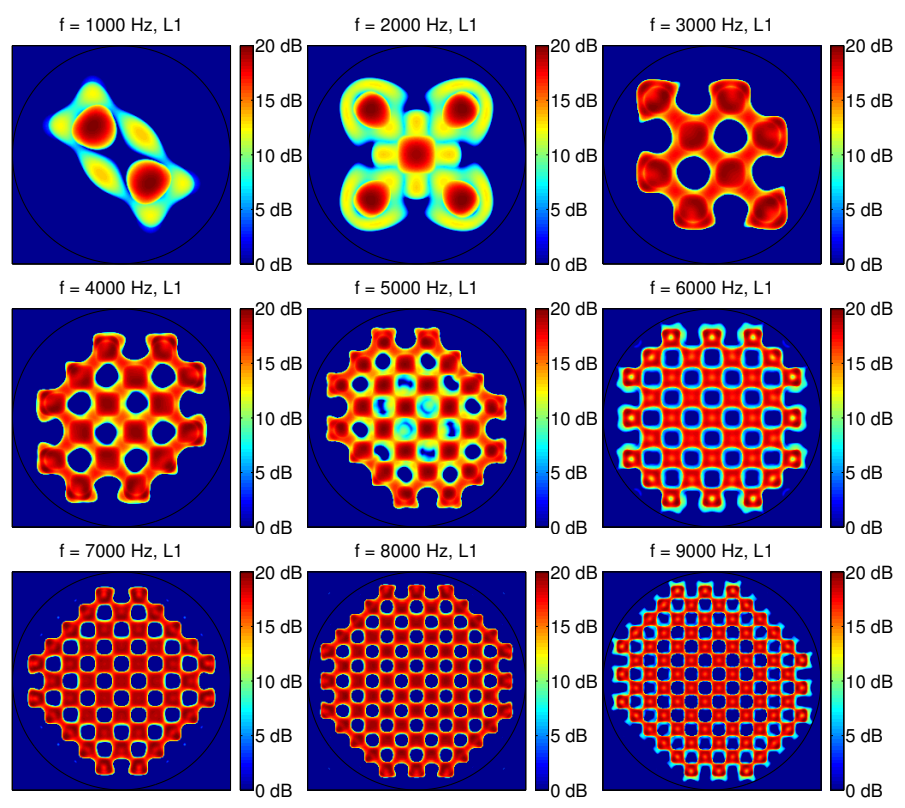

Figura 8.12: Reconstrução regularizada $\ell_{1}$, padrão de calibração, geometria separável

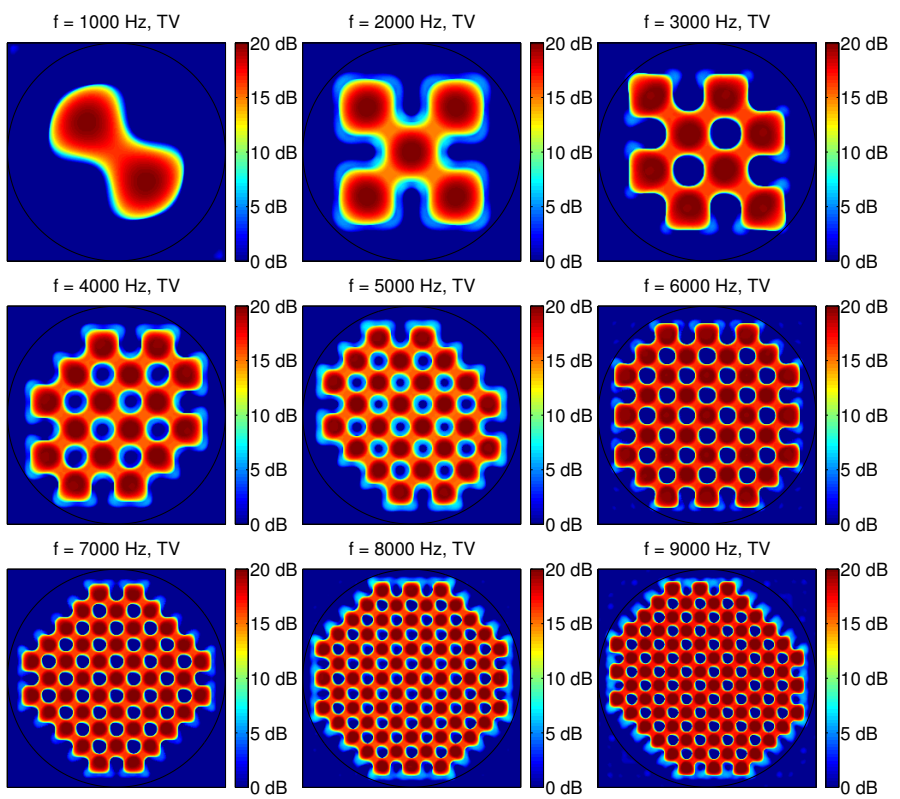

Figura 8.13: Reconstrução TV, padrão de calibração, geometria separável 


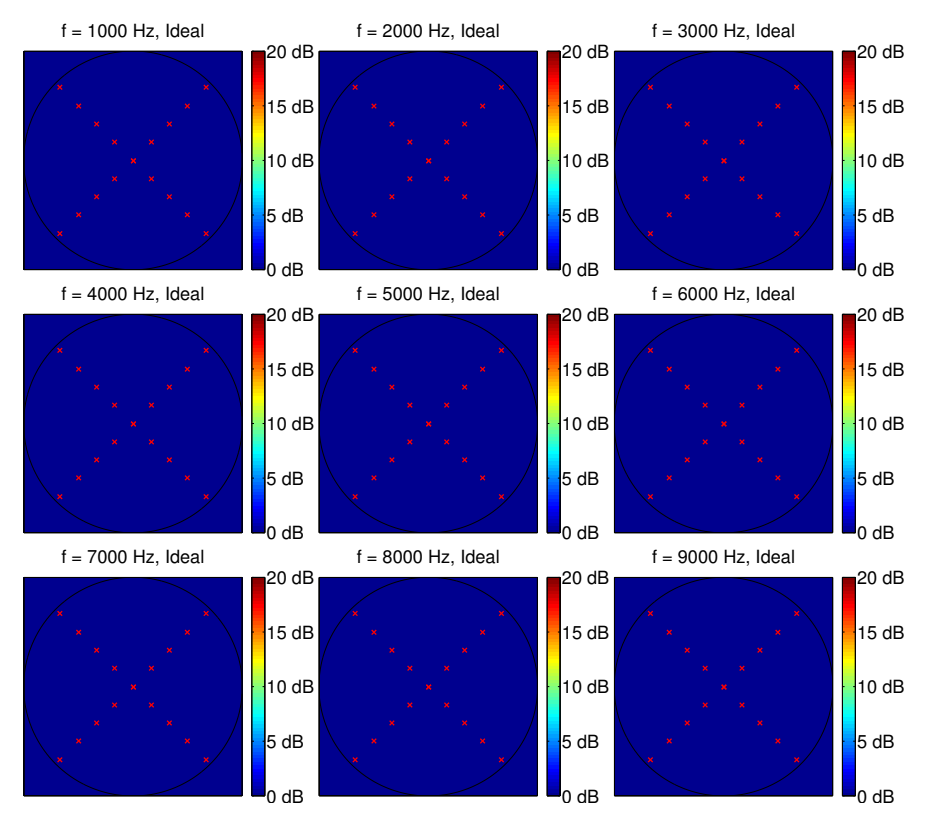

Figura 8.14: Distribuições ideais para o padrão impulsivo

\subsubsection{Padrões esparsos}

As Figuras 8.15-8.18 apresentam resultados de reconstrução para uma imagem de teste com 17 impulsos nas coordenadas $( \pm n / 6, \pm n / 6)$ do espaço $\mathrm{U}$, para $0 \leq n \leq 4$. Este teste foi projetado para avaliar a PSF equivalente para os métodos de reconstrução, e também detectar a presença de artefatos de aliasing. Como esperado, delay-and-sum tem a menor resolução espacial e apresenta lóbulos laterais significativos. DAMAS2 mostra resultados razoáveis, mas com artefatos devidos à falta de regularização. A reconstrução regularizada $\ell_{1}$ apresenta os melhores resultados, com fontes compactas e sem artefatos. Este é um resultado razoável, pois a imagem de interesse é de fato esparsa em sua representação canônica. Regularização TV também apresenta bons resultados, sem artefatos discerníveis mas maiores fontes que a regularização $\ell_{1}$.

\subsubsection{Padrão não-esparso}

Finalmente, as Figuras 8.20-8.23 apresentam resultados de reconstrução para um padrão não-esparso projetado especificamente para este experimento. Novamente, delayand-sum apresenta baixa resolução e fantasmas devido aos seus lóbulos laterais. DAMAS2 produz resultados muito melhores, mas apresenta alguns artefatos, especialmente para imagens de alta resolução. Os artefatos somem para a reconstrução regularizada $\ell_{1}$, que também apresenta melhor resolução que DAMAS2. No entanto, esta reconstrução não representa bem transições lisas, pois estas não são esparsas na representação canônica. Finalmente, a regularização TV produz as representações mais realistas, com formas corretas e a menor quantidade de ruído. 


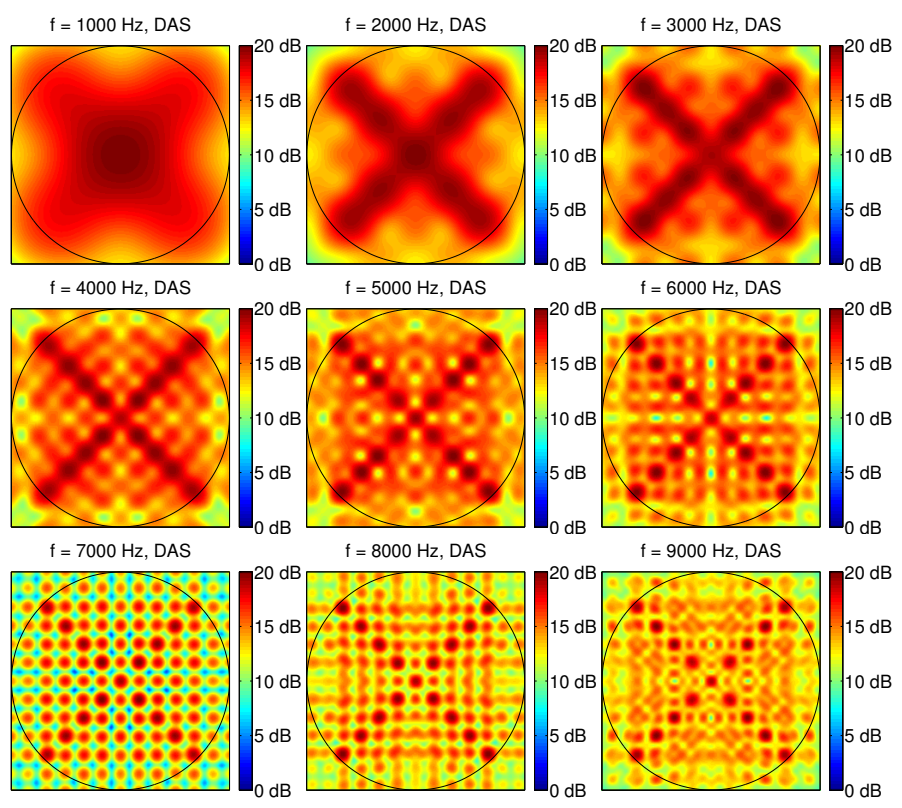

Figura 8.15: Reconstrução delay-and-sum, padrão impulsivo, geometria separável
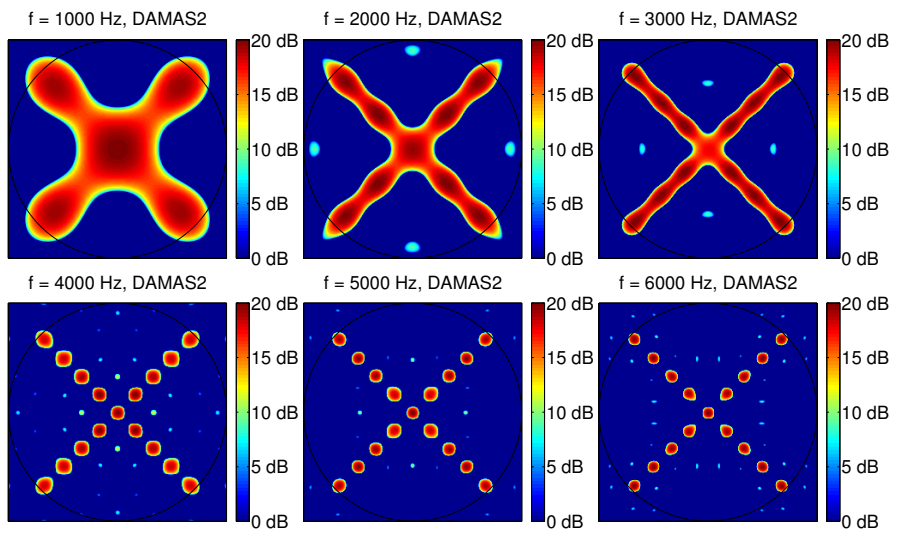

$\mathrm{f}=7000 \mathrm{~Hz}$, DAMAS2

$\mathrm{f}=8000 \mathrm{~Hz}$, DAMAS2

$f=9000 \mathrm{~Hz}$, DAMAS2
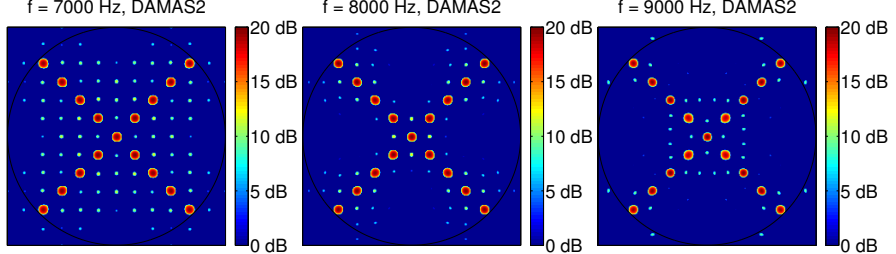

Figura 8.16: Reconstrução DAMAS2, padrão impulsivo, geometria separável 


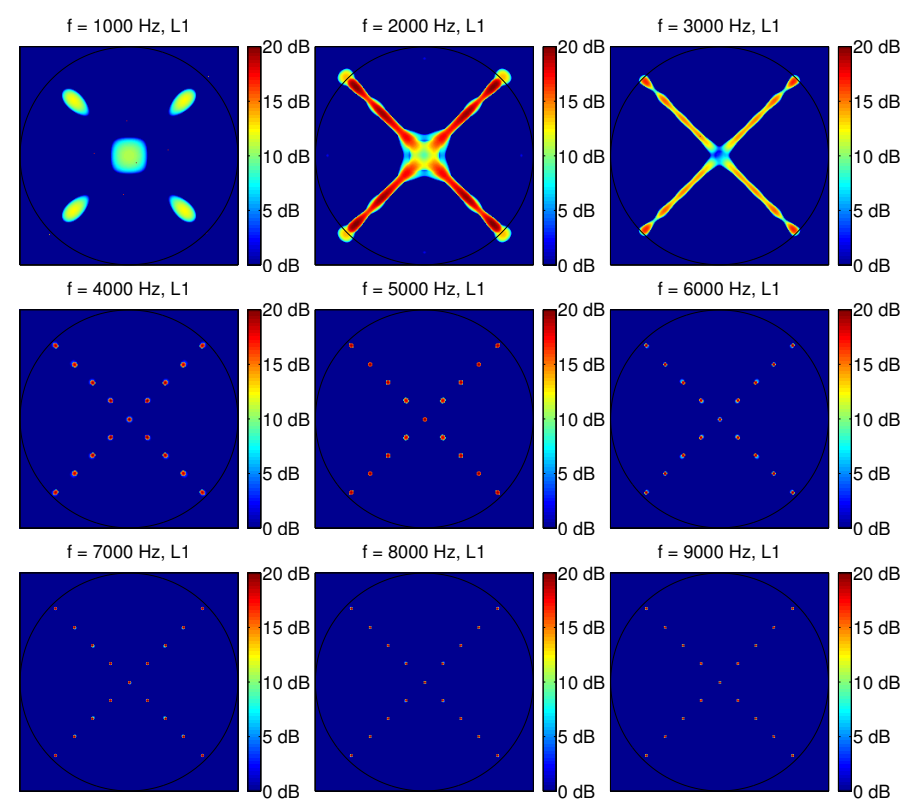

Figura 8.17: Reconstrução regularizada $\ell_{1}$, padrão impulsivo, geometria separável

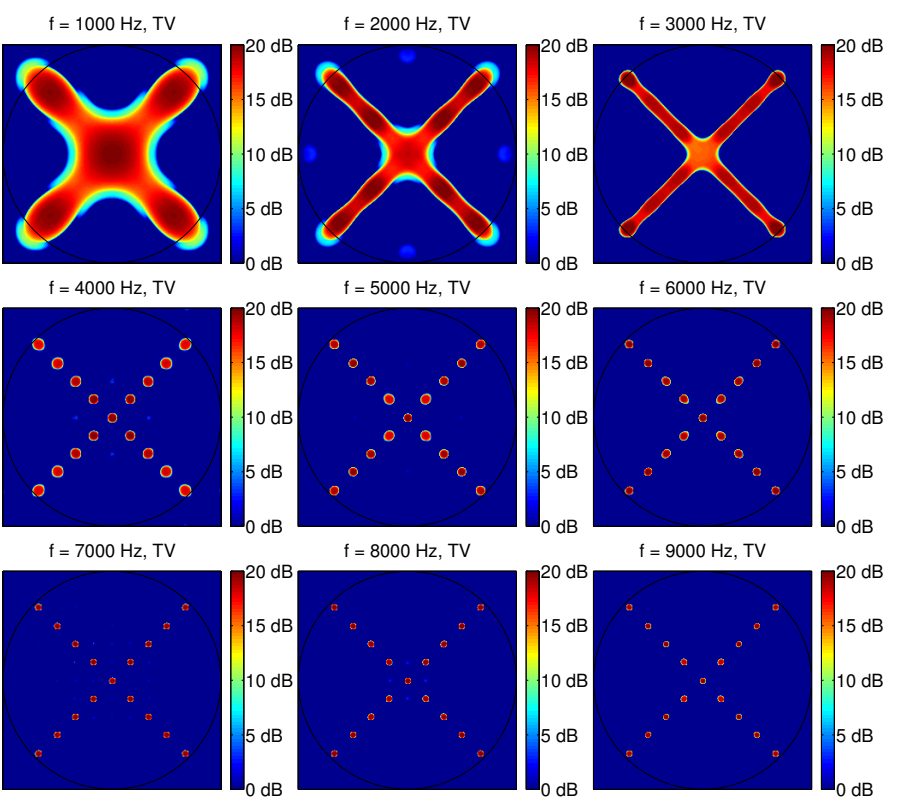

Figura 8.18: Reconstrução regularizada TV, padrão impulsivo, geometria separável 


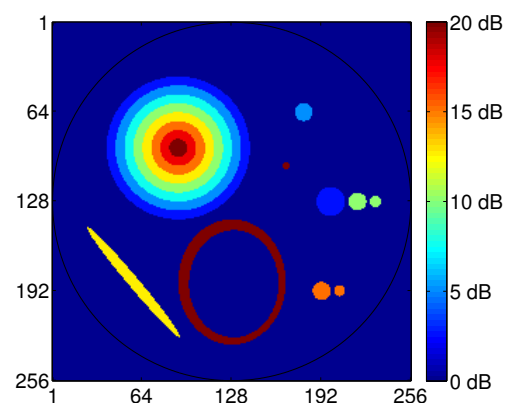

Figura 8.19: Padrão de testes não-esparso

\subsection{Comparação com geometrias espirais}

Arrays com geometria espiral logarítmica [85] têm pequenos lóbulos laterais para uma larga faixa de frequências. Como essa característica é crucial para síntese de imagens com beamforming, geometrias espirais se popularizaram em aplicações de imagens acústicas. Não obstante, lóbulos laterais têm pouca relevância quando se utilizam métodos de deconvolução ou mínimos quadrados. Nestes casos, geometrias ideais são aquelas com redundância zero e com mínimo de diferenças ausentes (que têm a maior largura de banda e alguns artefatos de reconstrução), ou redundância mínima e nenhuma diferença ausente (que teoricamente permite reconstrução ideal até uma frequência limite, sob a hipótese de campo distante e na ausência de ruído). Em geral, estas geometrias não produzem pequenos lóbulos laterais, mas os lóbulos são suficientemente pequenos para permitir reconstrução única.

Nesta seção, comparamos a geometria Cartesiana apresentada na Figura 8.8 com a geometria espiral logarítmica com 63 elementos apresentada na Figura 8.24. Esta geometria espiral tem $50 \times 50 \mathrm{~cm}$, que é a dimensão necessária para produzir imagens com resolução semelhante às de nosso array separável (que tem $30 \times 30 \mathrm{~cm}$ ). Além disso, seus parâmetros foram cuidadosamente escolhidos para produzir reconstrução ótima para as frequências de interesse. As Figuras 8.25-8.28 mostram resultados de reconstrução para esta geometria espiral (obtidos com a transformada exata, sem aceleração), sob as mesmas condições que as Figuras 8.20-8.23.

Enquanto a geometria espiral logarítmica produz melhores resultados para delay-andsum, as outras técnicas produzem imagens de qualidade comparável. Em particular, mínimos quadrados com regularização TV produz resultados muito parecidos para ambas geometrias. Isto não é surpreendente, pois a geometria Cartesiana foi escolhida para ter características ótimas (com mínimo de diferenças ausentes). Ainda que este exemplo não seja exaustivo, ele é destinado a convencer o leitor que dadas técnicas de reconstrução adequadas, geometrias Cartesianas podem produzir resultados de qualidade comparável às tradicionais geometrias espirais. Claramente, com arrays Cartesianos é possível aplicar transformadas rápidas e obter resultados com custo computacional muito inferior. Como 


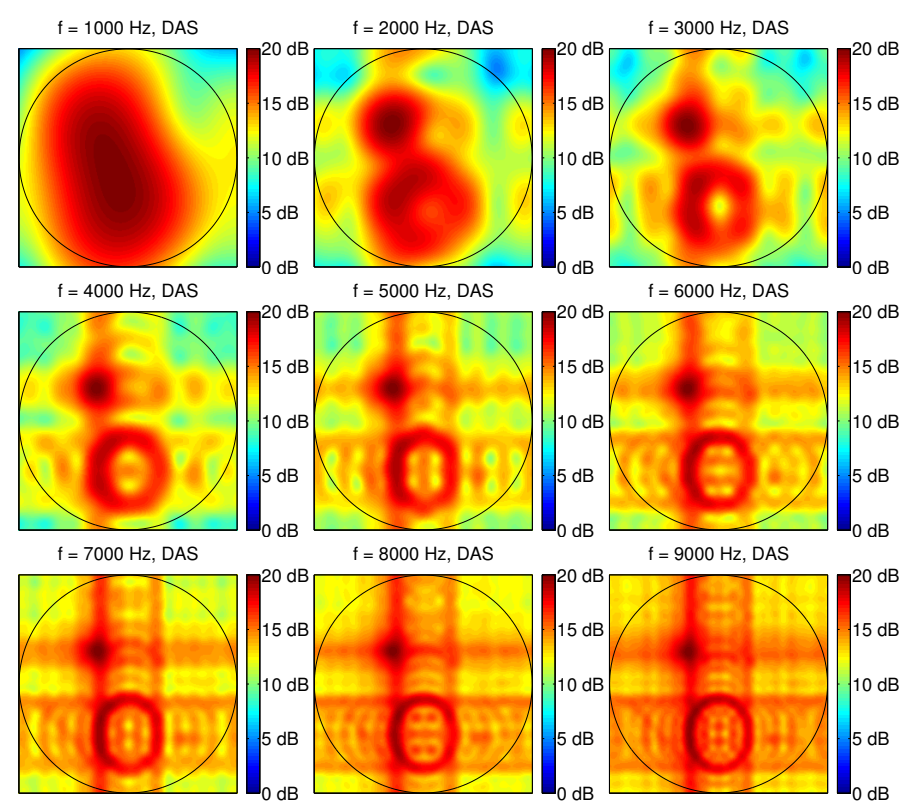

Figura 8.20: Reconstrução delay-and-sum, padrão não-esparso, geometria separável
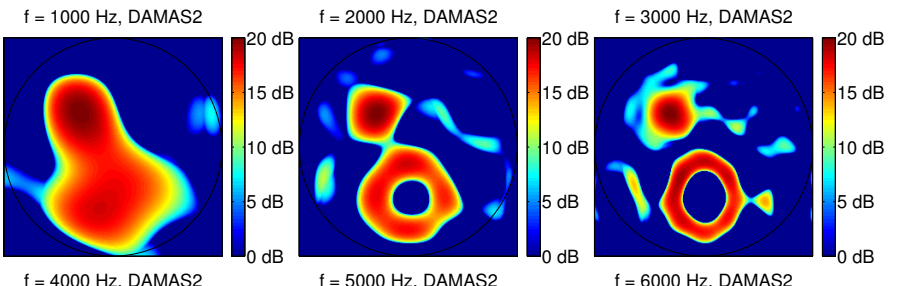

$\mathrm{f}=4000 \mathrm{~Hz}$, DAMAS2

$=5000 \mathrm{~Hz}$, DAMAS2
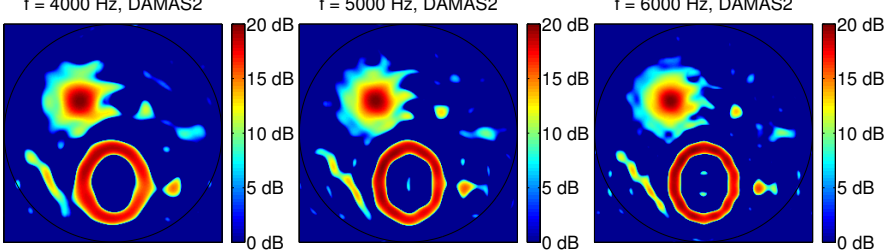

$\mathrm{f}=7000 \mathrm{~Hz}$, DAMAS2

$\mathrm{f}=8000 \mathrm{~Hz}$, DAMAS2

$f=9000 \mathrm{~Hz}$, DAMAS?
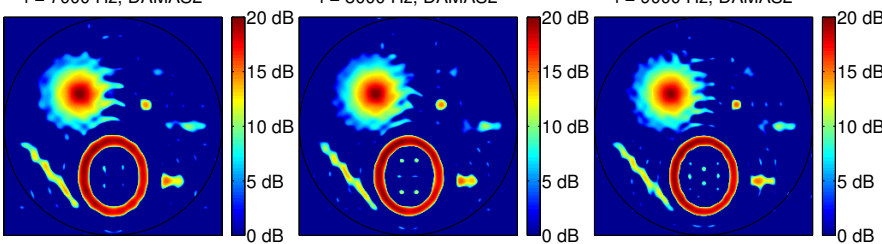

Figura 8.21: Reconstrução DAMAS2, padrão não-esparso, geometria separável 


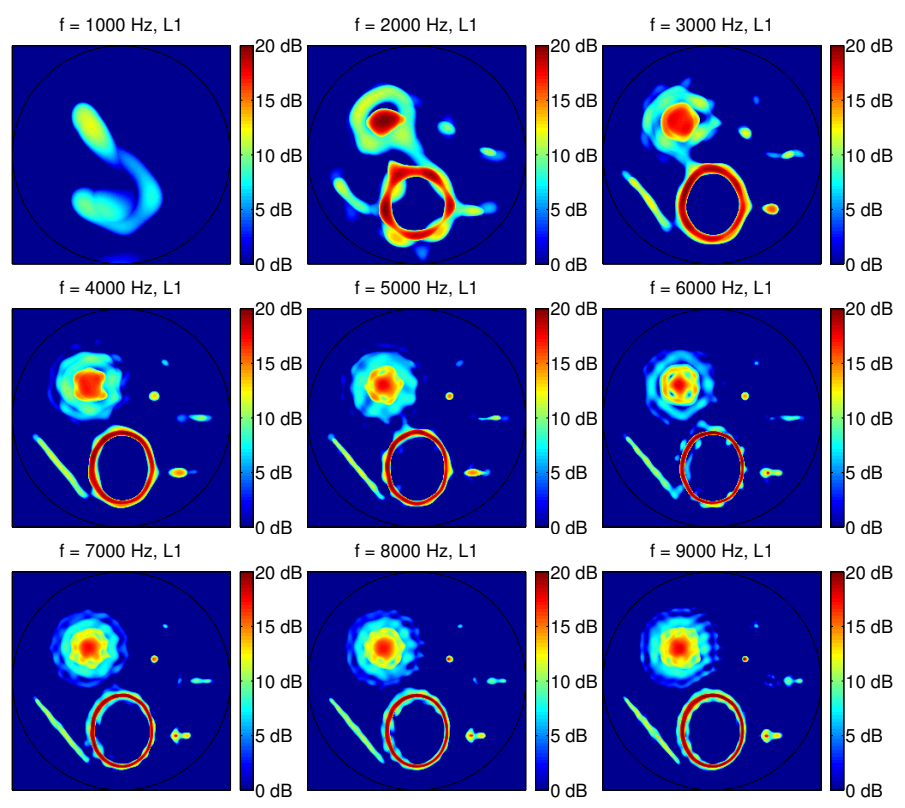

Figura 8.22: Reconstrução regularizada $\ell_{1}$, padrão não-esparso, geometria separável

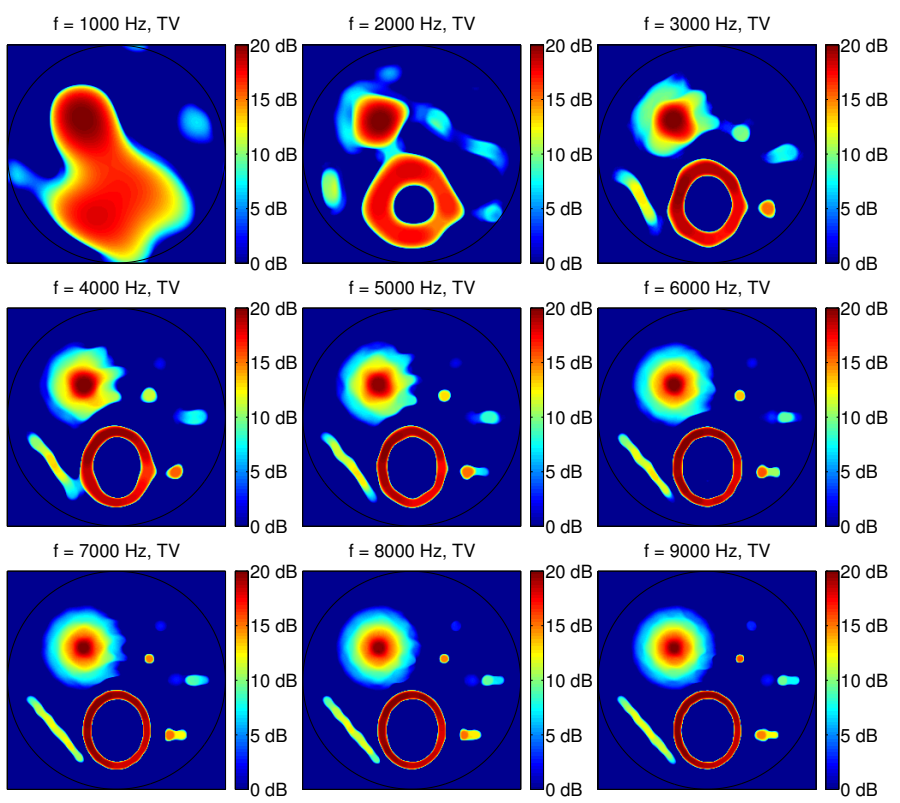

Figura 8.23: Reconstrução regularizada TV, padrão não-esparso, geometria separável 


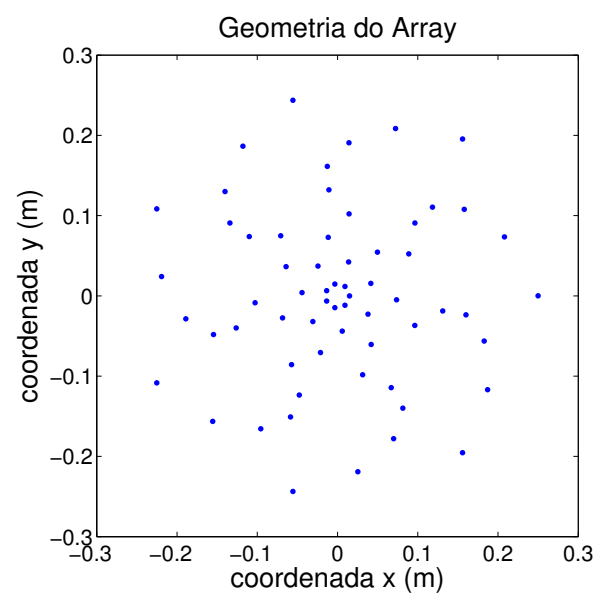

Figura 8.24: Geometria espiral logarítmica com 63 elementos, raio interno $r_{0}=1.5 \mathrm{~cm}$, raio externo $r_{\max }=25 \mathrm{~cm}, 9$ circunferências concêntricas e 7 braços, com cada braço realizando duas rotações completas.

é apresentado no Capítulo 9, a transformada proposta também pode ser estendida para modelar superfícies focais arbitrárias em campo próximo. As únicas transformadas rápidas que se aplicam a geometrias espirais são a NFFT e NNFFT, que exigem a hipótese de campo distante. 


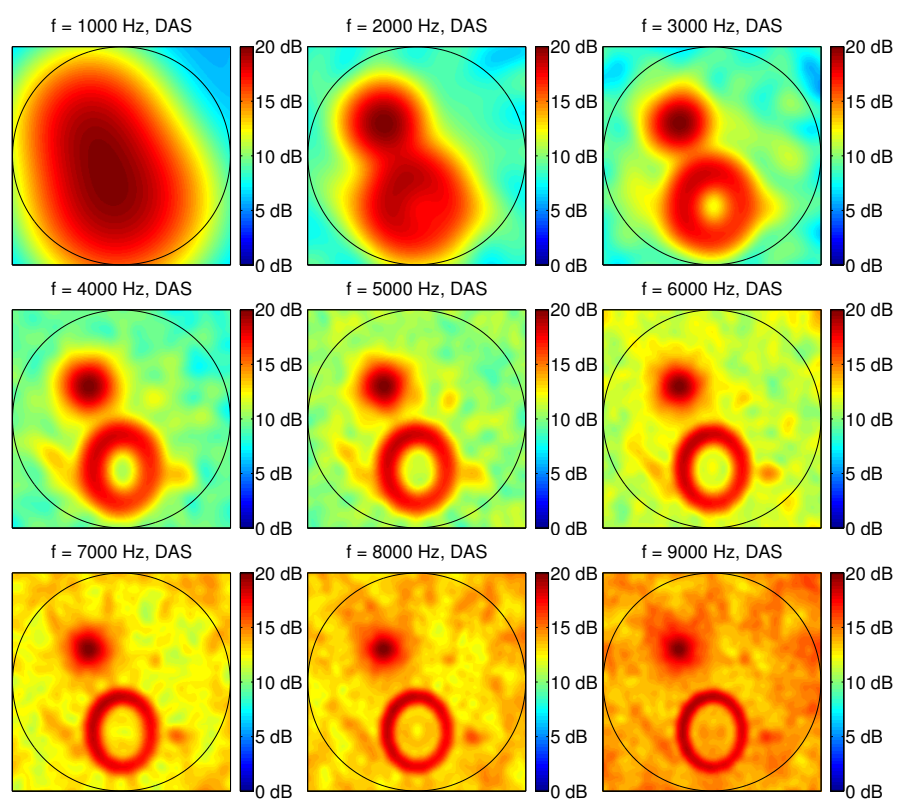

Figura 8.25: Reconstrução delay-and-sum, padrão não-esparso, geometria espiral

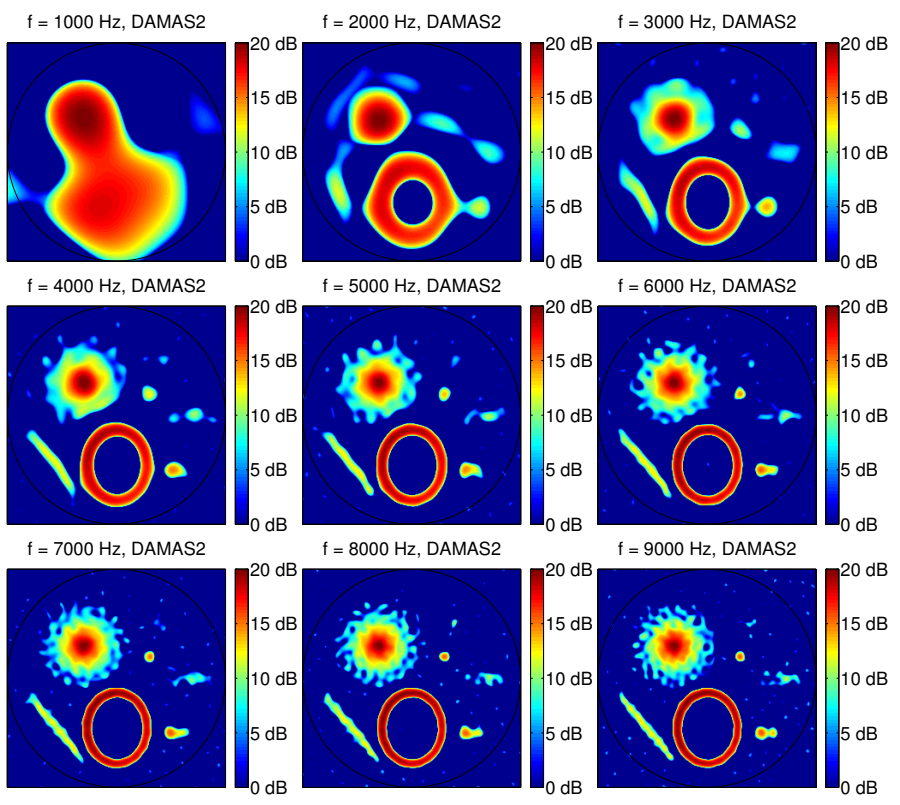

Figura 8.26: Reconstrução DAMAS2, padrão não-esparso, geometria espiral 


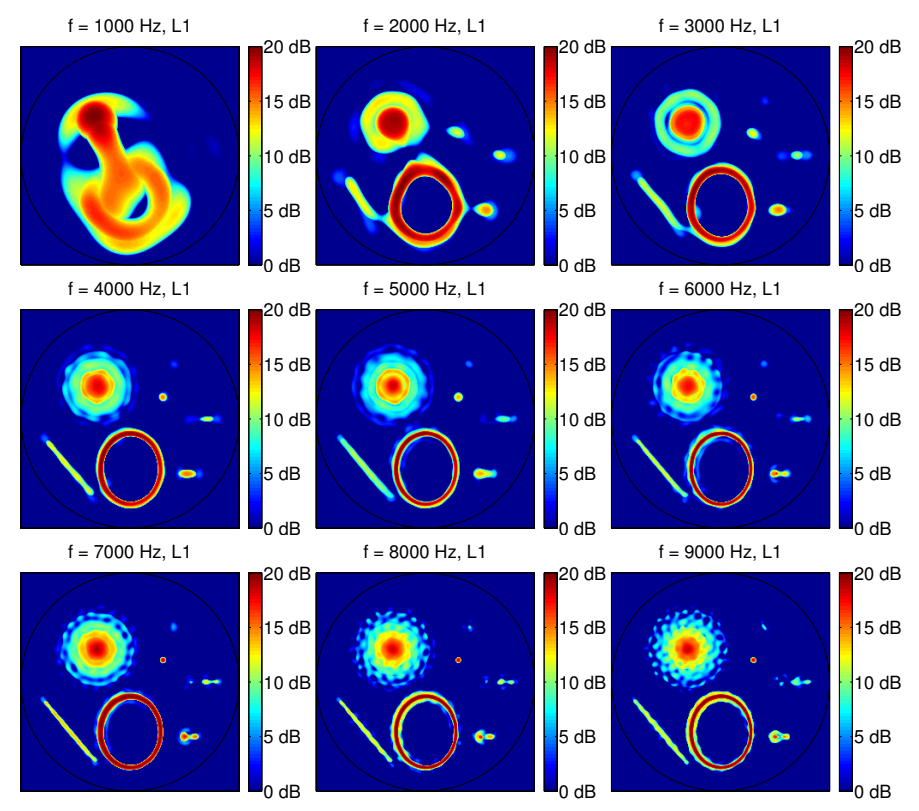

Figura 8.27: Reconstrução regularizada $\ell_{1}$, padrão não-esparso, geometria espiral

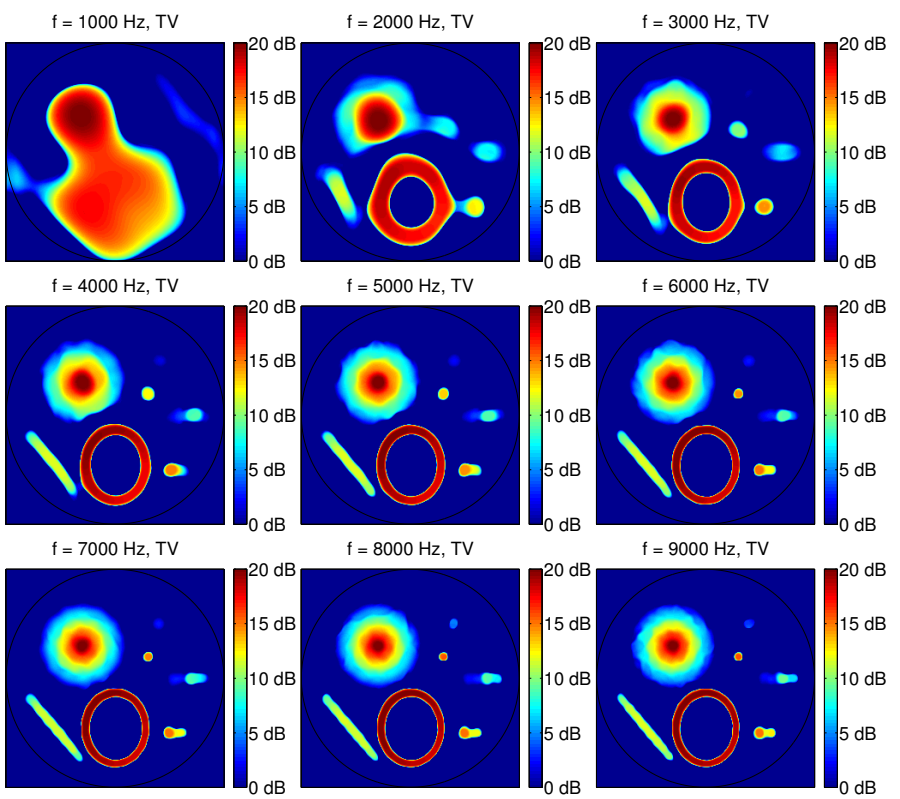

Figura 8.28: Reconstrução regularizada TV, padrão não-esparso, geometria espiral 


\section{Capítulo 9}

\section{Transformadas rápidas para campo próximo}

\subsection{Transformadas rápidas como aproximações de posto $\mathrm{K}$}

Até este ponto, trabalhamos sob a hipótese de que as fontes de interesse estão localizadas no campo distante do array. Portanto, usamos um modelo de ondas planas, com array manifold vector dado por

$$
\mathbf{v}\left(\mathbf{u}_{m}, \omega_{k}\right)=\left[\begin{array}{lll}
\mathrm{e}^{j \frac{\omega_{k}}{c}} \mathbf{u}_{m}^{T} \mathbf{p}_{0} & \cdots & \mathrm{e}^{j \frac{\omega_{k}}{c} \mathbf{u}_{m}^{T} \mathbf{p}_{N-1}}
\end{array}\right]^{T},
$$

admitindo parametrização no espaço U.

Neste capítulo mostramos como generalizar a transformada proposta e modelar frentes de onda esféricas, com array manifold vector

$$
\mathbf{v}\left(\mathbf{q}_{m}, \omega_{k}\right)=\left[\begin{array}{lll}
\frac{\mathrm{e}^{-j \frac{\omega_{k}}{c}\left\|\mathbf{p}_{0}-\mathbf{q}_{m}\right\|}}{\left\|\mathbf{p}_{0}-\mathbf{q}_{m}\right\|} & \cdots & \frac{\mathrm{e}^{-j \frac{\omega_{k}}{c}\left\|\mathbf{p}_{N-1}-\mathbf{q}_{m}\right\|}}{\left\|\mathbf{p}_{N-1}-\mathbf{q}_{m}\right\|}
\end{array}\right]^{T},
$$

onde $\mathbf{p}_{0}, \ldots, \mathbf{p}_{N-1} \in \mathbb{R}^{3}$ são as coordenadas dos $N$ microfones e $\mathbf{q}_{m} \in \mathbb{R}^{3}$ são as coordenadas de uma fonte de interesse.

Note que a transformada proposta não impõe nenhuma estrutura no array manifold vector além de sua separabilidade no sentido de Kronecker, que nos permite obter transformadas de campo distante com a forma

$$
\mathbf{A}=\mathbf{\Xi}\left(\mathbf{V}_{\mathbf{x}} \otimes \mathbf{V}_{\mathbf{y}}\right)
$$

onde $\Xi, \mathbf{V}_{\mathbf{x}}$ e $\mathbf{V}_{\mathbf{y}}$ dependem da geometria de interesse (uniforme ou Cartesiana).

$\mathrm{O}$ modelo de ondas planas e a parametrização em espaço $\mathrm{U}$ foram escolhidos por conveniência, por produzirem transformadas A com a estrutura acima. Porém, nada nos impede de escolher representações separáveis alternativas que sejam mais adequadas a casos de campo próximo. Neste capítulo, mostramos que o problema de encontrar representações separáveis ótimas (no sentido de mínimos quadrados) equivale a um problema de aproximação de posto 1 para uma versão rearranjada de $\boldsymbol{\Xi}^{-1} \mathbf{A}$. Através de uma aproximação de posto $K$ (para $K>1$ suficientemente grande), podemos obter modelos 
arbitrariamente precisos para propagação em campo próximo, mantendo baixos requisitos computacionais.

Adiantamos que a seguir, consideraremos apenas geometrias Cartesianas. Portanto, $\boldsymbol{\Xi}=\boldsymbol{\Xi}_{s}$ e $\boldsymbol{\Xi}$ é uma permutação. No desenvolvimento da transformada rápida para geometrias uniformes, admitimos que pares de sensores com linhas de base iguais produzem correlações iguais. Esta hipótese é falsa em campo próximo (ela implica, em particular, que a resposta de cada coluna de sensores é a mesma). Por um lado, esta hipótese efetivamente modela a redundância de arrays uniformes para fontes em campo distante, e reduz o custo computacional em uma ordem de grandeza. Porém, em campo próximo os arrays uniformes deixam de ser redundantes, e o modelo Cartesiano torna-se mais apropriado pelo fato de determinar as correlações para todos os pares de elementos.

Para simplificar a linguagem deste capítulo, usaremos a seguinte notação. Dadas matrizes $\mathbf{A}, \mathbf{B}, \mathbf{C}$, definimos a aplicação $\underline{\mathbf{A}}$ tal que $\mathbf{C}=\underline{\mathbf{A}}(\mathbf{B})$ se e somente se vec $\{\mathbf{C}\}=$ Avec $\{\mathbf{B}\}$.

Nosso objetivo é aproximar $\mathbf{A}$ usando $\breve{\mathbf{A}}=\mathbf{\Xi}\left(\sum_{k=1}^{K} \mathbf{C}_{k} \otimes \mathbf{D}_{k}\right)$, para pequenos valores de $K$. Note que $\mathbf{S}=\underline{\breve{A}}(\mathbf{Y})$ pode ser eficientemente implementado como $\mathbf{S}=$ $\underline{\Xi}\left(\sum_{k=1}^{K} \mathbf{D}_{k} \mathbf{Y} \mathbf{C}_{k}^{T}\right)$. Comparando esta expressão com (8.1.31), podemos concluir que estamos aproximando a transformada de campo próximo $\mathbf{A}$ através de uma série de $\mathrm{K}$ transformadas separáveis.

Consideremos o problema de aproximar uma matriz $\mathbf{B} \in \mathbb{C}^{m \times n}$ genérica, com $m=$ $m_{1} m_{2}$ e $n=n_{1} n_{2}$, usando uma soma de produtos de Kronecker, tal que

$$
\min _{\left\{\mathbf{C}_{k}\right\},\left\{\mathbf{D}_{k}\right\}}\left\|\mathbf{B}-\sum_{k=1}^{K} \mathbf{C}_{k} \otimes \mathbf{D}_{k}\right\|_{F},
$$

onde $\mathbf{C}_{k} \in \mathbb{C}^{m_{1} \times n_{1}}$ e $\mathbf{D}_{k} \in \mathbb{C}^{m_{2} \times n_{2}}$ para $1 \leq k \leq K$. Este problema é tratado em [86], onde os autores mostram ser equivalente a

$$
\min _{\left\{\mathbf{C}_{k}\right\},\left\{\mathbf{D}_{k}\right\}}\left\|\mathcal{R}(\mathbf{B})-\sum_{k=1}^{K} \operatorname{vec}\left\{\mathbf{C}_{k}\right\} \operatorname{vec}\left\{\mathbf{D}_{k}\right\}^{T}\right\|_{F},
$$

onde $\mathcal{R}(\cdot)$ é um operador que rearranja os elementos de uma matriz, tal que $\mathcal{R}(\mathbf{B}) \in$ $\mathbb{C}^{m_{1} n_{1} \times m_{2} n_{2}}$. Este problema de aproximação pode ser resolvido com a SVD de $\mathcal{R}(\mathbf{B})$.

Para nossos propósitos, aproximamos $\mathbf{B}=\boldsymbol{\Xi}^{-1} \mathbf{A}$. Destacamos que $\boldsymbol{\Xi}$ é a chave para uma decomposição precisa com pequeno posto. Como mostraremos a seguir, usar $\mathbf{B}=\mathbf{A}$ não é muito útil, pois $\mathcal{R}(\mathbf{A})$ possui muito valores singulares significativos.

Não é trivial computar os valores e vetores singulares dominantes de $\mathcal{R}(\mathbf{B})$, pois na prática $\mathcal{R}(\mathbf{B})$ é muito grande para ser armazenada explicitamente em memória. No entanto, a SVD pode ser computada com métodos de Lanczos [86,87], que somente exigem a implementação dos produtos matriz-vetor $\mathcal{R}(\mathbf{B}) \boldsymbol{\alpha}$ e $\mathcal{R}(\mathbf{B})^{H} \boldsymbol{\beta}$ para $\boldsymbol{\alpha}, \boldsymbol{\beta}$ arbitrários. 
Alternativamente, a SVD pode ser computada de forma aproximada, usando métodos que exigem poucas leituras sequenciais de $\mathcal{R}(\mathbf{B})$ (por exemplo, [88, 89]).

Usando a definição de $\mathcal{R}(\cdot)$ dada em [86], pode-se mostrar que

$$
\begin{aligned}
\mathcal{R}(\mathbf{B})^{T} & =\left[\begin{array}{ccc}
\mathbf{Z}_{0,0} & \cdots & \mathbf{Z}_{M_{x}-1,0} \\
\vdots & & \vdots \\
\mathbf{Z}_{0, M_{y}-1} & \cdots & \mathbf{Z}_{M_{x}-1, M_{y}-1}
\end{array}\right] \\
\mathbf{Z}_{m, n} & =\underline{\boldsymbol{\Xi}}\left(\mathbf{v}\left(u_{x_{m}}, u_{y_{n}}\right) \mathbf{v}^{H}\left(u_{x_{m}}, u_{y_{n}}\right)\right) .
\end{aligned}
$$

Como $\mathbf{v}\left(u_{x_{m}}, u_{y_{n}}\right)$ pode ser precomputada para $0<m<M_{x}$ e $0<n<M_{y}$ e $\boldsymbol{\Xi}$ é uma transformada muito rápida, $\mathcal{R}(\mathbf{B}) \boldsymbol{\alpha}$ e $\mathcal{R}(\mathbf{B})^{H} \boldsymbol{\beta}$ podem ser avaliados em tempo razoável. De fato, usando o método de Lanczos de [87], com $N=64$ e $M_{x}=M_{y}=256$ podemos resolver (9.1.1) para $K=8$ em 8 minutos em um processador Intel Core 2 Duo $2.4 \mathrm{GHz}$, usando um único core. Note que este procedimento precisa ser realizado somente uma vez.

A decomposição obtida com (9.1.1) é especialmente útil para $K>1$. De fato, mesmo na presença de fortes efeitos de campo próximo, $\mathcal{R}(\mathbf{B})$ pode ser bem aproximada por uma decomposição de posto pequeno. Ainda que o custo da transformada cresça de forma linear com $K$, devido à representação de Kronecker, o custo de cada aplicação de $\mathbf{C}_{k} \otimes \mathbf{D}_{k}$ é pequeno, tal que uma transformada com $K=8$ ainda é muito rápida.

Como discretizamos a superfície focal usando grids de amostragem Cartesianos, algumas imagens podem ter regiões inválidas. Por exemplo, podemos parametrizar uma casca semi-esférica com raio $r_{0}$ usando $u_{x}=r_{0} \sin \phi \cos \theta$ e $u_{y}=r_{0} \sin \phi \sin \theta$ para $\|\mathbf{u}\| \leq r_{0}$, tal que fontes com $\|\mathbf{u}\|>r_{0}$ sejam inválidas. Isto implica que alguns $\mathbf{Z}_{m, n}$ em $\mathcal{R}(\mathbf{B})$ não estão definidos. Referindo a (9.1.1), os $\mathbf{C}_{k}, \mathbf{D}_{k}$ agora são dados por

$$
\min _{\left\{\mathbf{C}_{k}\right\},\left\{\mathbf{D}_{k}\right\}}\left\|\mathbf{W} \odot\left(\mathcal{R}(\mathbf{B})-\sum_{k=1}^{K} \operatorname{vec}\left\{\mathbf{C}_{k}\right\} \operatorname{vec}\left\{\mathbf{D}_{k}\right\}^{T}\right)\right\|_{F},
$$

onde $\odot$ é o produto de Hadamard (ponto a ponto) e $\mathbf{W}$ é uma máscara binária definida em 1 para elementos válidos de $\mathcal{R}(\mathbf{B})$ e 0 para elementos inválidos. Esta SVD mascarada foi considerada em [90], e pode ser obtida iterando

$$
\mathbf{E}^{(i)}=\operatorname{LRA}_{\mathrm{K}}\left(\mathbf{W} \odot \mathcal{R}(\mathbf{B})+(\mathbb{1}-\mathbf{W}) \odot \mathbf{E}^{(i-1)}\right),
$$

onde $\operatorname{LRA}_{K}(\cdot)$ é uma aproximação de posto $K$ conforme computada pela SVD, $\mathbf{E}^{(i)}=$ $\sum_{k=1}^{K} \operatorname{vec}\left\{\mathbf{C}_{k}^{(i)}\right\} \operatorname{vec}\left\{\mathbf{D}_{k}^{(i)}\right\}^{T}$ e $\mathbf{E}^{(0)}=\mathbf{0}$. Em nossos experimentos, uma ou duas iterações mostraram-se suficientes para um bom ajuste.

Note que ao usar uma aproximação de posto $K$, obtemos uma transformada cujo custo computacional é $K$ vezes maior que o das transformadas apresentadas no capítulo anterior. 
Não obstante, como mostraremos a seguir, $K$ é pequeno o suficiente tal que esta penalidade não é significativa. De fato, mostraremos que é possível compensar fortes efeitos de campo próximo com $K=8$, o que torna a transformada proposta aproximadamente tão rápida quanto a NFFT, e a confere a capacidade única de modelar superfícies focais em arbitrárias em campo próximo.

\subsection{Calibração e foco}

Em aplicações práticas, a geometria do array pode desviar levemente de um grid Cartesiano ideal. Além disso, microfones são raramente casados, e requerem calibração. Enquanto estas características podem ser incorporadas na transformada para K suficientemente grande, é computacionalmente mais eficiente compensar desvios de separabilidade através de uma matriz de interpolação separada.

Por exemplo, consideremos o caso simples em que a calibração dos microfones requer apenas uma constante de ganho por microfone. Para ganhos arbitrários, a transformada A deixaria de ser separável (no sentido de Kronecker), mesmo para campo distante. No entanto, uma matriz de calibração (diagonal) pode ser usada para corrigir os manifold vectors, de forma a modelar os ganhos e permitir a implementação rápida de $\mathbf{A}$.

Para os propósitos de imagens acústicas em campo próximo, a matriz de calibração também pode ser projetada para alterar a superfície focal sem recomputar as matrizes $\left\{\mathbf{C}_{k}, \mathbf{D}_{k}\right\}$. Este método é conveniente para aplicações em tempo real, pois matrizes de interpolação/calibração podem ser computadas em alguns segundos, enquanto a obtenção de $\left\{\mathbf{C}_{k}, \mathbf{D}_{k}\right\}$ através de uma SVD requer tempos da ordem de minutos.

Sejam $\mathbf{v}_{\mathbf{u}}$ e $\breve{\mathbf{v}}_{\mathbf{u}}$ os manifold vectors ideais e desejados, respectivamente. Suponhamos que $\mathbf{v}_{\mathbf{u}}$ seja modelado através da transformada rápida, enquanto $\breve{\mathbf{v}}_{\mathbf{u}}$ potencialmente incorpore dados de calibração e ajustes à superfície focal. Nossa proposta envolve projetar uma matriz de interpolação $\mathbf{T}$ tal que $\mathbf{T}[\underline{\underline{A}}(\mathbf{Y})] \mathbf{T}^{H}$ se torna a transformada rápida.

Métodos prévios para interpolação de arrays [91,92] projetam $\mathbf{T}$ tal que $\mathbf{T v}(\varphi(\mathbf{u})) \approx$ $\breve{\mathbf{v}}(\varphi(\mathbf{u}))$, para $\mathbf{u}$ na região de interesse. Definindo

$$
\mathbf{V}=\left[\begin{array}{llll}
\mathbf{v}_{\mathbf{u}_{1}} & \mathbf{v}_{\mathbf{u}_{2}} & \cdots & \mathbf{v}_{\mathbf{u}_{M}}
\end{array}\right], \quad \quad \breve{\mathbf{V}}=\left[\begin{array}{llll}
\breve{\mathbf{v}}_{\mathbf{u}_{1}} & \breve{\mathbf{v}}_{\mathbf{u}_{2}} & \cdots & \breve{\mathbf{v}}_{\mathbf{u}_{M}}
\end{array}\right],
$$

uma matriz de interpolação tradicional é obtida resolvendo

$$
\underset{\mathbf{T}}{\operatorname{argmin}}\|\mathbf{T V}-\breve{\mathbf{V}}\|_{F}=\breve{\mathbf{V}} \mathbf{V}^{+}
$$

onde $\|\cdot\|_{F}$ é a norma de Frobenius e $\mathbf{V}^{+}$é a pseudoinversa de Moore-Penrose de $\mathbf{V}$.

Como nossa proposta de reconstrução utiliza a matriz espectral $\mathbf{S}$ e não o vetor de saída do array $\mathbf{x}$, a matriz $\mathbf{T}$ ótima tem mais graus de liberdade, e pode ser melhorada em relação aos métodos clássicos. De fato, segue de (7.3.5) que $\mathbf{S}=\underline{\breve{A}}(\mathbf{Y})$ é uma soma de produtos externos da forma $\mathbf{v}_{\mathbf{u}} \mathbf{v}_{\mathbf{u}}^{H}$. Como $\mathbf{v}_{\mathbf{u}} \mathbf{v}_{\mathbf{u}}^{H}=\left(\alpha \mathbf{v}_{\mathbf{u}}\right)\left(\alpha \mathbf{v}_{\mathbf{u}}\right)^{H}$ para qualquer $\alpha \in \mathbb{C}$ com 
$|\alpha|=1$, é suficiente impor que $\mathbf{T v}_{\mathbf{u}} \approx \alpha \breve{\mathbf{v}}_{\mathbf{u}}$ para $\alpha \in \mathbb{C}$ convenientemente escolhido, sob a restrição de que $|\alpha|=1$. Portanto, uma $\mathbf{T}$ melhor ajustada pode ser obtida resolvendo

$$
\min _{\mathbf{T}, \mathbf{U}}\|\mathbf{T V}-\breve{\mathbf{V}} \mathbf{U}\|_{F}=\min _{\mathbf{U}}\left\|\breve{\mathbf{V}} \mathbf{U} \mathbf{V}^{+} \mathbf{V}-\breve{\mathbf{V}} \mathbf{U}\right\|_{F}
$$

sob a hipótese de que U seja diagonal unitária.

Seja diag $\{\mathbf{U}\}=\boldsymbol{\alpha}$. Minimizamos (9.2.1) resolvendo para $\mathbf{U}$ e $\mathbf{T}$ alternadamente, com

$$
\begin{aligned}
\mathbf{T}(0) & =\mathbf{I} \\
\alpha_{i}(n) & =\underset{|\alpha|=1}{\operatorname{argmin}}\left\|\mathbf{T}(n) \mathbf{v}_{\mathbf{u}_{i}}-\alpha \breve{\mathbf{v}}_{\mathbf{u}_{i}}\right\|_{F} \\
& =\breve{\mathbf{v}}_{\mathbf{u}_{i}}^{H} \mathbf{T}(n) \mathbf{v}_{\mathbf{u}_{i}} /\left|\breve{\mathbf{v}}_{\mathbf{u}_{i}}^{H} \mathbf{T}(n) \mathbf{v}_{\mathbf{u}_{i}}\right| \\
\mathbf{T}(n+1) & =\breve{\mathbf{V}} \mathbf{U}(n) \mathbf{V}^{+} .
\end{aligned}
$$

Note que (9.2.1) não é convexa sob a restrição de que U seja diagonal unitária. Portanto, (9.2.1) pode ter múltiplos mínimos locais. Não obstante, em nossas simulações este método convergiu para o mínimo global ou para valores muito próximos dele.

Para aumentar a probabilidade de convergência para o mínimo global, podemos escolher um $\mathbf{U}(0)$ conveniente, com um maior custo computacional. Supondo que $\mathbf{U}$ seja diagonal unitária,

$$
\begin{aligned}
\| \breve{\mathbf{V}} \mathbf{U V}^{+} \mathbf{V} & -\breve{\mathbf{V}} \mathbf{U}\left\|_{F}^{2}=\right\| \breve{\mathbf{V}} \mathbf{U}\left(\mathbf{V}^{+} \mathbf{V}-\mathbf{I}\right) \|_{F}^{2} \\
& =\left\|\left[\left(\mathbf{V}^{+} \mathbf{V}-\mathbf{I}\right)^{T} \otimes \breve{\mathbf{V}}\right] \operatorname{vec}\{\mathbf{U}\}\right\|_{2}^{2} \\
& =\boldsymbol{\alpha}^{H}\left[\left(\mathbf{V}^{+} \mathbf{V}-\mathbf{I}\right)^{*}\left(\mathbf{V}^{+} \mathbf{V}-\mathbf{I}\right)^{T} \odot \breve{\mathbf{V}} \breve{\mathbf{V}}^{H}\right] \boldsymbol{\alpha}
\end{aligned}
$$

onde $\odot$ é o produto de Hadamard. Um $\boldsymbol{\alpha}(0)$ quase ótimo é o autovetor associado ao menor autovalor de $\left(\mathbf{V}^{+} \mathbf{V}-\mathbf{I}\right)^{*}\left(\mathbf{V}^{+} \mathbf{V}-\mathbf{I}\right)^{T} \odot \breve{\mathbf{V}} \breve{V}^{H}$, normalizado tal que cada uma de suas coordenadas esteja sobre a circunferência unitária.

Em nossos experimentos numéricos, este $\mathbf{U}(0)$ sempre esteve muito próximo do ponto fixo da iteração acima. Além disso, o mínimo de (9.2.1) assim encontrado nunca excedeu os mínimos (ocasionalmente locais) obtidos com outras escolhas de valores iniciais. Estas observações sugerem fortemente que o autovetor associado ao menor autovalor de $\left(\mathbf{V}^{+} \mathbf{V}-\mathbf{I}\right)^{*}\left(\mathbf{V}^{+} \mathbf{V}-\mathbf{I}\right)^{T} \odot \breve{\mathbf{V}} \breve{\mathbf{V}}^{H}$ encontra-se com alta probabilidade na vizinhança do mínimo global.

\subsection{Exemplos}

Nesta seção, apresentamos resultados de simulação para uma superfície focal retangular, mostrando como a precisão da reconstrução varia com K. Para isto, simulamos um array com a mesma geometria Cartesiana usada no capítulo anterior. A distri- 
buição de fontes é simulada sobre um retângulo paralelo ao array, localizado a $0.5 \mathrm{~m}$ de distância e com dimensões $0.5 \mathrm{~m} \times 0.5 \mathrm{~m}$. Este retângulo é parametrizado usando $\varphi\left(u_{x}, u_{y}\right)=\left[\begin{array}{lll}u_{x} & u_{y} & 0.5\end{array}\right]^{T}, \operatorname{com}\left(u_{x}, u_{y}\right) \in[-1,1]^{2}$.

As Figuras 9.1-9.3 mostram exemplos de reconstrução utilizando uma aproximação de campo distante, as transformadas propostas para $K=1,4,8,16$ e a transformada exata. Como a transformada para campo distante parametriza um hemisfério inteiro, o retângulo de $0.5 \mathrm{~m} \times 0.5 \mathrm{~m}$ ocupa apenas uma parte da região central da imagem. Para a parametrização de campo distante, o horizonte é representado por uma circunferência branca. Como a distribuição de fontes está muito próxima do array, a hipótese de campo distante produz uma reconstrução borrada, motivando o uso de nossa proposta.

Nossa referência é a transformada exata (e lenta) para campo próximo. Como a resolução do array diminui monotonicamente em direção ao horizonte, todas as reconstruções aparecem borradas em direção às bordas. Este não é um artefato da transformada rápida, e pode ser observado também com a transformada exata. Note que A é mais fácil de aproximar para frequências baixas.

A Figura 9.4 compara os primeiros 100 valores singulares (de um total de 16384) para $\mathcal{R}(\mathbf{A})$ e $\mathcal{R}\left(\boldsymbol{\Xi}^{T} \mathbf{A}\right)$. Consideramos $\mathbf{A}$ modelando a superfície focal retangular definida previamente, para frequências de $1 \mathrm{kHz}$ a $9 \mathrm{kHz}$. O decaimento acentuado da curva para $\mathcal{R}\left(\boldsymbol{\Xi}^{T} \mathbf{A}\right)$ destaca a importância de $\boldsymbol{\Xi}$ em permitir aproximações de posto reduzido. Para frequências baixas, os valores singulares mostram um decaimento ainda mais acentuado. Observamos que superfícies focais com campo de visada maior produzem um maior número de valores singulares significativos, e portanto exigem valores maiores de $\mathrm{K}$.

A Figura 9.5 mostra como usar erros de reconstrução para estimar distâncias focais. De fato, obtém-se o melhor ajuste quando a transformada modela a superfície focal real, com a distância focal correta. As linhas pontilhadas mostram que na ausência de uma transformada ótima projetada para a superfície focal real (neste caso, um retângulo a $0.5 \mathrm{~m}$ do array), é possível corrigi-la usando uma matriz de interpolação.

Tempos de reconstrução para $K=1,2,4,8$ e 16 são aproximadamente 4, 5, 6, 9 e 15 segundos por imagem, com implementações em MATLAB em um processador Intel Core 2 Duo T9400 em 64-bits, utilizando apenas um core (tempos de reconstrução exatos variam tipicamente \pm .5 segundo por imagem, dependendo da distribuição de fontes utilizada). Em contraste, os tempos de reconstrução são da ordem de 2000 segundos por imagem se for usada uma representação matricial explícita. 

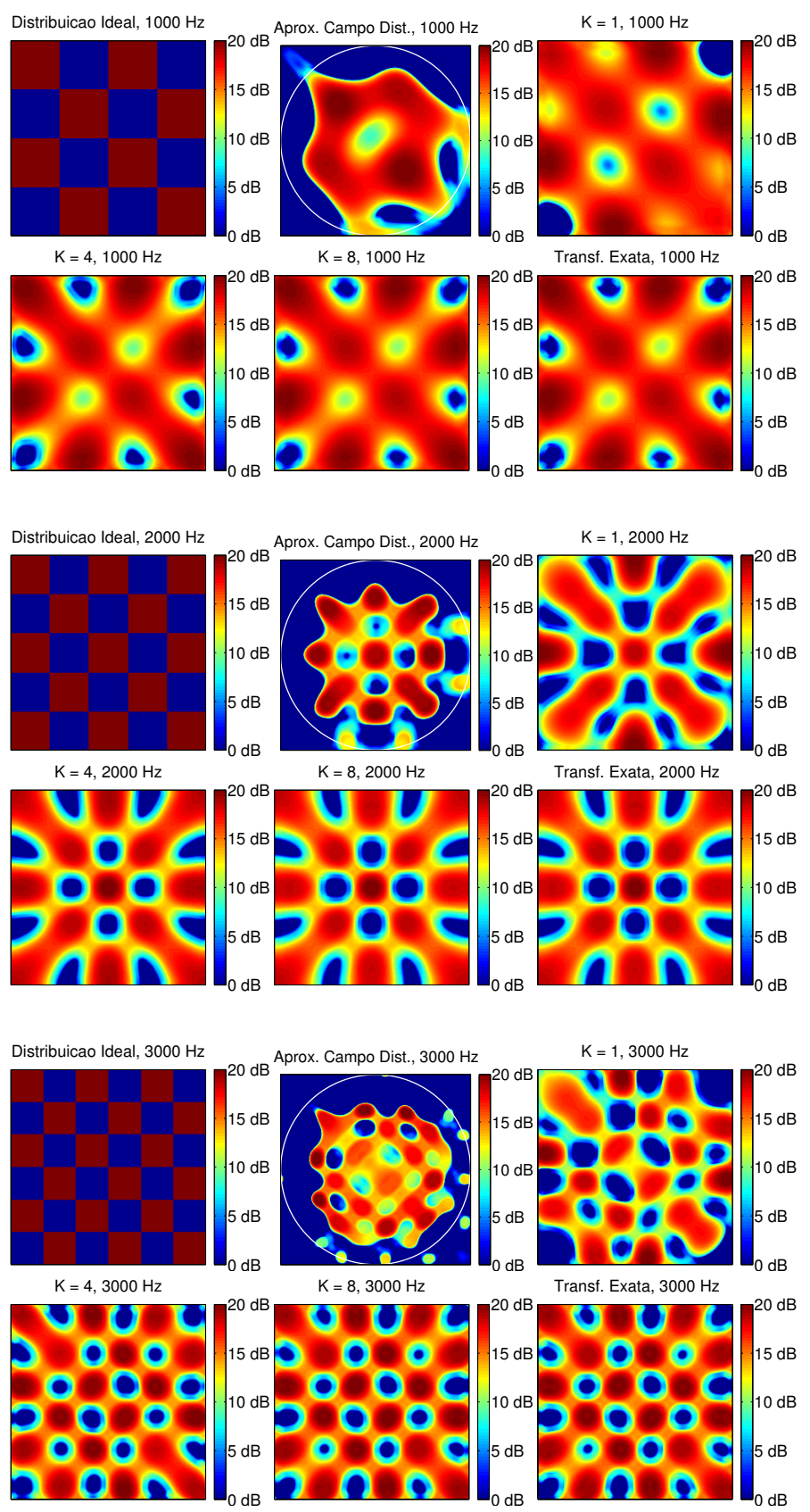

Figura 9.1: Reconstrução regularizada TV para uma aproximação de campo distante, para a decomposição de Kronecker ótima (figuras com K) e para a transformada exata (lenta). Fontes estão posicionadas sobre um retângulo de $0.5 \mathrm{~m} \times 0.5 \mathrm{~m}$ paralelo ao array, localizado a uma distância de $0.5 \mathrm{~m}$. Imagens para $1 \mathrm{kHz}-3 \mathrm{kHz}$. 

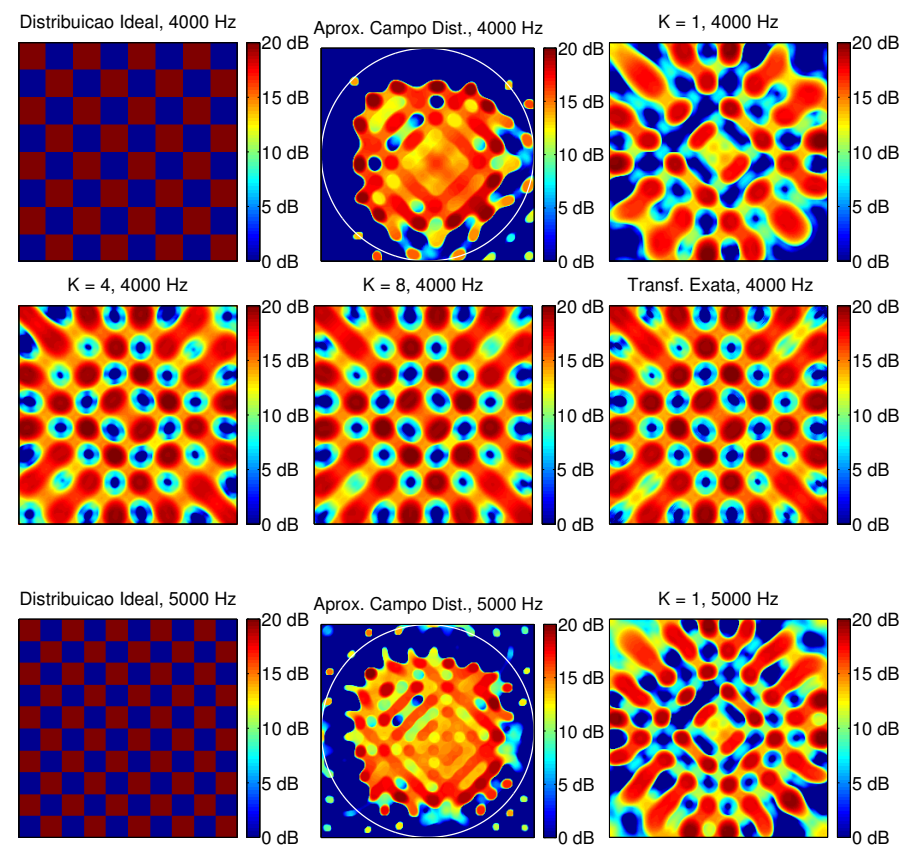

$\mathrm{K}=1,5000 \mathrm{~Hz}$
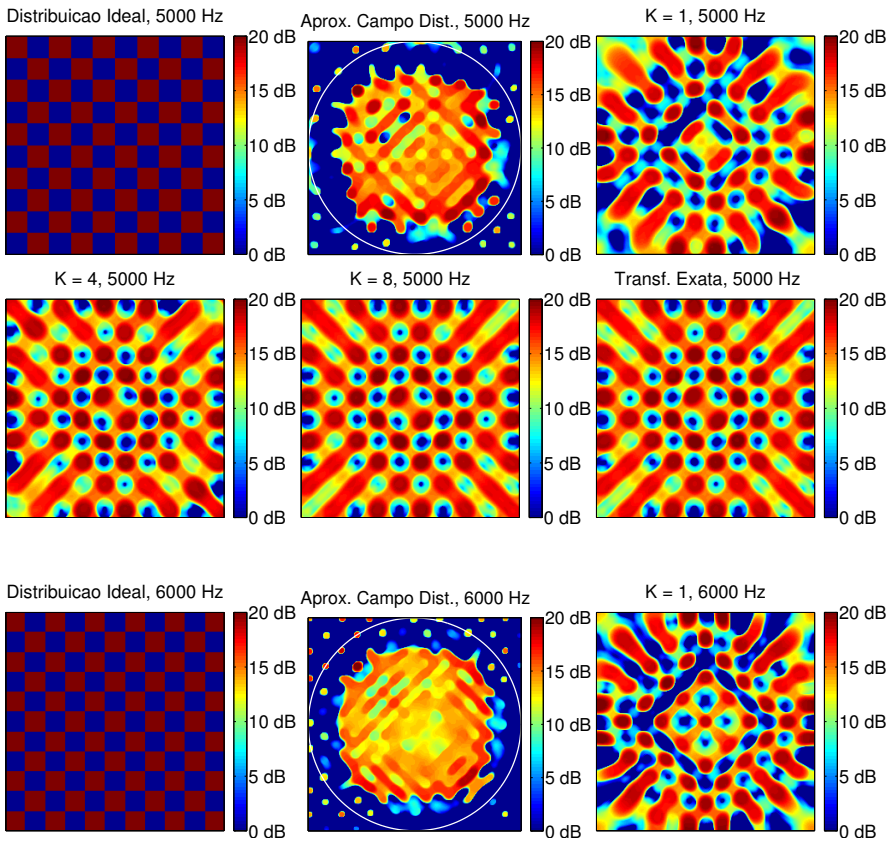

$\mathrm{K}=4,6000 \mathrm{~Hz}$

$\mathrm{K}=8,6000 \mathrm{~Hz}$

Transf. Exata, $6000 \mathrm{~Hz}$

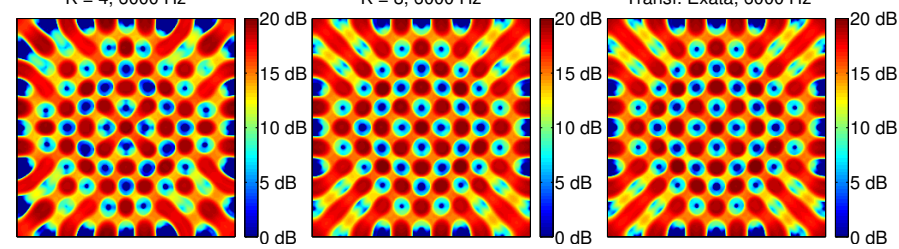

Figura 9.2: Reconstrução regularizada TV para uma aproximação de campo distante, para a decomposição de Kronecker ótima (figuras com K) e para a transformada exata (lenta). Fontes estão posicionadas sobre um retângulo de $0.5 \mathrm{~m} \times 0.5 \mathrm{~m}$ paralelo ao array, localizado a uma distância de $0.5 \mathrm{~m}$. Imagens para $4 \mathrm{kHz}-6 \mathrm{kHz}$. 

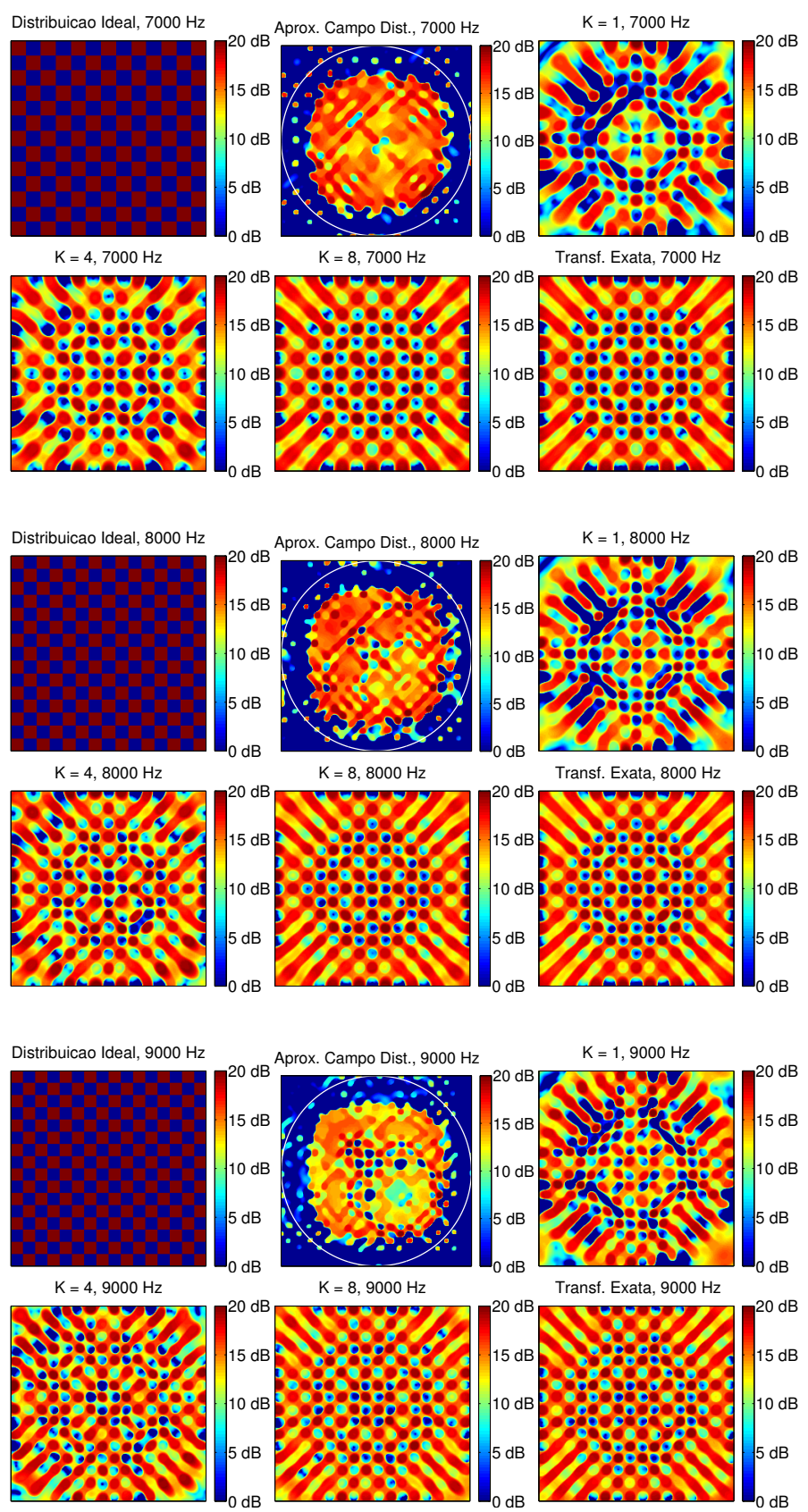

Figura 9.3: Reconstrução regularizada TV para uma aproximação de campo distante, para a decomposição de Kronecker ótima (figuras com K) e para a transformada exata (lenta). Fontes estão posicionadas sobre um retângulo de $0.5 \mathrm{~m} \times 0.5 \mathrm{~m}$ paralelo ao array, localizado a uma distância de $0.5 \mathrm{~m}$. Imagens para $7 \mathrm{kHz}-9 \mathrm{kHz}$. 
$f=1000 \mathrm{~Hz}$
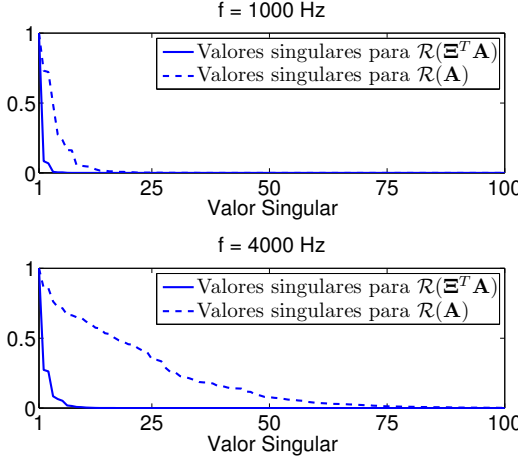

$f=7000 \mathrm{~Hz}$
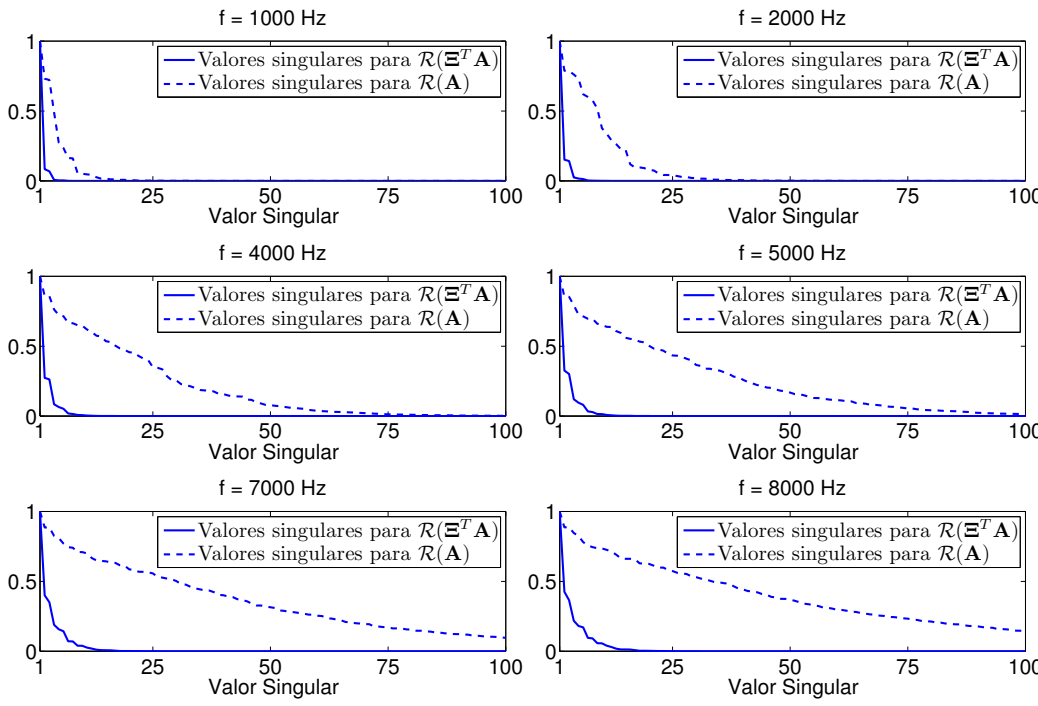

$\mathrm{f}=5000 \mathrm{~Hz}$

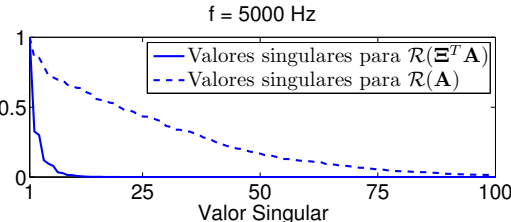

$\mathrm{f}=8000 \mathrm{~Hz}$

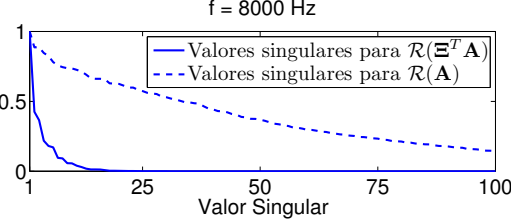

$f=3000 \mathrm{~Hz}$

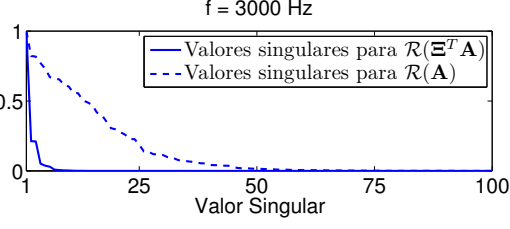

$f=6000 \mathrm{~Hz}$

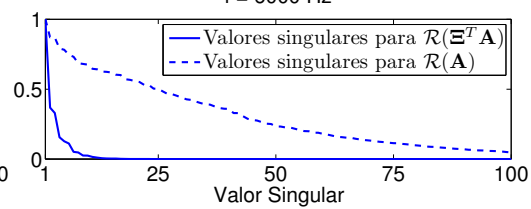

$\mathrm{f}=9000 \mathrm{~Hz}$

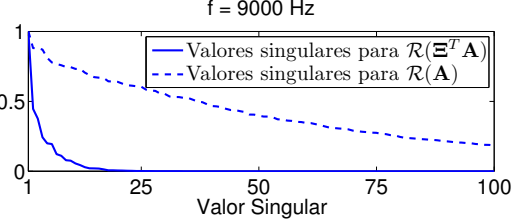

Figura 9.4: Primeiros 100 valores singulares de $\mathcal{R}(\mathbf{A})$ e $\mathcal{R}\left(\boldsymbol{\Xi}^{T} \mathbf{A}\right)$ (normalizados)
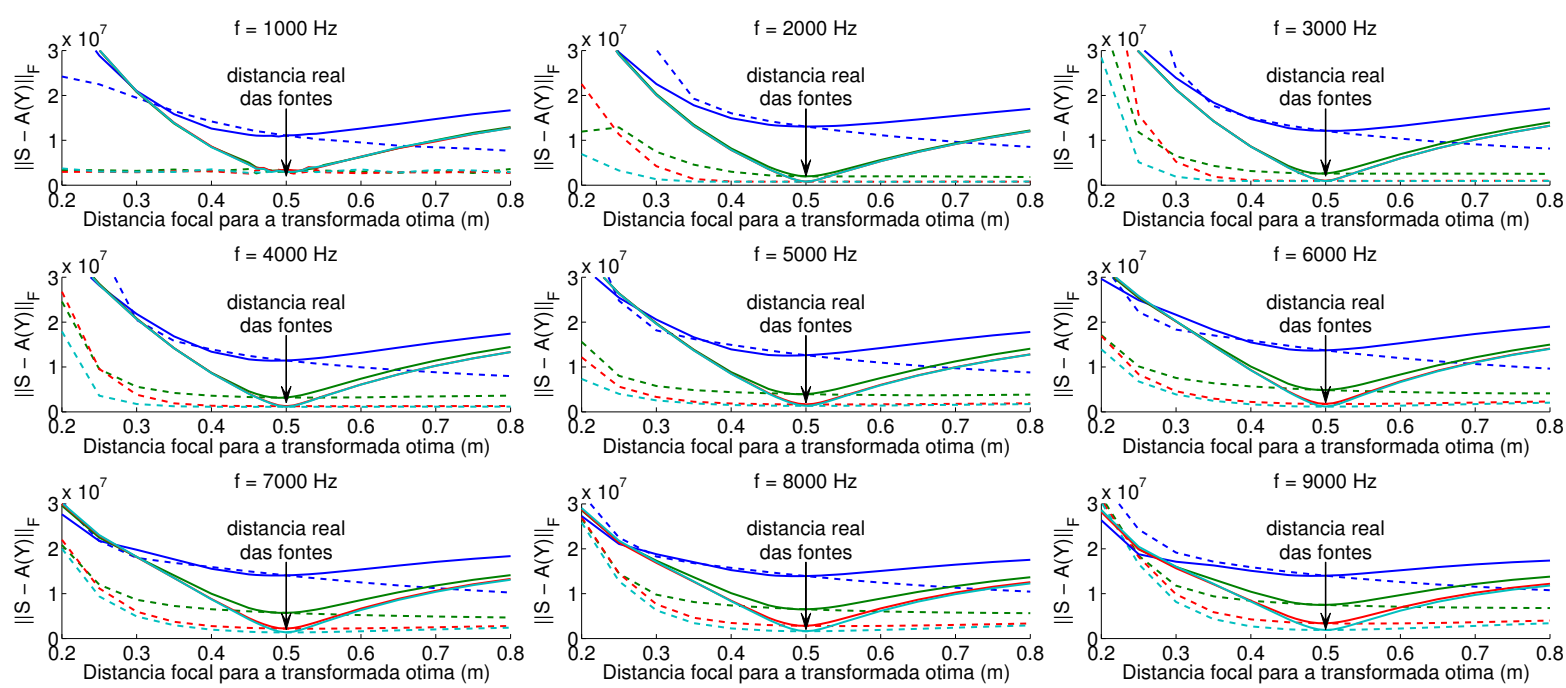

Figura 9.5: Erros de reconstrução em função de $K .-$ : $K=1$, sem foco; $--: K=1$, com $\mathbf{T} ;-: K=4$, sem foco; $--: K=4$, com $\mathbf{T} ;-: K=8$, sem foco; $--: K=8$, com $\mathbf{T} ;-: K=16$, sem foco; $--: K=16$, com $\mathbf{T}$. 


\section{Capítulo 10}

\section{Transformadas rápidas para imagens correlaci- onadas}

Até este ponto supomos que todas as fontes pontuais no mapa reconstruído fossem descorrelacionadas. Nesta seção abandonaremos esta hipótese. Portanto, dada uma distribuição com $M=M_{x} M_{y}$ fontes pontuais, teremos até $\frac{1}{2} M(M+1)$ correlações únicas. Mesmo para valores moderados de $M$, estas correlações serão muito numerosas para serem armazenadas, e em geral, tampouco seremos capazes de estimá-las com um array dotado de centenas de elementos.

No entanto, se a distribuição de fontes for esparsa, então torna-se possível armazenar e estimar somente as correlações entre as fontes ativas. Em uma aplicação prática, podese reconstruir um mapa aproximado supondo que a distribuição seja descorrelacionada, utilizando um dos métodos descritos anteriormente. Usando este mapa, as fontes dominantes podem ser selecionadas, e o problema pode ser resolvido novamente considerando os termos cruzados somente para estas fontes dominantes. Como os termos cruzados dependem do quadrado do número de fontes, este ainda não é um problema simples, mas é viável para distribuições simples e esparsas. Além disso, outras hipóteses a respeito da distribuição poderiam ser usadas para regularizá-la. Por exemplo, poder-se-ia considerar a variação total dos mapas de correlação.

Considere uma imagem acústica $M_{x} \times M_{y}$, defina $M=M_{x} M_{y}$ e seja $\mathbf{u}_{0}, \ldots, \mathbf{u}_{M-1}$ uma enumeração de todas as coordenadas de pixels no espaço U. Seja $\mathbf{v}\left(\mathbf{u}_{m}\right)$ o array manifold vector orientado na direção $\mathbf{u}_{m}$. O modelo do sinal e da matriz espectral são dados por (7.3.1) e (7.3.4), que repetimos abaixo por conveniência:

$$
\begin{aligned}
& \hat{\mathbf{x}}\left(\omega_{k}\right)=\mathbf{V}\left(\omega_{k}\right) \hat{\mathbf{f}}\left(\omega_{i}\right)+\hat{\boldsymbol{\eta}}\left(\omega_{k}\right) . \\
& \mathbf{S}\left(\omega_{k}\right)=\mathbf{V}\left(\omega_{k}\right) \mathrm{E}\left\{\hat{\mathbf{f}}\left(\omega_{k}\right) \hat{\mathbf{f}}^{H}\left(\omega_{k}\right)\right\} \mathbf{V}^{H}\left(\omega_{k}\right)+\sigma^{2} \mathbf{I} .
\end{aligned}
$$

Neste caso, não supomos que $E\left\{\hat{\mathbf{f}}\left(\omega_{k}\right) \hat{\mathbf{f}}^{H}\left(\omega_{k}\right)\right\}$ seja diagonal. Para obter $\mathbf{S}\left(\omega_{k}\right)$ a partir de $\mathrm{E}\left\{\hat{\mathbf{f}}\left(\omega_{k}\right) \hat{\mathbf{f}}^{H}\left(\omega_{k}\right)\right\}$ (i.e., implementar a transformada direta), devemos avaliar (10.0.2) $\operatorname{com} \sigma^{2}=0$. Este produto matricial requer $N M^{2}+N^{2} M$ MACs complexos. Para um array 
com $N=256$ elementos e uma imagem com $M=256^{2}$ pixels, temos aproximadamente $10^{12}$ MACs complexos, o que claramente não é prático.

Infelizmente, admitir a separabilidade não produz resultados tão dramáticos como antes. Além disso, admitir a uniformidade da geometria ou da amostragem em U não produz otimizações adicionais. Como qualquer implementação da transformada correlacionada exige o processamento de $M^{2}$ pontos, ela sempre terá custo computacional alto. Portanto, na prática somos obrigados a manter $M$ pequeno.

\subsection{Transformada rápida direta}

Para manter a consistência com a notação anterior, seja

$$
\mathbf{Y}=\mathrm{E}\left\{\hat{\mathbf{f}}\left(\omega_{k}\right) \hat{\mathbf{f}}^{H}\left(\omega_{k}\right)\right\}
$$

Escrevamos (10.0.2) como uma transformada linear A, tal que vec $\{\mathbf{S}\}=\operatorname{Avec}\{\mathbf{Y}\}$.

Por definição,

$$
\begin{aligned}
\operatorname{vec}\{\mathbf{S}\} & =\mathbf{A} \operatorname{vec}\{\mathbf{Y}\} \\
& =\left[\begin{array}{llll}
\operatorname{vec}\left\{\mathbf{v}_{\mathbf{u}_{0}} \mathbf{v}_{\mathbf{u}_{0}}^{H}\right\} & \operatorname{vec}\left\{\mathbf{v}_{\mathbf{u}_{1}} \mathbf{v}_{\mathbf{u}_{0}}^{H}\right\} & \cdots & \operatorname{vec}\left\{\mathbf{v}_{\mathbf{u}_{M-1}} \mathbf{v}_{\mathbf{u}_{M-1}}^{H}\right\}
\end{array}\right] \operatorname{vec}\{\mathbf{Y}\} \\
& =\left[\begin{array}{lllll}
\mathbf{v}_{\mathbf{u}_{0}}^{*} \otimes \mathbf{v}_{\mathbf{u}_{0}} & \mathbf{v}_{\mathbf{u}_{0}}^{*} \otimes \mathbf{v}_{\mathbf{u}_{1}} & \cdots & \mathbf{v}_{\mathbf{u}_{M-1}}^{*} \otimes \mathbf{v}_{\mathbf{u}_{M-1}}
\end{array}\right] \operatorname{vec}\{\mathbf{Y}\},
\end{aligned}
$$

onde a última igualdade é válida porque

$$
\begin{aligned}
\operatorname{vec}\left\{\mathbf{v}_{\mathbf{u}_{m}} \mathbf{v}_{\mathbf{u}_{n}}^{H}\right\} & =\left[\begin{array}{c}
\mathbf{v}_{\mathbf{u}_{m}} \mathbf{v}_{\mathbf{u}_{n}}^{0 *} \\
\mathbf{v}_{\mathbf{u}_{m}} \mathbf{v}_{\mathbf{u}_{n}}^{1 *} \\
\vdots \\
\mathbf{v}_{\mathbf{u}_{m}} \mathbf{v}_{\mathbf{u}_{n}}^{(N-1) *}
\end{array}\right] \\
& =\mathbf{v}_{\mathbf{u}_{n}}^{*} \otimes \mathbf{v}_{\mathbf{u}_{m}} .
\end{aligned}
$$

Para $0 \leq m, n<N_{x} N_{y}$, podemos escrever a linha $m \cdot N_{x} N_{y}+n$ de $\mathbf{A}$ como

$$
\left[\begin{array}{ccc}
\mathrm{v}_{u_{0}}^{m *} & \cdots & \mathrm{v}_{u_{M-1}}^{m *}
\end{array}\right] \otimes\left[\begin{array}{ccc}
\mathrm{v}_{u_{0}}^{n} & \cdots & \mathrm{v}_{u_{M-1}}^{n}
\end{array}\right] .
$$

Para $0 \leq m, n<N_{x} N_{y}$, um elemento arbitrário $\mathbf{S}_{n, m}$ de $\mathbf{S}$ pode ser escrito como o produto da linha $m \cdot N_{x} N_{y}+n$ de $\mathbf{A}$ e vec $\{\mathbf{Y}\}$, tal que

$$
\begin{aligned}
\mathbf{S}_{n, m} & =\left\{\left[\begin{array}{lll}
\mathrm{v}_{u_{0}}^{m *} & \cdots & \mathrm{v}_{u_{M-1}}^{m *}
\end{array}\right] \otimes\left[\begin{array}{ccc}
\mathrm{v}_{u_{0}}^{n} & \cdots & \mathrm{v}_{u_{M-1}}^{n}
\end{array}\right]\right\} \operatorname{vec}\{\mathbf{Y}\} \\
& =\left[\begin{array}{lll}
\mathrm{v}_{u_{0}}^{n} & \cdots & \mathrm{v}_{u_{M-1}}^{n}
\end{array}\right] \mathbf{Y}\left[\begin{array}{c}
\mathrm{v}_{u_{0}}^{m *} \\
\vdots \\
\mathrm{v}_{u_{M-1}}^{m *}
\end{array}\right],
\end{aligned}
$$


onde (10.1.8) e (10.1.9) são equivalentes porque $\left(\mathbf{A}^{T} \otimes \mathbf{B}\right)$ vec $\{\mathbf{C}\}=\operatorname{vec}\{\mathbf{B C A}\}$ sempre que BCA está definido [77]. Por definição, (10.1.9) é equivalente a $\mathbf{S}=\mathbf{V} \mathbf{Y V}^{H}$, que é dado por (10.0.2). Portanto, $\mathbf{S}=\mathbf{V Y} \mathbf{V}^{H}$ implementa $\mathbf{A}$ com a ordem de pixels induzida por vec $\{\cdot\}$.

Suponha que o array tenha geometria separável. Novamente, podemos escrever $\mathbf{v}(\mathbf{u})=$ $\mathbf{v}\left(u_{x}, u_{y}\right)=\mathbf{v}_{x}\left(u_{x}\right) \otimes \mathbf{v}_{y}\left(u_{y}\right)$, onde $\mathbf{v}_{x}$ e $\mathbf{v}_{y}$ têm manifold vectors com dimensão $N_{x} \times 1$ e $N_{y} \times 1$, elementos virtuais com a mesma separação do array principal, e estão localizados simetricamente em relação à origem.

Defina

$$
\begin{aligned}
& \mathbf{V}_{\mathbf{x}}=\left[\begin{array}{ccc}
\mathrm{v}_{x_{0}}^{0} & \cdots & \mathrm{v}_{x_{M_{x}-1}}^{0} \\
\vdots & & \vdots \\
\mathrm{v}_{x_{0}}^{N_{x}-1} & \cdots & \mathrm{v}_{x_{M_{x}-1}}^{N_{x}-1}
\end{array}\right] \\
& \mathbf{V}_{\mathbf{y}}=\left[\begin{array}{ccc}
\mathrm{v}_{y_{0}}^{0} & \cdots & \mathrm{v}_{y_{M_{x}-1}}^{0} \\
\vdots & & \vdots \\
\mathrm{v}_{y_{0}}^{N_{x}-1} & \cdots & \mathrm{v}_{y_{M_{x}-1}}^{N_{x}-1}
\end{array}\right] .
\end{aligned}
$$

Dada a separabilidade do array,

$$
\mathbf{V}=\mathbf{V}_{\mathbf{x}} \otimes \mathbf{V}_{\mathbf{y}}
$$

tal que

$$
\begin{aligned}
\mathbf{S} & =\mathbf{V} \mathbf{Y} \mathbf{V}^{H} \\
& =\left(\mathbf{V}_{\mathbf{x}} \otimes \mathbf{V}_{\mathbf{y}}\right) \mathbf{Y}\left(\mathbf{V}_{\mathbf{x}}^{H} \otimes \mathbf{V}_{\mathbf{y}}^{H}\right)
\end{aligned}
$$

Seja $\mathbf{z}$ um vetor arbitrário de tamanho $M \times 1$, e seja $\mathbf{Z}$ uma matriz $M_{y} \times M_{x}$ tal que $\mathbf{z}=\operatorname{vec}\{\mathbf{Z}\}$. Note que

$$
\left(\mathbf{V}_{\mathbf{x}} \otimes \mathbf{V}_{\mathbf{y}}\right) \mathbf{z}=\operatorname{vec}\left\{\mathbf{V}_{\mathbf{y}} \mathbf{Z} \mathbf{V}_{\mathbf{x}}^{H}\right\}
$$

Enquanto $\left(\mathbf{V}_{\mathbf{x}} \otimes \mathbf{V}_{\mathbf{y}}\right) \mathbf{z}$ requer $N M$ MACs para ser computado, $\left(\mathbf{V}_{\mathbf{y}} \mathbf{Z}\right) \mathbf{V}_{\mathbf{x}}^{H}$ requer somente $N_{y} M+N M_{x}$ MACs. O segundo produto dispensa qualquer reorganização de memória se matrizes forem armazenadas em memória em formato vetorizado (assim como no MATLAB, por exemplo). Portanto, $\left(\mathbf{V}_{\mathbf{x}} \otimes \mathbf{V}_{\mathbf{y}}\right) \mathbf{Y}$ pode ser implementado com $N_{y} M^{2}+$ $N M_{x} M$ MACs e $\left(\mathbf{V}_{\mathbf{x}} \otimes \mathbf{V}_{\mathbf{y}}\right) \mathbf{Y}\left(\mathbf{V}_{\mathbf{x}}^{H} \otimes \mathbf{V}_{\mathbf{y}}^{H}\right)$ requer $N_{y} M^{2}+N M_{x} M+N_{y} N M+N^{2} M_{x}$ MACs. Alterando a associatividade dos produtos matriciais é possível obter variações deste custo. Note que avaliar (10.1.13) com produtos matriciais requer $N M^{2}+N^{2} M$ MACs. 
Admitindo que $M \gg N$, a aceleração relativa é dada por

$$
\begin{aligned}
\frac{N M^{2}+N^{2} M}{N_{y} M^{2}+N M_{x} M+N_{y} N M+N^{2} M_{x}} & \approx \frac{N M^{2}}{N_{y} M^{2}+N M_{x} M} \\
& =\frac{N M}{N_{y} M+N M_{x}} \\
& =\frac{N_{x} M_{y}}{N_{x}+M_{y}} .
\end{aligned}
$$

Note que admitir uma geometria uniforme não resulta nas mesmas otimizações apresentadas na Seção 8.1. De fato, neste caso $\mathbf{V}_{\mathbf{x}}$ e $\mathbf{V}_{\mathbf{y}}$ não têm linhas redundantes no caso geral. Tampouco é possível acelerar os produtos por $\mathbf{V}_{\mathbf{x}}$ e $\mathbf{V}_{\mathbf{y}}$ usando NFFTs, pois elas têm poucas linhas.

\subsection{Transformada rápida transposta}

Sejam $\overline{\mathbf{S}} \in \mathbb{C}^{N \times N}$ e $\overline{\mathbf{Y}} \in \mathbb{C}^{M \times M}$ tal que vec $\{\overline{\mathbf{Y}}\}=\mathbf{A}^{T}$ vec $\{\overline{\mathbf{S}}\}$. Dados vetores $\mathbf{u}, \mathbf{v} \in \mathbb{C}^{n}$, definimos o produto escalar $\mathbf{u} \cdot \mathbf{v}=\mathbf{u}^{T} \mathbf{v}$ (observamos que ele não corresponde ao produto escalar usual, pois não é um produto interno).

Para $0 \leq m<M_{x}$ e $0 \leq n<M_{y}$, um elemento arbitrário $\overline{\mathbf{Y}}_{n, m}$ de $\overline{\mathbf{Y}}$ pode ser escrito como produto escalar da coluna $m \cdot M_{y}+n$ de $\mathbf{A}$ e vec $\{\overline{\mathbf{S}}\}$. Usando (10.1.4), podemos escrever

$$
\begin{aligned}
\overline{\mathbf{Y}}_{n, m} & =\left[\mathbf{v}_{\mathbf{u}_{m}}^{*} \otimes \mathbf{v}_{\mathbf{u}_{n}}\right] \cdot \operatorname{vec}\{\overline{\mathbf{S}}\} \\
& =\left[\mathbf{v}_{\mathbf{u}_{m}}^{H} \otimes \mathbf{v}_{\mathbf{u}_{n}}^{T}\right] \operatorname{vec}\{\overline{\mathbf{S}}\} \\
& =\mathbf{v}_{\mathbf{u}_{n}}^{T} \overline{\mathbf{S}} \mathbf{v}_{\mathbf{u}_{m}}^{*} .
\end{aligned}
$$

Portanto,

$$
\overline{\mathbf{Y}}=\mathbf{V}^{T} \overline{\mathbf{S}} \mathbf{V}^{*}
$$

Dada a separabilidade do array,

$$
\mathbf{V}=\mathbf{V}_{\mathbf{x}} \otimes \mathbf{V}_{\mathbf{y}}
$$

tal que

$$
\begin{aligned}
\overline{\mathbf{Y}} & =\left(\mathbf{V}_{\mathbf{x}} \otimes \mathbf{V}_{\mathbf{y}}\right)^{T} \overline{\mathbf{S}}\left(\mathbf{V}_{\mathbf{x}} \otimes \mathbf{V}_{\mathbf{y}}\right)^{*} \\
& =\left(\mathbf{V}_{\mathbf{x}}^{T} \otimes \mathbf{V}_{\mathbf{y}}^{T}\right) \overline{\mathbf{S}}\left(\mathbf{V}_{\mathbf{x}}^{*} \otimes \mathbf{V}_{\mathbf{y}}^{*}\right)
\end{aligned}
$$

Seja z um vetor arbitrário de dimensão $N \times 1$, e seja $\mathbf{Z}$ uma matriz $N_{y} \times N_{x}$ tal que $\mathbf{z}=\operatorname{vec}\{\mathbf{Z}\}$. Note que

$$
\left(\mathbf{V}_{\mathbf{x}}^{T} \otimes \mathbf{V}_{\mathbf{y}}^{T}\right) \mathbf{z}=\operatorname{vec}\left\{\mathbf{V}_{\mathbf{y}}^{T} \mathbf{Z} \mathbf{V}_{\mathbf{x}}\right\}
$$

Enquanto $\left(\mathbf{V}_{\mathbf{x}}^{T} \otimes \mathbf{V}_{\mathbf{y}}^{T}\right) \mathbf{z}$ requer $N M$ MACs para ser computado, $\left(\mathbf{V}_{\mathbf{y}}^{T} \mathbf{Z}\right) \mathbf{V}_{\mathbf{x}}$ requer $N M_{y}+$ 
$N_{x} M$ MACs. O segundo produto dispensa qualquer reorganização de memória se as matrizes forem armazenadas em memória em formato vetorizado (assim como no MATLAB, por exemplo). Portanto, $\left(\mathbf{V}_{\mathbf{x}}^{T} \otimes \mathbf{V}_{\mathbf{y}}^{T}\right) \overline{\mathbf{S}}$ pode ser implementado com $N^{2} M_{y}+N_{x} N M$ MACs e $\left(\mathbf{V}_{\mathbf{x}}^{T} \otimes \mathbf{V}_{\mathbf{y}}^{T}\right) \overline{\mathbf{S}}\left(\mathbf{V}_{\mathbf{x}}^{*} \otimes \mathbf{V}_{\mathbf{y}}^{*}\right)$ requer $N^{2} M_{y}+N_{x} N M+N M_{y} M+N_{x} M^{2}$ MACs. Alterando a associatividade dos produtos matriciais, podemos obter variações deste custo. Avaliar (10.2.4) usando produtos matriciais requer $N^{2} M+N M^{2}$ MACs.

Admitindo que $M \gg N$, a aceleração relativa é dada por

$$
\begin{aligned}
\frac{N^{2} M+N M^{2}}{N^{2} M_{y}+N_{x} N M+N M_{y} M+N_{x} M^{2}} & \approx \frac{N M^{2}}{N M_{y} M+N_{x} M^{2}} \\
& =\frac{N M}{N M_{y}+N_{x} M} \\
& =\frac{N_{y} M_{x}}{N_{y}+M_{x}} .
\end{aligned}
$$

\subsection{Transformada rápida adjunta}

Seja $\mathbf{A}$ a transformada direta, tal que $\mathbf{A}^{T}$ e $\mathbf{A}^{H}$ sejam sua transposta e adjunta, respectivamente. Como visto na subseção anterior, podemos implementar $\operatorname{vec}\{\overline{\mathbf{Y}}\}=$ $\mathbf{A}^{T}$ vec $\{\overline{\mathbf{S}}\} \operatorname{com} \overline{\mathbf{Y}}=\left(\mathbf{V}_{\mathbf{x}}^{T} \otimes \mathbf{V}_{\mathbf{y}}^{T}\right) \overline{\mathbf{S}}\left(\mathbf{V}_{\mathbf{x}}^{*} \otimes \mathbf{V}_{\mathbf{y}}^{*}\right)$. Como $\mathbf{A}^{H}=\left[\mathbf{A}^{T}\right]^{*}$, a adjunta rápida $\operatorname{vec}\{\breve{\mathbf{Y}}\}=\mathbf{A}^{H} \operatorname{vec}\{\overline{\mathbf{S}}\}$ pode ser determinada com

$$
\breve{\mathbf{Y}}=\left(\mathbf{V}_{\mathbf{x}}^{H} \otimes \mathbf{V}_{\mathbf{y}}^{H}\right) \overline{\mathbf{S}}\left(\mathbf{V}_{\mathbf{x}} \otimes \mathbf{V}_{\mathbf{y}}\right)
$$

\subsection{Transformada rápida direta-adjunta}

Dada uma entrada $\mathbf{Y}$, a versão rápida de vec $\{\breve{\mathbf{Y}}\}=\mathbf{A}^{H} \mathbf{A}$ vec $\{\mathbf{Y}\}$ pode ser obtida através da composição de (10.1.14) e (10.3.1). Isto produz

$$
\breve{\mathbf{Y}}=\left(\mathbf{V}_{\mathbf{x}}^{H} \otimes \mathbf{V}_{\mathbf{y}}^{H}\right)\left[\left(\mathbf{V}_{\mathbf{x}} \otimes \mathbf{V}_{\mathbf{y}}\right) \mathbf{Y}\left(\mathbf{V}_{\mathbf{x}}^{H} \otimes \mathbf{V}_{\mathbf{y}}^{H}\right)\right]\left(\mathbf{V}_{\mathbf{x}} \otimes \mathbf{V}_{\mathbf{y}}\right)
$$

Admitindo que $M_{x}, M_{y}>N_{x}, N_{y}$, a associatividade que produz o melhor desempenho é aquela apresentada acima. De fato, a identidade

$$
\left(\mathbf{V}_{\mathbf{x}}^{H} \otimes \mathbf{V}_{\mathbf{y}}^{H}\right)\left(\mathbf{V}_{\mathbf{x}} \otimes \mathbf{V}_{\mathbf{y}}\right)=\mathbf{V}_{\mathbf{x}}^{H} \mathbf{V}_{\mathbf{x}} \otimes \mathbf{V}_{\mathbf{y}}^{H} \mathbf{V}_{\mathbf{y}}
$$

produz uma grande matriz $M \times M$, cujo produto por $\mathbf{Y}$ é computacionalmente caro. Portanto, é melhor usar produtos por $\mathbf{V}_{\mathbf{x}}, \mathbf{V}_{\mathbf{y}}$ e suas transpostas, que produzem matrizes menores. 


\subsection{Aplicações}

As aplicações da transformada rápida correlacionada são as mesmas da transformada rápida descorrelacionada. Somente devemos considerar que no caso correlacionado, Y é uma matriz $M \times M$. Sua diagonal principal tem $M=M_{x} M_{y}$ elementos, e contém as potências das fontes em forma vetorizada. Portanto, para uma distribuição descorrelacionada, esta diagonal contém a imagem acústica vetorizada, enquanto os termos fora da diagonal serão nulos. Para distribuições correlacionadas, a diagonal principal contém as potências das fontes (que serão reais e não-negativas), e os termos fora da diagonal armazenarão as covariâncias cruzadas complexas.

Como um exemplo de aplicação, consideremos DAMAS-C [93], que é a variante de DAMAS para fontes correlacionadas. Assim como DAMAS, DAMAS-C resolve um sistema linear iterativamente usando o método de Gauss-Seidel. Sua formulação é idêntica a (7.5.10), exceto que a matriz $\mathbf{B}=\mathbf{A}^{H} \mathbf{A}$ é definida usando a transformada correlacionada A. Note que se as fontes forem correlacionadas, a distribuição suja $\breve{Y}$ não pode ser produzida convoluindo uma distribuição limpa Y com a PSF do array. Portanto, DAMAS-C não pode ser implementado usando uma convolução acelerada por FFT, para produzir uma versão análoga ao DAMAS2. Por outro lado, podemos obter $\breve{Y}$ a partir de $\mathbf{Y}$ com a implementação rápida de $\mathbf{A}^{H} \mathbf{A}$ descrita na Seção 10.4. Portanto, uma versão rápida de DAMAS-C pode ser obtida implementando (8.7.6) como escrito, mas com a transformada rápida correlacionada no lugar da transformada descorrelacionada.

Se a distribuição de fontes for esparsa, regularização $\ell_{1}$ pode ser usada com resultados melhores. Uma opção eficiente consiste em reconstruir uma imagem aproximada usando a transformada rápida descorrelacionada, aplicar um limiar para manter apenas as fontes dominantes e então re-estimar as potências e covariâncias cruzadas somente para as fontes significativas. Se a distribuição for suficientemente esparsa, então será possível determinar as potências e correlações. Porém, como já argumentamos, muitos problemas acústicos de interesse têm fontes distribuídas. Em particular, ressonâncias e reflexões criam fontes distribuídas com atrasos de fase não nulos.

A reconstrução de distribuições correlacionadas é atualmente um problema aberto. Em particular, não está claro como regularizar estes mapas para obter soluções únicas e estáveis sem restringir excessivamente o espaço de soluções potenciais. Como DAMAS e DAMAS2, DAMAS-C exige que Y seja real com elementos não-negativos. Portanto, DAMAS-C exige que todas as fontes correlacionadas irradiem em fase (em média), o que não é verdade em geral. Uma solução fisicamente correta deveria restringir $\mathbf{Y}$ a matrizes positivas definidas. Porém, esta restrição teria custo computacional proibitivo. 


\section{Capítulo 11}

\section{Conclusão}

Este trabalho introduz transformadas rápidas para modelar a relação entre distribuições de fontes e matrizes de covariância amostradas por arrays. Utilizando estas transformadas, pode-se utilizar algoritmos de mínimos quadrados regularizados para obter reconstruções com resolução muito superior à de métodos tradicionais, como beamforming.

Ainda que as transformadas propostas nas seções anteriores tenham considerável aplicabilidade, acreditamos que elas sejam apenas instâncias de uma classe de transformadas. De fato, tratamos apenas os casos de geometrias uniformes e Cartesianas, com uma extensão específica para campo próximo. Outras geometrias exigiriam outras transformadas, com permutações $\Xi$ distintas. As geometrias estudadas são convenientes pois são exatamente separáveis em campo distante. Como este tipicamente não é o caso, outras propostas de transformadas rápidas provavelmente exigirão aproximações ou não exigirão separabilidade.

Resta determinar condições sob as quais as transformadas A descritas nos Capítulos 8-10 atendem a condições suficientes para reconstrução estável de sinais esparsos, como por exemplo a restricted isometry property (RIP) [74]. Da Seção 8.5.1 sabemos que para $d=\frac{\lambda}{2}$, A corresponde a uma FFT sub-amostrada e permutada. Neste caso, A atende à RIP. Intuitivamente espera-se que $\mathbf{A}$ não atenda à RIP para arrays uniformes com $d \ll \frac{\lambda}{2}$ ou $d \gg \frac{\lambda}{2}$, pois no primeiro caso é impossível obter reconstrução única devido a aliasing, e no segundo caso o campo não é amostrado com diversidade suficiente para obter reconstruções detalhadas. Porém, não podemos afirmar nada quantitativamente conforme $d$ varia, e nada podemos afirmar para geometrias arbitrárias ou em campo próximo.

O problema inverso de imagens acústicas torna-se intratável se considerarmos as correlações entre todos os pares de fontes pontuais modeladas. De fato, uma distribuição com $256 \times 256$ fontes possui 4 bilhões de correlações cruzadas, que sequer podem ser armazenadas em memória. Portanto, qualquer proposta de estimação exigirá hipóteses mais fortes para regularização, de forma a obter reconstruções estáveis. Para casos em que o campo de ondas não é esparso em sua representação canônica, este é outro problema aberto. 
No exemplo de calibração da Seção 9.2, a matriz de calibração foi gerada usando uma superfície planar retangular. Tipicamente, as fontes acústicas que compõem uma imagem estarão a distâncias diferentes do array, tal que uma matriz de calibração que utilize uma única distância focal deixará certas fontes fora de foco. Caso as fontes de interesse possam se mover, este mecanismo deve ser adaptativo, de forma que a distância focal de cada região acompanhe as fontes dominantes.

Do ponto de vista de implementações, notamos que a ausência de uma plataforma de referência dificulta a validação dos métodos propostos pela academia. Os resultados experimentais presentes na literatura foram obtidos em cenários tão diversos que sua comparação é essencialmente impossível. Em particular, não existem datasets públicos para validação de algoritmos de imagens acústicas. Portanto, a divulgação de uma metodologia prática de como projetar e produzir arrays pode diminuir consideravelmente as barreiras de entrada a esta área de processamento de sinais. Com estes objetivos, desenvolvemos um sistema de aquisição de dados altamente escalonável e de baixo custo, descrito no Apêndice A.

Finalmente, destacamos que o uso de transformadas rápidas acelera em até 1000 vezes o processo de reconstrução de campos acústicos, além de diminuir os requisitos de memória em várias ordens de grandeza. Com seu uso, torna-se possível reconstruir imagens em tempo real e banda larga utilizando beamforming (por exemplo, delay-and-sum). Por outro lado, algoritmos de reconstrução regularizada ainda consomem muito tempo de processamento para aplicações em tempo real. Para acelerar a reconstrução, seria altamente desejável implementar os métodos propostos utilizando uma arquitetura de processamento paralelo (por exemplo, [94]). 


\section{Referências Bibliográficas}

[1] W. Hodgkiss e L. Nolte, "Covariance between Fourier coefficients representing the time waveforms observed from an array of sensors," The Journal of the Acoustical Society of America, vol. 59, pp. 582-590, 1976.

[2] H. L. Van Trees, Optimum Array Processing: Part IV of Detection, Estimation, and Modulation Theory. New York, NY: John Wiley \& Sons, 2002.

[3] R. G. Bartle, The Elements of Real Analysis, 2nd ed. New York: John Wiley \& Sons, 1976.

[4] J. G. Proakis e D. G. Manolakis, Digital Signal Processing: Principles, Algorithms, and Applications. Prentice Hall, 1996.

[5] A. V. Oppenheim, R. W. Schafer, e J. R. Buck, Discrete-Time Signal Processing, 2nd ed. Prentice Hall, 1999.

[6] D. Tufts e J. Francis, "Designing digital low-pass filters-comparison of some methods and criteria," IEEE Transactions on Audio and Electroacoustics, vol. 18, no. 4, pp. 487-494, 1970.

[7] A. Papoulis e M. Bertran, "Digital filtering and prolate functions," IEEE Transactions on Circuits and Systems, vol. 19, no. 6, pp. 674-681, 1972.

[8] S. Prasad, "On the index for array optimization and the discrete prolate spheroidal functions," IEEE Transactions on Antennas and Propagation, vol. 30, no. 5, pp. 1021-1023, 1982.

[9] D. Brandwood, "A complex gradient operator and its application in adaptive array theory," IEE Proceedings, Part F-Communications, Radar, vol. 130, no. 1 pt F, pp. 11-16, 1983.

[10] H. Steyskal, "Synthesis of antenna patterns with prescribed nulls," IEEE Transactions on Antennas and Propagation, vol. 30, pp. 273-279, 1982.

[11] A. N. Akansu e R. A. Haddad, Multiresolution Signal Decomposition. Academic Press, 2001. 
[12] N. S. Jayant e P. Noll, Digital Coding of Waveforms. Englewood Cliffs, NJ, USA: Prentice Hall, 1984.

[13] E. Vertatschitsch e S. Haykin, "Nonredundant arrays," Proceedings of the IEEE, vol. 74 , no. 1, pp. 217-217, 1986.

[14] A. Papoulis e S. Pillai, Probability, random variables, and stochastic processes. McGraw-Hill Companies, 2002.

[15] H. L. Van Trees, Detection, Estimation, and Modulation Theory, part I. Wiley Interscience, 2001.

[16] — Detection, Estimation, and Modulation Theory, part III. Wiley Interscience, 2001.

[17] A. B. Baggeroer, "Space/time random processes and optimum array processing," Navy Undersea Center, San Diego, CA, Tech. Rep. 506, 1976.

[18] T. S. Alexander, Adaptive Signal Processing: Theory and Applications. SpringerVerlag, 1986.

[19] S. S. Haykin, Adaptive Filter Theory, 4th ed. Prentice Hall, 2002.

[20] A. H. Sayed, Fundamentals of Adaptive Filtering. Wiley-Interscience, 2003.

[21] J. Capon, "High-resolution frequency-wavenumber spectrum analysis," Proceedings of the IEEE, vol. 57, no. 8, pp. 1408-1418, 1969.

[22] B. Carlson, "Covariance matrix estimation errors and diagonal loading in adaptive arrays," IEEE Transactions on Aerospace and Electronic Systems, vol. 24, no. 4, pp. 397-401, 1988.

[23] A. Neumaier, "Solving ill-conditioned and singular linear systems: a tutorial on regularization," SIAM Review, vol. 40, no. 3, pp. 636-666, 1998.

[24] O. Frost III, "An algorithm for linearly constrained adaptive array processing," Proceedings of the IEEE, vol. 60, no. 8, pp. 926-935, 1972.

[25] B. Van Veen e K. Buckley, "Beamforming: a versatile approach to spatial filtering," IEEE ASSP Magazine, vol. 5, no. 2, pp. 4-24, 1988.

[26] S. Applebaum e D. Chapman, "Adaptive arrays with main beam constraints," IEEE Transactions on Antennas and Propagation, vol. 24, no. 5, pp. 650-662, 1976.

[27] L. Griffiths e C. Jim, "An alternative approach to linearly constrained adaptive beamforming," IEEE Transactions on Antennas and Propagation, vol. 30, no. 1, pp. 27-34, Jan. 1982. 
[28] D. Abraham e N. Owsley, "Beamforming with dominant mode rejection," in Proc. of IEEE OCEANS, 1990.

[29] H. Cox e R. Pitre, "Robust DMR and multi-rate adaptive beamforming," Proc. of the 31st Asilomar Conference on Signals, Systems \&f Computers, vol. 1, 1997.

[30] A. Cantoni e L. Godara, "Resolving the directions of sources in a correlated field incident on an array," The Journal of the Acoustical Society of America, vol. 67, pp. 1247-1255, 1980.

[31] T. Shan, M. Wax, e T. Kailath, "On spatial smoothing for direction-of-arrival estimation of coherent signals," IEEE Transactions on Acoustics, Speech, and Signal Processing, vol. 33, no. 4, pp. 806-811, 1985.

[32] T. Shan e T. Kailath, "Adaptive beamforming for coherent signals and interference," IEEE Transactions on Acoustics, Speech, and Signal Processing, vol. 33, no. 3, pp. 527-536, 1985.

[33] V. Reddy, A. Paulraj, e T. Kailath, "Performance analysis of the optimum beamformer in the presence of correlated sources and its behavior under spatial smoothing," IEEE Transactions on Acoustics, Speech, and Signal Processing, vol. 35, no. 7, pp. 927-936, 1987.

[34] S. Pillai e B. Kwon, "Forward/backward spatial smoothing techniques for coherent signal identification," IEEE Transactions on Acoustics, Speech, and Signal Processing, vol. 37, no. 1, pp. 8-15, 1989.

[35] J. Evans, D. Sun, e J. Johnson, "Application of Advanced Signal Processing Techniques to Angle of Arrival Estimation in ATC Navigation and Surveillance Systems," M.I.T. Lincoln Laboratory, Tech. Rep., Jun. 1982.

[36] A. Gershman e V. Ermolaev, "Optimal subarray size for spatial smoothing," IEEE Signal Processing Letters, vol. 2, no. 2, pp. 28-30, 1995.

[37] M. Jansson e P. Stoica, "Forward-only and forward-backward sample covariances-a comparative study," Signal Processing, vol. 77, no. 3, pp. 235-245, 1999.

[38] M. Lanne, A. Lundgren, e M. Viberg, "Optimized beamforming calibration in the presence of array imperfections," in Proc. of ICASSP, 2007.

[39] A. Lundgren, M. Lanne, e M. Viberg, "Two-Step ESPRIT with compensation for modelling errors using a sparse calibration grid," in Proc. of ICASSP, 2007. 
[40] B. Ng e C. See, "Sensor-array calibration using a maximum-likelihood approach," IEEE Transactions on Antennas and Propagation, vol. 44, no. 6 Part 1, pp. 827-835, 1996.

[41] V. Raykar e R. Duraiswami, "Automatic position calibration of multiple microphones," in Proc. of ICASSP, 2004.

[42] R. Schmidt, "Multiple emitter location and signal parameter estimation," IEEE Transactions on Antennas and Propagation, vol. 34, no. 3, pp. 276-280, 1986.

[43] B. Rao e K. Hari, "Performance analysis of root-music," IEEE Transactions on Acoustics, Speech, and Signal Processing, vol. 37, no. 12, pp. 1939-1949, 1989.

[44] M. Pesavento, A. Gershman, e M. Haardt, "Unitary root-MUSIC with a real-valued eigendecomposition: a theoretical and experimental performance study," IEEE Transactions on Acoustics, Speech, and Signal Processing, vol. 48, no. 5, pp. 1306-1314, 2000.

[45] R. Roy, A. Paulraj, e T. Kailath, "ESPRIT-a subspace rotation approach to estimation of parameters of cisoids in noise," IEEE Transactions on Acoustics, Speech, and Signal Processing, vol. 34, no. 5, pp. 1340-1342, 1986.

[46] R. Roy e T. Kailath, "ESPRIT-estimation of signal parameters via rotational invariance techniques," IEEE Transactions on Acoustics, Speech, and Signal Processing, vol. 37, no. 7, pp. 984-995, 1989.

[47] M. Haardt e J. Nossek, "Unitary ESPRIT: how to obtain increased estimation accuracy with a reduced computational burden," IEEE Transactions on Acoustics, Speech, and Signal Processing, vol. 43, no. 5, pp. 1232-1242, 1995.

[48] M. Zoltowski, M. Haardt, e C. Mathews, "Closed-form 2-D angle estimation with rectangular arrays in elementspace or beamspace via unitary ESPRIT," IEEE Transactions on Acoustics, Speech, and Signal Processing, vol. 44, no. 2, pp. 316-328, 1996.

[49] J. Maynard, E. Williams, e Y. Lee, "Nearfield acoustic holography: I. Theory of generalized holography and the development of NAH," The Journal of the Acoustical Society of America, vol. 78, pp. 1395-1413, 1985.

[50] M. Park e B. Rafaely, "Sound-field analysis by plane-wave decomposition using spherical microphone array," The Journal of the Acoustical Society of America, vol. 118, pp. 3094-3103, 2005. 
[51] B. Rafaely, I. Balmages, e L. Eger, "High-resolution plane-wave decomposition in an auditorium using a dual-radius scanning spherical microphone array," The Journal of the Acoustical Society of America, vol. 122, pp. 2661-2668, 2007.

[52] A. O’Donovan, R. Duraiswami, e J. Neumann, "Microphone arrays as generalized cameras for integrated audio visual processing," in Proc. of IEEE CVPR, 2007.

[53] A. O’Donovan, R. Duraiswami, e D. Zotkin, "Imaging concert hall acoustics using visual and audio cameras," in Proc. of ICASSP, 2008, pp. 5284-5287.

[54] B. Rafaely, "Analysis and design of spherical microphone arrays," IEEE Transactions on Speech and Audio Processing, vol. 13, no. 1, pp. 135-143, 2005.

[55] W. Home, K. James, T. Arledge, P. Sodermant, N. Burnside, e S. Jaeger, "Measurements of 26\%-scale 777 Airframe Noise in the NASA Ames 40- by 80-Foot Wind Tunnel," in Proc. of the 11th AIAA/CEAS Aeroacoustics Conference, 2005.

[56] W. Humphreys e T. Brooks, "Noise spectra and directivity for scale-model landing gear," in Proc. of the 13th AIAA/CEAS Aeroacoustics Conference, 2007.

[57] S. Lee, "Phased-array measurement of modern regional aircraft turbofan engine noise," in Proc. of the 12th AIAA/CEAS Aeroacoustics Conference, 2006.

[58] S. Oerlemans, P. Sijtsma, e B. Mendez Lopez, "Location and quantification of noise sources on a wind turbine," Journal of Sound and Vibration, vol. 299, no. 4-5, pp. 869-883, 2007.

[59] F. Wang, H. Wassaf, A. Gulsrud, D. Delisi, e R. Rudis, "Acoustic imaging of aircraft wake vortex dynamics," in Proc. of the 11th AIAA/CEAS Aeroacoustics Conference, 2005.

[60] L. Brusniak, J. Underbrink, e R. Stoker, "Acoustic imaging of aircraft noise sources using large aperture phased arrays," in Proc. of the 12th AIAA/CEAS Aeroacoustics Conference, 2006.

[61] L. Rabiner e B. Gold, Theory and application of digital signal processing. Prentice Hall, 1975.

[62] P. Hughett, "Linearity and sigma-linearity in discrete-time linear shift-invariant systems," Signal Processing, vol. 59, no. 3, pp. 329-333, 1997.

[63] Z. Wang, J. Li, P. Stoica, T. Nishida, e M. Sheplak, "Constant-beamwidth and constant-powerwidth wideband robust Capon beamformers for acoustic imaging," The Journal of the Acoustical Society of America, vol. 116, pp. 1621-1631, 2004. 
[64] S. Yan, Y. Ma, e C. Hou, "Optimal array pattern synthesis for broadband arrays," The Journal of the Acoustical Society of America, vol. 122, pp. 2686-2696, 2007.

[65] J. Högbom, "Aperture synthesis with a non-regular distribution of interferometer baselines," Astronomy and Astrophysics Supplement, vol. 15, no. 3, pp. 417-426, 1974 .

[66] Y. Wang, J. Li, P. Stoica, M. Sheplak, e T. Nishida, "Wideband RELAX and wideband CLEAN for aeroacoustic imaging," The Journal of the Acoustical Society of America, vol. 115, pp. 757-767, 2004.

[67] R. Dougherty e R. Stoker, "Sidelobe suppression for phased array aeroacoustic measurements," in Proc. of the 4th AIAA/CEAS Aeroacoustics Conference, 1998, pp. $235-245$.

[68] R. Dougherty, "Extensions of DAMAS and benefits and limitations of deconvolution in beamforming," in Proc. of the 11th AIAA/CEAS Aeroacoustics Conference, 2005.

[69] K. Ehrenfried e L. Koop, "Comparison of iterative deconvolution algorithms for the mapping of acoustic sources," AIAA Journal, vol. 45, no. 7, pp. 1584-1595, 2007.

[70] T. Brooks e W. Humphreys, "A deconvolution approach for the mapping of acoustic sources (DAMAS) determined from phased microphone arrays," Journal of Sound and Vibration, vol. 294, no. 4-5, pp. 856-879, 2006.

[71] W. Richardson, "Bayesian-based iterative method of image restoration," Journal of the Optical Society of America, vol. 62, pp. 55-59, 1972.

[72] L. Lucy, "An iterative technique for the rectification of observed distributions," The Astronomical Journal, vol. 79, no. 6, pp. 745-754, 1974.

[73] T. Yardibi, J. Li, P. Stoica, e L. Cattafesta III, "Sparsity constrained deconvolution approaches for acoustic source mapping," The Journal of the Acoustical Society of America, vol. 123, pp. 2631-2642, 2008.

[74] E. Candès, J. Romberg, e T. Tao, "Stable signal recovery from incomplete and inaccurate measurements," Communications on Pure and Applied Mathematics, vol. 59, no. 8, pp. 1207-1223, 2006.

[75] B. Zimmermann e C. Studer, "FPGA-based real-time acoustic camera prototype," in Proc. of ISCAS, 2010.

[76] X. Huang, "Real-time algorithm for acoustic imaging with a microphone array," The Journal of the Acoustical Society of America, vol. 125, no. 5, 2009. 
[77] R. Horn e C. Johnson, Matrix analysis. Cambridge University Press, 1990.

[78] J. Keiner, S. Kunis, e D. Potts, "Using NFFT 3-a software library for various nonequispaced fast Fourier transforms," ACM Transactions on Mathematical Software (TOMS), vol. 36, no. 4, p. 19, 2009.

[79] D. Blacodon, "Spectral estimation method for noisy data using a noise reference," in Proc. BeBeC, 2010.

[80] M. Frigo e S. Johnson, "The design and implementation of FFTW 3," Proceedings of the IEEE, vol. 93, no. 2, pp. 216-231, 2005.

[81] S. Mallat e Z. Zhang, "Matching pursuits with time-frequency dictionaries," IEEE Transactions on Signal Processing, vol. 41, no. 12, pp. 3397-3415, 1993.

[82] E. van den Berg e M. Friedlander, "Probing the Pareto frontier for basis pursuit solutions," SIAM Journal on Scientific Computing, vol. 31, no. 2, pp. 890-912, 2008.

[83] L. Rudin, S. Osher, e E. Fatemi, "Nonlinear total variation based noise removal algorithms," Physica D: Nonlinear Phenomena, vol. 60, no. 1-4, pp. 259-268, 1992.

[84] C. Li, "An efficient algorithm for total variation regularization with applications to the single pixel camera and compressive sensing," Master's thesis, Rice University, 2009 .

[85] J. Underbrink e R. Dougherty, "Array design for non-intrusive measurement of noise sources," in Proc. of NOISE-CON, 1996, pp. 757-762.

[86] C. Van Loan e N. Pitsianis, "Approximation with Kronecker products," in Linear Algebra for Large Scale and Real Time Applications, M. Moonen e G. Golub, Eds. Kluwer Publications, 1992, pp. 293-314.

[87] J. Baglama e L. Reichel, "Augmented implicitly restarted Lanczos bidiagonalization methods," SIAM Journal on Scientific Computing, vol. 27, no. 1, pp. 19-42, 2006.

[88] P. Drineas, R. Kannan, e M. Mahoney, "Fast Monte Carlo algorithms for matrices II: Computing a low-rank approximation to a matrix," SIAM Journal on Computing, vol. 36, no. 1, pp. 158-183, 2007.

[89] N. Halko, P. Martinsson, e J. Tropp, "Finding structure with randomness: Stochastic algorithms for constructing approximate matrix decompositions," California Inst. Tech., Tech. Rep. ACM 2009-05, Sep. 2009.

[90] N. Srebro e T. Jaakkola, "Weighted low-rank approximations," in Proc. ICML, 2003, pp. $720-727$. 
[91] J. Pierre e M. Kaveh, "Experimental performance of calibration and direction-finding algorithms," in Proc. of ICASSP, 1991, pp. 1365-1368.

[92] M. Pesavento e A. Luo, "Robust array interpolation using second-order cone programming," IEEE Signal Processing Letters, vol. 9, no. 1, pp. 8-11, 2002.

[93] T. Brooks e W. Humphreys, "Extension of DAMAS phased array processing for spatial coherence determination (DAMAS-C)," in Proc. of the 12th AIAA/CEAS Aeroacoustics Conference, 2006.

[94] “Compute Unified Device Architecture Programming Guide," NVIDIA: Santa Clara, $C A, 2007$. 


\section{Apêndice A}

\section{Projeto de uma plataforma de referência}

\section{A.1 Introdução}

Acreditamos que a próxima fronteira em imagens acústicas dependerá fortemente de modelos. De fato, não é possível estimar bilhões de correlações cruzadas sem hipóteses fortes sobre sua dependência. Analogamente, algoritmos de foco e calibração online são problemas inversos de grande escala para os quais ainda não foram estudados métodos de regularização.

A determinação de modelos adequados exige o uso de uma quantidade significativa de dados experimentais. Observamos, no entanto, que não existem datasets públicos para imagens acústicas. E ainda que estes existissem, os arrays utilizados não teriam as geometrias Cartesianas que propusemos ao longo deste trabalho. Portanto, fomos motivados a desenvolver um array que pudesse servir como plataforma de referência.

Para dispensar o uso de conversores A/D (que representam o maior custo em um array acústico), utilizamos microfones MEMS (micro electrical-mechanical system), que possuem conversores $\mathrm{A} / \mathrm{D}$ sigma-delta de 1 bit integrados às cápsulas. Cada microfone tem como saída apenas um sinal digital de 1 bit à taxa de $2.4 \mathrm{MHz}$. Portanto, este sistema possui alta imunidade a radio-interferência e sequer exige cabos blindados, ao contrário de arrays produzidos com microfones analógicos.

Cada microfone MEMS é fabricado com processos de litografia, deposição e corrosão semelhantes aos que são usados para produção de circuitos integrados. Esta construção integra sobre um único substrato componentes mecânicos (como o diafragma de um microfone), circuitos analógicos e circuitos digitais. Os processos utilizados permitem a produção de microfones com tolerâncias melhores do que as presentes em cápsulas tradicionais de eletreto, reduzindo significativamente os problemas de casamento e calibração existentes em arrays tradicionais.

Como cada microfone produz um único sinal digital, um array de $\mathrm{N}$ elementos produz $\mathrm{N}$ linhas digitais a $2.4 \mathrm{MHz}$. Para realizar a aquisição de dados, utilizamos uma FPGA Altera Stratix III. Esta controla um módulo de 1 GB de memória DDR3, que é usado como buffer para armazenar os sinais adquiridos pelo array. A mesma FPGA também está 
conectada a uma interface USB 2.0, que é utilizada para transferir os dados da memória DDR3 para um computador, onde é executado o algoritmo de reconstrução de imagens.

A FPGA utilizada pode adquirir sinais de até 256 microfones. Arrays maiores podem ser construídos interconectando FPGAs, bastando que todos os microfones estejam sincronizados por um mesmo clock de $2.4 \mathrm{MHz}$ (utilizando buffers para atender aos requisitos de fan-out da tecnologia usada). Como os conversores A/D já estão integrados aos microfones, o custo dos arrays MEMS torna-se consideravelmente inferior ao de arrays tradicionais.

\section{A.2 Hardware projetado}

Para demonstrar as técnicas de reconstrução descritas anteriormente, projetamos um array planar de $8 \times 8$ elementos com $35 \times 35 \mathrm{~cm}$, utilizando a geometria separável descrita na Seção 8.8, e plotada na Figura A.1. O array é construído sobre uma única placa de circuito impresso. Utilizando soldagem por refusão, os microfones se posicionam naturalmente por efeito capilar, tornando desprezíveis os desvios em relação à geometria ideal. A Figura A.2 mostra o layout desenvolvido, sobre uma placa com 4 layers (os layers internos de $0 \mathrm{~V}$ e $3.3 \mathrm{~V}$ foram omitidos para facilitar a visualização).

Para a aquisição dos sinais, utilizamos uma placa de desenvolvimento Altera DE3 (ver Figura A.3), que integra uma FPGA Stratix III 3SL150, 1 GB de memória DDR3 e uma porta USB 2.0 implementada com a controladora ISP1761. O controle de todos estes dispositivos é feito por um processador NIOS II, sintetizado na FPGA. A taxa de aquisição é limitada pela taxa de transferência da interface ISP1761 com o processador Nios II, que é de aproximadamente $16 \mathrm{MB} / \mathrm{s}$. Como um array de 64 microfones operando a $2.4 \mathrm{MHz}$ requer uma banda de $64 \cdot 2.4 \cdot 10^{6} / 8=19.2 \mathrm{MB} / \mathrm{s}$, a memória DDR2 deve sempre ser usada como buffer. 


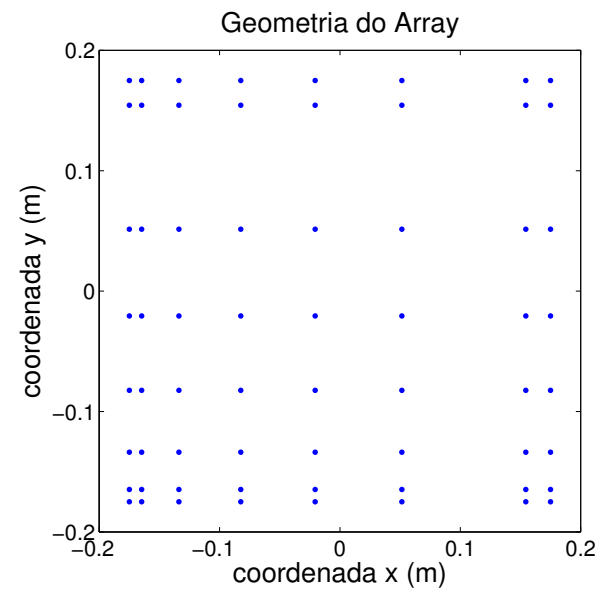

Figura A.1: Geometria do array projetado

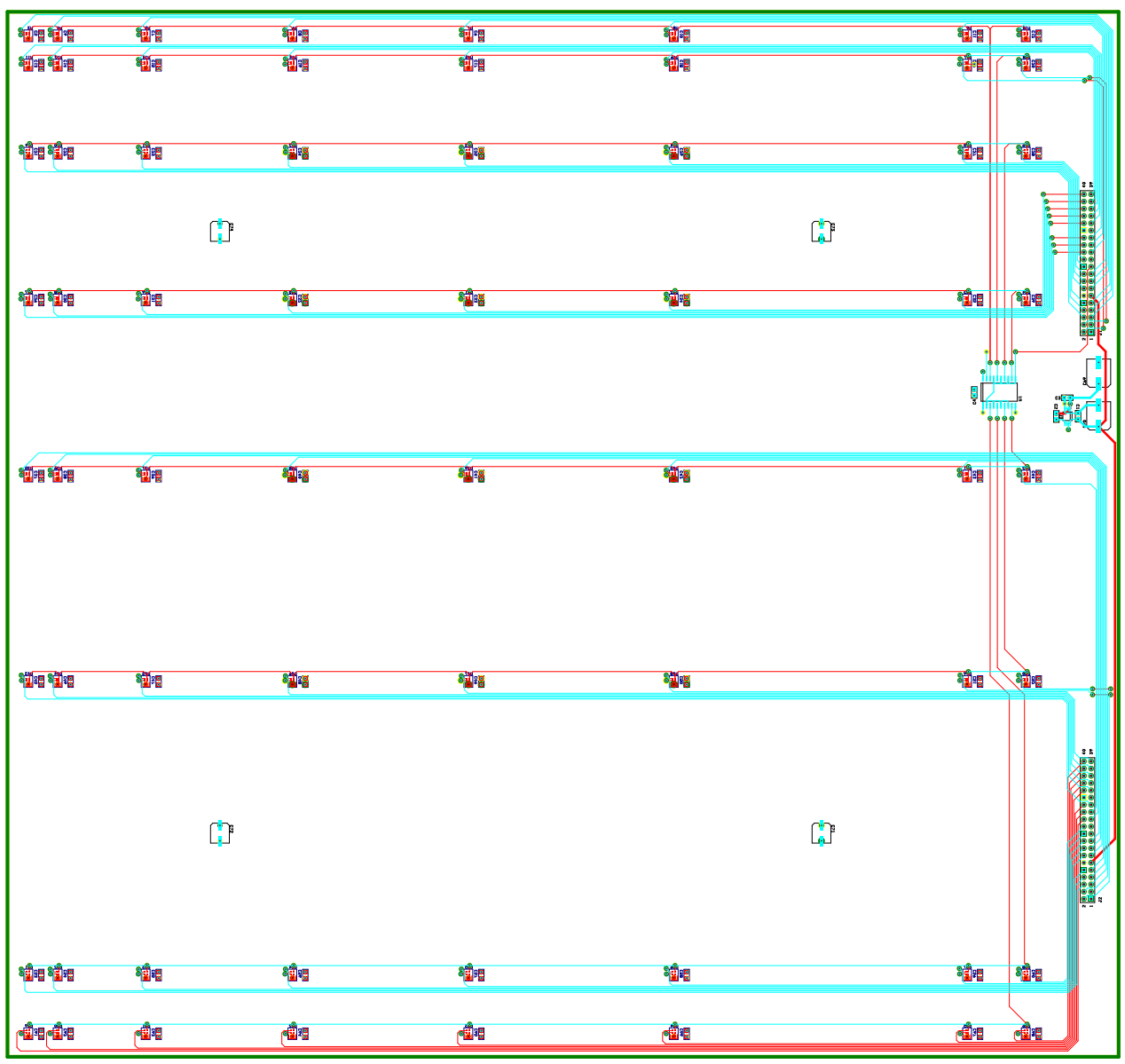

Figura A.2: Layout do array de microfones 


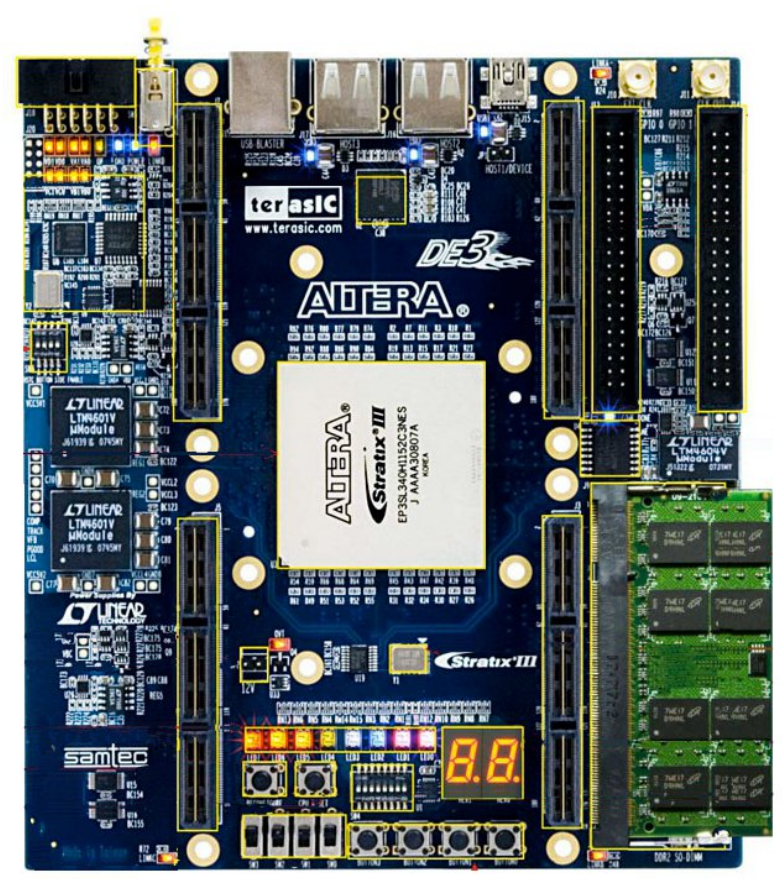

Figura A.3: Placa de desenvolvimento Altera DE3

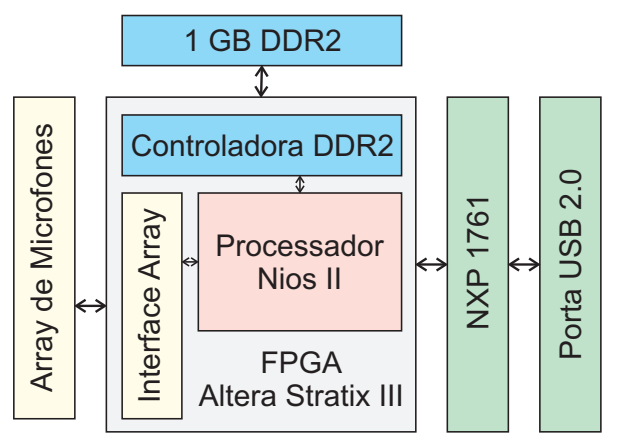

Figura A.4: Diagrama de blocos do sistema de aquisição 


\section{Apêndice B}

\section{Publicações relevantes}

1. F. Ribeiro e V. Nascimento, "Fast Transforms for Acoustic Imaging - Part I: Theory," IEEE Transactions on Image Processing, vol. 20, no. 8, pp. 2229-2240, 2011.

2. F. Ribeiro e V. Nascimento, "Fast Transforms for Acoustic Imaging - Part II: Applications," in IEEE Transactions on Image Processing, vol. 20, no. 8, pp. 2241-2247, 2011.

3. F. Ribeiro e V. Nascimento, "Fast Near-field Acoustic Imaging with Separable Arrays," in Proceedings of the IEEE Workshop on Statistical Signal Processing, 2011. 



\section{Fast Transforms for Acoustic Imaging-Part I: Theory}

Flávio P. Ribeiro, Student Member, IEEE, and Vítor H. Nascimento, Member, IEEE

\begin{abstract}
The classical approach for acoustic imaging consists of beamforming, and produces the source distribution of interest convolved with the array point spread function. This convolution smears the image of interest, significantly reducing its effective resolution. Deconvolution methods have been proposed to enhance acoustic images and have produced significant improvements. Other proposals involve covariance fitting techniques, which avoid deconvolution altogether. However, in their traditional presentation, these enhanced reconstruction methods have very high computational costs, mostly because they have no means of efficiently transforming back and forth between a hypothetical image and the measured data. In this paper, we propose the Kronecker Array Transform (KAT), a fast separable transform for array imaging applications. Under the assumption of a separable array, it enables the acceleration of imaging techniques by several orders of magnitude with respect to the fastest previously available methods, and enables the use of state-of-the-art regularized least-squares solvers. Using the KAT, one can reconstruct images with higher resolutions than was previously possible and use more accurate reconstruction techniques, opening new and exciting possibilities for acoustic imaging.
\end{abstract}

Index Terms-Acoustic imaging, array imaging, array processing, fast transform, regularized least squares, sparse reconstruction.

\section{INTRODUCTION}

A COUSTIC imaging refers to the problem of mapping the locations and intensities of sound sources over a region of interest using microphone arrays. For example, a microphone array can be positioned in a wind tunnel to determine the noise distribution over a model due to high velocity airflow [1], [2] These measurements are routinely used to design cars, trains, and aircraft, which are quieter to outside observers and to passengers. Microphone arrays have been employed to measure the noise generated by turbofan engines [3] and wind turbines [4] for similar noise reduction applications. Acoustic imaging has also been used to visualize the reverberant structure of concert halls [5]. We note that techniques for imaging the shapes of objects and structures via acoustic waves are sometimes called acoustic imaging, but we will not address these problems.

Manuscript received May 26, 2010; revised December 21, 2010; accepted February 09, 2011. Date of publication February 22, 2011; date of current version July 15,2011 . This work was supported in part by the São Paulo Research Foundation (FAPESP) and in part by the National Council for Scientific and Technological Development (CNPq). The associate editor coordinating the review of this manuscript and approving it for publication was Dr. John P. Kerekes.

The authors are with the Electronic Systems Engineering Department, Escola Politécnica, Universidade de São Paulo, São Paulo, SP 05508-900, Brazil (e-mail: fr@lps.usp.br; vitor@lps.usp.br).

Color versions of one or more of the figures in this paper are available online at http://ieeexplore.ieee.org.

Digital Object Identifier 10.1109/TIP.2011.2118220
Array imaging is possible because sensor arrays can be electronically steered toward arbitrary directions. One can define a grid over a region of interest, electronically steer the array over all elements of the grid, and, thus, create a map of estimated sound pressure levels. Each point in the grid can be represented as a pixel. The value of the pixel can be chosen to represent the estimated sound pressure level, thus creating an acoustic image.

Array imaging differs from source localization techniques, such as [6]-[10], because these usually produce a pseudospectrum of the wavefield, with maxima that indicate the location of dominant sources but with values that do not map to source powers. Thus, in this paper, we will assume that accurate power estimates are desirable.

The simplest and most common method for imaging uses delay and sum beamforming. This technique consists of delaying and summing the signals arriving at each sensor so that the sources located at a direction of interest are reinforced, and sources located in other directions are attenuated. Beamforming is simple, but unfortunately produces the lowest quality images. Indeed, under the assumption that the sources are in the far-field of the array, beamforming produces the source distribution of interest convolved with the array point spread function (PSF). Since a typical acoustic array has a relatively small aperture with respect to its operating wavelengths, its PSF can be quite large, so that delay and sum beamforming produces very smeared images. Alternative beamforming techniques have been developed to improve resolution by using data-dependent methods and numerical optimization of the beampattern [11]-[13], but they do not overcome the fundamental limitation that beamforming produces convolved images.

To overcome this limitation, several deconvolution techniques have been proposed [14]-[17]. They use as inputs the image obtained with delay and sum beamforming and the array PSF, and generally produce a much better approximation of the original source distribution. Nevertheless, deconvolution is an ill-conditioned inverse problem, and typically requires some knowledge of the solution to discriminate between different solutions which would be equally good fits for the measured data. The acoustic imaging methods proposed so far tend to use very simple types of regularization, such as low-pass filtering between iterations, or no regularization at all.

Regularized signal reconstruction has been a topic of interest for many decades, and gained significant momentum with the popularity of compressive sensing [18]-[20]. Indeed, many image reconstruction problems can be recast as convex optimization problems, which can be solved with computationally efficient iterative methods. While many of these techniques were designed for imaging applications, they have remained limited to fields, such as medical image reconstruction. Therefore, most of these developments have not been applied to acoustic imaging. 
A major reason for this separation between fields has been the absence of computationally efficient transforms for aeroacoustic imaging. For example, consider the generic nonlinear signal reconstruction problem given by

$$
\hat{\mathbf{y}}=\underset{\mathbf{y}}{\arg \min }\|\mathbf{\Psi} \mathbf{y}\| \text { such that } \mathbf{\Phi} \mathbf{y}=\mathbf{x}
$$

where $\mathbf{x}$ is the measured signal, $\hat{\mathbf{y}}$ is the reconstructed signal, $\Psi$ is a sparsifying transform, and $\Phi$ is a transform which models the measurement process. For an acoustic image, $\mathbf{y}$ would be a vectorized version of the image describing the true source distribution, and $\mathbf{x}$ would be a vectorized version of the array's sample covariance matrix.

Since, in practice, (1) is solved iteratively, one must be able to quickly evaluate $\boldsymbol{\Psi} \mathbf{u}$ and $\boldsymbol{\Phi} \mathbf{u}$ (and $\boldsymbol{\Psi}^{H} \mathbf{v}$ and $\boldsymbol{\Phi}^{H} \mathbf{v}$, as we will see in $[21$, Sec. II] of this paper) for arbitrary $\mathbf{u}, \mathbf{v}$. This is a very strong requirement, because the application of these transforms is the bottleneck of efficient convex optimization algorithms and completely determines their computational costs (regardless of whether the transform is fast or slow). While one can choose a convenient fast sparsifying transform $\boldsymbol{\Psi}$, the transform $\boldsymbol{\Phi}$ is determined by the physical measurement process. For example, for MRI applications, we naturally have $\boldsymbol{\Phi}=\mathbf{P} \mathcal{F}$, where $\mathcal{F}$ is a fast Fourier transform (FFT) and $\mathbf{P}$ is a subsampling operator Finite differences have been successfully used as the sparsifying transform $\Psi$ with $\|\cdot\|=\|\cdot\|_{1}$ [22], [23].

While sparsity-enforcing approaches have been proposed for the direction of arrival estimation [24] and acoustic imaging [25], to our knowledge, no method of acoustic imaging uses a fast implementation of $\boldsymbol{\Phi}$. To motivate the need for a fast transform, consider a naive matrix representation of $\Phi$. Given an array of $N$ sensors and an image with $M$ pixels, $\boldsymbol{\Phi}$ has $N^{2}$ rows and $M$ columns. For $N^{2}=M=256^{2}, \boldsymbol{\Phi}$ has 4 billion elements and the products $\boldsymbol{\Phi} \mathbf{u}$ and $\boldsymbol{\Phi}^{H} \mathbf{v}$ are computationally very expensive, making convex optimization methods intractable with current desktop computers. Thus, the naive implementation of $\boldsymbol{\Phi}$ is only practical for very small images and arrays, thus motivating the development of a fast transform.

In this paper, we develop the Kronecker Array Transform (KAT), a fast transform which implements $\boldsymbol{\Phi}, \boldsymbol{\Phi}^{H}$, and $\boldsymbol{\Phi}^{H} \boldsymbol{\Phi}$ for separable arrays. The KAT can be applied to many existing array imaging algorithms, with significant performance gains It also allows for the use of state-of-the-art solvers for acoustic imaging problems, obviating ad-hoc solutions which typically produce worse results. Indeed, with a fast transform, one can use most of the general purpose, state-of-the-art solvers developed for other imaging and compressive sensing applications By combining the KAT with these methods, we can accelerate reconstruction times by several orders of magnitude with respect to the fastest previously available implementations. In practica terms, an image which would take minutes to reconstruct can be obtained in a few seconds. Finally, while this transform was motivated by applications in aeroacoustics, it also applies to generic wave fields and separable sensor arrays.

To our knowledge, previous proposals for accelerated acoustic imaging are all based on beamforming. Zimmerman and Studer [26] propose offloading delay-and-sum beamforming to a field-programmable gate array (FPGA), which performs all of the computation and draws acoustic images over a framebuffer. While this approach makes beamforming faster, it does not reduce its underlying computational cost. Huang [27] uses a state observer model to recursively obtain an approximation of the acoustic image while acquiring data (in contrast to computing an image from a sample covariance matrix). While this method has the advantage of returning incremental results, it has the same computational cost as beamforming, and a comparable beampattern. In contrast, the KAT dramatically reduces the underlying computational costs of acoustic imaging, allowing more accurate reconstruction methods to be used instead of beamforming.

Part I is organized as follows: Section II gives several definitions and further motivates the need for fast transforms. Section III introduces the KAT, its adjoint and its direct-adjoint composition, under the assumption of far-field sources. Section IV presents connections with the fast Fourier transform, fast non-equispaced Fourier transform (NFFT), and fast non-equispaced in time and frequency Fourier transform (NNFFT) [28]. These connections are also a contribution, because to our knowledge, the NFFT and NNFFT have never been used for acoustic imaging. We show how the NFFT and NNFFT can also be used to accelerate acoustic imaging under a far-field approximation, despite being an order of magnitude slower than the KAT. Section $\mathrm{V}$ presents benchmarks comparing the KAT with the NFFT, NNFFT, and explicit matrix representations. Section VI shows how to extend the KAT for near-field imaging, modeling spherical wavefronts instead of planar wavefronts. This generalization is unique to our proposal, and produces a transform which is orders of magnitude faster than direct matrix multiplication (which becomes the only alternative, since the FFT, NFFT, and NNFFT require a far-field approximation). Section VII concludes this paper.

Reference [21, Sec. II] presents applications. Section I briefly reviews the results from Part I. In Section II, we use the KAT to significantly accelerate existing techniques and to enable the use of general-purpose solvers, obtaining more accurate reconstructions than possible with current state-of-the-art methods. Section III features examples and compares several reconstruction methods with respect to computational cost and accuracy. Section IV compares separable arrays with multiarm logarithmic spiral arrays, and shows that by requiring separable arrays, we are not trading reconstruction quality for speed. Finally, Section $\mathrm{V}$ consists of conclusions and final comments.

\section{PRELIMINARIES}

Consider a sensor array composed of $N$ microphones at Cartesian coordinates $\mathbf{p}_{0}, \ldots, \mathbf{p}_{N-1} \in \mathbb{R}^{3}$, and an arbitrary wavefield which we wish to estimate. Suppose that this wave field can be modeled as generated by the superposition of $M$ point sources located at coordinates $\mathbf{q}_{0}, \ldots, \mathbf{q}_{M-1} \in \mathbb{R}^{3}$, where $M$ may be a large number in order to obtain an accurate model. Let

$$
\mathbf{Q}=\left[\begin{array}{llll}
\mathbf{q}_{0} & \mathbf{q}_{1} & \cdots & \mathbf{q}_{M-1}
\end{array}\right]
$$

The time-domain samples of each microphone are segmented into frames of $K$ samples, and each frame is converted to the 
TABLE I

LIST OF SYMBOLS

\begin{tabular}{ll}
\hline Symbol & Meaning \\
\hline$T$ & matrix or vector transpose \\
\hline$H$ & matrix or vector Hermitian transpose \\
\hline$*$ & complex conjugate \\
\hline $\bmod (a, b)$ & remainder of $a / b$, for $a, b \in \mathbb{Z}_{+}$ \\
\hline$\lfloor x\rfloor$ & round-off of $x \in \mathbb{R}$ towards $-\infty$ \\
\hline $\operatorname{vec}\{\cdot\}$ & vectorization operator \\
\hline$\otimes$ & Kronecker product \\
\hline$\odot$ & Hadamard product \\
\hline$M=M_{x} M_{y}$ & number of image pixels \\
\hline$N=N_{x} N_{y}$ & number of array microphones \\
\hline$\omega$ & operating frequency in the Fourier domain \\
\hline $\mathbf{q} m$ & source coordinates, for $0 \leq m<M$ \\
\hline $\mathbf{u}_{m}$ & source coordinates in U-space, for $0 \leq m<M$ \\
\hline $\mathbf{p}_{n}$ & array element coordinates, for $0 \leq n<N$ \\
\hline $\mathbf{v}_{\mathbf{u}_{m}}(\omega)$ & $N \times 1$ array manifold vector for look direction $\mathbf{u}_{m}$ \\
\hline $\mathbf{v}_{x_{n}}(\omega)$ & $N_{x} \times 1$ separable component of $\mathbf{v}_{\mathbf{u}_{m}}(\omega)$ \\
\hline $\mathbf{v}_{y_{n}}(\omega)$ & $N_{y} \times 1$ separable component of $\mathbf{v}_{\mathbf{u}_{m}}(\omega)$ \\
\hline $\mathbf{v}_{x n}^{i}(\omega)$ & $i^{\text {th } \text { element of } \mathbf{v}_{x_{n}}(\omega)}$ \\
\hline $\mathbf{Y}(\omega)$ & $M_{x} \times M_{y}$ image at a frequency $\omega$ \\
\hline $\mathbf{S}(\omega)$ & $N \times N$ spectral matrix at a frequency $\omega$ \\
\hline $\mathbf{B}_{i, j}$ & $(i, j)$ entry of matrix $\mathbf{B}$ \\
\hline $\mathbb{1}$ & matrix of ones \\
\hline
\end{tabular}

frequency domain using a fast Fourier transform (FFT). In the presence of additive noise, the $N \times 1$ array output vector for a single frequency on a single frame can be modeled as

$$
\mathbf{x}\left(\omega_{k}\right)=\mathbf{V}\left(\mathbf{Q}, \omega_{k}\right) \mathbf{f}\left(\omega_{k}\right)+\boldsymbol{\eta}\left(\omega_{k}\right),
$$

where $0 \leq k<K / 2, \mathbf{V}\left(\mathbf{Q}, \omega_{k}\right)=$ $\left[\mathbf{v}\left(\mathbf{q}_{0}, \omega_{k}\right) \mathbf{v}\left(\mathbf{q}_{1}, \omega_{k}\right) \cdots \mathbf{v}\left(\mathbf{q}_{M-1}, \omega_{k}\right)\right]$ is the array manifold matrix, $\mathbf{f}\left(\omega_{k}\right)=\left[f_{0}\left(\omega_{k}\right) f_{1}\left(\omega_{k}\right) \cdots f_{M-1}\left(\omega_{k}\right)\right]^{T}$ is the frequency-domain signal waveform, and $\boldsymbol{\eta}\left(\omega_{k}\right)$ is the frequency-domain noise waveform.

The near-field array manifold vector for source $m$ is given by [29]

$$
\mathbf{v}\left(\mathbf{q}_{m}, \omega_{k}\right)=\left[\begin{array}{lll}
\frac{\mathrm{e}^{-j \frac{\omega_{k}}{c}\left\|\mathbf{p}_{0}-\mathbf{q}_{m}\right\|}}{\left\|\mathbf{p}_{0}-\mathbf{q}_{m}\right\|} \quad \ldots \quad \frac{\mathrm{e}^{-j \frac{\omega_{k}}{c}\left\|\mathbf{p}_{N-1}-\mathbf{q}_{m}\right\|}}{\left\|\mathbf{p}_{N-1}-\mathbf{q}_{m}\right\|}
\end{array}\right]^{T}
$$

where $c$ is the speed of sound.

Define $\mathbf{u}_{m}=\mathbf{q}_{m} /\left\|\mathbf{q}_{m}\right\|$, the look direction for source $m$. Under a far-field approximation (modeling a plane wave), the time differences of arrival is given by $-\mathbf{u}_{m}^{T} \mathbf{p}_{n} / c$, for $0 \leq n<$ $N$. Since the wavefront is not expanding, the $1 /\left\|\mathbf{p}_{n}-\mathbf{q}_{m}\right\|$ attenuation disappears, and the far-field array manifold vector for source $m$ is given by

$$
\mathbf{v}\left(\mathbf{u}_{m}, \omega_{k}\right)=\left[\begin{array}{lll}
\mathrm{e}^{j \frac{\omega_{k}}{c} \mathbf{u}_{m}^{T} \mathbf{p}_{0}} & \cdots & \mathrm{e}^{j \frac{\omega_{k}}{c} \mathbf{u}_{m}^{T} \mathbf{p}_{N-1}}
\end{array}\right]^{T} .
$$

Using spherical coordinates

$$
\mathbf{u}_{m}=\left[\begin{array}{c}
\sin \phi \cos \theta \\
\sin \phi \sin \theta \\
\cos \phi
\end{array}\right]
$$

where $\theta$ and $\phi$ are the azimuth and elevation angles, respectively. One can reparameterize the unit half-sphere by defining

$$
\begin{aligned}
& u_{x}(\theta, \phi)=\sin \phi \cos \theta \\
& u_{y}(\theta, \phi)=\sin \phi \sin \theta
\end{aligned}
$$

so that

$$
\mathbf{u}_{m}=\left[\begin{array}{c}
u_{x} \\
u_{y} \\
\sqrt{1-u_{x}^{2}-u_{y}^{2}}
\end{array}\right]
$$

for $u_{x}^{2}+u_{y}^{2} \leq 1$. Uniform sampling in $\mathrm{U}$-space (where $\mathrm{U}=$ $\left.[-1,1]^{2}\right)$ is convenient in many applications, because under a far-field approximation, it makes point-spread functions shiftinvariant. In this paper, it will enable us to decouple the $x$ and $y$ axes, producing the fast transform.

Since the optimizations presented in the following sections require Cartesian (not necessarily uniform) parameterizations in U-space and far-field approximations, we will assume that manifold vectors have the form (5). In Section VI, we extend our results for near-field sources, which will allow us to approximate (4) with arbitrary accuracy.

Using the assumption of far-field sources, we rewrite (3)as

$$
\mathbf{x}\left(\omega_{k}\right)=\mathbf{V}\left(\omega_{k}\right) \mathbf{f}\left(\omega_{k}\right)+\boldsymbol{\eta}\left(\omega_{k}\right)
$$

where $\mathbf{V}\left(\omega_{k}\right)=\left[\mathbf{v}\left(\mathbf{u}_{0}, \omega_{k}\right) \mathbf{v}\left(\mathbf{u}_{1}, \omega_{k}\right) \cdots \mathbf{v}\left(\mathbf{u}_{M-1}, \omega_{k}\right)\right]$. Let

$$
\mathbf{S}_{\mathbf{x}}\left(\omega_{k}\right)=\mathrm{E}\left\{\mathbf{x}\left(\omega_{k}\right) \mathbf{x}^{H}\left(\omega_{k}\right)\right\}
$$

be the array's narrowband cross spectral matrix for $0 \leq k<$ $K / 2$. If $\mathbf{x}_{0}\left(\omega_{k}\right), \ldots, \mathbf{x}_{L-1}\left(\omega_{k}\right)$ corresponds to $L$ frequency-domain frames (also known as snapshots), the spectral matrix can be estimated with

$$
\mathbf{S}_{\mathbf{x}}\left(\omega_{k}\right)=\frac{1}{L} \sum_{l=0}^{L-1} \mathbf{x}_{l}\left(\omega_{k}\right) \mathbf{x}_{l}^{H}\left(\omega_{k}\right) .
$$

We assume that the statistics of the signal and noise are stationary over the measured period, so that (12) is an unbiased estimator.

Processing $\mathbf{S}_{\mathbf{x}}\left(\omega_{k}\right)$ instead of each $\mathbf{x}_{l}\left(\omega_{k}\right)$ is typically more convenient, because $\mathbf{S}_{\mathbf{x}}\left(\omega_{k}\right)$ carries only the relative phase shifts between microphones and is the result of averaging, so that it has less noise content. Indeed, for each $0 \leq l<L$, $\mathbf{x}_{l}\left(\omega_{k}\right)$ has a phase shift which is equal for every element but unknown, which disappears when computing $\mathbf{S}_{\mathbf{x}}\left(\omega_{k}\right)$. To save space, in the following text, we will assume narrowband processing and omit the argument $\omega_{k}$. Also, the $\mathbf{x}$ subscript will be dropped, and $\mathbf{S}_{\mathbf{x}}\left(\omega_{k}\right)$ will be written as $\mathbf{S}$.

Substituting (10) into (11) and assuming that the noise is spatially white and uncorrelated with the sources of interest, we have

$$
\mathbf{S}_{\mathbf{x}}\left(\omega_{k}\right)=\mathbf{V}\left(\omega_{k}\right) \mathrm{E}\left\{\mathbf{f}\left(\omega_{k}\right) \mathbf{f}^{H}\left(\omega_{k}\right)\right\} \mathbf{V}^{H}\left(\omega_{k}\right)+\sigma^{2} \mathbf{I}
$$

where $\sigma^{2}=\mathrm{E}\left\{\eta_{i}\left(\omega_{k}\right) \eta_{i}^{*}\left(\omega_{k}\right)\right\}, 0 \leq i<N$.

Assume that the wavefield impinging on the array can be modeled as emitted by the superposition of uncorrelated point sources located in the array's far field. One can represent these sources by a collection of points at coordinates $\left\{\mathbf{u}_{i}\right\}_{i=0}^{M-1}$ located in a sufficiently fine grid in U-space. This representation is effectively a 2-D digital image, where the pixel coordinates 
correspond to locations in U-space, and the pixel values correspond to source intensities. Note that in (13), assuming that the sources are uncorrelated implies that $\mathrm{E}\left\{\mathbf{f}\left(\omega_{k}\right) \mathbf{f}^{H}\left(\omega_{k}\right)\right\}$ is diagonal. Furthermore, the diagonal of $\mathrm{E}\left\{\mathbf{f}\left(\omega_{k}\right) \mathbf{f}^{H}\left(\omega_{k}\right)\right\}$ is a vectorized version of the acoustic image.

Given an acoustic image where each pixel corresponds to a point source, one can easily obtain the array spectral matrix as long as all point sources (pixels) are assumed to be pairwise uncorrelated. If there were cross-correlations, one would drop the assumption that $\mathrm{E}\left\{\mathbf{f}\left(\omega_{k}\right) \mathbf{f}^{H}\left(\omega_{k}\right)\right\}$ is diagonal, and require the correlation coefficient for each pair of sources, whose determination would be clearly impractical even for small images (for example, a $64 \times 64$ pixel image would have $64^{2}=4096$ pixels (sources) and $(1 / 2) 4096 \cdot 4097 \approx 8$ million unique cross-correlations). Therefore, unless stated otherwise, we shall assume that sources are pairwise uncorrelated.

However, we note that a fast transform can also be obtained for correlated source distributions as a natural generalization of the KAT. It has special importance because one cannot apply Fourier methods to accelerate the reconstruction of correlated source maps. Nevertheless, since the number of cross-correlations scales quadratically, estimating every cross term is only viable for very simple source distributions. Thus, a proposal for imaging correlated sources should combine a fast transform and domain-specific regularization, the latter being an open problem. Due to space limitations, we will not address these topics in this text.

Recall that to solve (1) efficiently, one requires a fast method of obtaining $\mathbf{S}$ from a hypothetical image. Consider an $M_{x} \times M_{y}$ pixel acoustic image, define $M=M_{x} M_{y}$ and let $\mathbf{u}_{0}, \ldots, \mathbf{u}_{M-1}$ be an enumeration of all pixel coordinates in U-space. Let $\mathbf{v}\left(\mathbf{u}_{m}\right)$ be the array manifold vector when steered towards the look direction $\mathbf{u}_{m}$. For a single source at $\mathbf{u}_{m}$ radiating with power $\left|Y\left(\mathbf{u}_{m}\right)\right|^{2}$, the measured spectral matrix is $\mathbf{S}=\left|Y\left(\mathbf{u}_{m}\right)\right|^{2} \mathbf{v}\left(\mathbf{u}_{m}\right) \mathbf{v}^{H}\left(\mathbf{u}_{m}\right)$. Given the source powers for $\mathbf{u}_{0}, \ldots, \mathbf{u}_{M-1}$, one can reconstruct $\mathbf{S}$ by superposition, so that

$$
\mathbf{S}=\sum_{m=0}^{M-1}\left|Y\left(\mathbf{u}_{m}\right)\right|^{2} \mathbf{v}\left(\mathbf{u}_{m}\right) \mathbf{v}^{H}\left(\mathbf{u}_{m}\right) .
$$

Unless the image is very sparse, this expression becomes computationally intractable. For instance, consider a 256 element array and a $256 \times 256$ acoustic image. Each $\left|Y\left(\mathbf{u}_{m}\right)\right|^{2} \mathbf{v}\left(\mathbf{u}_{m}\right) \mathbf{v}^{H}\left(\mathbf{u}_{m}\right)$ outer product generates a $256 \times 256$ matrix. Neglecting the cost to scale $\mathbf{v}\left(\mathbf{u}_{m}\right)$ by $\left|Y\left(\mathbf{u}_{m}\right)\right|$, the outer product requires $2^{16}$ complex multiply-accumulate (MAC) instructions. ${ }^{1}$ This process must be repeated $M=2^{16}$ times, resulting in $2^{32}$ complex MACs. Since each outer product has Hermitian symmetry, it suffices to determine its upper or lower triangular part (including the main diagonal), which reduces the total complex MAC count to approximately $2^{31}$. Nevertheless, this computational cost is still excessive for a transform intended to be used in an iterative method. In

${ }^{1}$ Modern digital-signal-processing (DSP) architectures are able to implement a multiplication followed by an accumulation in the same clock cycle. This single cycle instruction is known as a MAC. Since the computational cost of performing a sum, product, or MAC is the same, for the purposes of estimating computational complexity, it suffices to estimate the total number of MACs. the following text, we describe how to implement an efficient transform to obtain $\mathbf{S}$ from $\left\{\left|Y\left(\mathbf{u}_{m}\right)\right|^{2}\right\}_{0 \leq m<M}$.

\section{KRONECKER ARRAY TRANSFORM}

Define $\mathbf{y}=\left[\left|Y\left(\mathbf{u}_{0}\right)\right|^{2} \cdots\left|Y\left(\mathbf{u}_{M-1}\right)\right|^{2}\right]^{T}$. Let us write (14) as a linear transform $\mathbf{A}$ so that $\mathbf{s}=\mathbf{A y}$, with $\mathbf{s}=\operatorname{vec}\{\mathbf{S}\}$. To save space, we will write $\mathbf{v}\left(\mathbf{u}_{m}\right)$ as $\mathbf{v}_{\mathbf{u}_{m}}$, and will denote its $i$ th element by $v_{u_{m}}^{i}$ (elements of array manifold vectors will be indexed using superscripts). Let $N$ be the number of microphones in the array. Note that

$$
\operatorname{vec}\left\{\mathbf{v}_{\mathbf{u}_{m}} \mathbf{v}_{\mathbf{u}_{m}}^{H}\right\}=\left[\begin{array}{c}
\mathbf{v}_{\mathbf{u}_{m}} \mathbf{v}_{\mathbf{u}_{m}}^{0 *} \\
\mathbf{v}_{\mathbf{u}_{m}} \mathbf{v}_{\mathbf{u}_{m}}^{1 *} \\
\vdots \\
\mathbf{v}_{\mathbf{u}_{m}} \mathbf{v}_{\mathbf{u}_{m}}^{(N-1) *}
\end{array}\right] .
$$

Therefore

$$
\begin{aligned}
& \mathbf{s}=\mathbf{A y} \\
& =\left[\begin{array}{cccc}
\mathbf{v}_{\mathbf{u}_{0}} \mathrm{v}_{\mathbf{u}_{0}}^{0 *} & \mathbf{v}_{\mathbf{u}_{1}} \mathbf{v}_{\mathbf{u}_{1}}^{0 *} & \cdots & \mathbf{v}_{\mathbf{u}_{M-1}} \mathrm{v}_{\mathbf{u}_{M-1}}^{0 *} \\
\mathbf{v}_{\mathbf{u}_{0}} \mathrm{v}_{\mathbf{u}_{0}}^{1 *} & \mathbf{v}_{\mathbf{u}_{1}} \mathbf{v}_{\mathbf{u}_{1}}^{1 *} & \cdots & \mathbf{v}_{\mathbf{u}_{M-1}} \mathrm{v}_{\mathbf{u}_{M-1}}^{1 *} \\
\vdots & \vdots & & \vdots \\
\mathbf{v}_{\mathbf{u}_{0}} \mathbf{v}_{\mathbf{u}_{0}}^{(N-1) *} & \mathbf{v}_{\mathbf{u}_{1}} \mathbf{v}_{\mathbf{u}_{1}}^{(N-1) *} & \cdots & \mathbf{v}_{\mathbf{u}_{M-1}} \mathbf{v}_{\mathbf{u}_{M-1}}^{(N-1) *}
\end{array}\right] \mathbf{y} \\
& =\left[\begin{array}{llll}
\mathbf{v}_{\mathbf{u}_{0}}^{*} \otimes \mathbf{v}_{\mathbf{u}_{0}} & \mathbf{v}_{\mathbf{u}_{1}}^{*} \otimes \mathbf{v}_{\mathbf{u}_{1}} & \cdots
\end{array}\right. \\
& \left.\mathbf{v}_{\mathbf{u}_{M-1}}^{*} \otimes \mathbf{v}_{\mathbf{u}_{M-1}}\right] \mathbf{y},
\end{aligned}
$$

where $\otimes$ is the Kronecker product.

Given a 2-D array, its array manifold vector $\mathbf{v}(\mathbf{u})=$ $\mathbf{v}\left(u_{x}, u_{y}\right)$ is said to be separable if $\mathbf{a}\left(u_{x}\right)$ exists and $\mathbf{b}\left(u_{x}\right)$ so that $\mathbf{v}\left(u_{x}, u_{y}\right)=\mathbf{a}\left(u_{x}\right) \otimes \mathbf{b}\left(u_{y}\right)$ for all valid $u_{x}, u_{y}$. Note that $\mathbf{a}\left(u_{x}\right)$ and $\mathbf{b}\left(u_{y}\right)$ need not be submanifold vectors. We say that an array is separable if and only if it has a separable manifold vector. We will show below how the array geometry relates to its separability under a far-field assumption.

To simplify the notation that follows, let us specify the enumeration $\mathbf{u}_{0}, \ldots, \mathbf{u}_{M-1}$ of look directions we are using. Suppose that $\mathbf{Y}$ is a $M_{x} \times M_{y}$ digital image representing the acoustic image. The rows of $\mathbf{Y}$ correspond to horizontal scan lines of arbitrarily sampled pixels, and the columns of $\mathbf{Y}$ correspond to vertical scan lines of arbitrarily sampled pixels. Let $\left\{u_{x_{m}}\right\}_{0 \leq m<M_{x}}$ and $\left\{u_{y_{n}}\right\}_{0 \leq n<M_{y}}$ be points which sample the U-space along the $x$ and $y$ axes, ordered from left to right and from top to bottom. We define $\mathbf{u}_{0}, \ldots, \mathbf{u}_{M-1}$ so that

$$
\mathbf{y}=\operatorname{vec}\{\mathbf{Y}\}=\left[\begin{array}{c}
\left|Y\left(\mathbf{u}_{0}\right)\right|^{2} \\
\left|Y\left(\mathbf{u}_{1}\right)\right|^{2} \\
\vdots \\
\left|Y\left(\mathbf{u}_{M-1}\right)\right|^{2}
\end{array}\right]
$$

Breaking $\mathbf{u}$ into components, this implies that

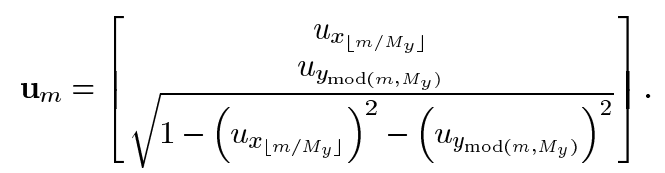




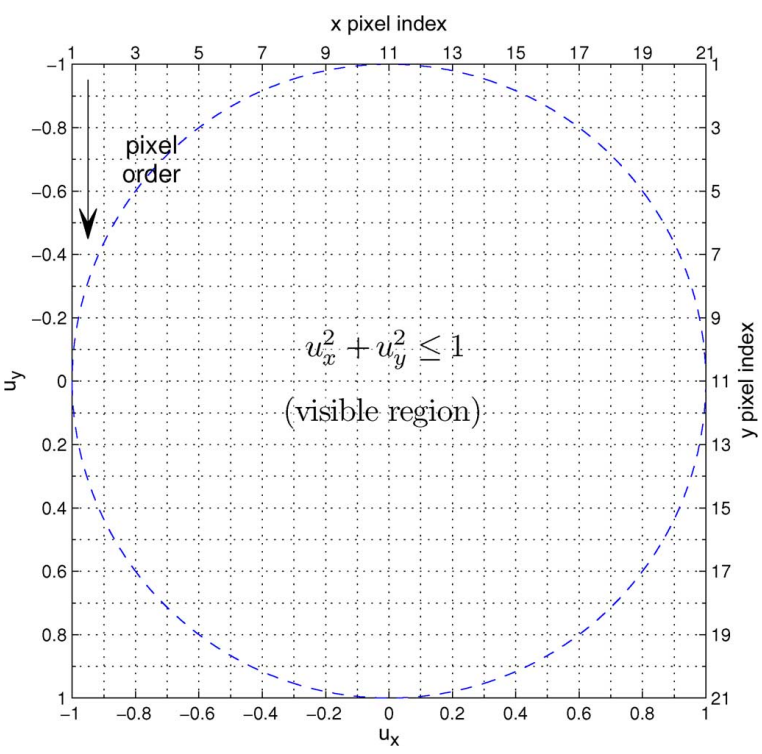

Fig. 1. Example of pixel order and U-space parameterization for an acoustic image, for $M_{x}=M_{y}=21$ and uniform sampling in the U-space.

Fig. 1 shows an example of how pixels are ordered and parameterized in U-space.

We now show that under the far-field parameterization given by (5), an array is separable if and only if it has elements positioned over a (potentially nonuniform) Cartesian grid.

To see this, consider an array with sensor coordinates $\mathbf{p}_{i} \in$ $\mathbb{R}^{3}$, for $0 \leq i<N$, with $x$ and $y$ coordinates drawn from $\left\{p_{x_{i}}\right\}_{i=0}^{N_{x}-1}$ and $\left\{p_{y_{i}}\right\}_{i=0}^{N_{y}-1}$, respectively, so that

$$
\mathbf{p}_{i}=\left[\begin{array}{lll}
p_{x_{\left\lfloor i / N_{y}\right\rfloor}} & p_{y_{\bmod \left(i, N_{y}\right)}} & 0
\end{array}\right]^{T} .
$$

Let $N=N_{x} N_{y}$ be the number of array elements. Define a horizontal array with sensor coordinates $\mathbf{p}_{x_{i}} \in \mathbb{R}^{3}$, for $0 \leq i<$ $N_{x}$ and a vertical array with sensor coordinates $\mathbf{p}_{y_{j}} \in \mathbb{R}^{3}$, for $0 \leq j<N_{y}$, so that

$$
\mathbf{p}_{x_{i}}=\left[\begin{array}{lll}
p_{x_{i}} & 0 & 0
\end{array}\right]^{T} \quad \mathbf{p}_{y_{j}}=\left[\begin{array}{lll}
0 & p_{y_{j}} & 0
\end{array}\right]^{T} .
$$

Let $\mathbf{v}_{x}$ and $\mathbf{v}_{y}$ be the $N_{x} \times 1$ and $N_{y} \times 1$ manifold vectors for these 1-D arrays. Then, for $0 \leq i<N_{x}$ and $0 \leq j<N_{y}$

$$
\begin{aligned}
& {\left[\mathbf{v}\left(u_{x}, u_{y}\right)\right]_{i \cdot N_{y}+j}} \\
& \left.=\mathrm{e}^{j \frac{\omega_{k}}{c}\left[u_{x}\right.} u_{y} \sqrt{1-u_{x}^{2}-u_{y}^{2}}\right] \mathbf{p}_{i \cdot N_{y}+j}
\end{aligned}
$$

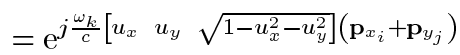

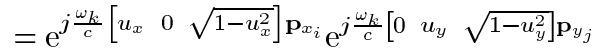

$$
\begin{aligned}
& =\left[\mathbf{v}_{x}\left(u_{x}\right)\right]_{i}\left[\mathbf{v}_{y}\left(u_{y}\right)\right]_{j}
\end{aligned}
$$

which, by definition, is equivalent to

$$
\mathbf{v}\left(u_{x}, u_{y}\right)=\mathbf{v}_{x}\left(u_{x}\right) \otimes \mathbf{v}_{y}\left(u_{y}\right) .
$$

Thus, arrays with Cartesian geometries are separable under $\mathrm{U}$-space parameterization. To prove the converse, note that

$$
\begin{aligned}
{\left[\mathbf{v}\left(u_{x}, u_{y}\right)\right]_{i} } & \left.=\mathrm{e}^{j \frac{\omega_{k}}{c}\left[u_{x}\right.} u_{y} \sqrt{1-u_{x}^{2}-u_{y}^{2}}\right] \mathbf{p}_{i} \\
& =\mathrm{e}^{j \frac{\omega_{k}}{c}\left(u_{x} \bar{p}_{x_{i}}+u_{y} \bar{p}_{y_{i}}\right)} \\
& =\mathrm{e}^{j \frac{\omega_{k}}{c} u_{x} \bar{p}_{x_{i}}} \mathrm{e}^{j \frac{\omega_{k}}{c} u_{y} \bar{p}_{y_{i}}}
\end{aligned}
$$

where $\mathbf{p}_{i}=\left[\bar{p}_{x_{i}} \bar{p}_{y_{i}} 0\right]^{T}$. By hypothesis, $\mathbf{a}\left(u_{x}\right)$ and $\mathbf{b}\left(u_{x}\right)$ exist so that $\mathbf{v}\left(u_{x}, u_{y}\right)=\mathbf{a}\left(u_{x}\right) \otimes \mathbf{b}\left(u_{y}\right)$. The term $u_{x} \bar{p}_{x_{i}}$ from (21) must belong to $\mathbf{a}\left(u_{x}\right)$, since it is a function of $u_{x}$, and $\bar{p}_{x_{i}}$ is constant. It follows that $\mathbf{a}\left(u_{x}\right)=\mathbf{v}_{x}\left(u_{x}\right)$ and $\mathbf{b}\left(u_{y}\right)=$ $\mathbf{v}_{y}\left(u_{y}\right)$, with $\mathbf{v}_{x}\left(u_{x}\right)$ and $\mathbf{v}_{y}\left(u_{y}\right)$ defined before, imply a Cartesian geometry.

\section{A. Fast Direct Transform}

To save space, we will use the shorthand notation

$$
\begin{aligned}
\mathbf{v}_{x}\left(u_{x_{m}}\right) & =\mathbf{v}_{x_{m}}=\left[\begin{array}{llll}
\mathrm{v}_{x_{m}}^{0} & \mathrm{v}_{x_{m}}^{1} & \cdots & \mathrm{v}_{x_{m}}^{N_{x}-1}
\end{array}\right]^{T} \\
\mathbf{v}_{\boldsymbol{y}}\left(u_{y_{n}}\right) & =\mathbf{v}_{y_{n}}=\left[\begin{array}{lllll}
\mathrm{v}_{\boldsymbol{y}_{n}}^{0} & \mathrm{v}_{\boldsymbol{y}_{n}}^{1} & \cdots & \mathrm{v}_{\boldsymbol{y}_{n}}^{N_{y}-1}
\end{array}\right]^{T} .
\end{aligned}
$$

Using the separability of the array in (17), we obtain

$$
\begin{aligned}
\mathbf{A}=[ & \left(\mathbf{v}_{x_{0}}^{*} \otimes \mathbf{v}_{y_{0}}^{*}\right) \otimes\left(\mathbf{v}_{x_{0}} \otimes \mathbf{v}_{y_{0}}\right) \cdots \\
& \left.\ldots\left(\mathbf{v}_{x_{M-1}}^{*} \otimes \mathbf{v}_{y_{M-1}}^{*}\right) \otimes\left(\mathbf{v}_{x_{M-1}} \otimes \mathbf{v}_{y_{M-1}}\right)\right] .
\end{aligned}
$$

For $0 \leq m, n<N_{x} N_{y}$, the separability of the array also allows row $m \cdot N_{x} N_{y}+n$ of $\mathbf{A}$ to be written as

$$
\left[\mathrm{v}_{x_{0}}^{i *} \mathrm{v}_{x_{0}}^{j} \cdots \mathrm{v}_{x_{M_{x}-1}}^{i *} \mathrm{v}_{x_{M_{x}-1}}^{j}\right] \otimes\left[\mathrm{v}_{y_{0}}^{k *} \mathrm{v}_{y_{0}}^{l} \cdots \mathrm{v}_{y_{M_{y}-1}}^{k *} \mathrm{v}_{y_{M_{y}-1}}^{l}\right. \text { (24) }
$$

where $i=\left\lfloor m / N_{y}\right\rfloor, j=\left\lfloor n / N_{y}\right\rfloor, k=\bmod \left(m, N_{y}\right)$, and $l=\bmod \left(n, N_{y}\right)$.

For $0 \leq i, j<N_{x}$ and $0 \leq k, l<N_{y}$, define

$$
c_{m}(i, j)=\mathrm{v}_{x_{m}}^{i *} \mathrm{v}_{x_{m}}^{j} \quad d_{n}(k, l)=\mathrm{v}_{y_{n}}^{k *} \mathrm{v}_{y_{n}}^{l} .
$$

For $0 \leq m, n<N_{x} N_{y}$, an arbitrary element $\mathbf{S}_{n, m}$ of $\mathbf{S}$ can be written as the inner product of line $m \cdot N_{x} N_{y}+n$ of $\mathbf{A}$ and $\operatorname{vec}\{\mathbf{Y}\}$. Define

$$
\begin{aligned}
\mathbf{c}(i, j) & =\left[\begin{array}{lll}
c_{0}(i, j) & \cdots & c_{M_{x}-1}(i, j)
\end{array}\right]^{T} \\
& =\left[\begin{array}{lll}
\mathrm{v}_{x_{0}}^{i *} \mathrm{v}_{x_{0}}^{j} & \cdots & \mathrm{v}_{x_{M_{x}-1}}^{i *} \mathrm{v}_{x_{M_{x}-1}}^{j}
\end{array}\right]^{T} \\
\mathbf{d}(k, l) & =\left[\begin{array}{lll}
d_{0}(k, l) & \cdots & d_{M_{y}-1}(k, l)
\end{array}\right]^{T} \\
& =\left[\begin{array}{lll}
\mathrm{v}_{y_{0}}^{k *} \mathrm{v}_{y_{0}}^{l} & \cdots & \mathrm{v}_{y_{M_{y}-1}}^{k *} \mathrm{v}_{y_{M_{y}-1}}^{l}
\end{array}\right]^{T} .
\end{aligned}
$$

Using (24), we have

$$
\begin{aligned}
\mathbf{S}_{n, m} & =\left[\mathbf{c}^{T}(i, j) \otimes \mathbf{d}^{T}(k, l)\right] \operatorname{vec}\{\mathbf{Y}\} \\
& =\mathbf{d}^{T}(k, l) \mathbf{Y} \mathbf{c}(i, j)
\end{aligned}
$$

where $i=\left\lfloor m / N_{y}\right\rfloor, j=\left\lfloor n / N_{y}\right\rfloor, k=\bmod \left(m, N_{y}\right)$, and $l=\bmod \left(n, N_{y}\right)$. Also, (26) and (27) are equivalent because 
$\left(\mathbf{A}^{T} \otimes \mathbf{B}\right) \operatorname{vec}\{\mathbf{C}\}=\operatorname{vec}\{\mathbf{B C A}\}$ whenever BCA is defined [30].

Note that $\mathbf{c}(i, j)$ and $\mathbf{d}(k, l)$ completely model the response of the $(n, m)$ pair of sensors, for all directions of arrival. This is already a more compact representation than before, since this model uses the separability of the array. All that is left is how to efficiently compute all of the responses for all pairs.

For $0 \leq i, j<N_{x}$ and $0 \leq k, l<N_{y}$, define

$$
(i, j) \diamond(k, l)=\mathbf{d}^{T}(k, l) \mathbf{Y} \mathbf{c}(i, j)
$$

and

$\mathbf{T}_{j, i}=\left[\begin{array}{ccc}(i, j) \diamond(0,0) & \cdots & (i, j) \diamond\left(N_{y}-1,0\right) \\ (i, j) \diamond(0,1) & \cdots & (i, j) \diamond\left(N_{y}-1,1\right) \\ \vdots & & \vdots \\ (i, j) \diamond\left(0, N_{y}-1\right) & \cdots & (i, j) \diamond\left(N_{y}-1, N_{y}-1\right)\end{array}\right]$

From the results above, it is easy to show that

$$
\mathbf{S}=\left[\begin{array}{cccc}
\mathbf{T}_{0,0} & \mathbf{T}_{0,1} & \cdots & \mathbf{T}_{0, N_{x}-1} \\
\mathbf{T}_{1,0} & \mathbf{T}_{1,1} & \cdots & \mathbf{T}_{1, N_{x}-1} \\
\vdots & \vdots & & \vdots \\
\mathbf{T}_{N_{x}-1,0} & \mathbf{T}_{N_{x}-1,1} & \cdots & \mathbf{T}_{N_{x}-1, N_{x}-1}
\end{array}\right]
$$

Even though one could determine $(i, j) \diamond(k, l)$ for $0 \leq i, j<N_{x}$ and $0 \leq k, l<N_{y}$ by directly evaluating (28), one should organize the computations to eliminate redundancy. Also, since in modern computer architectures the arithmetic units can process data faster than the main memory can provide via random accesses, one should maximize the locality of reference to ensure that the arithmetic operands are typically in the cache. In particular, the algorithm should promote sequential memory accesses so that the arithmetic units do not stall while waiting for a memory read. We will present this implementation below.

Let

$$
\begin{aligned}
\mathbf{t}_{i, j} & =\operatorname{vec}\left\{\mathbf{T}_{i, j}\right\} \\
\mathbf{Z} & =\left[\begin{array}{llll}
\mathbf{t}_{0,0} & \mathbf{t}_{1,0} & \ldots & \mathbf{t}_{N_{x}-1, N_{x}-1}
\end{array}\right] .
\end{aligned}
$$

Given $\mathbf{Z}$, it is very easy to obtain $\mathbf{S}$, since every block $\mathbf{T}_{i, j}$ of $\mathbf{S}$ can be obtained by unstacking $\mathbf{t}_{i, j}$.
Define (33) and (34), shown in the equation at the bottom of the page. In comparison with (28), one can verify that

$$
\mathbf{Z}=\mathbf{V}_{\mathbf{y}} \mathbf{Y} \mathbf{V}_{\mathbf{x}}^{T}
$$

Define $\boldsymbol{\Xi}$ so that $\operatorname{vec}\{\mathbf{S}\}=\boldsymbol{\Xi} \operatorname{vec}\{\mathbf{Z}\}$. (Note that $\boldsymbol{\Xi}$ is a permutation.) Thus, $\operatorname{vec}\{\mathbf{S}\}=\mathbf{\Xi}\left(\mathbf{V}_{\mathbf{x}} \otimes \mathbf{V}_{\mathbf{y}}\right) \operatorname{vec}\{\mathbf{Y}\}$ and

$$
\mathbf{A}=\mathbf{\Xi}\left(\mathbf{V}_{\mathbf{x}} \otimes \mathbf{V}_{\mathbf{y}}\right) .
$$

Since $\boldsymbol{\Xi}$ is a computationally efficient permutation and $\left(\mathbf{V}_{\mathbf{x}} \otimes\right.$ $\left.\mathbf{V}_{\mathbf{y}}\right) \operatorname{vec}\{\mathbf{Y}\}=\operatorname{vec}\left\{\mathbf{V}_{\mathbf{y}} \mathbf{Y} \mathbf{V}_{\mathbf{x}}^{T}\right\}$, (36) can be implemented as a fast transform (as follows).

From (30), it can be seen that each $\mathbf{T}_{i, j}$ contains the crosscovariance between two $N_{y} \times 1$ columns of sensors. Thus, $\mathbf{Z}$ is a reorganization of $\mathbf{S}$ which stacks these cross-covariances with a regularity that matches the row order of $\mathbf{V}_{\mathbf{x}} \otimes \mathbf{V}_{\mathbf{y}}$ (since $\left.\operatorname{vec}\{\mathbf{Z}\}=\left(\mathbf{V}_{\mathbf{x}} \otimes \mathbf{V}_{\mathbf{y}}\right) \operatorname{vec}\{\mathbf{Y}\}\right)$.

We now make some remarks regarding computational cost. The direct product $\mathbf{A y}$ in (16) requires approximately $(1 / 2) M_{x} M_{y} N_{x}^{2} N_{y}^{2}$ complex MACs when considering the Hermitian symmetry $\mathbf{S}$. Evaluating $\left(\mathbf{V}_{\mathbf{y}} \mathbf{Y}\right) \mathbf{V}_{\mathbf{x}}^{T}$ and $\mathbf{V}_{\mathbf{y}}\left(\mathbf{Y} \mathbf{V}_{\mathbf{x}}^{T}\right)$ requires $N_{y}^{2} M_{x} M_{y}+N_{x}^{2} N_{y}^{2} M_{x}$ and $N_{x}^{2} M_{x} M_{y}+N_{x}^{2} N_{y}^{2} M_{y}$ complex MACs, respectively. Since $\mathbf{Y}$ is real-valued, the first product can be optimized and the costs drop to $(1 / 2) N_{y}^{2} M_{x} M_{y}+N_{x}^{2} N_{y}^{2} M_{x}$ and $(1 / 2) N_{x}^{2} M_{x} M_{y}+N_{x}^{2} N_{y}^{2} M_{y}$ complex MACs, respectively. Using the first expression and neglecting the time to obtain $\mathbf{S}$ from $\mathbf{Z}$, the relative speedup in terms of MACs is given by

$$
\frac{\frac{1}{2} M_{x} M_{y} N_{x}^{2} N_{y}^{2}}{\frac{1}{2} N_{y}^{2} M_{x} M_{y}+N_{x}^{2} N_{y}^{2} M_{x}}=\frac{M_{y} N_{x}^{2}}{M_{y}+2 N_{x}^{2}} .
$$

If the array geometry is symmetric with respect to the $y$ axis, then $\mathbf{V}_{x}$ has conjugate symmetry with respect to its middle row. An analogous statement applies to $\mathbf{V}_{y}$. If applicable, these symmetries can be used to further reduce the computational cost.

Recall that we introduced $\mathbf{Y}$ as having scan lines which realize an arbitrary Cartesian sampling of U-space. If $\left\{u_{x_{i}}\right\}$ and $\left\{u_{y_{i}}\right\}$ uniformly sample $\mathbf{U}$-space, then $\mathbf{V}_{\mathbf{x}}$ and $\mathbf{V}_{\mathbf{y}}$ can be interpreted as DFT matrices for nonuniform frequency sampling. (This fact can be verified by explicitly writing $\mathbf{V}_{\mathbf{x}}$ and $\mathbf{V}_{\mathbf{y}}$ in terms of complex exponentials.) Therefore, for sufficiently large values of $N_{x}$ and $N_{y}$, a further optimization consists of using a

$$
\begin{array}{r}
\mathbf{V}_{\mathbf{x}}=\left[\begin{array}{ccc}
c_{0}(0,0) & \cdots & c_{M_{x}-1}(0,0) \\
c_{0}(0,1) & \cdots & c_{M_{x}-1}(0,1) \\
\vdots & & \vdots \\
c_{0}\left(N_{x}-1, N_{x}-1\right) & \cdots & c_{M_{x}-1}\left(N_{x}-1, N_{x}-1\right)
\end{array}\right] \\
\mathbf{V}_{\mathbf{y}}=\left[\begin{array}{ccc}
d_{0}(0,0) & \cdots & d_{M_{y}-1}(0,0) \\
d_{0}(0,1) & \cdots & d_{M_{y}-1}(0,1) \\
\vdots & & \vdots \\
d_{0}\left(N_{y}-1, N_{y}-1\right) & \cdots & d_{M_{y}-1}\left(N_{y}-1, N_{y}-1\right)
\end{array}\right] .
\end{array}
$$


fast nonequispaced Fourier transform (NFFT) [28] instead of each matrix product in (35). A rule of thumb obtained from numerical experiments is to use the NFFT for $N_{x}>8$ or $N_{y}>8$ and $M_{x}>2^{8}$ or $M_{y}>2^{8}$. Details regarding the performance with and without the NFFT are presented in Section V.

\section{B. Fast Adjoint Transform}

As we present [21, Sec. II], with a measured spectral matrix S, many computationally efficient image reconstruction methods require only fast implementations of $\mathbf{A}$ and $\mathbf{A}^{H}$ to estimate a source distribution $\hat{\mathbf{Y}}$. A computationally efficient reconstruction algorithm must have fast implementations of both; otherwise, the slow transform becomes the bottleneck for the solver.

Let $\overline{\mathbf{S}} \in \mathbb{C}^{N \times N}$ and $\overline{\mathbf{Y}} \in \mathbb{R}^{M_{y} \times M_{x}}$ so that $\operatorname{vec}\{\overline{\mathbf{Y}}\}=$ $\mathbf{A}^{H} \operatorname{vec}\{\overline{\mathbf{S}}\}$. It follows from (36) that:

$$
\mathbf{A}^{H}=\left(\mathbf{V}_{\mathbf{x}}^{H} \otimes \mathbf{V}_{\mathbf{y}}^{H}\right) \boldsymbol{\Xi}^{H} \text {. }
$$

Since $\boldsymbol{\Xi}$ is a permutation, $\boldsymbol{\Xi}^{-1}=\boldsymbol{\Xi}^{T}=\boldsymbol{\Xi}^{H}$. If $\operatorname{vec}\{\overline{\mathbf{Z}}\}=$ $\boldsymbol{\Xi}^{T} \operatorname{vec}\{\overline{\mathbf{S}}\}$, then

$$
\overline{\mathbf{Y}}=\mathbf{V}_{\mathbf{y}}^{H} \overline{\mathbf{Z}} \mathbf{V}_{\mathbf{x}}^{*}
$$

which is the fast implementation of $\mathbf{A}^{H}$. (Note that it has the same computational cost as the direct transform.)

If the U-space sampling is symmetric with respect to the $y$ axis, then $\mathbf{V}_{\mathbf{x}}$ has conjugate symmetry with respect to its center column. An analogous statement applies to $\mathbf{V}_{\mathbf{y}}$. If applicable, this symmetry can be used to further reduce the computational cost.

For separable arrays which are uniformly sampled in $\mathrm{U}$-space, multiplication by $\mathbf{V}_{\mathbf{x}}$ and $\mathbf{V}_{\mathbf{y}}$ can again be optimized by using NFFTs, under the same considerations presented for the direct transform.

\section{Fast Direct-Adjoint Transform}

Given the direct transform $\mathbf{A}$ and its adjoint $\mathbf{A}^{H}$, consider the transform given by $\mathbf{A}^{H} \mathbf{A}$. This composition will be used in [21, Sec. II] for image reconstruction, and in this section, we present a method of accelerating it further. Since $\Xi^{H}=\Xi^{-1}$, it follows from the previous results that $\operatorname{vec}\{\overline{\mathbf{Y}}\}=\mathbf{A}^{H} \mathbf{A} \operatorname{vec}\{\mathbf{Y}\}$ can be implemented as

$$
\overline{\mathbf{Y}}=\mathbf{V}_{\mathbf{y}}^{H} \mathbf{V}_{\mathbf{y}} \mathbf{Y} \mathbf{V}_{\mathbf{x}}^{T} \mathbf{V}_{\mathbf{x}}^{*}
$$

This implementation is especially interesting when $N_{x}$ and $N_{y}$ are sufficiently large in comparison to $M_{x}$ and $M_{y}$, because it can be evaluated as

$$
\overline{\mathbf{Y}}=\left(\mathbf{V}_{\mathbf{y}}^{H} \mathbf{V}_{\mathbf{y}}\right) \mathbf{Y}\left(\mathbf{V}_{\mathbf{x}}^{T} \mathbf{V}_{\mathbf{x}}^{*}\right)
$$

with precomputed versions of $\mathbf{V}_{\mathbf{y}}^{H} \mathbf{V}_{\mathbf{y}}$ and $\mathbf{V}_{\mathbf{x}}^{T} \mathbf{V}_{\mathbf{x}}^{*}$, which are real valued.

Implementing the direct-adjoint transform with (39) can be much faster than using a composition of the direct and adjoint KAT, because for large problems, one can precompute $\mathbf{V}_{\mathbf{y}}^{H} \mathbf{V}_{\mathbf{y}}$ and $\mathbf{V}_{\mathbf{x}}^{T} \mathbf{V}_{\mathbf{x}}^{*}$. Furthermore, (39) can be parallelized more effectively, since it avoids applying $\mathbf{\Xi}$.
TABLE II

APPLICABILITY OF THE FFT, NFFT, NNFFT, AND KAT

\begin{tabular}{|l|c|c|c|}
\hline Transform & Geometry & U-space sampling & Exact? \\
\hline \hline FFT & Uniform & Uniform & Yes \\
\hline NFFT & Arbitrary & Uniform & No \\
\hline NNFFT & Arbitrary & Arbitrary & No \\
\hline KAT & Separable & Separable & Yes \\
\hline
\end{tabular}

The implementations, which use the NFFT for further acceleration, are at a disadvantage for the direct-adjoint transform, since one cannot precompute the equivalent of $\mathbf{V}_{\mathbf{y}}^{H} \mathbf{V}_{\mathbf{y}}$ and $\mathbf{V}_{\mathbf{x}}^{T} \mathbf{V}_{\mathbf{x}}^{*}$. Therefore, one is forced to use a composition of the previously presented transforms.

\section{CONNECTIONS}

In this section, we briefly describe how the KAT relates to the 2-D FFT, NFFT, and NNFFT. To our knowledge, the NFFT and NNFFT have never been applied to acoustic imaging. With the exception of the NNFFT, each transform is only suitable for specific array geometries or U-space sampling patterns. Transforms, which make more restrictive assumptions about the array geometry and U-space pattern, can generally be more computationally efficient, so the choice of which transform to use depends on a series of tradeoffs, summarized in Table II.

Assume that the sampled wavefield is a zero-mean random process which is stationary in time and homogenous in space. ${ }^{2}$ Consider a sensor array consisting of $N$ microphones at coordinates $\mathbf{p}_{0}, \ldots, \mathbf{p}_{N-1} \in \mathbb{R}^{3}$. For a fixed frequency $\omega$, the crossspectral matrix $\mathbf{S}(\omega)=\mathrm{E}\left\{\mathbf{x}(\omega) \mathbf{x}^{H}(\omega)\right\}$ is, by definition, a covariance matrix. For $0 \leq m, n<N$, $[\mathbf{S}(\omega)]_{m, n}$ holds the frequency-domain cross-covariance of the wavefield between any two points whose coordinates differ by $\mathbf{p}_{m}-\mathbf{p}_{n}$. Let $P(\omega, \mathbf{k})=$ $|Y(\omega, \mathbf{k})|^{2}$ be the power spectral density when parameterized as a function of the wave number $\mathbf{k}=-(\omega / c) \mathbf{u} \in \mathbb{R}^{3}$, and $S(\omega, \Delta \mathbf{p})$ be the spectral covariance between two points whose coordinates differ by $\Delta \mathbf{p}$. It can be shown [29] that

$$
\begin{gathered}
P(\omega, \mathbf{k})=\int_{\mathbb{R}^{3}} S(\omega, \Delta \mathbf{p}) e^{+j \mathbf{k}^{T} \Delta \mathbf{p}} d \Delta \mathbf{p} \\
S(\omega, \Delta \mathbf{p})=\frac{1}{(2 \pi)^{3}} \int_{\mathbb{R}^{3}} P(\omega, \mathbf{k}) e^{-j \mathbf{k}^{T} \Delta \mathbf{p}} d \mathbf{k}
\end{gathered}
$$

which is essentially a generalization of the relationship between the cross-covariance and cross-spectral density for wide-sense stationary spatial-temporal processes, and is expressed as a Fourier transform.

Therefore, the knowledge of $S(\omega, \Delta \mathbf{p})$, limited to a finite set of baselines $\Delta \mathbf{p}$, allows us to approximate a discrete space version of $P(\omega, \mathbf{k})$, which is the image of interest. The following connections arise naturally from different ways of sampling these relations in order to evaluate them numerically for discrete space and discrete U-space.

${ }^{2}$ The random process model follows naturally from the fact that we do not know a priori what the source waveforms are. We model this process using its second-order statistics given by the cross-spectral matrix. Stationarity in time and homogeneity in space let us estimate the power spectral density of the process as a function of $\omega$ and $\mathbf{k}$, which is the acoustic image of interest. 


\section{A. NFFT Imaging}

A d-dimensional NDFT [28] (nonequispaced discrete Fourier transform) is defined by a set of arbitrary spatial nodes $\mathcal{X}$ and a frequency bandwidth vector $\mathbf{M} \in \mathbb{N}^{d}$. Each node $x_{j}$ belongs to the sampling set $\mathcal{X}=\left\{x_{i} \in[-(1 / 2),(1 / 2))^{d}: 0 \leq i<N\right\}$ so that $|\mathcal{X}|=N$, where $|\cdot|$ indicates set cardinality. The index set

$$
I_{\mathrm{N}}=\mathbb{Z}^{d} \cap \prod_{t=0}^{d-1}\left[-\frac{M_{t}}{2}, \frac{M_{t}}{2}\right)
$$

defines a rectangular grid over which a function of interest is sampled.

Given as input a set of samples $h_{\mathbf{k}} \in \mathbb{C}$ for $\mathbf{k} \in I_{\mathbf{N}}$, the NDFT is defined as

$$
\hat{h}_{i}=\sum_{\mathbf{k} \in I_{\mathbf{N}}} h_{\mathbf{k}} e^{-j 2 \pi \mathbf{k}^{T} \mathbf{x}_{i}}
$$

for $0 \leq i<N$.

The NFFT is a fast implementation of the NDFT obtained by interpolating an oversampled FFT. It is an approximate method which provides a very good compromise between accuracy and computational complexity.

Let $\mathbf{Y} \in \mathbb{C}^{M_{y} \times M_{x}}$ be an image obtained by uniform rectangular sampling of U-space with even $M_{x}$ and $M_{y}$, and sampling coordinates drawn from

$$
\tilde{\mathrm{U}}=\left\{\frac{2 i}{M_{x}}\right\}_{i=-M_{x} / 2}^{M_{x} / 2-1} \times\left\{\frac{2 j}{M_{y}}\right\}_{j=-M_{y} / 2}^{M_{y} / 2-1} .
$$

We now show that $\mathbf{S}$ can be obtained from $\mathbf{Y}$ by using a 2-D NFFT. Due to the linearity of the NFFT, it suffices to show that this transform is exact for an image containing one arbitrary unit impulse at coordinates $\mathbf{u}_{0}$, which must be in the U-space sampling grid.

Using (9), for arbitrary $-\left(M_{x} / 2\right) \leq m_{0}<\left(M_{x} / 2\right)$ and $-\left(M_{y} / 2\right) \leq n_{0}<\left(M_{y} / 2\right)$, define

$$
\mathbf{u}_{0}=\left[\begin{array}{c}
\frac{2 m_{0}}{M_{x}} \\
\frac{2 n_{0}}{M_{y}} \\
\sqrt{1-\left(\frac{2 m_{0}}{M_{x}}\right)^{2}-\left(\frac{2 n_{0}}{M_{y}}\right)^{2}}
\end{array}\right]
$$

and

$$
\mathbf{Y}_{n, m}= \begin{cases}1, & \text { if }(m, n)=\left(m_{0}+\frac{M_{x}}{2}, n_{0}+\frac{M_{y}}{2}\right) \\ 0, & \text { otherwise. }\end{cases}
$$

By definition

$$
\begin{aligned}
\mathbf{S}_{r, s} & =\left[\mathbf{v}\left(\mathbf{u}_{0}\right) \mathbf{v}^{H}\left(\mathbf{u}_{0}\right)\right]_{r, s} \\
& =\mathrm{e}^{j \frac{\omega}{c} \mathbf{u}_{0}^{T}\left(\mathbf{p}_{r}-\mathbf{p}_{s}\right)} \\
& =\mathrm{e}^{j 2 \pi \mathbf{u}_{0}^{T} \frac{\left(\mathbf{p}_{r}-\mathbf{p}_{s}\right)}{\lambda}} \\
& =\mathrm{e}^{j 2 \pi \frac{\mathbf{u}_{0}^{T}}{2} \frac{2\left(\mathbf{p}_{r}-\mathbf{p}_{s}\right)}{\lambda}} .
\end{aligned}
$$

To obtain (51) by using the NFFT, rectangular U-space sampling and an arbitrary geometry of $N$ microphones, we use

$$
\begin{aligned}
& \mathbf{M}= {\left[\begin{array}{ll}
M_{x} & M_{y}
\end{array}\right]^{T} } \\
& I_{\mathbf{N}}=\mathbb{Z}^{2} \cap {\left[-\frac{M_{x}}{2}, \frac{M_{x}}{2}\right) \times\left[-\frac{M_{y}}{2}, \frac{M_{y}}{2}\right) } \\
& \mathcal{X}=\left\{\mathbf{x}_{i}=\frac{2}{\lambda}\left(\mathbf{p}_{\lfloor i / N\rfloor}-\mathbf{p}_{\bmod (i, N)}\right)\right. \\
&\left.\odot\left[\begin{array}{l}
M_{x}^{-1} \\
M_{y}^{-1}
\end{array}\right]: 0 \leq i<N^{2}\right\}
\end{aligned}
$$

where $\odot$ represents the pointwise (Hadamard) product, and the baselines $\mathbf{p}_{r}-\mathbf{p}_{s}$ are represented only by their $x$ and $y$ coordinates. We now show that this parameterization of the NFFT produces the direct transform.

Once again

$$
\begin{aligned}
\mathbf{S}_{r, s} & =\mathrm{e}^{j 2 \pi \frac{\mathbf{u}_{0}^{T}}{2} \frac{2\left(\mathbf{p}_{r}-\mathbf{p}_{s}\right)}{\lambda}} \\
& =\mathrm{e}^{j 2 \pi\left(\mathbf{u}_{0}^{T} \odot\left[\frac{M_{x}}{2} \frac{M_{y}}{2}\right]\right)\left(\frac{2}{\lambda}\left(\mathbf{p}_{r}-\mathbf{p}_{s}\right) \odot\left[M_{x}^{-1} M_{y}^{-1}\right]^{T}\right)}
\end{aligned}
$$

where $\mathbf{u}_{0}$ is also represented only by its $x$ and $y$ components.

Comparing (55) with (44), the first term in parentheses clearly belongs to $I_{\mathbf{N}}$. Since for $0 \leq i<N^{2},\left(\mathbf{p}_{\lfloor i / N\rfloor}-\mathbf{p}_{\bmod (i, N)}\right)$ spans all possible baselines, the second term in parentheses belongs to $\mathcal{X}$. The enumeration given by $(\lfloor i / N\rfloor, \bmod (i, N))$ indexes the elements of $\mathbf{S}$ row by row. Given the Hermitian symmetry of $\mathbf{S}$, this is equivalent to conjugating (55) and indexing the elements of $\mathbf{S}$ column by column (in the order of vec $\{\cdot\}$ ), making (55) equivalent to (44).

It is possible to show that for uniform rectangular arrays with horizontal and vertical interelement spacings $d_{x}=d_{y}=\lambda / 2$ (where $\lambda$ is the wavelength of the signal of interest), this NFFT reduces to a 2-D FFT. This implementation is not convenient for aeroacoustic imaging, since: 1 ) the constraint $d_{x}=d_{y}=\lambda / 2$ can only be satisfied for one frequency, and we are interested in wideband operation; 2) the 2-D FFT is inefficient, since it ignores that image pixels significantly outnumber array sensors, and determines covariances for sensors that do not exist; 3) the 2-D FFT requires uniform rectangular geometries, which have their upper operating frequency constrained by the Nyquist-Shannon sampling theorem.

The NFFT has the advantage of allowing arbitrary array geometries, but as we will see in Section V, it is one order of magnitude slower than the KAT. Furthermore, as will be shown in Section VI, the KAT can be generalized to approximate the spherical wavefronts due to near-field sources. In contrast, the FFT, NFFT, and NFFT require a far-field assumption.

Finally, the KAT has the advantage of allowing separable (as opposed to uniform) U-space sampling grids. Acoustic images are often formed by clusters of distributed sources (for example, located over a model in a wind tunnel) and large regions with no significant sources. Thus, the KAT allows one to oversample the regions which are expected to have sources and undersample quiet regions, while maintaining low computational requirements. 


\section{B. NNFFT Imaging}

By dropping the uniform sampling constraint (45), one obtains the far-field array transform in its fullest generality. This transform can be accelerated with the nonequispaced in time and frequency fast Fourier transform (NNFFT). While the NNFFT is significantly slower than the KAT and the NFFT, it requires much less memory than the matrix representation of $\mathbf{A}$, which makes it useful for smaller problems that can be solved offline.

Given as input a set of samples $h_{l} \in \mathbb{C}$ for $0 \leq l<L$, the NNDFT is defined as

$$
\hat{h}_{i}=\sum_{l=0}^{L-1} h_{l} e^{-j 2 \pi\left(\mathbf{v}_{l} \odot \mathbf{M}\right)^{T} \mathbf{x}_{i}}
$$

for $0 \leq i<N$, and arbitrary $\mathbf{v}_{l}, \mathbf{x}_{i} \in[-(1 / 2),(1 / 2))^{d}$. The NNFFT is a fast approximation of the NNDFT.

To obtain (51) using the NNFFT, arbitrary U-space sampling and an arbitrary geometry of $N$ microphones, we use $x_{j} \in \mathcal{X}$ and $v_{l} \in \mathcal{V}$, with

$$
\begin{aligned}
& \mathbf{M}= {\left[\begin{array}{ll}
M_{x} & M_{y}
\end{array}\right]^{T} } \\
& \mathcal{V}=\left\{v_{l}=\left(\frac{u_{x_{l}}}{2}, \frac{u_{y_{l}}}{2}\right) \in\left[-\frac{1}{2}, \frac{1}{2}\right)^{2}: 0 \leq l<M\right\} \\
& \mathcal{X}=\left\{\mathbf{x}_{i}=\frac{2}{\lambda}\left(\mathbf{p}_{\lfloor i / N\rfloor}-\mathbf{p}_{\bmod (i, N)}\right)\right. \\
&\left.\odot\left[\begin{array}{l}
M_{x}^{-1} \\
M_{y}^{-1}
\end{array}\right]: 0 \leq i<N^{2}\right\}
\end{aligned}
$$

which has the same form as (52)-(54), but allows arbitrary U-space sampling.

\section{Computational Cost}

\section{A. Asymptotic Complexity}

To simplify the following formulas, we will assume that $N_{x}=N_{y}$ and $M_{x}=M_{y}$. We will present the asymptotic complexity for the direct and adjoint transforms.

The product Ay in (16) requires approximately (1/2)MN $N^{2}$ complex MACs to compute and, thus, has complexity $O\left(M N^{2}\right)$. For the KAT, the cost of computing $\boldsymbol{\Xi}$ can be neglected. Evaluating $\left(\mathbf{V}_{\mathbf{y}} \mathbf{Y}\right) \mathbf{V}_{\mathbf{x}}^{T}$ with matrix multiplication requires $(1 / 2) M N+M^{1 / 2} N^{2}$ complex MACs and, thus, has complexity $O\left(M N+M^{1 / 2} N^{2}\right)$.

One can also evaluate $\left(\mathbf{V}_{\mathbf{y}} \mathbf{Y}\right) \mathbf{V}_{\mathbf{x}}^{T}$ by interpreting each matrix product as a 1-D NFFT. Evaluating $\mathbf{V}_{\mathbf{y}} \mathbf{Y}$ requires $M_{x}$ 1-D NFFTs, each with cost $O\left(M_{y} \log M_{y}+N_{y}^{2}\right)$ [28]. The second product requires $N_{y}^{2}$ 1-D NFFTs, each with cost $O\left(M_{x} \log M_{x}+N_{x}^{2}\right)$. Assuming that $M^{1 / 2}>N$, the total complexity becomes $O\left(M \log M+M^{1 / 2} N\right)$. The direct NFFT and NNFFT implementations have complexity $O\left(M \log M+N^{2}\right)$ [28].

Table III summarizes these results. Note that since the asymptotic complexity is similar for most of the fast transforms, it simply guarantees that these methods will scale about as well as an FFT. Nevertheless, the constants hidden in the $O(\cdot)$ notation are significant. As we show next, the direct NNFFT im-
TABLE III

ASYMPTOTIC COMPLEXITY OF THE KAT, FFT, NFFT, NNFFT, AND EXPLICIT MATRIX REPRESENTATION

\begin{tabular}{|l|l|}
\hline Transform & Complexity \\
\hline \hline KAT with matrix multiplication & $O\left(M N+M^{1 / 2} N^{2}\right)$ \\
\hline KAT with 1-D NFFTs & $O\left(M \log M+M^{1 / 2} N\right)$ \\
\hline 2-D FFT & $O(M \log M)$ \\
\hline 2-D NFFT & $O\left(M \log M+N^{2}\right)$ \\
\hline 2-D NNFFT & $O\left(M \log M+N^{2}\right)$ \\
\hline Explicit matrix representation & $O\left(M N^{2}\right)$ \\
\hline
\end{tabular}

plementation is much slower than a KAT with 1-D NFFTs, despite having similar asymptotic complexity. Also, since the A matrix is very large, memory bandwidth becomes the limiting factor for the explicit matrix representation. Thus, the constant hiding in the $O(\cdot)$ notation for the explicit matrix representation is greater than the MAC count suggests. Furthermore, for practical problem sizes, one does not have enough memory to store a full matrix representation and is forced to recompute the rows of $\mathbf{A}$ every time a matrix-vector product is required. This can dramatically increase the computational cost of the explicit matrix representation.

\section{B. Numerical Benchmarks}

This section presents experiments to assess the execution times for the KAT, the NFFT, and the NNFFT. Even though the relative performance of algorithms based on matrix multiplication can be easily estimated in terms of MACs, actual runtimes can deviate significantly from these estimates for certain problem sizes. Indeed, for modern architectures, performance is strongly dependent on the interaction of parallel arithmetic units, memory bandwidth, cache size, and branch prediction, so that the number of floating-point operations only serves as an approximate measure of computational complexity.

The runtimes presented in Figs. 2 and 3 are averages collected over $10 \mathrm{~s}$ for each algorithm and problem size. All simulations were run on an Intel Core 2 Duo T9400 processor in 64-bit mode, using only one core. The permutation $\Xi$, which obtains $\mathbf{S}$ from $\mathbf{Z}$, was written in ANSI C, the NFFT library was compiled with default optimizations as used by its authors, and all other functions were written in M-code for MATLAB $\mathrm{R} 2008 \mathrm{~b}$. Since the code does not feature time-consuming loops, and MATLAB uses the Intel Math Kernel Library for matrix and vector arithmetic, the proposed transforms run very much like machine-specific tuned code. MATLAB and the NFFT use FFTW [31] for computing FFTs, so that they also run like machine-specific code. Thus, having the code written in MATLAB actually incurs negligible computational overhead when compared to an optimized implementation in $\mathrm{C}$ and machine-specific assembly code.

The computational cost of efficient convex optimization methods (and, in particular, of the regularized least-squares methods presented in $[21, \mathrm{Sec}$. II] is completely dependent on the cost of applying $\mathbf{A}, \mathbf{A}^{H}, \boldsymbol{\Psi}$, and $\boldsymbol{\Psi}^{H}$. Since $\boldsymbol{\Psi}$ depends on the regularization method and can be chosen to be very fast, the bottleneck is on applying $\mathbf{A}, \mathbf{A}^{H}$, and possibly $\mathbf{A}^{H} \mathbf{A}$. Thus, from the runtimes of $\mathbf{A}, \mathbf{A}^{H}$, and $\mathbf{A}^{H} \mathbf{A}$ presented in this section, one can assume with good approximation that a $K$-fold 

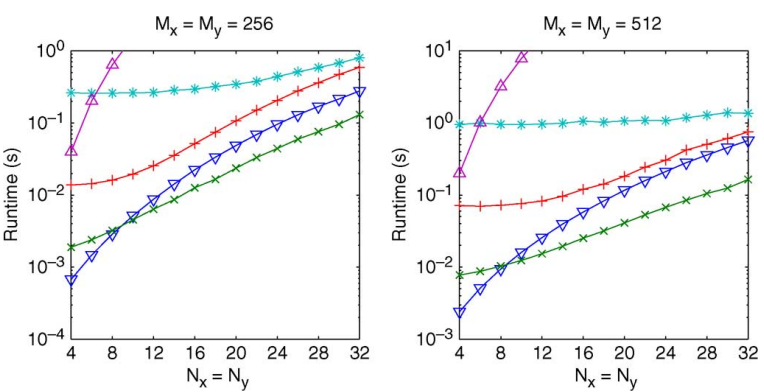

Fig. 2. Runtimes for the direct transform. $\nabla:$ KAT implemented with matrix multiplication, $x$ : KAT implemented with 1-D NFFTs replacing matrix multiplication, + : direct NFFT implementation with (52)-(54), $*$ : direct NNFFT implementation with (57)-(59), $\triangle$ : explicit matrix representation.
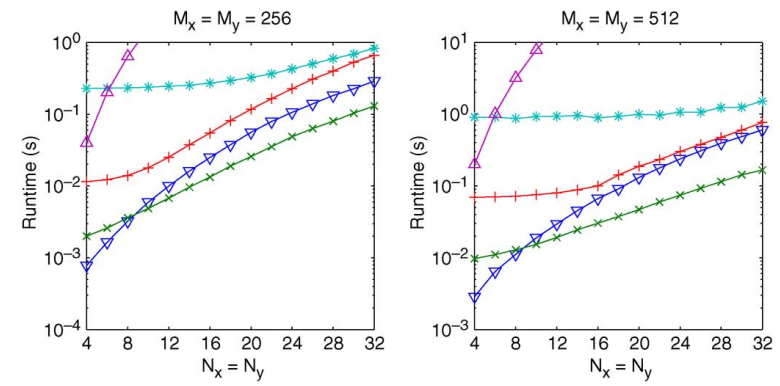

Fig. 3. Runtimes for the adjoint transform. $\nabla$ : KAT implemented with matrix multiplication, $\times$ : KAT implemented with 1-D NFFTs replacing matrix multiplication, + : direct NFFT implementation with (52)-(54), *: direct NNFFT implementation with (57)-(59), $\triangle$ : explicit matrix representation.

decrease in computational time translates to an algorithm which reconstructs an image $K$ times faster.

It is clear that the KAT with the NFFT optimization is the fastest transform for arrays with more than 64 elements $\left(N_{x}>\right.$ 8 and $N_{y}>8$ ). This is the case because as $\mathbf{V}_{\mathbf{x}}$ and $\mathbf{V}_{\mathbf{y}}$ grow, the NFFT scales better than matrix multiplication. The direct implementation with the NFFT is useful if one must have an arbitrary array geometry, but it has the drawback of being around an order of magnitude slower and requiring a far-field approximation. (As we show in Section VI, the KAT can be extended for near-field imaging.) The direct implementation with the NNFFT is by far the slowest. For all implementations, the direct and adjoint transforms perform similarly.

Fig. 4 presents runtimes for the direct-adjoint composition. The implementation using (39) analyzes the problem size and automatically selects the optimal order for matrix multiplication. For large values of $N_{x}$ and $N_{y}$, it also uses precomputed versions of $\mathbf{V}_{\mathbf{y}}^{H} \mathbf{V}_{\mathbf{y}}$ and $\mathbf{V}_{\mathbf{x}}^{T} \mathbf{V}_{\mathbf{x}}^{*}$, which makes the computational complexity depend only on $M_{x}$ and $M_{y}$. The NFFT implementation uses a composition of the direct and adjoint transforms, without any additional optimizations.

As shown in [21], under a far-field assumption and uniform U-space sampling, the direct-adjoint composition reduces to a 2-D convolution of the input image with the array point-spread function. Thus, it can be accelerated with a 2-D FFT (with zero padding to prevent edge effects). Fig. 4 shows that the direct-adjoint composition implemented with the KAT also outperforms
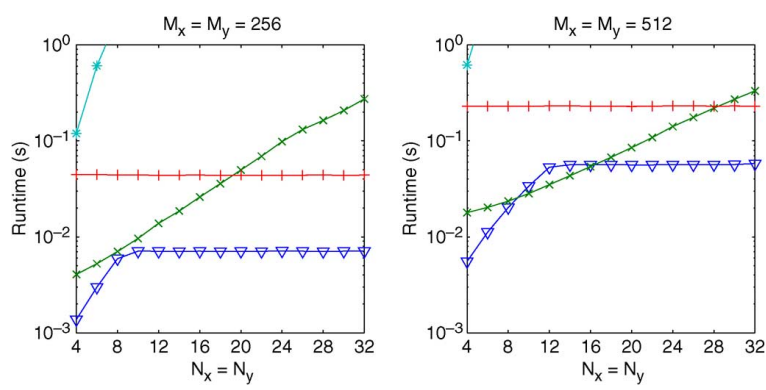

Fig. 4. Runtimes for the direct-adjoint composition. $\nabla:$ KAT implemented with (39), $\times$ : KAT implemented with the composition $\mathbf{A}$ and $\mathbf{A}^{H}$, with the 1-D NFFT optimization, + : 2-D FFT-accelerated convolution, $*$ : composition of the explicit matrix representation.

2-D FFT accelerated convolutions. As we show in the following section, the KAT can be generalized to near-field scenarios, allowing us to drop the far-field assumption. Note that for nearfield cases, the direct-adjoint composition no longer reduces to a convolution, and KAT becomes the only fast transform suitable for imaging.

Finally, the KAT has the additional advantage of being easy to implement and parallelize, since it only requires relatively small matrix multiplications and computationally efficient permutations.

\section{NEAR-FIELD IMAGING}

Up to this point, we have assumed that the sources were located in the far field. Thus, we used a plane-wave model. In this section, we show how to generalize the KAT and address near-field scenarios, where one has spherical wavefronts.

Note that the KAT does not impose any structure onto the array manifold vector other than its separability. The specific far-field representation was only chosen for convenience, since for any Cartesian geometry, the far-field array manifold vector is separable. Nothing prevents us from choosing a different separable representation that is more suitable for the near-field case. In this section, we show that the problem of finding the best separable representation can be recast as a rank-1 approximation of a rearranged version of $\boldsymbol{\Xi}^{-1} \mathbf{A}=\boldsymbol{\Xi}^{T} \mathbf{A}$. By using a rank- $K$ approximation (for $K>1$ ), one is able to obtain an arbitrarily accurate model for near-field propagation, while maintaining low computational requirements.

To simplify the language in this section, we will use the following notation. Given suitably sized matrices $\mathbf{A}, \mathbf{B}, \mathbf{C}$, we use $\mathbf{C}=\underline{\mathbf{A}}(\mathbf{B})$ to denote vec $\{\mathbf{C}\}=\mathbf{A} \operatorname{vec}\{\mathbf{B}\}$.

We approximate $\mathbf{A}$ by $\mathbf{A}=\Xi\left(\sum_{k=1}^{K} \mathbf{C}_{k} \otimes \mathbf{D}_{k}\right)$, for small values of $K$. Note that $\mathbf{S}=\underline{\breve{A}}(\mathbf{Y})$ can be efficiently implemented as $\mathbf{S}=\boldsymbol{\Xi}\left(\sum_{k=1}^{K} \mathbf{D}_{k} \underline{\mathbf{Y}} \mathbf{C}_{k}^{T}\right)$. Compared with (36), we are approximating the near-field transform $\mathbf{A}$ with a series of $K$ separable transforms, to which we can apply the KAT.

Let us consider the problem of approximating a generic $\mathbf{B} \in$ $\mathbb{C}^{m \times n}$ with $m=m_{1} m_{2}$ and $n=n_{1} n_{2}$ with a sum of Kronecker products, so that

$$
\min _{\left\{\mathbf{C}_{k}\right\},\left\{\mathbf{D}_{k}\right\}}\left\|\mathbf{B}-\sum_{k=1}^{K} \mathbf{C}_{k} \otimes \mathbf{D}_{k}\right\|_{F}
$$


where $\mathbf{C}_{k} \in \mathbb{C}^{m_{1} \times n_{1}}$ and $\mathbf{D}_{k} \in \mathbb{C}^{m_{2} \times n_{2}}$ for $1 \leq k \leq K$. This problem is addressed in [32], where it is shown to be equivalent to

$$
\min _{\left\{\mathbf{C}_{k}\right\},\left\{\mathbf{D}_{k}\right\}}\left\|\mathcal{R}(\mathbf{B})-\sum_{k=1}^{K} \operatorname{vec}\left\{\mathbf{C}_{k}\right\} \operatorname{vec}\left\{\mathbf{D}_{k}\right\}^{T}\right\|_{F}
$$

where $\mathcal{R}(\cdot)$ is a matrix rearrangement operator so that $\mathcal{R}(\mathbf{B}) \in$ $\mathbb{C}^{m_{1} n_{1} \times m_{2} n_{2}}$. This is a low-rank approximation problem which can be solved with the SVD of $\mathcal{R}(\mathbf{B})$.

For our purposes, we approximate $\mathbf{B}=\Xi^{-1} \mathbf{A}$. We note that $\Xi$ is the key to a successful low-rank decomposition. As we show later in this section, using $\mathbf{B}=\mathbf{A}$ is not useful, since $\mathcal{R}(\mathbf{A})$ has too many significant singular values.

Computing the dominant singular values and vectors of $\mathcal{R}(\mathbf{B})$ is not trivial, since, in practice, $\mathcal{R}(\mathbf{B})$ is too large to be stored explicitly in memory. Nevertheless, one can use the Lanczos methods [32], [33] which only require the implementation of the matrix-vector products $\mathcal{R}(\mathbf{B}) \boldsymbol{\alpha}$ and $\mathcal{R}(\mathbf{B})^{H} \boldsymbol{\beta}$ for arbitrary $\boldsymbol{\alpha}, \boldsymbol{\beta}$. One can also use approximate SVD methods which are designed to require a small number of passes over $\mathcal{R}(\mathbf{B})$ (e.g., [34] and [35]).

Using the definition of $\mathcal{R}(\cdot)$ from [32], it can be shown that

$$
\begin{aligned}
\mathcal{R}(\mathbf{B})^{T} & =\left[\begin{array}{ccc}
\mathbf{Z}_{0,0} & \cdots & \mathbf{Z}_{M_{x}-1,0} \\
\vdots & & \vdots \\
\mathbf{Z}_{0, M_{y}-1} & \cdots & \mathbf{Z}_{M_{x}-1, M_{y}-1}
\end{array}\right] \\
\mathbf{Z}_{m, n} & =\Xi\left(\mathbf{v}\left(u_{x_{m}}, u_{y_{n}}\right) \mathbf{v}^{H}\left(u_{x_{m}}, u_{y_{n}}\right)\right) .
\end{aligned}
$$

Since $\mathbf{v}\left(u_{x_{m}}, u_{y_{n}}\right)$ can be precomputed for $0<m<M_{x}$ and $0<n<M_{y}$ and $\boldsymbol{\Xi}$ is a very fast permutation, $\mathcal{R}(\mathbf{B}) \boldsymbol{\alpha}$ and $\mathcal{R}(\mathbf{B})^{H} \boldsymbol{\beta}$ can be evaluated with relative efficiency. Indeed, using the Lanczos method from [33], $N=64$ and $M_{x}=M_{y}=$ 256, we can solve (60) for $K=8$ in $8 \mathrm{~min}$ on an Intel Core 2 Duo $2.4-\mathrm{GHz}$ processor, using only one core. Note that this procedure only has to be performed once.

The decomposition obtained with (60) is especially useful for $K>1$. Indeed, even in the presence of strong near field effects, $\mathcal{R}($ B $)$ can be well approximated by a low-rank decomposition Even though the transform cost grows linearly with $K$, due to the Kronecker representation, the cost of applying each $\mathbf{C}_{k} \otimes \mathbf{D}_{k}$ is very small, so a transform with $K=8$ is still very fast.

Since we discretize the focal surface with Cartesian sampling grids, some images may have invalid regions. For example, one can parameterize a spherical half-shell with radius $r_{0}$ by using $u_{x}=r_{0} \sin \phi \cos \theta$ and $u_{y}=r_{0} \sin \phi \sin \theta$ for $\|\mathbf{u}\|<r_{0}$, so that source locations for $\|\mathbf{u}\|>r_{0}$ are invalid. Note that this implies that some $\mathbf{Z}_{m, n}$ in $\mathcal{R}(\mathbf{B})$ are not defined. Referring to (60), the optimal $\mathbf{C}_{k}, \mathbf{D}_{k}$ are now given by

$$
\min _{\left\{\mathbf{C}_{k}\right\},\left\{\mathbf{D}_{k}\right\}}\left\|\mathbf{W} \odot\left(\mathcal{R}(\mathbf{B})-\sum_{k=1}^{K} \operatorname{vec}\left\{\mathbf{C}_{k}\right\} \operatorname{vec}\left\{\mathbf{D}_{k}\right\}^{T}\right)\right\|_{F}
$$

where $\odot$ is the pointwise (Hadamard) product and $\mathbf{W}$ is a binary mask set to 1 for valid elements and 0 for invalid elements of $\mathcal{R}(\mathbf{B})$. This masked-SVD problem was considered in [36], and can be solved by iterating

$$
\mathbf{E}^{(i)}=\operatorname{LRA}_{K}\left(\mathbf{W} \odot \mathcal{R}(\mathbf{B})+(\mathbb{1}-\mathbf{W}) \odot \mathbf{E}^{(i-1)}\right)
$$

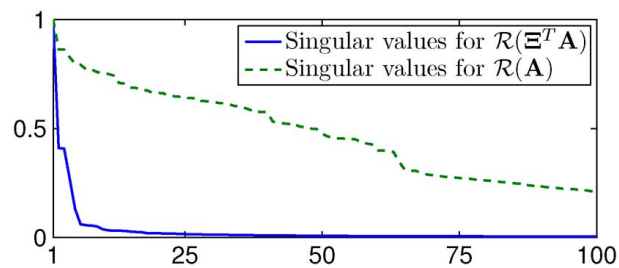

Fig. 5. First 100 singular values for $\mathcal{R}(\mathbf{A})$ and $\mathcal{R}\left(\boldsymbol{\Xi}^{T} \mathbf{A}\right)$, out of a total of 16384 , normalized to 1 .

where $\operatorname{LRA}_{\mathrm{K}}(\cdot)$ is a rank- $K$ approximation as computed by the $\mathrm{SVD}, \mathbf{E}^{(i)}=\sum_{k=1}^{K} \operatorname{vec}\left\{\mathbf{C}_{k}^{(i)}\right\} \operatorname{vec}\left\{\mathbf{D}_{k}^{(i)}\right\}^{T}$ and $\mathbf{E}^{(0)}=\mathbf{0}$. In our experiments, 1 or 2 iterations have shown to be sufficient for a good fit.

Fig. 5 compares the first 100 (out of a total of 16384) singular values for $\mathcal{R}(\mathbf{A})$ and $\mathcal{R}\left(\boldsymbol{\Xi}^{T} \mathbf{A}\right)$. In this experiment, $\mathbf{A}$ models $256 \times 256$ sources radiating at $9 \mathrm{kHz}$, located over a spherical half-shell with a radius of $1.0 \mathrm{~m}$, as measured by an $8 \times 8$ separable array with a $30 \times 30-\mathrm{cm}$ aperture. (This configuration is the same used in the examples from [21].) The sharp decay of the curve for $\mathcal{R}\left(\boldsymbol{\Xi}^{T} \mathbf{A}\right)$ highlights the importance of $\boldsymbol{\Xi}$ in enabling an accurate low-rank approximation. For lower frequencies, the singular values show an even sharper roll-off. Also, this transform models the complete hemisphere. By modeling a smaller field of view, near-field effects are not as severe, and the singular values also decay faster.

Note that by using a rank- $K$ approximation, we obtain a transform with a computational cost that is $K$ times larger than the far-field KAT presented in the previous sections. Nevertheless, as we show in [21], $K$ will be small enough that this penalty is not significant. In fact, we will show that it is possible to compensate for strong near-field effects with $K=$ 8 , which makes the KAT about as fast as the NFFT, while being able to model arbitrary near-field focal surfaces.

\section{CONCLUSION}

This paper presents the KAT, which was designed to enable computationally efficient and accurate acoustic imaging. To obtain fast implementation, we assumed a separable microphone array, source parameterization in U-space, and far-field sources. The KAT transforms back and forth between a hypothetical source distribution and the corresponding covariance matrix which would be measured by the array. Another contribution is the novel application of the NFFT and NNFFT to acoustic imaging, which we used as baselines against which our proposal was compared.

The KAT is orders of magnitude faster than equivalent implementations using explicit matrix representations. Despite the fact that the NFFT and NNFFT are already fast transforms, we have shown in Section V that our proposal is at least one order of magnitude faster than them. In contrast with FFT- and NFFTbased transforms, the KAT allows arbitrary Cartesian samplings of the source distributions, which let one oversample regions with sources and undersample silent areas without performance degradation. Also, in contrast with the NFFT, the KAT makes no numerical approximations, and can be more easily implemented and parallelized, since it only requires relatively small matrix products and simple permutations. 
Even though the KAT was motivated with the far-field assumption, it does not impose any structure onto the array manifold vector other than its separability. We have used this fact to extend it for near-field imaging, providing a computationally efficient approximation of the exact near-field transform.

Future work involves developing fast transforms for other array geometries. In contrast with the KAT, which is exact for far-field sources, transforms for other geometries will most likely require approximations to obtain good performance. Furthermore, the KAT can be generalized for correlated distributions, which we also intend to address in future work.

\section{REFERENCES}

[1] W. Home, K. James, T. Arledge, P. Sodermant, N. Burnside, and S. Jaeger, "Measurements of $26 \%$-scale 777 airframe noise in the NASA Ames 40 - by 80 -foot wind tunnel," presented at the 11th AIAA/CEAS Aeroacoust. Conf., Monterey, CA, 2005.

[2] W. Humphreys and T. Brooks, "Noise spectra and directivity for scalemodel landing gear," presented at the 13th AIAA/CEAS Aeroacoust. Conf., Rome, Italy, 2007.

[3] S. Lee, "Phased-array measurement of modern regional aircraft turbofan engine noise," presented at the 12th AIAA/CEAS Aeroacoust. Conf., Cambridge, MA, 2006

[4] S. Oerlemans, P. Sijtsma, and B. Mendez Lopez, "Location and quantification of noise sources on a wind turbine," J. Sound Vibr., vol. 299 no. 4-5, pp. 869-883, 2007.

[5] A. O'Donovan, R. Duraiswami, and D. Zotkin, "Imaging concert hall acoustics using visual and audio cameras," in Proc. ICASSP, 2008, pp. 5284-5287.

[6] M. Wax and T. Kailath, "Optimum localization of multiple sources by passive arrays," IEEE Trans. Acoust., Speech Signal Process., vol. ASSP-31, no. 5, pp. 1210-1217, Oct. 1983.

[7] R. Schmidt, "Multiple emitter location and signal parameter estimation," IEEE Trans. Antennas Propag., vol. AP-34, no. 3, pp. 276-280, Mar. 1986.

[8] R. Roy and T. Kailath, "ESPRIT-estimation of signal parameters via rotational invariance techniques," IEEE Trans. Acoust., Speech, Signal Process., vol. 37, no. 7, pp. 984-995, Jul. 1989.

[9] C. Zhang, D. Florencio, D. Ba, and Z. Zhang, "Maximum likelihood sound source localization and beamforming for directional microphone arrays in distributed meetings," IEEE Trans. Multimedia, vol. 10, no. 3 , pp. $538-548$, Apr. 2008

[10] M. Brandstein and H. Silverman, "A robust method for speech signal time-delay estimation inreverberant rooms," in Proc. ICASSP, 1997, vol. 1 , pp. $375-378$

[11] Z. Wang, J. Li, P. Stoica, T. Nishida, and M. Sheplak, "Constant-beamwidth and constant-powerwidth wideband robust Capon beamformers for acoustic imaging," J. Acoust. Soc. Amer., vol. 116 p. $1621,2004$.

[12] S. Yan, Y. Ma, and C. Hou, "Optimal array pattern synthesis for broadband arrays," J. Acoust. Soc. Amer., vol. 122, p. 2686, 2007.

[13] J. Li, Y. Xie, P. Stoica, X. Zheng, and J. Ward, "Beampattern synthesis via a matrix approach for signal power estimation," IEEE Trans. Signal Process., vol. 55, no. 12, pp. 5643-5657, Dec. 2007.

[14] Y. Wang, J. Li, P. Stoica, M. Sheplak, and T. Nishida, "Wideband relax and wideband clean for aeroacoustic imaging," J. Acoustic. Soc. Amer. vol. 115 , p. 757,2004

[15] R. Dougherty and R. Stoker, "Sidelobe suppression for phased array aeroacoustic measurements," in Proc. 4th AIAA/CEAS Aeroacoust.s Conf., 1998, pp. 235-245.

[16] R. Dougherty, "Extensions of DAMAS and benefits and limitations of deconvolution in beamforming," presented at the 11th AIAA/CEAS Aeroacoustics Conf., Monterey, CA, 2005.

[17] K. Ehrenfried and L. Koop, "Comparison of iterative deconvolution algorithms for the mapping of acoustic sources," AIAA J., vol. 45, no. 7, p. $1584,2007$.

[18] E. Candès, J. Romberg, and T. Tao, "Stable signal recovery from incomplete and inaccurate measurements," Commun. Pure Appl. Math. vol. 59 , no. 8, p. $1207,2006$.

[19] E. Candes, J. Romberg, and T. Tao, "Robust uncertainty principles Exact signal reconstruction from highly incomplete frequency information," IEEE Trans. Inf. Theory, vol. 52, no. 2, pp. 489-509, Feb. 2006.

[20] D. Donoho, "Compressed sensing," IEEE Trans. Inf. Theory, vol. 52 no. 4, pp. 1289-1306, Apr. 2006.

[21] F. Ribeiro and V. Nascimento, "Fast transforms for acoustic imaging-Part II: Applications and extensions," IEEE Trans. Image Process., vol. 20, no. 8, pp. XXX-XXX, Aug. 2011.
[22] L. He, T. Chang, S. Osher, T. Fang, and P. Speier, "MR image reconstruction by using the iterative refinement method and nonlinear inverse scale space methods," UCLA CAM Rep., vol. 6, p. 35, 2006.

[23] M. Lustig, D. Donoho, and J. Pauly, "Sparse MRI: The application of compressed sensing for rapid MR imaging," Magn. Resonan. Med., vol. 58 , no. 6 , pp. $1182-1195,2007$

[24] D. Malioutov, M. Cetin, and A. Willsky, "A sparse signal reconstruction perspective for source localization with sensor arrays," IEEE Trans. Signal Process., vol. 53, no. 8, pp. 3010-3022, Aug. 2005.

[25] T. Yardibi, J. Li, P. Stoica, and L. Cattafesta, III, "Sparsity constrained deconvolution approaches for acoustic source mapping," J. Acoust. Soc. Amer., vol. 123, p. 2631, 2008.

[26] B. Zimmermann and C. Studer, "FPGA-based real-time acoustic camera prototype," in Proc. ISCAS, 2010, p. 1419.

[27] X. Huang, "Real-time algorithm for acoustic imaging with a microphone array.," J. Acoust. Soc. Amer., vol. 125, no. 5, 2009.

[28] J. Keiner, S. Kunis, and D. Potts, "Using NFFT 3-A software library for various nonequispaced fast fourier transforms," ACM Trans. Math. Softw., vol. 36, no. 4, p. 19, 2009.

[29] H. L. Van Trees, Optimum Array Processing: Part IV of Detection, Estimation, and Modulation Theory. New York: Wiley, 2002.

[30] R. Horn and C. Johnson, Matrix Analysis. Cambridge, U.K.: Cambridge Univ. Press, 1990.

[31] M. Frigo and S. Johnson, "The design and implementation of FFTW 3," Proc. IEEE, vol. 93, no. 2, pp. 216-231, Feb. 2005.

[32] C. Van Loan and N. Pitsianis, "Approximation with Kronecker products," in Linear Algebra for Large Scale and Real Time Applications, M. Moonen and G. Golub, Eds. Norwell, MA: Kluwer, 1992, pp. M. Moonen-314.

[33] J. Baglama and L. Reichel, "Augmented implicitly restarted Lanczos bidiagonalization methods," SIAM J. Scientif. Comput., vol. 27, no. 1, pp. $19-42,2006$

[34] P. Drineas, R. Kannan, and M. Mahoney, "Fast Monte Carlo algorithms for matrices II: Computing a low-rank approximation to a matrix," SIAM J. Comput., vol. 36, no. 1, pp. 158-183, 2007.

[35] N. Halko, P. Martinsson, and J. Tropp, "Finding structure with randomness: stochastic algorithms for constructing approximate matrix decompositions," California Inst. Tech., Tech. rep. ACM 2009-05, Sep. 2009.

[36] N. Srebro and T. Jaakkola, "Weighted low-rank approximations," in Proc. ICML, 2003, pp. 720-727.

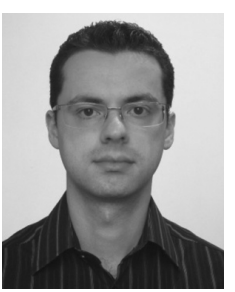

Flávio P. Ribeiro (S'09) received the B.S. degree in electrical engineering from Escola Politécnica, University of São Paulo, São Paulo, Brazil, in 2005, and the B.S. degree in mathematics from the Institute of Mathematics and Statistics, University of São Paulo, in 2008. He is currently pursuing the Ph.D. degree in electrical engineering from the Escola Politécnica, University of São Paulo.

From 2007 to 2009, he was a Hardware Engineer with Licht Labs, where he developed controllers for power transformers and substations. In the Summers of 2009 and 2010, he was a Research Intern with Microsoft Research Redmond. His research interests include array signal processing, multimedia signal processing, and computational linear algebra.

Mr. Ribeiro was a recipient of the Best Student Paper Award at ICME 2010.

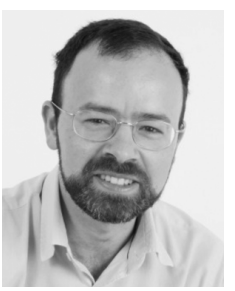

Vítor H. Nascimento (M’01) was born in São Paulo, Brazil. He received the B.S. and M.S. degrees in electrical engineering from the University of São Paulo, in 1989 and 1992, respectively, and the Ph.D. degree from the University of California, Los Angeles, in 1999.

From 1990 to 1994 , he was a Lecturer with the University of São Paulo, and in 1999 he joined the faculty at the same school, where he is now an Associate Professor. His research interests include signalprocessing theory and applications, robust and nonlinear estimation, and applied linear algebra.

Prof. Nascimento was a recipient of the IEEE Signal Processing Society (SPS) Best Paper Award in 2002. He served as an Associate Editor for IEEE SigNAL PROCESSING LETTERS from 2003 to 2005, for the IEEE TRANSACTIONS ON SignAL PROCESSING from 2005 to 2008, and for the EURASIP Journal on Advances in Signal Processing from 2006 to 2009. He is currently a member of the IEEE-SPS Signal Processing Theory and Methods Technical Committee. 


\title{
Fast Transforms for Acoustic Imaging-Part II: Applications
}

\author{
Flávio P. Ribeiro, Student Member, IEEE, and Vítor H. Nascimento, Member, IEEE
}

\begin{abstract}
In Part I ["Fast Transforms for Acoustic Imaging-Part I: Theory," IEEE TRANSACTIONS ON IMAGE PROCESSING], we introduced the Kronecker array transform (KAT), a fast transform for imaging with separable arrays. Given a source distribution, the KAT produces the spectral matrix which would be measured by a separable sensor array. In Part II, we establish connections between the KAT, beamforming and 2-D convolutions, and show how these results can be used to accelerate classical and state of the art array imaging algorithms. We also propose using the KAT to accelerate general purpose regularized least-squares solvers. Using this approach, we avoid ill-conditioned deconvolution steps and obtain more accurate reconstructions than previously possible, while maintaining low computational costs. We also show how the KAT performs when imaging near-field source distributions, and illustrate the trade-off between accuracy and computational complexity. Finally, we show that separable designs can deliver accuracy competitive with multi-arm logarithmic spiral geometries, while having the computational advantages of the KAT.
\end{abstract}

Index Terms-Acoustic imaging, array imaging, array processing, fast transform, regularized least-squares, sparse reconstruction.

\section{INTRODUCTION}

A S DESCRIBED in [1], array imaging requires solving the inverse problem of finding the best estimate for a source distribution, given wavefield statistics sampled by a sensor array. This is not a trivial problem, since in general one must rely on arrays with less than 100 elements to reconstruct source distributions modeled with tens of thousands of point sources. To obtain accurate reconstructions, regularization is required to narrow the space of possible wavefields which result in essentially the same data at the sensors.

Let $\mathbf{S} \in \mathbb{C}^{N \times N}$ be a narrowband sample covariance matrix acquired using a planar sensor array with a separable geometry. Let $\mathbf{Y} \in \mathbb{R}^{M_{y} \times M_{x}}$ be a discretization of the source distribution at the same frequency. Assume for the sake of this argument that the true source distribution is represented exactly by $\mathbf{Y}$, and that the sources are uncorrelated. If $\mathbf{s}=\operatorname{vec}\{\mathbf{S}\}, \mathbf{y}=\operatorname{vec}\{\mathbf{Y}\}$, and $\mathbf{A}$ is the KAT presented in [1], in the absence of noise we have

Manuscript received May 26, 2010; revised December 21, 2010; accepted February 09, 2011. Date of publication February 22, 2011; date of current version July 15, 2011. This work was supported in part by the São Paulo Research Foundation (FAPESP) and the National Council for Scientific and Technological Development $(\mathrm{CNPq})$. The associate editor coordinating the review of this manuscript and approving it for publication was Dr. Brian D. Rigling.

The authors are with the Electronic Systems Engineering Department, Escola Politécnica, Universidade de São Paulo, São Paulo 05508-900, Brazil (e-mail: fr@lps.usp.br; vitor@lps.usp.br).

Digital Object Identifier 10.1109/TIP.2011.2118219 that $\mathbf{A y}=\mathbf{s}$. The generic image reconstruction problem then becomes

$$
\hat{\mathbf{y}}=\underset{\mathbf{y}}{\arg \min }\|\mathbf{\Psi} \mathbf{y}\| \quad \text { such that } \mathbf{A y}=\mathbf{s}
$$

where $\boldsymbol{\Psi}$ is a sparsifying transform for $\mathbf{y}$. For example, if $\mathbf{Y}$ is known to be sparse in its canonical representation, then one could consider minimizing $\|\mathbf{\Psi} \mathbf{y}\|=\|\mathbf{y}\|_{1}$, which turns (1) into an instance of basis pursuit [2].

In the presence of noise, the constraint $\mathbf{A y}=\mathbf{s}$ no longer applies, motivating the formulation

$$
\hat{\mathbf{y}}=\underset{\mathbf{x}}{\arg \min }\|\mathbf{\Psi} \mathbf{y}\|+\mu\|\mathbf{A y}-\mathbf{s}\|_{2}^{2}
$$

which is a regularized least-squares problem.

The problem of seeking sparse approximations to underdetermined systems has received significant attention in the recent years with the advent of compressive sensing [3]-[5]. Recently, many exact and approximate methods have been proposed for solving variations of (1) for specific instances of $\boldsymbol{\Psi}$ and $\|\cdot\|$, such as [6]-[11].

The computational bottleneck for solving (1) or (2) with efficient convex optimization methods lies exclusively in the implementations of $\mathbf{A}, \mathbf{A}^{H}, \boldsymbol{\Psi}$, or $\boldsymbol{\Psi}^{H}$. For imaging applications, $\boldsymbol{\Psi}$ can be a fast wavelet transform, fast Fourier transform or a finite difference operator, which can all be evaluated quickly. Therefore, the potential bottleneck lies in the implementations of $\mathbf{A}$ and $\mathbf{A}^{H}$. However, the KAT makes $\mathbf{A}$ and $\mathbf{A}{ }^{H}$ orders of magnitude faster than competing transforms (and in particular, much faster than explicit matrix representations), allowing the use of regularized least-squares methods for acoustic imaging.

This Part II describes applications of the KAT for image reconstruction. In Section II, we present several methods for acoustic imaging using a common language based on the transform. Using the KAT, we accelerate these techniques without compromising quality. We also propose applying the fast transforms to state-of-the-art, general purpose regularized least-squares solvers, and obtain more accurate reconstructions than what was possible with previous methods. Section III features examples, comparing the performance of the different approaches. Section IV compares the reconstruction accuracy using a separable array and a logarithmic spiral array. We show that by using regularized reconstruction methods, separable arrays can match logarithmic spiral arrays in terms of reconstruction accuracy, while allowing the computational benefits provided by the KAT. Finally, Section V has our conclusions and final comments. 


\section{IMAGE RECONSTRUCTION APPLICATIONS}

\section{A. Delay and Sum Imaging}

Given a spectral matrix $\mathbf{S}$, its corresponding image is traditionally approximated using delay-and-sum beamforming with

$$
\left|Y\left(u_{x_{m}}, u_{y_{n}}\right)\right|^{2} \approx \frac{\mathbf{v}^{H}\left(u_{x_{m}}, u_{y_{n}}\right) \mathbf{S v}\left(u_{x_{m}}, u_{y_{n}}\right)}{\left[\mathbf{v}^{H}\left(u_{x_{m}}, u_{y_{n}}\right) \mathbf{v}\left(u_{x_{m}}, u_{y_{n}}\right)\right]^{2}}
$$

where the approximation is due to convolution effects.

We can rewrite

$$
\begin{aligned}
\mathbf{v}^{H} & \left(u_{x_{m}}, u_{y_{n}}\right) \mathbf{S v}\left(u_{x_{m}}, u_{y_{n}}\right) \\
& =\left[\mathbf{v}^{T}\left(u_{x_{m}}, u_{y_{n}}\right) \otimes \mathbf{v}^{H}\left(u_{x_{m}}, u_{y_{n}}\right)\right] \mathbf{S} \\
& =\left[\mathbf{v}^{*}\left(u_{x_{m}}, u_{y_{n}}\right) \otimes \mathbf{v}\left(u_{x_{m}}, u_{y_{n}}\right)\right]^{H} \operatorname{vec}\{\mathbf{S}\}, \\
& =\left[\mathbf{A}^{H} \operatorname{vec}\{\mathbf{S}\}\right]_{m \cdot M_{y}+n}
\end{aligned}
$$

where (4) is true because $\left(\mathbf{A}^{T} \otimes \mathbf{B}\right) \operatorname{vec}\{\mathbf{C}\}=\operatorname{vec}\{\mathbf{B C A}\}$ whenever BCA is defined, and (6) follows by comparing (5) with $[1,(17)]$. Thus, delay-and-sum imaging can be implemented with the KAT adjoint.

It follows that the direct-adjoint composition $\mathbf{A}^{H} \mathbf{A}$ is a transform that obtains the delay-and-sum image from a clean (ideal) image. If we assume that the sources are in the far-field and that U-space is sampled uniformly, this delay-and-sum image is simply the clean image convolved with the beamformer's point spread function (PSF). Since it represents a convolution, under these assumptions $\mathbf{A}^{H} \mathbf{A}$ can also be accelerated with a 2-D FFT. But as presented in Section V of [1], the KAT can always be used to implement $\mathbf{A}^{H} \mathbf{A}$ more efficiently than an equivalent FFT-accelerated convolution.

\section{B. MVDR Imaging}

Imaging using minimum variance distortionless response (MVDR) beamforming [12] is often preferable to delay-and-sum imaging, given that the MVDR beamformer can get very fine resolution for point sources (as long as the noise is not excessive, and the regularization parameter is chosen correctly). Recall that the MVDR processor steered towards $\mathbf{v}_{T}=\mathbf{v}\left(u_{x_{T}}, u_{y_{T}}\right)$ is given by

$$
\mathbf{w}_{\mathrm{MVDR}}^{H}\left(u_{x_{T}}, u_{y_{T}}\right)=\frac{\mathbf{v}_{T}^{H} \mathbf{S}_{\mathbf{n}}^{-1}}{\mathbf{v}_{T}^{H} \mathbf{S}_{\mathbf{n}}^{-1} \mathbf{v}_{T}}
$$

where $\mathbf{S}_{\mathbf{n}}$ is the noise spectral matrix.

To obtain $\mathbf{S}_{\mathbf{n}}$ for acoustic imaging, one should perform a separate measurement (for example, with the model removed from the wind tunnel) [13]. If this is not possible, one can obtain the minimum power distortionless response (MPDR) processor [14] by using $\mathbf{S}+\lambda \mathbf{I}$ instead of $\mathbf{S}_{\mathbf{n}}$, where $\lambda$ is a suitably chosen regularization parameter, such that

$$
\mathbf{w}_{\mathrm{MPDR}}^{H}\left(u_{x_{T}}, u_{y_{T}}\right)=\frac{\mathbf{v}_{T}^{H}[\mathbf{S}+\lambda \mathbf{I}]^{-1}}{\mathbf{v}_{T}^{H}[\mathbf{S}+\lambda \mathbf{I}]^{-1} \mathbf{v}_{T}}
$$

where $\mathbf{S}$ is the spectral matrix of the whole signal, including the sources of interest and noise.

Let $\mathbf{x}$ be the frequency domain signal at the array output, such that $\mathbf{S}=\mathrm{E}\left\{\mathbf{x x}^{H}\right\}$. The acoustic image can be approximated by the power at the output of the MVDR beamformer, such that for $\mathbf{w}=\mathbf{w}_{\mathrm{MVDR}}$

$$
\begin{aligned}
\left|Y\left(u_{x_{T}}, u_{y_{T}}\right)\right|^{2} & \approx \mathrm{E}\left\{\left|\mathbf{w}^{H}\left(u_{x_{T}}, u_{y_{T}}\right) \mathbf{x}\right|^{2}\right\} \\
& =\mathbf{w}^{H}\left(u_{x_{T}}, u_{y_{T}}\right) \mathrm{E}\left\{\mathbf{x} \mathbf{x}^{H}\right\} \mathbf{w}\left(u_{x_{T}}, u_{y_{T}}\right) \\
& =\frac{\mathbf{v}_{T}^{H} \mathbf{S}_{\mathbf{n}}{ }^{-1} \mathbf{S S}_{\mathbf{n}}{ }^{-1} \mathbf{v}_{T}}{\left[\mathbf{v}_{T}^{H} \mathbf{S}_{\mathbf{n}}{ }^{-1} \mathbf{v}_{T}\right]^{2}}
\end{aligned}
$$

From the results of the previous section, one can obtain $\mathbf{v}_{T}^{H} \mathbf{S}_{\mathbf{n}}{ }^{-1} \mathbf{S S}_{\mathbf{n}}{ }^{-1} \mathbf{v}_{T}$ simultaneously for all look directions by evaluating $\mathbf{A}^{H}$ vec $\left\{\mathbf{S}_{\mathbf{n}}{ }^{-1} \mathbf{S S}_{\mathbf{n}}{ }^{-1}\right\}$. Likewise, one can compute $\left[\mathbf{v}_{T}^{H} \mathbf{S}_{\mathbf{n}}{ }^{-1} \mathbf{v}_{T}\right]^{2}$ for all directions with the pointwise square of $\mathbf{A}^{H} \operatorname{vec}\left\{\mathbf{S}_{\mathbf{n}}{ }^{-1}\right\}$. By dividing one by the other, one can efficiently perform imaging with an MVDR beamformer. MPDR imaging follows similarly.

\section{DAMAS2}

DAMAS2 [15] is a state of the art deconvolution method for aeroacoustic imaging. By using a far-field approximation, it assumes that the convolved image produced by delay-and-sum beamforming is equal to the clean image convolved with the beamformer's PSF. These convolutions are the bottleneck of the algorithm, but if uniform $\mathrm{U}$-space sampling is used, they can be significantly accelerated with 2-D FFTs.

Let $\breve{Y}$ be the image obtained with delay-and-sum beamforming, $\mathbf{P}$ the array PSF for delay-and-sum imaging, $\mathbf{Y}$ the clean image and $\hat{\mathbf{Y}}^{(k)}$ the reconstructed image at iteration $k$. By definition, $\breve{\mathbf{Y}}=\mathbf{P} * \mathbf{Y}$, where $*$ represents 2-D convolution.

DAMAS2 solves for $\mathbf{Y}$ by iterating

$$
\hat{\mathbf{Y}}^{(k+1)}=\max \left\{\hat{\mathbf{Y}}^{(k)}+\frac{1}{a}\left[\breve{\mathbf{Y}}-\left(\mathbf{P} * \hat{\mathbf{Y}}^{(k)}\right)\right], \mathbf{0}\right\}
$$

where $\max \{\cdot, \cdot\}$ returns the pointwise maximum, $a=$ $\sum_{i, j}|\mathbf{P}|_{i, j}, \mathbf{Y}^{(0)}=\mathbf{0}$ and the convolution is implemented with a 2-D FFT and zero-padding.

Given the fast transform, it is possible to implement a faster version of the already FFT-accelerated DAMAS2. Indeed, from Section II-A we have that $\breve{\mathbf{y}}=\operatorname{vec}\{\breve{\mathbf{Y}}\}=$ $\operatorname{vec}\{\mathbf{P} * \mathbf{Y}\}=\mathbf{A}^{H} \mathbf{A} \operatorname{vec}\{\mathbf{Y}\}=\mathbf{A}^{H} \mathbf{A y}$. Similarly, $\operatorname{vec}\left\{\mathbf{P} * \hat{\mathbf{Y}}^{(k)}\right\}=\mathbf{A}^{H} \mathbf{A} \hat{\mathbf{y}}^{(k)}$, where $\mathbf{A}^{H} \mathbf{A}$ can be implemented with the fast direct-adjoint KAT, described in [1, Sect. III-C].

Thus, (9) becomes

$$
\hat{\mathbf{y}}^{(k+1)}=\max \left\{\hat{\mathbf{y}}^{(k)}+\frac{1}{a}\left[\breve{\mathbf{y}}-\mathbf{A}^{H} \mathbf{A} \hat{\mathbf{y}}^{(k)}\right], \mathbf{0}\right\}
$$

where $a$ has the same definition as before and $\hat{\mathbf{y}}^{(k)}=$ $\operatorname{vec}\left\{\hat{\mathbf{Y}}^{(k)}\right\}$.

Since convolutions are the bottleneck of DAMAS2, the performance improvement of (10) with the fast transform with respect to (9) as conventionally implemented is given by the runtime of $\mathbf{A}^{H} \mathbf{A}$ when compared to that of an FFT accelerated convolution. By referring to [1, Fig. 4], one can see that significant improvements can be obtained for all problem sizes. In particular, for the examples shown in Section III, the KAT is 8 times faster than an FFT accelerated convolution. 
Even though DAMAS2 is considered to be a state-of-the-art method for computationally efficient acoustic imaging, it does not use any regularization other than forcing pointwise nonnegativity. Thus, it does not incorporate a prior model of the source distribution. Furthermore, DAMAS2 is a deconvolution approach that relies on restoring detail from very smeared delay and sum images. We have shown that delay-and-sum imaging is equivalent to the application of $\mathbf{A}^{H} \mathbf{A}$ or to convolution by the array PSF, which is a low pass filter. The low-pass characteristic implies that $\mathbf{A}^{H} \mathbf{A}$ has many small singular values. Applying $\mathbf{A}^{H} \mathbf{A}$ significantly attenuates input basis vector components corresponding to these small singular values, such that solving $\breve{\mathbf{y}}=\mathbf{A}^{H} \mathbf{A y}$ for $\mathbf{y}$ (as proposed by DAMAS) is not trivial. On the other hand, the singular values of $\mathbf{A}$ are the square roots of the singular values of $\mathbf{A}^{H} \mathbf{A}$. Thus, the application of $\mathbf{A}$ only attenuates input basis vector components by the square root of the previous factors, making it preferable to solve $\mathbf{s}=\mathbf{A y}$ for $\mathbf{y}$ (as proposed by least-squares formulations). For these two reasons, we favor regularized least-squares methods.

\section{D. $\ell_{1}$-Regularized Least-Squares}

To avoid deconvolution, [16] proposes a covariance fitting technique. Since in the absence of noise, $\operatorname{vec}\{\mathbf{S}\}=\mathbf{A v e c}\{\mathbf{Y}\}$, the authors propose solving

$$
\min _{\hat{\mathbf{Y}}, \sigma^{2}}\left\|\operatorname{vec}\{\mathbf{S}\}-\mathbf{A} \operatorname{vec}\{\hat{\mathbf{Y}}\}-\sigma^{2} \operatorname{vec}\{\mathbf{I}\}\right\|_{2}^{2}
$$

subject to $\hat{\mathbf{Y}}_{i, j} \geq 0, \sigma^{2} \geq 0$, and $\|\operatorname{vec}\{\hat{\mathbf{Y}}\}\|_{1} \leq \lambda$, where $\sigma^{2}$ is the white noise power and $\|\operatorname{vec}\{\hat{\mathbf{Y}}\}\|_{1} \leq \lambda$ is a sparsity constraint. This method assumes that the source distribution is sparse and that only a small number of $\mathrm{U}$-space points have radiating sources. Equation (11) is a convex optimization problem, and can be solved with reasonably efficient numerical methods.

The $\ell_{1}$ constraint serves to regularize the problem, and to permit the inversion of an otherwise ill-conditioned system. Thanks to the $\ell_{1}$ regularization, the authors of [16] show using numerical examples that by solving (11) one can indeed reconstruct sparse images with very high accuracy. Their proposal outperforms DAMAS regarding reconstruction accuracy due to the use of regularization and because no deconvolution was involved.

However, as we have detailed, $\mathbf{A}$ can be a very large matrix, such that solving (11) with a matrix representation of $\mathbf{A}$ (as implemented previously) is very computationally intensive. Of course, the KAT replaces the multiplications by $\mathbf{A}$ and $\mathbf{A}^{H}$, which is all that most convex optimization algorithms require.

In order to obtain a fast formulation that is amenable to existing solvers, we propose recasting (11) as a basis pursuit with denoising problem (BPDN), which has the form

$$
\min _{\hat{\mathbf{Y}}}\|\hat{\mathbf{Y}}\|_{1} \quad \text { subject to }\|\operatorname{vec}\{\mathbf{S}\}-\mathbf{A} \operatorname{vec}\{\hat{\mathbf{Y}}\}\|_{2} \leq \sigma
$$

and has been studied in detail in the compressive sensing literature. In the examples, we solve (12) with SPGL1 [10], which is a state-of-the-art solver designed for large scale problems. The use of the fast transform not only makes this problem tractable, but makes it competitive with our already very efficient variation of DAMAS2, despite using a more robust method for reconstruction. Note that the FFT acceleration is not applicable to (12).

\section{E. Total Variation (TV) Regularized Least-Squares}

To address scenarios where the acoustic images are not sparse in their canonical representations, we propose reconstructing acoustic images with TV regularization.

Given $\mathbf{Y} \in \mathbb{C}^{M_{y} \times M_{x}}$, define its isotropic total variation as

$$
\|\mathbf{Y}\|_{\mathrm{BV}}=\sum_{i, j} \sqrt{\left[\nabla_{x} \mathbf{Y}\right]_{i, j}^{2}+\left[\nabla_{y} \mathbf{Y}\right]_{i, j}^{2}}
$$

where $\nabla_{x}$ and $\nabla_{y}$ are the first difference operators along the $x$ and $y$ dimensions with periodic boundaries, for $0 \leq i<M_{y}$ and $0 \leq j<M_{x} \cdot\|\cdot\|_{\mathrm{BV}}$ is called the bounded variation (BV) semi-norm.

We propose solving

$$
\min _{\hat{\mathbf{Y}}}\|\hat{\mathbf{Y}}\|_{\mathrm{BV}}+\mu\|\operatorname{vec}\{\mathbf{S}\}-\mathbf{A v e c}\{\hat{\mathbf{Y}}\}\|_{2}^{2}
$$

subject to $\hat{\mathbf{Y}}_{i, j} \geq 0$. The first term measures how much an image oscillates. Therefore, it is smallest for images with plateaus and monotonic transitions, and tends to privilege simple solutions with small amounts of noise. The second term ensures a good fit between the reconstructed image and the measured data. This formulation was first proposed for image denoising by Rudin, Osher, and Fatemi [17], for $\mathbf{A}=\mathbf{I}$. It was later generalized and applied successfully to many image reconstruction problems.

To solve (14), we have chosen TVAL3 [11], which uses the augmented Lagrangian method and variable splitting to decouple the TV-minimization and covariance fitting problems. TVAL3 compares very favorably to other solvers in terms of processing time and reconstruction quality, and with the fast transform it becomes practically as efficient as our accelerated version of DAMAS2, while providing more accurate and stable reconstructions with guaranteed convergence.

\section{RECONSTRUCTION EXAMPLES}

In the following we show image reconstruction examples illustrating the use of delay and sum beamforming, DAMAS2, $\ell_{1}$ regularization, and TV regularization, all implemented with the KAT. We simulate a 64-element separable array, with $N_{x}=$ $N_{y}=8$, and with horizontal and vertical apertures of $30 \mathrm{~cm}$. Each $N_{x} \times 1$ and $N_{y} \times 1$ linear subarray is chosen to be a nonredundant array with minimum missing lags [18], with interelement spacing .1.3.5.6.7.10.2. (where the dots represent elements, and the numbers represent interelement distances). This geometry is plotted in Fig. 1.

In this section, we present results comparing delay and sum beamforming, DAMAS2, $\ell_{1}$-regularized reconstruction with SPGL1 [10] solving (12), and TV-regularized reconstruction with TVAL3 [11] solving (14). All methods were accelerated with exact versions of the KAT (not using the NFFT), and the images were reconstructed with $M_{x}=M_{y}=256$. 


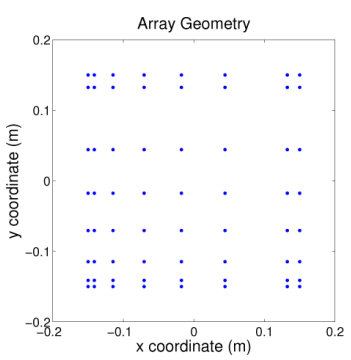

Fig. 1. Simulated array geometry.

DAMAS2, SPGL1, and TVAL3 used 1000, 200, and 100 iterations, respectively, which provide a good compromise between computational cost and image quality. Thanks to the KAT, the reconstruction times for delay-and-sum, DAMAS2, $\ell_{1}$ regularized reconstruction and TV regularized reconstruction were approximately $5 \mathrm{~ms}, 1.5 \mathrm{~s}, 8 \mathrm{~s}$, and $4 \mathrm{~s}$ per image, respectively. DAMAS2 requires no parameters. SPGL1 used $\sigma=0.01\|\mathbf{S}\|_{F}$ in (12). TVAL3 used $\mu=10^{3}$ in (14). The signal model is given by $\mathbf{S}=\mathbf{V E}\left\{\mathrm{ff}^{H}\right\} \mathbf{V}^{H}+\sigma^{2} \mathbf{I}$, with $\sigma^{2}$ set to obtain $20 \mathrm{~dB}$ $\mathrm{SNR}$. Since the intent of these simulations is not to analyze the noise sensitivity of each method, only one SNR is used.

\section{A. Checkerboard Patterns}

Fig. 2 shows reconstructed checkerboard patterns. This reconstruction clearly shows the deficiencies of delay-and-sum imaging. The images are quite smeared, and all images present artifacts due to sidelobes. DAMAS2 produces very good results, reproducing the checkerboard patterns correctly, with small artifacts outside the checkerboards. $\ell_{1}$-regularized reconstruction shows better defined edges, but some artifacts, since the patterns are not sparse. Note that some checkerboard squares have dots where the sound pressure level has been underestimated. Artifacts of this kind are common when using $\ell_{1}$ regularization to reconstruct signals with plateaus, because they present a compromise between sparsity and fitness to the measured data. TV regularization produces reconstructions similar to DAMAS2, but with some smearing around the edges of the visible region.

\section{B. Sparse Patterns}

Fig. 3 presents reconstruction results for a test image with 17 unit impulses at U-space coordinates $( \pm n / 6, \pm n / 6)$, for $0 \leq$ $n \leq 4$. This test is designed to evaluate the equivalent PSF for the reconstruction methods, as well as detect the presence of aliasing artifacts. As expected, delay and sum has the lowest spatial resolution and shows significant sidelobes. DAMAS2 shows some artifacts, which result from its lack of regularization. $\ell_{1}$-regularized reconstruction presents the best results, with very small sources and no artifacts. This is a reasonable result, since the image of interest is indeed very sparse. TV-regularization also presents good results, with no discernible artifacts but with larger sources than $\ell_{1}$ regularization.

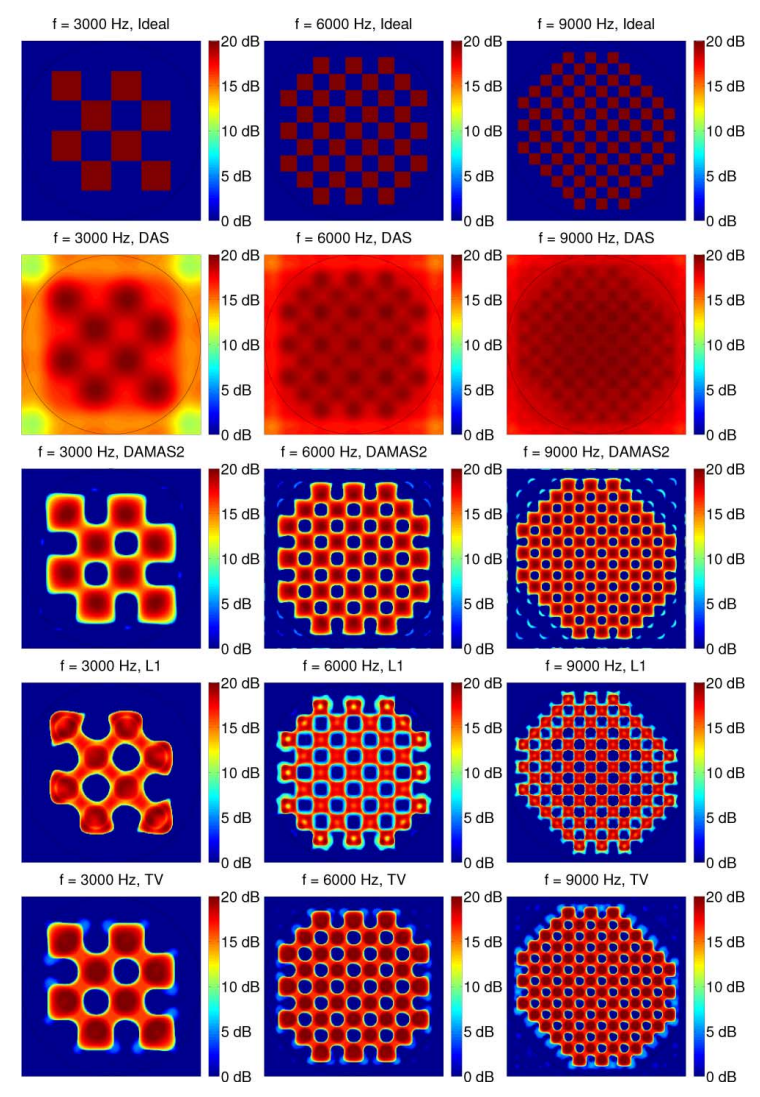

Fig. 2. Reconstruction of the checkerboard patterns, for $M_{x}=M_{y}=256$. First row: ideal distribution; second row: delay-and-sum; third row: DAMAS2; fourth row: $\ell_{1}$-regularized least-squares; fifth row: TV-regularized least-squares.

\section{Non-Sparse Test Pattern}

Fig. 4 shows reconstruction results for a non-sparse test pattern designed for this experiment. Once again, delay-and-sum has low resolution and rectangular smearing due to the separable geometry's sidelobes. DAMAS2 produces much better results, but still shows some artifacts, especially for high frequency images. The artifacts are gone with $\ell_{1}$-regularized reconstruction, which also has better resolution than DAMAS2. Nevertheless, it does not represent smooth transitions well, since they are not sparse. Finally, TV-regularized reconstruction produces the most accurate representations, with correct shapes and low noise.

\section{Near-Field Imaging}

In this section, we show how the far-field assumption can break down, and how the near-field extension of the KAT can be used to model near-field effects. We simulate the checkerboard source distribution positioned over a hemispherical shell with a radius of $1.0 \mathrm{~m}$. Both the array and the shell are centered at $(0,0,0)$. 

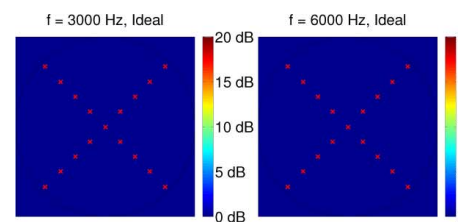

$f=9000 \mathrm{~Hz}$, Idea

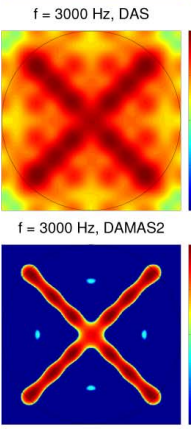

$\mathrm{f}=6000 \mathrm{~Hz}$, DAS
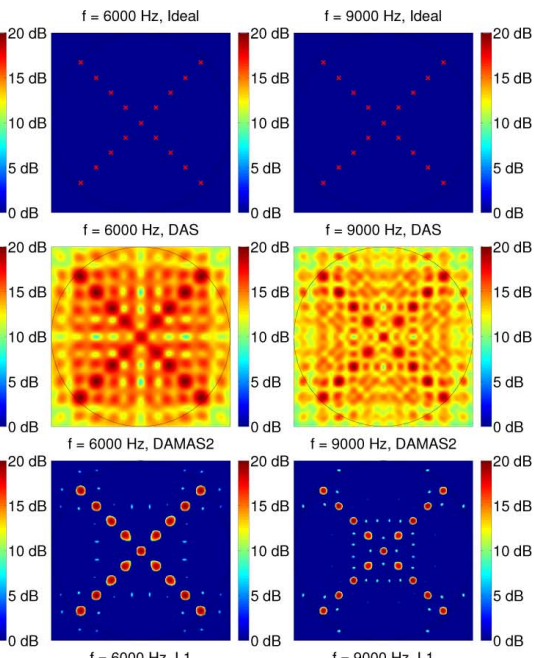

$\mathrm{f}=3000 \mathrm{~Hz}, \mathrm{LI}$ $f=6000 \mathrm{~Hz}, \mathrm{~L}$ $=9000 \mathrm{~Hz}, \mathrm{~L}$
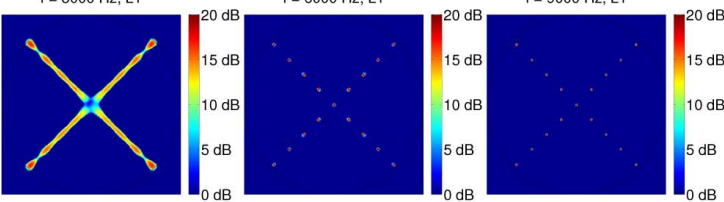

$\mathrm{f}=3000 \mathrm{~Hz}, \mathrm{TV}$
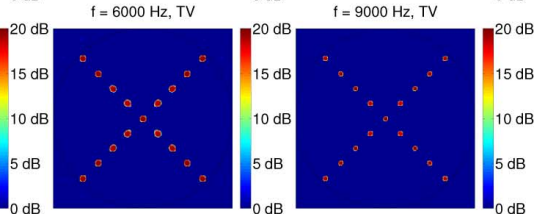

Fig. 3. Reconstruction of the impulsive patterns, for $M_{x}=M_{y}=256$. First row: ideal distribution; second row: delay-and-sum; third row: DAMAS2; fourth row: $\ell_{1}$-regularized least-squares; fifth row: TV-regularized least-squares.

The top row of Fig. 5 presents the checkerboard images reconstructed with the exact (slow) near-field transform. Reconstruction results are very similar to the far-field ones, indicating that the transform did not degenerate. The second row shows the reconstruction using a far-field approximation. The estimated distributions are very smeared and show significant artifacts. The other rows show reconstruction results for $K=1,4$, and 8 , as prescribed in [1, Sect. VI]. The artifacts are essentially gone, and the smearing has been significantly reduced. Note that the computational cost for implementing a rank- $K$ KAT is $K$ times larger than implementing a far-field KAT. Nevertheless, even for $K=8$ this approach is about as fast as a direct NFFT implementation (which cannot be used in this case), while accurately modeling strong near-field effects. Indeed, the reconstruction times for Fig. 5 (using TVAL) were 4.0, 4.9, 6.4, and $10.0 \mathrm{~s}$ for $K=$ $1,2,4$, and 8 . In contrast, explicit matrix multiplication requires approximately $2000 \mathrm{~s}$.

\section{HOW GoOd ARE CARTESIAN ARRAYS?}

Multi-arm logarithmic spiral arrays [19] have been shown to have low sidelobes over a wide range of frequencies. Since the low sidelobe characteristic is crucial when performing imaging with beamforming, these geometries have found widespread use. Nevertheless, sidelobes have little relevance if one can effi-

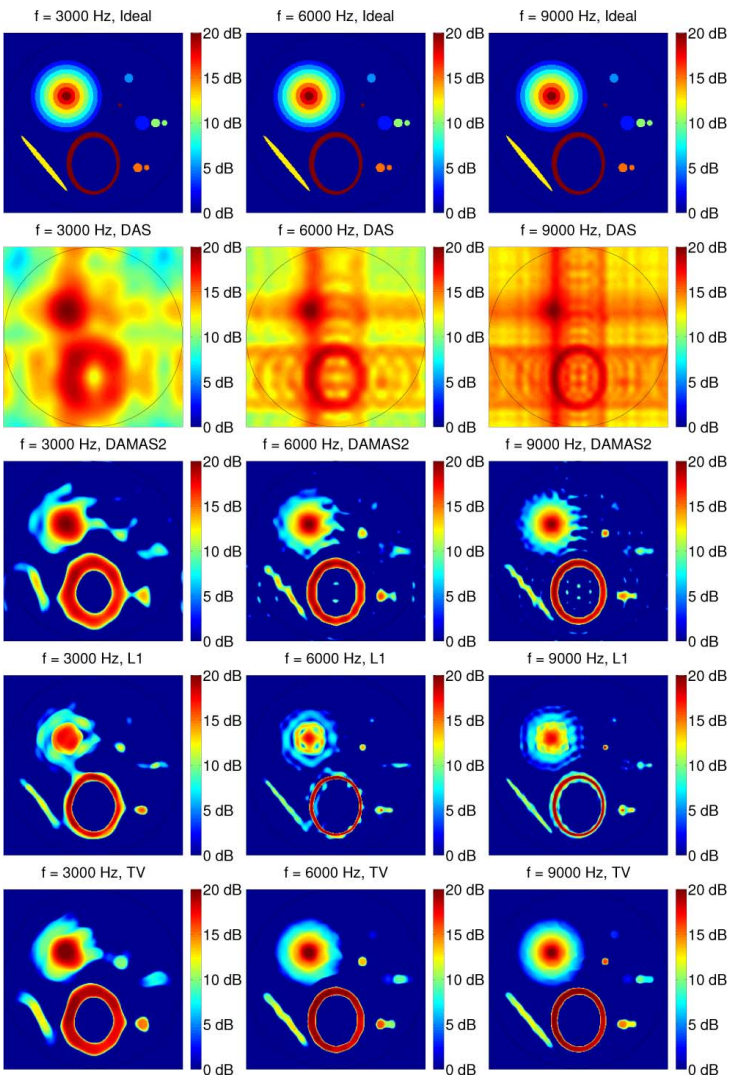

Fig. 4. Reconstruction of the non-sparse test pattern, for $M_{x}=M_{y}=256$. First row: ideal distribution; second row: delay-and-sum; third row: DAMAS2; fourth row: $\ell_{1}$-regularized least-squares; fifth row: TV-regularized least-squares.

ciently use deconvolution or regularized least-squares methods. In this case, ideal geometries become the ones with zero redundancy and minimum missing lags (which give highest bandwidth and some reconstruction artifacts) or minimum redundancy and zero missing lags (which theoretically allow ideal reconstruction up to a given frequency, under a far-field assumption and in the absence of noise). In general, these geometries do not produce low sidelobes, but the sidelobes are low enough to allow nonambiguous reconstruction.

In this section, we compare the Cartesian geometry presented in Fig. 1 and the 63-element logarithmic spiral geometry presented in Fig. 6. This spiral array has an aperture of $50 \times 50$ $\mathrm{cm}$, which was chosen to produce images with resolution similar to those of our separable array (which has a $30 \times 30 \mathrm{~cm}$ aperture). Furthermore, its parameters were carefully chosen to produce optimal reconstruction for the frequencies of interest. Fig. 7 shows reconstruction results for this logarithmic spiral geometry, under the same conditions as Fig. 4.

While the logarithmic spiral geometry produces better results for delay-and-sum, the other techniques produce results of comparable quality. In particular, TV-regularized least-squares produces very similar results for both geometries. This is not surprising, since the Cartesian geometry was chosen to have op- 


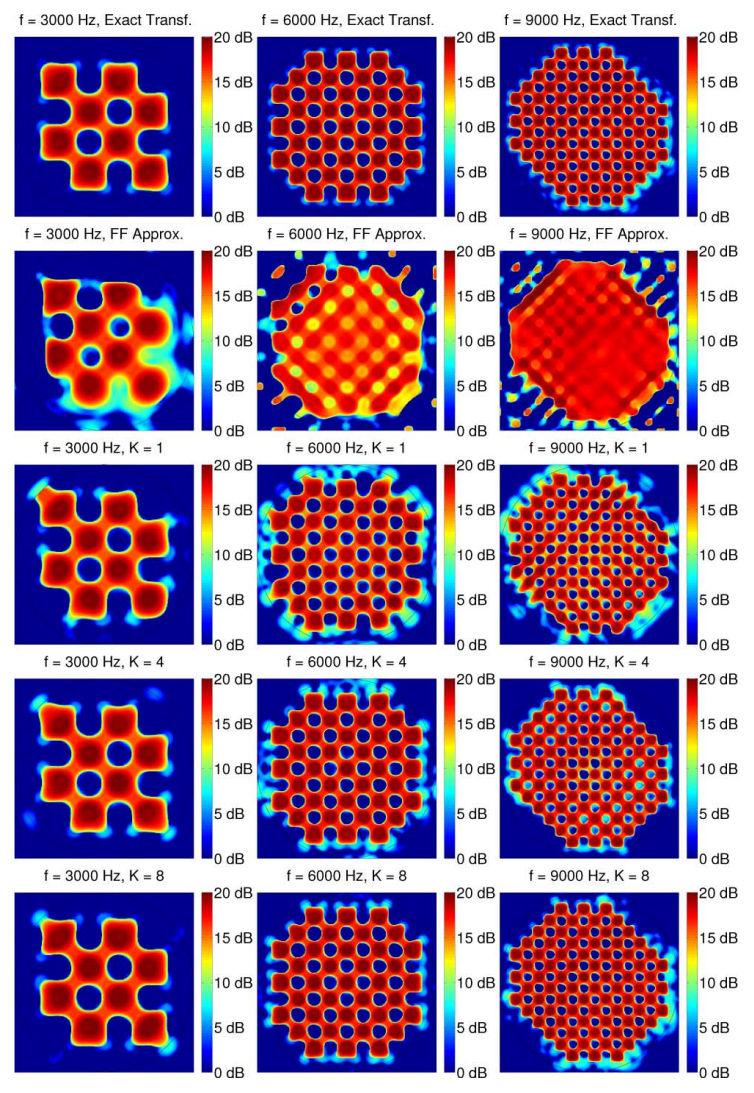

Fig. 5. TV-regularized reconstruction of the checkerboard patterns. The real source distribution is located over a hemispherical shell with a radius of $1 \mathrm{~m}$. From top to bottom: reconstruction using the exact (slow) transform; reconstruction using a far-field approximation; reconstructions using the best Kronecker approximation for varying values of $K$.

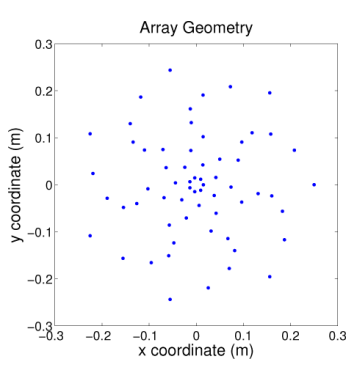

Fig. 6. Logarithmic spiral array geometry with 63 elements, inner radius $r_{0}=$ $1.5 \mathrm{~cm}$, outer radius $r_{\max }=25 \mathrm{~cm}, 9$ concentric circles, 7 arms, and having each arm perform 2 full rotations.

timal characteristics. While this example is by no means exhaustive, it is meant to convince the reader that given appropriate image reconstruction techniques, Cartesian geometries can be nearly as powerful as more traditional logarithmic spiral geometries. Of course, with Cartesian arrays one can apply the KAT and obtain extremely fast and accurate near-field reconstruction for arbitrary focal surfaces. With logarithmic spiral geometries, one has no such option.

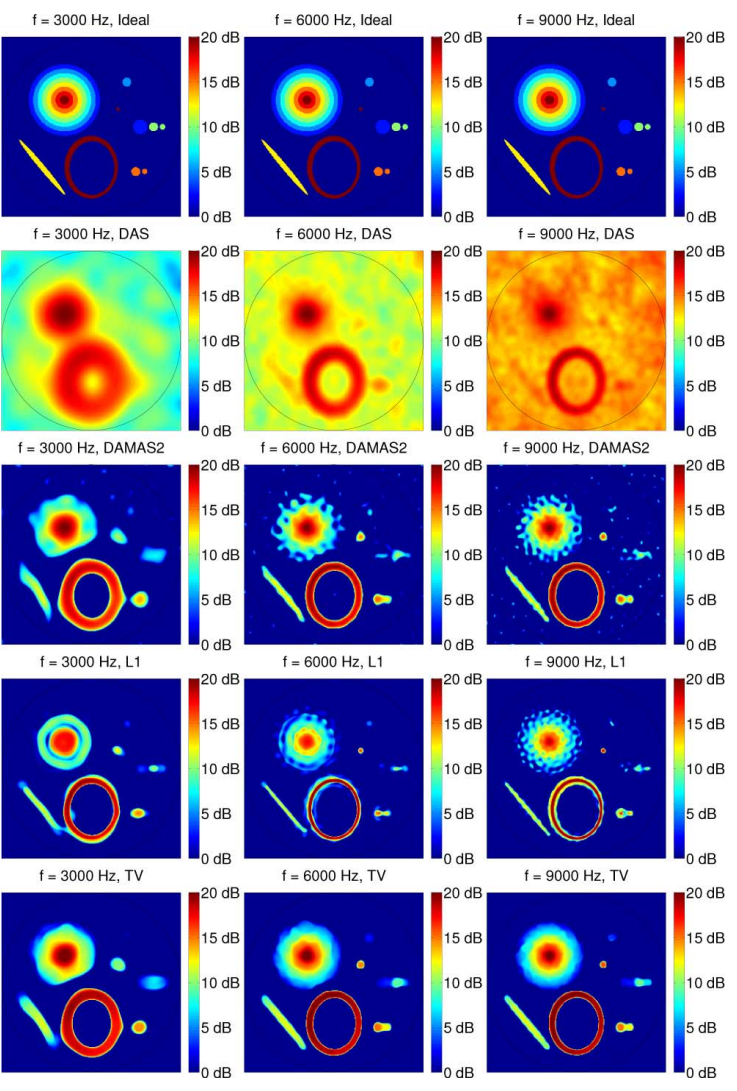

Fig. 7. Reconstruction of the non-sparse test pattern with a logarithmic spiral array, for $M_{x}=M_{y}=256$. First row: ideal distribution; second row: delayand-sum; third row: DAMAS2; fourth row: $\ell_{1}$-regularized least-squares; fifth row: TV-regularized least-squares.

\section{CONCLUSION}

Using the assumption of a separable array geometry, in [1] we presented the KAT, which can efficiently transform back and forth between a hypothetical source distribution and its corresponding spectral matrix, under the assumption of a separable array. This transform is orders of magnitude faster than explicit matrix multiplication, and one order of magnitude faster than NFFT-based approaches. Furthermore, the KAT can be generalized for near-field imaging, while the NFFT must use a far-field approximation.

In this Part II, we have recast delay-and-sum beamforming, MVDR beamforming and the DAMAS2 deconvolution algorithm [15] as applications of our transform. Thus, we have shown how the computational benefits from [1] can be realized in practice. Furthermore, the KAT allows the efficient use of general purpose regularized least-squares solvers. To demonstrate this application, we used it to recast acoustic imaging as least-squares problems with $\ell_{1}$ and total variation regularization. With the KAT, regularized reconstruction becomes straightforward, elegant and computationally efficient. With this approach, image reconstruction can be delegated to third party solvers, saving the time and effort of developing ad hoc methods. 
Finally, we have shown that by using carefully chosen separable arrays one does not have to compromise on reconstruction quality. Thus, the KAT does not require a tradeoff between accuracy and reconstruction time.

\section{REFERENCES}

[1] F. Ribeiro and V. Nascimento, "Fast transforms for acoustic imaging-Part I: Theory," IEEE Trans. Image Process., vol. 20, no. 8, pp. XXX-XXX, Aug. 2011

[2] S. Chen, D. Donoho, and M. Saunders, "Atomic decomposition by basis pursuit," SIAM Rev., vol. 43, no. 1, pp. 129-159, 2001

[3] E. Candès, J. Romberg, and T. Tao, "Stable signal recovery from incomplete and inaccurate measurements," Commun. Pure Appl. Math., vol. 59, no. 8, p. 1207, 2006.

[4] E. Candes, J. Romberg, and T. Tao, "Robust uncertainty principles: Exact signal reconstruction from highly incomplete frequency information," IEEE Trans. Inf. Theory, vol. 52, no. 2, pp. 489-509, Feb. 2006.

[5] D. Donoho, "Compressed sensing," IEEE Trans. Inf. Theory, vol. 52, no. 4, pp. 1289-1306, Apr. 2006.

[6] D. Donoho and J. Tanner, "Sparse nonnegative solution of underdetermined linear equations by linear programming," in Proc. Nat. Acad. Sci. USA, 2005, p. 9446

[7] E. Hale, W. Yin, and Y. Zhang, "A fixed-point continuation method for 11-regularized minimization with applications to compressed sensing," Rice Univ., Houston, TX, Tech. Rep. CAAM R07-07, 2007.

[8] M. Figueiredo, R. Nowak, and S. Wright, "Gradient projection for sparse reconstruction: Application to compressed sensing and other inverse problems," IEEE J. Sel. Topics Signal Process., vol. 1, no. 4, pp. 586-597, Dec. 2007.

[9] S. Kim, K. Koh, M. Lustig, S. Boyd, and D. Gorinevsky, "An interiorpoint method for large-scale 11-regularized least squares," IEEE J. Sel. Topics Signal Process., vol. 1, no. 4, pp. 606-617, Dec. 2007.

[10] E. van den Berg and M. Friedlander, "Probing the pareto frontier for basis pursuit solutions," SIAM J. Scientif. Comput., vol. 31, no. 2, pp. 890-912, 2008.

[11] C. Li, "An efficient algorithm for total variation regularization with applications to the single pixel camera and compressive sensing," M.S thesis, Dept. Computat. Appl. Math., Rice Univ., Houston, TX, 2009.

[12] J. Capon, "High-resolution frequency-wavenumber spectrum analysis," Proc. IEEE, vol. 57, no. 8, pp. 1408-1418, Aug. 1969.

[13] D. Blacodon, "Spectral estimation noisy data using a reference noise," presented at the BeBeC, Berlin, Germany, 2010.

[14] H. L. Van Trees, Optimum Array Processing: Part IV of Detection, Es timation, and Modulation Theory. New York: Wiley, 2002

[15] R. Dougherty, "Extensions of DAMAS and benefits and limitations of deconvolution in beamforming," presented at the 11th AIAA/CEAS Aeroacoust. Conf., Monterey, CA, 2005.
[16] T. Yardibi, J. Li, P. Stoica, and L. Cattafesta, III, "Sparsity constrained deconvolution approaches for acoustic source mapping," J. Acoust. Soc. Amer., vol. 123, p. 2631, 2008

[17] L. Rudin, S. Osher, and E. Fatemi, "Nonlinear total variation based noise removal algorithms," Phys. D: Nonlinear Phenomena, vol. 60, no. $1-4$, pp. $259-268,1992$

[18] E. Vertatschitsch and S. Haykin, "Nonredundant arrays," Proc. IEEE, vol. 74, no. 1, p. 217, Jan. 1986.

[19] J. Underbrink and R. Dougherty, "Array design for non-intrusive measurement of noise sources," in Proc. NOISE-CON, 1996, pp. 757-762.

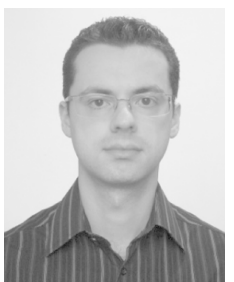

Flávio P. Ribeiro received the B.S. degree in electrical engineering from Escola Politécnica, University of São Paulo, São Paulo, Brazil, in 2005, and the B.S. degree in mathematics from the Institute of Mathematics and Statistics, University of São Paulo, São Paulo, Brazil, in 2008. He is currently pursuing the $\mathrm{Ph} . \mathrm{D}$. degree in electrical engineering from the Escola Politécnica, University of São Paulo.

From 2007 to 2009, he was a Hardware Engineer with Licht Labs, where he developed controllers for power transformers and substations. In the Summers of 2009 and 2010, he was a Research Intern with Microsoft Research Redmond. His research interests include array signal processing, multimedia signal processing, and computational linear algebra.

Mr. Ribeiro was a recipient of the Best Student Paper Award at ICME 2010.

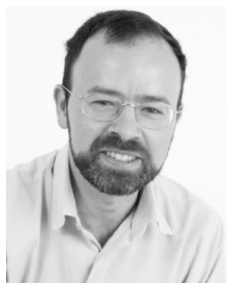

Vítor H. Nascimento was born in São Paulo, Brazil. He received the B.S. and M.S. degrees in electrical engineering from the University of São Paulo, São Paulo, Brazil, in 1989 and 1992, respectively, and the Ph.D. degree from the University of California, Los Angeles, in 1999.

From 1990 to 1994 , he was a Lecturer with the University of São Paulo, and in 1999 he joined the faculty at the same school, where he is now an Associate Professor. His research interests include signal processing theory and applications, robust and nonlinear estimation, and applied linear algebra.

Prof. Nascimento was a recipient of the IEEE SPS Best Paper Award, in 2002. He served as an Associate Editor for Signal Processing Letters from 2003 to 2005, for the Transactions on Signal Processing from 2005 to 2008, and for the EURASIP Journal on Advances in Signal Processing from 2006 to 2009. He is currently a member of the IEEE-SPS Signal Processing Theory and Methods Technical Committee. 



\title{
FAST NEAR-FIELD ACOUSTIC IMAGING WITH SEPARABLE ARRAYS
}

\author{
Flávio P. Ribeiro, Vítor H. Nascimento
}

Electronic Systems Engineering Dept., Universidade de São Paulo

$\{$ fr,vitor\}@lps.usp.br

\begin{abstract}
Acoustic imaging is a computationally intensive and ill-conditioned inverse problem, which involves estimating high resolution source distributions with large microphone arrays. We have recently shown how to significantly accelerate acoustic imaging under a far-field approximation using fast transforms. This paper generalizes our previous work to obtain computationally efficient and accurate transforms for near-field imaging with separable arrays. We show that under a suitable permutation, the imaging transform can be made nearly separable, even when modeling strong near-field effects. We exploit this quasi-separability to obtain a computationally efficient and accurate low-rank representation, allowing the design of fast transforms for near-field operation with arbitrary focal surfaces and arbitrary accuracy. We combine these transforms with calibration matrices, which compensate non-separable characteristics and allow one to quickly reshape focal surfaces without having to recompute the optimal transforms.
\end{abstract}

Index Terms - array processing, fast transform, acoustic imaging, low-rank approximation, kronecker approximation.

\section{INTRODUCTION}

Acoustic imaging with microphone arrays has become a standard tool for studying aeroacoustic sources. It is routinely used to measure the noise generated by engines, turbines, vehicles and aircraft for aerodynamic design and noise reduction purposes [1].

Due to its relatively low computational cost, beamforming remains a popular method for acoustic imaging. Unfortunately, it produces the source distribution of interest convolved with the array point spread function. Deconvolution algorithms [2] have been proposed to enhance beamformed images. More recently, regularized covariance fitting techniques [3] have been shown to deliver even better results. However, in their original formulations, these methods have high computational costs, because they have no means of efficiently transforming back and forth between the image under reconstruction and the measured data.

Motivated by this observation, we proposed a fast transform designed to enable computationally efficient and accurate imaging with separable ${ }^{1}$ planar arrays [4]. Using this transform, one can perform deconvolutions an order of magnitude faster than with FFTbased methods. In related work [5], we use such transforms to recast acoustic imaging as regularized least-squares covariance fitting. While these formulations would be ordinarily intractable, by using fast transforms we are able to accelerate their solution by many orders of magnitude. Indeed, this approach delivers more accurate results than competing FFT-based deconvolution methods, and in a smaller amount of time.

To our knowledge, previous work for acoustic imaging involving fast transforms has always assumed sources located in the far-field

${ }^{1}$ An array with manifold vector $\mathbf{v}\left(u_{x}, u_{y}\right)$ is said to be separable if there exist $\mathbf{a}\left(u_{x}\right)$ and $\mathbf{b}\left(u_{y}\right)$ such that $\mathbf{v}\left(u_{x}, u_{y}\right)=\mathbf{a}\left(u_{x}\right) \otimes \mathbf{b}\left(u_{y}\right)$ for all valid $u_{x}, u_{y}$, $\mathbf{a}\left(u_{x}\right)$ and $\mathbf{b}\left(u_{y}\right)$ such that $\mathbf{v}\left(u_{x}, u_{y}\right)=\mathbf{a}\left(u_{x}\right) \otimes \mathbf{b}\left(u_{y}\right)$ for all valid $u_{x}, u_{y}$,
where $\otimes$ is the Kronecker product. For example, arrays with elements positioned over where $\otimes$ is the Kronecker product. For example, arrays with elements positioned o
(not necessarily uniform) Cartesian grids are separable under a far-field assumption. of the array. Indeed, FFT-based deconvolution approaches such as DAMAS2 [2] require a shift-invariant point spread function, which in turn implies a far-field assumption.

Our transform for separable arrays was also derived under a farfield assumption [4]. Nevertheless, it does not impose any structure onto the array manifold vector other than its separability. Thus, we anticipated its use for near-field imaging, as long as one could produce suitable separable approximations to exact (non-separable) near-field manifold vectors.

In this paper we go a step further. We show that a suitable permutation and rearrangement of the transform matrix makes it easily approximable by a truncated series of Kronecker products, even when modeling strong near-field effects. This quasi-separability (in the Kronecker sense) is equivalent to the existence of a low-rank approximation, which we use to obtain a computationally efficient and accurate transform.

This paper is organized as follows: Section 2 provides definitions and reviews our fast transform for separable geometries. Section 3 generalizes the far-field transform by modeling spherical wavefronts and arbitrary focal surfaces. We show how to compute the optimal low-rank fast transform, and how to incorporate interpolation matrices for calibration and fast focusing. Section 4 features examples and Section 5 has our conclusions.

\section{PRELIMINARIES}

We use the superscripts $\cdot^{T},{ }^{H}$, and $*^{*}$ to denote transposition, Hermitian transposition, and complex conjugation, respectively. The remainder of $a / b$, for $a, b \in \mathbb{Z}_{+}$is written as $\bmod (a, b)$. Round-off of $x \in \mathbb{R}$ towards $-\infty$ is denoted by $\lfloor x\rfloor$. Given suitably sized matrices $\mathbf{A}, \mathbf{B}, \mathbf{C}$, we use $\mathbf{C}=\underline{\mathbf{A}}(\mathbf{B})$ to denote vec $\{\mathbf{C}\}=\mathbf{A v e c}\{\mathbf{B}\}$.

Consider a planar array of $N$ microphones with coordinates $\mathbf{p}_{0}$, $\ldots, \mathbf{p}_{N-1} \in \mathbb{R}^{3}$. Suppose the wave field of interest can be modeled by the superposition of $M$ point sources with coordinates $\mathbf{q}_{0}, \ldots$, $\mathbf{q}_{M-1} \in \mathbb{R}^{3}$, where $M$ is usually large. The $N \times 1$ array output for a frequency $\omega$ is modeled as

$$
\mathbf{x}(\omega)=\mathbf{V}(\omega) \mathbf{f}(\omega)+\boldsymbol{\eta}(\omega),
$$

where $\mathbf{V}(\omega)=\left[\mathbf{v}\left(\mathbf{q}_{0}, \omega\right) \cdots \mathbf{v}\left(\mathbf{q}_{M-1}, \omega\right)\right]$ is the array manifold matrix, $\mathbf{f}(\omega)=\left[f_{0}(\omega) f_{1}(\omega) \cdots f_{M-1}(\omega)\right]^{T}$ is the frequency domain signal waveform and $\boldsymbol{\eta}(\omega)$ is uncorrelated noise.

The near-field array manifold vector models a spherical wavefront, and is given by

$$
\mathbf{v}\left(\mathbf{q}_{m}, \omega\right)=\left[\frac{\mathrm{e}^{-j\left\{\frac{\omega}{c}\left\|\mathbf{p}_{0}-\mathbf{q}_{m}\right\|\right\}}}{\left\|\mathbf{p}_{0}-\mathbf{q}_{m}\right\|} \cdots \frac{\mathrm{e}^{-j\left\{\frac{\omega}{c}\left\|\mathbf{p}_{N-1}-\mathbf{q}_{m}\right\|\right\}}}{\left\|\mathbf{p}_{N-1}-\mathbf{q}_{m}\right\|}\right]^{T} \cdot(2)
$$

Let $\mathbf{S}_{\mathbf{x}}(\omega)=\mathrm{E}\left\{\mathbf{x}(\omega) \mathbf{x}^{H}(\omega)\right\}$ be the array cross spectral matrix. If $\mathbf{x}_{0}(\omega), \ldots, \mathbf{x}_{L-1}(\omega)$ correspond to $L$ frequency domain snapshots, the spectral matrix can be estimated with

$$
\mathbf{S}_{\mathbf{x}}(\omega)=\frac{1}{L} \sum_{l=0}^{L-1} \mathbf{x}_{l}(\omega) \mathbf{x}_{l}^{H}(\omega)
$$


It is usually better to work with $\mathbf{S}_{\mathbf{x}}(\omega)$ instead of directly with each $\mathbf{x}_{l}(\omega)$, because $\mathbf{S}_{\mathbf{x}}(\omega)$ carries only the relative phase shifts between microphones and is the result of averaging, such that it has less noise content. We will assume narrow-band processing and omit the argument $\omega$. To simplify the notation, $\mathbf{S}_{\mathbf{x}}(\omega)$ will be written as $\mathbf{S}$.

Assuming that the noise is spatially white and uncorrelated with the sources of interest, we have

$$
\mathbf{S}=\mathbf{V E}\left\{\mathbf{f f}^{H}\right\} \mathbf{V}^{H}+\sigma^{2} \mathbf{I},
$$

where $\sigma^{2}=\mathrm{E}\left\{\eta_{i} \eta_{i}^{*}\right\}, 0 \leq i<N$. Assuming that the sources are uncorrelated (a nearly universal assumption for acoustic imaging) implies that $\mathrm{E}\left\{\mathbf{f f}^{H}\right\}$ is diagonal.

For the purposes of acoustic imaging, the source coordinates $\left\{\mathbf{q}_{i}\right\}_{i=0}^{M-1}$ are chosen to discretize a surface of interest (the focal surface). We consider surfaces parameterized in two coordinates, such that $\mathbf{q}_{i}=\varphi\left(\mathbf{u}_{i}\right)$, for $\mathbf{u}_{i} \in \mathbb{R}^{2}$ and a parameterization $\varphi$. Furthermore, we discretize the focal surface using a Cartesian grid, such that $\left\{\mathbf{u}_{i}\right\}_{i=0}^{M-1}=\left\{u_{x_{m}}\right\}_{m=0}^{M_{x}-1} \times\left\{u_{y_{n}}\right\}_{n=0}^{M_{y}-1}$ and

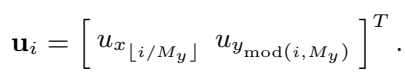

Using this parameterization, the ideal acoustic image is given by $\mathbf{Y} \in \mathbb{R}^{M_{y} \times M_{x}}$ with $\mathbf{y}=\operatorname{vec}\{\mathbf{Y}\}=\left[\left|f_{0}\right|^{2} \cdots\left|f_{M-1}\right|^{2}\right]^{T}$. Thus, the pixel coordinates of $\mathbf{Y}$ correspond to source locations, and the pixel values correspond to source powers.

Note that $\operatorname{diag}\left[\mathrm{E}\left\{\mathbf{f} \mathbf{f}^{H}\right\}\right]=\mathbf{y}$, so we can rewrite (4) as

$$
\mathbf{S}=\sum_{m=0}^{M-1} y_{m} \mathbf{v}\left(\varphi\left(\mathbf{u}_{m}\right)\right) \mathbf{v}^{H}\left(\varphi\left(\mathbf{u}_{m}\right)\right) \text {. }
$$

Many reconstruction algorithms iteratively compute an estimated image $\hat{\mathbf{Y}}$ and compare the corresponding $\hat{\mathbf{S}}$ obtained through (6) with the measured values obtained from (3). Unless the image and its iterates are very sparse, (6) becomes computationally intractable, motivating the need for fast transforms.

Let $\mathbf{s}=\operatorname{vec}\{\mathbf{S}\}, \mathbf{y}=\operatorname{vec}\{\mathbf{Y}\}$ and $\mathbf{v}_{\mathbf{u}_{m}}=\mathbf{v}\left(\varphi\left(\mathbf{u}_{m}\right)\right)$. Note that vec $\left\{\mathbf{v}_{\mathbf{u}_{m}} \mathbf{v}_{\mathbf{u}_{m}}^{H}\right\}=\mathbf{v}_{\mathbf{u}_{m}}^{*} \otimes \mathbf{v}_{\mathbf{u}_{m}}$, where $\otimes$ is the Kronecker product. Thus, we can write (6) as (note that $\mathbf{A}$ below is $N^{2} \times M$ )

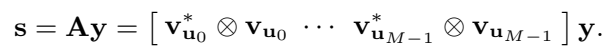

Let $N=N_{x} N_{y}$ be the number of array elements. In [4], we show that when the array manifold vector is separable such that $\mathbf{v}(\varphi(\mathbf{u}))=\mathbf{v}\left(\varphi\left(u_{x}, u_{y}\right)\right)=\mathbf{v}_{x}\left(\varphi_{x}\left(u_{x}\right)\right) \otimes \mathbf{v}_{y}\left(\varphi_{y}\left(u_{y}\right)\right)$, where $\mathbf{v}_{x}\left(\varphi_{x}\left(u_{x}\right)\right)$ and $\mathbf{v}_{y}\left(\varphi_{y}\left(u_{y}\right)\right)$ are $N_{x} \times 1$ and $N_{y} \times 1$ manifold vectors, the computation of (7) may be accelerated as follows. Let

$$
\begin{aligned}
& \mathbf{S}=\left[\begin{array}{ccc}
\mathbf{T}_{0,0} & \cdots & \mathbf{T}_{0, N_{x}-1} \\
\vdots & & \vdots \\
\mathbf{T}_{N_{x}-1,0} & \cdots & \mathbf{T}_{N_{x}-1, N_{x}-1}
\end{array}\right], \quad \mathbf{T}_{i, j} \in \mathbb{C}^{N_{y} \times N_{y}} \\
& \mathbf{Z}=\left[\begin{array}{llll}
\mathbf{t}_{0,0} & \mathbf{t}_{1,0} & \ldots & \mathbf{t}_{N_{x}-1, N_{x}-1}
\end{array}\right], \quad \mathbf{t}_{i, j}=\operatorname{vec}\left\{\mathbf{T}_{i, j}\right\} .
\end{aligned}
$$

Define $\boldsymbol{\Xi}$ such that vec $\{\mathbf{S}\}=\boldsymbol{\Xi} \operatorname{vec}\{\mathbf{Z}\}$ (note that $\boldsymbol{\Xi}$ is a permutation). As shown in [4], the separability of $\mathbf{v}(\varphi(\mathbf{u}))$ implies that there exist $\mathbf{V}_{\mathbf{x}} \in \mathbb{C}^{N_{x}^{2} \times M_{x}}$ and $\mathbf{V}_{\mathbf{y}} \in \mathbb{C}^{N_{y}^{2} \times M_{y}}$ such that $\mathbf{A}=\boldsymbol{\Xi}\left(\mathbf{V}_{\mathbf{x}} \otimes \mathbf{V}_{\mathbf{y}}\right)$. Since $\left(\mathbf{A}^{T} \otimes \mathbf{B}\right) \operatorname{vec}\{\mathbf{C}\}=\operatorname{vec}\{\mathbf{B C A}\}$ whenever BCA is defined [6], $\mathbf{S}=\underline{\mathbf{A}}(\mathbf{Y})$ can be efficiently implemented as $\mathbf{S}=\boldsymbol{\Xi}\left(\mathbf{V}_{\mathbf{y}} \mathbf{Y} \mathbf{V}_{\mathbf{x}}{ }^{T}\right)$ (see Section 3 for the gain in computational cost). We remark that, under a far-field approximation, the manifold vector will be separable if the microphones are disposed in a (possibly nonuniform) Cartesian grid [4].

\section{NEAR-FIELD IMAGING}

\subsection{Low-rank extension for non-separable arrays}

Near-field manifold vectors are not in general separable. As we show in Section 4, in the absence of compensation, a (separable) far-field approximation can cause numerous artifacts. In this section we show how to approximate $\mathbf{A}$ using a sum of separable transforms, allowing us to dramatically improve reconstruction quality.

Recall that if $\mathbf{v}(\varphi(\mathbf{u}))$ is separable, there exist $\mathbf{V}_{\mathbf{x}}$ and $\mathbf{V}_{\mathbf{y}}$ such that $\mathbf{A}=\boldsymbol{\Xi}\left(\mathbf{V}_{\mathbf{x}} \otimes \mathbf{V}_{\mathbf{y}}\right)$. In the general case, we approximate $\mathbf{A}$ by $\breve{\mathbf{A}}=\boldsymbol{\Xi}\left(\sum_{k=1}^{K} \mathbf{C}_{k} \otimes \mathbf{D}_{k}\right)$, for small values of $K$ and certain matrices $\mathbf{C}_{k}$ and $\mathbf{D}_{k}$, allowing $\mathbf{S}=\underline{\breve{A}}(\mathbf{Y})$ to be efficiently implemented as $\mathbf{S}=\boldsymbol{\Xi}\left(\sum_{k=1}^{K} \mathbf{D}_{k} \mathbf{Y} \mathbf{C}_{k}^{T}\right)$.

Let $\mathbf{B} \in \mathbb{C}^{m \times n}$ be a generic matrix with $m=m_{1} m_{2}$ and $n=$ $n_{1} n_{2}$. Consider approximating $\mathbf{B}$ as a sum of Kronecker products in terms of $\mathbf{C}_{k} \in \mathbb{C}^{m_{1} \times n_{1}}$ and $\mathbf{D}_{k} \in \mathbb{C}^{m_{2} \times n_{2}}, 1 \leq k \leq K$, obtained by solving

$$
\min _{\left\{\mathbf{C}_{k}\right\},\left\{\mathbf{D}_{k}\right\}}\left\|\mathbf{B}-\sum_{k=1}^{K} \mathbf{C}_{k} \otimes \mathbf{D}_{k}\right\|_{F} .
$$

In [7], this problem is shown to be equivalent to

$$
\min _{\left\{\mathbf{C}_{k}\right\},\left\{\mathbf{D}_{k}\right\}}\left\|\mathcal{R}(\mathbf{B})-\sum_{k=1}^{K} \operatorname{vec}\left\{\mathbf{C}_{k}\right\} \operatorname{vec}\left\{\mathbf{D}_{k}\right\}^{T}\right\|_{F},
$$

where $\mathcal{R}(\cdot)$ is a matrix rearrangement operator. This is a low-rank approximation problem which can be solved with the SVD of $\mathcal{R}(\mathbf{B})$.

For our purposes, we approximate $\mathbf{B}=\boldsymbol{\Xi}^{-1} \mathbf{A}=\boldsymbol{\Xi}^{T} \mathbf{A}$ (since $\boldsymbol{\Xi}$ is a permutation, $\boldsymbol{\Xi}^{-1}=\boldsymbol{\Xi}^{T}$ ). Note that $\boldsymbol{\Xi}$ is the key to a successful low-rank decomposition. As shown in Section 4, using $\mathbf{B}=\mathbf{A}$ is not useful, since $\mathcal{R}(\mathbf{A})$ has too many significant singular values.

\subsection{Computing the low-rank fast transform}

Computing the dominant singular values and vectors of $\mathcal{R}(\mathbf{B})$ is not trivial, since in practice $\mathcal{R}(\mathbf{B})$ is too large to be stored explicitly in memory. Nevertheless, one can use Lanczos methods [7, 8] which only require the implementation of the matrix-vector products $\mathcal{R}(\mathbf{B}) \mathbf{u}$ and $\mathcal{R}(\mathbf{B})^{H} \mathbf{v}$ for arbitrary $\mathbf{u}, \mathbf{v}$. One can also use approximate SVD methods designed to require a small number of passes over $\mathcal{R}$ (B) (e.g. [9, 10]).

It can be shown that

$$
\begin{aligned}
\mathcal{R}(\mathbf{B})^{T} & =\left[\begin{array}{ccc}
\mathbf{Z}_{0,0} & \cdots & \mathbf{Z}_{M_{x}-1,0} \\
\vdots & & \vdots \\
\mathbf{Z}_{0, M_{y}-1} & \cdots & \mathbf{Z}_{M_{x}-1, M_{y}-1}
\end{array}\right] \\
\mathbf{Z}_{m, n} & =\boldsymbol{\Xi}\left(\mathbf{v}\left(\varphi\left(u_{x_{m}}, u_{y_{n}}\right)\right) \mathbf{v}^{H}\left(\varphi\left(u_{x_{m}}, u_{y_{n}}\right)\right)\right) .
\end{aligned}
$$

Since $\mathbf{v}\left(\varphi\left(u_{x_{m}}, u_{y_{n}}\right)\right)$ can be precomputed for $0<m<M_{x}$ and $0<n<M_{y}$ and $\boldsymbol{\Xi}$ is a very fast permutation, $\mathcal{R}(\mathbf{B}) \mathbf{u}$ and $\mathcal{R}(\mathbf{B})^{H} \mathbf{v}$ can be evaluated with relative efficiency. Indeed, using the Lanczos method from [8], $N=64$ and $M_{x}=M_{y}=256$, we can solve (8) for $K=8$ in 8 minutes on an Intel Core 2 Duo $2.4 \mathrm{GHz}$ processor, using only one core. This is a very reasonable runtime for an offline procedure which must only be run once.

Even in the presence of strong near field effects $\mathcal{R}(\mathbf{B})$ can be well approximated by a low-rank decomposition (see Section 4). Even though the transform cost grows linearly with $K$, due to the Kronecker representation, the cost of applying each $\mathbf{C}_{k} \otimes \mathbf{D}_{k}$ is very small, so a transform with $K=8$ outperforms an explicit matrix representation of $\mathbf{A}$ by several orders of magnitude. 
In [4] we showed that $\mathbf{A}=\mathbf{\Xi}\left(\mathbf{V}_{\mathbf{x}} \otimes \mathbf{V}_{\mathbf{y}}\right)$ can be implemented with $\frac{1}{2} N M+N^{2} M^{1 / 2}$ complex MACs (multiply-accumulate operations). Thus, $\breve{\mathbf{A}}=\mathbf{\Xi}\left(\sum_{k=1}^{K} \mathbf{C}_{k} \otimes \mathbf{D}_{k}\right)$ can be implemented with $\frac{1}{2} K N M+K N^{2} M^{1 / 2}$ MACs, as opposed to the $N^{2} M$ complex MACs required by an explicit matrix representation of $\mathbf{A}$. To our knowledge, there is no other fast transform that can model nearfield propagation, so the explicit matrix representation is the only alternative to our proposal. Considering that the explicit matrix representation is too large to be stored in memory for realistic problem sizes, in practice its implementation is much slower, since its elements must be recomputed when applying $\mathbf{A}$.

As we show in Section 4, even when very strong near-field effects are present, $K=8$ or $K=16$ are sufficient for accurate reconstructions, such that this proposal is at least 100 times faster than explicit matrix multiplications for practical problem sizes.

\subsection{Calibration and focusing}

In practical applications, the array geometry might deviate slightly from an ideal Cartesian grid. Furthermore, microphones are seldom well matched, and require calibration. While these characteristics may be incorporated into the transform with a suitable choice of $\mathrm{K}$, it is more computationally efficient to compensate departures from separability using a separate interpolation matrix. For the purposes of near-field imaging, this matrix can also be designed to make moderate changes to the focal surface without having to recompute the $\left\{\mathbf{C}_{k}, \mathbf{D}_{k}\right\}$ matrices. This approach is convenient for real-time imaging, since one can compute interpolation matrices in a few seconds, while obtaining $\left\{\mathbf{C}_{k}, \mathbf{D}_{k}\right\}$ requires several minutes.

Let $\mathbf{v}_{\mathbf{u}}$ and $\breve{\mathbf{v}}_{\mathbf{u}}$ be the ideal and desired manifold vectors, respectively. Assume that $\mathbf{v}_{\mathbf{u}}$ is modeled by the fast transform, while $\breve{\mathbf{v}}_{\mathrm{u}}$ potentially incorporates calibration data and changes to the focal surface. We propose designing an interpolation matrix $\mathbf{T}$ such that $\mathbf{T}[\underline{\breve{A}}(\mathbf{Y})] \mathbf{T}^{H}$ becomes the fast transform.

Previous methods for array interpolation $[11,12]$ design $\mathbf{T}$ such that $\mathbf{T} \mathbf{v}(\varphi(\mathbf{u})) \approx \breve{\mathbf{v}}(\varphi(\mathbf{u}))$, for $\mathbf{u}$ in a region of interest. Defining

$$
\mathbf{V}=\left[\begin{array}{llll}
\mathbf{v}_{\mathbf{u}_{1}} & \mathbf{v}_{\mathbf{u}_{2}} & \cdots & \mathbf{v}_{\mathbf{u}_{M}}
\end{array}\right], \quad \breve{\mathbf{V}}=\left[\begin{array}{llll}
\breve{\mathbf{v}}_{\mathbf{u}_{1}} & \breve{\mathbf{v}}_{\mathbf{u}_{2}} & \cdots & \breve{\mathbf{v}}_{\mathbf{u}_{M}}
\end{array}\right],
$$

a traditional interpolation matrix is computed as

$$
\underset{\mathbf{T}}{\arg \min }\|\mathbf{T V}-\breve{\mathbf{V}}\|_{F}=\breve{\mathbf{V}} \mathbf{V}^{+},
$$

where $\|\cdot\|_{F}$ is the Frobenius norm and $\mathbf{V}^{+}$is the Moore-Penrose pseudoinverse of $\mathbf{V}$.

We can improve this approach when imaging with transforms. It follows from (6) that $\mathbf{S}=\underline{\breve{\mathbf{A}}}(\mathbf{Y})$ is a weighed sum of $\mathbf{v}_{\mathbf{u}} \mathbf{v}_{\mathbf{u}}^{H}$ outer products. Since $\mathbf{v}_{\mathbf{u}} \mathbf{v}_{\mathbf{u}}^{H}=\left(\alpha \mathbf{v}_{\mathbf{u}}\right)\left(\alpha \mathbf{v}_{\mathbf{u}}\right)^{H}$ for any $\alpha \in \mathbb{C}$ with $|\alpha|=1$, it suffices that $\mathbf{T}_{\mathbf{u}} \approx \alpha \breve{\mathbf{v}}_{\mathbf{u}}$ for a conveniently chosen $\alpha \in \mathbb{C}$ with $|\alpha|=1$. Thus, a more accurate $\mathbf{T}$ can be obtained by solving

$$
\min _{\mathbf{T}, \mathbf{U}}\|\mathbf{T V}-\breve{\mathbf{V}} \mathbf{U}\|_{F}=\min _{\mathbf{U}}\left\|\breve{\mathbf{V}} \mathbf{U} \mathbf{V}^{+} \mathbf{V}-\breve{\mathbf{V}} \mathbf{U}\right\|_{F},
$$

under the constraint that $\mathbf{U}$ is unitary diagonal.

Let $\operatorname{diag}\{\mathbf{U}\}=\boldsymbol{\alpha}$. We minimize (9) by solving for $\mathbf{U}$ and $\mathbf{T}$ alternatively, with

$$
\begin{aligned}
& \mathbf{T}(0)=\mathbf{I} \\
& \alpha_{i}(n)=\underset{|\alpha|=1}{\arg \min }\left\|\mathbf{T}(n) \mathbf{v}_{\mathbf{u}_{i}}-\alpha \breve{\mathbf{v}} \mathbf{u}_{i}\right\|_{F} \\
& =\breve{\mathbf{v}}_{\mathbf{u}_{i}}^{H} \mathbf{T}(n) \mathbf{v}_{\mathbf{u}_{i}} /\left|\breve{\mathbf{v}}_{\mathbf{u}_{i}}^{H} \mathbf{T}(n) \mathbf{v}_{\mathbf{u}_{i}}\right|
\end{aligned}
$$

$$
\mathbf{T}(n+1)=\breve{\mathbf{V}} \mathbf{U}(n) \mathbf{V}^{+} .
$$

Note (9) is not convex under the constraint that $\mathbf{U}$ is unitary diagonal, and may have multiple local minima. Nevertheless, in our simulations this approach converged to the global minimum or to solutions which are very close to it.

To increase the likelihood of converging to the global minimum, one can choose a more accurate $\mathbf{U}(0)$ at a higher computational cost. Assuming $\mathbf{U}$ is unitary diagonal,

$$
\begin{aligned}
\left\|\breve{\mathbf{V}} \mathbf{U V}^{+} \mathbf{V}-\breve{\mathbf{V}} \mathbf{U}\right\|_{F}^{2}=\left\|\breve{\mathbf{V}} \mathbf{U}\left(\mathbf{V}^{+} \mathbf{V}-\mathbf{I}\right)\right\|_{F}^{2} \\
=\left\|\left[\left(\mathbf{V}^{+} \mathbf{V}-\mathbf{I}\right)^{T} \otimes \breve{\mathbf{V}}\right] \operatorname{vec}\{\mathbf{U}\}\right\|_{2}^{2} \\
=\boldsymbol{\alpha}^{H}\left[\left(\mathbf{V}^{+} \mathbf{V}-\mathbf{I}\right)^{*}\left(\mathbf{V}^{+} \mathbf{V}-\mathbf{I}\right)^{T} \odot \breve{\mathbf{V}} \breve{\mathbf{V}}^{H}\right] \boldsymbol{\alpha},
\end{aligned}
$$

where $\odot$ is the pointwise (Hadamard) product. A nearly optimal $\boldsymbol{\alpha}(0)$ is the eigenvector associated with the smallest eigenvalue of $\left(\mathbf{V}^{+} \mathbf{V}-\mathbf{I}\right)^{*}\left(\mathbf{V}^{+} \mathbf{V}-\mathbf{I}\right)^{T} \odot \breve{\mathbf{V}} \breve{\mathbf{V}}^{H}$, normalized such that each of its coordinates lies on the unit circumference.

\section{EXAMPLES}

In this section we present simulation results for a rectangular focal surface, showing how reconstruction accuracy varies with $K$. We simulate a 64-microphone non-uniform planar array with a separable geometry, operating at $4 \mathrm{kHz}$ and $8 \mathrm{kHz}$, with elements having $\mathrm{x}$ and $\mathrm{y}$ coordinates drawn from $\{ \pm 2.8, \pm 7.8, \pm 12.2, \pm 15.0\}(\mathrm{cm})$. Each image has $256 \times 256$ pixels, and was reconstructed by solving the total-variation regularized least-squares problem given by $\min _{\hat{\mathbf{Y}}}\|\hat{\mathbf{Y}}\|_{B V}+\mu\|\operatorname{vec}\{\mathbf{S}\}-\mathbf{A} \operatorname{vec}\{\hat{\mathbf{Y}}\}\|_{2}^{2}$. We used the solver TVAL3 [13] with $\mu=10^{3}$ and 100 iterations, and set $\sigma$ in (4) to provide a $20 \mathrm{~dB}$ SNR, following the methodology from [5].

Fig. 1 shows examples reconstructing a checkerboard source distribution, which was chosen to illustrate how artifacts are influenced by source coordinates. We note that its symmetry does not provide any advantage to the proposed methods. The source distribution is located over a $0.5 \mathrm{~m} \times 0.5 \mathrm{~m}$ rectangle parallel to the array plane, simulated at $0.5 \mathrm{~m}$ from the array and parameterized by $\varphi\left(u_{x}, u_{y}\right)=\left[\begin{array}{lll}u_{x} & u_{y} & 0.5\end{array}\right]^{T}$, with $\left(u_{x}, u_{y}\right) \in[-0.5,0.5]^{2}$.

Since the far-field parameterization models a complete hemisphere, the $0.5 \mathrm{~m} \times 0.5 \mathrm{~m}$ rectangle does not fill its respective acoustic image. For the far-field parameterization, we indicate the horizon using a white circumference. The source distribution is very close to the array, so reconstructing it under a plane wave assumption produces smeared images, motivating the use of our proposal.

Our benchmark is the exact (and slow) near-field transform. Since the array resolution decreases monotonically towards the horizon, all reconstructions present smearing toward the edges of the focal rectangle. This is not an artifact of the fast transform, and can also be observed when using the exact transform. Note that $\mathbf{A}$ is easier to approximate for low frequencies. For this particular example, $K=4$ and $K=8$ are sufficient to deliver accurate reconstructions at $4 \mathrm{kHz}$ and $8 \mathrm{kHz}$, respectively.

Fig. 2 compares the first 100 singular values (out of a total of 16384) for $\mathcal{R}(\mathbf{A})$ and $\mathcal{R}\left(\boldsymbol{\Xi}^{T} \mathbf{A}\right)$. We consider $\mathbf{A}$ modeling the rectangular focal surface defined previously, for $8 \mathrm{kHz}$ sources. The sharp decay of the curve for $\mathcal{R}\left(\boldsymbol{\Xi}^{T} \mathbf{A}\right)$ highlights the importance of $\boldsymbol{\Xi}$ in enabling accurate low-rank approximations.

Fig. 3 shows how one can use reconstruction errors to estimate focal distances. Indeed, one obtains the best fit when using a transform which matches the true focal surface, with a correct focal distance. The dashed lines show that if one does not have an optimal transform designed for the true focal surface (in this case, a rectangle at $0.5 \mathrm{~m}$ ), one can correct it using an interpolation matrix. 


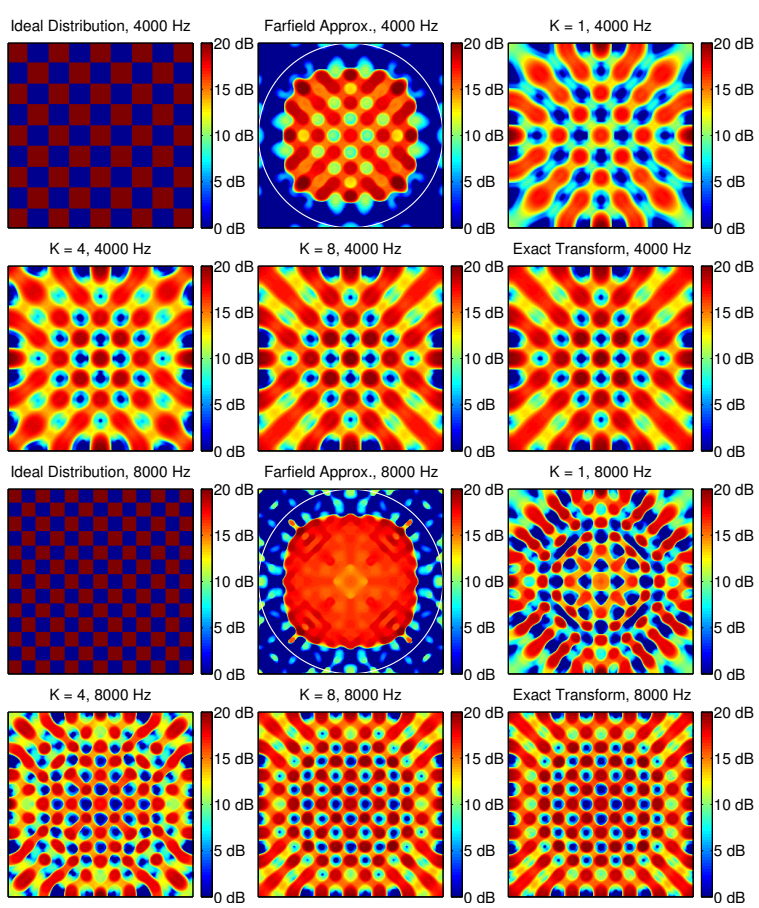

Fig. 1. TV-regularized reconstruction using a far-field approximation, the best Kronecker decomposition (figures with $\mathrm{K}$ ) and the exact (slow) transform. Sources are positioned over a $0.5 \mathrm{~m} \times 0.5 \mathrm{~m}$ rectangle parallel to the array, located at a distance of $0.5 \mathrm{~m}$.

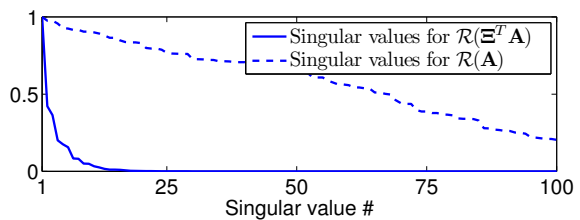

Fig. 2. First 100 singular values for $\mathcal{R}(\mathbf{A})$ and $\mathcal{R}\left(\boldsymbol{\Xi}^{T} \mathbf{A}\right)$, normalized to 1 .

Reconstruction times for $K=1,2,4,8$ and 16 are approximately 4, 5, 6, 9 and 15 seconds per image, with MATLAB implementations running on an Intel Core 2 Duo T9400 processor in 64-bit mode, using only one core (exact reconstruction times usually vary by \pm .5 seconds, depending on the source distribution). In contrast, using an explicit matrix multiplication requires around 2000 seconds per image.

\section{CONCLUSION}

In this paper we have proposed a method for computationally efficient near-field imaging. As shown in Section 4, we can achieve very accurate reconstructions with computational costs at most one order of magnitude above those of the far-field fast transform. This is a notable result, since it still makes the proposed near-field transforms about as fast as FFT2-accelerated deconvolutions (see results from [4]), which degrade quality by using a far-field approximation. Furthermore, it enables the use of practical use of regularized least-
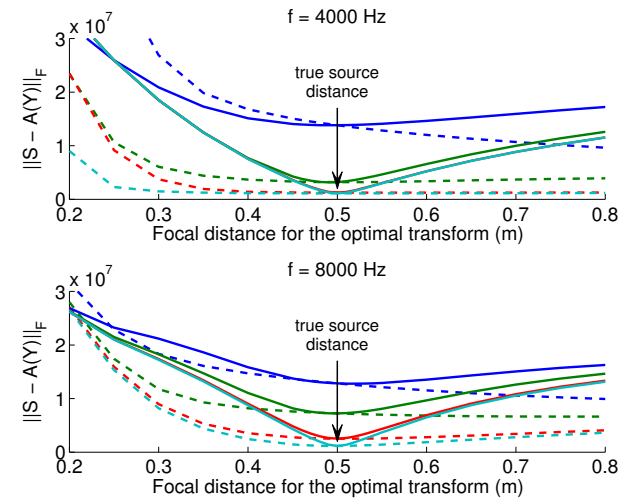

Fig. 3. Reconstruction errors for varying $K$. - : $K=1$, no focusing; - -: $K=1$, with $\mathbf{T} ;-: K=4$, no focusing; $--: K=4$, with $\mathbf{T} ;-: K=8$, no focusing; $--: K=8$, with $\mathbf{T} ;-: K=16$, no focusing; --: $K=16$, with $\mathbf{T}$.

squares methods, which produce better results than deconvolution methods.

Future work involves extending the Kronecker approximation for more diverse array geometries, for which other permutations $\boldsymbol{\Xi}$ will be required to promote separability.

\section{REFERENCES}

[1] T.J. Mueller, Ed., Aeroacoustic measurements, Springer Verlag, 2002

[2] R.P. Dougherty, "Extensions of DAMAS and Benefits and Limitations of Deconvolution in Beamforming," in Proc. of the 11th AIAA/CEAS Aeroacoustics Conference, 2005.

[3] T. Yardibi, J. Li, P. Stoica, and L.N. Cattafesta III, "Sparsity constrained deconvolution approaches for acoustic source mapping," The Journal of the Acoustical Society of America, vol. 123, pp. 2631, 2008.

[4] F.P. Ribeiro and V.H. Nascimento, "A fast transform for acoustic imaging with separable arrays," in Proc. of ICASSP, 2011.

[5] F.P. Ribeiro and V.H. Nascimento, "Computationally efficient regularized acoustic imaging," in Proc. of ICASSP, 2011.

[6] R.A. Horn and C.R. Johnson, Matrix analysis, Cambridge University Press, 1990.

[7] C.F. Van Loan and N. Pitsianis, "Approximation with Kronecker products," in Linear Algebra for Large Scale and Real Time Applications, ucts," in Linear Algebra for Large Scale and Real Time Applications, tions, 1992.

[8] J. Baglama and L. Reichel, "Augmented implicitly restarted Lanczos bidiagonalization methods," SIAM Journal on Scientific Computing, vol. 27 , no. 1 , pp. $19-42,2006$

[9] P. Drineas, R. Kannan, and M.W. Mahoney, "Fast Monte Carlo algorithms for matrices II: Computing a low-rank approximation to a matrix," SIAM Journal on Computing, vol. 36, no. 1, pp. 158-183, 2007.

[10] N. Halko, P.G. Martinsson, and J.A. Tropp, "Finding structure with randomness: Stochastic algorithms for constructing approximate matrix decompositions," Tech. Rep. ACM 2009-05, California Inst. Tech., Sept. 2009.

[11] J. Pierre and M. Kaveh, "Experimental performance of calibration and direction-finding algorithms," in Proc. of ICASSP, 1991, pp. 13651368.

[12] M. Pesavento, AB Gershman, and Z.Q. Luo, "Robust array interpolation using second-order cone programming," IEEE Signal Processing Letters, vol. 9, no. 1, pp. 8-11, 2002.

[13] C. $\mathrm{Li}$, "An efficient algorithm for total variation regularization with applications to the single pixel camera and compressive sensing," M.S. thesis, Rice University, 2009. 